


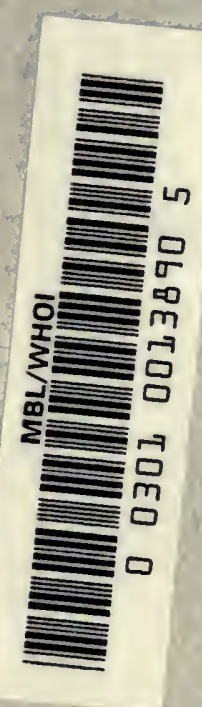





\section{DIATOVEES DU MONDR ENTIER}

\section{TEXTE}

\section{COLLECTION}

Tempere et Peragallo

(2e EDITION)

1915

Prix du texte seul: 35 franes

Chez J. THMPËRE, villa Andrée-Lucie à A rcachon (Gironde) 


\section{COLLECTIONS}

Les Diatomées du Monde entier (2e Edition) se composent de mille préparations étendues de récoltes et de dépôts de tous les pays du monde avec texte analytique.

Cette collection catalogue 266 genres dont 6 nouveaux et 6059 espèces ou variétés dont 347 lui sont propres.

Prix de la collection complète $\mathbf{1 . 0 0 0 ~ f r}$. (Nota. - Trois collections seulement sont disponibles).

Les Diatomées marines de France, en préparations d'espèces types isolées, correspondant au travail de MM. Peragallo, par séries mensuelles de 25 types.

Chaque série, franco : $35 \mathrm{fr}$.

Les Diatomées du Monde entier, en préparations de types isolés choisis parmi les plus beaux, et contenant un grand nombre de formes rares, par séries niensuelles de 25 types.

Chaque série, franco : $35 \mathrm{fr}$.

Les genres des Diatomées, en préparations de types isolés, de 1 à 3 espèces par genre sur une même plaque, par séries de 25 genres.

Chaque série, franco : $40 \mathrm{fr}$.

Les Diatomées d'eau douce de France, en préparations de types isolées ou purs, par séries mensuelles de 25 types.

Chaque série, franco : $30 \mathrm{fr}$.

Chez J. TEMPẼRE, villa Andrée-Lucie à A reachon (Gironde) 


\title{
DIATOMÉES DU MONDE ENTIRR
}

COLLECTION

\section{Tempère et Peragallo}

\author{
(2 EDITION)
}





\section{DIATOMÉES}

Collection Tempère et Peragallo

(2e EDITION)

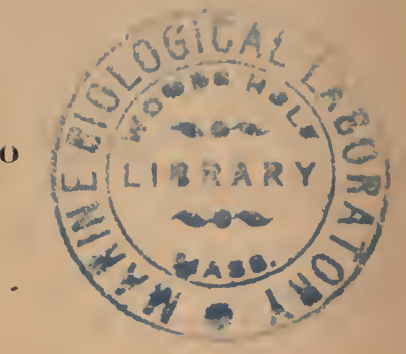

Depuis notre première édition, rapidement épuisée etqui date d'une quinzaine d'années, notre collection de Diatomées s'est enrichie d'un assez grand nombre de matériaux, tant récents que fossiles, d'un très grand intérêt, nombre d'entre eux contenant des espèces nouvelles décrites et figurées par les auteurs.

D'autre part, un certain nombre de demandes nous ayant été adressées depuis cette époque, exprimant le désir de voir publier une seconde édition de cette collection, nous nous somme décidé, malgré l'énorme travail que comporte une sembrable publication, à entreprendre cette seconde édition, qui, revue, corrigée et augmentée constituera une des plus belles, des plus intéressantes et des plus utiles collections de ce genre qui ait paru jusqu'ici.

Le plan général adopté pour la première édition sera suivi pour la seconde, avec toutefois cette différence que tous les matériaux riches en espèces seront représentés par deux, trois et dans certains cas quatre préparations comprenant autant de densités différentes, ce qui permettra d'y rencontrer un bien plus grand nombre des espèces qu'ils contiennent et qu'une seule préparation en mélange ne peut que rarement contenir; de plus, l'analyse des matériaux étant faite sur un plus grand nombre de préparations, la liste des espèces en sera plus complète.

A ces analysès, nous avons l'intention d'ajouter la liste complète de toutes les espèces décrites et figurées par les auteurs et rencontrées par eux dans les matériaux riches et classiques, tels que ceux des Barbades d'Oamaru, 
de Russie, de Hongrie, de Virginie, du Maryland, etc., etc., avecréférences aux meilleures figures publiées.

Enfin, grâce à l'excellent coucours de MM. H. et M. Peragallo, qui ont bien voulu se charger des reproductions, nous donnerons au fur et à mesure que nous aurons réuni les matériaux nécessaires, des planches représentant toutes les espèces nouvelles que nous aurons observées dans nos préparations, en y ajoutant celles des Diatomées Marines de France qui n'auront pas été reproduites dans ce travail; ces planches seront accompagnées d'un texte spécial donnant la description des espèces qu'elles contiendront. Nous espérons ainsi satisfaíre à toutes les observations et à tous les désiderata qui nous ont été signalés et remplir le but que nous nous sommes proposé, c'est-à-dire de satisfaire en tous points nos abonnés.

J. TeMPÈre.

NOTA. Le nom des espéces prédominantes sont imprimés en caractère gras; les rares en italique, ot les autres en caractère courant.

Les étiquettes portent les abréviations suivantes.
M. R.
Diatomées marines récentes.
S. R.
S. F.
M. F.
- d'eau douce récentes.
D. R.
Pel.
Diátomẻes saumâtres récentes.
D. F.
fossiles.
- pélagiques (plankton). 


\title{
DIATOMÉES DU MONDE ENTIER
}

\section{COLLECTION TEMPĖRE \& PERAGALLO}

\author{
(Deuxième Édition, 1907)
}

\section{$\mathrm{N}^{\circ} 1,2$ JACKSON'S PADDOCK OAMARU \\ (Nouvelle-Zélande) \\ (Dépôt fossile marin - période oligocène)}

BIBLIOGRAPHIE. - On a fossil marine Diatomaceous deposit from Oamaru New Zealand by E. Grove aud G. Sturt - voir aussi l'atlas de Ad. Schmidt.

Arachnoidiscus Ehrenbergii Bail.

$$
\text { - Gr. et St. - v. deflciens }
$$

Asterolampra decora Grev.

Aulacodiscus amœnus Grev.

- Janischii Gr. et St.

- margaritaceus $\nabla$. Debyi

Ratt.

- - v. undosa A.S.

- Novæ Zelandiæ Gr. et St.

- patulus Grun.

- subrimosus Grun.

Auliscus Oamaruensis Gr. et St.

- Hardmanianus $\nabla$. bifurcata Ratt.

Biddulphia elegantula Grev.

- punctata Grev.

- Tuomeyi Bail.
Coscinodiscus argus Ehr.

- Barbadensis Grev.

- $\quad$ curvatulus Grun var.

- elegans Grev.

- excentricus Ehr.

- griseus v. gallopagensis Grun.

- incqualis Gr. et St.

- marginatus Ehr.

- Oamaruensis Gr. et St.

- radiatus Ehr.

- subtilis Ehr. var.

- symbolophorus Grun.

Eunotogramma Weissii v. producta Gr. et St.

Hemiáulus ternuicornis Grev.

- ornithocephalus Grev.

Iyalodiscus punctatus $A$. S. 
Ilyalodiscus radiatus Ehr.

- - v. maxima P.P.

Isthmia nervosa Ehr.

Molosira clavigera Grun.

- sol Ehr.

- sulcata Ktz.

Podosira argus Grun.

- hirsutus Gir. et St.

- hormoides Grun.

Rutilaria radiata Gr. et St.

Stephanopyxis turris Grun.

Stictodiscus Hardmanianus Grov.

- $\quad$ - v. megapora A.S.

- . nitidus Gr. et St.

- parallelus v. gibbosa Gr. et St.

Triceratium castellatum West.

- castelliferum Grun.
Triceratium cornutum Gr. et $S t$. coscinoides $G r$. et St. crenulatum Gr. et St, favus Ehr. favus v. quadrata. fractum IV. et $\mathrm{Ch}$. glandiferum Grun. grande $\mathrm{Br}$. lineatum Grev. majus Gr. et St. Morlandii Gr. et St. Nọæ Zelandiæ Gr. et St. Oamaruensis Gr. et St. pseudo-nervatum Gr. et St. pulvinar A. S. secedens A. S. scitulum $B r$. trisulcum v, cuneata A. S. Weissflogii Gr. et St.

Trinacria simulacrum Gr. et $S t$.

\section{N³ SUCCESSFUL BAY KERGUELEN LAND (Océan Austral)}

\section{(Sondage)}

Biddulphia Roperiana Grev.

Campylodiscus Schleinitzii Jan.

Cocconeis costata v. kerguelensis Grev.

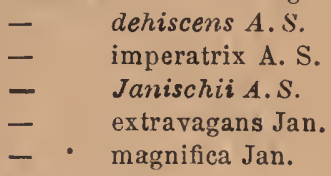

Coscinodiscus centralis Eh.

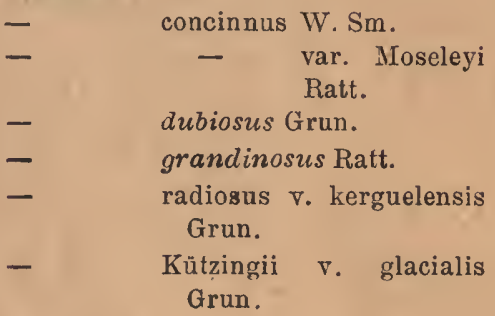

Coscinodiscus lentiginosus Jan.

- $\quad$ odontodiscus Grun.

Entopyla australis Eh.

Gramnatophora.

Hyalodiscus radiatus O'meara.

Melosira curvatula Jan. v. radiata.

- sol. Ktz

Navicula aspera Eh. - Crabro Eh.

orthosira interjecta Jan. .

Podosira Oliveriana Grun.

Stauroneis oblonga Bail.

Surirella Kerguelensis Grun.

Triceratium antarcticum Jan. 
No 4 WIMEREUX (Pas-de-Calais - France)

\section{Attheya Decora}

Cette espèce a été isolée vivante d'une belle récolte faite par M.

Giard, puis fixée par le formol et colorée au rouge diamant.

N०5

HUSO (Norvège)

Plankton (collection Gran)

Chatoceros boreale Bail.

- cochlea Schütt.

- criophilum Cast.

- didymus Fh.

- laciniosum $\mathrm{Cl}$.

Ditylium Brightwellii West.

Lauderia compressa $H$. P.
Licmophora Lyngbyei Ktz.

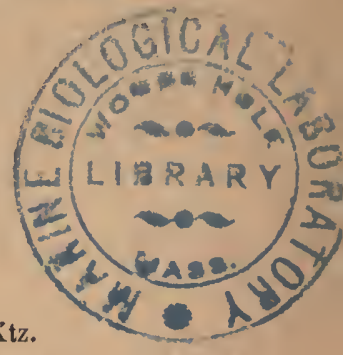

Rhizosolenia alata var. curvirostris. setigera Bright.

Skeletonema costatum Grun. Striatella unipunctata Ag.

Thalassiosira Nordenskioldii Cl.

\section{Plankton (collection Gran)}

Biddulphia aurita Bréb.

Chatoceros balticus $\mathrm{Cl}$.

- boreale Bail.

- cochlea Schütt.

- constrictus Gran.

- convolutum Cast.

- debilis Cl.

- decipiens Cl.

- didymus var. hiemalis.

- gracile Schútt,

- seriacanthum Gran.

- simile (il.
Chætoceros socialis Laud.

$$
\text { - vermiculus Schütt. }
$$

Coscinodiscus leptopus var. irregularis.

Leptocylindrus danicus $\mathrm{Cl}$.

vitzgchia seriata $\mathrm{Cl}$.

$$
\text { - - var. fraudulenta. }
$$

Rhizosolenia alata var.gracillima.

$$
\text { - setigera Bright. }
$$

Thalassiosira Nordenskioldii Cl.

Thalassiothrix elorgata Grun.

\section{$\mathrm{N}^{\circ} 7$ DEMERARA (Guinée Anglaise)}

(Sur les algues) 
$\mathrm{N}^{\circ} 8,9$

SZENT PETER (Hongrie)

(Marne tertiaire marine)

Bibliographie. -- J. Pantocsek: Fossilen Bacillarien Ungarns vol. I

Actinocyclus Ehrenbergii Ralfs.

$$
\text { - Ralfsii var. W. Sm. }
$$

Actinoptychus bifrons $A$. 'S.

$$
\begin{array}{ll}
- & \text { Grundlerii A. S. } \\
- & \text { splendens Grun. } \\
- & -\quad \text { var. halionyx. } \\
- & \text { stella A.S. } \\
- & \text { - var. Thumii A.S. } \\
\text { - } & \text { undulatus Eh. } \\
\text { - } & \text { vulgaris Schum. }
\end{array}
$$

Arachnoüliscus Ehrenbergii Bail.

Aulacodiscus amonus var. Hungarica Pant.

- Grunowii CI.

Auliscus colatus Bail.

$$
\text { - Hauchii Pant. }
$$

Blddulphia Tuomeyi Roper.

$$
\text { - homala Pant. }
$$

Cerataulus Johnsonianus Greo.

$$
\text { - polymorphus Ktz. }
$$

Cocconeis pellucida $K t z$.

Coscinodiscus apiculatus Ehr.

$\begin{array}{ll}\text { - } & \text { Argus Ehr. } \\ - & \text { asteromphalus Ehr } \\ \text { - } & \text { crassus Bail. } \\ \text { - } & \text { marginatus Ehr. } \\ \text { - } & \text { moravicus Grun. } \\ \text { - } & \text { obscurus A.S. } \\ & \text { oculus iridis Ehr. }\end{array}$

Coscinodiscus radiatus Ehr. undulatusGrun.

Endictya oceanica Ehr.

Goniothecium odontella Ehr.

IIyalodiscus radiatus (O'Meara) Grun.

Isthmia Szaboi Pant.

Melosira biharensis Pant.

- sol Ehr.

- sulcata Ehr.

Navicula aspera Ehr.

- gemmata var. fossilis Pant.

- lyra Ehr.

- O' Sroaldii Arn.

- prætexta Ehr.

- Schaarschmidtii Pant.

- Smithii Bréb.

Pantocsekia clivosa Grun.

Stephanodiscus Kanitzii Grun.

Stephanogonia turris Ehr.

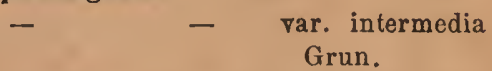

Stictodiscus parallelus fa trigona Pant.

Triceratium arcticum Bright.

-. Lavus Ehr.

- Pantocsekii A.S.

- $\quad$ radiato punctatum A.S.

- Stocksianum Grev.

- Thumii A.S.

- trisulcum Baîl. 
Chatoceros debilis $\mathrm{Gl}$.

- didymum Eh.

- mamillanum CI.

- protuberans Laud.

Leptocylindrus Danicus Cl.
Vitzschia seriata Cl.

Rhizosolenia alata var. gracillima. - setigera Bright.

Thalassiosira Nordenskioldii Cl.

Thalassiothrix nitzschioïdes Grun.

Nos 11 et 12 ANANINO-SIMBIRSK (Russie)

(Dépôt fossile marin)

BIBliographiE. - o.' Witt. - Ueber den Polierschiefer von Archangelsk gouvern. de Simbirsk (Russie) 1885. - J. Pantocsek. - Fossilen Bacillarien Ungarns. Vol II,1889. - Voir aussi Ad. Schmidt atlas.

Actinoptychus beterostrophus A.S.

- seductilis A.S.

- Simbirskianus A. S.

Aporodiscus Simbirskianus Ratt.

Arachnoidiscus Simbirslianus Pant.

Aulacodiscus crux Ehr.

$\begin{array}{ll}- & \text { - var tenera O.W. } \\ - & \text { excavatus A. S. } \\ - & \text { hystrix Pant. } \\ - & \text { Lahusenii O. W. } \\ \text { - } & \text { prominens Kitt. } \\ \text { - } & \text { quadrans A. S. } \\ \text { - } & \text { Weissflogii Pant. }\end{array}$

Biddulphia ruthenica $O$. W.

Cheloniodiscus ananinensis Pant.

Coscinodiscus excentrtcus Eh.

- Mölleri v. macroporus

Grun.

- simbirskianus Grun.

- subconcavus Grun.

- sublineatus Grun.

- symbolophorus Eh.

- vigilans A.S.

Cyclotella asiatica J. Br.

Eunotogramma Weissii Eh.

IIemiaulus elegans Heib.

- includens Eh.

Hyalodiscas laevis Eh.
Lepidodiscus elegans 0 . W.

Melosira sulcata Ktz.

- - var, coronata Grun.

- Thumii Pant.

Odontotropis hyalina $O . W$.

Pyxilla baltica Grun.

Raphoneis Fuchsii Pant.

Stephanogonia danica Eh.

Stephanopyxis ferox Grev. ,

$$
\text { - } \quad \text { turris var aretica Grun. }
$$

Triceratium Archangelskianum $\mathrm{O}$. W.

$$
\begin{array}{ll}
\text { - } & \text { blandum O.W. } \\
\text { - } & \text { caudatum O. W. } \\
\text { - } & \text { distiguendum var. Grun. } \\
\text { - } & \text { duplicatum A.S. } \\
\text { - } & \text { fenestratum O. W. } \\
\text { - } & \text { flos Cl. } \\
\text { - } & \text { - } \\
\text { - } & \text { Heibergii Grun. } \\
\text { - } & \text { Wobile o. W. } \\
& \text { Weissii Grun. }
\end{array}
$$

Trinacria coronata $0 . W$.

- excavata car producta Pant.

- insipiens 0. W.

- pileolus Grun.

- regina Heib. var obtusa.

- Weissflogii O. W. 


\section{No 13 ETANG DE SAINT-LOUP (Puy-de-Dôme)}

\section{Bibliogra Phie. - Frère J. Héribaud. - Les Diatomées d'Au- vergne 1893.}

Achnanthes lanceolata Grun.

Diatoma anceps Grun.

$$
\begin{array}{ll}
\text { - } & -\quad \text { v. anomalum W. Sm. } \\
\text { Eunotia lunaris Grun. } \\
\text { - } \\
\text { - } \quad \text { majoris } \nabla . \text { excisa. } \\
\text { - minor Rab. }
\end{array}
$$

Fragilaria capucina Desm.

$$
\text { - virescens Ralfs. }
$$

Gomphonema angustatum $\mathbf{v}$. intermedia Grun.

$\begin{array}{ll}\text { - } & \text { capitatum. } \\ \text { - } & \text { constrictum } \\ \text { Grun. subcapitata } \\ \text { - } & \text { parvulum Kitz. } \\ \text { - v.lanceolata Ehr. }\end{array}$

Gomphonema subclavatum Grun.

Meridion circulare Ag.

- constrictum Ralfs. .

Melosira crenulata $\mathrm{Ktz}$.

Navicula bacilliformis Grun.

- gentilis Donk.

- gibba Ehr.

- $\quad$ - v. hyalina M.P. et F.H.

- major Ktz.

- nobilis Ehr.

- viridis $\mathrm{Ktz}$.

Stauroneis amphilepta Ehr.

Synedira ulna Eh.

- - v. amphirynchus Eh.

- - v. vitrea Ktz.

$N^{\circ} 14,15$

\section{MACKINTOSH LAKE (Canada)}

(Dépôt fossile d'eau douce)

\section{Cymbella cuspidata Ktz.}

- heteropleura Ralfs var.

- lanceolata.Ehr.

Eunotia arcus Ehr.

- formica Ehr.

- gracilis Ehr.

- major Rab.

- robusta (Ehr.) Ralfs.

- - v. diadema.

- - v. polyodon.

Navicula amphigomphus Ehr.

- Brebissonii Ktz.
Navicula Dactylus Ehr.

- dilatata Ehr.

- elliptica Ehr.

- major Ehr.

- gigas Ehr.

- transversa A.S.

- viridis $\mathrm{Ktz}$.

Stauroneis gracilis $\mathrm{Sm}$.

- Phœnicenteron Ehr-

Surirella bifrons $\mathrm{K} \backslash \mathrm{z}$.

- biseriata Brèb.

- splendida $f^{2}$ minor. 
Amıpiprora pulchra var. pulchella.

Chrotocoros affinis Laud.

- curvisetum Cl.

- danicum Cl.

- didymus Eh.

- Lorensianus Grun.
Guinardia flaccida H. P.

Fhahdonema arcuatum Ktz. Ihizosolenia alata Bright.

$$
\text { - - var. gracillima. }
$$

Rhizosolenia setigera Bright.

Thalassiothrix nitzschioides Grun.

No 17

KUNDSHOVED (Danemark)

Plankton (collection Gran)

Biddulphia aurita Bréb.

Chretoceros borealo var. densa.

- cochlea Schütt.

- curvisetum Cl.

- danicum Cl.

- distans Cl.

- gracile Schütt.

- Grœnlandicum Cl.

- $\quad$ - var. leptopus.

- laciniosum Cl.

- Lorenzianus Grun.

- paradoxum Cl.

- simile Cl.

- socialis Laud.

Coscinodiscus leptopus var irregularis.

Tout à fait semblable au Cosc. leptopus mais dent la cellulation ne forme pas des lignes régulières ot ressemble à celle du Cosc. tumidus mais toutes les cellules sont de même dimension.

Coscinodiscus Oculus iridis Ehr.

Navicula fusiformis var. Cl.

Vitzschia seriata var fraudulenta $\mathrm{Cl}$.

Podosira glacialis Cl.

Pyxilla baltica Grun.

Rhizosolenia setigera Bright.

Skeletonema costatum Grun.

Synedra crystallina Ktz.

Thalassiosira Nordenskioldii Cl.

Thalassiothrix nizschioides Grun.

BIBLIOGRAPHIE. - De Brébisson. Diatomées de la Mousse de Corse (Revue des Sciences naturelles, sept. 1872).

Achnanthes longipes Ag. - subsessilis Eh. Actinoeyclus Ehrenbergii Ralfs.
Actinoptychus senarius $E h$.

$$
\text { - undulatus Eh. }
$$

Amphiprora maxima Grun. 
Amphiprora pusilla Greg.

Amphitetras antediluviana ot vars. Eh.

Amphora arenaria Donk.

$\begin{array}{ll}\text { - } & \text { costata W Sm. } \\ \text { - } & \text { crassaGreg. } \\ \text { - } & \text { elongata Greg. } \\ \text { - } & \text { granulata Greg. } \\ \text { - } & \text { lineata Greg. } \\ \text { - } & \text { marina W.Sm. } \\ \text { - } & \text { obtusa Greg. } \\ \text { - } & \text { spectabilis Greg. } \\ \text { - } & \text { sulcata Bréb. }\end{array}$

Asterolampra marylandica Eh.

Auliscus sculptus W. Sm.

\section{Bidldulphia pulchella Greg.}

Tuomeyi Bail.

Campylodiscus angularis Greg.

- clypeus Eh.

- decorus Bréb.

- eximius Greg.

- limbatus Bréb.

- parvulus W.Sm.

- Ralfsii W. Sm.

- Samcensis Grun.

- Thuretii Bréb.

- _ - v. simulans Greg.

Climacosphenia moniligera Eh.

Cocconeis diaphana W. Sm.
- dirupta Greg.
- $\quad$ - $\quad$. flexella.
- heteroidea Ktz.
- pellucida Grun.
- pseudomarginata Greg.
- scutellum Eh.
- - v. major.
- $\quad$ - v. orbiculare.

Coscinodiscus centralis Eh.

$\begin{array}{ll}\text { - } & \text { concarus Eh. } \\ \text { - } & \text { excentricus Eh. } \\ \text { - } & \text { minor Eh. } \\ \text { - } & \text { nitidus Greg. } \\ & \text { radiatus Eh. }\end{array}$

Donkinia carinata Ralfs.

Endyctia oceanica Eh.

Epithemia gibberula Eb.

$$
\begin{aligned}
& \text { - } \quad \text { musculus Ktz. } \\
& \text { - } \quad \text { sorex Ktz. }
\end{aligned}
$$

Grammatophora gibba Eh. .
Grammatophora macilenta $\mathrm{E} h$.

- marina Eh.

$\begin{array}{ll}\text { - } & \text { serpentina Eh. } \\ \text { - } & \text { subtilissima Eh. } \\ \text { undulata Eh. }\end{array}$

Homoeocladia Vidovichii Grun.

Licmophora gracilis Eb.

Mastogloia apiculata W. Sm.

- Erythrea Grun.
- $\quad$ lanceolata Thw.
- ovata Grun.

Melosira Borreri Grev.

Navicula aspera Eh.

- bombus Eh.

- clavata Greg.

- Crabro Eh.

- didyma Eh.

- liber W.Sm.

- - v. maxima.

- lineata Donk.

- littoralis Donk.

- Iyra et vars. Eh.

- marina Ralfs.

- notabilis v. expleta A.S

- pandura A.S.

- - v. elongata Greg.

- retusa Bréb.

- spectabilis Greg.

- Smithii et vars. Brèb.

- vetula A.S.

Nitzschia navicularis Grun.

- panduriformis Greg.

- sìgma W. Sm.

- $\quad$ - $\quad$. intercedens.

- sigmoidea W. Sm.

Orthoneis binotata Roper.

- fimbriata Bright.

- splendida Grun.

Plagiogranma Gregorianum Éh.

Pleurosigma angulatum W. Sm.

- decorum W. Sm.

- delicatulum W..Sm.

- formosum W. Sm.

- rigidum W. Sm.

Podocystis adriatica $K$ tz.

Podosira hormoides Mont.

- maculata W.Sm.

- Montagnei Ktz.

Raphoneis gemmifera Eh. 
Ihabdonema adriaticum kitz. arcuatum $\mathrm{K} t z$.

Rhoicosigma Reichardtii Grun.

Stauroneis anceps $E h$.

- pulchella IV.Sm.

- salina W.Sm.

Striatella unipunctata $\mathrm{Ktz}$.

Surirella fastuosa et vars. Eh. - lata W.Sm.

Synedra affinis $K t z$.
Syaedra baculus Greg.

- formosa Hantz.

- fulgens W. Sm.

- pulcherrima Hantz.

- robusta Ralis.

- superbakiz.

Toxarium undulatum Bail.

Triceratium formosum Bright.

- orbiculatum Shadb.

- Shadboldtianum Grev.

Tryblionella punctata W. Sm.

N०20

POTONICO (Salvador)

(Dépôt fossile d'eau douce)

Cocconema mexicanum Ehr.

Cerataulus lævis Ehr.

Epithemia gibba Ktz.

- zebra $\nabla$. ventricosa.

Fragilaria capucina Desm.

- ungeriana Grun.
Melosira crenulata $\mathrm{K} t z$.

- distans $\mathrm{Ktz}$.

- granulata Ehr.

- undulata Ktz.

Navicula sculpta Ehr.

Surirella tenera $\nabla$. nervosa.

Terpsinoe americana Bailey.

$N^{\circ} 21$

SOOS (Bohême)

(Dépôt fossile saumâtre)

BIBLIOGRAPHIE. - Grunow Beitrege zur Kenntniss der fossilen Diatomeen œsterreich Ungarns 1882.

Campylodiscus clypeus Ehr.

Navicula seulpta et vars.

- bohemica.
Navicula major $\mathrm{Ktz}$.

- viridis $\mathrm{K} t z$.

Nitzschia spectabilis Ralf. 
Nos 22 à 25

SENDAI (Japon)

(Calcaire argileux et bitumineux tertiaire)

BIBLIOGRAPHIE. - Diatomées fossiles du Japon, par MM. Tempère et Brun, Genève 1889. - J. Brun, Espèces nouvelles, 1891. - Ad. Schmidt, atlas.

NOTA. - Le No 12 contient les grandes formes de Triceratium, Arachnoidiscus, Coscinodisçus; les ${ }^{03} 23$ et 24 ne contiennent presque que les Coscinodiscus qui sont magnifiques et généralement très typiques, le $n^{0} 25$ contient les autres formes.

Achnanthes Lendugeri Temp et $B r$.

Actinocyclus ellipticus $\nabla$ Sandaiana $\mathrm{Br}$.

tlos J. Br.

asiaticus Temp.et $\mathrm{Br}$. glabratus Grun. interpositus J. Br. nitidus Grev. var. perisetosus J. Br. undulatus et var. Eh. turgidus Temp. et Br.

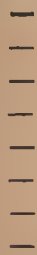

Alloioneis antillarum Cl. et Grun.

Amphora crassa Greg.

- proteus Greg.

- spectabilis Greg.

Arachnoidiscus Ehrenbergii Bail.

$$
\text { - _ - v. californicus. }
$$

Asterolampra stellaris Temp. et Br.

Asteromphalus Brockei Wall.

- senectus Temp.et Br.

Auliscus asiaticus $J . B r$.

- trigemmis A.S.

Auricula japonica $J$. Br.

Biddulphia aurita Bréb.

$\begin{array}{ll}\text { - } & \text { calamus Temp. et Br. } \\ \text { - } & \text { Edwardsii Feb. } \\ \text { - } & \text { obtusa Ralfs. } \\ \text { - } & \text { primordialis } J . B r . \\ \text { - } & \text { Roperiana Grev. } \\ \text { - Tuomeyi Bail. }\end{array}$

Campylodiscus Ralfsii Sm.
Campylodiscus scalaris Temp. et $\mathrm{Br}$. striolatus Grun. ritricatus Temp. et $\mathrm{Br}$.

Cerataulus japonicus Pant.

$$
\text { - turgidus Ehr. }
$$

Cestodiscus japonicus $\mathrm{Cl}$.

$$
\begin{array}{cl}
\text { - } & \text { Johnsonianus Grev. } \\
\text { - } & \text { pulcliellus Grev. } \\
\text { Chatoceros } & \text { clavigerum Grun. } \\
\text { - } & \text { didymur gemmifer. } \\
\text { - } & \text { javanicum } \mathrm{Cl} . \\
\text { - } & \text { pliocenum J. Br. } \\
\text { - } & \text { sigmo calamus Temp. et Br. }
\end{array}
$$

Clavicula robusta $J . B r$.

Cocconeis antiqua Temp. et $\mathrm{Br}$.

- costata Greg.

- dirupta Grev.

- scuteillum Eh.

- splendida v. crucifera'Temp. et $\mathrm{Br}$.

- cersicolor J. Br.

Coscinodiscus asteroides $T$ et $0 . W$.

- asteromphalus Eh.

- borealis Bail.

- crassus Bail.

- elegans Grev.

- Euryoma A.S.

- excentricus Eh.

- $\quad$ fimbrio-limbatus Eh. gigas Eu. 
Coscinodiseng heteroporus Eh.

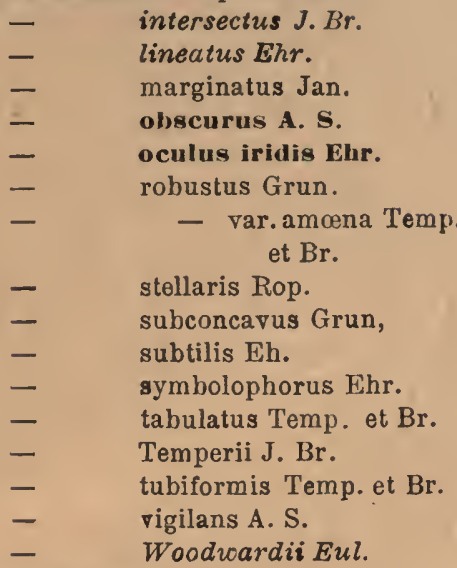

Cyclotella asiatica J. Br.

Dicladia capreolus $E h$.

Euodia gigantea $J . B r$.

- inornata var. curvirotunda Temp. et $\mathrm{Br}$.

- ventricosa Cast.

Ethmodiscus vitrifacies Temp. et Br.

Fragilaria pliocena $J . B r$.

Gephyria gigantea Greo

- - var. minor Grev.

Lithodesmium californicum Grun.

- $\quad$ - var.tigrina Temp. et $\mathrm{Br}$.

Navicula crabro v. japonica $A . S$. - scintillans Temp. et Br.

Nitzschia asiatica Temp. et $B r$.

- pennata Temp. et Br.

Pyxilla Barboi J. Br.

Rhablonema japonicum Temp. et $\mathrm{Br}$.

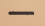

- var. recta.
Rhabdonema japonicum var. sparsi costata.

Rhaldonema japonicum var. valdelata. valdelatum Temp. et $\mathrm{Br}$.

Rutilaria capitata Temp. et $\mathrm{Br}$.

- hexagona v. cornuta Temp. et $\mathrm{Br}$.

- longicornis Temp. et Br.

Staurosigma asiaticum Temp. et Br.

Stephanopyxis limbata $v$ cristagalli Temp. et Br.

- nidulus Temp. et Br.

- Peragalli Temp. et $\mathrm{Br}$.

- , Weissflogii A. S.

Synedra affinis v. hybrida Grun.

- parva fa major CI. et Grun.

Thallasïothrix Franenfeldii $v$.javanica.

- longissima $\mathrm{Cl}$.

Triceratium albifrons $J$. $B$ r .

- $\quad$ arcticum Bright.

- $\quad$ - v.lucida Temp. et Br.

- Bergonii Temp. et Br.

- cultum A.S.

- cyclamen J.Br.

- interjectum A.S.

- Kinkerianum J. Br.

-- Peragalli J. Br.

- quadrangulare Grev.

- radians Temp. et $\mathrm{Br}$.

- Sendaiense A.S.

- $\quad$ - v. quadrata A.S.

- Stokesianum v. moravica Grun.

- $\quad$ tripolaris Temp. et $\mathrm{Br}$.

- versicolor J. Br.

- $\quad$ - v. cuneata J.Br.

Xauthiopyxis umbonatus Grev.

Zygoceros circinus $\nabla$. trapezoidalis Temp. et Br. 
No 27, 29

MORS (Jutland)

(Dépôt fossile Marin)

BIBLIOGRAPHIE. - Heïberg Conspectus criticus Diatomacearum danicarum (J. Q. M. S. Vol. IV 1864 - Kitton. - On diatomaceous deposits from Jutland (J. Q. M. S. Vol. 2 1870-71). Voir aussi Ad. Schmidt atlas et Van Heurck Synopsis.

Actinoptychus Klausenii A. S.

Aulacodiscus excavatus $\nabla$. apiculata.

$$
\begin{array}{ll}
\text { - } & \text { Jutlandicus Kitton. } \\
\text { - } & \text { Klauserii A. S. }
\end{array}
$$

Corinna elegans Heib.

Coscinodiscus antiquus Grun.

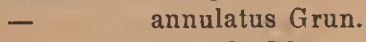

- centralis Eh.

- crassus var. morsiana Grun.

- excentricus Eh.

- exiguus v. æqualis Ratt.

- Oculus iridis $\nabla$. morsiana Grun. radiatus Eh.

- $\quad$ subtilis var. linoolața Ratt. - symbolophorus Grun.

Craspedodiscus Klavsenii Grun.

$$
\text { - } \quad \text { Mólleri A.S. }
$$

Goniothecium odontella var.danica Grun.

IIemiaulus affinis Grun.

$$
\begin{aligned}
& \text { - danicus Grun. } \\
& \text { - } \quad \text { hostilis Heib. } \\
& \text { - } \quad \text { mittonii Grun. } \\
& \text { - } \quad \text { polymorphus } \\
& \quad \text { Grun. } \\
& \quad \text { Weissii Grun. }
\end{aligned}
$$

Janischia antiqua Grun.
Melosira concentrica A.S.

- recedens A.S.

- sulcata Eh.

$$
\text { - } \quad \text { - } \text {. biseriata fa coronata }
$$
Grun.

Odontotropis carinata Grun.

$$
\text { - cristata Grun. }
$$

Omphalopelta jutlandica Grun.

Pseudo-stictodiscus angulatus Grun.

Pyrgodiscus Kinkerii Pant.

Pyxilla dubia Grun.

Solium exsculptum Heib."

Stephanopy $x$ is appendiculata $\vee$. parvispina Grun. v. paueispina Grun.

v. tenuispina Grun.

Triceratium Abyssorum Grun.

- maculatum Kitton.

- mammiferum Grun.

Trinacria excavata Heib.

- Heibergii Kitton.

- $\quad$ - $\quad$ F. sparsi punctata

- Jordanii A.S.

- regina Heib.

- - $\quad$ r. tetragona Grun.

- Wittii A.S.

Trochosira mirabilis Kitton.

- spinosa Kitton. 
Nos 30,32

PUY-DE-MUR (Puy-de-Dôme-France)

(Dépôt fossile saumâtre)

BIBLIOGRAPHIE. - Frère Iléribaud. Les diatomées d'Auvergue, 1890.

Amphora acutiuscula Kitz.

$$
\text { - ovalis Kitz. }
$$

Cocconeis lineala Grun.

Coscinodiscus pygmæus .I. Br. et M. P. Fragilaria brevistriata Grun.

$$
\begin{aligned}
& \text { - - v. laponica Grun. } \\
& \text { - pacifica Grun. } \\
& \text { - } \quad \text { - } \quad \text {. trigona J. Br. et } \\
& \text { F. H. }
\end{aligned}
$$

Je respecte la détermination de M. Brun; mais, la Fragilariạ pacifica Grun (Opephora pacifica P. P.) est beaucoup plus grande et plus robuste(V. Heurck. Syn. 41/20-22). Je rapporte plutôt cette forme au septroneis marina var. parva (V. Heurck Syn. 45/18-19). Il fandrait également y rapporter la variété. (M. P.)

Melosira Borreri Grev.

- $\quad$ - v. ignimontana J. Br. et F. H.

- crenulata v. ambigua Grun.

- granulata Eh.

- Heribauli J. Br.

Navicula Aqnitania .J. Br. et F. II.

- $\quad$ - o.undulataJ.Br.et F.H.
Navicula basaltæproxima J. Br. et F. H.

- $\quad$ - v. Iongistriata M. P. et F. H.

- bomboïdes A. S.

- $\quad$ - v. limanenșe F.H.

- $\quad$ - $\quad$ v. media Cl. et Grun.

- $\quad$ - v. minor F. I. et J. Br.

- digito-radiata Greg.

Vitzschia sigmoidea IV. $S m$.

- socialis v. basaltica J. I3r. et F. H.

- spectabilis Rab.

Periptera saxogallica I. Br. et F. Il.

Raphoneis amphiceros $E h$.

Striatella Girodi $F$. $H$. et $J . B r$.

Surirella Brunii F. H.

- striatula var. biplicata Grun.

- - $\quad$ v. Gautieri F. II. et J. Br.

Synedra affinis var. subarcuata Grun .

- - $\quad$ v. subtilis Grun.

Tetraeyclus decoratus $J$. Br. et $F$. $H$.

\section{No33 TOURBIERE-DE-BRIERE (Saint-Nazaire - France)}

(Vase saumâtre)

Bibliographie. - I. et M. Peragallo. Diatomées marines de France.

Achnanthes subsessilis Kiz.

Actinoptychus splendens Shad.
Actinoptychus undulatus Eh.

Auliscus sculptus Ralfs. 
Biddulphia rhombus W. Sm. Campyloneis Grevillei W. Sm.

Corataulus Sinithii Ralfs.

Coscinodiscus excentricus Eh.

$$
\text { - oculus iridis Eh. }
$$

Cyclotella striata Ǩtz.

Cymatopleura elliptica W. Sm.

Cymbella tumida Bréb.

Epithemia gibba $\mathrm{Ktz}$.

Grammatophora serpentina Eh.

Ilyalodiscus radiatus O'Meara.

- $\quad$ stelliger Bail.

- subtilis Bail.

Melosira sulcata Eh.

Navicula elliptica Ktz.

- formosa Greg.

- interrupta Ktz.

- liburnica Grun.

- major Ktz.

- nebulosa Greg.

- peregrina Eh.

- pusilla v. lanceolata Grun.
Navicula seulpta Eh.

- Smithii Bréb.

- subsalina Donk.

- viridis Eh.

Nitzschia acuminata W. Sm.

- circumsuta Bail.

- hungarica v. linearis Grun.

- paradoxa Grun.

- scalaris Greg.

- Tryblionella Hantz.

- - - v. levidensis Grun.

Pleurosigma Spencerii W. Sm.

$$
\text { - strigosum W. Sm. }
$$

Surirella ovata $\mathrm{Ktz}$.

- striatula Turpin.

Synedra affinis $\mathrm{Ktz}$.

$\begin{array}{lll}- & - & \text { v. hybrida Grun. } \\ \text { - } & - & \text { v. tabulata V. H. }\end{array}$

Terpsinoe americana Eh.

Triceratium antediluvianum Eh.

- alternans Eh. favus Eh.

\section{No34 EMBOUCHURE DU BRIVET à Saint-Nazaire}

(France)

(Vase saumâtre)

Bibliographie. - H. et M. Peragallo. Diatomées marines de France.

Achnanthes longipes Ag.

- $\quad$ subsessilis K'tz.

Actinocyclus nebulosus M. Per.

Actinoptychns splendens Ralfs.

- undulatus Eh.

- vulgaris Schum.

Amphiprora lepidoptera Greg.

Amphora acutiuscula Ktz.

- proteus Greg.

Auliscus sculptas Eh.

Biddulphia aurita W. Sm.
Biddulphia pulchella Greg.

- rhombus Eh.

- Tuomeyi Bail.

Campylodiscus hibernicus Eh.

- limbatus Bréb.

- Thuretii Bréb.

Campyloneis Grevillei Grun.

Cerataulus laevis Eh.

- polymorphus V.H.

- Smithii Ralfs.

- turgidus Eh. 
Cyclotella striata Ktz.

- $\quad$. intermedia Grun.

Cocconeis dirupta Greg. - pseudo-marginata Greg.

Coscinodiscus boliviensis Grun.

$\begin{array}{ll}\text { - } & \text { devius A.S. } \\ \text { - } & \text { oculusingii A.S. } \\ \text { - } & \text { radiatus Eh. }\end{array}$

Cymatopleura elliptica W. Sm.

$$
\text { -- solea Eh. }
$$

Cymbella cistula Hemph.

- lanceolata Eh.

Encyonema prostratum Ralfs.

Epithemia gibba $\mathrm{Ktz}$.

- sorex Ktz.

Eupodíscus argus W. Sm.

Fragilaria brevistriata Grun.

- construens Grun.

- $\quad$ - $\quad$ v. binodis Grun.

Grammatophora macilenta IV. Sm.

- serpentina Eh.

Ifyalodiscus stelliger Bail.

Isthmia enervis $\mathrm{Ktz}$.

Melosira Borreri Ag. fa minuta.

- Jurgensii Ag.

- sulcata Eh.

- - v. coronata Grun.

Navicula ambigua $\mathrm{K} t \mathrm{z}$.

- aspera Éb.

- bacillum Eh.

- bombus Eh.

- clavata Greg.

- crabro Eh.

- didyma Ktz.

- elegans W.Sm.

- elliptica Kitz.

- Hennedyi v. cuneata Grun.

- liber W. Sm.

- lyra v. elliptica A. S.

- major Ktz.

- nobilis Ktz.

- nebulosa Greg.

- oblonga Ktz.

- palpebralis Brèb.

- pennata A. S.

- peregrina Eh.

- placentula Eh.
Navicula prætexta Eh.

- radiosa v. acuta Grun.

- Smithii Bréb.

- sculpta Eh.

- semen Eh.

- subsalina Donk.

- spectabilis Greg.

- viridis Ktz.

- viridula ₹. slesvicensis Grun.

Nitzschia armoricana Grun.

- circumsuta Bail.

- hungarica Grun.

- ligeriana n. sp. H Per.

- navicularis Bréb.

- panduriformis Greg.

- punctata Grun.

- - - v. minor.

- rigida W.Sm.

- $\quad$ - v. rigidula H Per.

- scalaris Greg.

- tryblionella Hantz.

- - v. levidensis Grun.

- vermicularis Hantz.

- vitrea Norm.

Pleurosigma attenuatum W. Sm.

- $\quad$ egtuarii Bríb.

- distortum W.Sm.

- littorale W. Sm.

- Spencerii W Sm.

Raphoneis belgica W. H

- amphiceros Eh.

- rhombus Eh.

Scoliopleura tumida Rab

Stauroneis anceps Eh.

- salina W. Sm.

Surirella Brightwellii W. Sm.

- crumena Bréb.

- fastuosa Eh.

- gemma Eh.

- hybrida Grun.

- medulica H. Per.

- Mölleriana Grun.

- tenera v. nervosa A.S.

Synedra lanceolata Kitz.

- pulchella Ktz.

- tabulata fa elongata II. Per.

- ulna Eh.

Triceratium alternans Eh.

- antediluvianum Eh.

farus Eh. 
(Vase du port)

On trouve dans cette récolte les mêmes espèces que contiennent le $\mathbf{n}^{0} 34$, mais avec une répartition différente, elle complète l'étude des espèces de cette partie des côtes de France.

$\mathrm{N}^{\circ \mathrm{s}} 36,37$

BIRGE'S POND, BRISTOL, Connecticut (U. S. A.)

Amphiprora ornata Bail.

Amphora ovalis $K t z$.

- pediculus Grun.

Cyclotella Meneghiniana Ktz.

Cymbella americana A. S.

- anglica Lag.

- cuspidata Kitz.

- cymbiformis Eh.

- Ehrenbergii Ktz.

- gastroïdes Kitz.

- heteropleura Ralfs.

- maculata Kitz.

- sp. A. S. atlas $9 / 52$.

Encyonema gracile $\mathrm{Eh}$

Epithemia gibba Ktz.

Eunotia diodon Eh.

- formica Eh.

- incisa Greg.

- lunaris Grun.

- minor Rab.

- mododon Eh.

- parallela Th.

- pectinalis Rab.

- - v. ventricosa Grun.

- robusta Ralfs. $\nabla$. tetraodon V.II.

Fragilaria brevistriala Grun.

- capucina v. mesolepta Rab.

- construens Grun.
Gomphonema acuminatum Eh.

- affine v. major Ktz.

- augustatum Ktz.

- $\quad$ - - producta P.P. - apicatum Cl: ? (Forme assez commune ayant tout à fait le contour de G.apicatum Cl. mais lo dessin de Clevenemontre pas la strie médiane isolée comme ici, qui rapproche cette forme de $G$. augur auquel elle passo progressivement par la var. Gautieri M. P.)

Gomphonema augur Eh.

$\begin{array}{ll}\text { - } & \text { - } . \text { Gautieri V.H. } \\ \text { - } & \text { capitatum Eh. } \\ \text { - } & \text { constrictum Eh. } \\ \text { - } & \text { elongatum W. Sm. } \\ \text { - } & \text { intricatum Ktz. } \\ \text { - } & \text { sagitta A. S. } \\ & \text { turris El. }\end{array}$

Melosira distans v. alpigina Gr.

Meridion cirulare $A g$.

Navicula acrosphaeria $R a b$.

- - var. dilata var. $n$.

Caractérisée par une forte dilatation de la valve à la partie médiane.

Navicula affinis $f^{2}$ maxima $E h$.

- amphirynchus Elı.

- anglica var. snbsalsa Gr. 
Navicula bacilliformis Grun.

- bacillum Eh.

- columnaris Eh.

- cuspidata Ktz.

- dactylus Eh.

- Dariana A.S.

- elegantissima M. P. n. sp.

- elliptica Ktz.

- flanatica Grun.

- gigas Eh.

- heroïna A. S. variété. in termédiaireavec le N. transversa.

- Hitchcockii Eh.

- iridis Eh.

- latevittata Cl.

- limosa Ktz.

- major Ktz.

- $\quad-\quad \mathrm{f}^{\mathrm{a}}$ maxima.

- $\quad$ - v. dilatata. M.P.

- mesolepta $E h$.

- $\quad$ - var. stauroneiformis. Gr.

- mesostyla Eh.

- nobilis Ktz.

- peripunctata J. B́r.

- producta W. Sm.

- pupula v. minor Ktz.

- radiosa $\mathrm{Ktz}$.

- - var. acuta Grun.

- rhyncocephala Ktz.

- trinodis Lewis var.

- ventricusa Ktz.
Navicula viridis $\mathrm{K} t z$.

- - var. fallax Cl.

- viridula Ktz.

Nitzschia paradoxa Grun.

- sigmoidea W. Sm.

- spectabilis Ralfs.

Stauroneis anceps Eh.

- - var. linearis Eh.

- Baileyi En.

- gracilis Eh.

- macrocephala Ktz.

Surirella Cardinalis Kit.

- el sgans Eh.

- $\quad$ forme anormale perforata.

Cette curieuse forme a èté décrite et figuréedans le Micrographe préparateur. Volume XIII, page 57.

Surirella linearis W. Sm.

- $\quad$ - $\quad$. constricta Grun.

- robusté Eh.

- saxonicá Auers.

- tenera var splendidula A.S.

Synedra ulna $\nabla$. danica V. H.

- - v. Lanceolata Grun.

Tabellaria fenestrata Ktz:

- flocculosa Ktz.

Van Ileurckia rhomboides var. Bréb. amphipleuroides $\mathrm{Gr}$. viridula Bréb.
Achnarthes coarctataGrun. - inflata Grun.

Amphora oralis $\mathrm{Ktz}$.

Epithemia argusvar. amphicephala Grun. - gibba Ktz.

- $\quad$ - fa curta.

- - - ventricosa Grun.
Eacyonema ventricosum Ktz.

Fragilaria elliptica Grun.

Gomphonema affine $\mathrm{Ktz}$.

- augustatum $\nabla$. intermedia

Grun.

- $\quad$ - $\quad$ v. producta

P. P. 
Gomphonema constrictum Eh.

Melosira varians Ag.

Navicula Braunii var.

- sculpta Eh.

- slesvicensis Grun.

Nitzschia acutiuscula Grun.

- brevissima Grun.
Nitzschia linearis W. Sm.

Stephanodiscus Astraea Grun.

Surirella Chiliensis Jan. var.

- linearis W. Sm.

- ovalis Bréb.

- ovata Ktz.

Synedra acus? Grun.

Nos 39,40

ROYAN (collection II. Peragallo)

(Pélagique)

Cette récolte, fixée par l'acide osmio-chromique, a été colorée par le carmin alcoolique et montée dans le baume du Canada.

Aetinoptychus undulatus Eh.

- splendens Eh.

Bacteriastrum varians Laud.

Bidlulphia moliliensis Bail.

Chretoceros curvisetuu cl.

- decipiens Grun.

- didymum Gl.

- socialis Laud.

Cocconeis scutelium fa parva.

Coscinodiscus gigas Eh.

- oculus-iridis Eh.

- radiatus $\mathrm{Eh}$.
Ditylium intricatum West.

Eucampia zodiacus EL.

Guinardia flaccida $H$. Per.

Lauleria borealis Cl.

Melosira sulcata Kiz.

Pleurosigma delicatulım IV. Sm.

- strigosum WV. Sm.

Raphoneis rhombus Eh.

Rhizosolenia Stolterfothii H. Per.

Stephanopyxis turgida Grev.

Surirella gemma Kitz.

Triceratium alternans Eh.

No 41

EDSFJORD (Norvège)

Plankton (collection Gran)

Biddulphia aurita Brẻb.

Chatoceros balticus Cl.

- cellulosum Laud.

- convolutum Cast.

- Ostenfeldii Cl.
Nitzschia lineola Gl.

' - seriata var. fraudulenta $\mathrm{Cl}$.

Rhizosolenia alata var. gracillima Grun.

- styliformis Bright.

Thalassiosira Nordenskioldii CI. 
$N " 42$

NORVEGE (Côte Ouest)

Plankton (collection Gran)

Chatoceros danicum Cl.

- lecipiens $\mathbf{C l}$.

Chretoceros Glevei Schūtt?

LA ROCHELLE (France)

(Vase du port)

Bibliogra PhiE. - H. et M. Peragallo. - Diatomées marines de France.

Actinoptychus adriaticus Grun.

$\bar{\beth}$ glabratus A. S. splendens Ralfs. undulatus Eh.
- v. balearica Grun.

Amphiprora elegans W. Sm.

Asteromphalus flabellatus Breb.

Auliscus colatus Bail.

- sculptus Eh.

Biddulphia mobiliensis Bail.

- rhombus El.

Campylodiscus limbatus Bréb. Thuretii Bréb.

Campyloneis Grevillei Grun.

Cerataulus Smithii Bréb.

Dans une récolte d'Arcachon, j'ai observẻ tout récemment des exemplaires de cette espèce ou les deux cellules nouvellement formées ne sont pas encore séparées. Les appendices ne sont pas en contact comme dans les Gerataulus et Biddulphia ordinaires, mais entrecroisés, les valves étant en contact direct. Ce n'est donc pas un véritable Cerataulus, ni même un Biddulphia : c'est un Coscinodiscus, muni d'appendices. H. P.

Cocconeis pseudo-marginata Greg.
Coscinodiscus boliviensis Grun.

$$
\begin{array}{ll}
\text { - } & \text { excentricus Eh. } \\
\text { - } & \text { gigas Eh. } \\
\text { - } & \text { heteroporus Eh. } \\
\text { - } & \text { oculus-iridis Eh. } \\
& \text { radiatus Eh. }
\end{array}
$$

IIyalodiscus stelliger Bail.

$$
\text { - subtilis Bail. }
$$

Licmophora Ehrenbergii Ktz.

Lithodesmium undulatum Eh.

Melosira nummuloïdes Ag.

- sulcata Ktz.

Navicula aspera Eh.

- Hennedyi W. Sm.

- humerosa Brèb.

- liburnica Grun.

- lyra Eh.

- - v. elliptica A. S.

- maxima v. bicuneata Grun.

- nebulosa Greg.

- Smithii Brèb.

- - $\quad$ v. major H. Per.

Nitzschia insignis Greg.

- - v. mediterranea Grun.

-- sigina W. Sm.

Pleurosigma affine Grun.

- balticum W. Sm.

- decorum W. Sm. 
Pleurosigma delicatulum W. Sm.

- distortum WV. Sm.

- elongatum TV. Sm.

- formosum WV. Sm.

- littorale IV. Sm.

- naviculaceum Bréb.

- Normanni Grun.

- rigidum IV. Sm.

- strigosum IV. Sm.

- Wansbeckii Donk.

Rhabdonema adriaticum $\mathrm{Ktz}$.
Roperia tessellata Ralfs.

Scoliopleura latestriata Grun.

$$
\text { - tumida Bréb. }
$$

Surirella armoricana $\mathrm{H}$. Per.

- fastuosa Eh.

- gemma Eh.

- hybrida Grun.

Triceratium allernans Eh. antediluvianum Eh. favus Elı.

No 44 RIVIERE SAINT-DENIS (Ile de la Réunion) (Vase de l'embouchure)

Achnauthes ventricosa Eh.

Actinoptychus undulatus Eh.

Amphora libyca Eh.

$$
\text { - ovalis } K t z \text {. }
$$

Campyloneis Grevillei Grun.

Cocconeis lineata Eh.

- pseudo-marginala Greg.

Epithemia gibberula $\mathrm{K} t z$.

Eunotia monodon Eh.

Gomplionema commutatum Grun. - gracile var. lata Grun.
Ityalodiscus subtilis Bail.

Melosira roeseana Rab.

- sulcata Elı.

Navicula dactylus $E h$.

$$
\begin{aligned}
& \text { - } \quad \text { esox Eh. } \\
& \text { - major Ktz, } \\
& \text { - } \quad \text { spectabilis Greg. }
\end{aligned}
$$

Vitzschia Frauenfeldii Grun v, major.

Surirella tenera $\nabla$. nervosa $A$. S.

Syneira Goulardi Bréb.

- - v.elongatavar. n. M.P.

Nos 45 à 47 REDONDO-BEACH, Californie (U. S. A.)

(Dépôt fossile Marin)

Actinocyclus Ehrenbergii Ralfs.

- incertus Grun var.

Actinoptychus areolatus Eh.

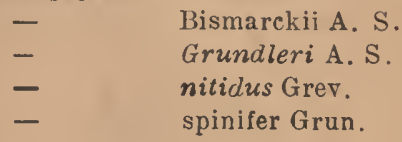

Actinoptychus splendens Ralfs.

- undulatus Eh.

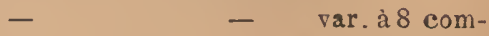

partiments.

- var. compressa

De forme obtusément triangulaire, les 
Navicula lyra Eh. var.

- O'Swaldii Jan.

- pratexta Eh.

- rhombica var. Greg.

- spectabilis Greg. var.

- splendida Greg. var.

- suborbicularis Greg. var.

- sp. A. S. atlas 6/32 var.

Orthoneis barbadensis Grev.

$$
\text { - }
$$

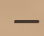
จ. tenuipunctata
J. Br.

- var.? Plus petite et plus allongée.

Periptera clamidophora Eh.

- tetracladia Eh.

Plagiogramma atomus Grev. var.

- tessellatum Grun.

Ploiaria petasiformis Pant. var.

Porpeia quadrata Grev.

Pyxilla carinifera Grun.

- dubia Grun.

- subulata Grun.

Rhabdonema adriaticum Elı.

- japonicum Temp. et Br.

Rhizosolenia alata Bright var.

- Barboi J. Br.en fragments.

- hastata Grun.
Rhizosolenia laevis H. P. pileolus Eh.

Rutilaria hexagona Grun.

Stephanogonia polygona Eh.

Stephanopyxis appendiculata var. intermedia.

- $\quad \operatorname{aristata} \mathrm{J} . \mathrm{Br}$.

- corona Eh.

- spinosissima Grun.

Stictodiscusi californicus v. ecostata Grun - Hardmanianus Grev.

Thalassiothrix nitzschioides $v$. japonica $f$ a elongata Grun.

Trachyneis aspera $v$. vulgaris Cl.

$$
\text { - oblonga Bail. }
$$

Triceratium arcticum var. californica Grun.

- elegans Grev.

- montereyi Bright.

- punctatum 4 Gona. Bright.

Xanthiopyxis alata Eh.

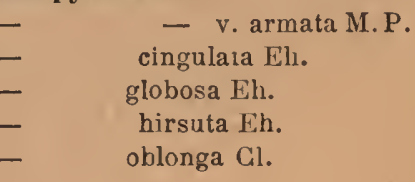

\section{Nos 48 et 49 CALIFORNIE (Lavage d'Haliotis)}

Actinocyclus Ehrenbergii Ralfs.

$$
\text { - splendens Ralfs. }
$$

Actinoptychus undulatus Eh.

Arachnoïliscus Ehrenbergii v. califor-

$$
\text { nica. }
$$

Aulacodiscus oregonus Bail.

Biddulphia aurita Bréb.

$$
\begin{array}{ll}
\text { - } & \text { obtusa Bail. } \\
\text { - } & \text { pulchella Grun. } \\
\text { - } & \text { regina W. Sm. } \\
\text { reticulata Roper. }
\end{array}
$$

Campylodiscus biangulatus Grev.
Campylodiscus teniatus $A$. S. Thuretii Bréb.

Cocconeis circumcincta A. S. - pellacida Grun.

Coscinodiscus nitidus Greg.

$$
\text { - } \quad \text { radiatus Eh. }
$$

\section{Entopyla australis Eh.}

Granmatophora marina Eh.

$$
\begin{aligned}
& -\quad-\quad \text { v. gihba. } \\
& -\quad \text { uncina. Leud. Fort. } \\
& \text { Hyalodiscus lievis Eh. }
\end{aligned}
$$


Ilyalodiscus stelliger Bail.

Isthmia nervosa $\mathrm{K} t z$.

Navicula aspera var. minor?

Melosira sol Eh.

Navicula crabro Eh.

- Hennedyi W. Sm.

- lyra var Eh.
Orthoneis fimbriata Bright.

Pollosira maxima v. californica Grun.

Rhablonema adriaticum Ktz.

Surirella fastuosa $\mathrm{Eh}$.

Triceratium arcticum Bright.

- antediluvianum Eh.

\section{No 50 EMBOUCHURE DU SADO à Sétubal (Portugal)}

\section{(Vase saumâtre)}

BIBLIOGRAPHIE. - La presque totalité des espèces marines contenues dans cette récolte sont décrites el figurées dans Les Diatomées Marines de France de MM. H. et M. Peragallo.

Achnanthes brevipes Ag.

- longipes Ag.

Actinoptychus splendons Sliadb.

- undulatus Eh.

Amphiprora decussata Grun.

- lepidoptera Greg.

Amphora acuta $\nabla$. arcuata A. S.

- proteus Greg.

- $\quad$ - v. oculata H. Per.

Auliscus coelatus Bail.

- $\quad$ - $\quad$. rhipis H. Per.

Biddulphia pulchella Gray.

- regina W. Sm.

Campylodiscus Clevei H. Per.

$\begin{array}{ll}- & \text { decorus Bréb. } \\ \text { - } & \text { Echeneis Eh. } \\ \text { - } & \text { eximius Greg. } \\ - & \text { Gregorii H. Per. } \\ - & \text { hibernicus Eh. } \\ - & \text { impressus Grun. } \\ - & \text { parvulus W. Sm. } \\ - & \text { samoensis A. S. } \\ - & \text { Thuretii Brëb. }\end{array}$

Cerataulus turgidus Eh.

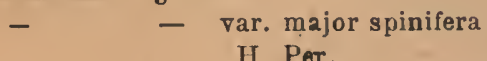

Cocconeis distans Greg.

$$
\text { - lineata Eh. }
$$

- ornata Greg.

- placentula Eh.

- pseudo-marginata Greg.

Coscinodiscus heteroporus Eh.

$\begin{array}{ll}\text { - } & \text { marginatus Eh. } \\ \text { - } & \text { nitidus Greg. } \\ \text { - } & \text { nodulifer A. S. }\end{array}$

Dimeregramma marinum Ralfs fa recta.

Epithemia gibba Ktz.

- musculus Ktz.

- - turgida Kitz.

- Hyndmannii W.Sm.

Fragilaria viresceus Ralfs v. exigua?

Grammatophora serpentina Eh.

$$
\begin{array}{ll}
- & - \\
& \text { v. elongata } \\
\text { H. Per. }
\end{array}
$$

IIantzschia marina Grun.

Ilyalodiscus laevis Eh.

- $\quad$ stelliger Bail.

Iastogloia Smithii v. amphicephala Grun.

Nelosira granulata Ralfs.

- sulcata kitx.

- - fa radiata Grun.

- $\quad$ v. coronata Gruu. 
Navicula abrupta Greg.

- arenaria Donk.

- allantica. A. S

- Baileyana A. S.

- Beyrichiana A S.

- bombus Eh.

- clavata Greg.

- $\quad$ - v. elongata H. Per. crabro Eh.

- didyma Ehr.

-

- v. lusitanica H. Per.

- var? Diatomées marines de France pl. 19 fig. 12.

- digito-radiata Greg.

- Donkinii A. S.

- elliptica Ḱtz.

- eudoxia A.S.

- - - v. elongata H. Per.

- formosa Greg.

- fusca Greg.

- $\quad$ - $\quad$. delicata Cl.

- granulata Bréb.

- Hennedyi IV. Sm.

- - $\quad$ v. cuneata Grun.

- $\quad$ - $\quad$ v.granulata Grun.

- $\quad$ - $\quad$ v.minuta Cl.

- interrupta Kitz.

- latissima Greg.

- liber W. Sm.

- _ v. linearis Grun.

- $\quad$ - . *tenuistriata II. Per.

- lineata Donk.

- lyra Eh.

- - v. elliptica A. S.

- marina v. producta O'Meara.

- nebulosa Greg.

- palpebralis Brèb.

- pandurả Bréb.

- pennata A. S.

- permagna Bail.

- scandinavica Lag.
Navieula Smithii Brér.

$$
\begin{array}{lll}
- & - & \text { v. delicata Cl. } \\
- & - & \text { v. permagna Pant. } \\
- & - & \text { v. scutellum O'Meara. } \\
\text { - } & \text { - var. (A.S. atlas } 12 / 28 \text { ). }
\end{array}
$$

Nitzschia acuminata IV. Sm.

-. insignis v. mediterranea Grun

- macilenta Greg.

- navicularis Bréb.

- punctata W. Sin.

- rigida H. Per.

- $\quad$ - v. rigidula H. Per.

Orthoneis splendida Grun.

Pleurosigma ætuarii Bréb.

- balticum W. Sm.

- decorum W.Sm.

- formosum W. Sm.

- longum. v. lanceolata H. Per.

- rigidum W. Sm.

Raphoneis liburnica Grun.

Rhahdonema adriaticum Ktz.

Roperia tesselata Grun.

Scoliopleura tumida Rab.

Stanroneis Gregorii Ralfs.

Surirella collare var. A. S.

- íactuosa Eh.

$$
\text { - } \quad \text { - } \quad \text { v. cuneata A.S. }
$$

Syaedra dalmatica Ḱtz.

Terpsinue americana Bail.

Tracheneis aspera $\mathrm{Cl}$.

$$
\begin{array}{lll}
- & - & \text { } \\
- & \text { - minuta H. Per. }
\end{array}
$$

Triceratium antediluvianum Eh.

- favus Eh. spinosum Bail.

erratum. - Page 15, dernière ligne, lisez: Ciomphonema acnminatum et non Cieminatum, imprimé par erreur. 
$N^{\circ} 51$ à 53

JOURSAC (Cantal - France)

(Dépôt fossile d'eau douce)

BIBLIOGRAPHIE. Frère J. Héribaud. Les Diatomées fossiles d'Auvergne 1903.

Achnanthes Joursacence $F$. $H$.

Amphora distincta F. H.

- lybica Eh.

- pediculus Grun.

- - var. major Grun.

Cocconeis lineata Grun.

- $\quad$ - var. euglypta Grun.

- - var. rotunda F. II.

- placentula Eh.

- Rouxii var. minor M. P. et F. H.

Coseinolliscus dispar M.P. et F. H.

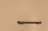

$-$

Cyclotella iris J. Br. et F.H.

- - var. integra.

- perforata $F$. $H$.

Cymatopleura apiculata IV. Sm.

Cymbella affinis Ktz.

- aspera Eh.

- Brevieri F. H.

- Creguti F. H.

- cymbiformis Eh.

- Laubyi F. H.

- leptoceros Ktz.

- meniscus F.H.

- Pagesi F. II.

- turgidula Grun.

Diploneis elliptica Cl.

- - v. minutissima Grun.

Encyonema cespitosum var. Anerswaldii W. H.

- intermodium F.H.
Epithemia gibba Eh.

- - v. parallela Grun.

- - v. ventricosa Grun.

- gibberula Eh.

- - var.producta Grun.

- Ilyndmanni W.Sm.

- $\quad$ - v. perlonga F. II.

- sorex Ktz.

- turgida Kitz.

- - var. granulata Grun.

- Westermanni Kiz.

- sebraKtz.

- $\quad$ - var. minor F.H.

- - var. proboscidea.

Eunotia impressa $E h$.

- - var. angusta Grun.

- gracilis Rab.

- polyglyphis Grun.

Fragilaria brevistriata Grun.

- $\quad$ - var. pusilla Grun.

- construens Grun.

- - var. circulare F.H.

- - var. venter Grun.

- elliptica Sclium.

- - - var. minor Grun.

- Gustavei F.H.

- Zeilleri F. H.

Gomphonema abbreviatum $\mathrm{K} t z$.

- accessum F. II.

- arcticum Grun.

- brevistriata M. P. et F.H.

- commutatum Grun.

- erienser. acuminata F. H.

- exiguum Kitz.

- exscissum H.H.

- insigne v. minor Grun.

- intricatum Ktz.

- - varpumileGrun. 
Gomphonema micropus Kitz.

- $\quad$ v. major F. H.

- parvulum Ktz.

- subclaratum Grun.

Grunowia tabellaria V. H.

llantzschia amphyoxis Grun.

- _ - $\quad$. intermedia Grun

Melosira Boulayiana M. P.

- Camusi F. H.

- $\quad$ - var conica F. H.

- crenulata Kitz.

- - var ambigua Grun.

- granulata Eh.

- $\quad$ - $\quad$. australiensis V:H.

- minuta F. H.

- tenuissima Grun.

- undulata var. producta A.S.

Navicula acrosphæria Bréb.

- - var. loevis M. P et F. II.

- amphibola Cl.

- amphigomphus Eh.

- bacillum Eh.

- - var. minor V. H.

- Berriati F. H.

- Brebissonii Ktz.

- cincta Ktz.

- Lorbieri F. H.

- Costei F. II.

- $\quad$ - v. bacillaris F. H.

- decurrens Eh. V. curtecostata

- dubitala F. H. F. H.

- esox Eh.

- $\quad$ - var. recta F. H.

- firma Ktz.

- gastrum F. H.

- Gendrei F. H.

- major Ktz.

- $\quad$ - .convergentissima F.H.
Navicula nobilis $E h$.

- Olivieri $F . H$.

- peregrina var. fossilis M. P. et F. $\mathrm{H}$.

- placentula Eh.

- pseudo-bacillum var. Grun.

- elapsa F. H.

- radiosa Kitz.

- - var. acuta Grun.

- Reinhardtii Grun.

- $\quad$ - var. elliptica F. H.

- Renauldi F. $H$.

- rupestris $K t z$.

- sculpta Ehr.

- triangulifera F. H.

- rentricosa $K t z$.

Opephora cantalense $\mathrm{F}$ : H.

- $\quad$ - car. capitata F.H.

- Marlyi F. H.

- $\quad$ - var. capilata F. H.

Ithoicosphenia curvata Grun.

Stauroneis gracilis Eh.

- phoenicenteron Eh.

Surirella bifrons Ktz.

- gracilis Grun.

- $\quad$ - var. constricta F. H.

- Pagesi F. H.

- robusta $\mathrm{Eh}$.

- tenera Greg.

Synedra capitata $E h$.

- Joursacensis F. H.

- ulna Eh.

Tetracyclus emarginatus Eh.

- $\quad$ Pagesi F. H et M. P.

- Peragalli F. $\boldsymbol{H}$.

- $\quad$ - var. eximia F. H.

- $\quad$ - var. major F.H. stellare F. H.

$\mathrm{N}^{\text {os }} 54,55$

ILE-RODRIGUE (Océan Indien)

(Sur les algues)

Achnanthes indica $\mathrm{J} . \mathrm{Br}$.

- subsessilis var.
Actinocyclus Ehrenbergii Ralfs.

- Ralfsii Pritch. 
Actinoptychus indicus $\mathrm{J}$. Br.

Ara'chnoüliseus ornatus Eh.

Asterionella Bleakeleyi var. Grun.

Bidllulphia aurita Bréb.

$$
\begin{array}{ll}
\text { - } & \text { balaena Eh. } \\
\text { - } & \text { obtusa Ralfs. } \\
\text { - } & \text { pulchella Greg. }
\end{array}
$$

Campyloneis notabilis $\mathrm{J}$. Br.

Cocconeis heteroïdea.

$$
\text { - } \quad \text { pellucida Hantz. }
$$$$
\text { - } \quad \text { - var. obliqua J. Br. }
$$

Epithemia hirundinella J. Br.

- musculus Ktz.

Gephyria media Arnott.

Grammatophora marina $\mathrm{K} t z$.

Isthmia enervis Eh.

Mastogloia indica J. Br.

- $\quad \begin{gathered}\text { Peragalli Grun var. circom- } \\ \text { nodosa J. Br. }\end{gathered}$
- $\quad$ quinquecostata Grun.
- $\quad$ squamosa $\mathrm{Cl}$.
- De Toni J. Br.
- $\quad$ tortilis J. Br.

Melosira Borreri v. octogona.

Navicula aspera Eh.

- didyma Kitz. var.

- lyra Eh. et vars.

- multicostata Grun.

- notabilis Grev. var.

Nitzschia fluminensis Grun.

- paradoxa Grun.

- panduriformis Greg.

Orthoneis Cilevei Grun.

- fimbriata Grun.

Plagiodiscus nervatus Grun.

Pleurosigma formosum Grun.

- speciosum Sm. var.

Podosira ambigua Grun.

Rhablonema adriaticum Ktz.

$$
\text { - } \quad \text { mirificum W. Sm. }
$$

Synedra formosa Hantz.

- fulgens W. Sm.

- Hennedyatwa Greg.

Triceratum arcticum Bright.

- dubium Bright.

- pentacrinus Wall.

- Shadboldtianum Grev.

- sculptum Shadb.

- zonulatum Grev.

BIBLIOGRAPHIE. - Diatomées des Alpes et du Jura, par le professeur J. Brun, qui a fait l'analyse de cette récolte.

\section{Campylodiscus hibernicus Eh.} noricus Eh.

Cymatople ura solea Eh.

$$
\begin{aligned}
& \text { - } \quad \text { elliptica W. Sm. } \\
& \text { - } \quad \text { - } \quad \text { v. constricta. }
\end{aligned}
$$

Fpithemia Hyndmannii W. Sm.

Navicula nobilis Eh.

$$
\text { - patula W. Sm. }
$$

\section{Navicula viridis Elı.}

Nitzschia sigmoïdea W. Sm.

Pleurosigma attenuatum W. Sm.

Surirella biseriata Bréb.

$$
\begin{aligned}
& \text { - } \quad \text { - var acuminata. } \\
& \text { - - var minor. } \\
& \text { - Capronii Brél.. } \\
& \text { - elegans v. elongata Eb. }
\end{aligned}
$$




\section{No 57 LAC DES QUATRE-CANTONS (Suisse)}

\section{(Sur les plantes aquatiques)}

BIBLiogra PhiE. - J. Brun. Diatomées des Alpes et du Jura, 1888.

Achnanthes minutissima Kitz.

- $\quad-\quad$ v.

Cocconeis pediculus Ktz.
$-\quad$ placentula Ktz.

Cyclotella Meneghiniana litz.

Cymatopleura solea Eh.

Cymbella cistula Hemph.

- cymbiformis Eh.

- Ianceolata Eh.

Diatoma elongatum litz.

- vulgaris Bory.

Encyonema cœepitosa Kitz.

- ventricosa Ktz.
Eunotia arcus Eh.

Fragilaria capucina Desm.

Gomphonema capitatum Eh.

$\begin{array}{ll}\text { - } & \text { commutatum Grun. } \\ \text { - } & \text { constricturn El. } \\ & \text { intricatum Ktz. }\end{array}$

Melosira varians $\mathrm{l} t \mathrm{z}$.

Navicula cryptocephala $\mathrm{K} t z$.

- radiosa Kiz.

Synedra capitata Kitz.

- ulna Eh. v. Iongissima.

Tabellaria fenestrata $\mathrm{K} t z$.

- flocculosa Kítz.

$N^{\circ} .58$

BOULOGNE-SUR-MER (France)

(Vase du port)

BIbliographie. - H. et M. Peragallo. Diatomées marines de France.

Actinocyclus Roperii Kitt.

Actinoptychus glabratus Grun.

$$
\text { - } \quad \text { splendens Ralf. }
$$

Amphiprora lepidoptera f. minor Greg, Billdulphia aurita Bréb.

$$
\begin{array}{ll}
\text { - } & \text { granulata Rop. } \\
\text { - } & \text { pulchella Grun. } \\
\text { - } & \text { rhombus Eh. }
\end{array}
$$

Campyloneis Grevillei IV. Sm.
Coseinodiseus devius A. S.

$$
\text { - } \quad \text { excentricus Eh. }
$$

A part sa petite taille, cette forme est bien typique; elle se montre fixée à des particules étrangères par une enveloppe muco-siliceuse. Il doit en êtı'e de même des autres formes qui ne sont pélagiques en grande partie que par occasion; elles sont en effet très aisément flottables. H. P.

Coscimodiscus lineatus Eh. 
Coscinodiscus radiatus Eh.

Cymatopleura elliptica W. Sm.

- solea Eh.

Eupodiscus argus W. Sm.

Hantzschia marina Grun.

$$
\text { - virgata Roper. }
$$

Iyalodiseus stelliger Bail.

Melosira nummuloïdes Ag.

- Westii WV. Sm.

- $\quad$ - fa pustulata.

Navicula ambigua Ktz.

- amplirynchus Eh.

- bomburs Eh.

- cancellata Donk.

- clavata $\nabla$. elongata H. Per.

- didyma Ktz.

- fusca Greg.

- Hennedyi v. cuneata Grun.

- - $\quad$ v. granulata I. Fort.

r. niceaensis H. Per.

- littoralis Donk.

- humerosa Brèb.

- lyra v. elliptica A.S.

- oblonga $K$ lz. fa minor.

- palpebralis Bréb.
Navicula smithii Bréb.

- spectabilis Greg.

- Weissflogii A. S.

Nitzsehia angularis Greg.

- - v. affinis Grun.

- spathulata Bréb

Pleurosigma balticum W. Sm.

- hippocampus W. Sm

- Normanni Grun.

Itaphoneis amphiceros Eh.

- belgica $\mathrm{V} . \mathrm{H}$.

- rhombus Eh.

- surirella Grun.

- v. elongata H Per.

achizonema comoïdes $\mathrm{Ag}$.

- crucigerum IV. Sm.

- $\quad$ Grevillei Ag.

- ramosissimum $\mathrm{Ag}$.

Gurirella biseriata Bréb.

- hybrida Grun.

Synedra affinis $\mathrm{K} t z$.

- decipiens Grun.

Triceratiuu alternans Eh. favus Eh.

$\mathrm{N}^{\circ 8} 59,60$ LE BOSPHORE ('Turquie d'Europe)

(Sur les Algues)

Achnanthes brevipes $\mathrm{Ag}$.

- longipes Ag.

- subsessilis Ktz.

J'ai observé un frustule entier oủ la valve inférieure appartenait à l'A. longipes et la valve supérieuro à l"A. brevipes ॥.P.

Cocconeis maxima $H$. Per.

- - fa parva H. Per.

- $\quad$ - v. niceaensis fi. Per.

- scutellum Eh.

- $\quad$ - fa parva H.P.

- $\quad$ v. Morrissii H. P.

Epithemia musculus Eh.
Graumatophora macilenta W. Sm.

$$
\text { - } \quad \text { marina Ktz. }
$$

Ifyaloniscus scoticus IV. Sm.

Licmophora Ehrembergii v.angustata $H$. Per.

Melosira Jurgensii Ag.

- nummuloides Ag.

Navicula aspera El.

- cancellata fa minor Donk.

- lanceolata Ktz.

Pleurosigma delicatulum W. Sm. 
Pleurosigma rigidum W. Sm.

Bhabdonema adriaticum $\mathrm{Ktz}$.

Surirella fastuosa Eh.

Synedra affinis Kitz.

- v. fasciculata Grun.
Synedra v. hybrida Grun.

- baculus v. Greg.

- Gaillonii Eh.
- - v. elongata H. Per
- - v. macilenta Grun.

Triceratium antediluvianum Eh.

\section{Nos 61,63 ILE SANDWICH ou ILE VATÉ}

\section{(Nouvelles-Hébrides)}

\section{(Sur les algues)}

Actinocyclus Ebrenbergii Ralfs.

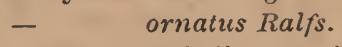

- Ralsii v. monicce Grun.

- splendens Ralfs.

- subtilis Ralfs.

Actinoptychus splendens $v$. halionyx Ehr.

Amphora crassa $v$. minor Pant.

Alloioneis antillarum Cl, et Grun.

Iulacodiscus argus (Eh) A.S.

$$
\text { - orientalis Grev. }
$$

Biddulphia crenulata W. et Ch.

- pulchella Grer.

- reticulata Roper.

- rhombus W.Sm.

Campylodiscus ambiguus Grer.

$$
\begin{array}{ll}
- & \text { Brightvellii Grun. } \\
- & \text { latus v. superba Eul. } \\
- & \text { teniatus A.S. }
\end{array}
$$

Climacosphenia moniligera Ehr.

Cocconeis eximia A.S.

$$
\begin{array}{ll}
\text { - } & \text { heteroidea Hantz. } \\
\text { - } & \text { pellucida Grun. } \\
\text { - } & \text { - v. minor Grun. }
\end{array}
$$

Coseinodiseus dimorphus Cast.

$$
\text { - Marginatus Eh. }
$$$$
\text { - nitidus Grev. }
$$

Coscinodiscus radiatus $E h r$.
Epithemia musculus $\mathrm{K} t z$.

Gephyria media Arnott.

Granmatophora adriatica Grun.

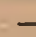

marina Ehr. var. subundulata Grun. var. undulata Ehr. maxima Grun. subtilis Ehr.

IIomoeocladia Vidovichii Grun.

Ilyalodiscus Laevis Wh.

- stelliger Bail.

Licmophora Ehrenbergii Grun.

Nastogloia citrus $\mathrm{Cl}$.

$$
\text { - decussata Grun. }
$$

- - interrupta Hantz.

Navicula advena A.S.

$$
\begin{aligned}
& \text { - apis Donk. } \\
& \text { - aspera Elir. } \\
& \text { - bicuneata Grun. } \\
& \text { - coaretata A.S. } \\
& \text { - consurs.A.S. } \\
& \rightarrow \quad \text { digito-radiata Greg. } \\
& \text { - elliptica Kitz. } \\
& \text {-. fusca Rulfs. } \\
& \text { - gemmatula A.S. } \\
& \text { - imperfecta Cl. var. } \\
& \text { - impressa Lag. } \\
& \text { - lacustris Grev. } \\
& \text { - longa } \text { v. Greg. }
\end{aligned}
$$$$
\text { - crabro v. multicostata Grun. }
$$ 
Vavicula lyra Ehr.

- - v. dilatata A.S.

- maxima Greg.

- nicobarica Grun.

- notabilis v. expleta Pant.

- separabilis A.S.

- sublyrata Grun.

- velata A.S.

- Weissflogii A.S.

- zostereti Grun.

Vitzschia acuta Hantz.

- angularis Greg.

- constricta Greg.

- longissima var. Ralfs.

- panduriformis Greg.

-- var. minor Grun.

- scalaris W. Sin.

- $\quad$ - socialis Greg.

Orthoneis fimbriata Grun.

Pleurosigma O'Mearii Grun. rigidum $\mathrm{WV} . \mathrm{Sm}$.

Podosira ambigua Grun.

- hormoïdes Ktz.
Podosira maxima Grun.

$$
\text { - } \quad \nabla \text {. intermedia H.P. }
$$

- Montagnei Ktz.

Porodiscus calyciflos Temp. et $\mathrm{Br}$.

Rhabdonema mirificum W. Sm.

Rhoicosphenia Reichardtii Grun.

Surirella anfractuosa A.S.

- eximiavar. Grun.

- fastuosa Ehr.

- - v. opulenta Grun.

Synedra formosa Hantz.

- undulata Greg.

Triceratium arcticum Bright.

- bullosum Witt (T. dubium).

- elongatum Grun.

- favus Ehr.

- gibbosum $\nabla$. crenulata

Grun.

- gibbosum $\nabla$. excisa Grun.

- papillatum Eitton.

- pentacrinus IVall.

- zonulatum fa triangularis Grev.
Actinocyclus alienus v. arctica Cl.

Amphora Pusio $\mathrm{Cl}$.

Chretoceros atlanticus Cl.

- var. nova (spinosa).

Caractérisée par ses soies toutes courtes et trẻs épineuses M. Per.

Chretoceros boreale Bright.

- contortum Schütt.

- convolutum Cast.

- decipiens Cl.

- distans $\mathrm{Cl}$.

- furcellatum Bail.

- Groenlandicus Cil.

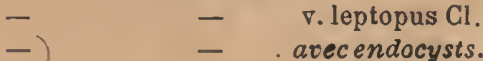

Chatoceros mitra $\mathrm{Cl}$ avec endocysts.

- rostratum Laudervar.

- socialis Bright (dissociè).

Coscinodiscus asteromphalus Eh. - . oculus iridis Eh.

Diploneis littoralis $v$ arctica $\mathrm{Cl}$.

Eucampia Groenlandica $\mathrm{Cl}$.

Fragilaria cylindrus Grun.

$$
\text { - oceanica Cl. }
$$

Navicula gelida v. perpusilla Grun. - septentrionalis Ost.

Nitzschia rigida Grun.

- polaris Grun.

- seriata $\nabla$ fraudulenta $\mathrm{Cl}$. 
Podosira glacialis $\mathrm{Cl}$.

Rhizosolenia obtusa.

- setigera Bright.

- styliformis Bright.
Skelotonema costatum $\mathrm{Cl}$.

Thalassiosira gravida Cl.

- Nordenskioldii Gl.

Thalassiothrix longissima Cl. et Grun.

No 65

SPITZBERG

Plankton (Collection Cleve)

Chatoceros atlanticum $\mathrm{Cl}$.

- boreale Bright.

- compactum Schütt.

- contortum Scbült.

- convolutum Cast.

- criophilum Cast.

- currens Ci.

- decipiens Gl.
Chatoceros gracile Schütt.

- Tanischianum var. Cast.

- solitaria.

Rhicosolenia alata Bright.

- - v. gracillima Grun.

- - setigera Bright.

- - styliformis Bright.

Skeletonema costatum $C l$.

N॰66 AIN HAMANN-OULED ALI, dép. de Constantine (Algérie)

Denticula tenuis var. inflata Grun

No67 HAMMAM MESKRONTINE, dép. de Constantine (Algérie)

\section{Denticula thermalis Ktz.}


N. 68, 69 PRE COHENDY (Puy-de-Dôme - France)

(Dépôt fossile d'eau douce)

BIBliogra PHIE. - Frère J. Héribaud. Les Diatomées d'Auvergne 1893.

Cocconeis lineata Grun.

Cymbella amphicephala Næy.

- aspera Ehr.

Diatoma anceps Grun.

- mesodon $\mathrm{Ktz}$.

Encyonema ventricosum $\mathrm{K} t z$.

Epithemia gibba Ehr.

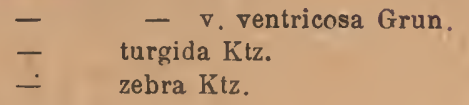

Eunotia lunaris Grun.

$$
\text { - } \quad \text { - } \quad \text { - } \text { - excisa Grun. }
$$

Fragilaria construens Grun.

$$
\begin{gathered}
-\quad-\quad \text { v. venter Grun. } \\
\text { - virescens fa elongata } \\
\text { M.P et F.II. }
\end{gathered}
$$

Gomphonema capitatum Ehr.

$$
\text { - constrictum } \nabla \text {. subcapita- }
$$$$
\text { ta Grun. }
$$

Hantzschia amphioxys Grun.
Meridion circulare Ag.

- constrictum Ralfs.

Melosira crenulata $\mathrm{K} t z$.

- $\quad$ - v. undulata M.P.et F.H.

- tenuis Grun.

Navicula amphigomphus Ehr.

$$
\begin{array}{ll} 
& \multicolumn{2}{c}{\text { v. major M.P. et }} \\
\text { - } & \text { } \text { bisulcata fa major M.P. et F.H. } \\
\text { - } & \text { gentilis Donk. } \\
\text { - gibba Ehr. } \\
\text { - } \quad \text { limosa Ktz. } \\
\text { - } \quad \text { - var. major M.P. et H.P. } \\
\text { - } \quad \text { major Ktz. } \\
\text { - } \quad \text { rupestris Ktz. } \\
\text { - } \quad \text { viridiš Ktz. } \\
\text { - } \quad \text { - v. commutata Grun. }
\end{array}
$$

Stauroneis amphilepta Ehr.

- phœnicenteron Ehr.

Synedra ulna Ehr.

Tabellaria tlacculosa $\mathrm{Ktz}$.

\section{$N^{\circ} 70$ MONTCOURT (Seine-et-Marne - France) (Flaques d'eau des carrières de sable)}

Amphora ovalis $K t z$.

Cocconels intermedia M.P. et F.H.

Cymbella cistula Hantz.
Cymbella cuspidata $K t z$.

- Ehrenbergii ta curta.

- maculata Ktz.. 
Epithemia turgida Ktz.

Gomphonema acuminatum v. clavus

Ehr.

angustatum v. producta

P.P.

- obtusatum Ktz.

- sarcophagus Greg.

Hantzschia amphioxys Grun.

- - v. major Grun.

Navicula affinis Eh.

- ambigua Ehr.
Navicula ambigua fa craticula.

- Brebissonii Ktz.

- cymbula Donk.

- firma v. subampliata Grun.

- oblongata v. directa Pant.

- rupestris Hantz.

Nitzschia commutata fa minor Grun.

- sigmoidea W. $\mathrm{Sm}$.

Stanroneis anceps Eh.

- - - v. linearis Eh.

Synedra $u \ln a E h$.

Nos 71,72

ARCACHON (France)

(Vase de la Canelette)

BIbliographie. - H. et M. Peragallo. Diatomées marines de France.

Achnanthes longipes Ag.

Actínooyclus nebulosus M.Per.

$$
\text { - } \quad \text { sparsus Greg. }
$$

Actinoptychus adriaticus Grun.

$\begin{array}{ll}\text { - } & \text { - var. balearica Grun. } \\ \text { - } & \text { areolatus Eh. } \\ \text { - } & \text { delectus A.S. } \\ \text { - } & \text { Janischii A.S. } \\ \text { - } & \text { splendens Ralfs. } \\ & \text { undulatus Eh. }\end{array}$

Amphiprora elegans W. Sm.

$$
\text { - lepidoptera Greg. }
$$

Amphora proteus Greg.

- - var. major.

Auliscus coelatus Bail.

- reticulatus var. Grev. .

- sculptus Eh.

Bidrlulphia aurita Bréb.

- granulata Roper.

- mobiliensis Bail.

- pulchella Greg.

- rhombus W. Sm.
Campylodiscus adriaticus v. massiliensis Grun.

$$
\begin{array}{ll}
\text { - } & \text { biangulatus Grov. } \\
\text { - } & \text { Echeneis Eh. } \\
& \text { Thuretii Bréb. }
\end{array}
$$

Cerataulus Smithii Ralfs.

$$
\text { - turgidus Eh. }
$$

Cocconeis scutellum Eh.

Coscinodiscus concavus Eh.

$\begin{array}{ll}\text { - } & \text { concinnus W. Sm. } \\ \text { - } & \text { excentricus Eh. } \\ \text { - } & \text { marginatus Eh. } \\ \text { - } & \text { nitidus Greg. } \\ \text { - } & \text { radiatus Eh. } \\ & \text { subtilis Eh. }\end{array}$

Uantzschia amphioxys var. major Grun.

llyalodiscus stelligèr Bail.

- subtilis Bail.

Melosira Borreri Grev.

- nummuloïdos Ag.

- sulcata Ktz.

- var. coronata Grun. 
Navicula abrupta Greg.

- atlantica A.S.

- aspera Eh.

- - var. pulchella.

- Baileyana Grun.

- Beyrichiana A.S.

- bombus Eh.

- didyma Eh.

- formosa Greg.

- fusca Greg.

- humerosa Bréb.

- liber W. Sm.

- lineata Donk,

- lyra Eh.

- - var. constricta H.P.

- - var. elliptica A.S.

- - var. intermedia H.P.

- marina Ralis.

- multicostata Bréb.

- scopulorum Bréb.-

- Smithii Bréb.

- Trevelyana Donk.

Vitzschia acuminata Grun.

- constricta Ralfs.

-- bilobata W. Sm.

- circumsuta Grun.

- insignis Greg.

- - var.mediterranea Grun.

- longa Grun.

- punctata Grun.

- sigma W. Sm.
Nitzschia sigma var. intercedens Grun. - spectabilis Ralfs.

Orthoneis splendida Grun.

Pleurosigma affind Grun.

$$
\text { - } \quad \text { balticum W. Sm. }
$$

Podosira hormoides Mont.

Rhabdonema adriaticum Ktz .

Raphoneis surirella Grun.

Scoliopleura latestriata Grun.

- tumida Rab.

Śchizonema crucigerum W. Sm .

Stauroneis salina W. Sm.

Sturirella armoricana H.P.

- fastuosa $\mathrm{Eh}$.

- $\quad$ - var. opulenta Grun.

- flnminensis Grun.

- gemina Eh.

- hybrida Grun.

Synedra Gaillonii var. macilenta Grun.

Terpsinoe americana Ralfs.

Triceratium alternans Bail.

- antediluvianum Eh.

- favus Eh.

- punctatum WVall.

- $\quad$ spinosum Bail.

\section{Nos 73-75 GOLFE DE NAPLES (Italie)}

(Le $\mathrm{n}^{0} 75$ (sondage) contient le plus grand nombre d'espèces)

Achnanthes longipes Ag.

Actinoptychus splendens Shad. Actinocyelus Ralfsii W. Sm.

Amphora crassa Greg.

- - var. punctata Grun.

- egregia Eh.

- mexicana A.S.

- obtusa Greg.
Amphora pellucida Greg. var.

- proteus Greg.

- robusta Greg.

- spectabilis Greg.

Asterolampra marylandica Eh.

Aulacodiscus Petersii Eh.

Auliscus colatus v. major A.S.

- sculptus Eh. 
Auliscus splendidus Ratt.

Auricula amphitritis Cast.

Biddulphia pulchella Gran. regina W. Sm.' Tuomeyi Bail.

Campylodiscus adriaticus $\vee$. massiliensis Grun. biangulatus Grov.

$\begin{array}{ll}\text { - } & \text { biangulatus Grov. } \\ \text { - } & \text { decorus Bréb. } \\ \text { - } & \text { fluminins Grev. } \\ \text { - } & \text { horologiuim Grev } \\ \text { ranea Grun. } \\ \text { - } & \text { impressus Grun. } \\ \text { - } & \text { limbatus Bréb. } \\ \text { - } & \text { samoensis Grun. } \\ \text { - } & \text { Thuretii Bréb. }\end{array}$

Corataulus turgidus Eh.

Cocconeis pseudo-marginata Greg. - regalis Greg.

Coscinodiscus concavus Grev. - curvatulus Grun.

-. lineatus Eh.

- oculus iridis Eh.

- radiatus Eh.

Glyphodesmis Williamsonii Grun.

Grammatophora gibberula $\mathrm{K} t z$. - macilenta W. Sm. - marina var. tropica Grun.

Hyalodiscus radiatus Grun.

Licmophora Ehrenbergii Grun.

- Jurgensii Ag. var. ovata Grun.

Mastogloia reticulata Grun.

Melosira Westii IV. Sm.

Navicula apis Donk.

- aspera Eh.

- Beyrichiana A.S.

- Bleischiana Jan.

- bomboides A.S.

- bombus Eb.

- californica v. ellipticaH.P.

- carinifera A.S.

- Chersonensis Grun.

- clavata Greg.

- $\quad$ - var. elongata H.P.
Navicula didyma Eh.

- fusea W.Sm.

- geminata v. spectabilis A.S.

- Hennedyi W. Sm.

- - var. granulata A.S.

- - var. niceaensis H.P.

- incus Grun.

- liber W. Sm.

- longa Greg.

- lyra El.

- - var. dilatata A.S.

- - var. elliptica A.S.

- - var, recla Greg.

- - var. subcarinata Grun.

- maxima Grev.

- multicostata Grun.

- pandura Bréb.

- prætexta Eh.

- pristiophora Jan.

- quadriseriata Grun.

- Sandriana Grun.

- seductilis var. H.P.

- Smithii Bréb.

- $\quad$ - fa maxima A.S.

- spectabilis Grev.

Nitzschia insignis Grun.

- - - var. mediterranea Grun.

- panduriformis Greg.

- sigma W.Sm.

- valida Cl. el Grun.

Orthoneis splendida Grun.

Pleurosigma affine Grun.
-

$-$

\author{
formosum W. Sm. \\ rigidum $W$. Sm.
}

Podosira adriatica (rrun.

- hormuides Mont.

- stellulifera Grun.

Rhabdonema adriaticum Eh.

Rhoicosplienia robustum Grun.

Surirella Baljickii Norm.

- tastuosa Eh.

- - var. abludens Grun.

- - var. cuneala A.S.

- - var. opulentaGirun.

- $\quad$ - var. panduriformis

H.P.

var. suborbicularis

Grun. 
Surirella hybrida Grun.

- intercedens Grun.

- lata W. Sm.

synedra affinis $\mathrm{Ktz}$.

- baculus Greg.
Synedra robusta Ralfs.

- undulata Grev.

Triceratium antediluvianum Eh.

- articum Bright.

favus $\mathrm{Eh}$.

N $* 3,77$ PONTEIX (Puy-de-Dôme - France)

(Dépôt fossile d'eau douce)

Amphora affinis Ktz.

- ovalis Ktz.

Cocconeis intermedia M.P. et F. $H$.

- lineata Grun.

- Rouxii F.H. et J. Br.

- - v. euglypta.

Cyclotella comta v. arverna M.P. et F.H.

Cymbella anglica Lag.

- aspera Eh.

- cistula Hэmp.

-. delecta A.S.

- Ehrenbergii v. minor Ktz.

- helvetica Ktz.

- gastroides Ktz.

- lanceolata Ktz.

- - Ehr.var.

- maculata Ktz.

- naviculiformis Auers.

- norregica Grun.

- turgidula Grun.

Encyonema gracile Rab.

- lunula Grun.

Fpithemia argus v. alpestris IV. sm.

- gibba falongissima M.P. et F.H.

- turgida Ktz.

- zebra Ktz.

Eunotia gracilis Rab.

- compressa v. angusta Grun.

- major Rab.

- - v. bidens W. Sm.

- parallela Ehr.
Fragilaria brevistriata Grun.

- capucina Desm.

- elliptica fa minor Grun.

- virescens halfs.

- - v. exigua Grun.

Gomphonema acuminatum $v$. coronata Eh.

- $\quad$ - v. intermedia

Grun.

- _ $\quad$ v. laticeps

Grun.

- $\quad$ Brebissonii Ktz.

- capitatum Ehr.

- constrictum Ehr.

Uantzschia amphioxys Grun.

Navicula borealis $\mathrm{Ktz}$.

- $\quad$ - v. major M.P. et F.H.

- cardinalis Ktz.

- dilatata Ehr.

- dubia Ehr.

- elliptica Ktz.

- gentilis Donk.

- gibba Ehr.

- globiceps Greg.

- hemiptera Ktz.

- iridis Ehr.

- legumen fa vix undulatus V.H.

- limosa Ktz.

- - v. undulata Grun.

- major v. interrupta M.P. et F.H.

- mesostyla Ehr.

- nobilis Ehr.

- $\quad$ - v. gracilis M.P. et F.H.

- nodosa Ktz. 
Vavicula parva Grun.

- placentula Ehr.

- radiosa $\mathrm{Ktz}$.

- $\quad$ - v. acuta Grun.

- rupestris $\mathrm{Ktz}$.

- stauroptera Gran.

- subacuta Ehr.
Navicula subcapitata Grun.

- termes Ehr.

Stauroneis phœnicenteron Ehr.

$$
\begin{gathered}
\text { - } \quad \text { v. gracilis J. Br. } \\
\text { et F.H. }
\end{gathered}
$$

Synedra ulna v. longissima W. Sm.

\section{No 78 ICE POND - NEW BRITAIN (Connecticut U.S.A)}

Achnanthes coarctata Grun.

Amphora libyca Ehr.

- ovalis Ktr.

- pediculus Ehr.

Cymbella amphicephala Næg.

- Ehrenbergii $K \iota z$

- gastroides $\mathrm{Ktz}$.

- naviculæformis Auers.

Encyonema lunatum Grun.

Epithemia argus v. amphicephala Grun.

- gibha $\mathrm{Ktz}$.

- $\quad$ - v. ventricosa Ktz.

- zebra v. proboscida Grun.

Eunotia diodon Eh $f^{\alpha}$ minor.

- impressa Grun.

- lunaris Grun.

- major Rab.

- $\quad$ - v. ventricosa $\mathrm{Cl}$.

- monodon Eh.

- pectinalis Rab.

- $\quad$ - v. ventricosa Grun.

- proerupta v. bidens Grun.

- ternaria Eh.

Fragilaria virescens Ralfs.

Gomphonema acuminatum Eh.

$\begin{array}{ll}\text { - } & \text { dichotonum W. Sm. } \\ \text { - } & \text { intricatum Ktz. } \\ \text { - } & \text { montanum Shum. } \\ \text { - } & \text { montanum v. suecica Grun. }\end{array}$

llantzschia amphioxys Grun.

$$
\text { - elongata Grun. }
$$

Meridion constrictum Ralfs.
Navicula acrosphæria Bréb.

- affinis Ktz.

- amphirynchus Eh.

- bicapitata v. hybrida Grun.

- Braunii Grun.

- - v. interrupta.

- Brebissonii Ktz.

- cryptocephala Ktz.

- cuspidata ktz.

- dubia Greg.

- gigas Ehr.

- iridis Eh.

- limosa $\mathrm{Ktz}$.

- - v. subundulata.

- major Ktz.

- mesogongyla v. interrupta Cl.

- mesolepta v. stauroneiformis Gr.

- mesotyla Eh. var

- nobilis Ktz.

- nodosa v. curta Rab.

- pupula $\nabla$. lineare $\nabla$. n. avec

la striation de N. pupula et la forme bacillaire à extrémités arrondies $\mathrm{M}$. $\mathrm{P}$.

Navicula trinodis Lewis.

- - var.

- $\quad$ viridis $\mathrm{Ktz}$.

- - $\quad$ - commutata Grun.

Vitzschia sigmoidea W. Sm.

Stauroneis anceps $\mathrm{Eh}$.

$$
\begin{aligned}
& \text { - } \quad \text { vracilis Eh. } \\
& \text { - legumen Ktz. La forme repré- } \\
& \text { sentée dans V. H. Syn. 4/11 n'en est } \\
& \text { qu'une variétè très étroite. }
\end{aligned}
$$


Surirella 82xonica Auersw.

- splendida Eb.

- tenera $\nabla$. splendidula A. S.

- Terryi Wari n. sp.
Synedra capitata $E h$. - ulna Eh.

Van lleurckia rhomboides $\nabla$. amphipleuroides Grun.

No 79

\section{EISS FJORD (Norvège) \\ Plankton (Collection Gran)}

Chrtoceros angulatum Schütt. (avec endocysts).

- balticum Cl.
- $\quad$ boreale var. Bright.
- $\quad$ convolutum Cast.
currens Cl.

Nitzchia seriata var. fraudulenta $\mathrm{Cl}$. Rhizosolenia alata v. gracillima Grun.

- corpulenta.

- inermis Cast.

- styliformis Bright.

Skeletonema costatum Cl.

Nitzschia Lineola Cl.

No 80 BANYULS-SUR-MER (Pyrénées-Orientales - France)

(Dans un bac du laboratoire Arago)

Bibliogra PhiE. - H. et M. Peragallo. Diatomées Marines de France.

Achnanthes longipes Ag.

Ictinoptychus Mölleri A. S.

Amphora arenaria Donk.

- arcus Greg.

- arenicola Grun.

- obtusa Greg.

- proteus Greg.

- rhombica Kitton (typique).

- sulcata Greg.

Auricula insecta Grun.

Biddulphia membranacea Cl.

$$
\text { - pulchella Greg. }
$$

Campylodiscus biangulatus Grev. decorus Bréb.
Compylodiseus impressus Grun.

- limbatus Bréb.

- samoensis A. S.

Coscinodiscus boliviensis A. S.

- centralis Cireg.

- oculus iridis El.

- radiatus Eh.

- gigas $\mathrm{Eh}$.

Gephyria media Arnott.

Grammatophora serpentina Eh.

IIyalotiscus subtilis Bail.

Isthmia nervosa $K t z$.

Licmophora flabellata Ag.

- paradowa Ag.


Melosira sulcata $\mathrm{Ktz}$.

Navicula aspera Eh.

- hombus Eh.

- $\quad$ - v. densestriata A.S.

- crabro Eh.

- $\quad$ - $\quad$. limitanea A. S.

- dalmatica Grun.

- exemta A. S.

- Kũtzingii Grun

- liber IV. Sm.

- - v. bicuneata Grun.

- multicostata Bréb.

- muscæformis v. constricta Grun.

- Smithii Bréb.

- _ - v. major Cl.

- - v. permagna Pant.

- splendida Greg.

vitzschia incerta Grun.

- insignis Grun.

- - v. mediterranea Grun.

- - $\quad$ v. notabilis Grun.

- macilenta Grun.

- maxima Grun.
Nitzochia panduriformis Greg.

- punctata W. Sm.

- - - v. elongata Grun.

- valida Cl. et Grun.

Orthoneis fimbriata Grun.

Pleurosigma decorum W. Sm. - elongatum W. Sm.

Pyxidicula adriatica $\mathrm{Ktz}$.

Rhoicosigma compactum Grun.

Surirella fastuosa Eh.

- - - $\quad$. opulenta Grun.

- - v.suborbicularisGrun.

- fluminensis Grun.

Synedra baculus Greg.

- cristallina Lyngh.

- - $\quad$ v dalmatica Ktz.

- formosa Hantz.

- fulgens $\mathrm{Ktz}$.

- Hennedsana Greg.

- tabulata $\mathrm{Ktz}$.

- undulata Greg.

\section{No 81 WRANGEN-CHRISTIANIAEJORI) (Norvège)}

\section{Plankton (Collection Gran)}

Amphora ostrearia Bráb.

Asterionella formosa Hass.

Auricula insecta Grun.

Biddulphia mobiliensis Bail.

Chrtoceros affine Laud.

- angulatum Schñtt.

- cochlea Schütt.

- constrictum Gran. avec endoeysts.

- contortum Schütt.

- curvisetum Cl. (avec endocysts).

- didymum v. longicornis.

- Groenlandicum Cl.
Chrteceros mamillanum Laud.

- radians Schült. (dissocié)

- seiracanthum Sctütt.

- socialis Laud. (dissocié).

Ditylun Brightwellii West.

Guinardia flaccida $H$. $P$.

Licmophora flabellata $A g$.

-- Jurgensit Ag.

Rhizosolenia inermis Cast.

- styliformis Bright.

Skeletonema costatum Cl.

Striatella interrupta Heib.

- unipunctata Ag. 
No 82

EMMINGEN (Hanovre)

(Dépôt fossile d'eau douce)

Amphora ovalis $\mathrm{Ktz}$.

Campylodiscus hibernicus Eh.

Cocconeis pediculus Eh.

Cyclotella comta v. radiosa Grun.

Cymatopleura elliptica $\mathbf{K} t z$.

$$
\text { - solea Ktz. }
$$

Cymbella cistula Hempr.

- Ehrenbergii Ktz.

-- lanceolata Eh.

Gomphouema acuminatum Kitz.

Epithemia gibba Ktz. capitatum Ktz.

- $\quad$ sorex Ktz.

- turgida. Ktz.

- - v. vertagus Grun. zebra Ktz.

Melosira arenaria moore.
Melosira crenulata $\mathrm{K} t \mathrm{z}$.

- granulata Ralfs.

Navicula elliptica Kiz.

- firma Ktz var.

- limosa Ktz.

- major $\mathrm{K} \mathrm{tz}$.

- oblonga Ktz.

- viridis $\mathrm{K} t \mathrm{z}$.

- viridula $\overline{\mathrm{n}} \mathrm{z}$.

Pleurosigma attenuatum W. Sm.

Stauroneis acuta IV. Sm.

- phoenicenteron Eh.

Stephanodiscus astræa Grun.

Surirella bifrons $\mathrm{K}$.

$\rightarrow \quad$ elegans Eh.

Synedra ulna Ktz.

Tabellaria fenestrata Ktz.

N॰ 83

ILES MARQUISES (Polynésie)'

A ulacodiscus Johnsonii Arnott

No 84 SING-SING-HUDSON RIVER (États-Unis)

(Dépôt fossile d'eau douce)

Cymbella lanceolata Ktz.

Runotia gracilis Rab.

- major Eh.
Navicula acrosphoeria Bréb.

- dactylus Eh.

- dilatata Eb. 
Navicula tirma v. subundulata Grun.

- gentilis Donk.

- major ktz.

- iridis Eh.

- pachyptera Eh.

- semen Eh.
Navicula tabellaria Eh.

Nitzschia Brébịsonii W. Sm.

Melosira crenulata $\mathrm{Ktz}$.

Stauroneis phœnicenterou Eh.

Stephanodiscus astraæa Grun.

No 85

MONTE-AMIATO (Italie)

(Dépôt fossile d'eau douce)

Amphora ovalis Ktz.

- pediculus Ḱtz.

Cyclotella operculata $\mathrm{K} \mathrm{z}$.

Cymatopleura solea $f^{2}$ elongata.

Cymbella cymbiformis Eh.

- Ehrenbergii ktz.

- lanceolata Eh.

Epithenia gibba ḱtz.

- turgida $\mathrm{Ktz}$.

- $\quad$ - v. granulata Grun.

- _ - v. vertagus Grun.

Fragilaria capucina v. acuta Desm.

- construens Grun.

- . - v. binodis Grun.

- _ $\quad$ - . minuta Grun.

- - v. venter Grun.

- mutabilis Grun.
Gomphonema acuminatum Eh.

Melosira crenulata.

Navicula amphigomphus Eh.

- amphirynchus Eh.

- cardinalis Eh.

- cuspidata Ktz.

- major Ktz.

- mesotyla Eh. var.

- nobilis Eh.

- gentilis Donk.

- papula $\mathrm{Ktz}$.

- radiosa $\mathrm{K} t z$.

- subacuta Eh.

- viridis Eh.

Stauroneis phœnicenteron Eh.

Synedra capitata Ktz.

- ulna var. longissima.

BIBLIOGRA PHIE. - Ce dépôt d'eau douce ou farine fossile a été cité par Ehrenberg.

Cymbella lanceolata Ehr.

Eunotia diadema Ralfs.
Vavicula cardinalis Eh. dilatata Eh. 
Navicula gentilis Donk.

- gigas Eh.

- major kitz.

- nobilis Ktz.

- stomatophora Grun:
Surirella elegans Eh.

- robusta Eh.

- tenera Greg.

Tetracyclus lacustris $\mathrm{Ktz}$.

\section{No 87 RIVER MOBILE - ALABAMA (États-Unis)}

L'espèce qui domine dans cette récolte est une variété du Terpsinoë intermedia Grun. On y trouve quelques auxospores telles qu'elles sont figurées dans l'atlas de Ad. Schmidt, pl. 199, fig. 1-6. Le Terpsinoë interinedia se distingue du T. musica par sa structure aréolée.

Actinocyclus Barkleyi Grun.

Canpylodiseus Echeneis Eh.

Coscinodiscus biangulatus A.S.

- $\quad$ nobilis Grun.

Navicula dactylus Eh.

- Dariana A.S.

gigas Eh.

Nitzschia circumsuta Bail.

Terpsinoë intermedia Grun. var.

Triceratium favus Eh.

No 88

CHRISTIANSTAD (Suède)

Amphora robusta.

Campylodiscus bicostatus W. Sm.

- costatus W. Sm.

- clypeus Eh.

- . Echeneis Eh.

Cymbella gastroides Ktz.

Epithemia turgida $\mathrm{K} t z$.

- zebra Kiz.
Navicula crucicula Donk.

- cuspidata Ktz.

- peregrina Eh.

- pusilla Ralfs.

- sculpta Eh.

Nitzschia scalaris W. Sm.

- circumsuta Bail.

Pinnularia distinguenda $\mathrm{Cl}$. viridis. 
№ 89 Environs d'AIX-EN-PROVENCE (France)

Amphora ovalis $\mathrm{Ktz}$.

Cymatopleura solea Eh.

elliptica Eh.

Encyoneua prostratum Ralfs.

Navicula ambigua Eh.

- amphisbœna Bory.

- eryptocephala Ktz.

- - v. exilis.

- gracilis $\mathrm{Ktz}$.

- radiosa $\mathrm{Kt}$. v. acuta.

vitzschia angustata W. Sm.

- curvula Dippel.
Vitzschia dubia W. Sm.

- linearis W. Sm.

- palea W. Sm.

- sigmoidea W. Sm.

- tryblionella Grun.

- vermicularis W. Sm.

Pleurosigua acuminatum W. Su

- attenuatum W. Sm.

Stauroneis anceps $\mathrm{Ktz}$.

- Smithii Grun.

Surirella minuta Bréb.

- ovalis Bréb.

-. splendida fa minor.

No 90

TUKURO-ILE YESO (Japon)

(Dépôt fossile marin)

BIBLIOGRAPHIE. - Tempère et Brun. Diatomées du Japon J. Pantocsek. Diatomées de Hongrie, vol. III.

Actinoptychus biformis J. $B r$. undulatus Eh.

Arachnoidiscus Ehrenbergii Bail.

- $\quad$ v. californica.

- indicus Eh.

Middulphia Tuomeyi Bail. var.

Cestodiscos superbus Hard. var

Chateceros pliocenum J. Br.

- Wighamii Bright.

Dicladia capreolus Eh.

Coscinodiscus elegans Grev.

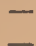

Coscinodiscus oc. irid. v. morsianus Gr. radiatus Eh et var. robustus Grev. subtilis Eh.

- subtil

- symbolophorus Gr.

- cribrosus Truan et Witt.

Goniothecium decoratum $J . B r$.

- , vitripons J.Br.

Grammatophora monilifera Temp.et $B r$. IIyalodiscus loevis tih.

Isthmia enervis Eh. car.

- nervosa $\bar{k}$.

Melosira sol Eh. 
Rhabdonema japonicum Temp. et Br.

Rutilaria capitata Temp. et $\mathrm{Br}$.

Stephanopyxis appendiculata Eh.

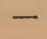

$-$

$-$ corona Eh. var. Grunowii Grov. et St. nidulus Temp. et $\mathrm{Br}$.
Stophanopyxis superba Grev. fa minor. Stictodiscus Hardmanianus Grev. Syndendrium Diadema Eh.

Triceratium versicolor $\mathrm{J}$. $\mathrm{Br}$.

Xantbiopyxis umbonatus Grev.

\section{No $91 \quad$ F́ELSO ESTERGALY (Hongrie) \\ (Dépôt fossile argileux tertiaire)}

BIBliographie. - J. Pantocsek. Diatomées de Hongrie, vol: I

Actinòcyclus Ehrenbergii Ralfs.'

$$
\text { - Ralfsii W.Sm. }
$$

Actinoptychus'amblyoceros $A$. $S$.

$\bar{z}$
$\bar{z}$
$\overline{-}$
$\overline{-}$ areolatus Eh. bifrons A.S. kymalodes Pant. mo.onensis $\mathrm{Cl}$. splendens f' partita Pant. stella A. S. undulatus Eh.

Anisodiscus Pantocsekii Grun.

Auliscus pulvinatus $\mathrm{Cl}$.

Bicldulpuia elegantula Grev.

- regina W.Sm.

- Tuomeyi Bail.' .

Campylodiscus limbatus Bréb. obsoletus $\mathrm{Cl}$.

Cerataulus Johnsonianus Grev.

Clavicula polymorpha $\nabla$. amphilepta Pant.

Cocconeis neogradensis Pant.

- pellucida Grun.

- pracellens Pant.

- pseudomarginata Greg.

Coscinodiscua actinocycloides Pant.

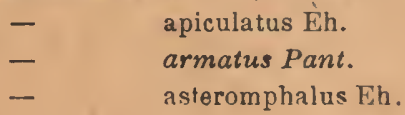

Coscinouliscus clivosus Pant.

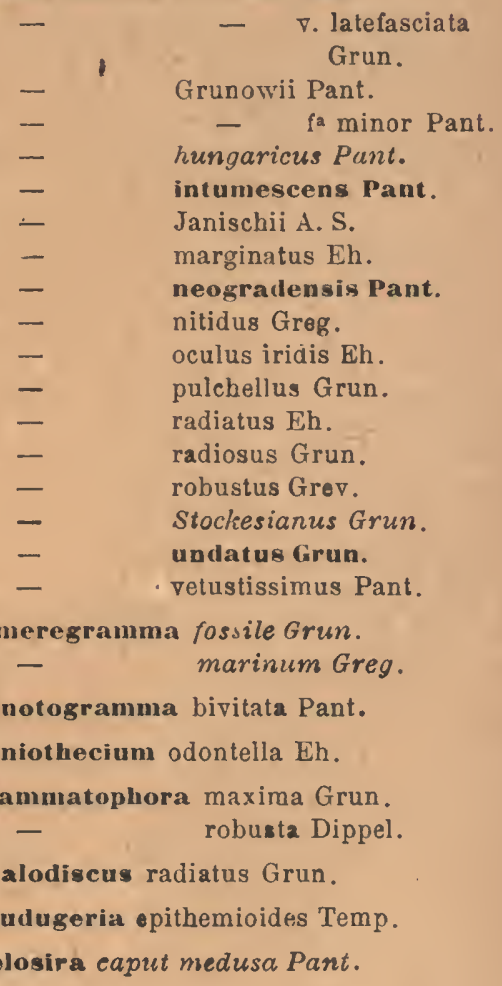


Melosira clavigera Grun.

- granulata Ralís. omma Cl. sol $\mathrm{Ktz}$.

- sulcala Eh.

Navicula apis Donk.

- aspera Eh.

- crabro Eh.

- didyma Eh.

- excavata Greg.

- fusca Ralfs.

- gemmataGrev.

- granulata Bréb.

- Hennedyi W. Sm.

- bumerosa Bréb.

- Lyra Eh.

- mikrotatos Pant.

- prætexta Pant.

- Sandriana Grun.

- Szontaghii Pant.
Orthoneis splendida Grun.

Pleurosigma Normanii Ralfs.

Raphoneis delicatula Pant.

- gemmifera Eh.

- subtrlissima Pant.

Rhabdonema adriaticum $K t z$.

Stephanopyxis corona Grun. turris Eh.

Stictodiscus Estergalyensis Pant.

Triceratium acutangulum Grun.

- arcticum Bright.

- balearicum Grun.

- favus Eh.

- Grovei Pant.

- Pantocsekii A.S.

- Stockesianum Grev.

Nanthiopyxis oblonga Eh.
Actinoptychus undulatus Eh.

Arachnoïdiscus Barbadensis Grer..

Asterolampra affinis Grev.

$$
\text { - Marylandica Eh. }
$$

\section{Cestodiscus pulchellus Grev.}

Coscinodiscus centralis Greg.

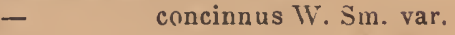

- curvatulus Eh var.

- decrescens Grun.

- excavatus Eh.

$\overline{-}$

$$
\begin{aligned}
& \text { - v. monophtalma } \\
& \text { - v. semilunaris } \\
& \text { marginatus Eh. }
\end{aligned}
$$

Coscinodiseus odontodiscus Grun. var. oculus iridis Eh.

- radiatus Eh. var. Cette variété qui est la forme principale du dépôt, se rapproche beaucoup de la fig. $13, \mathrm{pl} .6 \mathrm{l}$ de l'atlas de Ad. Schmidt que Rattray rapproche du C. radiatus type. C'est une forme intermédiaire entre les C. radiatus et marginatus. Tous les Coscinodiscus forment une chaine ininterrompue.

Craspedodiscus elegans var.

Stephanopyxis. Barbadensis Grev. 
Nos $94,95 \quad$ BORY - HQNGRIE

(Dépôt fossile tertiaire sarmatique marin)

\section{Bibliographie. - J. Pantocsek. Diatomées de Hongrie. Volu- mes II et III.}

Achnanthe brevipes $\boldsymbol{A} g$.

Actinooyclus Boryanus Pant.

- Ehrenbergii Ralfs.

- subtilis Ralfs.

Actinoptychus Boryanus Pant.

- $\quad$ Petıtii Pant.

- Szontagii Pant.

- undulatus v. subtilis Pant.

Alloioneis Castracanei Pant.

- Grunowii Pant.

Amphora Boryana Pant.'

- crassa Greg.

- $\quad$ - v. vninor Pant.

- - v. punctata Grun.

- egregia Eh.

- Grundleri Grun.

Auliscu Uauckii Punt.

Biddulphia elegantula Grev.

- permagna Pant.

-. Tuomeyi Bail.

- $\quad$ - v. boryana Pant.

- tridentata v. andesitica Pant.

Campylodiscus angularis Greg.

- $\quad$ - v. punctata Pant.

$$
\begin{array}{ll}
- & \text { Boryanus Pant. } \\
\text { - } & \text { striolatus Grun. } \\
\text { - } & \text { Thuretii Bréb. }
\end{array}
$$

Cerataulus Boryanus Pant.

- turgidus Eli.

Clavicula Kinkerii Pant.

Climacosphenia moniligera v. hungarica Pant.

Cocconeis Raeana Pant.

- scutellum Eh.
- _ - v. fossilis̀ Pant.
Coscinodiscus argus $A . \$$.

$\begin{array}{ll}\text { - } & \text { astęromphalus Eh. } \\ \text { - } & \text { Boeckei Pant. } \\ \text { - } & \text { oculusinatus Eh. } \\ \text { - } & \text { radiatus Eh. } \\ \text { - } & \text { undatus Grun. }\end{array}$

Dyctioneis Pantocsekil $\mathrm{Cl}$.

Dimeregramna Boryanum Pant.

Endyctia boryana Pant.

- oceanica Eh.

Grammatophora biharensis Pant.

$$
\text { - } \quad \text { insignis Grun. }
$$

Hyalodiscus radiatus Grun.

- scoticus Grun.

IIydrosera Boryana Pant.
-
v. hexagona Pant.

Mastogloia Szontagii Pant.

Melosira clavigera Grun.

- sol Ktz.

- sulcata Eh.

Navicula andesitica Pant.

- bimaculata Pant.

- Boryana Pant.

- didyma Eh.

- Fushsii Pant.

- fusca v. permagna Pant.

- granulata Bréb.

- halionata Pant.

- Haynaldii Pant.

- Hennedsi W. Sm.

- - $\quad$ v. fossilis Pant.

- humerosa Bréb.

- - v. elongata Pant.

- latissima Greg.

- - v. capitata Pant.

- Le Tourneurii Pant. 
Navicula Lyra Eh.

- maxima Greg.

- perlonga Pant.

- sectilis A. S.

- - $\quad$. Boryana Pant.

- Smithii Bréb.

- - v. minor Pant.

Nitzechia Loczyi Pant.

- spectabilis Grun.

- tryblionella $\vee$. biharensis Pant. Plagiogranima bih̆arense Pant.

- Boryanum Pant.

Podosira Boryana Pant.

Raphoneis Boryana Pant.

Rhabdonema adriaticum $\mathrm{kitz}$.
Rhabdonema adriaticun $v$. fossilis Pant.

Salacia Boryana Pant.

Surirella Baldjickii A. S.

- fastirosa Eh.

$$
\text { - v. fossilis Pant. }
$$

Synedra crystallina $\mathrm{Ktz}$.

$$
\text { - } \quad \text { - v. fossilis Pant. }
$$

Terpsinoë americana Ralfs.

- intermedia Grun.

Triceratium biquadratum Jan.

- horridum Pant.

- Iætum Pant.

- Loczyi Pant.

- pileus Eh.

- Sturtii Pant

\section{$\mathrm{N}^{\text {To }}$ 96-100 TAMATAVE - MADAGASCAR}

\section{(Sondages)}

Les numéros 96 à 98 proviennent d'un sondage fait dans le port même de Tamatave et les numéros 99 et 100 de la rade à 60 mètres de profondeur. Ces préparations contiennent de belles et rares espèces dont plusieurs sout nouvelles.

\section{Actinocyclus (nebulosus var?) spiralis} M. Per. - Diffère de l'A. nebulosus M. P. (Les Diat. mar. de France, p. 416, planche $113 / 11$ ) par sa zone marginale plus ètroite et moins nettement délimitée, son nombre de compartiments beaucoup plus grand et l'arrangement de ses pointes en spirales plus nettement définies.

Actinocyclus Ralfsii W. Sm.

Actinoptychus concentricus $A$. $S$.

$\begin{array}{ll}\text { - } & \text { hexagonas Grun. } \\ \text { - } & \text { - v.decumana A.S. } \\ \text { - } & \text { japtermeduus A. S. } \\ \text { - } & \text { splendens Shadb. } \\ \text { - } & \text { trilingulatus Bright. } \\ \text { - } & \text { - v. halyonyx } \\ \text { - } & \text { v. major. }\end{array}$

Actinoptychus undulatus Eh.
- vulgaris Schum.

A. Stella et A. Truanii.

Alloioneis antillarum $r$. Castracanei.

Ampliora areolata $v$ elegans $M$. Per.v.n. à extrémités plus longues, plus fines et élégamment récurvèes vers le bord dorsal.

- egregia Eh./A. S.

- formosa Cl.

- Grevilleana Greg.

- Grundleri Grun.

- pecten J. Br.

Aulacodiscus affinis Grun.

- Macraeanus Grev.
- $\quad$ Petersii Eh.
- $\quad$ v. crucifer A. S.


Auliscus colatus Bail.

- - $\quad$ v. rhipis A. S.

- splendidus Ratt. fa minor.

- sculptus Ratt.

- translucidus J. Br.

Bidduiphia pulchella et var Greg.

- Roperinna Grev.

- Tuomeyi et var Bail.

Campylodiscus adornatus $A . S$.

$\bar{z}$
$\bar{z}$
$\bar{z}$
$\bar{z}$
$\bar{z}$
$\overline{-}$ biangulatus Grev. crebrecostatus Grun. Daemelianus Grun. Heufleri Grun. horologium Williams ornatus Grev.

- v. amphileia Grun. - v. mesoleia Grun.

Kittonianus Grev. Grun.

toniatus A. S.

Cocconeis heteroidea Hantz. pseudo-marginata Greg.

Coscinodiscus excentricus Eh.

- lineatus Eh.

- nitidulus Grun.

- nitidus Greg.

- - v. sparsa Ralf.

- radiatus Eb.

-. Rothii Grun.

-- symmetricus Grev.

Grammatophora serpentina $E h$.

IIyalodiscus granulosus M. Per. n. sp. Bord large et lisse, centre grand à contour irrégulier, partie intermédiaire portant des lignes rayonnantes de 10 en $10 \mu$ formées de granules assez gros nıais peu robustes. Diamètre: $50 \mu$.

- maculatus Cl.

- stelliger Bail. var.

Leudugeria epithemioides Temp.

- _ - var. obtusa Temp.

Mastogloia panduriformis $\mathrm{Cl}$.

- reticulata Grun.

Melosira nummuloïdes Ag.

- sulcata v. coronata Grun.

- - v. radiata Grun.
Navícula abrupta Greg.

- - - compressa M. Per. De formeallongée et à bords latéraux en partie rectilignes.

- bomboïdes A.s.

- bombus Ktz.

- bullata Norm.

- californica Grev.

- clavata Greg.

- coarctata Eh?

- crabro v. pandura Brẻb.

- excarata Grev.

- expleta A. S. var.

- geminata Grev.

- Grunowii Rab.

- Hennedyi W. Sm.

- - v. cuneata A.S.

- - v. granulata Grun.

- humerosa Bréb.

- indefinita M. Per. n. sp. (Lyra

var 9. A la forme extérieure du Nav. I yra var. subtypica, mais les aires latérales, beaucoup plus longues, sont nettement délimitées, les granules qui sont sur les bords, des deux côtés, étant progressivement plus écartées que ceux qui forment la strie, et ne s'arrêtent pas à une ligne régulièrement définie.

Navicula liber W. Sm.

- Lyra Elı.

- $\quad$ - elliptica A. S.
- $\quad$ - recta Grev.
- Madagtypica A. S.
- nebulosa Gregsis $C l$.
- notabilis Grev.
- nummularioïdes M. Per. n. sp.

A la forme etl'aspect de $N$. nummularia Grev., mais entre les côtes, qui sont au noinbre de 6 à 7 en $10 \mu$, on aperçoit une ligne de gros granules assez peu marqués. Longueur : $50 \mu$, presque orbiculaire.

Navicula polita J. Br.

- polysticta Grev.

- prætexta Eh.

- proxima Jan.

- Robertsiana Grev.

- $\quad$ rugosa Jan = Nav. Hennedyi v. bacillifera A. S.? 
Navicula Smithii Bréb.

- spectabilis Greg.

- $\quad$ falata.

- Yarrensis Grun.

- Zanzibarica Grev.

Nitzschia Jelineckii Grun.

- panduriformis Greg.

- - fa acuta.

Plagiogramma Ceylanense Leud Fort.

Podosira argus Grun.

Pseudauliscus diffusus Ratt.

Pyxidicula cruciata Eh.

Rhabdonema mirificum W. Sm.

Stephanopyxis superba Grev.

Surirella fastuosa Eh.

$$
\begin{aligned}
& \text { - } \quad \text { - } \quad \text { v. robusta A. S. } \\
& \text { - } \quad \text { v. suborbicularis Grun. } \\
& \text { - } \quad \text { Sentis Eh. }
\end{aligned}
$$

Temperea Mephist,pheles M. Per. n. $g$. et $n s p$. Valve circulaire, bombée, ayant l'aspect général du Skeletonema mediterranea (V. H. syn. Pl. 90/5), mais le bord porte une rangée de perles ponctuées de forme allongée dans le sens du rayon; la zone intermédiaire, ètroite, porte de fines stries segmentées, et, de la partie centrale, grande et finement ponctueje. sortent deux soies divergentes dont les directions sont parallèles mais ne passent pas par le centre de la valve. Diamètre : $50 \mu$ (nos 98 et 100$)$.

Triceratium bicorne $\mathrm{Cl}$.

$$
\begin{aligned}
& - \\
& = \\
& = \\
& = \\
& = \\
& = \\
& = \\
& = \\
& = \\
& = \\
& = \\
& = \\
& -
\end{aligned}
$$

compar A.S.

dubium v. irregularis.

favus Eh.

Grunowii Jan. punctum A. S. latum Grev. Madagascarense Grun. megastomum Eh. pallidum Grev. parallelum Grev. pantacrinum Wall. scitulum Bright. sculptum Rhab. spinosum Bail. sublime.A.S. Temperei J. Br. zonulatum Grev. sp. A. S. atlas $93 / 5$.

No 101

ODENSE-FJORD - DANEMARK

(Vase saumatre)

Achnantbes brevipes Ag.

- longipes $\mathrm{Ag}$.

- subsessilis Ehr.

Actinocyclus Ebrenbergii Ralfs.

- nebulosus M. Per.

- sparsus Ratt.

- tenellus Bréb.

Amphlprora renusta Grer.

Amphora proteus Greg.

Biddulphia aurita Bréb.

Campylodiscus elypeus Eh.

echeneis Eh.
Cocconeis grata A.S.?

- scutellum Eh.

Coscinodiscus excentricus $E h$. - $\quad$ radiatus $E h$.

Cyclotella striata ? v. radiosa M. Per.

8 côtes en $10 \mu$. Diffère du Cocc. radiata par son centre à granulations fines formant des lignes rayonnantes al lieu de granules épars.

Epithemia gibba.v. ventricosa Ktz.

- musculus Ktz.

Melosira Borreri Grev. 
Navieula amphisbona Bory.

- Baileyana Grun.

- bombus Ktz.

- - v. egena A. S.

- claviculus Greg.

- cyprinus Ktz.

- digito radiata Greg.

- elliptira Eh.

- peregrina Eh.

- puella A. S.

- - pusilla W. Sm.

- Smithii Bréb.

- subsalina Donk.

Nitzschia bilobata W. Sm.

- littoralis Grun.

- navicularis Bréb var.

- punctata W. Sm.
Nitzschia rigìa Per.

- sigma W. Sm.

- Tryblionella Hantz

- $\quad$ - $\quad$ v.salinarum Grun.

- vivax W. Sm.

Pleurosigma hippocampus fa major.

$$
\text { - longum Cl. }
$$

Stauroneis salina W. Sm.

Surirella Brightwellii W. Sm.

- ovata Ktz.

- striatula Turp.

Synedra affinis Ktz.

- cristallina $\mathrm{Ktz}$.

- fulgens W. Sm.

- pulchella Ktz.

Tropidoneis lepidoptera Greg.
Achnanthes brevipes $\mathrm{A} g$.

Amphora mexicana $A$. S.

Epithemia musculus $\bar{K} t z$.

Hantzsehia amphioxys Grun.

Mastogloia angustata Leucis.

Melosira sulcata $\mathrm{Ktz}$.

$$
\text { - } \quad \text { - } \quad \text { - } \quad \text { } \text {. coronata } \mathrm{Gr} \text {. }
$$

Navicula bombus o. gemina A.S.

- brevis Greg.

- lyra Eh.

- - var. elliptica A. S.

- peregrina Eh.

- sculpta T. major Cl.
Navicnla spectabilis Greg.

- subsalina v. major V. H.

Nitzschia panduriformis Greg.

- sigma W. Sm.

- valida $\mathrm{Cl}$.

Pleurosigma angulatum W. Sm.

- balticum W. Sm.

Stauroneis salina W. Sm.

Surirelia striatula Turp.

Synedra affinis $\mathrm{K} t z$.

- crystallina Ktz.

-. undulata Greg.

Trieeratium antediluvianum Elı. 


\section{Nos. 103, 104 FALL MOUNTAIN \\ BRISTOL - CONNECTICUT no I (Etats-Unis) \\ (Sources et ruisseaux à 800 pieds d'altitude)}

Achnanthes lanceolata Bréb.

$$
\text { v. Haynaldii? }
$$

Cymbella gastroides $\mathrm{K} t z$.

Diatoma anceps v. capitata M. Per. à extrémités resserrées et fortement capitées.

- anceps v. linearis M. Per. très ètroite, lineaire à extrémitès atténuées.

- hyemalc: fa curta.

Encyonema ventricosum Grun.

Eunotia arcus $E h$. $v$, minor $V . H$.

- - v. tenella Grun.

- minor Rab.

- pectinalis v. stricta Rab.

Fragilaria oqualis $v$. producta Lag.

Comphonema angustatum $\mathrm{K} t \mathrm{z}$.

- $\quad$ - $\quad$. elongata M. Per. plus long que le type, biconique à extrémités à peine un peu produites. Longueur $60 \mu$.

- angustatum v.producta P.P.

- dichotomum W. Sm.

- micropus $\mathrm{Ktz}$.

- tenellum $\mathrm{Ktz}$.

IIantzechia amphioxys Grun.

$-$

- fa major Grun. vivax $\nabla$. granulata M. Per. très grand, porte à une certaine distance de la caréne une ligne de points analogues aux points carénaux Longueur $250 \mu .13$ striès en $10 \mu$.

Meridion constrictum fa elongata, très long et ètroit. v. Zinkenii Grun.
Navicula acrosphæria v. minor $\mathrm{II}$. Per. et F.H.

- appendiculata $\mathrm{Ktz}$.

- bisulcata Lag.

- Bogotensis $\nabla$. ininterrupta M.P. à striation non interrompue au milieu de la valve.

- Bogotensis $\nabla$. undulata M. Per. diffère de A.S. 44/30 par lés bords triondulés.

Cari Eh. v. angusta Grun. decurrens Eh. dicephala Eh.

- - v. lata M. Per. $\operatorname{larg\theta -}$ ment elliptique, rostrée, capitée et striation du Nav. dicephala.

- divergens W. Sm.

- elegantissima M. Per. Petite, largement ellıplique à extrémités rostrées, capitées; structure formée de granules fines disposées en élégantes lignes courtes décussées comme chez les Orthoneis.

- elliptica Ktz.

- fasciata Lag.

-- gibba Ktz.

- hemiptera Ktz.

- heroina A.S.

- major Kitz.

- parva Eh.

- Smithii v. dilatata M. Per. fortement,dilatée, presque circulaire; aire centrale développèe, côtes fines et isolées, peu distinctem $\mapsto$ nt granulées.

- stomatophora Grun.

- subcapilata $\nabla$. stauroneiformis.

- viridis $\mathrm{Ktz}$. 
Navicula viridis $\mathrm{v}$. commutata Grun

$$
\text { - - } \quad \mathrm{v} \text {. fallax } \mathrm{Cl} \text {. }
$$

Pleurosigma attenuatum W.Sm.

Stauroneis anceps $\mathrm{Eh}$.

$$
\text { - } \quad \text { - } \quad \text {. linearis. }
$$$$
\text { - - v. capitata M. Yer }
$$

Semblableà la variété a mphicephala ma is plus petit, á burds droits et à extrémitès relativement plus larges et plus capitèes .

Long $22 \mu$ largeur $7 \mu$.

Synedra ulna Eh.

$$
\text { - - v. vitræa V.H. }
$$

Tabellaria fenestrata $\mathrm{Ktz}$.

$$
\text { - flocculosa Ktz. }
$$

- $\quad$ - v. ventricosa Grun.

Van Ileurckia vulgaris $\mathrm{Thw}$.

\section{No 105, 106 BUNNEL'S POND \\ BRISTOL - CONNECTICUT (Etats-Unis)}

\section{(Dépôt fossile d'eau douce)}

Amphora Lybica Eh.

$$
\text { - pediculus Grun. }
$$

Cocconeis placentula Eh.

$$
\text { - - - v. lineata V.H. }
$$

Cymbella cuspitata Ktz.

- Ehrenbergiiv. minor V. H.

- gastruides $\mathrm{Ktz}$.

- beteropleura Kiz.

-. producta M. Per. De forme elliptique, à extrémités productes, semblable à celle figuréedans A. S. atlas $9 / 52$ mais plus grande. à aire centrale plus dèveloppèe et stries distinctement divisées en travers Long $74 \mu ; 7$ stries dorsales, 8,5 ventrales en $10 \mu$.

Encyonema ventricosum Kítz.

Epithemia turgida $k t \tilde{t}$.

- Zebrakitz.

Eunotia arcus Eh.

- $\quad$ - v. plicata J. B. et F. H.

- diodon Eh.

- $\quad$ fa minor Grun.

- formica Eh.

- gracilis o. nodosa? les extrémitès sont rondes, différentes de celles de $E$. formica.

- incisa Greg.

- lunaris Grun.
Ennotia monodon Eh.

- pectinalss v. rentricosa Grun.

- prærupta Eh.

- robusta v, tetraodon V.H.

Fragilaria Harrissonii Grun.

- virescens Ralfs.

Gomphonema acuminatum Eh.

- angustatum Kitz.

- augur Eh.

- commutatum Grun.

- constrictum v. capitata Eh.

- - - v. subcapitata Els.

- Herculaneum Eh.

- micropus Ktz.

Mastogloia Smithii Thwo.var.

Meridion constrictum Ralfs.

Navicula acrosphæria Ktz.

- - var. dilatata M. Per, à centre très fortement dilaté en forme de cercle et à extrém!tés capitées.

- affinis v. amphirynchus Eh.

- - fa maxima.

- americana $\nabla$. bacillaris M. P. et F. H.

- amphigomphus Eh.

- bisulcata Lag.

- Bogotensis Grun. var

- brevicostata Eh.

- commutata Grun. 
Navicula dactylus $f_{a}$ curta Ktz.

- Dariana A.S.

- divergens W.Sm.

- $\quad$ - $\quad$. bacillaris M. Per. fout à fait bacillaire à extrèmités exactement hémicirculalles. - $\quad{\text { à partie ventrale resserrée } e^{t}}$ v. constricta M. Per à extrémitès atténuées arrondies.

- elegantissima M. Per.

- fasciata Lag.

- gentilis Donk.

- gibba El.

- Ilitehcockii Eh.

- iridis Eh.

- legumen Eh.

- lindosa Ktz.

- linearis Greg.

- major $\mathrm{Ktz}$.

- mesolepta v. stauroneiformis Grun.

- mesostyla Ktz.

- nobilis Eh.

- rhyncocephala Ktz.

- rupestris Hantz.

- transversa A.S.

- viridis $\mathrm{Ktz}$.

- sp. A. S. atlas $44 / 44$.

- sp. A. S. atlas $49 / 40$. (N. producta v. acuta.)

Nitzschia spectabilis Ralfs.

Stauronois acuta W. Sur.
Stauroneis anceps Eh.

- - - v. gracilis Eh.

- gracilis Eh.

- lanceolata Ktz.

- Phonicenteron Eh.

- Pteroidea Eh.

Stephanodiscus astrae Grun.

Surirella Guatematensis Eh. $=\mathrm{Sm}$ Curdinalis Kitt.

- Kittoni ? A. S. v. elliptica.

- linearis W.Sm.

- - v. constricta Grun.

- pseuro cruciata M. Per., de structure tout à fait semblable à celle de $\mathrm{A}$. S. 56/15, 16 mais ne présentant pas le renforcement de la partie centrale.

- ^ spléndida Eh.

- tenera Greg.

- - v. nerrosa A. S.

- $\quad$ - . splendidula A. S.

- valida A. S.

Synedra splendens Ktz.

- ulna Eh.

- - v. lanceolata fa brevis Grun

- v. undulata Greg.

Tabellaria fenestrata Ktz.

- flocculosa Ktz.

_ - - v. ventricosa Grun.

Van Heurckia rhomboides Brèb.

roïdes Grun.

v. amphipleu-

vulgaris Thw.

\section{N*6 107, 108 TACOMA - WASHINGTON (Etats-Unis)}

(Vase du réservoir de la Ville)

Achnanthes lanceolata v. Haynaldii Sclum. Amphiprora ornata Bail.

Amphora ovalís $\mathrm{Ktz}$. var.

Campylodiscus costatus W. Sm.
Cocconeis placentula Eh.

$$
\text { - - v. lineata V.H. }
$$

Cymatopleura apiculata W. Sm.

$$
\text { - solea Brèb. }
$$


Cymbella anglica Lag.

- cistula Hemp.

- cuspirata Ktz.

- Ehrenbergii Ktz.

- gastroides $\mathrm{Ktz}$.

- gibba Ktz.

- heteropleura Ralfs.

- mexicana Eh.

- producta M. Per.

Diatoma hiemalo Heib.

Encyonensa cœspitosum Ktz. - ventricosum Grun.

Lipithemia gibba $K t z$.

- $\quad$ - v. ventricosa Grun.

- Hyndmanii W. Sm.

- - v. perlonga Pant.

- sorex $\mathrm{Ktz}$.

- turgida v. granulata Grun.

- zebra Ktz.

Eunotla minor Rab.

- monodon Eh.

- proerupta v. bidens Grun.

- ternaria Eh.

\section{Fragilaria Ilarrisonii Grun.}

- lancettula Schum.

- marina v. parva iOpephora. Septroneis). On observe toules les formes intermédiaires entre ces trois espèces.

Gomphonema acuminatum Eh.

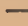

$-$

-

$-$

$\overline{-}$

$-$ constrictum Eh.

- v. capitata Eh.

- v. subcapitata Eh. Herculaneum Eh.

- v. robusta Grun. montanumv.suecica Grun. ventricosum Greg.

IIantzschia amphioxys v. elongata Grun.

Melosira crenulata $\mathrm{K} t z$.

- undulata Ktz.

Meridion constrictum Ralfs.

Navicula acrosphæria $\mathrm{Ktz}$. fa parva.

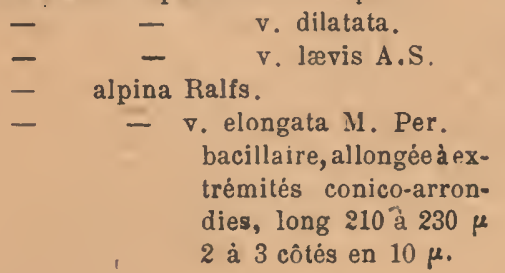

Navicula amphirynchus Eh.

- anglira Rab.

- antinitescens M. Per, intermédiaire entre $N$. Smilhii et $N$. fusca, mais, à l'inverse $d u$ $N$. nitescens, elle porte une seule 1 angée de grosses perles entre deux côles extérieurement au sillon et deux rangées de fins granules extérieurement au sillon. - très largement elliptique.

- cryptocephala Ktz.

- dilatata Eh.

- divergens $v$. bacillaris.

- elliptica Ktz.

- gentilis Donk.

- gigas Eh.

- Hitchcockii Eh.

- humilis Donk.

- iridis v. ampliata Eh.

- limosa Ktz.

- - v. gibberula Ktz.

- Ludlowiana A.S.

$\rightarrow \quad$ major Ktz.

- megaloptera Eh.

- nobilis Eh.

- pachyptera Eh.

- peregrina $\nabla$. truncata M. Per. peulancéolée, extrémités larges et tronquées; intermédiaire entrele type etle N. Bernhardtii.

- pseudo bacillum Grun.

- pupula Ktz.

- radiosa Ktz.

- Reinhardtii Grun.

- transversa A.S.

- trinodis Lewis.

- viridis $\mathrm{K} \mathrm{l}$.

Nitzschia linearis W. Sm.

Rhoicophenia curvata Grun.

Stauroneis acuta W. Sm.

- gracilis Eh.

- phænicenteron Eh. var.

- pteroidea Eh.

Surirella bifrons Eh.

- biseriata Brèb.

- elegans Eh. fa minor.

- elliptica Terry.

$=$ S. regina Jan. A.S. atlas. 21/5. 
Surirella Kittonii A.S.

$\begin{array}{cc}- & -\quad \text { v. asperula M. Per. } \\ \text { Intermèdiaire entre } \\ \text { le } S \text { Kittonii et le } \\ \text { S. bifrons, possède } \\ \text { un areacentral et des } \\ \text { côtes épineuses. } \\ \text { - } \quad \text { linearis W. Sm. } \\ \text { - } \quad \text { v. constricta Grun. } \\ \text { - Mölleriana Grun. } \\ \text { - } \quad \text { robusta Eh. } \\ \text { - } \quad \text { splendida Ktz. } \\ \text { - tenera Greg. } \\ \text { - } \quad \text { v. nervosa A.S. }\end{array}$

Surirella tenera v. robusta.

valida Eh.

$$
\begin{aligned}
& \text { - } \quad \text { - v.erosa (A.S. atlas 22/4). } \\
& \text { - } \quad \text { v.triumphanus (A.S). } \\
& \text { atlas 206/20). }
\end{aligned}
$$

Synedra Goulardi Grun.

- ulna Eh.

- $\quad$ - v. æqualis Rab.

- $\quad$ - v. danica V.H.

- - v. lanceolata Grun.

Tabellaria fenestrata $K t z$.

Van Ileurckia rhomboides Brèb.

- amphipleuroides Grun

BIBLIOGRAPHIE. - Kitton in monthly micro. journ. XIV 1875. - Cleve et Mïller préparation $n^{\circ} 164$. - Ad. Schmidt Atlas.

Actinocyclus alienus v, californica Grun.

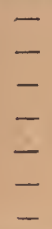
confluens Grec. Lhrenbergii $\mathrm{ii}^{\prime} \mathrm{v}$. intermedia.

- $\quad$ Easciculatus axt.

- incertus Grun.

- moniliformis Ralfs.

- Ralfsii v. monicæ Grun.

- subtilis Ralfs.

Actinoptychus areolatus Eh.

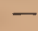

$-$

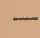

$-$

$-$

$-$

$-$

$-$ Bismarckii A. S. glabratus Grun. - v. angelorum Grundleri A. S. spiniferus Grun. splendens $\nabla$. californica. undulatus Eh.

- fa maxima. vulgaris v. monicæ Grun.

Annellus californicus Temp. n. g. et n. sp. Valve à silice replite sur ellemême sous forme d'anneau tubulaire re. couvert de grosses ponctuations régulièrement disposées. Diamètre de la valve : 70 à $80 \mu$; épaisseur de l'anneau : 10 à $15 \mu$; largeur : 30 à $40 \mu$. Pas rare dans le $n^{\circ} 112$.

Arachnoidiscus Ehrenbergii $\nabla$.. Monteryana.

- ornatus v. Monteryana.

Asterolampra variabilis Grev.

Asteromphalus Brookei Bail.
-
Darwinii Eh.
$-$
Moronensis Ratt.
-
Wallichianus Ralfs.

Aulacodiscus affinis Grun.

- Brownei Norm.

- decorus Grev.

- Kinkerii A $S$.

- Kittonii Arnott.

- inflatus Greo.

- margaritaceus Ralf. 
Aulacodiscus margaritaceus v. robusta.

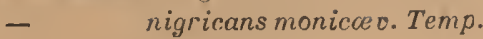

- pulcher Norm.

- Thumii A. S.

Auliscus antiquus Ratt.

- Biddulphia Kitt.

- cœla!us v. major.

- Grunowii A. S. v. californica.

- pruinosus v. subreticulata Grev.

- punctatus Bail.

-, textilis A. S.

Isicldulphia aurita Bréb.

- longispina Grun.

- Tuomeyi var.

Campylotliscus coronilla J. $\mathrm{Br}$.

- ecclesianus Greo.

Chatoceros californicum Grun.

$\begin{array}{ll}\text { - } & \text { distans } \mathrm{Cl} . \\ \text { - } & \text { incurvum Bail. } \\ \text { monicre Grun. }\end{array}$

Cladogramma californicum Eh.

Climacosphenia moniligera $\mathrm{Eh}$.

Cocconeis dirupta Grég. var.

- $\quad$ Kinkeri A.S.
- $\quad$ Montereyi Grun.
- $\quad$ oculus catis J. Br.
- $\quad$ praestans A. S.
- $\quad$ regalis var. Grev.
superba Jan.

Coscinodiscus anastomosans Grun.

$\begin{array}{ll}- & \text { apiculatus Eh. } \\ - & -\quad \text { v. monicæ } \\ - & \text { argus Grun. } \\ - & \text { asteromphalus Eh. } \\ - & - \text { v. eximia Grun. } \\ - & - \text { v. Brightwelloïds } \\ - & \text { v. pulchra Grun. } \\ - & \text { biradiatus Greo. } \\ - & \text { blandus A. S. } \\ - & \text { Bolioiensis Grun. } \\ - & \text { borealis Bailey. } \\ - & \text { centralis Eh. } \\ - & \text { concavus Greg. var. } \\ - & \text { curvatulus Grun. } \\ - & \text { depressus Grun. } \\ - & \text { diorama Grun. } \\ - & \text { elegans Grev. var. } \\ - & \text { excentricus Ih. } \\ - & \text { floridulus } A . S .\end{array}$

Coseinodiscus gigas Eh. griseus Grev. implicatus Ratt. impressus Grun. incretus $A$. $S$. lineatus $\mathrm{Eh}$.

\section{- v. minor} marginatus Eh. monirce Ratt. obscurus A. S. oculus iridis Eh. pilosus A.S. radiatus Eh. robustus Grev. subtilis Eh. symbolophorus Grun. tuberculatus v. monicw

Craspedolliscus elegans Eh.

Ditylium undulatum Eh.

Endictya oceanica Eh.

Entopyla australis v. gigantea Grun.

Vuotlia gibba Bail.

Eupodiscuscalifornicus fa bioculata Grun.

Glyphotiscus stellatus (irun.

Goniothecium odontella Grun.

Grammatophora robusta Eh.

IIemiaulus februatus Heib. var.

Leudugeria epithemiö̈es Temp.

Lithodesmium minusculum Grun.

Melosira clavigera Grun.

- polaris Grun.

- sol Eh.

- sulcata Kı́z.

Navicula aspera Eh.

- bombus Eh.

- crabro Eh. var.

- excávata Grev. var.

- Eudoxia A. S. car.

- formosa Grez.

- incuroata Greg.

- lyra Eh.

- navigans J. Br.

- nitescens Greg.

- ornata o. spirifera A. S.

- pedalis $J B r$.

- Smithii Bréb.

- cagabunda J. Br.

Orthoneis splendida Grun. 
Plagiogramma Nancoorense Grun.

Podosira variegata $A . S$.

Polymyxus pulchellus Grun.

Porpeia quadrata Greo.

- quadriceps Bail.

Pterotheca subulata Grun.

Pyxilla americana Grun.

Rutilaria epsilon o. longicornis $\mathrm{Tp}$. et $\mathrm{Br}$.

- hexagonus Grer.

- pulchraA.S.

Stephanogonia actinoptychus Eh.

Stephanopyxis corona Eh.

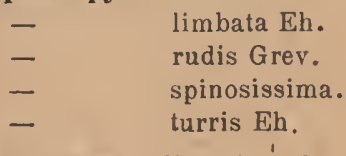

Stictodiscus californicu's Grun.
Stictodiscus Hardmanianus Grun.

$$
\text { - fa minor. }
$$

Triceratium arcticum v. californica fa tetragona.

- californicum Grun.

- consimile Grun.

- elegans Grev. v. major.

- _ fa pusilla.

- favus Eh.

- heteroporum Grun.

- inelegans Greo.

- $\quad$ - o.yucatensis Grun.

- Montereyi Bright.

- obliquum Grun.

- quadrangulare Grev.

- receptum A. S.

- strabo A. S. fragments.

- validum Grun.

Zygoceros circinus Bailey.

\section{No 113 SAINT-NECTAIRE - PUY-DE-DOME (France)}

(Source minérale)

\section{BIBLIOGRAPHIE. - Frère J. Héribaud. Les Diatomées d'Auver gne 1893}

Achnanthes lanceolata Grun.

- minutissima Ktz.

Amphora acutiuscula $K t z$.

- affinis v. Ergadensis Greg.

- ovalis $\mathrm{Ktz}$.

- pediculas Grun.

- $\operatorname{salina} W . S m$.

Cocconeis placentula Eh.

Cymatopleura elliptica W. Sm.

- solea Bréb.

Cymbella parva W. Sm.

Denticula elegans $\mathrm{Ktz}$.

$$
\text { - } \quad \text { - } \quad \text {. thermalis } \mathrm{K} t z \text {. }
$$

Encyonema lunula Grun.

- prostratum Ralfs.
Epithemia gibba v. parallela Grun.

- gibberula Eh.

- $\quad$ - v. producta Grun.

- sorex Ktz.

- zebra Grun.

Fragilaria construens v'. pumila Grun.

Gomphonema micropus v. minor Grun.

- montanum Schum.

- - - v. pumila Grun.

Melosira distans $\nabla$. alpigena Grun.

- Rœseana Moor.

- tenuis Grun.

Navicula amphisbcna Bory.

- appendiculata $\mathrm{K} \mathrm{z}$.

- - $\quad$ v. irrorata Giun. 
Navicula cincta Ktz.

- gracilis Eh.

- hemiptera Ktz.

- Heutleri Grun.

- loevissima Grun.

- producta Ehr.

- radiosa $\nabla$. acuta Grun.

- viridis $\mathrm{Ktz}$.

Vitzschia acuminata Grun.

- Brebissonii W. Sm.

- commutata Grun.

- frustulum Grun.
Nitzschia microcephala Grun.

- vitrea Norm.

- - v. gallica J. Br.

Rhoicosphenia curvata Grun.

Surirella elegans Ehr.

- ovalis Bréb.

- patula Ehr.

- splendida Ehr.

- tenera Greg.

Synedra ulna Eh.

- - $\quad$ v. vitrea Ktz. gracilis Grun.

\section{No 114 LA BOURBOULE - PUY.DE-DOME (France)}

(Dépôt fossile d'eau douce)

BIBliogra PhiE. - Frère J. Héribaud. Les Diatomées d'Auvergue 1902.

Cocconeis placentula Eh.

$$
\text { - lineata Grun. }
$$

Cyclotella Temperei M.P. et F.II.

Cymbella lanceolata Kirch.

Epithemia Hyndmanni Ktz.

- sorex $\mathrm{Ktz}$.

- turgida $\nabla$. vertagus.

- Westermanni Ktz.
Fragilaria elliptica Schum.

Melosira arenaria Moor.

- granulata Eh.

- varennarum M.P et F.II.

Navicula crassinervia Bréb.

- major $\mathrm{Ktz}$.

- oblonga Ktz.

- peregrina Ehr.

Rhoicosphenia curvata Grun.

No 115 MONTEREY - CALIFORNIE (Etats-Unis)

(Sur le sable)

Aulacodiscus Kittonil Arnott

No 116 CONSTANTINE - ALGÉRIE

(Sur les algues tapissant une rigole d'écoulement des bains) de Sidi M' Sid 


\section{$N^{\text {os }}$ 117-119 PORT TOWVEND - WASHINGTON (Etats-Unis)}

\section{(Sonda.ge)}

Achnanthes brevipes $\mathrm{Ag}$.

Actinocyclus americanus M. Per.

- $\quad$ nebulosus M. Per.
- $\quad$ tenellus Breb.

Actinoptychus areolatus Eh.

$$
\begin{array}{ll}
\text { - } & \text { undulatus Eh. } \\
\text { - } & \text { vulgaris Shum. }
\end{array}
$$

Amphora acuta Greg. var.

- angusta Greg v. ventricosa.

- arcuata A.S. var.

- costata W. Sm. var. inflata?

- mexicana A.S.

- obtusa Greg.

- ostrearia Bréb. $\nabla$. granulata.

- ovalis Ktz.

- proteus Greg. v. oculata.

Arachnoidiscus Elırenbergii v. californica.

$$
\text { - indicus Eh. var. }
$$

Aulacodiscus oregonus Bail.

$$
\text { - } \quad \text { sp. A.S. atlas } 133 / 7
$$

Auliseus cœlatus v. Aucklandica Grun.

- - var. gigas W. et Ch.

- - var. latecostata A.S.

- rhipis A.S.

- sp. (auxospore de A. cœlatus?)

Biddulphia aurita Brèb.

- granulata Roper.

- obtusa Rilfs.

- rotunda M. Per.

Cocconeis antiqua $\mathrm{J} . \mathrm{Br}$.

- Britannica Næg. et var.

- Baldjickiana Grun.

- costata v. pacilica Grun.

- dirupta v. flexella Jan.

- $\quad$ - vo californica $\mathrm{Cl}$.

- maxima H. Per.

- pellucida Haniz.

- scutellum Eh.
Cocconeis sigmoradians $J . B r$.

- versicolor J. Br. var.

Cerataulus turgidus Eh.

Coscinodiseus asteromphalus var. hybrida.

$-$

- denarius A.S.

- Biorama A.S. var.

intermediaire avec $C$ fulgularis.

- excentricus Eh.

- lineatus'Eh. var.

- oculus iridis Eh.

Cyclotella striata Grun.

- Transylvanica Pant.var.

Dicladia capreolus $E h$.

Emlictya oceanica Eh.

Fpithenia turgida Kitz.

- Westermannii Ḱlz.

Grammatophora robusta El.

Ilyalodiscus subtilis Bail.

Isthmia nervosa $\mathrm{Ktz}$.

Nelosira sol Eh.

- sulcata Kiz.

- - var. coronata Grun.

Navicula Baileyana A.S. var.

- brevis Greg. v. elliptica.

- bohemica Eh.

- bombus Eh.

- clavata Greg.

- elliptica Kitz.

- Hennedyi v. californica Cl.

- humerosa Breb.

- longa Grev.

- Lyra Eh.

- - v. dilatata A.S.

- $\quad$ - V. elliptica A.S.

- pennata A.S.

, - riombica Greg.

- Smithii Bréb.

- spectabilis Greg.

- transversa A.S. 
Vitzschla insignis Greg. $\nabla$. mediterranea. pennata Temp. et Br.

- plana W. Sm. var.

Plagiogramma pulchellum Grev.

Pleurosigma affine Grun.

- formosum $\nabla$. longissima.

- longum $\mathrm{Cl}$.

Podosira hormoides Mont.

Pyxilla baltica Grun.

Rhablonema arcuatum Ktz.

- biquadratum $J . B r$.

Rhoicosphenia curvata v. marina. Grun.

Scoliopleura tumida Bréb.

skeletonema mediterraneum Grun.
Stephanodiscus astræa $\nabla$. spinulosa.

fa maxima

Syndendrium diadema Eh.

Surirella americana Terry (roir n• 119).

- var. intermédiaire au

S. Comis A.S.

Trachyneis aspera Eh.

- - var. intermedia Cl.

- - var, pulchella Cl.

Triceratium antediluvianum $\mathrm{Eh}$, var.

- $\quad$ arcticum Bright.

- - v. quadrata.

alternans Bail.

Montereyi Bright.

\section{No 120 PORT TOWSEND - WASHINGTON (Etats-Unis)}

\section{(Sur les algues $[\mathrm{N} \cdot 1]$ )}

\section{Achnanthes longipes Ag.}

Actinoeyclus americanus M. Per. $\mathrm{n}$. sp. (subtilis var. ?). Intermediaire entre A. Barkleyi et $\mathrm{A}$. subtilis, dont il a la structure, mais plus distinctement fasciculé et à granules plus gros; 12 en $10 \mu$ au milieu; 16 en $10 \mu$ dans la zone submarginale qui la sépare difficilement du reste de la valve.

Actinoptychus undulatus Eh.

Ampbora Graeffei Grun fa minor.

latecingulata M. Per. n- sp

Differe de l'A. cingulata par sa forme plus bacillaire, à extrẻmités tronquées arrondies, et son stauros très large. Longueur: 100 à $115 \mu$; largeur ; $14 \mu$, cello du stauros ; 5 \%.

Amphora bistriata Per.

- cingulata $\mathrm{Cl}$.

- mexicana A. S. fa minor.

- obtusa Greg.

- - - v. crassa n. v. très largo relativement à sa longueur.
Longueur : $65 \mu$; largeur : $20 \mu$.

Amphora ostrearia Bréb. var.

- granúlata n. var. à stries écar.

tées ot fortement granulèes;

16 stries en $10 \mu$.

- proteus Greg. v. oculata Per.

Auliscus rhipis A. S.

Biddulphia rotunda M. Per. (Roperiana var?) à contour presque circulaire (voir no 121).

- sp. A. S. $120 / 25$ var.

Cerataulus turgidus Eh.

Cocconeis Baldjickiana Grun.

- costata Grev.

- pellucida Hantz.

- sp. nov.

Coscinodiscus asteromphalus Eh.

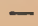

$$
\begin{aligned}
& \text { eximia } \\
& \text { excentricus } \mathrm{Eh} \text {. }
\end{aligned}
$$$$
\text { sp.? }
$$ 
Ilantzsohia virgata Grun.

Melosira sulcata Ktz.

Navicula Báileyana A. S. var.

- cancellata Donk.

- crucæformis Donk var.

- digito-radiata Greg.

- interrupta Ktz.

- lineata o. contracta Donk.

- Juxuriosa Grev. var. abrupta

M. Per. Semblable à la var. cuneata J. Br. (Esp. nouv. $189116 / 3$ ), mais ayant la partie centrale surélevée et 4 lignes de granules seulement sur cette partie.

- Lyra Eh.

- $\quad$ - v. dilatata A. S.

- $\quad$ - o. densestriata Per.

- - var?

- mediterranea $\mathrm{Cl}$. et $\mathrm{Br}$.
Navicula pennata A. S.

- rhombica Greg. var.

- Smithii Bréb.

- Stuxbergii Cl.

Nitzschia acuminata W. Sm.

- Tryblionella Grun. oar.

Plagiogramma pulcliellum.

Pleurosigma balticum Sm. var.

$$
\text { - Olympianum Terry. }
$$

Rhablonema arcuatum Kitc.

Scoliotropis latestriata Bréb.

Stauroneis salina W. Sm.

Surirella hybrida var.

Trachyneis aspera Eh.

$$
\text { - } \quad \text { - v. pulchella Cl. }
$$

Tropidoneis lepidoptera $\nabla$. proboscidea.

Xanthiopyxis hirsuta Eh?

\section{No 121 PORT TOWSEND - WASHINGTON (Etats-Unis)}

\section{(Sur les algues [No 2] )}

Actinocyclus americanus $\mathrm{M}$. Per.

Actinoptychus undulatus Eh.

Amphora arcuata A.S.

- arenaria Donk.

- bistriata Per.

- obtusa Greg.

- ostrearia var. granulata Bréb.

A uliscus rhipis A. S.

Biddulphia rotunda M. Per. (Roperiana var?) Intermédiaire en tre B. Roperiana et B. obtusa, comme granulation 8 à 9 lignes de granulos en $10 \mu$. Face valvaire à contours presque circulaires. Ocelles larges et étroits et 2 ou 3 èpines diagonales à environ moitié du rayon, face connective montrant une valve presque plate a appendices peu prononcés.

Cocconeis Baldjickiana Grun.

Coscinodiscus asteromphalus $k h$. $v$. hybrida.

llantzschia amphioxys Grun. v. major.

Navicula brevis Greg.

- lyra $\nabla$. dilatata.

- mediterranea $\mathrm{Cl}$. et $\mathrm{Br}$

- pennata A.S.

Vitzschia sigma W. Sm.

- socialis Greg.

Pleurosigma xstuarii W. Sm.

- balticum W. Sm.

Rhabdonema arcuatum Ktz.

Scoliotropis latestriata. Bréb.

Trachyneis aspera $\mathrm{Eh}$.

- $\quad$ - v. pulchella Cil.

Tropidoneis lepidoptera Grun. 

d'Auvergne 1902.

Actinella pliocenica F.H et M.P.

Amphora affinis $K t z$.

Asterionella antiqua F.H. et M.P.

Campylodiscus costatus W. Sm.

Cocconeis lineata $\nabla$. euglypta Grun.

Cyclotella iris J. Br. et F.H.

- $\quad$ - v, integra M.P. et F.II.

Cymatoploura elliptica W. Sm.

- v. constricta Grun.

Cymbella aspera Eh. solea W. Sm?

- cymbiformis Ehr.

- scotica W. Sm.

Encyonema Girodi F.H.

Epithemia argus Ktz. v. amphicephala

- gibba Ehr.

- Hyndmannii W. Sm.

- $\quad$ sorex $\mathrm{Ktz}$.

- turgida $\mathrm{Ktz}$.

- zebra Kítz.

Eunotia impressa Ehr.v. angusta Grun.

- pectinalis v. stricta Rab.

- polyglyphis Ehr.

Fragilaria brevistriata Grun.

$$
\text { - intermedia Grun. }
$$

Gomphonena acuminatum Eh. biventralis $F . H$. et M.P. geminatum $\mathrm{A} g$. insigne $v$. acuminata $M . P$. et $F . H$. intricatum $K t z$. subclavatum Grun.

- v. major F.H. et M.P.
Melosira granulata Eh.

- - $\quad$ - arcuata F.H.

- spiralis $\mathrm{Ktz}$.

$$
\begin{aligned}
& \text { - } \quad \text { - v. homispharia } \\
& \text { M.P et F.II. } \\
& \text { - v. spharica F.H. } \\
& \text { et M.P. }
\end{aligned}
$$

Navicula acrospharia v. lavis Breb.

- Cellesensis F.H. et M.P.

- elliptica Ktz.

- Gomontiana F.H.

- limosa o. gibberula Grun.

- major Ktz.

- nobilis Ehr.

- Pagesi F.H.

- rhomboides Ehr.

- rupestris $\mathrm{Ktz}$.

- transversa A.S.

- viridis $K t z$.

Nitzschia tabellaria Grun.

- sigmoidea Nitz.

Pleurosigma attenuatum $K t z$.

Synedra capitata Ehr.

- ulna Ehr.

Surirella norvegica Ehr.

- robusta.

Tabellaria fenestrata Ktz.

Tetracyclus costellatus.

- elegans Eh.

- $\quad$ - v. eximia F.H. et M.P.

- emarginatus Ehr.

- $\quad$ - v. crassa F.H. et M.P.

- Pagesi F.H. stella Ehr. 


\section{SAINT-MALO (France)}

(Sur la vase)

Achnanthes brevipes Ag.

- longipes Ag.

- $\quad$ subsessilis Eb.

Actinocyclus subtilis Ralfs.

Actinoptychus undulatus Eh.

Amphiprora alata W. Sm.

- elegans W. Sm.

Amphora commutata Grun.

Bacillaria paradoxa Gmel.

Biddulphla aurita Brẻb.

- mobiliensis Bailey.

Campylodiscus ecclesianus var Greg.

Thuretii Bréb.

Cerataulus Smithii Ralfs.

Cocconeis dirupta Greg.

- psoudo-marginata Grun.

- scutellum Eh.

Coscinodiscns excentricus Eh.

Cyclotella Meneghiniana Ktz.

Hantzschia amphioxys Ktz.

- marina Donk.

Hyalodiscus scoticus Grun.

- stelliger Bailey.

Licmophora flabellata Ag.

Mastogloia lacustris Grun.

Melosira nummuloides $\mathrm{Ag}$.

- sulcata Eh; petite variété se rapprochant de $\mathbf{M}$. siberica Grun.

Navicula amphisboena Bory.

- aspera Eh.

- crabro Eh. ४. minor.

- cyprinus W. Sm.
Navicula elegans W. Sm.

- elliptica Ktz.

- humerosa Bréb.

- peregrina Eh.

Nitzschia acuminata Grun.

- sigma.

- spathulata Bréb.

Orthoneis binotata Roper.

Plagiotropis Van Heurckii Grun.

Pleurosigma æstuarii W. Sm.

- angulatum W. Sm.

- balticum W. Sm.

- fasciola W. Sm.

- hippocempus W. Sm.

- littoralo W. Sm.

- quadratum W. Sm.

- Spencerii W. Sm.

- strigosum W. Sm.

- Wansbeckii Donk.

Podocystis adriaticum Ktz.

Podosira Montagnei Ktz.

Rhabdonema adriaticum Ktz. arcuatum Ktz.

Raphoneis amphiceros Eh.

Schizonema Grevillei Ag.

Scoliopleura latestriata Grun.

$$
\text { - tumida Grun. }
$$

Surirella gemma Eh.

Synedra affinis $\mathrm{K} t z$ :

- fasciculata Ktz.

- pulchella $\mathrm{Ktz}$.

- undulata Greg.

Triceratium antediluvianum (Eh) V.H. 
Chretoceros cellulosum Laud.

- convolutum Castr.

- curvisetum $\mathrm{Cl}$.

- debilis Cl.

- decipienś Cil.

- distans.Cl.

- laciniosum Schütt.

- procerum Schütt var.

- vermiculus Schūtt.
Leptocylindrus danicus $C l$.

vitzschia seriata var. fraudulenta $\mathrm{Cl}$.

\section{- lineola Cl.}

Rhizosolenia alata Bright.

- Shrubsolii Cl.

Skeletonema costatum $\mathrm{Cl}$.

Thalassiosira Nordenskioldii Cl.

Thalassiothrix Nitzschioides Grun.

No 125

HONFLEUR - CALVADOS (France)

(Sur le sable)

Navicula minima Grun

Nor 126 a 129 SZAKAL - HONGRIE

(Dépôt fossile tertiaire marin méditerranéen;

Bibliographie. - J. Pantocsek. Diatomées de Hongrie, vol. I et 11 .

Actinocyclus circumdatus Pant.

- Ehrenbergii Ralfs var. minuta Pant. subcrassus Ratt.

Actinoptychus amblyoceros A. S.

- bifrons A. S.

- bolioiensis Jan.

- kymatoides Pant.
Actinoptychus moronensis $\mathrm{Cl}$.

$\begin{array}{ll}\text { - } & \text { neogradensis Pant. } \\ \text { - } & \text { reticulatus Pant. } \\ & \text { splendens v. glabrata } \\ \text { Grun. } \\ \text { - } & \text { splendens v. halionyx Grun. } \\ \text { - } & \text { stella A. S. } \\ & \text { Truanii A. S. }\end{array}$


Actinoptyehus undulatus Eh. oulgaris Schum.

Arachnoïliscus Ehrenbergii Bail.

- v. indica Grun.

Asterolanpra marylandica $E h$.

Aulacodiscus Grunowii $\mathrm{Cl}$.

\section{- o. subsquamosa} Pant.

Auliscus confluens $A . S$.

- Hauckti Pant.

- pulvinatus Cl.

- sculptus Ralfs.

Biddulphia elegantula Grev.

- regina Greg.

- Tuomeyi Bail.

Campylodiscus Neogradensis Pant:

$$
\text { - } \quad \text { Szakalensis Pant. }
$$

Cerataulus Johnsonianus Grev.

- Weissflogii Pant.

Chretoceros affinis Laud.

$$
\text { - gastridium Eh. }
$$

Clavicula Szakalensis Pant.

Cocconeis sigma Pant.

$$
\text { - vitrea } J . B r \text {. }
$$

Coscinodiscus actinocycloides Pant.

$\begin{array}{ll}\text { - } & \text { apiculatus Eh. } \\ \text { - } & \text { asteromphalus Eh. } \\ \text { - } & \text { clivosus Pant. } \\ \text { - } & \text { elegans Grev. } \\ \text { - } & \text { hungaricus Pant. } \\ - & \text { intumescens Pant. } \\ - & \text { leptopus Grun. } \\ - & \text { lineatus Eh. } \\ - & \text { marginatus Eh. } \\ \text { - } & \text { neogradensis Pant. } \\ \text { - } & \text { nitidus Greg. } \\ - & \text { oculus iridis Eh. } \\ \text { - } & \text { radiatus Eh. } \\ \text { - } & \text { robustus Grev. } \\ \text { - } & \text { symbolophorus Grun. } \\ \text { - } & \text { undatus Grun. }\end{array}$

Craspedodiscus Weissfogii Pant.

Craspedoporus Truanii Pant.

Cyclotella Szakalensis Grun.

Endyctia oceanica A.S.

Entopyla australis Eh.
Leudugeria epithemioides Temp.

Goniothecium $S$ zalialense Pant.

Grammatophora maxima Grun. robusta Dippel.

Hemiaulus hungaricus Pant.

$$
\text { - petasiformis Pant. }
$$

Iyalodiscus radiatus Grun.

Isthmia Szaboi Pant.

Mastogonia crux Eh.

Melosira biharensis Pant.

- cincta Pant.

- Peragalloi Pant.

- sol. Ktz.

- sulcata Ktz.

Navicula crabro Eh.

- inhalata A.S.

- lyra Eh.

- milerostatos Pant.

- prætexta Eh.

- Sandriana Grun.

- spectabilis Greg.

Vitschia antediluoiana $f a$ interrupta Pant.

Odontella neogradensis Pant.

Odontotropis vitrea Pant.

Orthoneis splendida Grun.

Pantocsekia clioosa Grun.

Periptera tetracladia Eh.

Pyxidicula cruciata Eh.

Pyxilla americana Grun.

- cornuta Pant.

- vasta Pant.

Raphoneis amphiceros Grun.

- gemmifera Eh.

- hungarica Pant.

- Szakalensis Pant.

Rhabdonema adriaticum $\mathrm{Ktz}$.

Rutilaria ventricose Grun.

Skeletonema hungaricum Pant.

Stephanogonia actinoptychus Grun.

$$
\text { - polygona Eh. }
$$

Stephanopyxis corona Grun.

- turris Eh.

Stictodiseus californicus Greo.

Surirella Baldjikii Norm.

Synclendrium Diadema Eh. 
Triceratinm favus Eh.

$\begin{array}{ll}\text { - } & \text { Grocei Pant. } \\ \text { - } & \text { Pantocsekii A. S. } \\ \text { - } & \text { radiato-punctatum A.S. } \\ \text { - } & \text { Stoksianum Grev. } \\ \text { - } & \text { Szakii Pant. } \\ & \end{array}$

Triceratium Thumii $A . S$. trisulcum Bail.

Xanthiopyxis oblonga $E h$. panduroformis Pant.

Zygoceros circinus Bail. - Weissflogii Pant.

\section{No 130 VILLEFRANCHE - ALPES-MARITIMES (France)}

(Sondage fait à $400 \mathrm{~m}$. entre Nice et Villefranche)

BIBLIOGRA PHIE. - Les Diatomées marines de France par MM. H. et M. Peragallo.

Actinoptychus adriaticus car. balearica Grun.

Auliscus colatus Bail.

- sculptus Ralfs.

- splendidus Ratt.

Amphiprora lepidoptera Greg.

$$
\text { - elegans W. Sm. }
$$

\section{Biddulphia pulchella Greg.}

$$
\text { - regina W. Sm. }
$$

Campylodiscus adriaticus Grun.

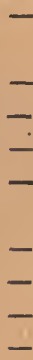

- v. massiliensis Grun.

decorus Bréb.

- eximius Grev.

- , fluminensis Grun.

- horologium W. Sm. var. mediterranea Grun.

- limbatus Breb.

- $\quad$ samoensis Grun.

- Thuretii Brèb.

- - var. simulans. Greg.

Cerataulus polymorphus Grun.

Climacosphenia elongata Bail.

Coscinodiscus radiatus Eh.

$$
\text { - nodulifer A.S. }
$$

Endietya oceanica $E h$.
Mastogloia reticulata Grun.

Navicula aspera $\mathrm{Eh}$.

$$
\begin{aligned}
& \text { - Beyrichiana A. S. } \\
& \text { - } \quad \text { bombus Eh. } \\
& \text { - clacata Greg. o. elliptica. } \\
& \text { - crabro Eh. } \\
& \text { - liber W. Sm. } \\
& \text { - } \quad \text { - v. linearis V.H. } \\
& \text { - lyra v. elliptica A. S. } \\
& \text { - } \quad \text { - v. suptypica A.S. } \\
& \text { - pardura Bréb. } \\
& \text { - pretexta Eh. } \\
& \text { - } \quad \text { separabilis A. S. } \\
& \text { - Smithii Bréb. }
\end{aligned}
$$

Vitzschia notabilis Grun.

- panduriformis Greg.

Orthoneis splendida Grun.

Pleurosigma decorum W.Sm.

-1 naviculaceum Brèb.

- robustum Grun.

- formosum W. Sm.

- elongatum W. Sm.

- balticum W.Sm.

Surirella collare A. S.

- comis var. A. S.

- fastuosa Eh.

- - car. abludens Grun.

- - oar, opulenta Grun. 
Surirella fastuosa var. sentis Pant.

- $\quad$ - Guinardi H. P.

Synedra baculus Greg.

- undulata Greg.

- robusta Ralfs.

Triceratium antediluvianum V.H.
Triceratium arcticum Bright. Balearicum Grun. - favus Eh. v. major. - fimbriatum Bright. pentacrinus Wall. Shadboldtianum Grun. spinosum Bail.
Chatoceros angulatum Schütt (avec endocyst).

- boreale Bright.

- $\quad$ var. caracterisée par des cellules beaucoup plus larges et des cloisons légèrement tordues.

\section{Chatoceros cochlea Schuitt.} contortum Schütt.

- curvisetum Cl.

- Danicum fa solitaria $C l$.

- $\quad$ didymum (aree endocyst) Eh.

- distans Cl.

- laciniosum Schütt.

- paradoxum Cl. (aoec endo. cysts).

- procerum Schüitt.

- protuberang Laud (avec endocysts).

- seiracanthum Schütt?
Chatoceros spinosum Laud. Coscinodiscus excentricus $\mathrm{Eh}$. Ditylium Brightwellii West. Eucampia Zodiacus Eh.

Guinardia flaccida H.P.

Leptocylindrus danicus $\mathrm{Cl}$.

Nitzschia seriata $C l$.

Rhizosolenia alata Bright.

- _ - $\quad$. gracillima Grun.

- gracilis H.L. Sm. (serait à assimiler au R. Iongispina Schūtt, si cette espèce pourait être considérée comme marine).

- setigera Bright.

- styliformis Bright.

Skeletonema costatum Cl.

Thalassiothrix nitzchoides Grun

LE HAVRE (France)

(Sur les algues)

Melosira Borreri Grev. 
Nor 133 à 137 AUXILLAC - CANTAL (France)

(Dépôt lacustre).

BIBliogra PhiE. - Frère J. Héribaud. Les Diatomées fossiles d'Auvergne, 1893 et 1902.

Achnanthes lanceolata Grun. var. elliptica $\mathrm{Cl}$.

Amphora ovalis $\mathrm{Ktz}$.

- pediculus Grun.

Cocconeis lineata 0 . euglypta Grun.

- lineata Grun.

- pediculus Ehr.

- $\quad$ - v.rotunda J.Br. et F.H.

- placentula fa minor J. Br et

- F.H.

speciosa Greg.

- trilineatus M.P. et F. H.

Coscinodiscus Chambonis M. P. et F.H - pygmæus $\nabla$. crassipunctata J. Br. et F..H.

Cyclotella comta Ehr.

- iris J. Br. et F. H. (n॰ 136).

- $\quad-v$. cocconeiformis (n॰136).

- $\quad-$ v. ovalis ( $n^{\circ}$ 136).

- Kützingiana W. Sm.

- Meneghiniana Ktz.

- $\quad$ - $\quad$. rectangulata

Cymbella alpina minor J. B. et F. H.

- aspera Ehr.

- Bouleana F.H. et J.Br.

- cistula Hemp.

- conifera J. Br. et F. H.

- cymbiformis Ehr.

- gastroides Kitz.

- lanceolata Ehr.

- leptoceros Ktz. fa curta.

- paroa W. Sm.

- maculata Ktz.

Encyonema prostratum Ralis.

- turgidum Grun.
Bpithemia argus $\mathrm{Ktz}$.

- gibba Eh.

- - v. parallela Grun.

- - v. ventricosa Grun.

- Hyndmannii W. Sm.

- $\quad$ sorex Ktz.

- turgida Ktz.

- $\quad$ - $\quad$ granulata Grun.

- zebra Ktz.

- $\quad$ - v. pruboscidea Grun.

Eunotia linearis Grun.

- polyglyphis Grun.

Fragilarla binodis Ehr.

- brevistriata Grun.

- $\quad$ - v. laponica Grun.

- P. mormorum Grun.

- $\quad$ - v. subacuta V. H.

- construens $\nabla$. pumila Grun.

- elliptica Schum.

- Harrisonii Grun.

- mutabilis Grun.

Gomphonema cantalicum J. Br. et $\mathrm{F}$. H. - v. costalonga F.H. et J. Br.

- v. lepida F. H. et M. P.

- fa major J. Br. et F. H.

- $\quad$ capitatum Grun.

- olivaceum Ehr.

- subclavatum Grun.

Nelosira canalifera $\mathrm{J} . \mathrm{Br}$. et $\mathrm{F}$. H.

- granulata Ktz (n' 135$)$.

- imperfecta F, H, (n॰ 137).

- solKtz.

- tenuis Ktz. 
Melosira tenuissima Grun.

- varennarum M.P. et F.H.

Navicula amphibola $\mathrm{Cl}$. 0 . staurone formis $M$. P. et $F$. $H$.

- dicephala W. Sm.

- elliptica $\mathrm{Ktz}$.

- $\quad$ - v. oblongella Næg.

- Heufleri Grun.

- Hitchcockii Ehr.

- limosa $\mathrm{Ktz}$.

- major $\bar{n} t z$.

- menisculus Schum.

- nodosa Ktz.

- placentula Ehr.

- perpusilla Grun.

- radiosa $\mathrm{Ktz}$.

- Reinhardtii Grun.

- Renauldi F.H.

- ventricosa Donk.

- - fa minuta Grun.

Nitzechia acutiuscula Grun.

- fossilis Grun.

- ignimontana J. Br. ot F. H.

Rhoicosphenia curvata Grun.

Stauroneis Phonicenteron Eh.
Surirella biseriata Bréb.

- robusta Eh.

- saxonica Auersw.

Synedra acus $\nabla$. fossilis Grun.

- $\quad-\quad$. subtilis $\mathrm{Ktz}$.

- $\quad$ - $\nabla$, ventricosa J.Br. etF.H.

- capitella Grun.

- Closterioides Grun v. fossilis

H.P. et F.H.

- crotonensis Edw.

- delicatissima W. Sm.

- - $\quad$ - angustissima Grun.

- _ - v. Mauleri Grun.

- hyperborea Grun.

- rumpens Grun.

- ulna Ehr.

- vaucherix $\mathrm{Ktz}$.

- $\quad$ - v. parrula Ktz.

Tetracyclus ellipticus M. P.

- - . minutissima $F . H$. et $M . P$.

lancea Ehr.

rhombus Ralfs.

tripartitus J. Br. et F. H.

No 138

LE HAVRE (France)

(Sur les algues-saumâtres)

Achnanthes brevipes Ag.

Actinoptychus undulatus Eh.

Campylodiscus parvulus W. Sm.

Cocconeis costata Greg.

- distans Greg.

- scutellum Eh.

Grammatophora marina Ktz.
Grammatophora nodulosa Grun.

Melosira Borreri Grev.

- sulcata fa minima.

Navicula Bottnica Gruń.

Nitzschia angularis W. Sm.

Rhoicosphenia curvata Ktz. var. marina.

Synedra affinis Ktz. 


\section{N` 139 à 141 BAIN’S FARM-OAMARU (Nouvelle-Zélande)}

(Dépôt fossile tertiaire marin)

BIBLIOGRAPHIE. - On a fossil marine Diatomaceous deposit from Oamaru (New-Zealand) by E. Grove et G. Sturt.

Actinodiscus barbadensis Grev.

Actinoptychus undulatus fa parva. vulgaris var. maculata Grove et St.

Wittianus Jan.

Amphora Sturtii Grun.

Anthodiscus floreatus Grooc et St. Aporodiscus 'Oamaruensis Ratt. Arachnoidiscus Ehrenbergii Bail.

Asterolampra affinis Grev.

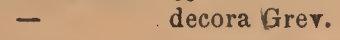

$$
\text { - vulgaris Grev. }
$$

Aulacodiscus amœnus Grev.

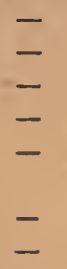
angulatus Grev. cellulosus Grove et St. crux $\nabla$. squamosa Grun. Janischii Grove et St. margaritacous v. Debyi. Ratt. Rattrayi Grove et St. sollitianusv. nova zelandiæ Grove et St.

Auliscus lacunosus Grove et St.

- Oamaruensis Grove et St.

- Stöckardtii Jan.

Biddulphia Oamaruensis Grove et St.

- Tuomeyi Bail.

- $\quad$ - v. rigida A. S.

- tenera Grove et St.

Brightwellia coronata Ralis.

Cerataulus subangulatus Grove et St.

Coscinodiscus angulatus Grev.

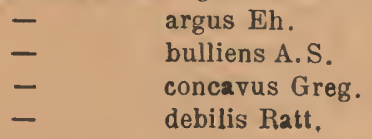

Coscinodiscus decrescens v. repleta Grun. excentricus Eh. marginatus Eh. Oamaruensis Gr. et St. partitus $G r$. et $S t$. radiatus Eh.

Rothii Grun. spiniferus Gr. et St. vetustissimus Pant.

Eunotogramna productum Grove et $\mathrm{St}$.

Eunotia striata Grove et St.

Goniothecium odontella Eh.

IIemiaulus barbadensis Grun.

- includens Grun.

- ornithocephalus Grev.

- polymorphus Grun.

- tenuicornis Grev.

Hyalodiscus permàculatus Gr. et St.

- $\quad$ radiatus $P . P$.

- robustus Grove.

Iuttonia alternans Grove et St.

Isthmia enervis Eh.

- tenuicornis Grun.

Kittonia elaborata Grove et St.

Liradiscus ovalis Grev.

Melosira Oamaruensis $\mathrm{Gr}$. et St.

- sulcata Ktz.

- truncata Grovo.

- Westii W. Sm.

Navicula apis Donk.

- Oamaruensis Grun.

Podosira argus Grun var.

- hormoides Ktz. var.

Pseudauliscus anceps Ratt. diffusus Ratt. 
Pseudorutilaria monile Grove et St.

Pyxidicula cruciata Eh.

Pyxilla .Tohnsoniana Grev.

- reticulata Grev.

- dubia Grun.

Rutllaria epsilón v. tenuis Gr. et St.

- radiata Gr. et $\mathrm{St}$.

Stephanogonia nova zelandica Grun.

Stephanopyxis barbadensis Grun.

- $\quad$ corona Ehr.

- $\quad$ Grunowii Gr. ot St.

stíctodiscu californicus Grev.
-
Hardmannianus Grev. var.
-

Syndetoneis amplectans Grun.

Triceratium americanum Ralfs.

- arcticum Bright.

- _ - fa quadrata Grun.
Triceratium arcticum fa quinquelobata Grun.

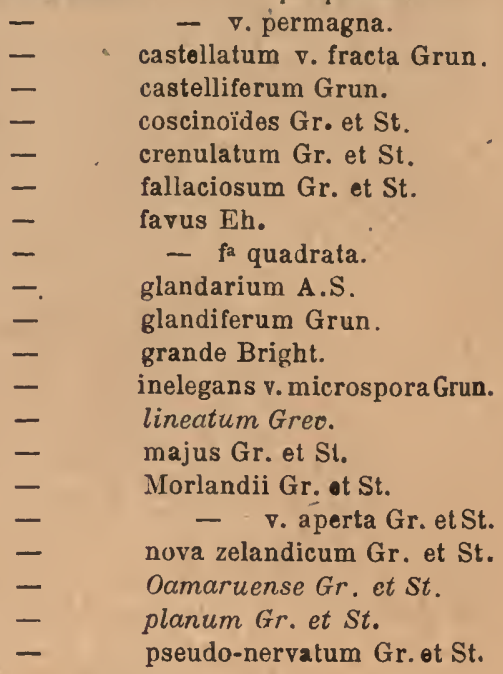

Trinacria ligulata $\mathrm{Gr}$. et St.

- ventricosa Gr. et St.

Xanthiopyxis constricta $\mathrm{Eh}$.
Biddulphia aurita Brêb.

Cerataulina Bergonii H. Per.

Chatoceros balticus $\mathrm{Cl}$.

- boreale Bright.

- contortum Schütt.

- convolutum Cast.

- curvisetum Gl.

- danicum Cl.

- decipiens Gl.

- didymus Eh.

- gracile Schūtt.

- paradoxum Gl. (arec endocyst).

- Schïttii Cl. (avec endocyst).

- scolopendra Gl.
Chretoceros simile $\mathrm{Cl}$. (avec endocyst). socialis Laud.

Cocconels scutellum Eh.

Coscinodiscus asteromphalus Eh. - excentricus Eh.

Fragilaria islandica Grun.

Lauderia glacialis Grun.

Leptocylindrus danicus (il.

Navicula Wanhöffenii Grun.

Nitzschia closterium W. Sm.

- dolícatissima Gl.

- seriata Gl.

- - v. fraudulenta Cl.

- Wankaromæ Gl. 
Pyxilla baltica (ondocyst du Rhizosolenia setigera).

Raphoneis amphiceros v. rhombica Grun. Rhizosolenia alata Bright.
Rhizosolenia alata v. Betigera Bright. Skeletonema costatum Eh.

Thalassiosira Nordenskioldii Cl.

Thalassiothrix nitzschioides 'Grun.

Nos 143,144 EMBOUCHURE DE LA ȘEINE - LE HAVRE (Sur la vase)

Nitzschla acuminata W. Sm. - sigma W. Sm.

Plagiotropis vitrea Grun.

Pleurosigma æstuarii W. Sm.

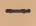

$\overline{-}$ angulatum W. Sm. (n• 143).

balticum W. Sm. Brebissonii Donk. hiprocampus W. Sm.
Pleurosigma littorale W. Sm.

- quadratum W. Sm (No 144).

- sulcatum Grun (N• 144).

- strigosum W. Sm.

- Wansbeckii Donk.

Scoliopleura latestriata Grun.

- tumida Grun.

Surlrella gemma Bh.

\section{Nos 145 à 147 ATLANTIC/CITY - NEIV-JERSEY}

\section{(Etats-Unis)}

BibliographiE. - Henry Kain et E. A. Schultze. On a fossil marine Diatomaceous deposit from Atlantic City.

Actinocyclus Ehrenbergii var. Ralfs.

- interpunctatus Ralfs. .

- Ralfsii W. Sm.

Actinodíscus atlanticus $\mathrm{K}$. et $\mathrm{Sch}$.

Actinoptychus areolatus Ehr.

$\begin{array}{ll}\text { - } & \text { cathedralis J. Br. } \\ \text { - } & \text { heliopelta o. oersicolor } \\ \text { - } & \text { J. Br. } \\ \text { - Kraussii A.S. } & \text { mosaica J. Br. }\end{array}$

Actinopt ychus notabilis $J . B r$.

$\begin{array}{ll}\text { - } & \text { ranunculus } J . B r \\ \text { - } & \text { segmentatus } J . B r\end{array}$

$-$

- _ - v. halionyx Grun.

- undulatus Eh.

- vulgaris Schum.

- $\quad$ - v.virginica Grun.

Amphitetra minuta Grev.

Anaulus birostratus Grun. 
Asterolampra marylandica Eh.

Aulacodiscus Crux Eh.

- (Crux? var) atlanticus Temp.

Auliscus prúinosus Bail.

- spinosus Christian.

Bidldulphia aurita Bréb.

- Brittoniana K. et Sch.

- Baileyi W. Sm.

- Cookiana K. et Sch.

- decipiens Grun.

- elegantula Grev.

- longispina Grun.

- pulchella Greg.

- pustulata J. Br.

- rhombus W. Sm.

- seticulosa Grun.

- Tuomeyi Bail.

- Weissflogii Grun.

- Woolmanii $K$ et Sch.

Cerataulus Smithii Ralfs var. antiqua

- turgidus Eh.

Cestodiscus ovalis Greg.

Cocconeis oculus catis $J . B r$.

Coscinodiscus argus Eh.

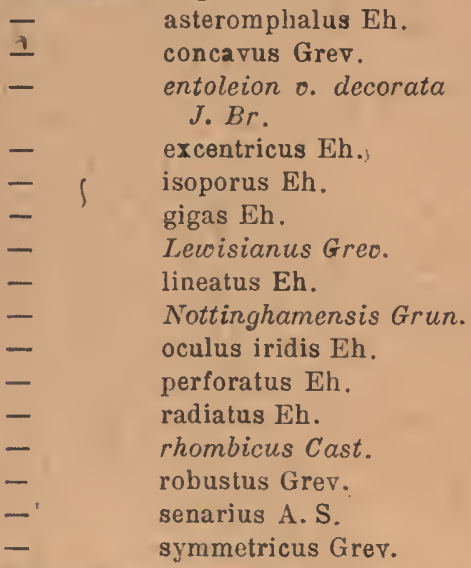

Craspedodiseus Coscinodiscus Eh.

Cyclotella operculata $\mathrm{Ktz}$.

- v. nankoorensis.

- physoplea Eh.

Dimeregramma fulvum Ralfs.

novæ cæsaræa K. et Sch.

- . obtusa K. et $\mathrm{S}$.
Ditylium Brightwellii West.

Eucampia virginica Grun.

Eunotia americana K. et Sch.

Eupodiscus Rogersii Eh.

$\mathrm{sp}$.

Goniothecium odontella Eh.

- Rogersii kh.

Graumatophora serpentina $E h$.

Grunowiella geminata V. H.

Ilemiaulus affinis Grun.

Ilyalodiscus lævis Eh.

- stelliger Bail.

Hydrosera triquetra Wall. .

Liradiscus minutus Grev.

Mastogonia actinoptychus Eh.

Melosira fausta A.S.

- ornata Grun.

- - sulcata Ktz.

Navicula crabro Eh.

- didyma Eh.

- elliptica $\mathrm{Ktz}$.

- entomon Eh.

- forcipata Grev.

- gracilis Ktz.

- Lewisiana Grev.

- lyra et var. Eh.

- macilenta Eh.

- major Kitz.

- prætexta Eh.

- permagna Bail.

- Schultzei Kain.

- viridis $\mathrm{Ktz}$.

- de Wittiana K. et Sch.

Plagiogramma Gregorianum Grev.

Pleurosigma affine v. fossilis Grun.

Pseulauliscus radiatus Bail.

Pyxidicula cruciata Eh.

Pyxilla americana Grun.

Raphoneis ampliceros Eh.

- belgica Grun.
- $\quad$ fluminengis Grun.
- $\quad$ gemmifera Eb.
-

Rhalsionema atlanticum Kain.

Rhizosolenia styliformis Bright.

Septroneis caducous $\mathrm{Eh}$. 
Stephanogonia actinoptychus Eh. polygona Eh.

Stephanopyxis apiculata Eh.

$\begin{array}{ll}\text { - } & \text { corona Grun. } \\ \text { - } & \text { ferox Ralfs. } \\ \text { - } & \text { limbata Eh. }\end{array}$

Stictodiscus Kittonianus Grev.

Surirella Febigerii Lewis.

Terpsinoë americana var. Grun.

Triceratium americanum Ralfs. - r condecorum Bright.
Triceratium Fischerii A. S.

$$
\begin{array}{ll}
\text { - Heilprinianum } K \text {. et Sch. } \\
\text { - } & \text { indentatum } K \text {. et Sch. } \\
\text { - } & \text { Kainii Schultze. } \\
\text { - } & \text { - v. constrictum. } \\
\text { - } & \text { marylandicum Bright. } \\
\text { - } & \text { obtusum El. } \\
\text { - } & \text { robustum Grev. } \\
\text { - } & \text { semicirculare Bright. } \\
\text { - } & \text { solenoceros Rab. } \\
& \text { spinosum Bail. }
\end{array}
$$

Triblionella Hantzschiana Grun.

- scutellum W. Sm.

No 148

COLOMBO - ILE DE CEYLAN

(Sondage)

BIBLIOGRa PHiE.- Leuduger Fortmorel. Diatomées de Ceylan.

Actinocyclns ovalis Roper.

$$
\text { - Ralfsii W. Sm. }
$$

Actinoptychus splendens Ralf.

$$
\text { - trilingulatus Bright. }
$$

Amphiprora elegans TV. Sm.

Amphora gigantea Grun.

Aulacodiscus Macraeanus Grev'.

Auliscus colatus Bail.

$$
\text { - formosus Leud. }
$$

Bacteriastrum varians Lauder.

Bichlulphia granulata Roper.

$$
\text { - } \quad \text { pulchella Greg. }
$$

Campylodiscus crebrecostatus Grev.

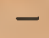

Coscinodiscus apiculatus Eh.

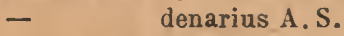

- excentricus Eh.

- fimbriatus Eh.

- lineatus Eh. var.
Cosoinodiseus Janischii A. S. nitidus Greg.

- radiatus $\mathrm{Eh}$.

- . subtilis Eh.

Climacosphenia moniligera Eh.

Euodia gibba Bail.

IIyalodiscus stelliger Bail.

Mastogloia lemniscata Leud.

Melosira sulcata Ktz.

Navicula aspora Eh.

- Beyrichiana A. S.

- caribor $\mathrm{Cl}$.

- carinata A. S.

- Craveni Leud.

- cruciata Leud.

- diplosticta Grun.

- fusca Greg.

- indica Grev.

- Lyra Eh. et var.

- polysticta Grov. 
Navicula prætexta Eh.

- Smithii Bréb.

- spectabilis Grev.

Nitzschia granulata Leud.

- panduriformis Greg.

- $\quad$ sigma W. Sm:

Pleurosigma balticum Var.

- elongatum Grun.

- rhombicum Grun.

- rigidum W. Sm.
Pseudauliscus Debyi Leud.

- nebulosus Leud.

Petitii Leud.

Surirella fastuosa $\mathrm{Eh}$. ot var.

Stephanopyxis turris Eh.

Triceratium favus Eh.

- pentacrinus Wall.

- sculptum Shad.

- $\quad$ scitulum Bright.

$\mathrm{N}^{\text {os }} 149,150$ VERNEUGE (Puy-de-Dôme - France)

(Dépôt fossile d'eau douce)

BIBliographie. - Frère J. Héribaud. Les Diatomées fossiles d'Auvergne 1893.

Cocconeis intermedia $M, P$. et $F, H$. Cymatopleura solea $\nabla$. apiculata Pritch. Cymbella anglica Lag.

- aspera Eh.

- cuspidata Ktz.

- cymbiformis Ehr.

Diatoma anceps Grun.

- - $\quad$. anomalum W. Sm.

Epithemia gibba $\nabla$. ventricosa Grun.

- turgida Ktz.

Eunotia lunaris Grun.

- - v. excisa Grun.

- - 10. subarcuata Grun.

- minor Rab.

Fragilaria elliptica Schum.

- intermedia Grun.

- producta Grun.

- virescens Ralfs.

- $\quad$ fa elongata M. P. et F. II.

Gonphonema acuminatum Eh. $\mathrm{v}$. gigantea F. H. et M. P.

- $\quad$ auritum C. Br.
Gomphonema parvulum Ktz.

subclavatum $\nabla$, acuminata

H. P. et F. H.

Melosira crenulata 0 . undulata $M . P$. et $F . H$.

Meridion circulare $\mathrm{Ag}$.

Navicula acrosphæria Bréb.

- ampliata Ehr.

- $\quad$ - o. minor M. P. et F.H.

- amphigomphus Enr.

- arrerna $\nabla$. stauroneiformis M.

$P$. et $F$. $H$.

- bacillum v. minor V.H.

- borealis Ktz.

- $\quad$ - $\quad$. minorM. P. etF. H.

- elliptica Ktz.

- esox Ehr.

- gigas $\nabla$. gracilis F. H.

- gentilis Donk.

- hybrida M.P. et F. H.

- limosa KIz.

- maera Grun.

- mesolepta Ehr.

- $\quad$ - v. Saintignyi F.H.

- nobilis Ehr.

- rupestris $\mathrm{Ktz}$. 
Navicula seminulum $\nabla$. fragilarioides

- stomatophora Grun.

- viridis fa anomala.

- - v. commutata Grun.

Stauroneis anceps Ehr.
Surirella elegans Ehr.

- spiralis Ḱtz.

Synedra capitata Ehr.

- ulna Ehr.

Tabellaria fenestrata $\mathrm{Ktz}$.

- flocculosa Kítz.

BIBLIOGRAPHIE. - Ehrenberg Microgéologie Pl. 31. Le dépôt d'Oran est un des plus anciennement connu, toutefois, l'étude qu'én a faite Ehrenberg dans sa Microgéologie est loin d'être complète et n'a dú porter que sur quelques échantillons peu riches. Nous donnons ici l'analyse de six localités de l'arrondissement d'Uran, auxquelles nous ajoutons celle de Mascara qui s'y rattache.

Dans le résidu provenant des lavages des échantillons provenant de Misserghin, et composé de sable, de spicules et de quelques Radiolaires, nous y avons rencontré quelques beaux spécimens d'une jolie variété de l'Aulacodiscus Grunowii et du Triceratium Thumii var. quadrata rencontrés récemment dans les terres fossiles de Hoñgrie, et qui n'avaient pas encore été signálés dans celle d'Oran.

Actinocyclus Ehrenbergii $f_{a}$ bisenarius.

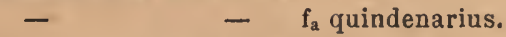

\footnotetext{
- $\quad$ Ralfsii W. Sm.

- rotula J. Br.fa minor.

- ' subtilis Ralfs.

Amphora crassa Greg.

- robusta Greg.

Arachnoidiscus ornatus Eh. 0 . Montereyana.
}

Aulacodiscus Grunowiio. africanaTemp.
Auliscus cœlatus Bail v. major.

- - v. rhipis A. S.

- pulvinatus $f^{2}$ inermis, ne diffère de A. S. atlas $108 / 4$, que par l'absence des épines marginales.

Biddulphia Tuomeyi Bail.

Cocconeis vitrea $\mathrm{J}$. $\mathrm{Br}$.

Coscinodiscus Argus Eh.

- crassus Bail. var. 
Coscinodiscus curvatulas Grun.

$\bar{\Xi}$
$\overline{ }$
$\overline{ }$
$\bar{z}$ diversus Grun.

excentricus Eh.

heteroporus 0 .moronensis

lineatus $E$ h.

marginatus Eh.

- v. intermedia.

nitidus Greg.

Oculus iridis Eh. var.

radiatus Eh.

symbolophorus Grun.

Dimeregramma marinum. Greg.

Endyctia oceanica Eh.

Grammatophora angulosa Eh.

pararella Eh.

Hyalodiscus radiatus Bail.

Isthmia (Zaboi Pant var?) gigantea M. Per. a comme l'Isth. Zaboi ses marques lobées, mais elles sont plutôt quadrangulaires, sont plus grosses et le frustule est beaucoup plus large, long $300 \mu$ environ 1 marque en $10 \mu$.

Mlelosira sulcata $\mathrm{Eh}$.

Navicula Beyrichiana A. S.

$-\quad$ bomboides $A$. S.

- clavata Greg.

- crabro Eh.

- fusca Ralfs v. major.

- gemmata Grer. v. spectabilis.

- Hennedyi W. Sm.

-

o. africana $n$. var.

intermédiaire entre le $N$ Hennedyi o. granulata et le $N$ Sandriana, quelques granules seulement dans les aires latėrales.

- Hennedyi o. neapolitana.

Lapeyrereana Temp. et M. Per. n. sp. Intermediaire entre le $N$ lyra et le $N$ prætexta de forme large souvent presque orbiculaire; les granules non uniformément distants sur les stries, forment une zone marginale et une seconde garnie de lignes longitudinales ondulées s'étendant jusqu'à l'aréa lyriforme.

Navicula lyra Eh. o. recta.

- nitescens Greg.

- prætexta Eh.

- spectabilis Greg.

- - - v. emarginata.

Orthoneis splendida Grun.

Rhabdonema adriaticum Ktz.

Raphoneis amphiceros Grun v. rhombica. nitida Grun.

Synedra baculus Greg.

Trachyneis aspera $\mathrm{Cl}$.

Triceratium antediluvianum Eh.

- spinosum $v$. tetragona.

Thumii A.S. o. quadrata.

\section{Nos 152,153 VILLAGE NEGRE - ORAN (Algérie)}

Achnanthes danica Flög fa maxima.

- Lapeyrerei Temp. et M. Per. n. sp. (A sp. A. S. atlas 198/40 var.).

Actinocyclus Ehreubergii Ralfs.

- moniliformis Ralfs.

- Ralfsii W. Sm.

- sparsus Ralfs.

- subtilis Ralis.

- tenellus Brẻb.
Actinoptychus splendens Ralfs.

- I undulatus Eh.

Amphora alata. Per.

- arcuata Greg.

- crassa Greg.

- cymbifera Greg.

- dubia Greg.

- eunotia Cl.

- Graeffei Grun car.

- ostrearia Bréb. 
Amphora proteus Greg.

- robusta Greg.

Biddulphia aurita Bréb.

Tuomeyi Bail.

Cestodicus pulchellus Grev. var.

Chretoceros didymus Eh.

Climacosphenia moniligera $\mathrm{Eh}$.

Cocconeis scutellum Eh.

$$
\text { - } \quad \text { sigmoradians J. Br. oar. }
$$

Coscinodiscus curvatulus Grun.

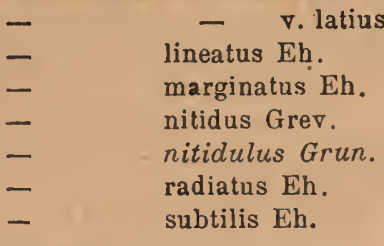

Dimeregramma marinum Grog.

Endictya oceanica Eh.

Euodia gibba Bail.

- - v. africana M. Per. - De forme rhomboido-orbiculaire et $\dot{a}$ cellules centrales libres.

Grammatophora africana $E h$.

\begin{tabular}{ll} 
- & angulosa Eh. \\
- & lyrata var. \\
- & marina v. undulata. \\
\hline & parallela Eh.
\end{tabular}

Hyalodiscus radiatus Bail.
Melosira sulcata Eh.

Navicula abrupta var. Ratt.

- bombus o. egena.

- cancellata Donk var.

- clavata Greg.

- crabro Eh.

- forcipata Grev.

- fusca Ralfs.

- gemmata Grev.

- - v. spectabilis.

- granulata Bréb.

-. Hennedyi W. Sm.

- $\quad$ - $\quad$ v. granulata.

- - $\quad$ v. neapolitana.

- Lapeyrereana Temp. et M. Per.

- lyra var. recta.

- - var. subtypica.

- nitescens Greg.

- prætexta Eh.

- polysticta $\nabla$. elliptica.

- Sandriana Grun.

- scintillans A. S.

- spectabilis Greg.

Podosira Hormoides Mont.

Rhabdonema adriaticum Ktz.

Raphoneis amphiceros t. rhombica. nitida Grun.

Stephanodiscus carconensis v. minor.

Synedra, superba Ktz.

Terpsino intermedia Grun.

Trachyneis aspera Cl. var.

Triceratium antediluvianum Eh.

\section{No 154}

RAZ et AIIN - ORAN (Algérie)

Actinocyclus Ehrenbergii Ralfs.

Actinoptychus undulątus Eh.

Biddulphia pulchella Greg.

$$
\text { - Tuomeyi Bail. }
$$

Cocconeis vitrea $\mathrm{J}$. B.

Coscinodiscus argus $\mathrm{Eb}$.

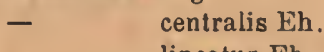

Coscinodiscus marginatus 'Eh.

- oculus iridis Eh.

- radiatus Eh.

- $\quad$ subtilis Elt.

Endictya oceanica $E h$.

Hemiaulus polycistinorum Grun.

Hyalodiscus radiala Bail.

Isthmia gigantea M. Per. 
Melosira sulcata Eh.

Navicula crabro Eh.

- fusca Ralf.

- gemmata o. spectabilis.

- Kittoniana A.S.

- Lapeyrereana Temp.et M. Per.

- lyra Eh. o intermedia fa

- lyra v. subelliptica.

- nitescens fa major long. $110 \mu$.

- prætexta Eh.
Podosira hormoides Mont.

Rhabolonema adriaticum $\mathrm{Ktz}$.

Synellra superba $K t z$.

Triceratium antediluvianum Eh. latum v. plana M. Per, à côtés à peine concaves et à ponctuations trés fines. parallelum Grev. spinosum Bail.
Actinocyclus Barklyi Grun.

$\begin{array}{ll}\text { - } & \text { Ehrenbergii Ralfs. } \\ \text { - } & \text { Ralfsii W. Sm. } \\ \text { tenellus Bréb. }\end{array}$

Actinoptychus undulata $\mathrm{Eh}$.

Amphora crassa Greg.

- exsecta Grun.

- robusta Greg.

Biddulphia Tuomeyi Bail.

Climacosphenia moniligera Eh.

Cocconeis vitrea $\mathrm{J}, \mathrm{Br}$.

Coscinodiscus argus Eh.

curvatulus Grun. lineatus $E h$. marginatus Eh. oculus iridis Eh. radiatus.

subtilis Eb. symbolophorus Grun.

Dimeregramma marinum Greg.

Endictya oceanica Eh.

Grammatophora marina $\nabla$. undulata.

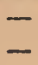
parallela Eh. robusta Dip.
Ilyalodiscus radiatus Bail.

Melosira sulcata Eh.

Navicula clavata Greg.

- crabro Eh.

- fusca Ralf.

- gemmata Greo.

- - o. spectabilis.

- Hennedyi W. Sm.

- - $\quad$ - cuneata.

- $\quad$ - v. neapolitana.

- lyrav.intermedia fa ambigua.

- - o. recta.

- , - o. subelliptica.

- nitescens Greg.

- prætexta Eh.

Rhabdonema adriaticum $\mathrm{Ktz}$.

Stictodiscus californicus o. Nancooren sis.

Synedra superba Ktz.

Toxarium undulatum Bail.

Trachyneis aspera $\mathrm{Cl}$. var.

Triceratium antediluvianum Eh.

- parällelum Grer.

- spinosum Bail.

Xantbiopyxis cingulata Eh. 
Actinoptychus areolatus Eh.

\section{undulatus Eh.}

Arachnoidiseus ornatus v. Montereyana.

Biddulphia pulchella Grun.

$$
\text { - Tuomeyi Bail. }
$$

Cocconeis maxima $H$. Per.

$$
\text { - vitrea J. Br. }
$$

Coscinodiscus argus Eh.

$\bar{z}$
$\bar{z}$
$\bar{z}$ curvatulus Grun.

- v. latius striata. lineatus Eh. marginatus Eh. . radiatus $\mathrm{Eh}$.
Euodia gibba Bail.

Gephyria media Arn.

Grammatophora angulosa $\mathrm{Eh}$. robusta Dip.

Hyalodiscus radiatus Bréb.

Isthmia gigantea M. Per.

Navicula crabo Eh.

- fusca Ralf.

- gemmata Grun.

- $\quad$ - $\quad$. spectabilis.

- Lapeyrereana Temp. et M. Per.

- prætexta Eh.

Triceratium antediluvianum Eh.

- parallelum Grun.
Amphora crassa Greg.

Actinocyclus Ehronbergii Ralfs.

Act Inoptychus splendens Ralfs. undulatus Eh.

Biddulphia Tuomeyi Bailey.

\section{Coscinodiscus argus Eh.} curvatulus Grun. hoteroporus Eh.

- lineatus Eh.

- marginatus Eh.

- Oculus iridis Eh.

- radiatus Eh.

- symbolophorus Eh.
Gephyria media Arnott.

Hyalodiscus radiatus Bailey.

Navicula Beyrichiana A. S.

- bombus Eh.

- fusca et var.

- Grunowii Rab.

- Hennedyi W. Sm.

- pandura Breb.

- spectabilis Grev. var.

Rhabdonema adriaticum $\mathrm{K}$.

Triceratium antediluvianum V. H.

- spinosum Bailey. 
Achnanthes longipes Ag.

Actinocyclus Ehrenbergii Ralfs.

Actinoptychus splendens Ralfs. undulatus Eh.

Biddulphia Tuomeyi Bailey.

Cocconeis pseudo marginata Greg.

Coscinodiseus argus Eh.

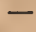

$-$ exentricus o. micropora Grun. flexilis Rattray var? forme grande et délicate, assez fréquente, se rapproche des Coscinodis- cus apiculatus et perforatus.

Coscinodiscus heteroporus Eh.

$\begin{array}{ll}\text { - } & \text { leptotus Grun. } \\ \text { - } & \text { marginatus Eh. } \\ \text { - } & \text { partitus Grove et St. } \\ \text { - } & \text { plicatus Grun. } \\ \text { - } & \text { radiatus Eh. } \\ \text { - } & \text { subglobosus } C l \text { et Grun. }\end{array}$

Navicula Hennedyi W. Sm.

spectabilis Greg.

Rhabdonema adriaticum Eh.

Triceratium antediluvianum V. H.

\section{No 159 BOLDPATTE POND - GEORGETOWN, MASSACHUSETTS (Etats-Unis)}

Amphora affinis $\mathrm{Ktz}$.

Cocconeis placentula Eh.

Cymbella americana A.S.

- aspera Eh.

- gastroides Ktz.

Encyonema ventricosum Grun.

Epithemia amphicephala Grun.

- $\quad$ zebra var. proboscidea.

Eunotia arcus Eh.

- formica Eh:.

- lunaris Grun.

- monodon Èh.

- pectinalis Rab.

- $\quad$ - var. ventricosa

- prærupta var. bidens.

Gomphonema acuminatum Eh.

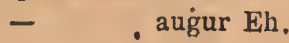

Gomphonema constrictum $\mathbf{v}$. capitata.

$$
\text { - } \quad \text { intricatum } \mathrm{Ktz} \text {. }
$$

Hantzschia amphioxys Grun.

Melosira laevis Grun.

Navicula acrosphceria o. minor.

- americana Eh.

$$
\begin{array}{lll}
- & - & \text { v. bacillaris M.Per. } \\
- & - & \text { v. minor M. Per. } \\
- & - & \text { v. moesta. Grande }
\end{array}
$$
forme à centre excavé et à area rugueuse dessinée par Wolle sous le nom de $N$. moesta A S., ce qui est évidemment une erreur, la forme de Schmidtẻtant un Diploneis. (Jo n'ai pu trouver oủ Wolle a pris son dessin.) 
Navicula amphigomphus Eh.

- amphirynchus Eh.

- bacilliformis Grun.

- borealis $K t z$.

- Brebissonii Ktz. var.

- commutata Ktz.

- firma Ktz.

- flammula A.S. var.

- gentilis Donk.

- gibba Eh.

- gracillima Greg var.

- instabilis A.S.

- iridis v. ampliata.

- legumen Eh.

- major Ktz.

- microstauron Eh.

- nobilis $\mathrm{Ktz}$.

- pseudo bacillum Grun.
Navicula rupestris Hantz.

- stauroptera Rab. var.

- viridis $\mathrm{Ktz}$.

Nitzschia sigmoidea W. Sm.

Stauroneis acuta v. maxima M. Per. Longueur $250 \mu .11$ stries transversales en $10 \mu$ dont les granules forment des stries longitudinales ondulées, silice jaune.

- anceps Eh.

- Brunii M. Per.

- gracilis Eh.

- lanceolata $\mathrm{Ktz}$.

- Phœnicenteron Eh.

Tabellària fenestrata $\mathrm{Ktz}$.

\section{ALBANY - KING GEORGE'S SOUND}

\section{(Australie Oecidentale)}

\section{Sur les algues}

Actinocyclus Ehrenbergii Ralfs.

- moniliformis Ralfs. sparsus Greg.

Actinoptychus glabratus Grun.

- Janischii Grun.

- $\quad$ splendens Ralf.

- vulgaris Schum.

Alloioneis amphora $J . \mathrm{Br}$.

Amphora crassa Greg.

- gigantea v. obscura.

- Graeffii $C l$.

- mexicana fa minor.

- obtusa Greg.

- proteus Greg.

- spectabilis Greg.

Auliscus cœlatus v. latecostata.

- - $\quad$ v. rhipis.

- Normanianus Greo. fa bioculata.

Bacteriastrum varians $f^{2}$ spirillum.
Biddulphia pulchella Greg.

$$
\text { - Tuomeyi Bail. }
$$

Campylodiscus crebrestriatus Greo.

$$
\text { - Robertianus Grov. }
$$

Campyloneis Grevillei Grun.

Climacosphenia elongata Bail.

$$
\text { - moniligera Eh. }
$$

Cocconeis contermina A.S. var.

- heteroidea Hantz.

Coscinodiscus nitidulus Greg.

- radiatus Eh.

- Simbirskianus Grun.

- symbolophorus v. Oama-

1. ruensii.

Cymatoneis sulcata Cl.

Cymatopleura elliptica W. Sm.

Endictya oceanica $\mathrm{K} t z$. 
Epithemia gibberula $K t$.

Granumatophora lyrata Grun var.

Hyalodiscus stelliger Bail.

- subtilis Bail.

Iydrosilieon mitra $\mathrm{J}$. Br.

Mastogloia Grunovii A.S.

- Peragalli v. circumnodosa.

Melosira sulcata Eh.

$$
\text { - } \quad \text { - v. coronata. }
$$

Navicula abrupta Greg.

- cancellata Donk.

- Chersonensis v, apiformis.

- crabro $\nabla$. multicostata.

- directa W. Sm.

- fusca Ralf.

- LyraEh.

- - v. atlantica.

- maxima Donk.

- $\quad$ - v. excentrica.

- pennata A.S.

- peregrina Eh.

- sectilis v. Boryana.

- splendida Grev.

- $\quad$ sp (A.S. atlas 6/31,33.)

Nitzschia angularis W. Sm.

- distans o. tumescens.

- panduriformis Greg.

- sigma $\nabla$. sigmatella.

- spectabilis Greg.

- vivax W. Sm.

Orthoneis aspera M. Per.

- - $\quad$ v. minor.

- binotata Grun.

- fimbriata Grun.
Orthoneis pacifica $J$. Br. var.

- splendida Grun.

- splendida Grun.

Pleurosigma speciosum v. Janceolata

M. Per. - A la même striation que le $\mathrm{Pl}$. speciosum W. Sm. mais est de forme plus large et lancéolèe.

Podosira hormoides Mont.

Pyxidicula cruciata Eh.

Rhablonema adriaticum Ktz.

Surirella fastuosa Eh. var.

Synedra baculus Greg var.

- formosa Hantz.

Trachyneis aspera $\mathrm{Cl}$.

- - v. intermedia.

- velata v. rhoinboidea M. Per, possède la structure du T. velata mais sa forme est beaucoup plus large et franchement rhomboidale.

Triceratium incisum A.S. var.

- punctulata. - Ne diffère de la fig. de A.S. Atlas 76/25 que par des points très visibles entreles granules.

- Pentacrinus Wall.

- - $\quad$ v. quadrata.

Tropidoneis lepidoptera var. intermedia M. Per. diffère du type et des autres variétés par son nombre de stries; 17 stries en $10 \mu$.

Weissflogia Macdonaldii Jan. 
Nos 162 à 164 LES QUEYRADES (Puy-de-Dôme - France)

(Dépôt fossile d'eau douce)

BIBliogra PhiE. - Frère J. Héribaud. Les Diatomées d'Au: vergne, 1893.

Achnanthes lanceolata Grun.

Amphora gracilis Ehr.

- pediculus Grun. ,

$$
\text { - - } \quad \text { v. major Grun. }
$$

Cymbella aspera Ehr.

- cymbiformis Ehr.

- helvetica $\mathrm{Ktz}$.

- maculata Ktz.

- naviculiformis Auerw.

Cocconeis lineata Grun.

- Rouxii $\nabla$. euglypta.

- tenuissima Næg.

Cyciotella stelligera Cl, et Grun.

Diatoma anceps Grun.

- - $\quad$ v. anomalum W. Sm.

- mesodon Ktz.

- pectinale fa elongata $\mathrm{Ktz}$.

- tenue Ag.

Encyonema gracile Rab.

$$
\text { - ventricosum Ktz. }
$$

Bpithemia gibba Ehr.

- - v. ventricosa Grun.

- turgida Ktz.

- V - v. vertagus Ktz.

- zebra $\mathrm{Ktz}$.

Eunotia faba Grun.

- gracilis Rab.

- lunaris Grun.

- - v. excisa Grun.

- minor Rab.

- parallela Ehr.

- pectinalis $\nabla$. striata Rab.

Fragilaria capucina Desm.

- $\quad$ - v. mesolepta Grun.

- elliptica Schum.

- Harissonii Grun.
Fragilaria parasitica Grun.

- virescens Ralfs.

- $\quad$ - fa elougata M.P. et F.H.

\begin{tabular}{|c|c|c|}
\hline- & - & o. clavus Rab. \\
\hline- & $\rightarrow$ & o. laticeps Grun. \\
\hline & & $\begin{array}{l}\text { o. trigonocepha- } \\
\text { lum Ehr. }\end{array}$ \\
\hline
\end{tabular}

Gomphonema acuminatum Ehr.

- abbreviatum Ktz.

- angustatum v. producta Grun.

- auritum A. Br.

- Brebissonii Ktz.

- capitatum Ehr.

- constrictum Ehr.

- _ - $\quad$ r subcapitata Grun.

- elongatum W. Sm.

- exiguum $\mathrm{Ktz}$.

- micropus Ktz.

- - $\quad$ - minor Grun.

- mustela v. curvata J. Br. et M. P.

- parvulum Ktz.

- $\quad$ - $\quad$. lanceolata Ehr.

- sarcophagus Greg.

- subclavatum Grun.

Hantzschia amphioxys Grun.

Meridion circulare $\mathbf{A g}$.

- constrictum Ralfs.

Melosira distans Ehr.

- granulata Ktz.

- nivalis W. Sm.

- orichalcez Mert.

- varians $\mathrm{Ag}$.

Navicula acrosphaeria Bréb.

- $\quad$ - v. minor M.P, et F.H.

- amphigomphus Ehr.

- ampliata Ehr. 
Navicula bacilliformis Grun.

- Bogotensis Grun.

- breoistriata Grun.

- cardinalis Kitz.

- Creguti F.H. et M.P.

- Dariana A.S.

- dicephala W. Sm.

- - o. minor W. Sm.

- elliptica Ktz.

- $\quad$ - $\quad$. major M.P et F.H.

- - $\quad$ v. minutissima Grun.

- firma Ktz.

- - gentilis Donk.

- gibba v. hyalina M.P. et F.H.

- gracillima Pritch.

- iridis $\nabla$. angustata M.P. ef.F.H.

- hemiptera v. Brelawskii M.P. et F.H.

- hybrida M. P. et F.H.

- limosa Ktz.

- $\quad$ - . major M.P. et F.H.

- macra Grun.

- major Ktz.

- nobilis Ehr.

- parva Grun.

- radiosa $\mathrm{Ktz}$.

- - v. acuta Grun.
Navicula Rotæana Grun.

- rupestris $\mathrm{Ktz}$.

- stauroptera Grun.

- $\quad$ - $\quad$ v. gracilis P.P.

- subcapitata Grun.

- - v. paucistriata Grun.

- tenella Bréb.

- viridis $\mathrm{K} \mathrm{tz}$.

- - - v. commutata Grun.

Nitzschia obtusa IV. Sm.

- / spectabilis Rab.

- tubicola Grun.

Stauroneis acuta W. Sm.

- amphilepta Ehr.

- Brunii M.P. et F.H.

- gallica M.P. et F.H.

- phónicenteron Ehr.

- - o. lanceolata fa major $J . B r$.

Synedra ulna Ehr.

$$
\begin{aligned}
& \text { - } \quad \text { - v. danica Ktz. } \\
& \text { - - v. longissima W. Sm. }
\end{aligned}
$$

Tabellaria fenestrata $\mathrm{Ktz}$.

- flocculosa Ktz.

\title{
No 165 THURSDAY ISLAND (Australie)
}

\author{
Lichmophora Ehrenbergii Ktz.
}

$\mathrm{N} \bullet 166$

GRUNDSTAT (Norvège)

Plankton (Collection Gran)

Achnanthes Hauckiana Grun var?

Amphora complanata Grun.

- pusilla H. Per.
Asterionella japonica $\mathrm{Cl}$.

Bacillaria paradoxa Gmel:

Biddulphia aurita Bréb. 
Chatocerus affine Land.

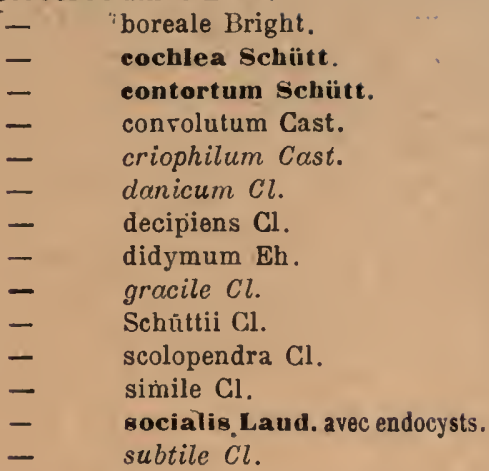

Cocconeis scutellum Eh.

Coscinodisens excentricus $E h$. lineatus $E h$.

Eucampia zodiacus $E h$.

Guinardia flaccida $H$. Per.

Landeria glacialis Grun.

Leptocylindrus danicus $\mathrm{Gl}$.
Melosira sulcata Ktz.

Navicula directa W. Sm.

- Vanhöffenii Grun.

vitzschia closterium W. Sm.

- seriata Cl.

- $\quad$ - v. fraudulenta.

Pleurosigma longum $\mathrm{Cl}$.

Rhizosolenia alata Bright.

- obtusa Hans.

- setigera Bright.

- Shrubsolei Cl.

Rhoicosigma arcticum $\mathrm{Cl}$.

skeletonema costatum Cl.

Stauroneis Growei.

Thalasiosira baltica Grun.

$$
\begin{array}{ll}
- & \text { condensata Cl. } \\
\text { - } & \text { decipiens Grun. } \\
\text { - } & \text { gravida } \mathrm{Cl} . \\
& \text { Nordenskioldii Gl. }
\end{array}
$$

Thalassiothrix Nitzschioïles Grun.

No 167

SOUND of MULL (Écosse)

Plankton (Collection Cleve)

Chretoceros curvisetum $\mathrm{Cl}$. (désagrégé).

- decipiens Cl.

Coscinodiscus nobilis Grun var.

Mölleria cornuta Grun.

Rhizosolenia alata Bright.
Rhizosolenia setigera Bright.

- Shrubsolei Cl.

Skeletonema costatum Cl.

stephanopyxis turgida Grev.

Thalassiosira gravida cil.

Nordenskioldi Cl.

No 168 VASSIVIERE (Puy-de-Dôme - France)

(Dépôt fossile d'eau douce)

BIBLlographie. - Frère J. Héribaud. Les Diatomées d'Au. vergne, 1893. 
Cymbella lanceolata Ehr.

- norcegica Grun.

Encyonema cospitosum Ktz.

$$
\text { - } \quad \text { gracile Rab. }
$$

Epithemia constricta $W$. Sm.

$$
\begin{aligned}
& \text { - } \quad \text { gibberula Ehr. } \\
& \text { - } \quad \text { succincta Bréb. } \\
& \text { - } \quad \text { zebra Ktz. } \\
& \text { arcus } \nabla . \text { bidens Grun. }
\end{aligned}
$$

Eunotia arcus v. hybrida.

- - $\quad$. plicata J. Br. et F.H.

- gracilis Rab.

- lunaris Grun.

- minor Rab.

- parallela Ehr.

- pectinalis $\nabla$. undulata Ralfs.

- - o. ventricosa Grun.

Gomphonema acuminatum Ehr.

- $\quad$ - c. intermedia

- auritum A. Br.

- hebridense Greg.

- parrulum Ktz.

- vibrio Ehr.

Hantzschia amphioxys Grun.

Melosira nivalis W. Sm.

Navicula affinis Ehr.

- Brebissonii Ktz.

- $\quad$ - v. elongata J. Br. et F.H.

- crassinervia Bréb.
Navicula divergens $\mathrm{W} . \mathrm{Sm}$.

- $\quad$ - $\quad$. prolongata J. Br. et F.H.

- exilis Grun.

- gibba Ehr.

- gregaria Donk.

- hemiptera Ktz.

- - var.

- limosa v. subintlata Grun.

- lineolata Ehr.

- major $\mathrm{Ktz}$.

- oblonga $\mathrm{Ktz}$.

- rupestris $\mathrm{Ktz}$.

- serians $\mathrm{Ktz}$.

- - v. minima Grun.

- - o. minor Grun.

- $\quad-\quad$ v. Peragalli J. Br. et F.H.

- tabellaria Ehr.

- viridis $\mathrm{Ktz}$.

Nitzschia fonticola Grun.

Peronia Heribaudi J. Br. et M.P.

Stauroneis pbonicenteron Ehr.

- $\quad$ - v. gracilis J. Br. et M.P.

Surirella angusta $\mathrm{Ktz}$.

- helvetica J. Br.

- splendida Ehr.

- splendidula o. minuta A.S.

Synedra ulna $\nabla$. subæqualis Grun.

Tabellaria fenestrata Ktz.

- $\quad$ flocculosa Ktz.

- $\quad$ - $\quad$ v. biceps Ehr.

No 169

LA BADE (Cantal - France)

Dépôt fossile d'eau douce

BIBLIOGR A PHIE. - Frère J. Héribaud. Diatomées d'Auvergne.

Amphora pediculus $K t z$.

Cyclotella Charetoni F.II.

- $\quad$ - . radiata F.H. et M.P

- - - v. scutiformis F.H.
Cymbella Charetoni F.H.

- Ehrenbergii Ktz.

- lanceolata Ehr.

- turgidula Grun. 
Epithemia Hyndmanni W.Sm.

- gibba Ktz.

- turgida Ktz.

- zebra Ktz.

Eunotia incisa Eh.

- lunaris Eh.

- polyglyphis Grun.

Fragilaria laponica Grun.

Gomphonema intricatum $\mathrm{K} t z$.

Melosira arenaria Moor.
Melosira undulata $\mathrm{K} t z$.

- - o. producta A.S.

Navicula acrosphceria 0 . badeana $F .11$.

$$
\text { et M.P. }
$$

- elliptica Ktz.

- major Kitz.

- peregrina Ktz.

- radiosa Kítz.

- - o. acuta Grun.

Surirella saxonica Auers.

Synedra pliocenica F.H. et M.P.

\section{No 170 VISCAINO BAY - CALIFORNIE (États-Unis)}

Sur les algues

\section{Isthmia nervosa Ktz.}

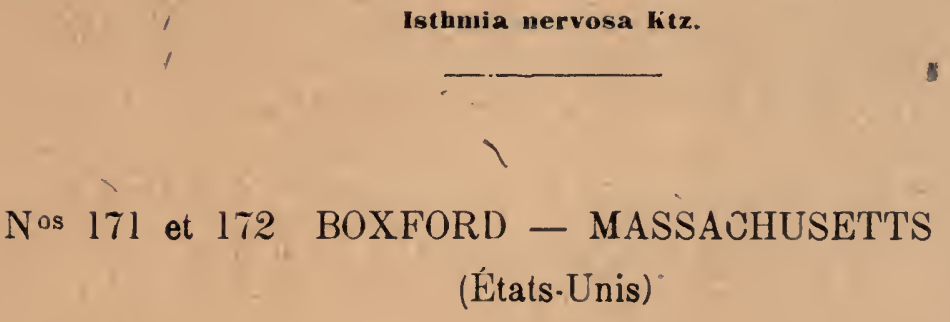

Amphora Libyca Eh.

Cocconeis placentula Eh.

Cymbella americana A.S.

- cuneata M. Per. n. sp. Formè de deux extrémitès fortement coniques et finement arrondies ; peu dissymétrique, replié, presque droit, aire axiale notable, lancéolée, stries granulées au nombre de 7 à 8 en $10 \mu$ au dos, 9 au ventre, au milieu, plus serrées aux extrémitès. Longueur 90 à $120 \mu$.

- gastroiiles kitz.

- heretopleura Ktz.
Epithemia amphicephala Grun.

- gibba Ktz.

- - var ventricosa.

- turgida Ktz.

Eunotia arcus Eh.

- formica Eh.

- gracilis Rab.

- - v. capitata M.P. et F.H.

- impressa Eh.

- incisa Greg.

- lunaris Grun.

- pectinalis Rab.

- - - $\quad$. ventricosa.

- - - fa arverna. Héribaud $19035 / 5$ bien assez diffèrente de la formo do Gru- 
Funotia prorupta $\nabla$. bidens.

now pour mériter une dénomination particulière.

Gomphonema acuminatum Eh.

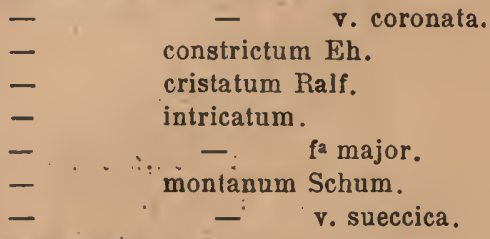

Hantzschia amphioxys Grun.

Melosira laevis Grun.

Meridion constrictum Ralfs.

Navicula acrosphaeria v. minor.

- americana Eh.

$$
\begin{aligned}
& \text { - } \quad \text { - } \quad \text { v. bacillaris. } \\
& \text { - - - } \quad \text { - minor. } \\
& \text { - } \quad-\quad \text { v. moesta. } \\
& \text { - amphigomphus Eh. } \\
& \text { - bacilliformis Grun. } \\
& \text { - commutata Grun. } \\
& \text { - conspicua A. S. oar. } \\
& \text { - cuspidata Ktz. } \\
& \text { - } \quad \text { fa.craticula. } \\
& \text { - esox Eh var. } \\
& \text { - flammula A. S. var. } \\
& \text { - gentilis Donk. }
\end{aligned}
$$

Navicula gibba Eh.

- - v. hyalina.

- gracillima Greg.

- hemiptera Rab.

- heroina A. S. var.

- instabilis A. S.

- iridis Eh.

- - v. ampliata.

- legumen Eh.

- limosa Ktz.

- macilentö oar.

- major Ktz.

- nobilis Eh.

- nodosa Ktz. A. S. atl. 45/56.

- peripunctata J. Br.

- producta W. Sm.

- semen Eh.

- viridis $\mathrm{Ktz}$.

Nitzschia sigmoidea W. Sm.

Stauroneis acuta W. Sm.

- Baileyi Eh.

- Brunii M. P. et F. H.

- gracilis Eh.

- lanceolata Ktz.

- Phœenicenteron Eh.

Synedra ulna $E h$.

Tabellaria fenestrata Ktz.

- flocculosa Ktz.

\section{Nor 173 à 175 NYERMEGY (Hongrie)}

Dépôt fossile saumâtre

Bibliographie. - J. Pantocsek. Diatomées de Hongrie, vol. III.

Achnanthes Baldjickii Grun.

Actinocyclus circumajatús Pant.

Actinoptychus undulatus Eh.

Amphiprora biharensis Pant.

punctata Pant.

striata Pant.
Anphora crassa Grev. v. punctata Grun.

- gigantea Grun var.

- Kossuthii Pant.

Asteromphalus Brookei Bail.

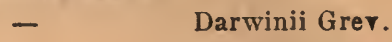

hungaricus Pant. 
Auricula Grunowii Pant.

Campylodiscus Bergonii Pant.

Climacosphenia' hungarica Pant.

Cocconeis scutellum Eh.

Coscinodiscus avalis Roper.

- . poseudolineatus Pant. symbolophorus Grun var.

Epithenia gibberula Ktz. v: protracta.

- tertiaria Pant.

Granmatophora hungarica Pant.

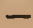

$$
\text { oceanica Eh. v. subtilis }
$$$$
\text { sima Grun. }
$$

Hyalodiscus scoticus Grun.

Mastogloia Braunii var.

- Debyi Cl.

- Kinkerii Pant.

- lanceolata Thw.

- neogena Pant.

- rhomboidalis Pant.

Melosira fungiformis Pant.
Navicula difficilis Pant.

- filiformis Pant.

- Hennedyi. IV. Sm. y. fossilis.

- irregularis Pant.

- lyra Eh. v. atlantica.

- Martoufli Pant.

- Orphei Pant.

- perducta Pant.

- robusta Pant.

- Trevelyana. Donkk v. Hungarica.

- Vaszaryi Pant.

Nitzschia costata Pant.

Orthoneis Pethoi Pant.

Plagiogramma Gregorianum Greo.

Pqdosira Loczyi Pant.

Rhabdonema adriaticum W. Sm.

- hamuliferum Kitt.

Rhizosolenia squamosa Pant.

Surirella Baldjickii Norm.

- Kinkerii Pant.

Synedra tæniata Pant.

\section{Nor 176 et 177 , SACO - MAINE (États-Unis)}

Amphora Libyca Eh.

Cocconeis pediculus $E h$.

Cymbella cistula Hemp.

- gastroides $\tilde{\mathrm{n}} \mathrm{tz}$.

- heteropleura Ktz.

- maculata $\mathrm{Ktz}$.

- pusilla Grun.

Diatoma anceps o. capitata.

Encyonema Auerswaldii Rab.

\section{- gracile Eh. \\ - intermedium M.P. et F.H. \\ - turgidum Grun. \\ - ventricosum Grun.}

Epithemia turgida $\hbar t z$.
-
-
○. granulata.

$$
\text { zebra v. proboscidea. }
$$

Eunotia arcus $\mathrm{Eh}$.

- - - minor.

- - v. uncinata.
Eunotia bidentula W. Sm. flexuosd o. pachycephala.

- lunaris Grun.

- monodon Eh.

- pectinalis Rab.

- - - v. stricta.

- - v. ventricosa.

- - v. biarcuata M. Per. à ventre proéminent et à face dorsale s'inflẻchissanl parallèlement à la face ventrale.

- polyglyphis $\vee$. hexaglyphis.

- - - v. heptoglyphis.

- robusta $v$. tetraodon.

- $\quad$ - $\quad$ เ. à $7,8,9,10,11,12$, $13,14,22$ odon etc.

- - oar. serra. 
Fragilaria brevistriata Grun.

$$
\text { -. virescens Ralfs. }
$$$$
\text { - undata W. Sm. }
$$

Gomphonema acuminatum Eh.

$$
\begin{array}{ll}
\text { - } & \text { - } \quad \text { vicatum Eh. coronata. } \\
\text { - } & \text { capitatum Lh. } \\
\text { - } & \text { constrictum Eh. } \\
\text { - } & \text { gracile Eh. } \\
\text { - } & \text { intricatum Ktz. } \\
\text { - } & \text { montanum Schum. } \\
\text { - } & \text { - } \quad \text { v. suecica. } \\
\text { - } & \text { subtilis Eh. }
\end{array}
$$

Melosira distans var.

Navieula amphigomphus Eh.

1- appendiculata Ktz.

- Brebissonii Ktz.

- Cesatii Rab.

- divergens W. Sm.

- dubia Eh.

- elliptica Ktz.

- firma Ktz.

- gibba Eh.

- gigas Ktz.

- gracillima Greg.

- iridis Eh.

- - $\quad$. ampliata.

- limosa? $\nabla$. dilatata $n$. var. H. Per. - Elliptique, allongéo; aire axiale lancéolèe no-
Navieula major Ktz.

table, élargie autour des nodules ; Long. 40 à $60 \mu$, larg. $14 \mu .14$ à 15 stries on $10 \mu$.

- mesolopta $\nabla$. stauroneiformis.

- nobilis Eh.

- parea Eh.

- producta W. Sm.

- pseudobacillum Grun.

- radiosa $\mathrm{Ktz}$.

- serians Eh.

- - v. minor.

- stauroptera Greg.

- subcapitata Greg.

- - - v. stauroneiformis.

- centricosa Eh.

- viridis $\mathrm{Ktz}$.

Stauroneis acuta $\nabla$. maxima.

- anceps $\nabla$. lineare.

- Brunii M.P. et F.H.

- gracilis Eh.

- lanceolata $\mathrm{Ktz}$.

- Phuenicenteron Eh.

Tabellaria fenestrata Ktz.

$$
\text { - flocculosa Ktz. }
$$

Van Ileurckia crassinervia. viridula $\nabla$. lineolata $n$. var. M. Per. - De forme lancéolée, à stries longitudinales ondulées bien visibles. Longueur $100 \dot{a}$ $110 \mu$.

$\mathrm{N}^{\circ} 178$ et 179 SHILOH - NEW JERSEY

(États-Unis)

Argile miocène provenant d'un puits artésien

Actinocyclus Ehrenbergii Ralf.

Actinoptychus heliopelta Grun.

$$
\begin{array}{ll}
\text { - } & \text { splendens Ralfs. } \\
\text { - } & \text { undulatus Eh. } \\
\text { vulgaris Schum. }
\end{array}
$$

Asteromphalus Dallasianus Grev.
Aulacoliscus crux Eh.

- margaritaceus Ralfs.

Auliscus cœlatus Bail.

- pruinosus Bail var.

- radiatus Grev.

Coscinodiscus apiculatus Eh. 
Cescinodiscus argus Eh.

$\begin{array}{ll}\text { - } & \text { asteromphalus Eh. } \\ \text { - } & \text { biangulatus A.S. } \\ \text { - } & \text { bulliens A.S. } \\ \text { - } & \text { concinnus W. Sm. var. } \\ \text { - } & \text { denarius A.S. } \\ \text { - } & \text { excavatus Eh. } \\ \text { - } & \text { fimbriatus Eh. } \\ - & \text { gigas Eh. var. } \\ \text { - } & \text { heteroporus Eh. } \\ \text { - } & \text { marginatus Eh. } \\ \text { - } & \text { oculus iridis Hh. } \\ \text { - } & \text { perforatis Eh. }\end{array}$

Coscinodiscus radiatus Eh.

$$
\text { - subtilis Eh. }
$$

Craspedodiscus elegans Eh.

Eupodiscus Rogersii Eh.

Hyalodiscus radiatus $O$. Witt.

Melosira sulcata $\mathrm{Ktz}$.

$$
\text { - - V. coronata. }
$$

Pseudauliscus Ralfsianus Grev.

Stephanopyxis corona Eh.

$$
\text { - turris Eh. }
$$

Terpsinoe americana fa trigona.

Triceratium americanum Ralfs. multifrons $\mathrm{J} . \mathrm{Br}$.

\section{Plankton (Collection Gran)}

Biddulphia aurita Bréb.

Chretoceros angulatum Schūtt avec endocysts.

$\begin{array}{ll}\text { - } & \text { boreale Bail. } \\ \text { - } & \text { cochlea Schütt. } \\ \text { - } & \text { criophilum Cast. } \\ \text { - } & \text { curvisetum Cl. } \\ \text { - } & \text { decipiens } \mathrm{Cl} . \\ \text { - } & \text { didymus Eh. avec endocysts. } \\ \text { - } & \text { mamillanum Cl. } \\ \text { - } & \text { scolopendra } \mathrm{Cl} . \\ \text { - } & \text { socialis Laud. } \\ & \text { teres Cl. }\end{array}$

Ditylium Brightwellii West.
Guinardia flaccida $H$. Per. Hyalodiscus stelliger Bail. Lauderia compressa H. Per. Leptocylindrus danicus $\mathrm{Cl}$.

Nitzschia seriata $C l$.

Rhizosolenla alata Bright.

$$
\begin{array}{ll}
\text { - } & \text { calcar avis Schulze. } \\
\text { - } & \text { setigera Bright. } \\
\text { Schrubsolii Cl. }
\end{array}
$$

Skeletonema costatum (il.

Thalassiosira Nordenskioldii Cl.

Thalassiothrix nitzschioïdes Grun.

$\mathrm{N}^{\circ} 181$

GULLIN - NORVEGE

Plankton (Collection Cleve)

Asterionella japonica $\mathrm{Cl}$. Biddulphia aurita Bréb.
Chatoceros boreale Bright. cochlea Schūtt. 
Chætoceros contortum Schütt.

- conoolutum Cast.

- danicum Gl.

- decipiens Cl.

- didymus Eh.

- scolopendra Cl.

- socialis Laud.

- Willei Grun.

Coscinodiscus asteromphalus Eh.

Eucampia zodiacus Eh.

Lauderia glacialis Grun.
Leptocylindrus danicus $\mathrm{Cl}$.

Nitzschia closterium W. Sm.

- seriata GI.

- - var. fraudulenta.

Rhizosolenia alata Bright.

- $\quad$ obtusa Hensen.

setigera Bright.

Skeletonema costatum Cl.

Thalasiosira gravida $\mathrm{Cl}$.

- Nordenskioldij Cl.

Thalassiothrix nitzchioïdes Grun.

\section{N* 182 et 18:3 RICHMOND - VIRGINIE (Etats-Unis)}

\section{Dépôt fossile marin}

BIBLIOGRAPHIE. - Ehrenberg. Microgéologie, PI. XVIII. Ad. Schmitd Atlas.

Actinoptychus amblyoceros Eh.

- Ebrenbergii Ralfs.

- $\quad$ ellipticus Grun.

- $\quad$ fasciculatus Cast.

- moniliformis Ralfs.

- splendens Schadb.

- undulatus Eh.

- vulgaris Schum.

Asterolampra marylandica $E h$.

Aulacodiscus argus $E \dot{h}$.

- crux Eh.

- Ehrenbergii Jan.

Bacteriastrum varians Laud.

Biddulphia seticulosa Grun.

- tridentata Eh. .

Brunia mirabilis, Temp. (en fragments).

Cestodiscus ovalis Grev.

Chatoceros gastridium Eh.

Cladogramma californicum Eh.

Cocconeis Febigeri $\mathrm{J}$. Br.

Coscinodiscus apiculatus Eh.

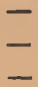

- v. maxima Grun. armatus Grev.
Coscinodiscus argus Eh.

asperulus Grun. asteromphalus Eh. biangulatus $\mathrm{A}$. S. centralis Eh. cinctus $K t z$. curvatulus Eh.

- var. gemina Grun. - var. minor Eh. - var. recta Ratt. epiphanes Ratt. excentricus Eh. gigas Eh.

- var. duplicata Grun. inclusus Ratt. Janischii A. S. lacustris o. septentrio. nales Grun. Lewisianus Greo. lineatus Eh. marginatus Fh. minor Eh. nobilis Grun. nodulifer Jan. Normannii Grev. 
Coscinodiscus Ocului iridis Bh.

$\begin{array}{ll}- & \text { odontodiscus Grun. } \\ \text { - } & \text { omphalanthus Eh. } \\ - & \text { perforatus Eh. } \\ \text { - } & \text { punctatus Eh. } \\ \text { - } & \text { radiatus Eh. } \\ - & \text { radiolatus Eh. } \\ - & \text { robustus Grev. } \\ - & \text { subtilis var. } \\ -\quad & \text { symbolophorus Eh. } \\ - & \text { turgidus Ratt. } \\ - & \text { oelatus Eh. } \\ \text { - } & \text { vetustissimus Pant. } \\ - & \text { Voodwardii Eul. }\end{array}$

Craspedodiscas coscinodiscus Eh.

- Eucampia virginica Grun.

Goniothecium odontella Eh.

Hyalodiscus subtilis Bail.

Mastogonia actinoptychus Eh.

Melosira suleata Ktz.

$$
\begin{array}{lll}
- & - & \text { v. minor Ktz. } \\
- & - & \text { v. separanda A. S. } \\
- & - & \text { fa radiata Grun. }
\end{array}
$$

Navicula confoederata Pant.

- distans W. Sm.

- prisca A. S.

Pleurosigma affine v. fossilis Grun.

Pyxilla americana Eh.

- dubia Grun.

Stephanogonia polygona Eh.

Stephanopyxis corona Grev.

$$
\text { - limbata Eh. }
$$$$
\text { - turris Grov. }
$$

Stictodiscus Kittonianus Grev.

Surirella alternans $A$. $S$.

Triceratium acutum Eh.

-- irregulare o. hebetata Grun

- membranaceum Bright.

- obtusum Eh.

- reticulatum Eh.

- sculptum v. petropolitana Grun.

- tridactylum Bright.

Zygoceros circinnus Ralfs.

No 184 VILLERS-SUR-MER (Calvados) France

Sur le sable

Chatoceros armatum West.

No 185 SAINT-LUNAIRE (Ille-et-Vilaine) France

Sur les algues

Isthnia enervis Kiz.

ABERDEEN (Écosse) 


\section{No 187 DAVIS PIT - QUININPIAC RIVER CONNECTICUT (États-Unis)}

Achnanthes subsessilis Ktz.

Temperei M. Per. n. sp. Frustule à valves diaphramées à leur base; valve supérieure à pseudo-stauros tout à fait marginal, présentant à chaque extrémité une petite area unilatérale. Valve inférieure à stauros assez large 8 à 10 lignes de granules en $10 \mu$.

Actinocyelus Barkleyi Grun.

$$
\text { - } \quad \text { crassus W. Sm. var. }
$$

Actinoptychus areolatus $\mathrm{Eh}$.

$$
\text { - } \quad \begin{aligned}
& \text { - } \\
& \text { - }
\end{aligned} \quad \text { untermedius A. S. var. }
$$

A mphiprora decussata $\mathrm{Cl}$.

$$
\text { - } \quad \text { lepidoptera Greg. }
$$$$
\text { - } \quad \text { - v. pulchella. }
$$

Amphora angusta Greg.

- angulata $\mathrm{Cl}$.

- mexicana A. S. var.

- ovalis Ktz.

Auliscus colatus Bail.

- moronensis Grun.

Biddulphia rhombus $W$. Sm.

Brebissonia Boeckii Grun.

Campylodiscus echeneis Eh. T

cocconeis scutellum Eh.

Cocconema gibbum $E h$.

Coscinodiscus asteromphalus Eh.

- blandus A. S.

- excentricus Eh.

- - v. minor.

- nitidus Greg.

- oculus iridis Eh.

- radiatus Eh.
Cyclotella striata Grun.

Cymatopleura elliptica W. Sm.

$$
\text { - solea W. Sm. }
$$

Epithemia amphicephala Grun.

$$
\begin{array}{lcl}
\text { - } & \text { gibberula Ktz. } \\
\text { - } & - & \text { var. producta. } \\
\text { - } & \text { - } & \text { var. protracta. } \\
\text { - } & \text { - }
\end{array}
$$

Fragilaria capucina Desm.

Grammatophora macilenta W. Sm.

llyalodiscus scoticus Grun.

- stelligera Bail.

Melosira Borreri Grev.

- sulcata $v$. coronata.

- - v. radiata.

- undulata Eh.

Navicula abrupta var, atlantica.

- Attwoodii M. Per. n. sp. (Pinnularia) bacillaire, à extrémités atténuées largement arrondies; area largement stauroneiforme évasée; côtes lisses 10 en $10 \mu$. Long. 45 à $60 \mu$.

- Brasiliensis Grun.

- brevis Greg.

- Campbellii M. Per. n. sp. (Caloneis) semblable à la forme de A. S. atlas, Pl. 50/48, dont elle diffère surtout par son area médiane strauroneiforme, 20 stries en $10 \mu$. Long. 50 à $70 \mu$.

- cancellata Donk.

- $\quad$ - var. Gregorii:

- clavata Greg.

- Dariana A.S.

- Delawarensís Grun v. robusta n. var. Plus trapu ot à stries plus écartées que dans le type (11 à 12 en $10 \mu$ ).

- elegans W.Sm. 
Navlcula elliptica Ktz.

- erythrea Grun.

- - var.

- formosa Greg.

- fortis Greg.

- fusca Rulfs.

- gigas Eh.

- Grundleri A. S.

- Hennedyi W. Sm.

- - $\quad$ - furcata.

- $\quad$ - . Niceaensis.

- - var.

- humerosa Bréb.

- icostauron Grun.

- interrupta Ktz.

- irrorata Grev. var.

- legumen Eh.

- Lyra Eh.

- - var. dilatata.

- - var. elliptica.

- - var. recta.

- major Eh.

- nobilis Eh.

- pachyptera Eh.

- peregrina Kitz.

- polysticta A. S. var.

- pretexta Eh.

- pusilla W. Sm.

- pygmæa Ktz.

- spectabilis Greg.

- ventricosa Eh.

- viridis Eh.

- - var. commutata.

Nitzschia acuminata W. Sm.

- bilolata W. Sm.

- circumsuta Grun.

- Fasciculata Grun.

- granulata Grun.

- littoralis Grun.

- obtusa W. Sm.

- plana W. Sm.

- scalaris W. Sm.

- sigma W. Sm.

- Tryblionella Hantz.

- - - - maxima.

Opephora Schwartzi P. P.
Plagiogramma pulchellum Greo.

Pleurosigma angulatum IV. Sm.

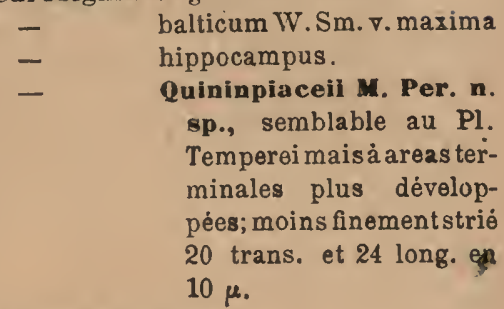

Pyxilla baltica Grun.

- dubia Grun.

Rhabdonema adriaticum Ktz.

Raphoneis amphiceros $\mathrm{Eh}$.

- - var. rhombica.

Skeletonema mediterraneum Grun.

Stauroneis acuta $W$. $\$ \$ m$.

- anceps var. Kinearis.

- Bakerii M.Per. n. sp. Lancéolè à extrémitès produites, lumen visible sur toute la longueur de la valve;

) stauros large; stries très radiantes, 20 en $10 \mu$. Long. $55 \mu$.

- phoenicenteron Eh.

stephanopyxis turris var. inermis.

Surirella crumena Bréb.

- fastuosa Eh. var.

- ovalis ver. Kotschiana.

- salina W. Sm.

- striatula Turp.

- tenera Greg.

Syndendrium Diadema Eh.

Synedra crystallina $K t$.

- pulchella Ktz.

- ulna Eh. o. oequalis.

Terpsinoe americana Bail.

Trachyneis aspera $\mathrm{Cl}$.

Triceratium alternans Bail.

farus Eh. 


\title{
No 188 QUININPIAC RIVER - CONNECTICUT
}

\author{
(Etats-Unis)
}

Sur les algues

Aohnanthes subsessilis $\mathrm{Ktz}$.

Actinocyclus Barkleyi Grun.

Actinoptychus undulatus Eh.

Amphiprora paludosa $W . S m$ pulchra v. pulchella.

Amphora angusta Greg. oar. - costata W. Sm.

Aulacodiscus Johnsonii Arn.

Brebissonia Boeckii Grun.

Cerataulus lavis var. robusta n. var., tout en ayant les valres absolument plates du $C$. loevis, il a la forte striation du C. polymorphus.

Cocconeis dirupta Greg.

- scutellum Eh.

Coscinodiscus excentricus Eh.

$$
\text { - } \quad 1 . \text { radiatus Eh. }
$$

Cyclotella striata Grun.

Epithemia gibba $\mathrm{Ktz}$.

$$
\text { - turgida Ktz. }
$$

Eunotia arcus $E h$.

Fragilaria capucina Desm.

Melosira Jurgensii Ag.

- mummuloides Ag.

- setosa Grev. var.

- sulcata v: radiata.

Navicula Baileyana var.

- Delawarensis v. robusta.

- elegans W. Sm. var.

- elliptica $K \Varangle z$.

- formosa Greg.

- furca Ralfs.

- humerosa Brèb.
Navicula icostauron $\mathrm{Cl}$.

- interrupta Ktz.

- Lyra Eh.

- major Ktz.

- peregrina Eh.

- pusilla W. Sm.

- viridis $\mathrm{Ktz}$.

- Campbellii M. Per.

Nitzschia bilobata W. Sm.

- incurva Grun.

- littoralis Grun.

- Lorenziana Grun.

- obtusa W. Sm. oar.

- paradoxa Gmel.

- scalaris W. sm.

- sigma W. Sm, var. rigida.

- - var, rigidulata.

- Tryblionella Hantz.

Pleurosigma angulatum W. Sm.

- hippocampus W. Sm.

- Karianum Grun.

- latum Grun.

- Quininpiaceii M. Per.

- Terryanum H. Per.

Rhabdonema adriaticum $\mathrm{Ktz}$.

Raphoneis amphiceros Eh.

Stauroneis gracilis $E h$.

- Gregorii Ralfs. var.

- phœnicenteron Eh.

Surirella Kittonii A. S.

- ovalis v. Kotschiana.

- - ovata Ktz.

- salina iv. Sm.

- tenera Greg.

Synedra affinis v. hybrida.

- pulchella Ktz. 


\title{
No 189 QUININPIAC RIVER - CONNECTICUT
}

\author{
(États-Unis).
}

\section{Estuaire (vase)}

Achnanthes Temperei M. Per.

Actinocyclus Barkleyi Grun.

$\begin{array}{ll}\text { - } & \text { crassus W. Sm. } \\ \text { - } & \text { nebulosus M. Per. } \\ \text { - } & \text { Ralfsii Pritch. } \\ \text { sp.? }\end{array}$

Actinoptychus amblyoceros Eh.

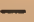

pseudoantistrophus $\mathrm{MI}$. Per.

n. sp. - Diffère de $A$. antistrophus. (A. S. atlas $156 / 11$ ) en ce que chaque compartiment porte une épino. Diam. $45 \mu$.

- racemosus A. S. var.

- undulatus Eh.

Amphiprora pulchra Bail.

Amphora cingulata Cl.

- var. pulchella.

- Glevei Grun.

- - var. splendida n. var. Diffère du type principalemerit par son nombre de stries 8 en $10 . \mu$

- mongolica Ost. oar.

- obtusa Greg.

- proteus Greg.

Adiscus colatus Bail.

- sculptus Eh.

Bacillaria paradoxa Gmel.

Biddnlphia aurita Bréb.

- rhombus W. Sm.

Brebissonia Boeckii Grun.

Campylodiscus echeneis Eh.

Cerataulus lævis var. robusta M. Per.

- turgidus Eh.

Cocconeis lineata $E h$.

- scutellum Eh.
Cocconema gibbum Eh.

Coscinodiscus excentricus Eh.

- nitidus Greg.

- oculus iridis Eh.

- radiatus $\mathrm{Eh}$.

Cyclotella striata Grun.

Cymatopleura elliptica W. Sm.

Cymbella cuspidata Ktz.

- gastroides $\mathrm{Ktz}$.

Denticula antillarum Cl. var.

Epithemia gibberula var. producta.

- musculus Ktz.

- turgida o granulata.

Buphyllodium spathulatum Shad.

Gomphonema sphcrophorum Eh.

Melosira sulcata Eh.

- - var. coronata.

Navicula acrosphoeria $K t z$.

- aestuarii Cl.

- approximata Grev.

- arabica Grun.

- Atwoodii M. Per.

- brasiliensis Grun.

- circumsecta Grun.

- clavata Greg.

- Delawarensis Grun.

- - var. robusta.

- digito-radiata Greg.

- elegans W. Sm.

- elliptica Ktz.

- excavata Greo.

- Fisheri var. gigas n. var. Long. $180 \mu, 5$ stries en $10 \mu$.

- formosa Greg.

- Pusca Ralrs.

- Grundleri A. S.

- hennedyi W. Sm.

- humerosa Brób. 
Navicula icostauron Grun.

- incurvata Greg.

- interrupta Eh.

- irrorata Grun.

- latissima Greg.

- limosa Ktz.

- Lyra Eh.

- - var. atlantica.

- - var. dilatata.

- - var. elliptica.

- - var. insignis.

- - var. subtypica.

- major Ktz.

- nobilis Eh.

- Noræ Guineensis Temp.

- palpebralis Breb.

- peregrina ktz.

- permagna Greg. var.

- pusilla W. Sm.

- pygmea Ktz.

- Smithii Bréb.

- streptoraphe Cl.

- viridis Ktz.

- yarrensis Grun.

Nitzschia acuminata W. Sm.

- circumsuta Grun.

- fasciculata Grun.

- granulata Grun.

- obtusa W. Sm.

- plana W. Sm.

- scalaris W. Sm.

- sigma W. Sm.

- Tryblionella Hantz.

- - var. maxima.

- $\quad$ - var.

- vitrea Norm
Plagiotropis seriata Cl.

Plaglogramma Gregorianum Grev

Pleurosigma affine Grun:

- angulatum W. Sm.

- balticum W. Sm.

- - var. maxima

- hippocampus W. Sm.

- Quininpiaceii M. Per.

- Spencerii W. Sm.

- $\quad$ strigilis W. Sm.

- strigosum W. Sm.

Pseudauliscus radiatus Ratt.

Pyxilla baltica Grun.

Rhabdonema adriaticum Ktz.

Raphoneis amphiceros Eh.

Scoliopleura tumida Rab.

Stauroneis Gregorii Ralfs.

- Phœnicenteron Eh.

Stephanopyxis turris Ralf.

Surirella cardinalis Kitt.

- crumena Bréb.

- fastuosa Eh.

- salina W. Sm.

- striatula Turp.

- - var.

Syndendrium diadema Eh.

Synedra affinis var. hybrida.

- crystallina $\mathrm{Ktz}$.

- pulchella Ktz.

Tabellaria fenestrata $K t z$.

Terpsinoe americana Ralf.

Triceratium alternans Bail.

Nos 190 et 191 " POURCHERES (Ardèche) France

Dépôt fossile d'eau douce

BIBliographiE. - Frère J. Héribaud. Les Diatomées fossiles d'A uvergne, 1903.

Cymbella alpina Grun.

- arctica $\nabla$. Lag.

Cymbella cistula Hemp.

- aspera Ehr.

- helvetica kitz.

- hungarica v. Grun. 
Cymbella maculata Ktz.

$$
\text { - parva W. Sm. }
$$

Cocconeis lineata Grun.

Diploneis elliptica Ktz.

Encyonema lunula Grun.

$$
\text { - n. sp. ? }
$$

Epithenia Hyndmanii W. Sm.

$$
\begin{array}{lll}
- & - & \text { v. interrupta P. H. } \\
\text { - } & \text { Westermanii ktz. }
\end{array}
$$

Eunotia polyglyphis Grun.

- - v. pentaglyphis Grun.

Gomphonema insigne Grun.
Gomphonema montanum Schum.

- subclavatum Grun.

- n. sp.

Melosira arenaria Moor.

$$
\begin{aligned}
& \text { - - - } \quad \text {. lævis F. H. } \\
& \text { - granulata Eh. } \\
& \text { - - } \text { - curvata Grun . } \\
& \text { - undulata Ktz. }
\end{aligned}
$$

Navicula major $\mathrm{Ktz}$.

- menisculus Schum.

- radiosa Ktz.

Surirella turgida W. Śm.

$N^{\circ} 192$

CHARAY (Ardèche)

Argile à Jiatomées

BIBLIOGRAPHIE. - Frère J. Héribaud. Les Diatomées fossiles d'Auvergne, 1903.

Amphora lybica Ehr.

Cocconeis californica Grun.

$$
\begin{aligned}
& \text { - } \quad \text { - } \quad \text { r. subcontinua Grun. } \\
& \text { - } \quad \text { placentula Ehr. }
\end{aligned}
$$

Coscinodiscus exasperans Ratt.

Cyclotelia comta Ktz.

- Meneghiniana var.

- striata v. ambigua Grun.
Cynubella helvetica $\mathrm{K} t z$.

Epithemia argus Ktz.

- Hyndmannii W. Sm.

- soréx $\mathrm{Ktz}$.

- zebra Ktz. $\nabla$. proboscideaGrun.

Melosina tenuissima Grun.

Pleurosigma Brebissonii Grun.

Synedra capitata Eh.

- ulna Eh.
Achitunthes brevipes Ag.

$$
\text { - longipes } \mathrm{Ag} \text {. }
$$

Actinocyclus crassus IV. Sm .

$$
\begin{aligned}
& \text { - } \quad \text { Ehrenbergii Ralfs. } \\
& \text { - Ralfsii Pritch. }
\end{aligned}
$$

Actinoptychus undulatus Eh.

Amphora cingulata $\mathrm{Cl}$.

Auliscus sculptus W. Sm.

Biddulphia aurita Bréb. 
Coscinodiscus oculus-iridis ELh .

Epithemia turgida Ktz.

Grammatophora marina $\mathrm{Ktz}$.

Ilyalodiscus scoticus W. Sm.

Navicula aspera Eh.

- brevis Greg.

- didyma Ktz.

- elegans W. Sm.

- forcipata Grun.

- formosa Greg.

- fusca Greg.

- Hennedyi W. Sm. var.

- liber W. Sm.

- lyra Eh. et var.

- maxima Greg.

- peregrina $\mathrm{Ktz}$.

- Smithii Bréb.
Navicula subsalina Donk.

Nitzschia acuminata W. Sm. - circumsuta Bail.

Pleurosigma affine Grun.

- balticum W. Sm.

- marinum Donk.

Pyxilla baltica Grun.

Rhabdonema minutum Ktz.

Scoliopleura tumida Bréb.

Stauroneis Gregori Ralfs.

Surirella striatula Turpin.

Synedra fulgens W. Sm.

- Hennedyana Greg.

- undulata Greg.

Triceratium favus Eh.

No 195 LE HAVRE (Seine-Inférieure) France Synedra fulgens $W$. Sm.

No 196 FONTAINE DE LAA PLACE DELILLE

Clermont-Ferrand (Auvergne)

Bibliographie. - Frère J. Héribaud. Les 'Diatomées d'Auvergne.

Diatoma hyemale var. mesodon.

- vulgare var. linearis.

Encyonema coespitosum Ktz.

Fragilaria intermedia Grun.

Gomphonema constrictum v. subcapitata.

Melosira varians Ag.

Navicula cryptocephala var. exllis.
Nitzschia communis var. abbreviata.

- fonticola Grun.

- frustulum Grun.

- minuta Bleish (palea var.).

- palea W. Sm.

Synedra ulna Eh.

- var. squalis. 
Cocconeis pediculus Eh.

Cymbella hungarica Grun.

- variabilis West.

Gomphonema angustatum $\mathrm{Ktz}$.
Gomphònema augustatum var. producta obtusatum $\mathrm{Ktz}$.

Synedra ulna Eh.

- var. æqualis.

\section{No 198 CARENAGE - TRINIDAD (Antilles)}

Achnanthes longipes var. minutissima $n$. var. - Très petite variété n'atteignant pas $25 \mu$ de longueur.

- subsessilis var. enervis P. $\mathbf{P}_{\text {. }}$

Biddulphia autita Bréb.

Coscinodiscus excentricus Eh.

$$
\text { - turgidus Ratt.? }
$$

Epithemia gibberula fa minor.

Grammatophora intermedia Grun.
Grammatophora serpentina fa minima.

Melosira Borreri fa minor.

Nitxschia panduriformis v. minor.

- sigma W. Sm.

Orthoneis binotata var. trinitatis $\mathrm{n}$. var. - Diffère du type par sa forme plus large à extrémités un peu coniques, une seule logette de chaque côté. Long. 22 , larg. $16 \mu$.

Synedra intermedia (affinis var.) Grun.
Achnanthes brevipes Ag.

$$
\text { - longipes Ag. }
$$

Aetinoptychus maculatus $\mathrm{Gr}$. et St.

Biddulphia aurita Brèb.

$$
\text { - pulchella Greg. }
$$

Coscinodiscus excentricus var. minor. Cyclotella striata Grun.

Dimeregramma nanum Grev.

Epithemia constricta Bréb. musculus $\mathrm{K} t \mathrm{x}$.
Grammatophora maxima fa minor. Mastogloia Grunowii A. S.

Navicula cancellata var. apiculata Greg. Plagiogramma Gregorianum Grev. Podosira Montagnei htz.

Raphoneis amphiceros var. rhombica. - Castracanei Grun.

Synedra hyalina M. Per. n. sp. - Très longuement lancéolée, à extrémitér quelquefois un peu ré- 
trécies et légèrement capitées. | Trachyneis aspera car. pulchella $\mathrm{Cl}$.

Pseudo raphé et stries invisi-

bles. Long. 350 à $500 \mu$.

Triceratium bicorne $\mathrm{Cl}$.

\title{
$\mathrm{N}^{\circ} 200$ PORT OF SPAIN - TRINIDAD (Antilles)
}

\section{Sur le sable}

\begin{abstract}
Amphiprora vitrea var. trinitatis $n$. var. - Diffère du type par sa forme plus large et ses stries plus fortes. Long. 80 à $100 \mu$. Larg. 25 à 30,16 stries en $10 \mu$.

Amphora proteus var. contigua $\mathrm{Cl}$.

Cocconeis scutellum Eh.

Coscinodiscus excentricus Eh.

Cyclotella striata Grun.

- stylorum Bright.

Navicula yarrensis var. Grun.

Nitzachia sigma var. sigmatella Grun. Plourosigma balticum var. trinitatis n. var. - Diffère du type dont il a la forme extérieure par son raphé et sa striation semblable à celle de la variété californica. Long. 170 à $250 \mu .14$ stries trans, 13 long. en $10 \mu$.

Stauroneis salina W. Sm.

Surirella Febigeri Lewis.
\end{abstract}

Nos 201 et 202 JÉRÉMIE - HAITI Dépôt fossile marin

Bibliographie. - A. Truau et O. Witt. Die Diatomaceen der Polycystinenkreide von Jérémie in Hayti, 1888.

C'est à titre d'échantillon que nous publions ce curieux dépôt, car les Diatomées y sont si rares qu'à peine en trouve-t-on quelques espèces dans chaque préparation. Ce n'est qu'en en examinant de grandes quantités que les auteurs sont parvenus à établir la liste suivante.

Il est à remarquer que la majorité des espèces que ce dépôt contient, lui sont spéciales; les formes qui y paraissent relativement les plus abondantes, appar . tiennent au genre Stictodiscus qui y est richement représenté. 
Aetinoptychus Hüttlingerianus T. et W.

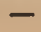

$-$ minutus Greo.

Wittianus Jan.

- $\quad$. hexagona.

Arachnoidiscus Ehrenbergii Bail.

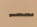

$-$ o. californicaA.S. Greoilleanus Hardm. ornatus Ehr.

Auliscus Hardmanianus Greo.

$$
\text { - - o. Haytiana T. }
$$

- punctatus Greo.

Biddulphia antiqua $\mathrm{T}$. et $\mathrm{W}$.

- caraibica T. et W.

- pulchella Greg.

Coscinodiscus angusto lineatus A. S.

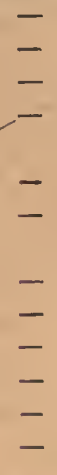
asteroides T. et W. caraibicus T. et $\mathrm{W}$. cribrosus T. et W. elegans Greo. oar. parsipunctata $\mathrm{T}$. et $\mathrm{W}$. Kinkerianus $\mathrm{T}$. et $\mathrm{W}$. lineatus var. tenera $\mathrm{T}$. et W.

lutescens Ratt.

- naviculoïdes T. et W.

- oblongus Greo.

- pauper T. et W.

- robustus Greo.

- subdioisus T. et W.

Cocconeis pellucida Hantz.

Craspedodiscus coscinodiscus Eh.

$$
\text { - elegans var. Eh. }
$$

Entogonia Daoyana Greo.

- - o. propincua fa.

- biangulata Bergon.

- Jeremiana Bergon.

- - - o. pentagona Berg-

- punctulata Greo.

Gephyria media Arnott.

Grammatophora stricta Eh.

Hemiaulus ornithocephalus Greo.

Isthmia $s p$.?

Melosira granulata o. Jeremice Grun. - sol Ktz.
Melosira solida o. Haittiensis Grun.

- sulcata Ktz.

Navicula Haïtiana T. et W.

- margaritifera T. et W.

- pandura Bréb.

- proetexta Eh.

orthoneis splendida Grun.

Porodiscus interruptus $G r$. et $S t$.

Porpeia robusta $\mathrm{T}$. et $\mathrm{W}$.

Stictodiscus adspersus T. et W.

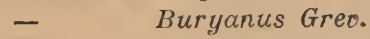

- $\quad$ - fa rotunda.

- $\quad$ - fatriangularis.

- $\quad$ fa subtriangularis.

- fa subquadrangularis.

et W.

o. gracilis $\mathrm{T}$.

- $\quad$ caraibicus T. et W.

- elaboratus T. et W.

- Eulensteinii Cast.

- Grunowii T. et W.

- Haitianus T. et W.

- Huttlingerianus T. et W.

- Jeremianus Cast.

- Johnsonianus Greo.

- $\quad$ Kinkerianus T. et W.

- multiplex Jan.

- pulchellus T. et TV.

- serpentinus T. et $\mathrm{WT}$.

- Truanii O. IV.

Triceratium arcticum oar. robusta $\mathrm{T}$. et W.

-
-
-
-
-
-
-
-
-
-
- arrogans $\mathrm{T}$. et $\mathrm{W}$.

Barbadense Greo.

biquadratum Jan.

Columbi O. W.

curoibaccatum J. Br.

Davidsonianum T. W.

elaboratum T. et IV.

faous Eh.

Godeffroyi Grun. grande Bright.

- o. pentagona Grun.

grave A.S.

Hardmanianum Greo.

imperator $\mathrm{T}$. et $\mathrm{W}$.

insuave $\mathrm{T}$. et $\mathrm{W}$. 
Triceratium Janischii T. et W.

$\begin{array}{ll}\text { - } & \text { Jordani T. et W. } \\ \text { - } & \text { pallidum Greo. } \\ \text { - } & \text { Perryanum T. et W. } \\ \text { - } & \text { rotundatum Greo. } \\ \text { - } & \text { Shadboltianum Greo. } \\ \text { - } & \text { Stolterfothii T. et W. } \\ \text { - } & \text { strabo A. } S . \\ \text { - } & \text { succinctum T. et W. }\end{array}$

Triceratium trisulcum o. producta $\mathrm{T}$. et W.

$$
\text { - } \quad \text { o. Haitiana T. }
$$

- turriferum T. et W.

- Wittianum Truan.

Trinacria Jeremice T. ot W. - subcapitata T. et W.

Bibliographie. - J. Pantocsek. Diatomées de Hongrie, vol. III.

Amphora argus Pant.

- Budayana Pant.

- sejuncta Pant.

- Staubii Pant.

- transylvanica fa minor Pant.

- verrucosa Pant.

Campylodiscus contortus Pant. hibernicus Eh.

v. transylvanica Pant.

Cocconeis lineata Eh. v. minor Pant.

\section{Cyclotella pygmea Pant.}

- radiato punctata Pant.

- transylvanica Pant.

$$
\text { - } \quad \text { v. disseminata }
$$

Cymatopleura elliptica W. Sm.

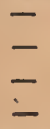
- $\quad$. subconstricta. gigantea Pant. gracilis $\mathrm{P}$ ant. Kinkerii Pant.

Cymbella Budayana Pant.

- - - v. gracilior Pant.

- lanceolata v. robusta Pant.

- Peragalloi Pant.

- præclara Pant.

- Rakoczyana Pant.

- suavis Pant.
Epithemia argus Ktz. v. fossilis Pant.

$$
\begin{array}{ll}
\text { - } & \text { Budayana Pant. } \\
\text { - } & \text { directa Pant. } \\
\text { - } & \text { Ehrenbergii Pant. } \\
\text { - } & \text { pibba Ktz. } \\
\text { - } & \text { striolata Pant. }
\end{array}
$$

Eunotia transylvanica Pant.

Fragilaria bituminosa v. elongata Pant Kochii Pant.

Gomphonema intricatum $\nabla$. vibrió Eh.

- Kinkerii Pant.

- subclavatum v. mustela Eh.

- transylvanicum Pant.

Melosira arcuata Pant.

- crenulata Ktz. var. hungarica Pant.

- Kochii Pant.

- Temperei Pant.

- transylvanicá Pant.

Naviçula bacillum Eh. var.

- basilica Pant.

- Bodosensis Pant.

- borealis Ktz. $\nabla$. fossilis Pant.

- Budayana Pant.

- conspersa Pant.

- curtestriatá Pant.

- decumana Pant.

- elliptica Ktz. 
Navicula Gurowii Pant.

- hasta Pant.

- Heribaudii M. Per. var.

- hilarula Pant.

- inculta Bant.

- latevittata Pant.

- Mauleri J. Br.

- præclara Pant.

- ostracodarum Pant.

- Toulac Pant.

- transylvanica Pant.

- trinotata Panl.
Vitzschia transylvanica Pant.

Pleurosigma Kochii Pant.

- transylvanicum Pant.

Podosira Mölleri Pant.

- transylvanica Pant.

Pseudocerataulus Kochii Pant.

Stauroneis Kochii Pant.

Stephanodiscus transylvanicus Pant.

Surirella biceriata Brẻb.

- Kellerii Pant.

Synedra transylvanica Pant.
Achnanthidium flexellum Bréb. minutum $\mathrm{Cl}$.

Amphora ovalis Ktz.

- pediculus Ktz.

Campylodiscus hibernicus Eh.

Cocconeis placentula Eh:

Cyclotella comta var. affinis Grun. Kützingiana Chauvin.

Cymatopleura elliptica W. Sm. solea var. W. Sm.

Cymbella anglica Lagerst.

- cistula Hemp.

- Ehrenbergii Ktz.

- helvetica Klz.

- gastroides Klz.

- gomphonemacea Cl.

- lacustris Ag.

- lanceolata Eh.

- lata Grun.

- leptoceros Grun.

- ventricosa $\mathrm{K}: z$.

Diploneis elliptica $\mathrm{Ktz}$.

- Didyma $\nabla_{4}$ subconstricta $\mathrm{Cl}$.

- Domblittensis Grun.

-. Mauleri J. Br.

Epithemia argus Eh.

- cistula Grun.
Epithemia gibba v. parallela Grun.

- Hyndmanii W. Sm.

- sorex Ktz.

Gomphonema acuminatum Ehr.

- constrictum Ehr.

- geminatum Ag.

Navicula gastrum Ehr.

- hebes Ralfs.

- lacustris Greg.

- oblonga Ktz.

- obtusa W. Sm.

- patula W. Sm.

- pseudo bacillum Grun.

- radiosa $\mathrm{Ktz}$.

- Schumaniana Grun.

- tuseula Eh.

- viridula Kítz.

- vulpina Ktz.

Mastogloia Smithii Thw.

Melosira granulata $\mathrm{Eh}$.

Neidium iridis $\mathrm{Eh}$.

- Hitchcockii Eh.

Niteschia ángustala $\mathrm{W}$. Sm.

Pinnularia hemiptera $\mathrm{Ktz}$.

- major Ktz.

Pleurosigma attenuatum Ktz.

Stephanodiscus astraea $\mathrm{Eh}$. 
Surirella bifrons Eh.

- robusta Eh.

- spiralis $\mathrm{Ktz}$.

Synedra capitata Eh.
Syuedra ulna Eh.

Tabellaria fenestrata $\mathrm{Ktz}$.

- flocculosa Rath.

\section{Nos 207 et 208 RANDANNE (Puy-de-Dôme) France}

\section{Dépôt fossile d'eau douce}

\section{BIBliogra PhiE. - Frère J. Héribaud. Les Diatomées d'Auver- gne, 1893.}

Amphora gracilis Ehr.

- ovalis Ktz.

- pediculus Grun.

Cocconeis placentula Ehr.

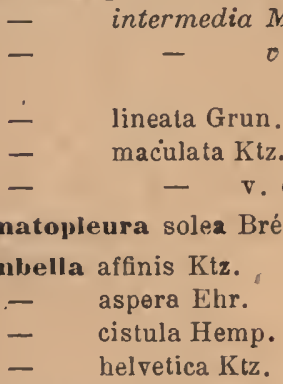

Diatoma anceps Grun.

Encyonema gracile v. minor Gruñ.

- ventricosum Ktz.

Epithemia gibba Ehr.

- gibberula Ehr.

- turgida Ktz.

- zebra Kiz.

- $\quad-f_{a}$ curta M. P. et F. H.

Eunotia lunaris Grun.

- prærupta fa curta Grun.

- Rabenhorstii v. monodon Cl. et Grun.

Fragilaria construens Grun.

Gomphonema acuminatum Eh.

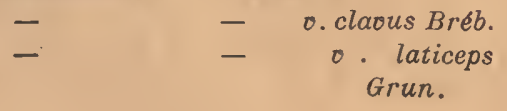

Goinphonema acuminatum o. trigonoce phalum Eh.

- $\quad$ auritum A. Br.

- angustatum จ. producta Grun.

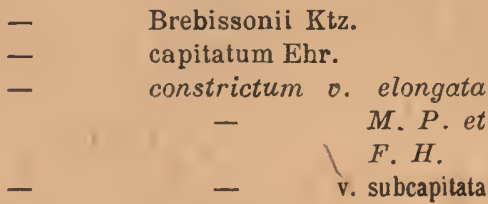

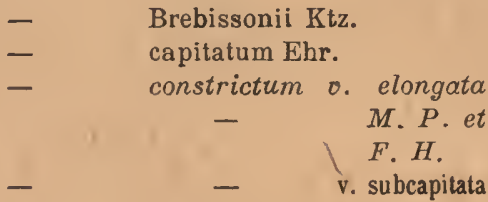

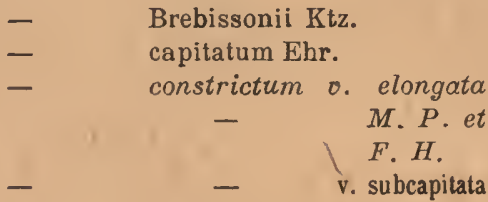

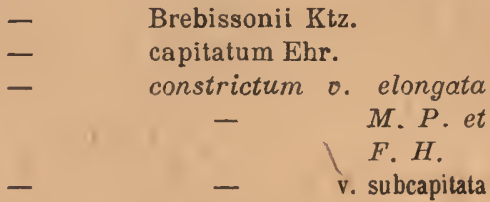

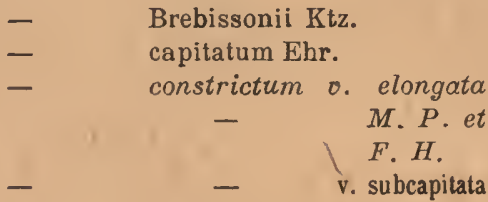

-

- cygnus Ehr.

- sarcophagus Greg.

- subclavatum Grun.

- vibrio Ehr.

Hantzschia dmphioxys Grun.

Melosira arenaria Moor.

- crenulata $\mathrm{Ktz}$.

- nivalis W. Sm.

- Rœseana Moor.

- Varennarum M. P. et F. H.

- varians Ag.

Meridion circulare Ag.

Navicula acrosphæria Bréb.

- affinis Ehr.

- ampliata Ehr.

- borealis Ktz.

- costata Ehr.

- Creguti F. H. et M. P.

- cuspidata Ktz.

- dactylus Ehr.

- elliptica Ktz. 
Navicala gracillima Pritch.

- hemiptera Ktz.

- limosa Ktz.

- - - undulata Grun.

- major Ktz.

- megaloptera Ktz.

- notata M. P. et F. H.

- pumila Grun.

- radiosa $\mathrm{Ktz}$.
Navicula rupostris Ktz.

- stauroptera Grnn.

- viridis Ktz.

Vitzachia spectabilis $B a b$.

Stauroneis amphilepta Ehr.

- Phcnicenteron Ehr.

Synedra nlna Ehr.

Tabellaria fenestrata Ktz.

No 209

\section{LE HAVRE (France)}

Sur la vase du port

Pleurosigna halticum W. Su.

No 210

BARRE DE LA BIDASSOA (France)

Sur les algues

Amphora cymbifera Greg.

$$
\begin{aligned}
& \text { - lineata (ireg. } \\
& \text { - marina W. Sm. } \\
& \text { - inflata Grun. }
\end{aligned}
$$

Achnanthes brevipes Ag.

Cocconeis dirupta var. flexella Grun.

- lineata Grun.

- scutellum var. ornata Grun.
Cocconeis scutellum var. stauroneiformis Rab.

Grammatophora angulosa var. mediterranea. macilenta W. Sm.

Hyalodiscus subtilis Bailey.

Ithoïcosphenia curvata var. marina. Synedra affinis $\mathrm{Ktz}$.
Achnanthes exigua Grun.

- lanceolata var. dubia Grun.

- - var. Haynaldii.

- Peragalli J. Br. var. fossilis $n$. $\nabla .$, diffère du type par ses extrẻmitén aiguës et savalve inférieure complètement striée 15 à $20 \mu$, longueur 8 a $10 \mu$. 
Amphora Libyca Eh. ovalis var. minor. V.-H.

Cocconeis placentula Eh.

Cyubella cistula Hemp.

- cuspidata Ktz.

- cymbiformis Eh.

- Ehrenbergii var. minor.

- gastroides Ktz.

- heteropleura kitz.

- maculata Ktz.

- naviculaeformis Auersw.

- parva W. Sm.

Diatuma hiemale var. mesodon.

- tenue Ag.

Encyouenıa crspitosum $\mathrm{K} t z$.

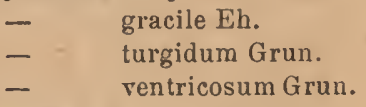

Epithemia amphicephala Grun.

$\begin{array}{lc}\text { - } & \text { gibla Ktz. } \\ \text { - } & -\quad \text { var. parallela. } \\ \text { - } & \text { - var. ventricosa. } \\ \text { - } & \text { - var. granulata. } \\ \text { - } & \text { - var. vertagus. } \\ \text { - } & \text { zebra Ktz. } \\ \text { - } & \text { - fa minor. }\end{array}$

Funotia arcus Eh.

- - var. minor.

- $\quad$ - var plicata.

- bidentula.

- diodon faninor.

- gracilis Rab.

- incisa Greg.

- lunaris Grun.

- major Rab.

- monodon Eh.

- - fa curtà.

- pectinalis Rab. fa. curta.

- - var. undulata.

- - var. ventricosa.

- prærupta var. bidens.

- tetraodon Eh.

Fragilaria æqualis Heib.

$\begin{array}{ll}- & \text { - var. producta. } \\ - & \text { brevistriata Grun. } \\ \text { - } & \text { construens Grun. } \\ \text { - } & \text { - } \quad \text { varr. binodis. } \\ \text { - } & \text { mutalilis Grun. }\end{array}$

Gomphonema acuminatum Eh.

\begin{tabular}{|c|c|}
\hline- & $\begin{array}{l}\text { - var. intermedia. } \\
\text { - } \quad \text { var. pusilla. }\end{array}$ \\
\hline - & $\begin{array}{l}\text { - var. pusilla. } \\
\text { angustatum Ktz. }\end{array}$ \\
\hline 一 & $\begin{array}{c}\text { - var. angustis } \\
\text { sima. }\end{array}$ \\
\hline - & var. producta. \\
\hline - & apicatum Eh. \\
\hline & augur Eh. \\
\hline - & Brebissonii Ktz. \\
\hline - & capitatum Eh. \\
\hline- & constrictum Eh. \\
\hline- & - var. subcapitata. \\
\hline- & dichotomum Ktz. \\
\hline 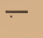 & intricatum var. pumila. \\
\hline- & montanum var. suecica. \\
\hline- & sagitta Schum. \\
\hline & sarcophagus Greg. \\
\hline & subtile Eh. \\
\hline & turris Eh. \\
\hline
\end{tabular}

IIantzchia elongata Grun.

Melosira crenulata $\mathrm{Ktz}$.

- distans Ktz.

Meridion constrictum Ralfs.

Navicula acrosphæria $\mathrm{Ktz}$.

- affinis Eh. var. minor.

- americana oar. bacillaris.

- amphigomphus Eh.

- ampliala Eh.

- bacilliformis Grun.

- bacillum Eh.

- bisulcata Lag.

- Brebissonii Ktz.

- commutata Grun.

- cryptocephala Ktz.

- - var. intermedia

- cuspidata $\mathrm{Ktz}$.

- $\quad$ - craticula.

- dactylus Ktz.

- dicepliala var. Ktz.

- digito radiata Greg.

- divergens W. Sm.

- elliptica Ktz.

- Falaisiensis Grun.

- Finnica Cl.

- firma $K t z$.

- gentilis Donk.

- gibba Ktz.

- gigas $\mathrm{Ktz}$.

- hemiptera Ktz. 
Navicula Hitcheockii Eh.

- inflata freg.

- iridis Eh.

- latevittata Cil.

- limosa $\mathrm{Ktz}$.

- macilenta Cl.

- major ktz.

- meniscus Schum.

- mesolepta Eh.

- - var.stauroneiformis. muralis Grun.

nobilis Eh.

pachyptera Eh. peregrina Ktz. pupula $\mathrm{Ktz}$.

- - fa minuta.

- - var. bacillaroides.

- radiosa $\mathrm{Ktz}$.

- - var. acuta.

- rhyncocephala Ktz.

- rupestris Hantz.

- salinarum Grun.

- scutelloides oar. minutissima.

- semen Eh.

- serians Ktz.

- subcapitata Greg.

- - var. stauroneiformis.

- tabellaria $\mathrm{Ktz}$.

- tenella Bréb.
Navicula transversa A. S.

- tuscula Eh.

- viridula Kitz.

- - var. arenacea.

Niteschia acutiuscula Grun.

- sigmoidea W. Sm.

- vitrea Norm.

Pleurosigma attenuatum W. Sm.

Pleurostauron acuta Rab.

- $\quad$ - var. robusta. paroula Grun?

Stauroneis anceps Eh.

- $\quad$ - var. amphicephala.

- - var. linearis.

- Baileyi Eh.

- gracilis Eh.

- lanceolata Ktz.

- Phonicenteron Eh.

Surirella elegans Eh.

- linearis vár. constricla.

- tenera Greg.

- - var. splendidula.

Syneira delicatissima var. mesoleia.

- rumpens var. familiaris.

- ulna var. danica.

Tabellaria fenestrata Ktz.

- flocculosa. Ag.

Nos 213 et 214 SANTA CRU\% - COLORADO

(Etats-Unis)

Dépôt fossile marin

Ce dépôt a été donné dans la première collection Tempère et Peragallo sous le nom erroné de Sta Suez, avec d'autres dépots de Californie : San Fernando, Sta Barbara, San Bernardino. - Dans les tables, l'erreur a été partiellement relevée et le dépôt désignécomme $S^{\text {ta }}$ Crux (non Suez) Cal. U.S.A.

Depuis, Brun (Le Diatomiste II, p. 209) en a décrit et figuré quelques. formes en attribuant le dépôt en Colorado.

Ce dépôt est en effet différent des dépôts californiens, ce fait m'avait déjá frappé dans l'étude assez sommaire que j'en avais faite tout d'abord (T. et P., 
n 397. La forme désignée par moi Triceratium coniferum? a été nommée par Brun: Lithodesmium cornigerum et est bien caractéristique de ce dépôt $)$.

Malheureusement, je n'ai pu trouver de Sta Cruz, sur les cartes que je possède, dans l'Etat de Colorado. La présence d'un dépot à l'intérieur du Continent, et différent du grand dépòt Californien est digne de remarque.

Actinocyclus Ehrenbergii Palfs.

Actinoptychus glablatus A. S.

\begin{tabular}{ll}
- & \multicolumn{1}{c}{- v. maculata H.P. } \\
- & areolatus Eh. \\
- & biformis J. Br. \\
- & vulgaris Schum. \\
- & splendens Ralfs. \\
- & undulatus Eh. \\
- & Janischii var. notata H.P. \\
- & crucitinus H. P. \\
- & coloradoï H. P. \\
- & sp. A. S. atl. 153/4. \\
- & sp. A. S. atl. 153/12.
\end{tabular}

Anaulus acutus $\mathrm{J}$. Br.

Arachnoidiscus Ehrenbergiiv. californica.

- Grevilleanus Hardm.

Asteromphalus Heptactis Ralfs.

- Hookeri Ehr.

Aalacodiscus Huttonii Gr. et St. var. (à 2 et 3 appendices).

Amphora crassa Greg.

- cymbifera Grog.

- egregia Eh.

- quadrisulcuin H. F.

Auliscus elegans var. coloradica H. P.

- Hardmanianus Grev.

- mirabilis Grev.

- punctatus Bail.

- sculptus Eh.

- Stöckardtii Jan.

- subreticulatus (Grun.) Ratt.

Biddulphia aurita Brẻb.

$$
\begin{array}{ll}
* \quad \text { - } & \text { hyalina H. P. } \\
\text { - } & \text { media H. P. } \\
\text { - } & \text { regina W. Sm. }
\end{array}
$$

"Campylodiceus omatus var. elaborata H. P.
Campyloneis regalis Grev.

Cocconeis dirupta var. A. S.

$\begin{array}{ll}- & \text { interrupta Grun. } \\ \text { - } & \text { Lorenziana Grun. } \\ \text { - } & \text { pellucida var. monicæ H. P. } \\ \text { - } & \text { sigma Pant. } \\ \text { - } & \text { sigmoradians J. Br. } \\ & \text { vitrea J. Br. }\end{array}$

Cestodiscus intersectus J. B. (C. Stokesianus, 8 rayons seulement).

Coscinodiscus apiculatus Eh.

$\begin{array}{ll}- & \text { asteromphalus Eh. } \\ - & \text { argus Eh. } \\ \text { - } & \text { decrescens Grun. } \\ \text { - } & \text { echinatus Ratt. var. } \\ - & \text { excentricus Eh. } \\ - & \text { gigas Eh. } \\ - & \text { heteroporus Eh. } \\ - & \text { marginatus Eh. } \\ - & \text { Mölleri A. S. var. } \\ - & \text { nitidus Greg. } \\ - & \text { nodulifer A. S. var. } \\ - & \text { odoutodiscus Greg. } \\ - & \text { oculus-iridis Eh. } \\ - & \text { Peruanus Eh. } \\ - & \text { radiatus Eh. } \\ - & \text { robustus Grun. }\end{array}$

Craspedodiscus elegans Eh.

Denticula nicobarica Grun. var.

Dieladia capreolus Eh. (à 2 et une corno).

Bntopyla australis Eh.

Epithemia gibba fa major elongata.

"Eunotiopsis (Leudugeria) Janischi var.? excavata $\mathrm{H}$. P.

Eunotogramma bivittata Grun.

Euodia gibba Bail. 
Goniothecium odontella Eh.

Glyphodiscus stellatus Grev.

- Grammatophora angulosa var.

$\begin{array}{ll}\text { - } & \text { fossilis H. P. } \\ \text { - } & \text { oceanica Ehr. }\end{array}$

Grunowiella gemmata (Grun.) V. H.

Heniaulus ambiguus Grun.?

sp. nov.?

- Hercotheca armata H. P.

Ilyalodiscus laevis Eh.

Ktonodiscus hungaricus Pant.

Lithodesmium cornigerum $\mathrm{J} . \mathrm{Br}$.

- minusculum Eh. biceps H. P.

Metosira clavigera Eh.

- Sanctae Crucis H. P.

- separanda A. S.

- sol Eh.

- sulcata Ktz.

Navicula angelorum $\mathrm{Cl}$.

- clavata var. subacuta H. P.

- crucitina H. P. (Fisheri var.)

- entomon Eh.

- fusea Greg.

- gemmatula Grun.

- Hennedyi $\nabla$. californica. Cl.

- - $\quad$ var.coloradica H. P.

- Lyra Eh.

- - var. atlantica fo elliptica.

- $\quad$ - var. lata H. P.

- var. pusilla.H. P.

- var. subtypica A. S.

musca Greg.

- nummularia Grev.

- pandura Bréb.

- pennata A. S.

- polysticta A. S.

- Powellii Lewis.

- prætexta Eh.

- Sancta Crux J. Br.

- scalarifer J. Br.

- semidecora J. Br.

- Smithii Bréb.

- var. fossilis H. P.
Navicula spectabilis Grov.

- splendida Greg.

- subeincta A. S.

Nitzschia sigma Eh.

- Tryblionella W. Sm.

Orthoneis splendida Grun.

Plasiogramma elongatum Grev. validum Grev.

Pleurosigma formosum. WV. Sm.

- sp. ?

Periptera totracladia Eh.

Porodiscus minutus $H$. P.

Psendo-Nitzschia pliocena $(\mathrm{Br}.) \mathrm{H}$. P.

Pterotheca subulata Grun.

- Raphoneis amphiceros var. coloradica

H. $P$.

$$
\begin{gathered}
\text { - } \quad \text { var. rhombica } \\
\text { - } \quad \text { fa elongata. } \\
\text { var. gemmi - } \\
\text { fera. }
\end{gathered}
$$

- - Sanctae Crucis H. P.

Rhabdonema mirificum W. Sm.

- Rouxia Peragalli J. Brun.

* - - var. elongata.

- Stephanogonia barbatula H. P.

Stephanopyxis appendiculata var.

- turis Eh.

$$
\text { - } \quad \text { - ta inermis. }
$$

Stictodiseus californicus Grev.

Septroneis caduceus Eh.

Syndendrium diadema Eh.

Thalassionema Nitzschioides Grun.

Truchyneis aspera $\mathrm{Cl}$.

, - oblonga $\mathrm{Cl}$.

Triceratium arcticum Bright.

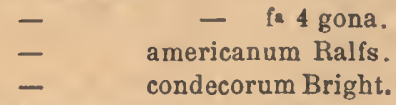

Iantbiopyxis umbonata Grev.

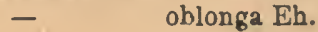


N० 215 TONTIN LAKE - NEW. BRUNSWICK

(Etats-Unis)

Cyclotella Bodanica $\mathrm{Cl}$.

- comta $\mathrm{Ktz}$.

- - - var. affinis.

Cymbella americana A. S.

- gastroïdes $\mathrm{K} t z$.

- heteropleura Ktz.

Epithemia turgida $K t_{z}$.

Eunotla arcus Eh.

- - var. uneinata.

- bidentata W. Sm.

- gracilis Rab.

- major Rah.

- - var. ventricosa.

- monodon Eh.

- pectinalis Rab.

- - var. undulata.

- - var. ventricosa.

- robusta var. tetraodon.

- - var. hezaodon.

- - var. duodecaodon.

- - var. serra.

- - var. polyodon.

Gomphonema acuminatum Eh. capitatum Eh.

Melosira distans $\mathrm{K} t z$.

Navicula amphigomphus Eh.

- Brebissonii Ktz.

- commutata Grun.
Navicula dactylus Ktz.

- divergens W. Sm.

- dubir Ktz.

- gibba Kitz.

- gigas Ktz.

- halophila Grun.

- hemiptera Ktz.

- iridis Eh.

- limosa Ktz.

- major Ktz.

- Paulensis Grun.

- pachyptera Eh.

- serians Ktz.

- Smithii Bréb.

- stomatophora Grun.

- streptoraphe Cl.

- transversa A. $\$$.

- viridis $\mathrm{Ktz}$.

Pleurostauron acuta var. robusta.

Stauroneis Baileyi Eh.

- Eracilis Eh.

- lanceolata Ktz.

- Phœnicenteron Eh.

Steropterobia anceps Lewis.

Surirella bifrons Eh.

- linearis var. constricta.

- oblonga Eh.

- Rattrayi A.S.

Van Heurckia rhomboides Bréb.

\section{No 216 ATKIN'S POND, BRISTOL - CONNECTICUT}

\section{(États-Unis)}

Achnanthes exigua Grun.

- lanseolata oar. Haynaldii.

- Peragalli J. Br. var. fossilis.
Cocconeis placentula Eh.

Coscinodiscus chambonis M. Per et F. H.

Cyclotella Meneghiniana Ktz. 
Cymbella americana A. S.

- anglica Lag.

- cuspidata Ktz.

- gastroīdes Ktz.

- gibba Bail.

Encyonema cœspitosum Ktz.

- ventricosum Grun.

- - var. stricta.

Epithemia amphicephala Grun.

- gibba Ktz.

- - var. ventricosa.

- turgida Ktz.

- - our. granulata.

- $\quad$ - faminor.

Eunotia arcus Eh.

- impressa Eh.

- incisa Greg.

- major Rab.

- minor V. H.

- monodon Ell.

- $\quad-\quad \mathrm{f}^{2}$ curta.

- pectinalis Rab.

- praerupta var. bidens Grun.

- tetraodon Eh.

Fragilaria capucina Desm.

- - - var. mesolepta.

- construens Grun.

- $\quad$ - var. binodis.

- $\quad$ - var. venter.

- elliptica Shum.

- Harrisonii Grun.

- mutabilis Grun.

Gomphonema acuminatum $\mathrm{Eh}$.

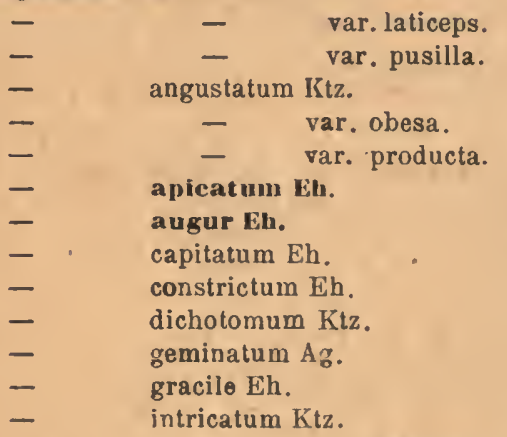

- maculata Ktz.

- turgidum Grun.

Gomphonema montanum var. suecica.

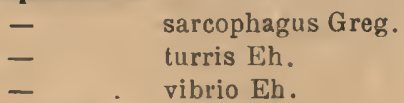

Uantzsehia amplioxys Grun.

Melosira crenulata $\mathrm{K} t z$.

- distans Ktz.

Meridion constrictum Ralfs.

- _ - cum valvis internis.

Navicula acrosphceria $K t z$.

- affinis Eh.

- ambigua fa craticula.

- americana Eh.

- - var, bacillaris.

- amphigomphus Eh.

- amphyrynchus Eh.

- ampliata Eh. var.

- bacilliformis Grun.

- bicapitata var. hybrida.

- biceps Greg.

- bipunctata Grun.

- bisulcata Lag.

- Bogotensis Grun.

- borealis Ktz.

- brevicostata Cl.

- Cardinalicus (il.

- Cari Eh.

- cominutata Grun

- cuspidata Ḱtz.

- $\quad$ - fa craticula.

- dactylus Ktz.

- Dariana A. S.

- dicephala Eh.

- divergens W. Sm.

- elegantissima var.

- elliptica $\mathrm{Ktz}$.

- gibba Ktz.

- gigas Ktz.

- hemiptera Ktz.

- interrupta W. Sm.

- - - var. stauroneiformis

- iridis Eh.

- - var. maxima.

- lata Bréb.

- legumen Eh.

- limosa $\mathrm{Ktz}$.

- - var. subinflata.

- - var. undulata.

- $\quad$ var. dichotoma 
Navleula major Ktz.

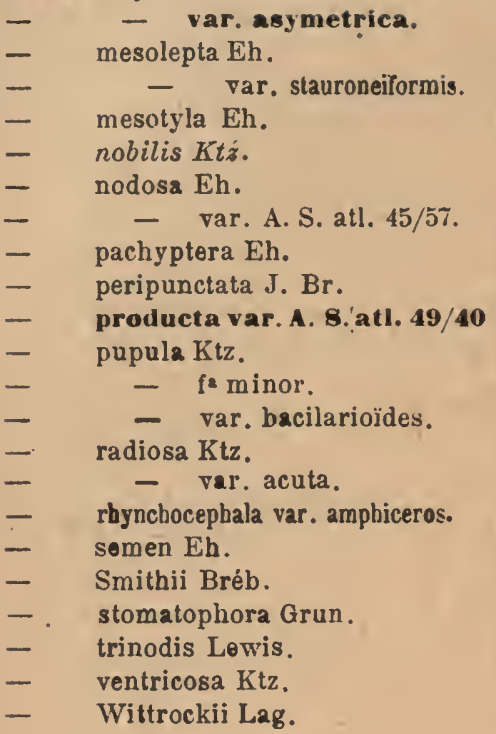

Nitzechla sigmoidea W. Sm.

Opephora pacifica $P . P$.

Sturoneis anceps Eh.

$$
\begin{array}{ll}
- & - \\
- & \text { var. amphicophala. } \\
- & \text { Baileyi Eh. linearis. } \\
\text { - } & \text { gracilis Eh. } \\
\text { Phcenicenteron Eh. }
\end{array}
$$

Stephanodiscus astraea Grun.

Surirella Baileyi Leu is.

$$
\begin{aligned}
& \text { - cardinalis Kitt. } \\
& \text { - elegans Eh. } \\
& \text { - Mölleriana Grun. } \\
& \text { - tenera Greg. } \\
& \text { - } \quad \text { - var. splendidula. }
\end{aligned}
$$

Synedra ulna Eh.

$$
\text { - - var. denica. }
$$

Tabellaria fenestrata Ktz.

$$
\text { - flocculosa } \mathrm{Ktz} \text {. }
$$

Van Beurckia rhomboides, car. amphipleuroides. oiridula Bréb.

\section{Nos 217-218 HOQUIAM - PUJET SOUND}

\section{(États-Unis)}

Aetinocyclu nebulosus $M$. Per.

Actinoptychus glabratus var. incisa.

$$
\text { - undulatus Eh. }
$$

Aulacodiscus Kittonii Arn.

Auliscu* pruinosus var. Zanzibarica.

Brebissonia Boechii Grun.

Campylodisens Echeneis Eh.

Corataulus turgidus Eh.

Cocconema mexicanum Eh.

Coscinodiscus excentricus Eh.

$\begin{array}{ll}\text { - } & \text { oculus-iridis Eh. } \\ \text { - } & \text { Peruanus Grun. } \\ \text { - } & \text { radiatus Eh. }\end{array}$

Cyclotella striata $\mathrm{Gr}$.
Endictya oceanica Eh.

Epithemia turgida $\mathrm{Ktz}$.

- Westermanii Ktz.

Isthmia neroosa $K t z$.

Melosira sulcata Ktz.

$$
\text { - - - var. coronata. }
$$

Navicula cancellata Donk.

- formosa lireg.

- fusia Ralfs.

- limosa Ktz.

- major Ktz.

- peregrina Eh.

Nitzschia sigma W. Sm.

- - var. Habirshawii.

- - var. rigida. 
Nitzschia Tryblionella Hantz.

Pleurosigma angulatum W. Sm.

Bennettii M. Per. n. sp.

Sigmoïde allongé, à ex-

trémités assez fines et arrondies; raphè à peu près mẻdian jusqu'aux extrémitès. Nodules terminaux renforcés en croissant; stries transversales bien visibles, 22 en $10 \mu$; longitudinales invisibles \ longueur 160 à $220 \mu$.

- hippocampus W. Sm.

- Olympianum Terry.

- IRyderil M. Per. n. sp. Contour analogue à celui du $P l$. balticum mais la partie médiane un peu moins large que les extrèmités. Raphé mèdian jusqu'aux extrémités ; stries fines, obliques, 16 en $10 \mu$. Longueur 180 à $250 \mu$.

Polymyxus sp.?

Wansbeckii Donk.

Scoliotropis iatestriata $\mathrm{Cl}$.

Scoliopleura tumida Rab.

Stauroneis acuta W. Sm. $\nabla$. maxima.

Stephanopyxis turris Ralfs.

Surireila gemma Eh. sp. A. S. atl. 24/25.

Triceratium bicorne Gl.

Van Heurckia Lewisiana Bréb.

- var. incomperta.

Nos 219-221

\section{MEJILLONES - BOLIVIE}

Terre fossile marine

BIBLIOGRAPHIE. - Un certain nombre de formes ont été données dans l'atlas de Ad. Schmidt.

Actinocyclus Ehrenbergii Ralfs.

$$
\begin{aligned}
& \text { - } \\
& \text { - }
\end{aligned}
$$

Actinoptychus Boliviensis Jan. splendens $v$. halionyx.

Asteromphalus Brookei Bail.

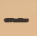

Roperinnus Ralfs.

Shadboltianus Ralfs.

Aulacodiscus catenarius Witt.

$\begin{array}{ll}\text { - } & \text { crux Eh. } \\ \text { - } & \text { Ehrenbergii Jan. } \\ \text { - } & \text { formosus Arnott. } \\ \text { - } & \text { margaritaceus Ralfs. } \\ \text { - } & \text { - } \quad \text { o. Debyi. }\end{array}$

Auliscus coelatus $\nabla$. constricta.

- - $\quad$ - major.

- gigas Grun.

- Greoillei Jan.

- ooalis Arnott.

- proelatus Ratt.

- sculptus Ralfs.

- Stöckardtii Jan.

Bidduiphia Baileyi car.?

Coscinodiscus angusto lineatus Grun.

- apiculatus Eh.

- argus Eh.

- asteromphalus Eh.

- . $\quad$ - v. princeps

- $\quad$ - . boliviensis Grun. 
Coscinodiscas boliviensis Grun.

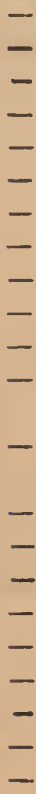
curvatulus Grun.

oculus iridis Eh. concavus Greg. concinnus W. Sm. elongatus Grun. excentricus Eh. gigas Eh. leptopus Grun. lineatus Eh. nobilis Grun.

$$
\begin{array}{cc}
-\quad & \text { v.loculifera } \\
\text { Ratt. } \\
\text { v. sabspi- } \\
\text { nosaGrun. }
\end{array}
$$

perforatus Eh. radiatus. Eh.

- v. centralis.

- $\quad$. media Grun. robustus Grev. stellaris v. mejillonis Grun. symbolophorus Eh. var. subtilis Eh.

Woodwardii Eul.
Endictya oceanica Eh.

- - var. A. S. 148/12.

Euodia gibba Bail.

Melosira sol Eh.

Navicula aspera Eh.

$$
\begin{aligned}
& \text { - } \quad \text { copiosa A. S. } \\
& \text { - } \text { - Heniosa J. Br. } \\
& \text { - multicostata Grun. } \\
& \text { - } \quad \text { Smithii Brèb. var. } \\
& \text { - } \\
& \text { spectabilis Grev. }
\end{aligned}
$$

Plagiogramma fenestrata $J . \mathrm{Br}$.

$$
\begin{array}{ll}
\text { - } & \text { Gregorianum o. robusta } \\
\text { J. Br. } \\
\text { - } & \text { obesum Greo. } \\
\text { - } & \text { pulchellum Greo. }
\end{array}
$$

Pleurosigma affine Grun. var.

Rhabdonema adriaticum $\mathrm{Kt}$.

Triceratium antediluvianum Eh. var.

- $\quad$ arcticum $\mathrm{Br}$.

- canalifer $J . B r$.

- patagonicum A.S.

No 222

Achnanthes minutissima $\mathrm{Ktz}$. - var. cryptocephala.

Achnanthidium flexellum Bréb.

- minutum Cl.

Amphora ovalis $\mathrm{Ktz}$.

Ceratoneis arcus $\mathrm{Ktz}$.

Cocconois lineata var. euglypta.

- placentula $f^{2}$ minor.

Cymbella affinis Ktz.

- cesatii Rab.

- cistula Hemp.

- cymbiformis Eh.

- delicatula Ktz.

- gibba Eb. A. S. atl. 10/27\&27. la forme pl. $9 / 39$ qui n'en diffère pas sensiblement s'y trouve aussi mais plus rarement.

Cymbella helvetica $\mathrm{Ktz}$.

- laeve Naeg.

- leptoceros var. obtusa.

- sp. A. S. atl. 9/57 fa minor.

Denticula tenuis $\mathrm{Ktz}$.

Diatoma gracillima Naeg.

- oulgare Bory?

Encyonema lunula Grun.

- ventricosum $\mathrm{f}^{2}$ minuta.

Epithemia argus $K t z$.

- gibba Ktz.

Eunotia pectinalis $\mathbf{f}^{2}$ curta.

Frugllaria intermedia Grun.

- mutabilis Grun. 
Fragilaria northumbrica Grun.

Gomphonena acuminatum Eh.

- Geminatum Ag.
-
-

Crunowia sinuata Grun.

Meridion constrictum Ralfs.

Vavicula cryptocephala var. exilis.

- elliptica Ktz.
$-\quad$ - var. minutissima.
- $\quad$ exilis Grun.
- Holstii $\mathrm{Cl}$.
- major $K t z$.

Navicula rostellata Ktz.

- - var. minor.

- rupestris Hantz.

- viridula Ktz.

- - var. arenaceun.

Nitxschía denticula Grun.

- palea W. Sm.

- - var. tenuirostris.

- thermalis oar. minor.

Synedra amphicephala Ktz.

- ulna Eh.

- - var. aqualis.

- - var. danica.

Tabellaria flocculosa $\mathrm{Ktz}$.

No 223 CABBAGE TREE SWAMP - AUCKLAND

\section{(Nouvelle-Zélande)}

Dépôt fossile d'eau douce

Achnanthes inflata Grun.

- lanceolata Bréb.

Cymbella pusilla Grun.

Encyonema turgidum Grun.

- ventricosum Giun.

Eunotia Burkartii. Eh.

- diodon Hh.

- lunaris var. subarcuata.

- major Rab.

- monodon Eh.

- - facurta.

- parallela Eh.

- pracrupta var. bideus.

Gomphonema affine $\mathrm{Ktz}$.

- subclavatum Grun.

- vibrio Eh.

Hantzschia amphioxys Grun.

$$
\text { - } \quad \text { - } \quad \text { var. elongata. }
$$

Melosira laevis Eh.

- Rosezna Rab var.
Melosira Roseana var. epidendron.

Navicula acrosphæria Bréb.

- affinis Eh.

- bacilliformis Grun.

- brevicostata var. interrupta.

- commutata Grun.

- divergens W. Sm.

- gibba Ktz.

- lacunarum Grun.

- major Ktz.

- transversa A. S.

- ventricosa Eh.

- viridis $\mathrm{Ktz}$.

Vitxschia acutiuscula Grun.

- sigmoidea W. Sm.

Pleurostauron juvanicunı Grun.

- scapulaeformis Grun.

Synedra rumpens Ktz.

- - var. fragilaroïdes.

- ulna ear. longissima. 
No 224

COTES DE SUEDE

Auricula Insecta $\mathrm{Cl}$.

$\mathrm{N}^{0} 225$

MEUDON (Seine-et-Oise) France

Hantzsehia amphioxys Grun.

Nos 226-228 SAINT-SATURNIN (Puy-de-Dôme) France

Dépôt fossile d'eau douce

BIBliographie. - Frère J. Héribaud. Les Diatomées d'Auvergne 1893.

Achnanthes exilis $\mathrm{K} \mathrm{z}$.

- lanceolata Grun.

- $\quad$ subsessilis Ktz.

Amphora ovalis $\mathrm{Ktz}$.

- pediculus Grun.

Asterionolla formosa o. gracillima Grun.

Campylodiscus costatus W. Sm.

Cocconeis californica $f^{2}$ subcontinuz Grun

- lineata Grun.

- placentula Ehr.

- Rouxii v. euglypta Grun.

Cyclotella Meneghiniana Ktz.

Cymatoploura elliptica W. Sm.

- hibernica W. Sm.

- $\quad$ - $\quad$ v. major M. P.

- solez Bréb.

Cymbella affinis Ktz.

- amphicephala Næg.

- aspera Ehr.

- cistula Hemp.

- Ehrenbergii Greg.

- gastroïdes Ktz.

- $\quad$ - $\quad$. minor Ktz.

- helrotica Ktz.
Cymbella lanceolata Ehr.

- maculata Ktz.

- parva W. Sm.

- turgidula Grun.

Diatoma anceps Grun.

- elongatum Ag.

- hyemale Heib.

- tenue Ag.

- vulgare Borz.

Encyonema lunula Grun.

Epithemia argus o. amphicephala Grun.

- gibba Ehr.

- gibberula Ehr.

- - o. producta Grun.

- sorex $\mathrm{Ktz}$.

- turgida Ktz.

- $\quad$ - $\quad$. vertagus Ktz.

- zebra Ktz.

- - $\quad$ - proboscidea Grun.

Eunotia arcus Eh.

- - $\nabla$. bidens Grun.

- faba Grun.

- flexuosa v. bicapitata Grun.

- gracilis Rab.

- lunaris Grun. 
Fragilaria bidens fa major Heib.

- - fa minor Heib.

- binodis Ehr.

- brevistriata v. subacuta

- capucina Desm.

- construens Grun.

- - $\quad$ v. venter Grun.

- intermedia Grun.

- mutabilis Grun.

- virescens Ralfs.

Gomphonema acuminatum Ehr.

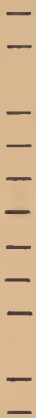

v. laticeps Grun.

v. trigonoce-

auritum $\mathrm{A}$. $\mathrm{Br}$. phalum Ehr.

- commutatum Grun.

- constrictum v. subcapitata Grun

- dichotomum W. Sm.

- intricatum Ktz.

- montanum Schum.

- semiapertum o. tergestina Grun.

subclavatum Grun.

vibrio Ehr.

Meridion circulare $\mathbf{A g}$.

- constrictum Ralfs.

Melosira arenaria Moor.

- crenulata Ktz.

- tenuis Grun.

- tenuissima Grun.

- varians Ag.

Navicula acrosphoeria Bréb. o. laevis

$M$. P. et $F, H$.

- ampliata Ehr.

- aponina Ktz.

- Brebissonii Ktz.

- borealis $\mathrm{Ktz}$.

- crJptocephala Ktz.

- divergens W. Sm.

- $\quad$ - o. undulata M.P. et F. $H$.

- elliptica 0. oblongella Nog.

- firma Ktz.

Navicula gastrum Donk. gibba Ehr.

- Gregaria Donk.

- limosa Ktz.

- - o. gibberula Grun.

- major Ktz.

- menisculus Grun.

- mesolepta Ehr.

- - v. stauroneiformis Grun

- mesostyla Ehr.

- nivalis v. interrupta W. Sm.

- nobilis Ehr.

- pseudobacillum v. major M.P.

- pupula v. minuta Ktz.

- radiosa $\mathrm{Ktz}$.

- stauroptera Grun.

- tabellaria Ehr.

- viridis $\mathrm{Ktz}$.

- - v. commutata Grun.

- vulgaris Heib.

Nitzsehia linearis W. Sm.

- sigmoidea Nitz.

Pleurosigma Spencerii W. Sm.

Rhoicosphenia curvata Grun.

Stauroneis acuta W. Sm.

- anceps Ehr.

- phœnicenteron Ehr.

- platystoma Ehr.

Synedra acus Grun.

- delicatissima W. Sm.

- ulna. Eh.

- ulna o. danica Ktz.

- - lanceolata fa brevis Kitz.

- - v. longissima W.Sm.

- - v. obtusa W. Sm.

Stephanodiscus astrcea $K t$.

Surirella angusta $\mathrm{Ktz}$.

- biseriata Bréb.

- norvegica Ehr.

- ovata v. minuta Bréb.

- saxonica Auers.

- turgida W. Sm. 


\section{Plankton (Collection Cleve)}

A aricula insecta $\mathrm{Cl}$.

Biddulphia aurita Bréb.

- mobiliensis Bail.

Chretoceros boreale Bail.

- curvisetum Cl.

- debilis Cl.

- didymus Eh.

- distans $\mathrm{Cl}$.

- protuberans Laud.

- similis $\mathrm{Gl}$.

Cocconeis scutellum Eh.

Coscinodiscus concinnus W. Sm.

$\begin{array}{ll}\text { - } & \text { excentricus Eh. } \\ \text { - } & \text { lineatus Eh. } \\ \text { - } & \text { oculus iridis Eh. } \\ \text { - } & \text { sol } \text { symbolophorus Grun. }\end{array}$

Ditylium |Brightwellii West.
Eucampla zodiacus Eh.

- - var. cornigera.

Guinardia flaccida $H$. P.

Lauderia compressa $H$. $P$.

Leptocylindrus danicus $\mathrm{Cl}$.

Mastogloia lanceolata Thw.

Podosira glarialis CI.

Rhizosolenia alata Bright.

- $\quad$ - var. gracillima.

Skeletonema costatum $\mathrm{Gl}$.

Stephanopyxls Palmeriana Grun.

- turgida Ralfs.

Striatella unipunctata Ag.

Thalassiosira Nordenskioldii Cl.

Thalassiothrix elongata Grun.

Nitzschioïdes Grun.

N॰230

HERO (Norvège)

Plankton (Collection Gran)

Biddulphia aurita Bréb.

Chrtoceros furcellatum Bail.

- socialis Laud.

- vermiculus Schūtt.

- Wighamii Bright.

Cocconeis scutellum Eh.
Lauderia glacialis Grun.?

vitzschia seriata var. fraudulenta $\mathrm{Cl}$.

Pleurosigma lanceolatum Donk.?

Thalassiosira Nordenskioldii Cl.

Thal:assiothrix Nitzschioïdes Grun. 
N*231 et 232 SAINTE-MARGUERITE (Puy-de-Dôme) France Source minérale

BIBLiographiE. - Frère J. Héribaud. Les Diatomées d'Auvergne, 1893.

Achnanthes exilis Ktz.

Amphora Normanii Rab.

Cocconeis pediculus Ehr.

$$
\text { - placentula Ehr. }
$$

Cyclotella operculata Ktz.

Cymatopleura solea v. apiculata Pritch.

Encyonema cœspitosum $\mathrm{Ktz}$.

$$
\begin{array}{ll}
\text { - } & \text { prostratum Ralfs. } \\
\text { - } & \text { ventricosum Ktz }
\end{array}
$$

Epithemia gibba v. parallela Grun.

$$
\begin{array}{ll}
\text { - } & \text { gibberula Ehr. } \\
\text { - } & \text { sorex Ktz. } \\
\text { - } & \text { turgida Ktz. } \\
& \text { Westermannii Ktz. }
\end{array}
$$

Fragilaria binodis Etr.

- $\quad$ - v. obliqua J. Br. et F. M.

- construens Grun.

$$
\text { - - - o. pumila Grun. }
$$

Gomphonema acuminatum Ehr.

- micropus Ktz.

$$
\text { - } \quad \text { - } \quad \text { o. minor Grun. }
$$

Navicula amphigomphus Ehr.

- amphisbona Bory.
Navicula Brebissonii $\mathrm{Ktz}$.

- cincta Ktz.

- cryptocephala Ktz.

- divergens W. Sm.

- gracilis $\nabla$. V. H.

- humilis Donk.

- limosa $\mathrm{Ktz}$.

- producta W. Sm.

- pusilla W. Sm.

- rhomboïdes Ehr.

- seminulum Grun.

- trinodis W. Sm.

- viridula $\mathrm{Ktz}$.

Nitzschia frustulum Grun.

- linearis W. Sm.

- sigmoidea Nitz.

- tenuis Grun.

- vitreá Norm.

Pleurosigma acuminatum W. Sm.

Rhoicophenia curvata Grun.

Surirella angusta $\mathrm{Ktz}$.

- patella Ehr.

- gracilis Grun.

Synedra ulna Ehr.

- - v. amphirynchus Ehr.

Nos 23:3-235 NOTTINGHAM - MARYLAND (États-Unis)

Dépôt fossile marin

BIBLIOGRAPHIE. - Ce dépôt, très étudié par Ehrenberg et dont la grande majorité des espèces qu'il contient ont été données dan l'atlas de Ad. Schmidt; est surtout intéressant par la variété de ses Coscinodiscus, sur- 
tout la série des formes qui unissent le C. apiculata type à mailles rondes et isolées au C. padiatus.

Actinocyclus Ehrenhergii Ralfs .

$\begin{array}{ll}\text { - } & \text { marylandicus Ratt. } \\ \text { - } & \text { moniliformis Ralfs. } \\ \text { - } & \text { partitus Grun. } \\ \text { Ralfsii Eh. }\end{array}$

Actinoptychus excellens Schm.

Amphitetras minuta. Grev.

Asterolampra Dallasiana Grev. marylandica Eh.

Asteromphalus Wallichianus Ralfs.

Aulacodiscus Mölleri Grun.

$$
\begin{array}{ll}
- & -\quad \text { o. distincta Ratt. } \\
- & \text { Rogersii A. S. } \\
- & \text { sollitianus Norm. }
\end{array}
$$

Auliscus pruinosus Bail.

Biddulphia angustata $\mathrm{A}$. S.

- decipiens Grun.

- suborbicularis Grun.

Cladogramma californicus Grun.

Coscinodiscus apiculatus Eh.

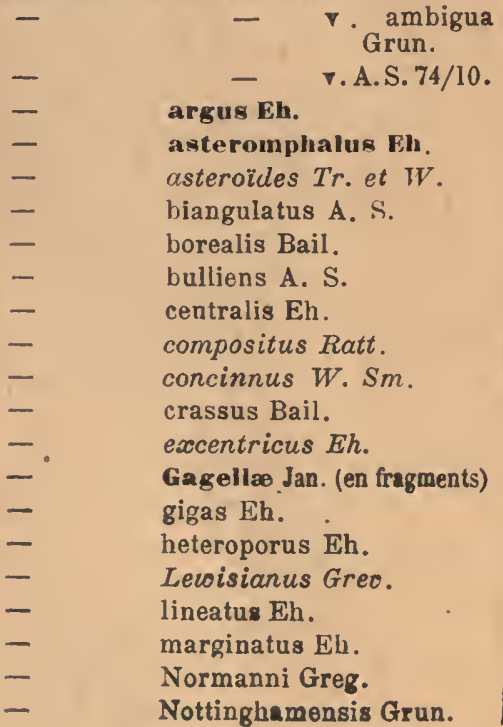

Coscinodiscus oculus-iridis Eh. adontophorus Grun. omphalanthus Eh. perforatus Eh. - - v. cellulosa Grun radiatus $\mathrm{Eh}$.

$\begin{array}{ll}- & \text { odontophorus Grun. } \\ \text { omphalanthus Eh. } \\ \text { - } & \text { perforatus Eh. } \\ - & \quad \text { v. cellulosa Grun } \\ - & \quad \text { radiatus Eh. } \\ -\quad \text { robustus Grun. var. } \\ - & \text { secernendus A. S. } \\ - & \text { subtilis Eh. } \\ - & \text { symbolophorus Eh. var. } \\ - & \text { velatus A. S. } \\ - & \text { Woodwardii Eul. }\end{array}$

Crasperlodíscus coscinodiscus Eh.

- elegans Eh.

- rhombus Grun. var.

Dictylium Ehrenbergii Grun.

- undulatum Eh.

Goniothecium odontella $\mathrm{Eh}$.

Grayia argonauta Grove et $\mathrm{J} . \mathrm{Br}$.

Ilemiaulus bifrons Grun.

Mastogonia crux Eh.

Melosira sulcata $\mathrm{Eh}$.

Navicula excacata Greg. oar.

Pleurosigma affine var. marylandica Grun.

Pseudauliscus radiatus Bail.

$$
\text { - Ralfsianus Ratt. }
$$

Pyxidicula cruciata Elı.

Pyxilla dubia Grun.

Septroneis caduceus Ehr.

Stophanogonia polygona $\mathrm{Eh}$.

Stephanopyxis corona Eh.

$$
\begin{array}{ll}
\text { - } & \text { limbata Eh. } \\
\text { - } & \text { Palmeriana Grev. }
\end{array}
$$

Stictodiscus Kittonianus Grev.

Triceratium acutum Eh.

- americanum Ralf.

- condecorum Bright.

- interpunctatum Grun.

- Kittonianum Grov. 
Triceratium margaritaceum Ralf.

- Marylandicum Bright.

- $\quad$ Montereyi Bright.

- multifrons J. Br.

- punctatum $B r$.

- robustum Greo.

- sculptum oar.

- secernendum A. S.
Triceratium semicirculare Bright.

- solenoceros $\mathrm{Br}$.

- subrotundatum A.S.

- tesselatum Grev. validum Grun.

Xanthiopyxis oblonga Eh.

Zygoceros quadricornis Grun.

Bibliographie. - J. Pantocsek. Diatomées de Hongrie, vol. I, II et III.

Achnanthes brevipes $\mathrm{Ag}$.

Actinocyclus Ehrenbergii Ralfs.

- Ralfsii W. Sm.

Actinoptychus amblyoceros A. S.

- areolatus Eh.

- $\quad$ bifrons A. S.

- Boliviensis, Jan.

- Clevei A. S.

- dilatatus Pant.

- gemminus A.S.

- Grundlerii A. S.

- bungaricus Pant.

- intermedius A. S.

- Janischii Grun.

- kymatodes Pant.

- Muronensis (il.

- neogradensis Pant.

- Pethoi Pant.

- Semseyi Pant.

- $\quad$ splendens Ralfs.

- - var. califor. nica Grun.

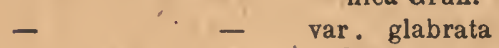

Grun.

- - var. halionyx Grun.

- var nicobarica Grun. undulatus Eh. vulgaris Schum.

Amphora arenaria Donk.

- crassa Greg.

- Grundlerii Grun.

- Lunyacsekii Pant.

- neogradensis Pant.

- oculus A. S.

- Szontaghii Pant.

- tertiaria Pant.

Arachnoidiscus Ehrenbergii Bail.

- indicus Eh.

- ornatus Eh.

Asterolampra Marylandica Eh.

Aulacodiscus affinis Grun.

:-

garica.
- Debyi Pant.

- $\quad$ Grunowii Gl.

$-$

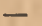

var mosa Pant.

- var. squamosa Pant.

Haynaldii Pant. hungaricus Pant. hyalinus Pant. Lunyacseckii fa maxima Pant. neogradensis Pant. notabilis Pant. reticulatus Pant. 
Aulacodiscus subangulatus Pant.

Auliscus celatus Bail.

$$
\begin{aligned}
& \text { - confluens Grun. } \\
& \text { - } \quad \text { Hauckii Pant. } \\
& \text { - } \quad \text { Noronensis Grev. } \\
& \text { - palvinatus Gl. fa rpiculata. } \\
& \text { - } \quad \text { - } \quad \text { fa inermis Pant. } \\
& \text { - } \quad \text { culptuas Ralfs. } \\
& \text { - } \quad \text { Stöckardtii Jan. } \\
& \text { Berkeleyia hungarica Pant. } \\
& \text { Biddulphia capucina A. S. }
\end{aligned}
$$

$\begin{array}{ll}\text { - } & \text { elegantula Grev. } \\ \text { - } & \text { homala Pant. } \\ \text { - } & \text { pulchella Greg. } \\ \text { - } & \text { regina W. Sm. } \\ \text { - } & \text { Tnomeyi Bail. } \\ \text { - } & \text { - var. elongata Pant. } \\ \text { - } & \text { vasta Pant. }\end{array}$

Campylodiscus Eulensteinii Pant.

$$
\text { - } \quad \text { limbatus Brèb. }
$$

Cerataulus hungaricus Pant.

$$
\begin{array}{ll}
- & \text { Johnsonianus Grev } \\
- & \text { Jinkerii A.S. } \\
- & \text { polymorphns Ktz. } \\
- & \text { turgidus Eh. }
\end{array}
$$

Chretoceros affine Laud.

$$
\text { - gastridium Eh. }
$$

Clavicula delicatula Pant.

- polymorphaGrun.

$$
\begin{array}{ccc}
- & - & \text { var. aspicephala } \\
\text { - } & \text { Pant. } & \text { var.pachycephala } \\
& & \text { Pant. } \\
\text { - } & \text { - } & \text { var. tumida Pant. }
\end{array}
$$

Cecconeis cruciata Pant.

$$
\begin{array}{ll}
\text { - } & \text { Lunyacsekii Pant. } \\
\text { - } & \text { neogradensis Pant. } \\
\text { - } & \text { pellucida Grun. } \\
\text { - } & \quad-\quad \text { var. fossilis Pant. } \\
\text { - } & \text { præcellens Pant. } \\
\text { - } & \text { pseudomarginata Greg. } \\
\text { - } & \text { sigma Pant. } \\
\text { de Toniana Pant. }
\end{array}
$$

Coscinoliscus actinocycloïdes Pant. apiculatus Eh. argus Eh.

Coscinodiscus armatus Pant.

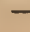

$-$

-

$-$

-

$-$

$-$

$-$

$-$

- fimbriatus Eh.

- fimbrio limbatus Eh.

- - hispidulus Pant.

- hungaricus Pant.

- intumescens Pant.

- Kockii Pant.

- Lewisianus Grev.

- lineatus Eh.

- marginatus Eh.

- moravicus Grun.

- neogradensis Pant.

- nitidus Greg.

- nitidulus Grun.

- oculus iridis Eh.

- pulchellus Grun.

- radiatus $\mathrm{Eh}$.

- $\quad$ - var. heterosticta.

- _ nar. subæqualis.

- robustus Grev.

- spiraliterpunctatus Pant.

- Stockesianus Grun.

- symbolophorus Grun.

- symetricus Grev.

- undatus Grun.

- Weisflogii Pant.

Craspedoporus Truanii Pant.

Dicladia capreolus Eh.

Dimeregramma fossile Grun.

Endictya Lunyacsekii Pant.

- minor A. S.

- oceanica Eh.

- Schmidtii Pant.

Entopyla australis Eh.

- hungarica Pant.

Grammatophora maxima Grun.

$$
\text { - robusta Dippel. }
$$

Gyrodiscus bungaricus Pant.

Hemiaulus hungaricus Pant.

- polymorphus v. frigida Grun.

Hyalodiscus radiatus Grun. 
Ifyalodiscus subtilis Bail.

- _ - v.australiensis Grun.

Isthmia Szaboi Pant.

Ktenodiscus hungaricus Pant.

Mastogonia crux Eh.

Melosira caput medusæ Pant.

- cincta Pant.

- clavigera Grun.

- granulata Ralfs.

- omnia Cl.

- sol Ktz.

Navicula aspera v. hungarica Pant.

- Baũmlerii Pant.

- Beyrichiana A. S.

- Bombus Eh.

- cancellata Donk.

- crabro Eh.

- didyma Eh.

- diplosticta A. S.

- excavata Greg.

- fusca Ralfs.

- gemmata Grev.

- Hennedyi W. Sm.

- humerosa Bréb.

- - var. elongata Pant.

- inhalata A. S.

- Kinkerii Pant.

- Kossuthii Pant.

- lacrymans A. S.

- lyra Eh.

- - var. acuta Pant.

- - var. elliptica A. S.

- - var. hungarica Pant.

- - var. producta Pant.

- mantichora Pant.

- margaritifora Pant.

- maxima Greg.

- nebulosa Greg.

- nitescens Greg.

- O'Swaldii A.S.

- porfecta Pant.

- pinnata Pant.

- pseudomarginata Pant.

- Sandriana Grun.

- Schaarschmidtii Pant.

- Smithii Bréb.

- Szontaghii Pant.

- Venus Pant.

Nitzschia antediluviana Pant.

Odontella neogradensis Pant.
Orthoneis splendida Grun.

Pantocsekia clivosa Grun.

Paralia sulcata $\mathrm{Cl}$.

Periptera letracladia Eh.

Plagiogramma neogradense Pant.

Pleurosigma neagradense Pant.

Ploiaria petasiformis Pant.

Pseudotriceratium cinnamoneum Grun.

Pyxidleula cruciata Eh.

Pyxilla americana Grun.

- baltica Grun.

- cornuta Pant.

- directa Pant.

- dubia Gran.

- hungarica Pant.

- Lunyacsekii Pant.

Rhadonéma adriaticum Ktz.

Raphonois angustata Pant.

- Debyi Pant.

- gemmifera Eh.

- _ - var. elegans Grun.

- $\quad$ var. noogradensis Pant.

- $\quad$ - var parcepunctata Grun.

- hungarica Pant.

- moravica Grun.

- rhombus Eh.

Rutilaria Szakalensis Pant.

- ventricosa Grev.

Scoliopleura Szakalensis Pant.

Stephanogonia actinoptychus Grun.
- aculeata Pant.
- cincta Pant.
- polygona Eh.
- striolata Pant.
- Szontaghii Pant.

Stephanopyxis corona Grun.

- turris var. gemina Grun.

Stictodiscus californicus Grev.

- $\quad$ var. nankooren-
sis Grun.

- parallelus Pant.

- $\quad$ - var. quadrigona

Pant.

Surirella Baldjickii Norm.

- fastuosa Eh.

- Neumayerii Jan. 
Syndendrium diadema Eh.

Synedra baculus Greg.

- crystallina Ktz.

- Nitzschioides var. acuminata Grun.

- - var. obtusa Grun.

Terpsinoë intermedia Grun.

Triceratlum acutangulum Grun.

- antiquum Pant.

- arcticum Bright.

- balearicum Cl. et Grun.

- biquadratum Jan.

- Brunii Pant.

- condecorum Bright.

- $\quad$ - var. neogradensis.

- elevatum Pant.

- favus Eh.

- grande Bright.

- Grovei Pant.

- latum Grev.

- lucidum Pant.

- madagascarense Grun.

- microtis Grun.

- Möllerii Pant.

- muricatum Bright . fossilis.
Triceratium Pantocsekii A. S.

- $\quad$ - fanvexa.

- Pethoi Pant.

- pilous Eh.

- pseudo arcticum Pant.

- radiato punctatum A. S.

- Rzehakii Pant.

- Stockesianum Grev.

- - var.moravica Grun.

- $\quad$ Sturtii Pant.

- $\quad$ Thumii A.S.

- $\quad$ trisulcum Bail

- var. moravica Pant.

undulatum Eh.

Wittii Jan.

- fa hexagona Pant.

Xantblopyxis angulata Eh.

- oblonga Eh.

- panduræformis Pant.

Zygocero circinus Bail.

- quadricornis Grun.

№ 239

SALT SEA-MANSFIELD (États-Unis)

(Vase saumâtre)

Campylodiscus bicostatus W. Sm. clypeas Eh.

echeneis Eh.

Cymatopleura elliptica var. rhomboidea.
Nitzschia circumsuta Bail. Surirella striatula Turp.

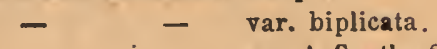

- $\quad$ - $\quad$ var. A.S. atlas 24/19.

\section{No 240 LEATE'S POND. - CONNECTICUT (États-Unis)}

\section{(Saumâtre)}

Actinocyelus crassus W. Sm. Actinoptychus areolatus $\mathrm{Eh}$.
Actinoptychus undulatus Eh.

Amphiprora pulcha Bail. 
Amphora proteus Greg.

Biddulphia aurita Brób. rhombus W. Sm.

Breblssonla Boeckii Grun.

Coscinodiscus excentricus Eh.

- $\quad$ oculus iridis Eh.

Cyclotella striata Grun.

Hyalodiscus stelliger oar.

Mastogloia pusilla Grun.

Melosira sulcata $\mathrm{Ktz}$.

- - var. coronata.

- - var, radiata.

Navicula brasiliensis Grun.

- elegans TV. Sm.

- elliptica var. oblongella.

- Flsherii A. $\mathbf{8}$.

- formosa Greg.

- icostzuron Grun.

- interrupta var.
Navicula lyra Eh.

- - var. elliptica.

- major Ktz.

- peregrina Ktz.

- permagna Bail.

- sculpta ish.

- Smithii Bréb.

- viridis $\mathrm{Ktz}$.

- Yarrensis Grun.

Nitzschia obtusa W. Sm.

- punctata var. coarctata.

- scalaria W. Sm.

Pleurosigma Terryanum H. Per.

Pyxilla baltica Grun.

Stauroneis Gregorii Ralfs.

Stephanopyxis turris Ralfs.

Surirella striatula Turp.

Synedra pulchella Ktz.

- ulna oar. rostrata.

Triceratium alternans Bail.

Tropidoneis lepidoptera var. proboscidea.

\section{N* 241-243 YARRA RIVER - MELBOURNE (Australie)}

(Saumâtre)

Achnanthes pennoformis Greo. Actinocyclus Barklyi Grun.

Amphlprora ornata Bail. var.

Amphora commutata Grun.

- mexicana $\mathrm{Cl}$.

- ooalis Ktz.

- pellucida Greg. var.

- proteus Greg.

- robusta Greg.

- sp. A. S. atlas 39/23.

BIddulphia aurita Bréb.

$$
\begin{aligned}
& \text { - } \quad \text { pulchella Greg. } \\
& \text { - } \quad \text { reticulata Rop. } \\
& \text { - } \quad \text { rhombus W. Sm. }
\end{aligned}
$$

Campylodiscus clypeus Eh. Echeneis Eh.
Campyloneis Greoillei Grun.

$$
\text { - - var. regalis. }
$$

Chatoceros Wighamii Bright (Spores).

Cocconeis heteroidea Hantz.

- lineata Eh. oar.

- pellucida Hantz.

Cocconema australicum $A$. S.

- gibbum Eh.

Coscinodiscus inclusus Ratt. var.

- Iacustris Grun.

- marginatus Eh.

Cyclotella Kützingiana?

- striata Grun.

Cymbella cymbiformis Bréb.

- gastroides Ktz.

- maculata Ktz. 


$$
-134-
$$

Cymatopleura elliptica var. rhomboides. Denticula thermalis Ktz.

Encyonema Auerswaldii Rab.

\section{Yarrense Grun.}

Epithemia gibba Ktz.

$$
\begin{aligned}
& \text { - } \quad \text { gibberula Ktz. } \\
& \text { - } \quad \text { - var. ventricosa. } \\
& \text { - } \quad \text { Hyndmannii W. Sm. } \\
& \text { - } \quad \text { zebra Ktz var. proboscidea. }
\end{aligned}
$$

Eunodia diodon $\mathrm{E} h$.

$$
\begin{aligned}
& \text { - eruca Eh. } \\
& \text { - formica Eh. } \\
& \text { - } \quad \text { - var. recta. } \\
& \text { - } \text { gracilis Rab. } \\
& \text { - lunaris Grun. } \\
& \text { - praerupta var. bidens. }
\end{aligned}
$$

Fragilaria virescens Ralfs.

Gomphonema acuminatum Eh.

$\begin{array}{ll}\text { - } & \text { commutatum Grun. } \\ \text { - } & \text { intricatum Ktz. } \\ \text { - } & \text { vibrio Eh. }\end{array}$

Grammatophor macilenta W. Sm.

Hyalodiacus laevis Eh.

Mastogioia citrus $\mathrm{Cl}$.

- Dansei Eh.

- quinquecostata Grun.

- Smithii var. amphicephala.

- $\quad$ var. lacustris.
Navicula acrosphoeria Bréb.

- ambigua Eh.

- amphirhynchus Eh.

- cuspidata Ktz.

- elliptica Ktz.

- gibba Ktz.

- lauta Grun.

- major Ktz.

- peregrina Ktz.

- producta W. Sm.

- pupula Ktz.

- sculpta Eh.

- Smithii Bréb.

- stauroptera var. parva.

- Yarrensis Grun.

Nitzschia Nooae Hollandice Grun.

- panduriformis Greg.

- scalaris W. Sm, var.

- Tryblionella var. Yarrensis.

Pleurosigma strigilis var.

Podosira hormoides Mont.

Rhabdonema mirificum W. Sm.

Stauroneis acuta W. Sm. var.

- gracilis Eh.

- pachycephala Cl.

- phœnicenteron var. lanceolata.

Stephanopyxis Grunowii oar.

Surirella fastuosa Eh.

- inducta A. S.

- laevigata Eh.?

Triceratium antediluoianum Eh.

Van lleurekia rhomboides var Oregonica.

\section{Embouchure de la Seine}




\section{No 248 et 249 LOCH KINNORD - ABERDEEN (Écosse)}

BIBLIOGRAPHIE. - Liste of Diatomaceæ found in Loch Kinnord Kieselguhr by the Rev. Georges Davidson. (Trans Geol. Soc. Edinburg. Vol. IV, part. 11, p. 212.)

Achnanthes Jackii Rab. lanceolata Bréb.

Achnanthidium flexellum Bréb.

Amphora lineatà Greg.

- ovalis $\mathrm{Ktz}$.

Campylodiscus noricus Eh.

Ceratoneis arcus $\mathrm{Ktz}$.

Cocconeis helvetica J. Br.

$$
\text { - placentula Eh. }
$$

Cyclotella antiqua W. Sm.

$$
\begin{aligned}
& \text { - } \quad \text { Kützingiana Thw. } \\
& \text { - } \quad \text { operculata Ktz. } \\
& \text { - } \quad \text { papillosa O’Meara. }
\end{aligned}
$$

Cymatoplenra apiculata Pritch.

$$
\text { - elliptica Bréb. }
$$

Cymbella acutiuscula $C l$.

- æqualis W.Sm.

- anglica Lag.

- angustata Grun.

- cistula Hemp.

- cuspidata Ktz.

- cymbiforme Eh.

- Ehrenbergii Ktz.

- gastroides $\mathrm{Ktz}$.

- helvetica Grun.

- hercynica A. S.

- naviculæformis Auers.

- 'Norvegica Grun.

- obtusiuscula Ktz.

- parva W. Sm.

- pisciculus Greg.

- scotica W. Sm.

- sp. A. S. atlas 9/56.

Denticula inflata W. Sm.

- tenuis Ktz.
Encyonema cœspitosum $\mathrm{Ktz}$.

$$
\begin{array}{ll}
- & \text { Cesatii Bréb. } \\
\text { - } & \text { gracile Rab. } \\
\text { - } & \text { lunula Grun. } \\
\text { - } & \text { turgidum Grog. }
\end{array}
$$

Epithemia alpestris $\mathrm{K} t \mathrm{z}$.

- gibba Ktz.

- - - ve ventricosa.

- gibberula Ktz.

- globifera Heib.

- Hyndmannii W. Sm.

- ocellata Ktz.

- sorex.Ktz.

- turgida $\mathrm{Ktz}$.

- $\quad$ - var. granulata.

- zebra Ktz.

- - var. proboscidea.

Bunotia arcus Eh.

- - var. minor.

- bigibba Greg.

- camelus Eh.

- diadema Eh.

- faba Eh.

- formica Eh.

- impressa var. minor.

- incisa Grez.

- lunaris Bréb.

- major W. Sm.

- monodon Eh.

- nodosa Eh.

- pectinalis Dillw.

- tetraodon Eh.

- triodon Eh.

- uncinata Eh.

- undulata W. Sm.

- sp.? 
Fragilaria capucina Desm.

- construens Grun.

- lapponica Grun.

- undata W. Sm.

- virescens Ralis.

- . - var. exigua.

Gomphonema acuminatum Eh.

\begin{tabular}{ll}
- & \multicolumn{1}{c}{$-\quad$ fa coronata. } \\
- & $\quad$ fa pusilla. \\
- & Brébissonii Grun. \\
- & constrictum Eh. \\
- & cygnus Eh. \\
- & dichotomum Ktz. \\
- & elongatum Grun. \\
- & gracile var. aurita. \\
- & geminatum Ag. \\
- & Hebridense Greg. \\
- & insigne Greg. \\
- & intricatum Ktz. \\
- & Lagerheimii Cl. \\
- & subtile Eh. \\
- & - var. sagitta Schum. \\
- & tenellum. \\
- & turris Eh. \\
- & vibrio Eh.
\end{tabular}

Mastogloia Grovillei W. Sm.

Melosira crenulata Thw.

vavicula acrospheria Bréb.

- acuta Ktz.

- affinis Eh.

- americana Eh.

- amphigomphus var.

- amphirynchus Eh.

- angustata WV. Sm.

- bacillum Eh.

- borealis Eh.

- Braunii Rab.

- Brebissonii Rab.

- cardinalis Eh.

- cocconeiformis Greg.

- commutata A. S.

- cuspidata Ktz.

- $\quad$ - fa craticula.

- dicephala Eh.

- - var. stauroneiformis.

- divergens W. Sm.

- - var. Mullensis.

- exilis Grun.

- firma Ktz.

- - - var. subampliata Grun.

- follis Eh.
Naricula fulva Eh.

- gibba Ktz.

- gracillima Pritch.

- Hebes Ralfs.

- hemiptera Ktz.

- Hitchockii Eh.

- inflata $\mathrm{Ktz}$.

- iridis Eh.

- lacustris var. $\beta$. Greg.

- laevissima Grun.

- leptogongyla.

- limosa Grun.

- major Ktz.

- mesolepta Eh.

- mesostyla Eh.

- mormonorum A. S.

- nobilis $\mathrm{Ktz}$.

- nodosa Eh.

- nodulosa Grun.

- oculata Bréb.

- ovalis W. Sm. fa elliptica.

- peregrina $\mathrm{Ktz}$.

- polyonca Bréb.

- pygmaea Ktz.

- radiosa Ktz.

- - var. acuta.

- rhoinbica Greg.

- rupestris Hantz.

- rhyncocephala Ktz.

- scutelloides W. Sm.

- semen Eh.

- semicruciata Eh.

- serians Ktz.

- var. minor.

Smithii Brèb.

- sphorophora Ktz.

- stauroptera Grun.

- - var.

- staumatophora Grun.

- termitina A. S.

- tumida W. Sm.

- tuscula Eh.

- undulata Greg.

- viridis Ktz.

- viridula Eh.

- - var. major.

- sp. ? A. S. atlas $43 / 25$.

- sp. ? A. S. atlas $44 / 51$.

- sp.? A. S. atlas 45/28.

- Zellensis Grun.

Nitzschia angustata Grun. 
Vitzschia denticula Grun.

- linearis W.Sm.

- parvula W. Sm.

- sigmoidea W. Sm.

Odontidium mutabile W. Sm.

Pleurosigma attenuatum $W . S m$.

- lacustre W.Sm.

- Spencerii W.Sm.

Stauroneis acuta $W$. Sm.

- anceps Eh.

- $\quad$ - var. linearis.

- dubia Greg.

- gracilis Eh.

- phœinicenteron Eh.

Stenopterobia anceps $G r$.

Stephanodiscus astrea Grun.

- rotula $\mathrm{Ktz}$.

- minutulus Grun.

Surirella constricta Eh.
Surirella elegans Eh.

- linearis W. Sm.

- robusta Eh. (= S nobilis).

- splendida Ktz.

- tenera.

synedra acus. var. tenuissima.

- biceps $K t x$.

- capilata Eh.

- linearis W. Sm.

- longissima W. Sm.

- lunaris W. Sm.

- radians $\mathrm{Ktz}$.

- ulna Eh.

Tabellaria fenestrata Lyng.

$$
\text { - flocculosa Ktz. }
$$

Tetracyclus lacustris Ralfs.

Tryblionella angustata W.'Sm:

Van lieurckla crassinervia Bréb.

rhomboides Bréb.

BIBLIOGRAPHIE. - Fossil Diatomaceous deposit from Oamaru NewZealand by E. Grove et G. Sturt 1886-1887. - Ad. Schmidt, atlas. Grunow dans le Bot-Centralblatt, $n^{\circ} 31,1887$; nos 15 et 16,1888 . - Le Diatomiste, vol. I et II.

Actinodiscus Barbudensis Greo.

Actinoptychus splendens v. fusca Gr. et St. - vulgaris $\nabla$. maculata Gr. et $\mathbf{S t}$.

Arachnoidiscus Ehrenbergii Bail.

Aulacodiscus angulatus Grev.

$$
-
$$

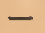
cellulosus Gr. et St. crux var. subsquamosa Grun.

- margaritaceus v. Debyi Ratt.

- Rattrayi Gr. et St.

- $\quad$ sollitianus v. Novæ-Zelandæ.

- subrimosus Grun.
Auliscus Oamaruensis Gr. et St.

Biddulphia Tuomeyi Bail.

- - rigida $\mathrm{A}$. $\mathrm{S}$.

Brightwellia coronata Ralfs.

Coscinodiscus argus Eh.

-

$-$

apollonis Eh.

$-$ concavus Greg.

- marginatus Eh.

- radiatus Eh.

- $\quad$ - var. media.

- Rothii Grun.

- spiniferus Gr et St. 
Coscinodiscus subtilis Eh.

$$
\text { - } \quad \text { symbolophorus Grun. }
$$

Cerataulus subangulatus $G r$. et St.

Eunotogramma productum Gr. et St.

- Melosira clavigera Grun.

- Oamaruensis Gr. et St.

- sol Eh.

- sulcate Ktz.

Podosira argus var. Grun:

Stephanopyxis Grunowii Gr. et St.
Stietodiscu californicus Grev. parallelus fa trigona.

[2 gibbosa.

Triceratium castellatum var, fracta Grun. castelliferum Grun. crenulatum Gr. et St. favus Eh. glandarium A. S. glandiferum Grun. grande Bright. majus Gr. et St. Nova-Zælandicum Gr. et St. rugosum Gr. et St.

\section{Nos 251-25:3 DEMERARA RIVER -'GEORGE TOWN (Guyane Anglaise)}

\section{Vase saumâtre}

Actinella Guyanensis Grun.

Actinoptychus splendens Shadb.

ín undulatus minor.

Ámphiprora conspicua Grev. - paludosa var. Nereis.

\section{Amphora ovalis var, affinis.}

$$
\text { - proteus Greg. }
$$

Auliscu coelatus var. rhipis.

Bacillaria paradoxa Grun.

Biddulphia obtusa Ralfs.

Campylodiscus ? cocconeiformis Grun.

Coscinodiscus asteromphalus Eh.

$$
\begin{array}{ll}
\text { - } & \text { concinnus W. Sm. } \\
\text { - } & \text { - var. Jonesiana. } \\
\text { - } & \text { curvatulus v. latiusstriata. } \\
\text { - } & \text { excentricus Eh. } \\
\text { - } & \text { lineatus Eh. } \\
\text { - } & \text { marginatus finor. } \\
\text { - } & \text { minor Eh. } \\
& \text { oculus-iridis Eh. }
\end{array}
$$

Cyclotella stylorum Bright.
Denticula Kittoniana Grun.

Epithemia argus $\mathrm{Ktz}$.

- gibberula Ktz.

- turgida Ktz.

$$
\text { - } \quad \text { - var. porcellus. }
$$

Euodia ? (Margaritacea var. ?) minor, M. Per. - Petite forme elliptique, $25 \mu$ : $13 \mu$, finement granulée sur les bords, un peu plus fortement au centre où les granules sont libres; une couronne submarginale d'èpines; pas d'ocelle visible.

Fragilaria construens Grun.

- viréscens. oar. binodis.

Gomphonema angustatum var. producta.

Ilantzschia amphioxys var. minor.

IIyalodiscus radiatus var. minor.

Melosira sulcata var. coronata.

- undulata Eh. 
Navlcula arenaria Donk.

- bombus Eh.

- - var. densestriata.

- brevicostata.

- cancellata var. Gregoryi (no 253).

- crabro fa minor.

- Dusenii Cl.

- elliptica Ktz.

- formosa Greg. var.

- gibba Ktz.

- liber W. Sm. fa curta (L. $43 \mu$ ).

- limosa Ktz.

- major Ktz.

- mulica var. Goeppertiana.

- permagna Edw.

- rostellata ktz. var. (n०253).

- salinarum var. intermedia.

- Stauntonii Grun.

Vitzschia angusta Grun.

- brevissima Grun.

- fasciculata Grun.

- - var. minor.

- granulata Grun.

- Ianceolata var. minor (n॰253).

- maxima fa minor.

- obtusa W. Sm.

- punctata Grun.

- sigma var. stigmatolla

- var. rigida.

- Tryblionellà $\vee$. maxima.

- - f minor.

- $\quad$ - var. Victoriæ.
Orthotropis lepidoptera,$\nabla$. proboscidea - $\quad$ - var. pusilla.

Plagiotropis zebra var.

Pleurodesmium Brebissonii Ktz.

Pleurosigma attenuatum W. Sm.

- spectabile Gr. var.

- Spencerii var. Arnotti.

Pleurostauron (sagitta var. ?) divisa, M. Per. - Différe du pleurostauron sagitta par sa forme moins rostrée et en ce que le stauros, limité par deux stries assez fortes est divisé par une strie médiane enoore plus forte; les striès au nombre de 16 en $10 \mu$ sont parallèles au milieu, plus serrées (18) et lègèrement convergentes aux extrémités. Long. 35 à $40 \mu$.

Stauroneis salina W. Sm.

Surirella biseriata Bréb. var.

- Davidsonii A. 8. (n॰251).

- Guyanensis M. Per. (A. S. atlas P1. 24/25).

- recedens $A$. S. (n॰252).

Syringidium americanum.

Van Ileurckia Lewisiana.

\section{Nos 254 et $245 \quad$ AROLLA (Alpes pennines)}

Tourbières situées à 2100 mètres d'altitude

Cette liste nous a été communiquée par M. le professeur J. Brun, de Genève, de qui nous tenons la récolte.

Achnanthidlum flexellum Bréb.

Cymbella cymbiformis Eh. - cistula Homp.
Cymbella delicatula Ktz.

- lævis Ktz.

Dlatoma ancops Eh. 
Diatoma mesodon $\mathrm{Eh}$.

Navicula amphisbœna $\nabla$. obtusa Bory. - tuscula Grun.

Cymbella Cesati Rab.
Vitzschia sinuata W. Sm. angusta $\mathrm{v}$. curta V. H.

Surirella Helvetica $\mathrm{J} . \mathrm{Br}$.

- spiralis $\mathrm{Ktz}$.

Totracyclus rupestris Braun.

No 256 MOISSAC - TARN-ET-GARONNE (France)

Cymatopleura olliptica W. Sm .

Nitzechla lunario W. Sm.
Surirella splendida Greg.

- tenora Greg.

N॰ 257

GUATEMALA (Etats-Unis)

Dépôt fossile d'eau douce

Melosira granulata Eh.

Stephanodiscus carconensis var. pusilla Cran.

$N^{08} 258-261$

KUSNETZK (Russie)

Dépôt fossile marin

Bibliographie. - J. Pantocsek. Diatomées fossiles de Hongrie.

\section{Volumes II et III.}

Acanthodisçs rugosus Pant.

Actinopiychus heterostrophus A. S.

Kusnetzkianus Pant.

Arachnoidiscus giganteus Pant.

- róssicus Pant.

Aulacodiscu* Darwinii Pant.
Aulacodisens Gurowii Pant.

- interruptus Pant.

- Kellerii Pant.

- Ledebourii Pant.

- nigrescens Pant.

- Peragalloi Pant.

- Tschestnowii Pant. 
Riddulphia crassiucula Pant. elegantula Grev. var. polycistina Pant.

\section{- $\quad$ - var. polygibba Pant.}

- - var. sarmatica Pant.

- fistula Pant.

- Gurowii Pant.

- pustulata J. Br.

- rossica Pant.

- Saratowiana Pant.

- Tschestnowii Pant.

Brightweliia rossica Pant.

Coscinodiscus Kusnetzkianus Pant.

$$
\text { - tenuis Grun. }
$$

Epithelion curvatum Pant.

- rossicum Pant.

- spinifer Pant.

Ethmodiscus carinatws Pant.

$$
\text { - } \quad \text { rossicus Pant. }
$$

Eunotogramma Weissii Eh.

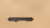

v. producta Grove et St.

Gyrodiscus vortex $\mathrm{O}$. W.

Hemiaulus caverna $J . B r$.

, - Grunowii Pant.

- perlongus Pant.

- Saratovianus Pant.

- Weissflogii Pant.

Koratophora nitida Pant.

- robusta Pant.

Ktenodiscus rossicus Pant.

Melosira clavigera Grun.

- irregularis Pant.

- Saratoviana Pant.

- sarmatica Pant.

Navicula curtestriata Pant.

- primordialis Pant.

Odontotropis birostrata Pant.

Pantocsekia rossica Pant.

Paralia polycistinea Pant.

Pyxilla carinifera v. rossica Pant.

- gracilis Temp. et Forti var. buccinalis Forti.

- - var. Saratoviana Temp. et Forti.

Shelotonema styliferum J. Br.

Stephanopyxis antiqua Pant.

maxima Pant.
Stephanepyxis rossica Pant.

casta Pant.

Stictodiscus Kossuthii Pant.

$\begin{array}{ll}\text { - } & \text { Pantocsekii Temp. } \\ \text { - } & \text { Tschestnou ii Pant. } \\ \text { - } & \text { tuberculatus Pant. }\end{array}$

Syndetoneis rossica Pant.

Triceratium abyssorum Grev. var.

- Saratovianum Pant.

- Armaseoskii Pant.

- caducum Pant.

- cristatum Pant.

- cucullatum Pant.

- var. latior.

- $\nabla$. dissemine punctatum.

v. validior.

-
-
-
-
-
-
-
-
-
-
-
-
-
-
-
-
-
-
-
-
-
-
-
-
-
-
-

\section{Debesii Pant.}

deformatum Pant.

deductum Pant.

delectabile Pant.

Dutertrei Temp.

Eulicherii Pant.

fasciatum Pant.

fragile Pant.

globulifer J. Br.

Gurowii Pant.

, hilaratum Pant.

idoneum Pant.

illustrum Pant.

jucundum Pant.

Kusnetzkianum Pant.

lanceolatum Pant.

Lahusenii Pant.

Mereskowski Pant.

notatum Pant.

Petitii Pant.

Peragalloi Pant. et Temp.

praferoso Pant.

proprium Pant.

protractum Pant.

Rattrayi Pant.

renunciatum Pant.

Saratovianum Pant.

sarmaticum Pant.

septum Pant.

Sokolowii Pant.

speciosum Pant.

squamatum Pant. 
Triceratium stigmaticum Pant.

- subcapitatum Pant.

- ruslandicum Temp.

- tectum Pant.

- tertiarium Pant.

- tetragonum Pant.

- triasicum Pant.

- Truanii Pant.

- undatum Pant.

- undosum Pant.

- vates Pant.

- ventriculosum A. S.

- oenustum 0 . W
Trinacria antiqua Pant.

$$
\begin{array}{ll}
\text { - } & \text { bystrix Pant. } \\
\text { - } & \text { palmipes J. Br. } \\
\text { - } & \text { proecellens Pant. } \\
\text { - } & \text { rossica Pant. } \\
\text { - } & \text { Semseyi Pant. } \\
\text { - } & \text { simulacroides Pant } \\
\text { - } & \text { sparsa Pant. } \\
\text { - } & \text { Tschestnowii Pant. } \\
\text { - } & \text { oetustissima Pant. }
\end{array}
$$

Zygoceros antiquus Pant.

Nor 262-264

\section{MOISSAC (Cantal France)}

Dépôt fossile d'eau douce

BIBLIO $¥$ RA PHIE. - Frère J. Héribaud. Les Diatomées fossiles d'Auvergne 1903.

Achnanthes hungarica Grun.

- Flahaulti F. Hérib.

- lanceolata Grun.

- - $\quad$ - elliptica Cl.

Amphora affinis Ktz.

- Borneti F. Hérib.

- distincta F. Hérib.

Cocconeis Bonnieri F. Hérib.

- lineata Grun.

- - v. euglypta Grun.

Cyclotella iris J. Br. et F. I.

- Kūtzingiana จ. gemmatula F. Hérib.

- perforata.F. Hérib.

Cymbella aspera Ehr.

- Brevieri F. Hèrib.

- capitata M. P. et F. H.

- cuspidata Ktz.

- cymbiformis Ehr.

- gastroides Ktz.

- hungarica Pant.

- Hauckii V. H. จ. fossilis F. H.

- Laubyi F. Hẻrib.
Cymbeila leptoceros Ktz. fa curta.

- Pagesi F: Hérib.

- Rhodesi F. Hérib.

Cymatopleura Martyi F. Hérib.

Diatuma pectinalis $\mathrm{Ktz}$.

Diploneis elliptica $\mathrm{Ktz}$.

- $\quad$ - $\quad$. oblongella Næg.

Epithemia Hyndmannii W. Sm.

- - sorex Ktz.

- turgida $\mathrm{Ktz}$.

- $\quad$ - . granulata Grun.

- zebra Ktz.

- - - v. proboscidea Grun.

Funotia impressa Ehr.

- parallela Eh.

- pectinalis Rab.

Fragilaria brevistriata Grun.

- - - v. elliptica F. H.

- construens Grun.

- - $\quad$ v. venter Grun.

- elliptica Schum. 
Fragilaria intermedia Grun.

- lancettula Schum.

- nitida F. H. et M. P.

- . delicatula F. H. ot M. P.

Zeilleri F. Hèrib.

Gomphonema affinis $\mathrm{KI}$.

$\begin{array}{ll}\text { - } & \text { clavatum fa curta V. H. } \\ \text { - } & \text { eriense Grun. } \\ \text { - } & \text { rigidum F. Hérib. } \\ & \text { vibrio Ehr.' }\end{array}$

Grunowla Moissacensis F. Hérib.

- tabellaria Grun.

Melosira canalifera $\mathrm{J}$. Br, et F. H.

- granulata Eh.

- lineolata v. robusta F. H.

- lævis Grun.

- Roseana Rab.

- undulata Ktz.

- - v. debilis F. H.

- - - v. producta A. S.

Navicala acrosphieria Bréb.

- - v. elongata F. H.

- - v. lævis F. H. et M. P.

- acephala F. Hérib.

- amphibola $\mathrm{Cl}$.

- $\quad$ - v. stauroneiformis F. H.

- amphigomphus Eh.

- bacillum Ehr.

- Berriati F. Hèrib.

- - $\quad$ v. minor F.H.

- bicapitata v. sulcata F. H.

- Braunii v. Moissacensis F.H.

- Chaberti F. Herib.

- cuspidata fa craticula Ktz.

- $\quad$ - v. Heribaudi M. P.

- decrecens $F$. Hèrib.

- decurrens $\nabla$. curtecostata F. $H$.

- digito-radiata Grog.

- divergens $\nabla$. undulata cuneata F. $\mathrm{H}$.

- esox Ehr.

- gustrum Donk.
Navicula Gendrei F. Hérib.

- gibbula Cl.

- - var, cantalica F. H.

- hemiptera Ktz.

- - $\quad$ - angusta F. H.

- $\quad$ v. Bulawskii F.H

- - v. gibba F. H.

- Huei F. Hérib.

- hybrida F. H et M. P.

- iridis v. subproducta F. H.

- major Ktz.

- mesolepta Ehr.

- - v. stauroneiformis Grun.

- microstauron Ehr.

- mutica $\nabla$. producta V. H.

- nobilis Ktz.

- placentula Ehr.

- producta W. Sm.

- radiosa $\mathrm{Ktz}$.

- - $\quad$ - acuta Grun.

- rostellata Ktz.

- sphærophora Ktz.

- tenella Bréb.

- termes Ehr.

- $\quad$ - v. stauroneiformis $\mathbf{V}: \mathrm{H}$.

- ventricosa $\nabla$. cuneata F. H.

Opephora Martyi F. Hérib.

- $\quad-\quad$ v. capitata F. H.

Stauroneis acuta WV. Sm.

- anceps Ehr.

- - v. linearis Grun,

- antediluviana F. Hérib:

- Baileyi Ehr.

- gallica M. P. et F. H.

- javanica $\nabla$. arvernense F.H.

- parrula Grun.

- Phœnicenteron Ehr.

Surirella gracilis Grun.

- splendida Ehr.

- tenera Greg.

Synedra ulna Ehr.

- - $\quad$. amphirynchus Eh.

Tetracyolus emarginatus W. Sm. 
No 265 BANYULS (France)

Dans les bacs de la Station Zoologique

Cyclophora tenuis Cast.

I Nitzsehia bicostata W. Sm.

\section{No 266 YAGIANA BAY OREGON (États-Unis)}

Amphora angusta Eh var.

Cocconeis scutellum Eh.

Epithemia musculus W. Sm.

Melosira Borreri Grev.

- nummuloides Ag.

Navicula didyma Ktz.

- humerosa Bréb.

Nitzschia longissima Ralfs.
Nitzschia sigmatella Greg.

Pleurosigma angulatum W. Sm.

- balticum W. Sm.

- elongatum W. Sm.

- formosum W. Sm.

- latum. Cl.

- strigosum W. Sm.

Synedra affinis Ktz.

N・ 267

MOJI (Japon)

Dépot fossile marin

Cestodiscus japonicus $\mathrm{Cl}$.

N• 268 NASSAU - ILES BAHAMA ou LUCAYES

Vase marine

Actinocyclus fasciculatus Gast.

- rotula J. Br. var.
Amphora (acuta var. ?) arcuata A. S. - bigibba Grun. var. 
Amphora corpulenta var. capitata $n$. $\nabla$. Dos un peu moins développé, en demi-cercle, extrémités capitées, recurvées vers le bord dorsal.

- cymbelloïles Grun. var.

- fusca A.S.

- Janischii A. S. var.

- lineata Greg.

- Nova Caledonica Grun. var.

- obtusa Greg.

- $\quad$ - v. rectangulata Per.

- porcellus Kitt.

- rhombica Kitt. var.

- sp. A. S. atlas 26/26.

Anaulus minutus Grun.

Auricula intermedia $\mathrm{Cl}$.

- minuta $\mathrm{Cl}$.

Biddulphia pulchella Greg.

Campylodiscus crebrestriatus Grev.

$\begin{array}{ll}- & \text { ecclesianus Grev. } \\ - & -\quad \text { var. } \\ - & \text { intermedius Grun. } \\ \text { - } & \text { limbatus Brób. } \\ \text { - } & -\quad \nabla . \text { chilensis. } \\ \text { - } & \text { samoaensis' Grun. } \\ \text { - } & \text { subangularis Greg. }\end{array}$

Climacosphenia (moniligera var. 9) Catera Shadb.

Cocconeis pseudomarginata Greg.

Coscinodiscus marginatus Eh.

$$
\text { - nodulifer Jan. }
$$

Cymatosira Lorenziana fa lata(Long. $10 \mu$ ). Dictyoneis marginata Cl.

Dimeregranma lanceolatum Per var. minor Ralfs.

Donkinia recta Grun. var. minuta.

Epithemia constricta Bréb.

$$
\begin{aligned}
& \text { - gibberula Ktz. } \\
& \text { - - - v. protracta. } \\
& \text { - musculus Ktz. }
\end{aligned}
$$

Euphyllodium spathulatum Shadb.

Glyphodesmis eximia Grev.

Grammatophorse marina var. tropica.

$$
\text { - ovalariensis Grun. }
$$

Mastogloia affinis $\mathrm{Cl}$. var. affirmata $\mathrm{Cl}$.
Mastogloia amaena $J, B r, \nabla$. turgida.

- angulata Lowis.

$$
\begin{aligned}
& \text { - } \quad \text { - var. pusilla. } \\
& \text { - asperula Grun. } \\
& \text { - Bahamensis Cl. oar. } \\
& \text { - bisulcata Grun. } \\
& \text { - citrus Cl. } \\
& \text { - corsicana Grun. } \\
& \text { - Craveni Leud Fort. } \\
& \text { - erythrea Grun. var. } \\
& \text { - - var. biocellata. } \\
& \text { - euxima Cl. var. } \\
& \text { - lineata } C l \text {. } \\
& \text { - Macdonaldii Grev. var. } \\
& \text { - paradoxa Grun. var. } \\
& \text { - Peragalli J. Br. } \\
& \text { - _ - var. circumnodosa. } \\
& \text { - pisciculus } \mathrm{Cl} \text {. } \\
& \text { - rhombus P. P. } \\
& \text { - rimosa Cl. } \\
& \text { - rostellata Grun. } \\
& \text { - Smithii Eh. } \\
& \text { - - var. abnormis. } \\
& \text { - - var. lacustris. } \\
& \text { - - } \quad \text { - var. lanceolata. } \\
& \text { - sp? }
\end{aligned}
$$

Melosira arenaria Moore.

- sulcata var. coronata.

- - var. radiata.

Navicula ambigua $E h$.

- apis Donkin.

- approximata Greo.

- Bleischii Jan. var.

- bombus Ktz.

- - var. dentestriata.

- campylodiscus Grun.

- coffeaeformis A. S.

- complanata Eh. oar.

- crabro Eh. var. separabilis.

- demta A. S.

- directa Ralfs.

- formosa Greg.

- gemmatula Grun. var Grunowii.

- irrorata Greo.

- liber W. Sm.

- . - vảr. linearis.

- liburnica Grun.

- lyra Eh.

- Powellii Lewis. 
Navicula Sausayana Grun.

- Smithii Bréb.

- transfuga Cl.

- sp. A. S. atlas 6/28.

- sp. A. S. atlás $8 / 14$.

Vitzschia augularis var. affinis.

- Jelinecki Grun.

- granulata Grun. var.

- panduriformis Greg.

- $\quad$ - var. minor.

- - var. peralbata.

- punctata v. coarctata.

- sigma fa maxima.

- , - var. rigida.

- vermieularis Hantz.

Orthoneis binotata Grun.

- Glevei Grun.

- cocconeiformis Grun.

- $\quad$ - var. polynesica.

- cribrosa Grun.

- fimbriata Grun.

- Howatiana Grun.

- ovata Grun.

- - var. intermedia.

- pacifica J. Br.

- splendida Grun.

Orthotropis lepidoptera Grun.

$$
\text { - var. pusilla. }
$$

Petitia Gen. nov, Valve bacillaire courbee, couverte de stries trans. versales interrompues par deux sillons latéraux (1).

- Temperei M. Per., extrémités elliptiques, long. 90 à $140 \mu$, larg.
11 à $12 \mu$ et 16 stries $10 \mu$, courbée aux extrémités.

Plagiogramma decussatum Grev.

Pleurosigma decorum W. Sm.

Rhabdonema adriaticum $\mathrm{K} \iota z$.

Rhoicosigma oceanicum H. P.

- Weissflogii Grun. v. minor.

Roperia tessellata Grun.

Stictodesmis australis Grev.

Surirella fastuosa Eh.

- manca Jan. var.

- mexicana A. S.

Synedra fulgens Grun.

- formosa Grun.

- superba Ktz.

Synedrosphenia baiculæformis M. Per., n. sp. - a la forme du $S$. cuneata et la striation du Synedra baculus.

Toxarium Hennedyanum Grun.

- undulatum Bail.

Trachyneis aspera $C l$.

$$
\begin{aligned}
& -1 \quad \text { - var. intermedia. } \\
& \text { Triceratium antillarum Cl. } \\
& \text { - } \quad \text { pentacrinus Wall. }
\end{aligned}
$$

(1) Le Septroneis intermedia(Cleve 1883) (Vega) p. 507, pl. 37, fig. 60, est une forme presque identique de structure, mais de forme à peu près droite.

\section{No 269 LA PETITE SOLE - MANCHE (France)}

Ce banc de la Manche est connu en Angleterre sous le nom de Shamlock Bank; les Diatomées qu'il a fournies à M. le docteur Leuduger-Fortmorel sont très précieuses pour la florule de la Manche.

Achnanthes longipes Ag.

Actinocycís crassus Bail.

$$
\text { - } \quad \text { moniliformis Ralfs. }
$$

Actinoptychus undulatus $\mathrm{Eh}$.

Amphiprora alata Eh.

$$
\text { - } \quad \text { elegans W. Sm. }
$$


Amphora acuta Greg.

- cingulata Gl.

- marina W. Sm.

- obtusaGreg.

- proteus Greg.

Auliscus cœlatus Bail.

- sculptus Ralfs.

Biddulphia aurita Bréb.

$$
\text { - Baileyi W. Sm. }
$$

Campylodiscus Lorenzianus. Thuretii Bréb.

Cerataulus Smithii Grun.

- turgidus Eh.

Coscinodiseus concinnus $W$. Sm.

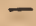

$-$ excentricus Eh.

$-$ gigas Eh.

$-$ lineatus $\mathrm{Eh}$.

oculus iridis Eh. radiatus $\mathrm{Eh}$.

Dimeregramma marinum $\mathrm{Rab}$.

Epithemia musculus Ktz.

Grammatophora macilenta W. Sm. - serpentina Eh.

Hyalodiscus scoticus Ktz.

- stelliger Bail.

- subtilis Bail.

Liemophora Ehrenbergii Grun.

Melosira sulcata $\mathrm{Ktz}$.

- Westii W. Sm.

Navicula abrupta Greg.

- apis Donk.

- aspera Eh.

- Baileyana Grun.

- blanda A. S.

- bomboïdes A. S.

- bombus Eh.

- brevis Greg.

- cruciformis Donk.

- cyprinus W. Sm.

- didyma ktz.

- digito-radiata Greg.

- exemta A. S. var.

- forcipata Greg.

- formosa Greg.

- fusca Greg.
Navicula Hennedyi W. $\mathrm{Sm}$.

- humerosa Bréb.

- incurvata Greg.

- libor W. Sm.

- - var. linearis WV. sm.

- longa Greg.

- Jyra Eh.

- - var. atlantica A. S.

- - var. elliptica A.S.

- maxima Greg.

- _ - var. bicuneata.

- nobilis v. explita A. S.

- prætexta fa minor.

- puella A. S.

- quadrataera A. S.

- rectangulata Grog.

- retusa Bréb. var.

- rhombica Greg.

- Smithii Bréb.

- subcincta A. S.

- Treweloyana Donk.

Nitzschia acuminata W. Sm.

- punctata W. Sm.

- sigma W. Sm.

- spectabilis W. Sm.

Pleurosigma æstuarii W. Sm.

- balticum W. Sm.

- angulatum W. Sm.

- formosum W. Sm.

- rigidum W. Sm.

Pyxilla baltica Grun.

Rhabdonema arcuatum $\mathrm{Ktz}$.

- minutum Ktz.

Scoliopleura latestriata Grun.

- tumida Grun.

Schizonema neglectum Thw.

Surirella fastuosa $\mathrm{Eh}$.

- - var. opulenta.

- flumimensis Grun.

Synedra baculus Greg.

- crystallina Ag.

- fulgens W. Sm.

- undulata Greg.

Triceratium antediluvianum Eh. punctatum Bright. 
Nos 270-272

KEKKO - HONGRIE

Diatomées fossiles marines

Bibliographie. - J. Pantocsek. Diatomées de Hongrie, volume I.

Actinocyclus Ehrenbergii Ralfs.

Actinoptychus Ralfsii IV. Sm.

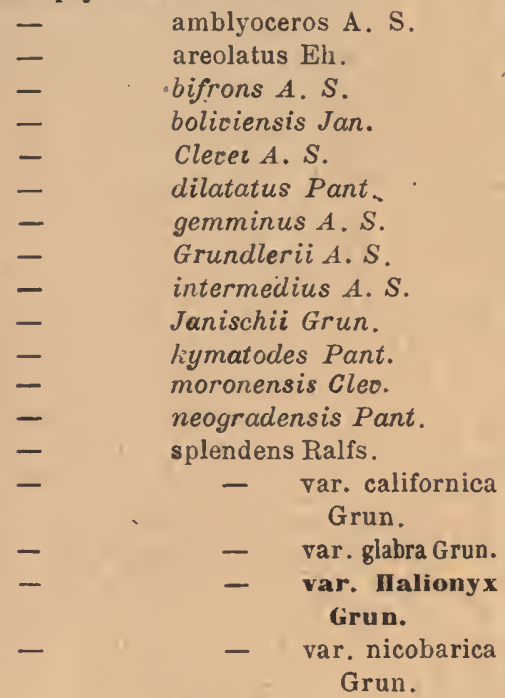

- $\quad$ Stella A. S.

- undulatus Eh. .

- vulgaris Grun.

Arachnoidiscus Ehrenbergii Bail.

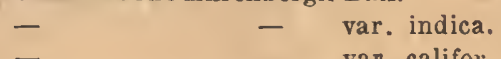

Aulacodiseus argus $E h$.

var. californica.

$\begin{array}{ll}- & \text { Grunowii Cl. } \\ - & -\quad \text { var . subsqua } \\ \text { - } & \text { mosa Pant. } \\ \text { - } & \text { Haberschawii Pant. } \\ & \text { hungaricus Pant. }\end{array}$

Aulacodiscus Lunyacsekii Pant. fa maxima.

$$
\begin{array}{ll}
\text { - } & \multicolumn{1}{c}{f^{2} \text { minor. }} \\
\text { - } & \text { neogradensis Pant. } \\
\text { - } & \text { reticulatus Pant. } \\
& \text { subangulatus Pant. }
\end{array}
$$

Auliscus colatus Bail.

- confluens Grun.

- Hauckii Pant.

- moronensis Grev.

- pulvinatus Cl. fa apiculata Pant.

- - fainermis Pant.

- sculptus Ralfs.

Biddulphia elegantula Grev.

- homala Pant.

- Regina IV. Sm.

- Tuomeyi Bail.

Campylodiscus adriaticus Grun.

$$
\text { - } \quad \text { limbatus Bréb. }
$$

Cerataulus Johnsonianus Greo.

- polymorphus Ktz.

- turgidus Eh.

Clavicula polymorpha Grun. car. delicatula Pant.

Cocconeis pellucida Grun.

- pseudomarginata Greg.

Coscinodiscus actinocycloïles Pant.

- apiculatus Eh.

- asteromphalus Eh.

- $\quad$ - var. hybrida

Grun.

clivosus Pant.

crassus Bail.

elegans Grev. 
Coscinodiscus fimbriatus Eh.

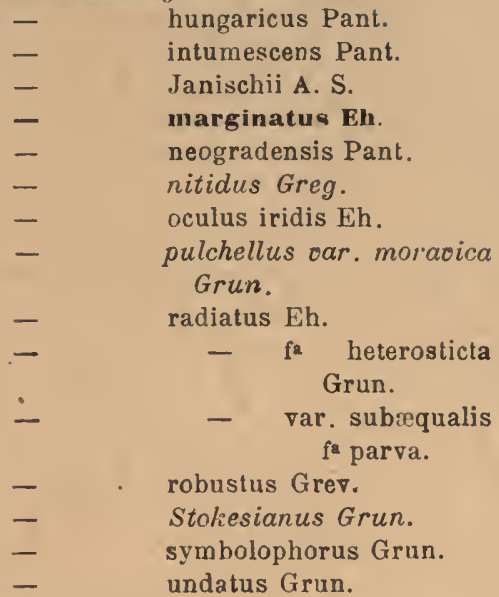

Endictya oceanica Eh.

Leudugeria Janischii Temp.

Goniothecium odontella Eh.

Grammatophora maxima Grun. - robusta Dippel.

liyalodiscus radiatus Grun.

- subtilis var. australiensis Grun.

Melosira biharensis Pant.

- caput medusce Pant.

- cincta Pant.

- clavigera Grun.

- granulata Ralfs.

- omma Cl.

- sol Ktz.

Navicula apis Dont.

- aspera Eh.

- Beyrichiana A. S.

- crabro Eh.

- didyma Eh.

- Elesdiana Pant.

- forcipata Greo.

- fusca Ralfs.

- gemmata Greo.

- - - oar. fossilis Pant.

- Hennedyi W. Sm.

- humerosa Bréb.

- latissima Greg.

- Lyra Eh.

- - var. elliptica.

- maxima Greg.
Navicula nebulosa Greg.

- protexta Eh.

- Sandriana Grun.

- Schaarschmidtii Pant.

- Smithii Breb.

- spectabilis Greg.

- Szontaghii Pant.

Orthoneis splendida Grun.

Pantucsekia clivosa Grun.

Paralia sulcata Cil.

Pseudotriceratium cinnamoneum Grun.

Pyxidicula cruciata Eh.

Pyxilla americana Grun.

Raphoneis angustata Pant.

- gemmifera Eh.

$$
\begin{array}{lll}
- & - & \text { fa breois. } \\
- & - & \text { oar. elegans Pant } \\
- & - & \text { oar. neogradensis } \\
& & \text { Pant. }
\end{array}
$$

Rhabdonema adriaticum $\mathrm{Ktz}$.

Stephanopyxis corona Grun. turris Eh.

- fa nuda Pant.

- fa intermedia Grun.

Stictodiscus californicus Greo.

- nankoorensis Grun.

Syndendrium diadema $E h$.

Synedra nitzschioides Grun. o. acuminata. oar. obtusa.

Triceratium acutangulum Grun.

- arcticum Bright.

- balearicum Grun.

- favus Eh.

- $\quad$ grande Bright. fa pentagona.

- Grovei Pant.

- microtis Pant.

'- Pantocsekii A. S.

- $\quad$ - fa convexa.

- $\quad$ - fa pentagona.

- radiato-punctatum.

- Stokesianum Grev.

- Thumii A. S.

- $\quad$ trisulcum Bail. - $\quad$ - $\quad$ Par hungarica

undulatum Eh.

Xanthiopyxis oblonga Eh.

Zygoceros circinus Bail. 
No 273 LAC ROTOREIA (Nouvelle-Zélande)

\section{Analysé par le professeur P.-T. Gleve}

Cocconeis placentula Eh.

Cymbella affinis $\mathrm{Ktz}$.

- anglica Lag.

- gastroïdes $\mathrm{Ktz}$.

Cyclotella Meneghiniana $\mathrm{Ktz}$. - stelligera Cl. et Grun.

Diploneis elliptica Ktz.

Encyonema verrucosa $\mathrm{K} t z$.

Epithemia sorex $\mathrm{Ktz}$.

Bunotia pectinalis Rab.

- diodon Eh.

- ophidocampa $\mathrm{Cl}$.

Fruetulia crassinervia Bréb.
Gomphonema Berggrenii Cl. gracile Eh.

Melosira granulata Eh. - crenulata Ktz.

Navicala amphigomphus Eh. - rhyncocephala $\mathrm{Ktz}$.

Vitzschia amphibia Grun.

Pinnularia legumen Eh.

- major $\mathrm{Ktz}$.

- - stauroptera Grun.

Stauroneis anceps Eh. var.

stephanodiscus Nova Zelandiæ $\mathrm{Cl}$.

Synedra ulna Eh.

\section{No 274 ARESKUTAN - JAMTLAND (Suède)}

\section{Analysé par le professeur P.-T. Cleve}

Cymbella amphicephala Nægel.

- anglica Lag.

- hebridicum Greg.

Eunotia diodon Eh.

- minor $\mathrm{Ktz}$.

- pentaglyphis Eh.

- robusta Ralfs.

- triodon Eh.

Gomphonema auritum Br.

- constrictum Eh.

Navicula pupula Ktz.

Noidium affine Eh.
Neidium sulcatum Lag.

Pinnulariá biceps Greg.

- commutata Grun.

- mesolepta Eh.

- mesostyla Eh.

- nobilis Eh.

- stauroptera Grun.

- viridis $\mathrm{K} t z$.

- - var. intermedia Eh.

Stauronelg anceps var. linearis Eh.

- phonicenteron fa minor Eh.

- legúmen Eh. 
Actinocyclus rotula $\mathrm{J}$. Br.

Aetinoptychus hexagonus var.? ou sp. nov. ?

$\begin{array}{ll}- & \text { spinifer Grun. } \\ \text { - } & \text { splendens Shadb. } \\ \text { - } & \text { subangulatus A. S. var. } \\ \text { - } & \text { trilingulatus Bright. } \\ & \text { undulatus Ehr. }\end{array}$

Amphitetras antediluoiana $\mathrm{Ehr}$.

Amphora arenaria Donk.

- arenicola Grun. o. major $\mathrm{Cl}$.

- areolata v. maxima J. Br.

- biconvexa Janisch.

- clara A. S. oar.

- cuneata A. S.

- egregia $\nabla$. ininterrupta Per.

- formosa CI.

- Graeffei Grun.

- Grundlerii Grun.

- obtusa Greg. var.

- porcellus Kitton.

- proteus Greg.

- spectabilis Greg.

Auliscus colatus Bail.

- compositus A. S, var.? A.zS. atlas $30 / 9$.

- reticulatus Grev. var.

- n. sp.?

Biddulphia aurita Bréb.

- granulata Roper. var.

- Mac Donaldii.
Biddulphia pulchella Greg.

- rhombus W. Sm.

- Tuomeyi Bail.

Campylodiscus ambiguus Grev.

- biangulatus Grev.

- $\quad$ crebrecostatus Grev.

- decorus Bréb. var. A. S. 208/8.

- ecclesianus Grev. var. $n$.

- Hodgsonii W. Sm. var.?

- Kinkerii A. S. = C. biangulatus var. Kittonii Grev. latus Shadb. Normanianus Greo. ornatus Grev. rioalis $A$. $S$. samoensis Grun. undulatus Grev. Wallischianus Grev.

Cerataulas turgidus Eh.

Cocconeis citrina A. S.

- curvirotunda Temp. et Br. var.

- - $\quad$ v. bifrons A. S.

- heteroïdea Hantzsch. var.?

- voluta J. Br. var.

Coscinodiscus nitidus Greg.

- symmetricus Grev.

- sp. A. S. 57/22.

Cyclotella stylorum Bright.

Cymatosira Lorenziana Grun. 
Dletyoneis marginata $\mathrm{Cl}$.

- _ - fa elongata.

Euphyllodium spathulatum Shadb.

Glyphodesmis Murrayana Cast.

Gomphonema Herculeana A. S. จ. robusta Grun.

Grammatophora marina. Ktz. oceanica $\nabla$. macilenta W. Sm.

Hyalodiscus stelliger Bail.

Lichmophora Ehrenbergii Ktz.

Mastogloia elongata Leud. Fort.

- Lemnistica Leud. Fort.

Melogira sulcata Ktz, et var.

Navieula affirmata Leud Fort.

- aspera $\mathbf{v}$. intermedia Eh.

- bombus Eh.

- brasiliensis Grun. var.? Atl., A. S., VI, 32/33.

- bullata Grev. var. A. S. atlas $3 / 2$.

- campylodiscus Grun.

- Chersonensis Grun.

- clavata Greg.

- crabro Eh.

- - $\quad$ v. multicostata Grun.

- cruciata Leud Fort.

- diplosticta Grun.

- forcipata $\nabla$. suborbicularis Grun.

- gemmata Grev. et var.

- lacrimans A. S.

- lyra $\nabla$. elliptica.

- - v. recta Grev.

- - $\quad$ . subcarinata Grun.

- mirabilis Leud. Fort.

- peripunctata $J . B r$.

- prætexta Eh.

- reticulo radiata Temp. et $B r$. var.

- robusta Grun.

- separabilis A. S.

- Smithii et var. Bréb.
Navicula spectabilis Greg et var.

- transfuga Grun. var.?

- velata A. S.

- Weissflogii A. S.

- noo.sp.?

Nitzschia angularis W. Sm.

- Gelineckii Grun. var.?

- marginulata Grun. v. Per.

(Diat. de France) 70/14.

- panduriformis Greg.

- punctata v. constricta Grun.

Orthoneis fimbriata Grun.

- splendida Grun.

Pleurosigma formosum W. Sin.

Raphoneis amphiceros $E$.

- surirella Eh. o. australis P. $P$.

Rhabdonema adriaticum $\mathrm{K} t z$.

Staurosira Harrisonii W. Sm.

Stephanodiscus astræa v. spinulosa Grun.

Stictodesmis australis Greo.

Surirella eximia Grev. v.

- fastuosa Eh.

- incurvata A. S.

- Lorenziana Grun.

- sp.n.(Fragment).

Synedra affinis v. tabulata Grun.

- formosa Hantch.

- undulata Greg. var.

Triceratum arcticum Bright. v, californica fa quadrangulata.

- dubium Bright.

- favus v. quadrata.

- $\quad$ - v. pentagona.

- latum Grev.

- Montereyi Bright.

- - fatrigona.

- pentacrinus Wall.

- scitulum fa quadrangulata.

- sculptum.

Tropidoneis lepidoptera Greg. 


\title{
Nos 278 et 279 CREUX MORTIER - PUY-DE DOME
}

\author{
(France)
}

Dépôt fossile d'eau douce

BIBLIOgRaphiE: - Frère J. Héribaud. Les Diatomées fossiles d'Auvergne 1909

Amphora gracilis Ehr.

- pediculus Grun.

- - v. major Grun.

Cocconeis californica Grun. fa subcontinua. Cyclotella comta v. arverna M. P. et F. H. Cymatopleura solea Bréb.

Cymbella cistula Hemp.

$$
\begin{aligned}
& \text { - } \quad \text { cymbiformis Ehr. } \\
& \text { - } \quad \text { obtusa Greg. }
\end{aligned}
$$

Diatoma anceps $\mathrm{\nabla}$. anomalum W. Sm.

Encyonema ventricosa $\mathrm{Ktz}$.

Epithemia turgida Ktz.

- zebra Ktz.

- - $\quad$ v. proboscidea Grun.

Fragilaria capucina Desm.

- $\quad$ elliptica Schum.

- intermedia Grun.

- producta Grun.

- virescens Ralfs.

- $\quad$ - $\quad$. ventricosa M. P. et F. H.

Gomphonema acuminatum o. claous Bréb.

constrictum v. subcapitata Grun. elongatum W. Sm.

- $\quad$ micropus Ktz.

- paroulum o. subcapitata V. $H$. sarcophagus Grun.

Hantzschia amphioxys Grun.

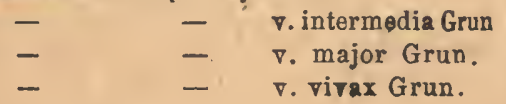

Melosira arenaria Moor.

- crenulata v. ambigua Grun.

- lævis Grun.

- tenuis Grun.

Meridion circulare $\mathrm{Ag}$.

Navicula amphirynchus Ehr.

- ampliata Eh.

- bisulcata Lag.

- borealis Ktz.

- cuspidata Ktz.

- $\quad$ - f $^{2}$ craticula M. P. et F. H.

- $\quad$ - v. Heribaudi M. P.

- dicephala W. Sm.

- dilatata Ehr.

- elliptica Ktz.

- $\quad$ - $\quad$ v. major M. P.et F.H.

- Falaisiensis Grun.

- gentilis Donk.

- gibba Ehr

- gracillum Pritch.

- limosa Ktz.

- $\quad$ - $\quad$ : undulata Grun.

- maera Grun.

- mesolepta $\nabla$. stauroneiformis Grun.

- minima Grun.

- Peisonis Grun.

- subcapitata $\vee$. stauroneiformis Grun.

- tenella Bréb.

- ventricosa fa minuta Donk.

- viridis v. commutata Grun.

Nitzschia ampbibia Grun.

Stauroneis acuta W. Sm.

- acutiuscula M. P. et F. H. 
Stauroneis Brunii $M . P$. et $F$. $H$.

- mesopachya Ehr.

- phœnicenteron Ehr.
Synedra nina v, subæqualis Grun. - - v. vitrea Ktz.

Surirella biseriata var.

\section{No 280 LE RUMMEL - CONSTANTINE (Algérie)}

Amphipleura pellucida Ktz.

Cocconeis placentula Eh.

Cocconema ventricosum Grun.

Cymatopleura solea W. Sm.

$$
\text { - - var. apiculata. }
$$

Cymbella affinis Ktz.

$$
\begin{aligned}
& \text { - cistula Hemp. } \\
& \text { - } \quad \text { maculata Ktz. } \\
& \text { - naciculceformis Auers. } \\
& \text { - } \quad \text { stomatophora Grun. }
\end{aligned}
$$

Diatoma tenue Ag.

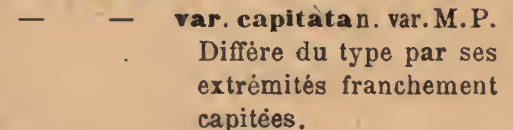

Eunotia pectinalis Rab.

Gomphonema capitatum Eh.

- $\quad$ constrictum Eh.

Melosira varians $\mathrm{Ag}$.

Navicula amphisbcena Bory.

- cryptocephala Ktz.

- elliptica o. minutissima.

- Falaisiensis Grun.

- limosa var. subinfata.

- pumila Ktz.

- radiosa Kitz.

Nitzschia dubia $W$. Sm.

- hungarica Grun.

- sigma var. rigidula.

- sigmoidea W. Sm.

Pleurosigna Spencerii var. Arnotti.

Rhoicosphenia curvata Gr.

Surirella gracilis $\mathrm{Gr}$.

- ovata Ktz.

Synedra acus $\mathrm{Ktz}$.

- pulchella Ktz.

- ulna Eh.

- - var. danica.

- - var. lanceolata fa brevis.

\section{$\mathrm{N}^{\circ}$ 281-282 WEST RIVER - NEWHAVEN, CONNECTICUT (États-Unis)}

Actinocyclus crassus V.H.

$$
\text { - nebulosus M. Per. }
$$$$
\text { - sparsus Ratt. }
$$

Actinoptychus areolatus Eh.

$$
\text { - delectus A. S, var. }
$$

Actinoptychus notabilis Pant.

- $\quad$ racemosus A. S. var.
undulatus Eh.

Amphiprora conspicua Greo.

- pulcha Bail. 
Amphiprora pulcha var. pulchella.

Amphora angusta Greg.

- Mlevei Grun.

- gigantea var. fusca.

- ocellata Donk.

- obtusa var. oceanica.

- proteus Gireg.

Aulacodiscus argus A. S.

Auliscus cælatus var. rhipis.

- - car. reticulata.

- Hauckii Panc. oar.

- pruinosus var. Zanzibarica.

- punctatus Bail.

Biddulphia $a$ urita $B r e b b$.

- pulchella Greg.

- rhombus WV. Sm.

Campylodiscus Echeneis Eh.

Cerataulus lævis Ralfs.

$$
\text { - turgidus Eh. }
$$

Cocconeis pseudomarginata Greg.

- scutellum Eh.

Coscinodiscus asteromphalus $\mathrm{Eh}$.
$-$

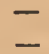

$\bar{z}$
$\overline{-}$ centralis Eh. concinnus W. Sm. curoatulus oar . latiusstriata. decipiens Grun. denarius A. S. excentricus Eh. oculus iridis Eh. radiatus $\mathrm{Eh}$.

Cyclotella striata Grun.

Cymbella gastroides Ktz.

Epithemia gibberula $K t z$.

- constricta Bréb.

- museulus Kfz.

- turgida Ktz.

- - var. granulata.

Eunotia monodon $E h$.

Glyphodesmis eximta Greo.

Grammatophora macilenta var. subtilis.

Hyalodiscus scoticus Grun.

- stelliger Bail.

- - fa minor.

Lithodesmium undulatum Eh.

Mastogloia angulata Lewis.

- Braunii Grun.
Melosira Borreri Greo.

- sulcata Ḱtz.

- - var. coronata.

- - var. radiata.

- undulata Eb.

Navicula abrupta Donk.

- - var. Rattrayi.

- adonis $J . B r$.

- æstiva Donk. var. $\beta$.

- ostuariicl.

- approximata Grev.

- Baileyana var.

- bombus Ktz.

- - var. gemina.

- breois Greg.

- claoata Greg.

- - var. Wrightii.

- dactylus Ktz.

- elliptica Ktz.

- formosa Greg.

- fusca Ratt.

- sigas Ktz.

- glacialis $\mathrm{Cl}$. var. intermédiaire à A. S. atlas 6,35.

- Grundleri A. S.

- Hennedyi W. Sm.

- - var. granulata.

- huinerosa Bréb.

- irrorata Grev.

- liburnica V. I.

- lineata Donk.

- lyra Eh.

- - oar. bacilliformis n. $\boldsymbol{c}$. $H$. $P$. Les areas latérales couvertes de petites stries en tous sens.

- - var. constricta.

- - var. elliptica.

- - var. recta.

- - var. recta fa elliptica.

- - var. subtypica.

- major Ktz.

- peregrina Kitz.

- permagna fa minor.

- protexta Eh.

- semen Eh.

- Smithii Brèb.

- Trevelyana Donk.

- Yarrensis Grun.

- sp. A. S. atlas 6/28.

Nitzschia acuminata Grun. 
Nitzschia circumsuta Grun.

- granulata Grun.

- Lorenziana Grun. var. subtilis.

- obtusa W Sm.

- $\quad$ - var. maxima var. N. 4

à $\dot{5}$ points en $10 \mu$ jusqu'à $410 \mu$ de'longueur.

- plana W. Sm.

- spectabilis Ralfs.

- sigma W. Sm.

- - - var. sigmatella.

- Tryblionella Ktz.

- - - var. maxima.

Plagiogramma antillarum $\mathrm{Cl}$.

Plagiotropis seriata $\mathrm{Cl}$.

Pleurodesmium Brebissonii $K{ }^{\prime} t_{z}$.

Pleurosigmaafine var. Normannii.

- decorum var. inflatum.

- balticun var.maxima fastricta.

Podocystis americana Bail.
Pyxilla baltica Gr.

Raphoneis amphiceros Eh.

- surirella Grun.

Rhabdonema adriaticum Ktz.

Rhizosolena styliform is Bright.

Seoliopleura tumida Rab.

Stephanopyxis turris $E h$.

Stauroneis Gregoryi Ralfs.

Surirellacrumena Bréb.

- elegans Eh.

- Febiger Lewis.

- recedens A. S.

- striatula Turp.

Syndendrium diadema Eh.

Synedra Gaillonii v. gigantea n. v. Longuement lancéolée, atteint $240 \mu$ de longueur.

Terpsinoë americana Ralfs.

Triceratium alternans Bail.

Van Heurekia Lewisiana car. entreposita.

No 283 SAVA RIVER - YAMAGUCHI (Japon)

Achnanthes minutissima $\mathrm{Ktz}$.

- var. cryptocephala

Cocconeis placentula Eh.

Cymbella stomatophora Grun.

- turgidula Grun.

Encyonema ventricosum Grun.

Gomphonema ventricosum Greg. val. olivaceum var. vulgaris.
Melosira varians $\mathrm{Ktz}$.

Nitzsehia Romana Grun.

Stephanodiscus Hantzschianus Grun.

Syneira acus Ktz. var.

- Goulardi Bréb.

- rostrata Eh.

- rumpens. var. fragilarioides.

- ulna Eb. et vars. 


\section{BANYULS-SUR-MER \\ PYRÉNÉES-ORIENTALES (France)}

\section{Sondages}

Achuanthes longipes Ag.

Actinocycluscrassus W. Sum.

$$
\text { - } \quad \text { Ehrenbergii Ralfs. }
$$$$
\text { - Ralfsii W. Sm. }
$$$$
\text { - _ var. sparsa Greg. }
$$$$
\text { - subtilis Greg. }
$$

Amphiprora complexa Greg.

$\begin{array}{ll}\text { - } & \text { decussata Greg. } \\ \text { - } & \text { - } \quad \text { rar. robusta H.P. } \\ \text { - } & \text { lepidoptera Greg. } \\ \text { - } & \text { maxima Greg. } \\ \text { - } & \text { pusilla Greg. }\end{array}$

Amphora acuta Greg.

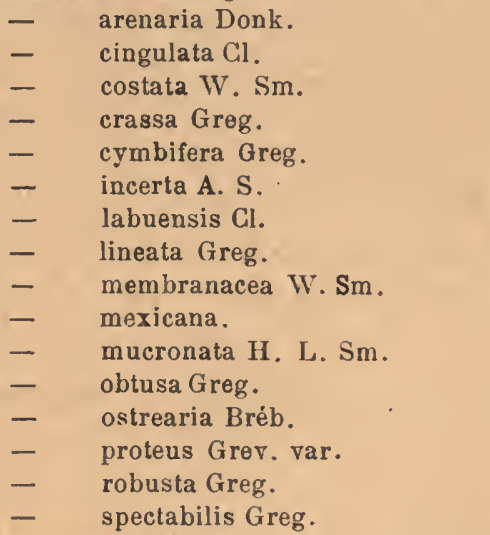

Asterolampra Grevillei var adriatica Grun.

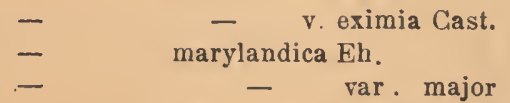

H. P.

Asteromphalus robustus Cast.

Aulacodiscus Ehrenbergii Jan. type.
Bacteriastrum parians Lauder.

Biddulphia aurita Bréb.

- mobiliensis Bail.

- pulchella Greg.

- Tuomeyi Bail.

Campylodiscus clypeus Eh.

- decorus Bréb.

- eximius Greg.

- $\quad$ - $\quad$ . Briocensis.

- fluminensis Grun.

- hibernicus Eh.

- horologium $\nabla$. mediterranea Grun.

- $\quad$ limbatus Bréb.

- Ralfsii W. Sm.

- Samoensis A. S.

$$
\text { - Thuretii Bréb. }
$$

Cerataulus Smithii Ralfs.

- turgidus Eh.

Chretoceros diversus $\mathrm{Cl}$.

- paradoxum $\mathrm{Cl}$.

- Peruvianum Bright.

Cocconeis pseudomarginata Ralfs.

- scutollum Eh.

- - var. ornata Grun.

Coscinodiscus concavus Greg.

- denarius A. S.

- excentricus Eh.

- gigas Eh.

- Janischii A. S.

- lineatus Eh.

- marginatus Eh.

- nodulifer A. S.

- oculus iridis Eh.

- radiatus Eh.

Ditylium intricatum West.

Donkinia recta Ralfs.

- Thumii H. P. 
Epithemia musculus Ktz .

Euodia gibba Bail.

Grammatophora adriatica $\mathrm{Ktz}$.

$$
\text { - macilenta Ktz. }
$$

Mastogloia angulata Lewis oar.

$$
\text { - quinquecostata Grun. }
$$

Melosira sulcata Ktz.

Navicula abrupta Greg.

$$
\begin{aligned}
& \text { - } \quad \text { var. lata. } \\
& \text { - } \quad \text { apis Donk. } \\
& \text { - } \quad \text { Beyrichiana A.S. } \\
& \text { - bomboides A. S. } \\
& \text { - } \quad \text { bombus Eh. } \\
& \text { - cistella Grev. } \\
& \text { - clavata Grog. } \\
& \text { - } \quad \text { - v. caribæa. } \\
& \text { - crabro Eh. }
\end{aligned}
$$$$
\text { -- } \quad \text { - var. A. S. atlas } 12 / 72 \text {. }
$$

- dalmatica Grun.

- didyma Ktz.

- elliptica Ktz. var.

- entomon Eh.

- forcipata Grev.

- formosa Greg.

- fusca Greg.

- gemina Ktz.

- granulata var.

- Hennedyi W. Sm.

- humerosa Bréb.

- incus Grun.

- lacustris Greg. var.

- liber W. Sm.

- lineata Donk.

- longa Greg.

- lyra Eh.

- - var. atlantisa.

- - var. dilatata.

- - - var. elliptica.

- - var. subcarinata.

- marina Ralfs.

- - v. bicuneata.

- multicostata Grun.

- musca Greg.

- nebulosa Greg.

- palpebralis Bréb.

- pandura Brób.

- pennata A. S.

- polysticta A. S.

- prætexta Eh.
Navicula rhombica Grev.

- Sandriana Grun.

- seductilis A. S.

- Smithii Bréb.

- suborbicularis Greg. var.

- - vetula A. S.

- Zanardiniana var.

- Zostereti A. S. var.

Nitzschia acuminata W. Sm.

- constricta Grun.

- - $\quad$ var. subconstricta.

- circumsuta Bail.

- dilatata H. P.

- distans Greg.

- hybrida Grun.

- insignis W. Sm.

- $\quad$ - var. mediterranea.

- macilenta W. Sm.

- marginulata $v$. didyma.

- notabilis Grun.

- oculata $\mathrm{Cl}$.

- paradoxa var. major.

- punctata Grun.

- plana WV. Sm.

- panduriformis Greg.

- - $\quad$ var. minor.

- sigma W. Sm.

- $\quad$ - var. rigida.

- $\quad$ - var. sigmatella.

- spathulifera Grun.

- valida Cl. et Grun.

- vermicularis Ktz.

Odontidium anomalum W. Sm. $\nabla$. longissima.

Orthoneis fimbriata Bright.

Pleurosigma affine Grun.

- angulatum W.Sm.

- - var. minor.

- rstuarii W. Sm. var.

- australe Grun.

- balticum W. Sm.

- $\quad$ - var. diminutum.

- decorum W.Sm.

- delicatulum W. Sm. var.

- $\quad$ elongatum W. Sm.

- formosum W. Sm.

- - $\quad$ var. adriaticum.

- latum Grun. var.

- longissimum Cl.

obsurum W. Sm. var. moditerranea. 
Pleurosigma prolongatum W. Sm.

$$
\begin{array}{lc}
\text { - } & \text { rigidum W. Sm. } \\
\text { - } & -\quad \text { v. gigantea. } \\
\text { - } & \text { speciosum W. Sm. } \\
\text { strigosum W. Sm. }
\end{array}
$$

Podosira Montagnei Ktz.

Rhabdonema adriaticum Ktz

Rhoicosigma lineare Grun.

$$
\begin{array}{ll}
\text { - } & \text { mediterranea } \mathrm{Cl} . \\
\text { - } & \text { oceanicum H. P. }
\end{array}
$$

Septroneis cuneata Grun.

Stauroneis spicula Hich.

Surirella fastuosa Eh.

$$
\begin{array}{lll}
- & - & \text { var. abludens. } \\
- & - & \text { var. opulenta. }
\end{array}
$$

Surirella fastuosa var. panduriformis.

- gemina Eh.

- Guinardii H. P.

- hybrida Grun.

- intercedens var.

- lata W. Sm.

Synedra acus $\mathrm{Ktz}$.

- baculus Greg.

- fulgens W. Sm:

- - var mediterranea.

- Gaillonii Eh.

- Hennedyana Greg.

- undulata Greg.

Toxonidea balearica Gl.

- Gregoriana Donk.

- insignis Ralfs.

Triceratium antediluvianum et var. Eh.
Biddulphia Edwardsii Feb.

Cocconeis scutellum Eh.

Coscinodiscus dubiosus Grun.

Epithemia turgida K'tz.

Iyalodiscus subtilis Bail.

Isthmia nervosa $\mathrm{Ktz}$.
Melosira sol Eh.

Nitzschia lanceolata W. Sm.

Podosira hormoïdes Mont.

Trachyneis aspera $\mathrm{Cl}$.

- $\quad$ - clepsydra Donk.

\section{No 289 RUISSEAU DE POINTE PIERRE TRINIDAD}

\section{(Antilles)}

Eunotia formica Eh. v, elongata. - - var. intermedia.
Gomphonema affine $\mathrm{K} t z$.

Navicula confervacea Grun. var. 
Achnanthes brevipes var. minor.

Amphora ovalis Ktz.

Coceoneis pediculus Eh.

Cymbella affinis $\mathrm{Ktz}$.

- cistula Hemp.

- helvetica $\mathrm{Ktz}$.

- leptoceros Rab.

- parva V.H.

Encyonema oentricosum var.

Epithemia sorex $\mathrm{Ktz}$.

Eunotia lunaris Grun.
Gomphonema angustatum Ktz.

- capitatum Eh.

- constrictum Eh.

Melosira varians Ag.

Meridion circulare Ag.

Navicula elliptica $\mathrm{Ktz}$.

Nitzschia linearis W. Sm.

Synedra oxyrhynchus Ktz.

- ulna Eh.

- $\quad$ - var. æqualis.

- - var. lanceolata fa brevis.

\section{Nos 291-292 SAN DIEGO - CALIFORNIE (États-Unis)}

Dépôt fossile marin

Actinocyclus Ehrenbergii Ralfs

Actinoptychus biformis $\mathrm{J}$. $\mathrm{Br}$.

$\begin{array}{ll}\text { - } & \text { Bismarckii A. S. } \\ \text { - } & \text { glabratus A. S. var. } \\ \text { - } & \text { spinifer Grun. } \\ \text {-' } & \text { spinulosus A. S. } \\ \text { - } & \text { splendens Ralfs. var: } \\ \text { - } & \text { oulgalatus Eh. }\end{array}$

Amphora gigantea Grun. var.

Arachnoidiscus indicus $\mathrm{Eh}$.

ornatus Eh.

Asterionella synedraeformis Grev.

Asteromphalus variabilis.

Auliscus Biddulphia Kitton. oar.

- californicus $J . B r$.
Auliseus Johnsonii A. $S$.

- mirabilis Greo.

- punctatus Bail.

- sculptus Ralfs.

Biddulphia longicornis Grun.

- Tuomeyi Bail.

Brunia mirabilis Temp. (fragments).

Campyloneis argus. Grun.

$$
\text { - regalis Grun. }
$$

Cerataulus turgidus $E$ h.

Cocconeis dirupta Grev. var. pseudomarginata Greo. oar.

Coscinodiscus asteromphalus Eh.

- biradiatus Greo. curtatulus Eh. $\mathrm{Ear}$. debilis Grun. diorama A. S. 
Coscinodiscus fimbriatus oar. californica Grun.

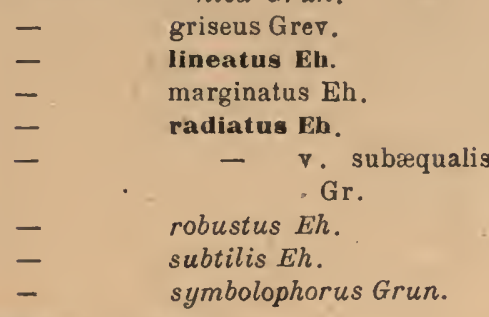

Dicladia capreolus Eh.

\section{Euodia gibba Bail.}

Gephyria constricta Arn.

$$
\text { - - costata Arn. }
$$

Goniothecium odontella Eh. var. Grammatophora robusta Dippel.

Isthmia nervosa $\mathrm{Ktz}$. (fragments)

Lithodesmium californicum Grun.

Melosira clavigera Grun.

minusculum Grun. var.
Melosira sulcata $\mathrm{Ktz}$.

Navicula aspera $E h$.

- crabro Eh. et var.

- lyra Eh.

- O'Swaldii Janisch.

- protesta Eh.

Orthoneis splendida Grun.

Podosira hormoides Mont.

Pterotheca subulata Grun.

Rhabdonema adriaticum Eh.

Rutilaria hexagona Grun.

Stephanogonla actinoptychus Eh.

Stephanopyxis appendiculata $\mathrm{Eh}$.

$$
\text { - } \quad \text { Grunowii Gr. et St. }
$$

Stictodiscus californicus Grev.

Tuiceratium arcticum Bright.

- elegans Greo.

- formosum Bright.

- Montereyi Bright. calidum Grun.

\section{No 293 VARENNES - PUY-DE-DOME (France)}

Dépôt fossile d'eau douce

\section{Bibliographie. - Frère J. Héribaud. Les Diatomées d'Auvergne 1893}

Cocconeis speciosa Greg var. Ktz.

$$
\text { - trilineatus } M \text {. P. et F. } H \text {. }
$$

Cuscinodiscus Chambonis M. P. et $\mathrm{F}$. $\mathrm{H}$.

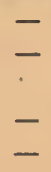

$$
\text { dispar M. P. et F. H. }
$$

- var. radiata M. P. et F. H.

pygmaens J. Br. et M. P.

$-$

- var. micropunctata

M. P. et F. H.

Cymatopleura elliptiça $\nabla$. subconstrieta Grun. solea Bréb.

Cymbella aspera Ehr.
Epithemia Hyndmanii W. Sm.

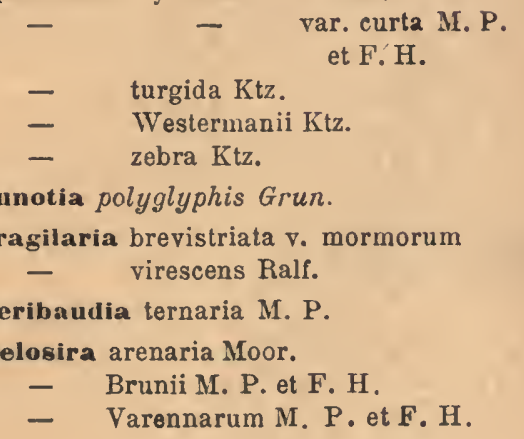


Navicula arverna M. P. et F. H.

- elliptica Ktz.

- gastrum fa elliptica M. P. et F. H.

- Heribaudi M. P.

- limosa Ktz.

- major Ktz.

- $\quad$ - o.horrida M. P.et F. H.

- peregrina Ehr.

- porrecta Ehr. var.

- radiosa $\mathrm{Ktz}$.

- Reinhardtii Grun.

- Smithii Bréb.
Nitzschia tabellaria Grun. - tubicola Grun.

Pleurosigma Spencerii W. Sm.

Stauroneis acuta W. Sm.

$$
\text { - phonicenteron Ehr. }
$$

Stenopterobia anceps Bréb.

Surirella biseriata Bréb.

Tetracyclus compressa M. P.

- elliptica M. P.

- emarginatus W. Sm.

- lamina M. P.

- lancea M. P.

No 294

LA SEINE - PARIS

Diatoma vulgare Bory

No 295

LE HAVRE (France)

Amphora proteus Greg.

\section{No 296 LAC D'AYDAT - PUY.DE-DOME (France)}

\section{BIBliogra PhiE. - Frère J. Héribaud. Les Diatomées. d'Auvergne 1893}

Achnanthes exilis $\mathrm{Ktz}$.

Amphora ovalis $\mathrm{Ktz}$.

Cocconeis pediculus Ehr.

- placentula Ehr.

Cyclotella bodanica Eul.

- comta Ktz.

- Kützingiana Thw.

- operculata'Ktz.

$$
\text { - - v. antiqua. }
$$

Cymbella amphicephala Nog.

- cistula Hemp.
Cymbella cuspidata $\mathrm{Ktz}$.

- cymbiformis Ehr.

- Ehrenbergii Greg.

- helvetica $\mathrm{Ktz}$.

- lanceolata Elir.

Denticula inflata W. Sm.

Diatoma Ehrenbergii v. grande W. Sm.

- tenue Ag.

Encyonema cospitosum $\mathrm{Ktz}$.

- gracile o. lunata W. Sm.

- prostratum Ralfs. 
Encyonema ventricosum Ktz.

Epithemia gibba Ehr.

- turgida Ktz.

- - v. granulata.

Fragilaria binodis Ehr.

- capucina Desm.

- virescens Ralfs.

Gomphonema Brebissonii $\mathrm{Ktz}$.

- constrictum Ehr.

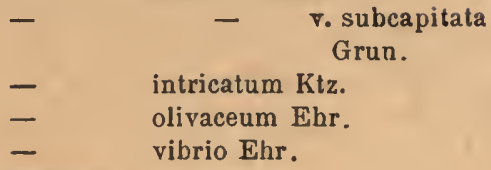

Mastogloia Smithii Thw.
Meridion circulare Ag.

Navicula ambigua Ehr.

- elliptica Ktz.

- major Ktz.

- patula W. Sm.

- radiosa $\mathrm{Ktz}$.

- viridis $\mathrm{Ktz}$.

Nitzschia angustata W. Sm.

- linearis W. Sm.

Pleurosigma Spencerii W. Sm.

Synedra capitata Eh.

- delicatissima W. Sm.

- gracilis Grun.

Tabellaria fenestrata $\mathrm{K} t z$.

- flocculosa Ktz.

Nos 297.299 CALVERT COUNTY - MARYLAND (États-Unis)

Dépôt fossile marin

Actinocyclus Ehrenbergii Ralfs. Actinoptychus Heliopelta $\mathrm{El}$. Asterolampra Marylandica Eh.

Aulacodiscus crux Eh.

Biddulphia Edwardsii Febig. - suborbicularis Grun.

Coscinodiscus asteromphalus Eh. oar. hybrida Grun.

apiculatus Eh.

biangulatus $\mathrm{A}$. S.

bulliens A.S.

centralis Eh.

concinnus W. Sm.

marginatus Eh.

oculus-iridis Eh.

obscurus Eh.

perforatus Eh..

radiolatus Eh.

robustus Grev.

subtilis Eh.

velatus Eh.
Craspedodiscus elegans Eh.

- coscinodiscus Eh.

Eupodiscus Rogersii Bail.

Goniothecium odontella Eh.

Mastogonia crux Eh.

Melosira sulcata Eh.

Pleurosigma affine var. Grun.

- Marylandica Grun.

Pyxilla americana Eh.

Stephanogonia actinoptychus Grun.

Stephanopyxis corona Eh.

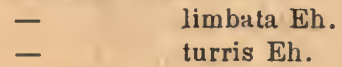

Triceratium americanum Ralfs.

- Ehrenbergii Grun.

- obtusum Eh.

- sermicirculare.

- solenoceros Bright.

- tessellatum Grev. var.

- undulatum Eh. 
No $: 300$

RADE DE YOKOHAMA (Japon)

\section{Sondage}

Aracbnoidiscus ornatus Els.

- Ehrenbergii Ralfs.

Aulacodiscus margaritaceus Ralfs.

affinis Grun

Auliscus rhipis A. S.

- pruinosus Bail.

Bidduiphia reticulata var. (A. S. utlas 8/21).

Campylodiscus bicinctus Cast.

$\begin{array}{ll}- & \text { Dcemelianus car. Grun. } \\ \text { - } & \text { Echeneis Eh. } \\ \text { - } & \text { Kittonianus. } \\ \text { - } & \text { Pfitzerii A. S. }\end{array}$

Cerataulus Johnsonianus Grun.

- turgidus Eh.

Coscinodiscus radiatus Eh.

Leudugeria epithemioïdes Temp.

Melosira sol Eh.

Navicula lyra Eh.

- - var. recta.

- multicostata Brẻb.

- puella A.S. fa major.

Triceratium favus $\mathrm{Eh}$.

- rivale A.S.

- grande $\nabla$.quadrangulata Bright.

- Robertsianum Grev.

No 301

YESO (Japon)

Dépôt fossile d'eau douce

Achnanthes delicatula $\mathrm{Ktz}$.

inopinata? var. elliptica $n . \nabla$. valve supérieure de même structure et de mêmes dimensions que celles de l'A. inopinata, mais do forme elliptique.

Amphora Lybica Eh.

Cocconeis costata Greg.

- molesta Ktz.

Coscinodiscus Barbadensis Greo.?

Cymbella cistula var. (fragments).

- Sp. (fragments).

Epithenia turgida Ktz.

Eunotia diodon fn minor.

- quinaria Eh.

- triodon $E h$.
Fragilaria oequalis Heil.

- - var. producta.

llantzschia amphioxys Grun:

Melosira arenaria var, lævis.

- granulata fa australiensis.

- _ fa curvata.

- $\quad$ - $f^{2}$ hemicyclus n. v. encore beaucoup plus courbée que la variètẻ précédente, un frustule entier atteint presque une demi-circonférence.

- undulata Eh.

Naviculạ gastrum fa maxima longueur $55 \mu$ Nitzschia linearis $W$. Sm. 
Stauroneis Tokuhisai $n$. sp. Temp. et M. Per. semblable au St. ventricosa Ktz., mais beaucoup plus rohuste. Le stauros est divisé en deux par une côte médiane forte avec un point unilatéral. Long. $40 \mu 10$ côtes en $10 \mu$.

Stephanodiscus astraea Grun.
Tetracyclus compressus Eh.

- ellipticus Eh.

glans $E h$.

lamina $\mathrm{f}_{\mathrm{a}}$ lata, long. $20 \mu$ au lieu de 15 . rhombus Eh.

var. producta n. v. extrémıtés productes.

\section{$N^{\text {os }} 302$ et 303 CLIMACUM VALLEY - ORCAS ISLANDS (États-Unis)}

Achnanthes ventricosa Eh.

Cocconeis lineata Eh.

Cyclotella perforata M. Per.

Cymbella americana A. S.

$$
\begin{array}{ll}
\text { - } & \text { cistula Kirch. } \\
\text { - } & \text { cuspidata.Ktz. } \\
\text { - } & \text { Ehrenbergii Ktz. } \\
\text { - } & \text { gastroides Ktz. } \\
\text { - } & \text { heteropleura Ktz. } \\
\text { - mexicana Eh. }
\end{array}
$$

Encyonema ventricosum Grun.

Epithemiagibba $\mathrm{Ktz}$.

$$
\begin{aligned}
& \text { - - var, ventricosa. } \\
& \text { - sorex Ktz. } \\
& \text { - turgida Kitz. } \\
& \text { - - var. granulata. } \\
& \text { - - var. vertagus. } \\
& \text { - Jestermannii Ktz. } \\
& \text { - zebra Kitz. } \\
& \text { - - var. proboscidea. }
\end{aligned}
$$

Eunotia formica Eh.

$$
\text { - gracilis Rab. }
$$

- Lunaris Grun.

- major Rab.

- tridentata Eh.

Fragilaria construens Grun.

$$
\text { - virescens Ralfs. }
$$

Gomphonema acuminatum Eh. constrictum Eh.
Gomphonema intricatum var. dichotoma. - montanum var. suecica.

Hantzschia amphioxys Grun.

\section{Melosira crenulata Kítz.}

Meridion constrictum Ralfs.

Vavicula acrosphæria $\mathrm{Ktz}$.

- americana var. minor.

- amphibola $\mathrm{Cl}$.

- amphigomphus.Eh.

- amphirynchus Eh.

- bacilliformis Grun.

- bacillum Eh.

- commutata Grun.

- costata Eh.

- cuspidata fa craticula.

- dactylus Ktz.

- divergens W. Sm. var.

- elliptica Ktz.

- - - var.

- flexuosa Cl. var. cuneata n. v.

Raphé flexueux, extrémitès cunéiformes. Long $310 \mu$; largeur $70 \mu 31 / 2$, côtes en $10 \mu$.

- hemiptera Gl.

- iridis Eh.

- latevittata Cl.

- limosa $\mathrm{Ktz}$.

- major Ktz.

- nobilis Ktz.

- nodosa Eh. 
Navicula nodosa var.

- oblonga Ktz.

- peregrina Ktz.

- rupestris $\mathrm{Ktz}$.

- termes var.

- transversa A. S.

- trinodis Lewis.

- ventricosa $\mathrm{Ktz}$.

- viridis $\mathrm{Ktz}$.

vitzschia sigmoïdea W. Sm.

- vitrea Norm (?).

- _ - var. major.
Stauroneis acuta var. major. - var.? gracilis Eh. phœnicenteron Eh.

Stephanodiscus astraea Grun. - var. spinulosa.

Surirella elegans Eh.

- oregonica fa minor.Long. $100 \mu$.

- robusta Eh.

- valida Eh.

\section{Nos 304 et 305 GYÖNGYÖS PATA (Hongrie)}

Dépôt fossile d'eau douce

BIBliogra PhiE. - J. Pantocseck. Diatomées de Hongrie, volume II.

Amphiprora dilatata Pant.

Amphora acutiuscula Ktz.

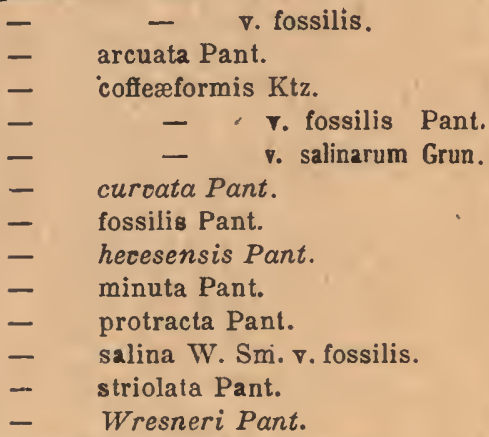

Cocconeis Californica $\nabla$. menilica Pant.

- pediculus Eh.

Cymbella hevesensis Pant.

- hungarica Pant.

- Neupauerii Pant.

- salina Pant.

Epithemia crucæformis Pant.

- inflexa Pant.

- Debyi Pant.

- multicostata Pant.
Epithemia salina Pant.

- - $\quad$ - nuda.

- vittata Pant.

Fragilaria bituminosa Pant.
- $\quad$ - $\quad$ v. curta.
- $\quad$ - $\quad$ v. elongata.
- $\quad$ - $\quad$ v. minor
- - $\quad$ - perlonga.
- $\quad$ - $\quad$ v. validior.
- brevistriata $\nabla$. fossilis Grun.
- minuta Pant.

Gomphonema olivaceum Eh. v. fossilis.

$$
\begin{aligned}
& \text { - } \quad \text { - } \quad \nabla \text {. salinarum. } \\
& \text { - - _ - } \quad \text { - staurophora. }
\end{aligned}
$$

Melosira bituminosa Pant.

- $\quad$ - v. dilatata.

- $\quad$ - $\quad \nabla$. interrupta.

- menilitica Pant.

- navicula aranariæformis Pant.

Navieula arenicola Grun.

- Beckii Pant.

- bituminosa Pant.

- $\quad$ - $\quad$ v. latecapitata.

- $\quad$ - $\quad$ - . signata.

- - - v. staurophora. 
Navicula oivittata Pant.

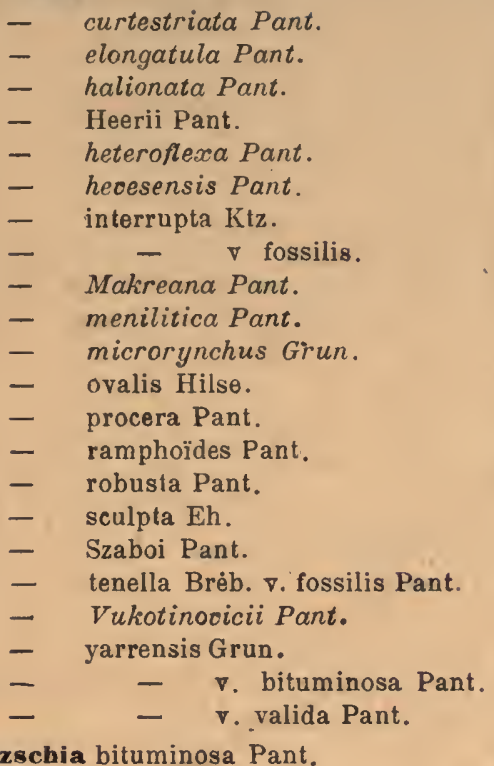

Nitzschia bituminosa $\nabla$. tenuior.

- frustulum Grun.

$$
\begin{array}{lll}
\text { - } & \text { - } & \text { v. acuta Pant. } \\
\text { - } & \text { - } & \text { v. curvata Pant. } \\
\text { - } & \text { - } \text {. minuta Pant. } & \text { v. obtusa Pant. } \\
\text { - } \quad \text { - } & \text { v. producta Pant. }
\end{array}
$$

- hevesensis Pant.

- Kittlii Grun.

- neogena Grun.

- spectabilis Grun.

- Szaboi Pant.

- Talyana Grun.

Podosira robusta Pant.

Staurosira mormonorum Grun.

- venter v. fossilis Grun.

Stephanodiscus Martensis Pant.

Surirella costata Neup.

- Neupauerii Pant.

- patella Eh.

- rotunda Pant.

- - $\quad$. minor.

Synedra salinarum Pant.

\section{Analysé par le professeur P.-T. Cleve}

Achnanthes lanceolata Bréb.

Amphora veneta $\mathrm{Ktz}$.

Cymbella tumidula Grun.

Epithemia argus $\mathrm{K} / \varepsilon$.

- gibba Ktz.

- gibberula Ktz.

Mastogloia amphibia Grun.
Navicula fonticola Grun.

- Hungarica Grun. var.

- semmoïdes $\mathrm{Cl}$.

Nitzschia denticula Grun.

- - linearis Ag.

Pinnularia viridis Eh.

Stauroneis anceps $\mathrm{Eh}$. 


\title{
$\mathrm{N}^{\text {os }} 307$ et 308 CEYSSAC - HAUTE-LOIRE (France)
}

\author{
Dépôt fossile d'eau douce
}

\section{BIBLIOgRAPHIE. - Frère J. Héribaud. Les Diatomées fossiles d'Auvergne 1903}

Achnanthes lanceolata Grun. v. elliptica. Gl.

$$
\text { - Ligeriana F. H. }
$$

Amphora libyca Ehr.

- pediculus Ktz.

Cocconeis lineata Grun. v. euglypta.

Cymatoplenra elliptica v. rhomboïdes Grun.

Cyclotella comta Ktz.v. decrescens F. H.

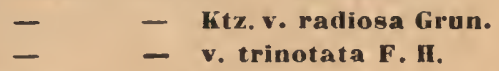

Cymbella cuspidata $\mathrm{Ktz}$.

- $\quad$ cymbiformis Ehr.
- $\quad$ gastroides Ktz.
- $\quad$ - $\quad$. minor V. H.
- lanceolata Ktz.
- leptoceros Ktz.
- $\quad$ maculata Ktz. . curta.

Diploneis elliptica Ktz.

- Smithii Bréb.

Encyonema cœspitosum Ktz.

$\begin{array}{ll}\text { - } & \text { prostratum Ralfs. } \\ \text { - } & \text { turgidum Grun. } \\ \text { - } & \text { ventricosum Ktz. }\end{array}$

Epithemia turgida Ktz.

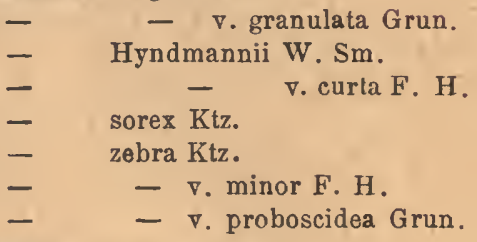

Eunotia polyglyphis Ehr.

Fragilaria bigibba F. H.

- brevistriata Grun. v. capitata F.H.
Fragilaria construens v. binodis Grun.

- $\quad$ - v. venter.

- Zeilleri v. densestriata F. H.

Gomphonema acuminatum. Eh.

$$
\begin{aligned}
& - \\
& \text { - }
\end{aligned}
$$

Ilantzschia amphioxys Grun.

Melosira arenaria Moor.

- bellicosa F. H.

- distans Ehr.

- - v. scalaris Grun.

- lineolata Grun.

- sphœrica F. H.

- tenuissima Grun.

- Varennarum M. P. et F. H.

- varians $\mathrm{Ag}$.

Navicula bacillum $\mathrm{Eh}$.

- $\quad-\quad$ v. major F. H.

- cuspidata Ktz.

- dicephala W. Sm.

- Gendrei F H.

- gracilis Ehr. v, neglecta Grun.

- halophila Grun. v. major. F.H.

- iridis Ehr.

- limosa v. gibberula Ktz..

- major $\mathrm{Ktz}$.

- mesolepta Ehr.

- $\quad$ - v. Alberti F. H.

- menisculus A. S.

- placentula Ehr.

- radians $F$. $H$.

- radiosa $\mathrm{Ktz}$.

- Reinhardtii Grun.

- sphœrophora Ktz.

- ventricosa Donk. 
Opephora Martyi F. H.

Rhopalodia gibba Ehr.

- - - ventricosa Grun.

Stauroneis acuta W. Sm.

Stephanorliscus astraa Ktz.

- — v. minutula Grun.

Surirella gracile Grun.

- ovata Ktz.
Synedra delicatissima v, angustissima .

- ulna $\nabla$. vitrea.

- rumpens Grun. v. fragilarioides.

- _ - Grun v. Meneghiniana.

- - $\quad$ - s. scotica.

Tetracyclus emarginatus IV.Sm.

- stella F. M. et M. P.

No 309

PITRUAMO GRANDE (Mexique)

Dépôt fossile d'eau douce

Achnanthes exigua Grun.

Cyclotella sp. (n. sp. ?).

Encyonema turgidum Greg.

Epithemia gibberula $\mathrm{Ktz}$.

Gomphonema affinis $\mathrm{Ktz}$.

- gracile.

Melosira granulata Ehr.
Navicula mutica $\mathrm{Ktz}$.

- pupula var. bacillaroïdes Grun.

Neidium amphirynchus Ehr.

Pinnularia viridis Ktz.

- brevicostata $\mathrm{Cl}$.

- major var. turgidula $\mathrm{Cl}$.

Stauroneis phœnicenteron $\mathrm{Eh}$.

Stephanodiscus astræa Grun.

Nos 310 et 311 ILE DE FÜR (Jutland-Danemark)

Dépôt fossile marin

BIBLIOGRAPHIE. - Heiberg. Compectus criticus Diatomacearum danicarum et de nombreuses figures dans l'atlas de Ad. Schmidt.

Corinna elegans Heib.

Coscinodiscus obscurus $A$. $S$.

$$
\text { - } \quad \text { oculus-iridis Eh. }
$$

Goniotheeium danicum Grun.

Hemiaulus affinis Grun.

$$
\text { - } \quad \text { februatus Heib. }
$$

Janischia antiqua. Grun.

Odontella Heibergii. Grun.

Odontotropis carinata Grun.

$$
\text { - cristata Grun. }
$$

Pseudo-stictodiscus angulatus Gran.

Pyxílla aculeifera Grun.

- carinifera Grun. 
Pyxilla dubia Grun.

- Kittoniana Grun.

Sceptroneis gemmata Grun.

Solium exsculptum Heib.

Stephanogonia danica Grun.

Stephanopyxls appendiculata Grun.

- var. paucispina.
Stephanopyxis appendiculata var, tenuispina. turris.

Trinacria excavata Heib.

- Heibergii Kitton.

- regina Heib.

Trochosira mirabilis Kitton.

$\mathrm{N} \cdot 312$

LAC D'OO (France)

Sur les Sphagnums

Tabellaria nocculosa Ktz

No 313 MEUDON - SEINE-ET-OISE (France)

Etang de Trivaux

Himantidium pectinale Ktz

No 314 MEUDON - SEINE-ET-OISE (France)

Fossés marécageux

Himantidum Soleirolii Ktz.

Tabellarla flocculosa Ktz.

Van Meurckia viridula Bréb.

No 315 MOISSAC. - TARN-ET-GARONNE (France)

Amphipleura pellucida Ktz 


\section{ANHALT (Suède) \\ Plankton (Collection Cleve)}

\begin{tabular}{|c|c|}
\hline Chato & balticus $\mathrm{Cl}$. \\
\hline - & boreale Bail: \\
\hline - & convolutum Cast. (type). \\
\hline- & curvisetum $\mathrm{Cl}$. \\
\hline- & Groenlandicus Cl. \\
\hline 一 & - $\quad$ v. leptopus. \\
\hline - & Lorenzianus Grun. \\
\hline 一 & socialis Leud. \\
\hline - & spinosum Leud. Fort. \\
\hline Coscin & us centralis Eh. \\
\hline- & excentricus Eh. \\
\hline
\end{tabular}

Guinardia flaccida $H$. P.

Leptocylindrus danicus $\mathrm{Cl}$.

Navicula directa $\nabla$. cuneata.

Nitzschia seriata var.

Rhizosolenia alata Bright. - semispina Hensen.

Skeletonema costatum Gl.

Thalassiosira Nordenskioldii $\mathrm{Cl}$.

Nitzschioïdes Grun.
Chretoceros atlanticum $\mathrm{Cl}$.

- convolutum var.

- criophilum var.
Chretoceros decipiens Cl.

Rhizosolenia inermis Cast.

- setigera Bright.

Nos 318 et 319

BOROSTELEK (Hongrie)

Dépôt fossile marin

Bibliographie. J. Pantocseck. Diatomées de Hongrie, volume III.

Achnanthes Baldjikii Grun. var. subquadrata Pant.

Actinocyclus circumdatus Pant. - knemoides Pant.
Actinoptychus Macraei Deby. pericavatus $J$. Br.

$-$ undulatus Eh.

Amphora acuta Greg. 
Amphoru cingulata $\mathrm{Cl}$. oar.

- Grundleri Grun.

- intersecta A. S.

- obtusa Greg.

- permagna Pant.

- porcellus Kitton.

Asteromphalus Brunii Pant.

- Grovei Pant.

Auricula Szontaghii Pant.

Biddulphia Tuomeyi Bail.

Campylodiscus ecclesianus Grev. var.

- reticulatus Pant.

- squamosus Pant.

- Thuretii Bréb. var. Bald-

Cocconeis Pethoi Pant. jikiana Grun.

- Raeana Pant.

Climacosphenia moniligera Eh. v. hungarica Pant.

Coscinodiscus marginatus Eh.

- oculus-iridis Eh.

- radiatus Eh.

Cymbella Szontaghii Pant.

Dictyoneis marginata $\mathrm{Cl}$.

Epithemia gibberula $\mathrm{Klz}$, var. producta Grun.

Grammatophora robusta Dippel. stricta var. biharensis Pant.

Hemiaulus biharensis Pant.
Hyalodiscus radiatus Prit.

- scoticus Grun.

- $\quad$ subtilis Bail.

Mastogloia Castracanei J. Br.

- Szontaghii Pant.

Melosira sulcata Ktz. v. coronata.

$$
\text { - - - v. radiata. }
$$

Navicula bimaculata Pant.

. - Boryana Pant.

- Fuschii Pant.

- humerosa Bail. var. elongata Pant.

- Hennedyi W. Sm. v. fossilis.

- irrorata Grev. v. fossilis.

- lineata Donk. var.

- Lyra Eh. eti var.

- perlonga Pant.

- pseudoaspera Pant.

- robusta Grun. var.

- sectilis $\nabla$. Boryana Pant.

- Smithii Brèb.

- splendida Greg.

Orthoneis splendida Grun.

Raphoneis Boryana Pant.

Stictodiscus californicus var. nankoorensis.

Stigmaphora hungarica Pant.

Surirella signata Pant.-

Synedra Juranyi Pant.

Triceratium biharensis Pant.

- elegans Grev. var.

- vastum Pant.

\section{$\mathrm{N}^{\circ} 320$ APPLEBY - WESTMORLAND (Angleterre) $\mathrm{N}^{\circ} 1$}

Ceratoneis arcus Ktz.

Cymbella cymbiformis Ktz.

- delicatula Ktz.
Cymbella gibba Brëb.

Synedra acus Ktz.

Tabellaria flocculosa $\mathrm{Ktz}$. 


\section{No 321 LA CASSIERE - PUY-DE-DOME (France)}

Dépôt fossile d'eau douce

\section{BIBliogra PHIE. - Frère J. Héribaud. Les Diatomées d'Auvergne 1893.}

Amphora affinis $K \mathrm{tz}$.

- pediculus Grun.

Cymbella cistula Hemp.

- naviculiformis Auersw.

Cocconeis placentula Ehr.

Cymatopleura solea v. apiculata Pritch.

Diatoma anceps Grun.

Encyonema ventricosum $\mathrm{Ktz}$.

Epithemia turgida v. granulata Grun.

$$
\text { - zebra Ktz. }
$$

Eunotia impressa v. angusta Grun.

- Iunaris Grun.

- major Rab.

- minor Rab.

- monodon o. diodon Ehr.

- tridenta $\nabla$. bidentata W. Sm.

Fragilaria binodis Ehr.

- capucina Desm.

- - - - mesolepta Grun.

- construens Grun.

- elliptica Schum.

- intermedia Grun.

Gomphonema acuminatum v.coronata Ehr.

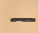
- o. trigonoce- phalum Ehr.
- angustatum v. producta Grun.
- Brebissonii Ktz.
- constrictum Eh.
- $\quad$ - v. subcapitata Grun.
dichotomum W. Sm.
elongatum 0. minor $M$. $P$. et $F$. $H$.
micropus $\mathrm{Ktz}$.

v. laticeps Grun.

Hantzschia amphioxys Grun.

Meridion circulare Ag.

Melosira striata M. P. ot F. H.

Navicula americana $E h r$.

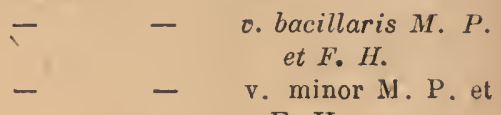
F. H.

- ampliata Ehr.

- bacilliformis Grun.

- bicapitata Lag.

- - v. hybrida Grun.

- biceps Greg.

- bisulcata Lag.

- borealis Ktz.

- Brebissonii Ktz.

- cryptocephala Ktz.

- dicephala W. Sm.

- - faminor W. Sm.

- elliptica $\mathrm{Ktz}$.

- - o. minutissima Grun.

- - O. oblongella Nag.

- gastrum fa major $M$. P. et F. $H$.

- gracillum Pritch.

- Gregoria Donk.

- loevissima Grun.

- lanceolata Ktz.

- lepida Greg.

- limosa Ktz.

- major $\mathrm{Ktz}$.

- mesolepta Ehr.

- - v. stauroneiformis Grun.

- pseudo bacillum Grun.

- pupula $\mathrm{v}$. minuta Ktz.

- radiosa $\mathrm{Ktz}$.

- rostellata fa minor.

- rupestris.

- subcapitata Grun. 
Navicula viridis Ktz.

- - $\quad$. commutata Grun.

Stauroneis acuta W. Sm.

- anceps Ehr.

- - v. amphicephala Ktz.

- mesopachya Ehr.
Surirella biseriata Bréb.

- elegans Ehr.

- saxonica Auersw.

Tabellaria flocculosa $\mathrm{Ktz}$.

\section{Sondage}

Actinocyclus undulatus Eh.

Amphora egregia Eh.

$$
\begin{aligned}
& \text { - } \quad \text { mexicana A. S. } \\
& \text { - } \quad \text { obtusa Greg. } \\
& \text { - } \quad \text { sulctabilis Greg. } \\
& \text { - }
\end{aligned}
$$

Amphiprora paradoxa Grev.

Asterolampra marylandica Eh.

Auliseus cœlatus Bail.

- sculptus Eh.

Biddulphia pulchella Graz.

Campylodiscus adriaticus Grun. var. massiliensis.

- decorus Bréb.

- eximius Greg.

- fluminensis Grun.

- limbatus Bréb.

- Lorenzianus Grun.

- Ralfsii W. Sm.

- $\quad$ samoensis Grun.

- Thuretii Bréb.

Coscinodiscus lineatus Eh.

nodulifer $A$. $S$. radiatus Eh.

Endyctia oceanica Eh.

Mastogloia reticulata Grun.

Navicula aspera Eh.

- Beyrichiana A. S.

- bombus Eh.

- californica Greo. o. campechiana.

- clavata Greg. ₹. elongata H. P.
Navieula carinifera Grun.

- Eudoxia A.S.

- forcipata Grev.

- Hennedyi Sm.

- liber W. Sm.

- longa Greg.

- lyra Eh.

- - var. dilatata A.S.

- - var. recta Grev.

- - var. subtypica.

- maxima Greg.

- multicostata Grun.

- pandura Bréb.

- Powellii Lewis.

- prætexta Eh.

- spectabilis Grev. var.

Nitzschla notabilis Grun.

- panduriformis Greg.

- valida Cl. et Gr.

Orthonele splendida Grun.

Pleurosigma affine Grun.

$$
\begin{array}{ll}
\text { - } & \text { balticum W. } \mathrm{Sm} . \\
\text { - } & \text { formosum W. } \mathrm{Sm} \text {. et var. } \\
\text { - } & \text { rigidum W. Sm. } \\
\text { - } & \text { - var. giganteum Grun. }
\end{array}
$$

Rbabdonema adriaticum Ktz.

Rhoicosigma oceanicum H. P.

- robustum Grev.

Surirella fastuosa $\mathrm{Ktz}$.

- - var abludens.

- - var. opulenta.

- gemma Eh.

- Guinardii H. P. 
Surirella intercedens Grun.

synedra baculus W. Sm.

- fulgens W. Sm.

- undulata Greg.
Triceratium antediluvianum Eh. arcticum Bright. favus Eh. spinosum Bailey.

No 323

MODENA (Italie)

Dépôt fossile d'eau douce

Cymatopleura solea Eh.

Cymbella cuspidata Ktz.

- Ehrenbergii Ktz.

- lanceolata Ktz.

- maculata W. Sm.

Epithemia gibba $\mathrm{Ktz}$.

- turgida Ktz.

Gomphonema clavus Eh.

$$
\text { - coronatum Eh. }
$$

Melosira crenulata $\mathrm{Ktz}$.

Navicula amphigomphus.

- Brebissonii.
Navicula bacillum Eh.

- firma Ktz.

- gibba Eh.

- legumen Eh.

- macilenta Eh.

- major Eh.

- nobilis $\mathrm{Ktz}$.

- viridis rar. commutata.

Stauroneis ancops Eh.

- gracilis Eh.

- phcenicenteron Eh.

Tabellaria flocculosa Eh.

\section{Nos 324 et 325 NOSSI.BE - MADAGASCAR}

\section{Sondage}

Achnanthes hexagona $\mathrm{Cl}$, et $\mathrm{Br}$.

Aetinoptychus Grundleri A. S.

- hexagonus Grun.

- - var. decumana A. S.

- splendens Schad.

$-$

Amphora nodosa J. Br.

- pecten J. Br.

Aulacodiscus inflatus Greo.

- margaritaceus Ralf.

- Petersii Eh.
Auliscus sculptus Eh.

- splendens Ratt.

Biddulphia pulchella Greg.

Campylodiscus allanticus var.

- biangulatus Grev.

- Heufleri Grun.

- borologium William.

- ornatus Greo.

- Ralfsii W. Sm.

- tænjatus A. S. var. oermicularis Temp.

Cosclnodiscus nididus Greg. 
Coscinodlscus sol Wall.

symmetricus Greg.

Endictya oceanica Eh.

Hyalodiscus radiatus Eh.

laevis Eh.

Leudugeria epithemioïdes Temp.

Mastogloia Clevei $J$. Br.

$$
\text { - rhombus } P \text {. P. }
$$

Melosira sulcata.

- - var. coronata.

Navicula aspera Eh.

- bullata Grer.

- Brunii Cl.

- californica Grev. var.

- clavata Greg.

- crabro Eh.

- Erythrea Grun. oar.

- gemmatula Grun.

- Hennedyi.
Navicula Hennedyi var. cuneata $f_{a}$ major. - lyra Eh.

- - var. elliptica.

- - var. insignis.

- - var. subtypica A. S.

- polita J.Br.

- polysticta Greo.

- prætexta Eh.

- separabilis A. S.

- Smithii Bréb.

- splendida Greg.

Vitzschia panduriformis Greg.

- valida fa minor.

Podosira argus Grun.

Surirella lepida A. S.

- Lorenziana Grun. var.

Triceratiun compar A. S.

- $\quad$ grande Bright.

- latum Grev.

- megastomum A. S. var. pentacrinus.

Nos 326 et 327 LA ROCHE LAMBERT - HAUTE-LOIRE (France)

Dépôt fossile d'eau douce

\section{BIBLIOGRAPHiE. - Frère J. Héribaud. Les Diatomées fossiles d'Auvergne 1903}

Ceratoneis antiqua F. $\mathrm{H}$.

Cocconeis lineata Grun.

Cyclotella antiqua W.Sm. v. Vernieri F. H.

- comta Ktz.

- $\quad$ - v. quadrinotata F. H.

- - v. quinquenotata F. H.

- Guignardi F. H.

- Temperei v. inermis F. H.

Cymatopleura elliptica W. Sm.

Cymbella affinis Ktz.

- aspera Ehr.

- australica A.S. v. fossilis F. H.

- Bruyanti F. H.

- cymbiformis Ehr.
Cymbella lanceolata Ehr.

- leptoceros Ktz.

Diploneis Smithii Bréb.

Encyonema prostratum Ralfs.

$$
\text { - ventricosum } \mathrm{Ktz} \text {. }
$$

Epithenia argus $K t z$.

- - v.amphiocephala Grun.

- Hyndmannii W. Sm.

- $\quad$ - v. perlonga F. H.

- ocellata Ehr.

- sorex $\mathrm{Ktz}$.

- turgida Ktz.

- - turgida Grun.

- zebra fa minor Ktz. 
Eplthemia zabra v. proboscidea Grun.

Eunotia pectinalis Rab.

- polyglyphis $\nabla$. excisa F. H.

Fragilaria brevistriata Grun.

- $\quad$ - - subcupitata Grun.

- construens lirun.

omphonema capitatum Ehr.

- brasiliense Grua V. fossilis F. H.

- exiguum Ktz.

Grunowia tabellaria Rab.

Melosira arenaria Moor.

- beliicona F. H.

- Boulaziana M. P.

- lineolata Grun.

- sphrerica F. II.

- tenuis Grun.

Navicula Gendrei F. H.
Navicula halophila Grun.

- Lambertensis F. H.

- major K'ı.

- nobilis Ehr.

- ventricosa Ktz.

- vulpina KIz.

Opephora Martyi F. H.

Pleurosigma attenuatum W. Su.

Rhopalodia gibba Elur.

Stauroneis acula W. Sm.

- Baileyi Eh.

Stephanodiscus astræa Ktz.

Tabellaria flocculosa $\mathrm{Ktz}$.

Terpsinoe trifoliata Cl.

Tetracyclus emarginatus W. Sm.

$$
\text { - stella Ehr. }
$$

Van Ileurckia rhomboïdes Bréb. v. am. phipleuroïdes Grun.

Nor 328 et 329 OLBY - PUY-DE-DOME (France)

l)épôt fossile d'eau douce

\section{BIBliograpHIE. - Frère J. Héribaud. Las Diatomées d'Auvergne 1893}

Amphora pediculus Grun.

Cymbella cistula Hemp.

Encyonema ventricosum $\mathrm{K} \mathbf{z}$.

Epithenia zebra Ktz.

Fragilarla capucina $\nabla$. mesolepta Grun.

- construens $\nabla$. genuina Grun.

- parasitica Grun.

- virescens Ralfs.

Gomphonema auritum A. Br.

- capitatum Ehr.

constrictum $\nabla$. subcapitata

Grun.
Melosira lineolata Grun.

Navicula bacillum Ehr.

- borealis Kłz.

- cuspidata Ktz.

- elliptica Ktz.

- Limosa Ktz.

- major Kız.

- mesolepta Ehr.

- radiosa Ktz.

- rupestris Ktz.

Synedra acuta $\mathrm{K} t z$.

- ulne Ehr. 


\section{Nos 330 et 331}

BAIE DE PENSACOLA - FLORIDE (Etats-Unis)

Achnanthe heteromorpha Grun. Actinocyclus Ralfsii W. Sm. Aotinoptychus splendens Ralfs.

$$
\text { - undulatus Ebr. }
$$

Amphiprora lepidoptera Greg.

Amphora angulata $\mathrm{Cl}$.

- arenaria Donk.

- areolata Grun.

- excisa Greg. var.

- intersecta A. S.

- obtusa Greg.

- proteus Grog var.

Autisens confluens Grun.

- colatus Bail.

- Moronensis Greo.

- pruinosu Grev.

- - var: Zanzibarica.

- punctatus.

- sculptus Eh.

Bacteriantrum varians Eh.

Biddulphia mobiliensis Bail.

- rhombus W. Sm.

Campylodiscus Echeneis Eh. punctulatus Grun.

Cerataulus Smithii Ralfs.

Cocconoin biradiata $J . B r$.

- Pensacoloe A.S.

- scutellum yar. dilatata A. S.

Coscinodiseas bulliens A. S.

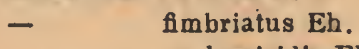

- oculus-iridis Eh.

Dietyonels marginata $C l$.

$$
\text { - naoiculacea } \mathrm{Cl} \text {. }
$$

Eupodiscus argus Eh.

- radiatus Bail.
Mastogloia Braunii Grun. Floridea $\mathrm{Cl}$. pisciculus $\mathrm{Cl}$.

Melogira Davidsonii A. S.

- Pensacolæ A. S.

- sulcata Ktz.

- - var. coronata.

Navicula aspera Elb.

- caribœa Cl.

- cruciata A. S.

- formosa Greg.

- longa Greg.

- lyra $\mathbf{r}$. dilatata.

- - v. elliptica.

- maxima Greg.

- multicostata Bréb.

- Kittoniana A. S.

- prætexta Eh.

- Roberteiana Grov.

- suborbicularis Greg.

Nitzschia circumsuta Bail.

- valida Cl. et Grun.

Plagiogramma tesselatum Grer. Pleurosigma balticum W. Sm.

Peeudauliscus radiatus Bail.

Rhabdonema adriaticum Ktz.

Stephanopyxis corona Eb.

Burirella Febigerii Lewis. - recedens A. S.

Bynedra fulgens W. Sm.

Terpeinoë musica $\mathrm{Eh}$.

Triceratium antediluvianum Eh.

$$
\begin{array}{ll}
\text { - } & \text { favus Eh. } \\
\text { - } & \text { sculptum Rab. } \\
\text { _ } & \text { scitulum Bright }
\end{array}
$$




\section{Nos 332 et 333 PUGET SOUND - WASHINGTON Co (Etat-Unis)}

\section{Sur les algues}

Arachnoldiscas Ehrenbergii Bail.

Aulacodiscus Oregonus Bail.

Billdulphia Edwardsii Feb.

Campylodiecus ecclesianus var.

Cocconeis costata Greg.

- - var. pacifica Grun. pellucida Grun.

Coscinodiscus oculus-iridis Eh.

Cyciotelia striata Grun.

Entopyla australis oar. gigantea.

Gralumatophora ambigua Grun.

Hyalodiscus laevis Eh.

$\begin{array}{ll}- & \text { subtilis Bail. } \\ \text { - } & \text { scoticus W. Sm. } \\ \text { - } & \text { - var. minor. }\end{array}$

Isthmia nervesa Ktz.

Melosira sol Eh.

- sulcata ra minor.

Navicula aspera Kitz.

Pleurosigma decorum W. Sm.

$$
\text { - intermedium W. Sm. }
$$

Podosira maxima Grun.

- hormoĩdes Grun.

- stellulifera Grun.

Rhabdonoma Crozierii Pritch, var.

- subrostratum fa paralle lo-lateralis.

Synedra pulchella var.

Triceratium arcticum Bail. - $\quad$ - var. \& Gona.

\section{ํ 334 BIG . LAKE, ARLINGTON - WASHINGTON C•}

\section{(Etats-Unis)}

Amphora Libyca Eh.

Campylodiscus costatus W.Sm.

Cocconols lineata Eh.

$$
\text { - placentula Eh. }
$$

Cymatoploura apiculata W. Sm.

$$
\text { - } \quad \text { elliptica var. }
$$

Cymbella americana A. S.

- vistula Kirch.

- cuspidata Ktz.

- Ehrenbergii Ktz.
Суйеlla gastroides Eh.

- heteropleura Ktz.

- lanceolata Kirch.

- maculata Ktz.

- mexicana Eh. var.

Bncyonema triangulum Kts.

- turgidum Grun.

Bpithemia amphicephala Grun.

- gibba Ktz.

- - var. ventricosa.

- turgida Ktz.

- - var. granulata. 
Epithemia turgida var. vertagus. zebra $\mathrm{Ktz}$.

Eunotia impressa Eh.

- major Rab.

- - var. ventricosa.

- præcupta var. bidens.

- tetraodon Eh.

Gomphonema acuminatum Ktz.

- angustatum $\mathrm{Ktz}$.

- apicatum Eh.

- constrictum Eh.

- $\quad$ intricatum $\mathrm{Ktz}$.

- montanum var. succisa.

- subtile Eh.

Bantzschia amphioxys Grun.

- - oar. major.

- elongata Grun.

Meiosira crenulata Grun.

Navicula acrosphoria $K t z$.

- aunericana Eh. fa minor.

- - - var. hacillaris.

- amphigomphus Eh.

- amphirynchus Eh.

- commutata Grun.

- costata Eh.

- dactylus $\mathrm{Ktz}$.

- divergens W. Sm.

- elliptica Ktz.

- gigas $\mathrm{Ktz}$.

- hemiptera Ktz.

- iridis Eh.

- Kefvigensil Eh. var.
Navicula limosa Ktz.

- major Ktz.

- megaloptera Eh.

- nobilis Ktz.

- nodora Eh.

- oblonga Ktz.

- peregrina $\mathrm{K} \mathrm{tz}$.

- Smilhii Bréb.

- transversa A. S.

- viridis $\mathrm{K} t \mathrm{z}$.

Nitzschia spectabilis Ralfs.

Pieurosigma attenuatum W. Sm.

Stuuroneis acula WV. Sm.

- amphilepta Eh.

- anceps Eh:

- Baileyi Eh.

- gallica M. Per.

- gracilis Eh.

- phœnicenteron Eh.

Stephanodiscus astraea Grun. var. spi- nulosa.

Carconensis var. pusilla.

Surirella elegans Eh.

- Rattrayi A.S.

- robusta Eh.

- tenera Greg.

- - var. nerrosa.

- valida A. S.

Synedra capitata Eh.

- biceps Ktr.

- ulna Eh. var. danica.

Tabellaria fenestrata Kts.

Van Hourckla oiridula Bréb.

No 335- SALINAS - MONTEREY (Etats-Unis)

(Dépot fossile)

Cyclotella Terryana Temp. et M. Per.n. sp. - var. major, plus grande que lo type et à granu. les contraux plus petito.
Cyclotella Terryana var. minor, plus petite et à granules centraux plus gros et parfois anguloux. 


\title{
No 336 BASSIN D'ARCACHON - GIRONDE (France)
}

\section{(Collection P. Bergon)}

\author{
Asterionella spathuilfera Cleve (As. Japonica Clevo)
}

(Voir Cleve Seasonal Distribution of Atlantic Plankton, etc., page 282)

No 337

LE HAVRE (France)

Berkeleya rutilane var. obtuea Gran.

No 338 LE TREPORT - SEINE-INF€RIEURE (France)

Amphiprora alata W. Sm.

- - lepidoptera Greg.

Coscinodiscus concinnus W. Sm.

Nitzschis acuminata Grun.

- sigma W. Sm.
Pleurosigma balticum W. Sm. var.

- hippocampus W. Sm.

- quadratum W. Sm.

Scoliopleura tamida Grun.

Surirolla gemma Eh.

No 339

KOGGIK (Hongrie)

Dépot fossile marin

Actinocyclua Knemeides Pant. rar.

- Loczyi Pant.

- moniliformis Ralfs.

- ovalis Grun. var.

- radiatus Ratt.

- tenellus Bréb.

Amphora acuta Greg.
Amphora costata W. Sm.

- gigantea Grun.

- int rsecta A. S. oar.

- Kossulhii Pant.

- Nooa Caledonica Grun.

- obtusa Greg.

- proteus Greg. var.

Aurlcula Szontaghii Pant. 
Campylodiscus Kidstonii Pant.

Climacosphenia hungarica? Pant.

Cocconeis scutellum Eh.

$$
\text { - - var. parva. }
$$

Coscinodiscus Bœkii. Pant.

Diatomelia Balfouriana Grev.

Epithemia Hirudinella $J$. Br. oar.

Grammatophora stricta Eh. var. fossilis. Mastogioia affinis $\mathrm{Cl}$.

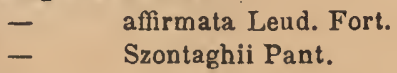

Melosira sulcata Ktz.

$$
\text { - - fa radiata. }
$$

Navicula abrupta Donk.

- $\quad$ var. Rattrayi.
- $\quad$ bisis Donk.
- $\quad$ Fischeri A. S.
- formosa Greg.
- $\quad$ Gorganowicii Pant.
- laterittata Pant.
- lyra Eh. var. atlantica.
- $\quad$ - var. connectans.
- $\quad$ - var. hungarica.
-

Navicula Martouni Pant.

- moesta A. S.

- notabilis Pant.

- pavida Pant.

- perducta Pant.(Mastogloia Debyi ?)

- perlonga Pant.

- Romanovii Pant.

- robusta Grun.

- splendida Greg.

Nitzechia scalaris $W . S m$.

- sigma W. Sm.

Pleurosigma angulatum W.Sm.

- balticum W. Sn.

- subrigidum Grun:

Podosira maculata W. Sm: var.

Pyxilla baltica Grun.

Rhabdonema musica J. Br.

Scoliopleura tumida Bréb.

Surirella Bibarensis Pant.

- fastuosa Eb.

-.. striatula Turp.

- torquata Pant.

- Toulae Pant.

Triceratium vastum Pant. (on fragments)

\section{Nos 340 et 341 HAMLIN'S POND - PLAINVILLE CONNECTICUT (États-Unis)}

Achnanthes lanceolata Brèb.

Amphora ovalis Ktz. var. minor.

Cocconets placentula Eh.

Cyclotella Meneghiniana Ktz.

Cymbella cistula Kirch.

- $\quad$ - var. fusidium.

- cymbiformis Bréb.

- Ehrenbergiifa minor.

- sastroides Ktz.

- gibba (Cocconema gibbum Eh.)

- heteropleura Ktz.

- maculata $\mathrm{Ktz}$.

- naviculæformis Auers.
Bncyonema ventricosum Grun.

Epithemia amphicephala Grun.

$$
\begin{aligned}
& \text { - gibba Ktz. } \\
& \text { - } \quad \text { - var. ventricosa. } \\
& \text { - turgida Ktz. } \\
& \text { - } \\
& \text { - } \\
& \text { - }
\end{aligned}
$$

Eunotia arcus oar. bidens.

- formica Bh.

- incisa Grog.

- minor Rab.

- praerupta oar. bidens. 
Eunotia tetraodon Eh.

Fragllaria cequalis Heib.

$$
\begin{aligned}
& \text { - - var. producta. } \\
& \text { - capucina var. mesolepta. } \\
& \text { - construens Grun. } \\
& \text { - } \quad \text { - var. binodis. } \\
& \text { - Harrisonii Grun. } \\
& \text { - intermodia. }
\end{aligned}
$$

Comphonema acuminatum Eh.

$$
\begin{aligned}
& \text { - - oar. laticeps. } \\
& \text { - - oar. pusilla. } \\
& \text { - angustatum } \mathrm{Ktz} \text {. } \\
& \text { - } \quad \text { - var. producta. } \\
& \text { - augur oar. Gautieri. } \\
& \text { - constrictum Eh. } \\
& \text { - - var. capitata. } \\
& \text { - gracile Eh. } \\
& \text { - - fomajor. } \\
& \text { - intermedium Grun. } \\
& \text { - } \quad \text { intricatum } \mathrm{Ktz} \text {. } \\
& \text { - sphærophorum Eh. } \\
& \text { - turris Eh. }
\end{aligned}
$$

Hantzwehia amphioxys oar. major.

Melosira oarians Ag.

Meridion constrictum Ralfs.

Navicula affinis $E h$.

- americana Eh.

- amphigomphus Eh.

- Bastianii M. Per. n. sp. - Intermédiaire entre le Nav. americana dont il a le raphé et l'area médian, et le Nav. pseudobacillum dont il a la striation centrale et la forme des nodules terminaux.

- biceps Eh.

- Brebissonii Ktr.

- eineta Eh.

- - var. Heufleri.

- commutata Grun.

- cuspidata $\mathrm{Ktz}$.

- dactylus Ktz.

- dilatata Ktz.

- elliptica Ktz.

- firma Ktz.

- gibba oar. hyalina.
Navicula gigas Eh.

- guttata J. Br.

- Hitchkockii Eh.

- instabilis A. S.

- iridis Eh.

- - var. ampliate.

- legumen Eh.

- limosa Ktz.

- major Ktz.

- - var. asymetrica.

- nodosa Eh.

- nobilis $\mathrm{Ktz}$.

- oblonga $\mathrm{Ktz}$.

- peregrina Ktz.

- pupula Ktz.

- radiosa $\mathrm{Ktz}$.

- streptoraphe Cl.

- subacuta Eh.

- transversa A. S.

- viridis $\mathrm{Ktz}$.

Nitzschia amphibia Grun.

- plana W. Sm.

Pleurosigma acuminatum Grun.

Btauroneis acuta W. Sm.

- anceps Eh.

- - var. nobilis.

- Baileyi Eh.

- gracilis Eh.

- Phyllodes Eh.

- quadrata M. Per. et F.H.

Surirella arcta A. S.

- bifrons Eh.

- cardinalis Kitt.

- cruciatr A. S.

- elegans Eb.

- splendida Ktz.

- tenera Greg.

- - var. nerrosa.

syuedra biceps Ktz.

- capitata Eh.

- crotonensis var. prolongata.

- rumpens var. fragilarioides.

- ulna Eh.

- $\quad$ - var. æqualis.

- vitrea Ktz.

Tabellaria fenestrata $\mathrm{Ktz}$.

- floceulosa $\mathrm{Ktz}$. 
No 342

\section{EXPEDITION DU CHALLENGER}

Sondage fait à une profondeur de $3872 \mathrm{~m}$. dans les mers Australes par 53`55' de latitude Sud et 108.35’ de longitude Ouest

BIBLIOGRA PHIE. - Castracane. - Expédition du Challenger, 1886

Actinoryclus complanatus Cast. - umbonatus Cast.

Asteromphalu Brookii Bail.

- Humboldtii Eb.

- Ralfsianus Grun.

Biddulphia Weissflogii Jan.

Coselnodiscus curvatulus Grun.

- decipiens Grun.

- denarius A. S.

- elegantulus Grov.

- griseus Grov.

- lentiginosus Jan.

- linertus Eh.
Coscinodiseus scintillans Grov.

- subtilis Grun.

- tumidus Jan.

Eacampia balaustium Cast.

Fragilaria antaretica Cast. $=$ Fr.

Castracanei.

Melosira sol var. (Cast. Exp. Chall. 17/13.)

Navicula nitescens Greg.

Thallasiothrix longissima $\mathrm{Cl}$. nitzschioïdes $\nabla$. lanceolata Grun.

$N \cdot 343$

SAINT-SEURIN - MEDOC (France)

Pleurosigma curralum Gran

$N^{\circ} 344$ et 345 LIGNITES DE SENDAI (Japon)

Actinella Braziliensis Grun.

Cymbella cuspidata $\mathrm{Ktz}$.

- gastroïdes Ktz.

- lanceolata Eh.

- turgidula Grun.

Bunotia diadema Ralfs.

- formica Eh.

- parallela E Eb.
Eunotla pectinalis Rab.

$$
\text { - } \quad \text { - var. stricta. }
$$

Gomphonema acuminatum $\nabla$. laticeps.

$$
\text { - intricatum Ktz. }
$$

Meloaira granulata.

Navicula acrosphœria Bréb.

- americana Eh. 
Navicula cardinalis oar.

- dactylus Eh.

- dirergens $\mathrm{Ktz}$.

- elliptica Ktz.

- firma Ktz.

- formosa var.

- gihba r major Eh.

- gigas Eh.

- major $\mathrm{Ktz}$.

- mesolepta $\mathrm{Ktz}$.

- - var. stauroneiformis.

- nobilis Eh.

- thorax J. Br.
Navicula riridis $\mathrm{K} t z$.

Nitzschia tryblionella Grun.

Stauroneis phoenicenteron Eb.

$$
\text { - anceps Eh. }
$$

Surirelia elegans var. constricta.

$$
\text { - splendida var. }
$$

Tabellaria fenestrala Ktz.

Terpainoes inflata J. Br.

Tetracyclus emarginatus W. Sm.

- japonicus P.P. = Stylobiblium japonicum.

\title{
Nor 346 à 349 KAVNA - BREMIA (Hongrie)
}

Dépots fossiles marins

\author{
Bibliographie. - J. Pantocseck. Diatomées de Hongrie, \\ volumes II ef III.
}

Achnanthes brevipes Ag.

- $\quad$ danica Grun.
- $\quad$ Loczyi Pant.
- $\quad$ pennæformis Grev. var.

Actinocyclus Bremianus Pant.

- disseminatus Pant.

- Ehrenberyit Ralfs.

- Knemeides Pant.

- labyrinthicus Pant.

- Loczyi Pant.

- rotula J. Br.

Actínoptychue elegans Ralfs.

- Petitii Pant.

- splendens Ralfs.

- Staubii Pant.

Amphiprora Posewitzii Pant.

Amphoracingulata Pant.

- crassa Grev.

- - v. minor Pant.

- - $\quad$ - punctata Grun.

- granulata Greg.

- Grundlerii Grun.
Amphora Loczyi Pant.

- proteus Greg.

- striata Pant.

- vittata Pant.

Asterolampra marylandica $\mathrm{Eh}$.

Asteromphaius hungaricus Pant.

Auliscus Hauckii Pant.

- Loczyi Pant.

- sculplus Ralfs.

Biddulphla capucina A. S.

- elegantula Grev.

- $\quad$ - v. polycistinea Pant.

- Elesdiana Pant.

- homala Pant.

- Loczyi Pant.

- permagna Pant.

- pulchella Gray.

- tridentata Eh.

- - fe minor Pant.

- Tuomeyí Bail.

- . - - - hungarica Pant.

Campylodiscus angularis Greg. Bremianu Pant. 
Campylodiscus clypous Eh.

- $\quad$ Grunowii Pant.

Ceratauius Peragalloi Pant.

Cocconeis Baldjikiana Grun.

$$
\begin{aligned}
& \text { - biharensis Pant. } \\
& \text { - - } \quad \text { v. minor Pant. } \\
& \text { - perpusilla Pant. } \\
& \text { - scutellum Eh. } \\
& \text { - - } \quad \text { v. Baldjicklana Grun. } \\
& \text { - - } \quad \text { - fossilis Pant. }
\end{aligned}
$$

Cuscinodiscus asteromphalus Eh.

$\begin{array}{ll}- & \text { Bremianus Pant. } \\ - & \text { concinnus A. S. } \\ \text { - } & \text { intumescens Pant. } \\ \text { - } & \text { marginatus Eh. } \\ \text { - } & \text { oculus-iridis Eh. } \\ - & \text { radiatus Eh. } \\ \text { - } & \text { robustus Grev. } \\ - & \text { tabulatus J. Br. } \\ \text { - } & \text { undatus Grun. }\end{array}$

Cymatosira biharensis Pant.

Bntopyla Rinnboeckii Pant.

Bpithemia gibberula $\nabla$. protracta.

Eunotia parallela Eh. var.

Grammatophora biharensis Pant.

$$
\begin{array}{ll}
\text { - } & \text { hungarica Pant. } \\
\text { - } & \text { maxima Grun. } \\
\text { - } & \text { oceanica Eh. } \\
\hline & \text { robusta v. gracilis Dip. }
\end{array}
$$

Hemialus Szaboi Pant.

Hyalodiscus radiatus Grun.

$$
\begin{array}{ll}
\text { - } & \text { scoticus Grun. } \\
\text { - } & \text { subtilis Bail. }
\end{array}
$$

Mastogluia obtusa Pant.

Melosira bituminosa $\nabla$. dilatata Pant.

- clavigera Grun.

- Loczyi Pant.

- nummuloides Ag. var. Elesdiana Pant.

- $\quad$ ol Ktz.

Navieula aradina Pant.

- aspera Eh. v. hungarica Pant.

- bacillifera Pant.

- Bäumberii Pant.
Navicula brasiliensis Grun, v. fosailis.

- cancellata Donk.

- claviculus Greg.

- didyma Eh.

- digrediens A. S.

- Döcyii Pant.

- egeria Pant.

- Elesdiana Pant.

-

- formosa Greg. $\nabla$. fossilis.

- Fuchsii Pant.

- fusca Ralfs.

- - v. major Pant.

- halionáta $\nabla$. minor Pant.

- Hantkenii Pant.

- Hennedyi W. Sm.

- $\quad$ - $\quad$ v. fossilis.

- Hoffmannii Pant.

- humerosa Brèb.

- - v. elongata Pant.

- ignobilis Pant.

- inflexa Greg. v. biharensis Pant.

- inhalata A. $\mathbf{S}$.

- - $\quad$. biharensis Pant.

- irrorata Grev. ₹. fossilis.

- lineata Donk.

- Loczyi Pant.

- lyra Eh.

- maxima Grev.

- parida Pant.

- phalangium Pant.

- pinnata Pant.

- polygibba Pant.

- sectilis A. S.

- sejuncta $A$. S.

- $\quad-\quad$ var. Baldjickiana Pant.

- Smitbii Brèb.

- suborbicularis Greg.

- venusta Pant.

- yarrensis Grun.

- - - v. valida Pant.

Nitzschia andesitica Pant.

$$
\text { - - Loczyi Pant. }
$$

Orthonele splendida Grun.

Paralia sulcata $\mathrm{Cl}$.

$$
\text { - - - } \quad \text { - } \text { - } \text {. hungeriata Grun. }
$$

Plagiogramma biharensis Pant.

- - Loczyi Pant.

- . salinaria Pent. 
Pleurosigma hippocempus W. Sm. - Wansbeckii Donk. var.

Podosira Baldjickiana Grun.

- hormoides Ktz.

- Loczyi Pant.

Pseudodictyoneis hungarica $\mathrm{Cl}$.

Pyxidicula cruciata Eh.

Pyxilla americana Eh.

Rbabdonema adriaticum Ktz.

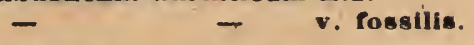

Raphoneis angustata Eh.

- gemmifera Eh.

$\begin{array}{lll}- & - & \text { v. biharensis Pant. } \\ \text { - } & \text { - } & \text { v. subtilior Pant. }\end{array}$
Staurosira Karnensis Pant.

Stephanodiscus Kanitzil Grun. Pant.

Surirelia antiqua Pant.

- fastuosa Eh.

- - $\quad$ - fossilis.

- striatula Turpin.

Synedra bibarensis Pant.

- Bremiana Pant.

Triceratium balearicum Cl. et Grun.

- lætum Pant.

- Loczyi Pant.

- - $\quad$ - confluens.

- pileus Eh.

\section{No 350 SAINT-FLORET - PUY-DE-DOME (France) \\ Source minérale}

Achnanthes lanceolata Bréb.

Cymbella parva W. Sm.

- pusilla Grun.

Denticula elegans Ktz.

Fragilaria construens Eh.

Gomphonema micropus var. minor.

$$
\text { - parvulum Eh. }
$$

Navicula appendiculata Ktz.

- atomoides Grun.

- viridis var. commutata f curta.
Nitzschia bilobata $\nabla$. hybrida Grun.

- communis Bab.

- - var. obtusa.

- commutata Grun.

- conticola Grun.

- minuta Bleish.

- frustulum Grun.

- Kittlii Grun.

- palea Ktz.

- vitrea Norm.

$\mathrm{N}^{*} 351$ et 352 BEDDINGTON MAINE (États-Unis)

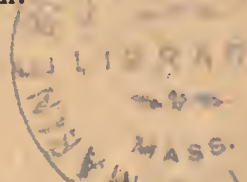

Dépôt fossile d'eau douce

Achnantidium flexellum Grun .

Cymbella anglica Lag.

- cuspidata Ktz.

- cymbiformis Eh.
Cymbella Ehrenbergli Ktz.

- incisa $\mathrm{Ktz}$.

Epithemia gibba Ktr..

Bunotla arcus Eh. 
Bunotla formica Eh.

- gracilis Eb.

- pectinalis Eh.

- - var. undulata.

- robusta et toutes ses variétés.

Gomphonema acuminatum Eh.

- capitatum Eh.

Navicula ambigua $\mathrm{Ktz}$.

- amphigomphus Eh.

- amphirynchus Eh.

- cardinalis Eh.

- dactylus Eh.

- dilatata Eh.

- divergens W. Sm.

- dubia Greg.

- elliptica Ktz.

- firma $\mathrm{Ktz}$

-. follis Eh.

- gigas Eh.

- hobes Ralfs.
Navicula iridis Eb.

- legumen Eh.

- macilenta Eh.

- major Ktx.

- nobilis Eh.

- rhomboides Eh.

- - var. major.

- verians Ktz.

- tabellaria Eh.

- - var. stauroneiformis.

- tumescens Grun.

- viridis En.

Stauronels phcenicenteron Eh.

- - var. minor.

- gracilis W. Sm.

Stenopterobia anceps Bréb. - intermedia Bréb.

Surirella linearis var. constricta W. Sm.

Tabellaria fenestrata Ktz.

\section{No 353 : ARTERN - THURINGE (Allemagne) \\ Lac salé}

Achnanthe brevipes $\mathbf{A g}$.

Epithemia musculus $\mathrm{K} \mathrm{tz}$.

- succinta Brèb.

Melosira nummuloides Ag.

Navicula mutica Ktz.

Pleurosigma delleatulum var. sallnarum Grun.

synedra affinis Ktz. var.

No 354 BUENOS-AYRES (République Argentine)

Sondage

Gomphonema constrictum Eh. Hyalodiscus laevis Ehr.

Nitzschia vitrea Norm.

Navicula formosa Greg.
Navicula interrupta Kı. var.

Rhopalodia gibberula var. Debyl Pant.

Surirelia striatula Turpin.

Synedra affinis Ktz. 


\title{
$N^{\circ s} 355$ et 356 CEYSSAT - PUY.DE-DOME (France)
}

Dépôt fossile d'eau douce

\author{
BIBLIOgRAPHIE. - Frère J. Héribaud. Les Diatomées \\ d'Auvergne 1893.
}

Achnanthes lanceolata Grun.

Amphora affinis $\mathrm{Ktz}$.

Amphora pediculus $\nabla$. minor Grun.

Cocconeis lineata Grun.

$$
\text { - placentula Ehr. }
$$

Cymatopieura solea Brèb.

Cymbeila anglica Lag.

$$
\begin{aligned}
& \text { - } \quad \text { aspera Ehr. } \\
& \text { cistula v. fusidium H. P. et } \\
& \text { F. H. } \\
& \text { - cymbiformis Ehr. } \\
& \text { - } \quad \text { helvetica Ktz. } \\
& \text { - lanceolata Ehr. } \\
& \text { - leptoceros Ktz. } \\
& \text { - parva W. Sm. } \\
& \text { - } \quad \text { Pauli M. P. et P. H. } \\
& \text { - turgidula Grun. }
\end{aligned}
$$

Denticula inflata $\mathrm{W}$. Sm.

Encyonema ventricosum $\mathrm{Ktz}$.

Epithemia gibba Ehr.

$$
\begin{aligned}
& \text { - - } \quad \text { - parallela Grun. } \\
& \text { - - v. ventricosa Grun. } \\
& \text { - gibberula Ehr. } \\
& \text { - sorex Ktz. } \\
& \text { - turgida Ktz. } \\
& \text { - } \quad \text { - fa crassa M.P. et F.H. } \\
& \text { - - } \text { - granulata Grun. } \\
& \text { - } \quad \text { - } \quad \text {. vertagus } \mathrm{Ktz} \text {. } \\
& \text { - zebra Ktz. } \\
& \text { - } \quad \text { - v. longicornis M. P. et } \\
& \text { F. H. } \\
& \text { - } \quad-\text { v. minor M. P. et F. H. } \\
& \text { - - v. undulata M. P. et }
\end{aligned}
$$

Eunotia minor Rab.

Fragilaria æqualis Lag.

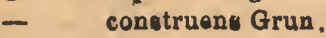

Fragilaria construens v. venter Grun.

$$
\begin{aligned}
& \text { - } \quad \text { intiptica Schum. } \\
& \text { - } \quad \text { striatula Lyngb. } \\
& \text { - } \quad \text { virescens Ralfs. }
\end{aligned}
$$

Gomphonema acuminatum Ehr.

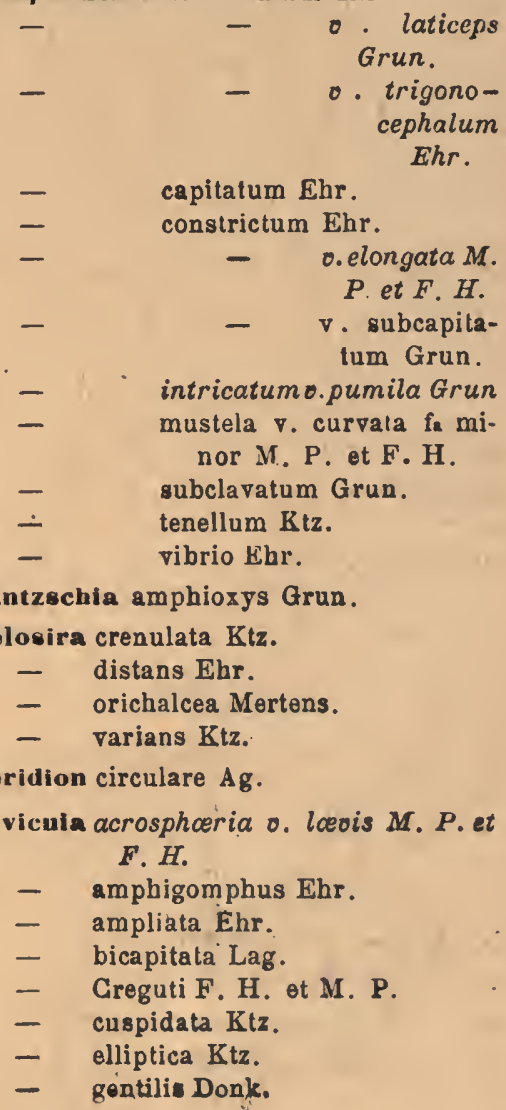


Navicula major Ktz.

- nobilis Ehr.

- pupula Ktz.

- radiosa $\mathrm{Ktz}$.

- viridis Ktz.

Nitzschia acutiuscula Grun.

- fossilis Grun.

- frustulum Grun.

- palea Ktz.

Rboícosphenia curvata Grun.

Stauroneis phcenicenteron Ehr.
Bynedra acus o. angustissima Grun.

- - v. fossilis Grun.

- acuta $\mathrm{Ktz}$.

- $\quad$ - v. oxyrynchus Ktz.

- capitata Ebr.

- delicatissima W. Sm.

- radians $\mathrm{Ktz}$.

- ulna Ehr.

- - $\quad$. danica Ktz.

- - T. obtusa W.Sm.

- $\quad$ - . snbæqualis Grun.

- $\quad$ - . vitrea $\mathrm{Ktz}$.

$\mathrm{N} \cdot 357$

ILE CAMPBELL (Nouvelle-Zélande)

Sondage

Bibliographie. - P. Petit. Diatomées de l'lle Ca:npbell et de la Nouvelle-Zélande (Fonds de la mer, 1877).

Actinoptychus areolatus $\mathrm{kh}$.

$$
\text { - } \quad \text { punctatus Eh. }
$$

Amphiprora decussata Grun.

Amphora Euleinsteinii Grun.

- proteus var. A. S. atl. 27/45.

Auliscus pruinosus var.

Campylodiscus eamoensis Grun.

Coceoneis pellucida Grun.

$$
\text { - - var. excentrica. }
$$$$
\text { - pseudo-marginata Greg. }
$$

Chretoceros dicladia (Eh) Cast. M. do Castracane a montré dans ses diatomées du Challenger que le Dicladia capreolus d'Ehrenberg n'était qu'une spore de Chrotoce. ros.
Entopyla australis Eh.

Hyalodiscus maximus P. P. $=H$, radiatus var. maxima.

$$
\text { - radiatus } 0^{\prime} \text { Meara. }
$$$$
\text { - - var. minor. }
$$

Melosira sulcata Eh.

Naviculu aspera Eh.

- bombus oar.

- digrediens $A . S$.

- Hennedyi W. Sm.

- protexta oar.

- Smithii Bréb.

Nitzschia panduriformis Greg.

Pleutosigma furmosnm W. Sm.

Rhabdonema adriáticum $\mathrm{Ktz}$.

Surirella fastuosa $\mathrm{Eh}$. 


\section{No: 358 à 360 MONTEREY - CALIFORNIE (États-Unis)}

\section{Dépôt fossile marin}

Actinocyclus confluens Grun.

$\begin{array}{ll}- & \text { Ehrenbergii Ralfs. } \\ - & \text { incertus Grun. } \\ - & \text { ingens Ratt. } \\ - & \text { moniliformis Ralfs. } \\ \text { - } & \text { abscurus Ratt. } \\ \text { - } & \text { radians Ratt. }\end{array}$

Actinoptyehus glabratus $\nabla$. Montereyi Grun.

$\begin{array}{ll}- & \text { Grundlerii A. S. } \\ \text { - } & \text { Laeoigatus Grun. } \\ \text { - } & \text { minutus Grun. } \\ \text { - } & \text { socius A. S. } \\ \text { - } & \text { undulatus Eh. } \\ & \text { vulgaris Schum. }\end{array}$

Amphitetras elegans Grev.

Arachnoidiscus Ehrenbergii $\nabla$. Montereyiana.

Greoilleanus Hardm.

$-$ indicus Eh. ornatus v. Montereyiana.

Asterolampra Brebissoniana Greo.

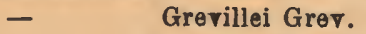

- Marylandica Eh.

- rotula Greo.

- variabilis Gręv.

Asteromphalue Darwinii Eh.

Aulacodiscus Brownei Norm.

- decorus Greo.

- Kittonii Arnott.

- minutus Ratt.

- Oregonus Bail.

- probabilis A. S.

- simplex Ratt.

Auliscus cœlatus Bail.

- - var.

- Hardmanianus.

- mirabilis Greo.

- punctatus Bail.

- Stöckarditi Jan.

Biddniphia aurita var.
Campylodiscus coronilla J. Br.

Cerataulus Johnsonianus Greo.

Cocconels dirupta v. californica $\mathrm{Cl}$.

$$
\begin{aligned}
& \text { - distans. } \\
& \text { - infleza A. S. } \\
& \text { - } \quad \text { notabilis A. S. } \\
& \text { - prisca Cl. }
\end{aligned}
$$

Concinodiscus antiquus Grun.

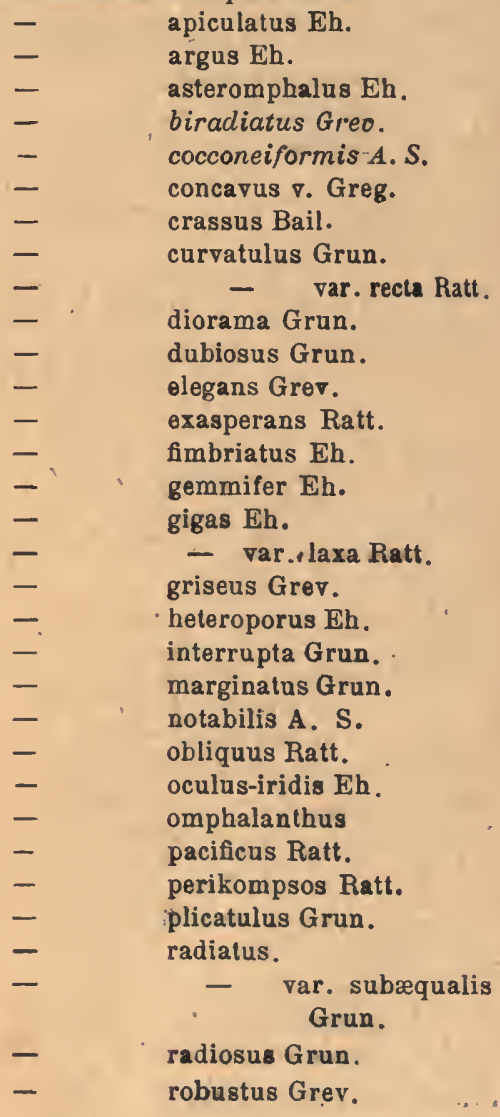


Cosclnodiscus sphcroidalis Ratt.

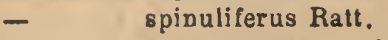

- subconcavus famajor Grun

- subtilis Eh.

- subvelatus.

- symmetricus Grev.

- Woodwardii Eul.

Cosmiodiscus elegans Greo.

- tenuis Grun.

Cranpedodiscus rhombicus Grun.

Cressivellia rudis Grev.

Bupodiscus oculatus Grev.

Gephyria gigantea Grev.

- constricta Grer.

Glyphodiscus stellalus Grev.

Iyalodiscus reticulatus A. $S$.

Liıhodesmium minusculum Grun.

Melosira clavigera Grun.

- separanda A. S.

- sol Eh.
Navicula angelorum $\mathrm{Cl}$.

- lyra El.

- ornata A. S.

- oscitans A.S.

- prætexta var. abundans A. S. spectabilis Grev. var.

Poussira hormoides var. Muntereyi Grun.

Rutiiaria epsilon Grev.

- $\quad$ - v. longicornis Temp. et $\mathrm{Br}$.

Stictorfiscus californicus Grev.

$$
\begin{array}{cc}
- & \text { var . ecostata } \\
\text { - } & \text { Grun. } \\
\text { - } & \text { Hardmanianum Grer. }
\end{array}
$$

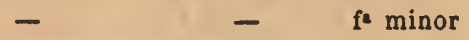

Stephanopyxis corona Grun.

- crassispina Grun.

- turris Eh.

Triceratium arcticuin $\nabla$. californica Grun

- inelegans Grev.

- Monteroyi Bright.

Xanthlopyxis umbonatus Grev.

\section{Nos 361 et 362 WERNAMO - SMALAND (Suède)}

\section{Analysé par le professeur P.-T. Cleve}

Achnanthe microcephala Grun.

Achnantidium flexellum Bréb.

Anomceoneis serians Bréb.

$$
\text { - follis Elr. }
$$

Cyclotella comta Ehr.

Cymbella æqualis W. Sm.

$$
\text { - cesati Rab. }
$$

Dipioneis finnica Cl.

Eneyonema cœspitosum Ktz.

- gracile Rab.

- hebridicum Greg.

Eunotia gracilis Rab.

- pectinalis Rab.

- robusta Ralfs.

- major var. bidens W. Sm.

Fragllarla virescens Ralls.
Frustulia crassinervia Bréb.

- rhomboides Bréb.

Gomphonema acuminatum Ehr.

- $\quad$ constrictum Ehr.

Navicula depressa $\mathrm{Cl}$.

- radiosa $\mathrm{Ktz}$.

- - var. angustata Grun.

Neidium affine Ehr.

Nitzschia angustata Grun.

- denticula Grun.

Pinnularia biceps Greg.

- Brebissonii Kts.

- dactylus Ehr.

- divergens W. Sm.

- gracillima Greg.

- major Ktz.

- nobilis Ehr.

- stauroptora Grun. 
Pinnularia subsolaris Grun.

- undulata Greg.

- viridis Ehr.

- var. major $\mathrm{Cl}$.
Stauroneis phœnicenteron Eh.

Stenopterobia intermedia Bréb.

Surirella robusta Eh.

Tabellaria fenestrata Ktz.
Ilantzschia amphioxys Grun.

Navieula cuspidata Kt. .

- Heufleriana Grun.
Nitzechia hungarica Grun. Surirella ovalis Bréb.

No 364

VILLEFRANCHE-SUR-MER (France)

Licmophora gabellata As.

Nor 365 et 366 SWAN LAKE KLAMATH Cty - OREGON (États-Unis)

Amphora Libyca Eb.

- ovalis Ktz.

Cocconeis amyǵdalina Brẻb.'

- lineata Eh.

- placentula Eh.

Cyclotella Terryana Temp. ot M. Per.

Cymatoploura apiculata W. Sm.

- Mannii M. Per. n. sp., robuste, forme extérioure et structure du Cy. apiculata, mais plus large; ondulations concentriques comme dans Cy. Martyi. 6 côtes en $10 \mu$ et perles intercostales peu visibles. Long. 120 à $150 \mu$, larg. - 45 à $60 \mu$.

- solea W. Sm.
Cymbella capitata M. Per.

- cistula Hirch.

- - var. crassa n. var. Do presque semi-circulaire relativement large. Long. 70 à $75 \mu$, larg. 20 à $25 \mu$; 9 stries du cỏté dorsal, 10 du côté ventral en $10 \mu$.

- cucumis A. S. var. delicata n. var. Intermédiaire entre Cym. cucumis et lo Cym. delecta.

- cuspidata $\mathrm{Ktz}$.

- dissimilis M. Per. n. sp. Rappelle la forme du Cym. Ehrenbergii, mais 27 stries en $10 \mu$ du côté dorsal et 10 du côté ventral. Long. de 75 à $85 \mu$.

- Janischli A. B. 
Cymbelia maculata Ktz.

mexicana Eh.

Bncyonema parallelum M. Per. n. sp. En segment de cercle, à angles fortement arrondis ; raphé droit, bifide vers le nodule central. Stries fortemont granulées, parallèles dans la partie dorsale. Long. 50 à $60 \mu ; 9$ stries en $10 \mu$.

turgidum Grun.

- 8p.9 A. S. atl. 10/60

Bpithemia gibba $\mathrm{Ktz}$.

- $\quad$ - var. ventricosa.

- - var. capitata

n. var. Peu aruué et à extrémités recurvées et capitées

- - oar. perlonga.

- sorex $\mathrm{Ktz}$.

- truncata M. Per. u. ep. Presque droit ou peu arqué à extrémités tron quées, quelque. fois un peu capitẻes, 3 à 4 côtès et 10 lignes do granules on $10 \%$.

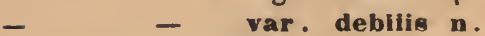

var. Plus grêle ot moins franchement tronqué que le type, les granules s'espacent vers le bord contral et ont moins fortement marqués.

- turgida $\mathrm{Ktz}$.

- - var. granulata.

- Westermannii var. stricta $\mathbf{n}$. var. De même forme mais plus étroit que lo type.

- zebra Ktz.

- _ fa minor.

- var. proboscidea.

Fragilaria aqualio var. major n. var.

Semblable à la var. producta mais atteignant $75 \mu_{\text {. }}$
Fragilaria brevistriata Grun.

$$
\begin{aligned}
& \text { - - var. mormorans. } \\
& \text { - construens Grun. } \\
& \text { - - var. venter. }
\end{aligned}
$$
forme extèrieure triondulèe comme le Nitz. sinuata; 11 a 12 stries en $10 \mu$ laissant un area central assez large. Long. 35 à $45 \mu$.

Gomphoneis elegans M. S.

$$
\begin{array}{ccc}
- & H e r c u l a n e a & \text { Eh. } \\
- & - & \text { var. clavata. } \\
- & - & \text { var robusta. } \\
& - & \text { var. rostrata } \\
& & \text { n. var. Extré- } \\
& \text { mité supérieure } \\
& \text { fortement atté- } \\
& \text { nuée, rostrée. } \\
& & \text { var. sepiceps. }
\end{array}
$$

- Mamilla Eh.

Gomphonema Eriense Grun oar.

- $\quad$ Puiggarianum Grun.
- $\quad$ semiopertum Grun. oar.
- $\quad$ moblaoatum Grun. oar.
montan Eh.

Hantzschia amphioxys Grun.

Melosira crenulata $\mathrm{Ktz}$.

- Varennarum M. Per.

Navicula acrospheria Bréb. oar. breois.

- ambigua fa craticula.

- amphibola Cl.

- - var. stauroneiformis.

- amphigomphus Eh.

- Arverna M. Per.

- Bohemica Eh.

- cuspidata Ktz.

- cyprinus W. Sm.

- Dactylus Ktz.

- Dariana A. S. var.

- dicephäla Eh. var.

- elegans W. Sm. v. cuspidata.

- elliptica Ktz.

- - var. ostracodarum.

- gastrum Eh. fa maxima. (Long. $50 \mu$.

- gentilis Donk. .

- interrupta $\nabla_{\bullet}$ stauroneiformis. 
Navicula iridis $\mathrm{v}$. ampliata.

- major $\mathrm{Ktz}$.

- mesolepta var. stauroneiformis.

- microstauron v. stauroneiformis.

- nobilis $\mathrm{Ktz}$.

- peregrina $\mathrm{Ktz}$.

- $($ - var ?) Peticolasii M. Per. n. sp. Les stries centrales courtes, entremêlées de plus couries, sont enveloppées par les roisines qui sont genouillées à cet effet Stries fortement linéolées 7 en $10 \mu$, au milieu, fortement convergentes aux extrémités, aire centrale unilatéralement renforcé $\theta$. Long. 250 à $300 \mu$.

- psendo bacillum Grun.

- radiosa $\mathrm{Ktz}$.

- Reinhardtii Grun.

- $\quad$ - var. elliptica.

- scutelloides var. Mocarensis.

- ventricosa $\mathrm{Ktz}$.

- - var, undulata.

Nitzschia sigmoidea $W$. Sm.

Opephora americana M. Per. n. sp. De

forme elliptique, à extrẻmitès atténuées, légèrement capitulées. Long. de 25 à $40 \mu, 5$ à 6 côtes en $10 \mu$.

Stauroneis anceps Eh.

-
-
-
-
- var. amphicephala.

Baileyi Eh.

gracilis Eh.

Stephanodiscus astraea $\mathrm{Ktz}$.

$-$

Surirella bifrons Eh.

- biseriata Bréb.

- gracilis A. S. var. gigantea n. var. Semblable au type mais plus robuste et beaucoup plus grand. Long. 200 à $210 \mu, 4$ côtés en $10 \mu$.

- splendida Ktz.

- tenera Greg.

- - var. nerrosa.

Tetracyclus emarginatus Ralfs.

- stella Eh.

rhombus var. maxima.

Nos 367 à 369 MARMORITO-ALESSANDRIA - PIEMONT (Italie)

Dépot fossile marin

Ce superbe ét riche dépôt a été étudié par M. le docteur Achille Forti, de Vérone, dont nous le tenons, et qui a bien voulu nous communiquer l'analyse suivante.

Actinocyclus fuscus Norm.

- moniliformis Ralfs.

$$
\text { - splendens Ratt. }
$$

Actinoptychus crepido $\mathrm{A}$. S.

- glabratus Grun.

- Heliopelta Grun et var.

- Janischii Pant.

- neogradensi Pant.
Actinoptyehus Petitii. Pant.

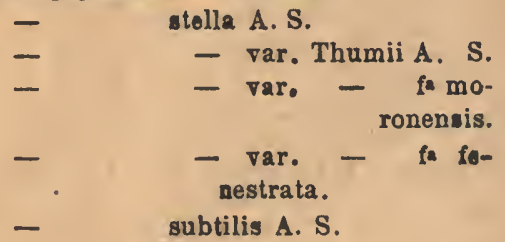


Actinoptychus Szontaghii Pant.

- undulatus Eh. var.

$$
\text { - } \quad \text { vulgaris Schum. }
$$

Arachnoidiscus ornatus Eh. var.

$$
\text { - - var. obscura. }
$$

Asterolampra Grevillei Wall.

$$
\begin{aligned}
& \text { - marylandica Eh. } \\
& \text { จ. fragilis Pant. }
\end{aligned}
$$

Aulacodlscus affinis Grun.

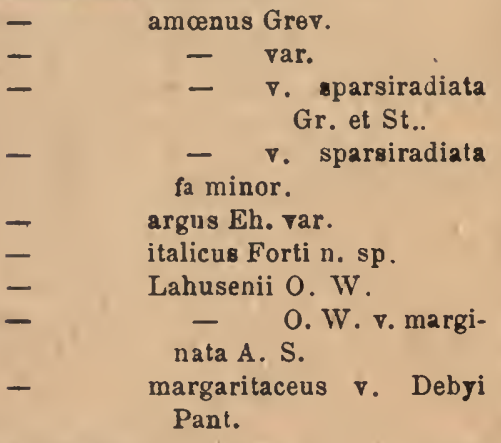

Auliscus Grunowii A. S. v. Pantocsekii v. $n$.

- Loczyi Pant. fa major v. n.

- Normanianus Grev.

- pruinosus Bail.

- punctatus Bail.

Blddulphia pulchella Gray.

$$
\begin{array}{lrl}
- & \text { Tuomeyi Bail. } \\
\text { - } & \text { - } & \text { v. centrospinusa n. v. } \\
\text { - } & \text { v. elongata Pant. } \\
\text { - } & \text { - v. globiceps Grun. } \\
\text { - } & \text { Sp.? } & \text { v. media n. }
\end{array}
$$

Campylodiscas Greenlezfianus Grun.

\section{Ceratauius Thumii A. S.}

Cladogramma conicum Grev.

$$
\text { tum } \mathbf{r} \text {. } \mathrm{n} \text {. campanula- }
$$

Coceoneis curvirotunda Temp. ot $\mathrm{Br}$.

- Lanzi Forti n. sp.

- præcellens Pant.

- - Pant. fa.

- splendida Grun.

Coscinodiscas apiculatus $\nabla$. Woodwardii Eul.

- argus Eh.
Cescinodiscus armatus Grev. fa Barbadensis.

asteroides Tr. et Witt.

asteromphalus Eb.

biangulatus A. S.

concavus Greg.

Lewisianus Grev.

marginatus Eh.

micans A. S.

Moronensis Ratt.

nitidus Greg.

odontophorus Grun.

omphalantus Eh.

papulosus Forti, n. sp.

polyacanthus Grun. v. intermedia.

psoudolineatu: Pant.

radiatus kih.

- Eh. var.

rhombicus Gr. var. italicus n. v.

robustus Grev.

Rothii. Grun.

superbus Hardm. var. trinitatis Gr. turgidus. Ratt.

Craspedodiscus coscinodiscus Eh.

- elegans Eh.

Craspedopora Pantocsekii J. Br.

Ditylium n. sp.?

Endictya oceanica. Eh.

Gephyria media Arn. v. Ardisoniæformis $\mathrm{n}, \mathrm{v}$.

Goniothecium odontella Eh.

Grammatophora marina Eh. var.

Hemiaulus Weissii Grun.

Hyalodisous radiatus Grun. $\nabla$. minor - - $\quad$ - arctica Grun.

Isthmla Squinaboli Forti.

- Szaboi Pant.

Leudugoria Janischii Temp. var. brovis $n$. v.

Melosira clavigera Grun.

$$
\begin{aligned}
& -\quad-\quad \text { fa trinotata } \nabla . n . \\
& -\quad \text { fa crassiradiata } \nabla . n . \\
& \text { - } n \text { sol Ktz. } \\
& -\quad \text {. breviradiata n. } \nabla .
\end{aligned}
$$


Melosira sulcata Eh. v. coronata Grun.

- - var. radiata.

Müileriella limbata V. $H$.

Navicula Debyi Pant. var. elliptica Cl.

- gemmata Grev. var. spectabilis Grun.

- Hennedyi W. Sm. $\nabla$. brachylopleura n. $\mathbf{v}$.

- - $\quad$ - neapolitana Cl.

- lyra var. intermedia fa ambigua $\mathrm{H}$. P.

- maculata Bail.

- Peragalli J. Br.

- Platessa Cl. ot Grove.

- præstes A. S.

- prætexta Eh.

- suborbicularis Grog.

Pseudopyxilia capreolus Forti.

- hungarica (Pant.) Furti.

- obliquepileata Forti.

- rossica (Pant.) Forti.

- Temperiana Forti.

Rhabdonema adriaticum Hantz.

Stephanogonia actinoptychus Eh.

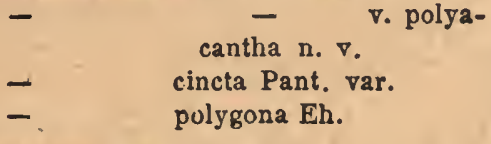

stophanopyxis corona $\mathrm{Eh}$.

- _ - v. monicæ Grun.

- grosse cellulata Pant.

- Joynsonii A. S.

- pediastriformis Forti. n. sp.

- turris Grun. $\quad$. intermodia.

Stictodiscus californicus Grev. จ. ecostata Grun.

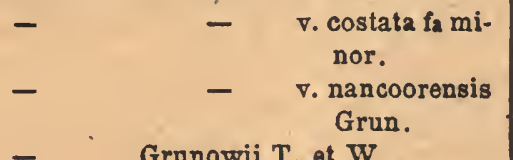

stictodisens Johnsonianus $\mathrm{T}$. et W. ta tri gona.

Haïtianus $T$. et W.

Triceratium acutum Eh.

- antediluvianum Eh.

- archangelskianum O. W. var.

- $\quad$ cristatum Pant. var. ta $n . \nabla$.

latum Grev. var.

- madagascarense Grun.

- nancoorense Grun.

- - var. italica.

- Pantocsekii Grun.

- - faconvexa Pant.

- pentacrinus Wall. $\nabla$. quadrata.

radiato punctatum $A$. $S$.

- secedens A. S.

- - var. quadrata.n r.

- Seychellense Grun.

- solenoceros Eh.

- Stokesianum Grun v. moravica.

$\nabla$. moravica fa parvula $n$.

suborbicularis Pant.

- $\quad$ - $\quad$ v. latilimba n. $\nabla$.

- $\quad$. subcycli. ca n. $\mathbf{r}$.

- Thumii Pant. var.
- $\quad$ trisulcum Bail. $\nabla$. acutiloba n. v.

- - $\quad$ - v. cuneata Bail.

- Weissii Grun. $\quad$. spinosa n. v.

Trinacria palmipes $\mathrm{J} . \mathrm{Br}$.

Truania?

Xanthiopyxis cingulata Eh.

- diaphana Forti. n. sp.

- globosa Eh.

- lacera Forti. n. sp,

- panduræformis Pant.

sp. sp. $a . n$. 
No 370 SHELL BEACH - LEATE'S ISLAND - CONN. (États-Unis)

Achnanthes brevipes Ag.

Actinocyclus crassus W. Sm.

- moniliformis Ralfs.

- nebulosus M. Per.

- Ralfsii var. sparsus.

- sp. Diat. M. de France $118 / 9$.

Actinoptychus areolatus Eh.

$\bar{z}$
$\bar{z}$
$\overline{-}$

racemosus A. S. var.

ranunculus $\mathrm{J}$. Br. var.

tummissus $A$. S.

undulatus Eh.

- var. minor.

- var. à 8 com-

partiments.

Wollei M. Per. n. sp. analogue à $l^{\prime}$ 'A. bifor. mis $J . B r$. mais avec un area circulaire marginale dans les compartiments non épineux; caractérisé surtout par l'arrangement en lignes circulaires des cellules de la couche inférieure.

Amphiprora pulchra var. pulchella.

Amphora angusta Greg.

- arcuata A. S.

- Clevei A. S. oar.

- crassa Greg.

- egregia Eh.

- proteus Greg.

- sp.? A. S. all. $27 / 45$ var.

Auliscus colatus Bail. $v$. latecostata.

- pruinosus Greo. o. zanzibarica.

- rhipis A. S. var.

Biddulphia aurita Bréb.

- rhombus W. Sm.

Campylodiscus Echeneis Eh.

Thuretii Breb.
Cerataulas turgidus $W$. Sm.

Cocconeis pseudomaginata Greg.

- scutellum Eh.

Coscinodisons blandus A. S.

- centralis Eh.

- excentricus Eh.

- heteroporus Eh.

- marginatus Eh.

- nitidus Greg.

- oculus iridis Eh.

- radiatus Eh.

Cyclotella striata Grun.

Denticula antillarum Cl.

Dicladia capreolus Eh.

Dimeregramma costatum Greo. var. rhomboides n. var. -

De forme rhombique. Long. $35 \mu$, larg. $14 \mu$, 3 côtes $1 / 2$ en $10 \mu$.

Diploneis bombus Eh.

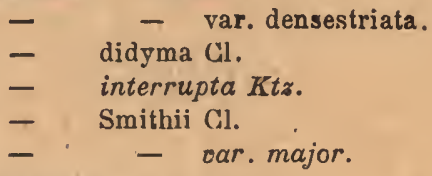

Bncyonema turgidum Grun.

Epithemia amphicephala Grun.

- gibberula Ktz.

- musculus $\mathrm{Ktz}$.

Eunotia diodon $E h$.

- monodon Eh. fa minor.

Hyalodiscus scoticus Grun.

- stelliger Bail.

Lithodesmium undulatum $E h$.

Melosira sulcata $\mathrm{Ktz}$.

- - var. coronata.

- - var. radiata.

Navicula Baileyana Grun. 
Navicula brevis Greg.

- clavata Greg.

- digito radiata Ralfe.

- directa Ralfs.

- Fischeri A. S.

- Fromentarice Cl. oar.

- formosa Greg.

- Fortis Greg.

- Griffithii M. Per. n. sp. De structure analogue à la forme f. 31 A. S. atlas pl. 6, mais de forme elliptique à extrémités fortement rostrées, area central beaucoup plus grand, plus large d'un côté que de l'autre. Long. $75 \mu$, larg. $35 \mu$, 9 stries granulées en $10 \mu$.

- Henredyi W. Sm.

- - var. rostrata n. var. Intermédiaire entre le N. Hennedyi etle $N$. Kittoniana, diffère du $N$. Hennedyi 0 . niceensis par sus extrémités rostrées. Larg. $87 \mu, 8$ stries en $10 \mu$.

- bumerosa Bréb.

- liburnica Grun.

- lyra Eh.

- $\quad$ - fa subtypica.

- - rar. recta.

- peregrina Ktz.

- quadrataera A.S.

- yarrensis Grun.

- sp.? A. S. allas $6 / 31$.

- 8p.? A. S. atlas 6/32.

Nitzschia acuminata Grun.

- circumsula Grun.

- granulata Grun.

- lanceolata W. Sm. var.

- panduriformis Greg.

- sigma W. Sm.

- - var. rigida.

- - var. sigmatella.
Nitzechia Tryblionella var. maxima.

Omphatotheca Jutlandica? var.

Opephora Schwarzii P. P.

Plagiogramma tesselatum Grun.

Plagiotropis seriata Cl.

- vitrea W. Sm.

Pleurosigma affine $\nabla$. Normannii.

- angulatum W. Sm.

- balticum W. Sm.

- naviculaceum Bréb.

Podocystis americana Bail.

Pyxilla baltica Grun.

Rhabdonema adriaticum W. Sm.

- arcuatum Ktz.

Raphoneis amphiceros Eb.

- - var. rhombica

- surirella oar. australis.

Scoliopleura latestriata Greo.

- tumida.

- - var. adriatica.

Stauronels Baileyi Eh.

- Grogoryi Ralfs.

- salina W. Sm.

Stigmaphora capitata Grun.

Surirella Febigerii Lowis.

- fluminensis Grun.

- gemma Eh.

- striatula Turp.

Syndendrium diadema Eh.

Synedra formosa Hantz.

- Gaillonii Ktz.

Trachyneis aspera $\mathrm{Cl}$.

Triceratium alternans Bail.

- antediluvianum Eh.

- favus Eh.

Trinacria regina Heib.

Xanthiopyxis alata Eh.

- aculeata? Eh. 
Achnamthes brevipen Ag.

- danica Flög.

Actinocyclus Ehrenbergii Ralfs.

- moniliformis Ralfs.

- ovalis Grun.

Aetinoptychus undulatus $\mathrm{Eh}$.

- vulgaris Schum.

Amphora crassa Greg.

- proteus Greg.

- - var. oculata.

- sarmiensis Grev. var.

Biddulphia Tuomeyi Rop.

Campylodiscus tæniatus A. S. var.

Chætoceros californicum Eh. (Endocyst).

- Ralfsii? Cl. (Endocyst).

- seiracanthu Cl. (Endocyst).

Coceonels costata var. A. S. atl. $189 / 8$.

- heteroidea Hantz.

- scutellum Eh.

Coscinodiscus marginatus Eh.

oculus-iridis Eh.

Dletyoneis marginata $\mathrm{Cl}$.

Dlploneis didyma $\mathrm{Eh}$.

- elliptica Ktz.

- Smithii Brèb.

Gephyria media Arn.

Grammatophora angulosa Eh.

-
Grammatophora oceanica fa commuris.

$$
\text { - robusta Dipp. }
$$

Hyalodiscus laevis Eh.

- scoticus Grun.

Mastoglola Braunii Grun.

- quinquecostata Grun.

Molosira sulcata Eh.

- - var. coronata.

- - var. radiata.

- Westii W. Sm.

Navicula abrupta Donk.

- Fischeri A. S.

- Hennedyii W. Sm.

- - var. granúlata.

- Kamorthensis Grun.

- lyra Eh. var. connectens.

Nitzschia littoralis Grun var.

- punctata Grun $\nabla$. olongata.

Orthoneis splendida Eh.

Plagiogramma Gregoryanum Greo.

Rhabdonema adriaticum W. Sm.

Raphoneis amphiceros Eh. var. rhombica.

- gemmifera Eh. var. elegans.

Rhizosolenia barbatula Eh.

- pileolus Cl.

Stephanogonia actinoptychus $E h$.

Burirelia fastuosa $\mathrm{Eh}$. oar.

- striatula Turp.

Van Heurckia Martoufli Pant.

Xanthiopyxis aculeata Eh.

No 372 HIGH POND-PICTON Co NOVA SCOTIA

(Canada)

Amphora ooalis $K t z$. fa minor.

I Cyclotella comta Kts. 
Cyclotella Temperil F. Herib. rar.

Eneyonema gracile Rab.

\section{- Innula Grua.}

Bunotla arcus Eh.

- - var. hybrida.

- bidentula W. Sm.

- fiezuosa Bréb. var.

- - pachycephala.

- incisa Greg.

- lunaris Grun.

- - $\quad$ v. bilunaris.

- major Rab.

- pectinalis Rab.

- $\quad$ v. undulata.

- - - v. ventricosa.

- robusta Ralfs, var.

- - v. tetraodon..

- - - - hexaodon.

- - v. polyodon.

Comphonema capitatum Eh.

gracile var. A. S. atlas 236/31. parvulum $\mathrm{Ktz}$.

Navienla affinis Eh.

- amphigomphus Eh.

- Brebissonii Ktz

- cuspidata $K t$ s.

- dilatata Eh.

- divergens W. Sm.

- dubia Greg.

- firma Ktz.

- - var. subundulata.

- gibba Eh.

- gigas Ktz.

- iridis Eh.
Naricala macilenta Eh.

- mesolepta Eh.

- - oar. stauroneiformis

- Paulonsis Grun.

- serians Ktz.

- - v. minor.

- subsolaris Grun.

- viridis $\mathrm{Ktz}$. v. fallax.

Pseudo-Eunotia hemicyclus Grun.

Stauronels anceps. Eh.

- $\quad$ Baileyi Eh.
- $\quad$ gracilis. Eh.
- $\quad$ Phœnicenteron Eh.
- $\quad$ Stodderi Greenl.var. superbo n. var. - Area stauroneiforme large et évasèe ver les bords sur laquelle on aperçoit les côtes longitu. dinales. Long. 90 à $125 \mu$, 9 côtes longitudinales, 15 stries transversales.

Stenopterobia ancepe Bréb.

Gurirella Baileyi Laevis.

- bifrons $\mathrm{Ktz}$. v. A. S. atl. $23 / 2$.

- cruciata A. S. var.

- linearis W. Sm. V. constricta.

- oblonga Eh.

- saxonica Auers.

- tenera Greg.

- Thuringiaca Hantz.

Tabollaria fenestrata $K t \boldsymbol{s}$.

Van lleurckia rhomboïdes Brỏb. - ra major.

\section{N• 373 SWAMPS POND-PICTON Co NOVA SCOTIA}

\section{(Canada)}

Amphora ooalls $K t z$.

Cocconeis placentula Eh.

Cymbella cistula Kirch. - cuspidata Ktz.

Diplonels Smithii Bréb.
Eunotia gracilis Rab.

- incisa Greg.

- major Rab.

- monodon Eh.

- pentaglyphis Eh. 
Bunotia robusta Ralfs. v. tetraodon.

- - var. octogon.

- - var. polyodon.

Molosira granulata Ralfs.

Navicuia amphigomphus Eh.

- bisulcata Lag.

- columnaris Eh.

- conspicua A. S.

- dactylus Ktz.

- dilatata.Eh.

- eximia $\mathrm{Cl}$.

- gibba Ktz.

- gigas kitz.

- iridis Eh. var. ampliata.

- legumen Eh.

- limosa Ktz. var. gibberula,
Navicula macilenta Eh.

- major $\mathrm{Ktz}$.

- - oar. asymetrica.

- - oar. subacuta.

- nobilis $\mathrm{Ktz}$.

- Paulensis Grun.

- serians Eh.

- subacuta fa minor.

- transversa A.S.

Pleurostauron acuta W. Sm. var. maxima.

Stauroneis Balleyi Eh.

- gracilis Eh.

Surirelia bifrons Ktz. phœnicenteron Eh.

- saxonica Auers.

\section{N 374 GRANT LAKE-PICTON Co NOVA SCOTIA}

\section{(Canada)}

Achnanthidium flexellum Bréb.

Amphora libyca Eh. var.

Cocconeis placentula Eih.

Cymbella acutiuscula $\mathrm{Cl}$.

- capitata M. Per, et F. H. Her.

- cistula Kirch.

- - var. crassa.

- cuspidata Ktz.

- $\quad$ fo major (Longueur $120 \mu)$.

- cymbiformis Breb.

- Ehrenbergii Ktz.

- - var. crassa $n$. var. Plus courte ot à extrémités plus rostrées tronquées que celle figurée dans $\mathrm{A}$. S. atlas $9 / 7$

- gaströdes Ktz.

- hetoropleura Ralis.

- naviculoeformis Auers.

Diploneis elliptica Ktz.

- Smithii Bréb.

Bncyonema ventricosum Grun.
Epithemia turgida Ktz.

$$
\begin{aligned}
& \text { - } \quad \text { - var. granulata. } \\
& \text { - var. porcellus fo ex- } \\
& \text { - cavata. } \\
& \text { - } \quad \text { ebra Ktz. }
\end{aligned}
$$

Eunotia arcus Eh.

- diodon Eh.

- impressa Eh.

- incisa Greg.

- major Rab.

- minor V. H.

- monodon Eh.

- - $\mathrm{f}_{\mathrm{a}}$ curta.

- obtusiuscula (incisa v.) Grun.

- pectinalis var. venter.

- robusta Ralfs var. tetraodon.

- - var. polyodon.

Gomphonema acuminatum Eh.

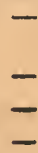

var. intermedia. var. pusilla. constrictum Eh. intricatum $\mathbf{K} t z$. 
Comphonema montanum var. succisa.

Melosira crenulata $K t z$.

- granulata Ralfs.

Navicula affinis Eh.

- - var. amphirynchus.

- americana Eh. v. bacillaris.

- amphirynchus Eh.

- - f curta.

- bacilliformis Grun.

- Braunii Grun.

- cardinalis Cl.

- commutata Grun.

- costata Eh.

- cuspidata Ktz.

- dactylus Ktz.

- divergens W. Sm.

- esox Eh.

- firma Ktz.

- flexuosa Cl.

- follis Eh.

- gibba Ktz.

- gigas Ktz.

- hemiptera Ktz.

- Hitchcockil Eh.

- iridis Eh.

- - var. ampliata.

- Legendrei F. H. et M. Per.

- legumen Eh.

- limosa Ktz.

- major Ktz.

- - var. asymetrica.
Navicala mesolepta Eh.

- - var. stauroneiformis.

- mesotyla Eh. var.

- microstauron O'Mea.

- nobilis Ktz.

- occidentalis Cl.

- pachyptera Eh.

- parva Eh.

- Paulensis Grun.

- peregrina Ktz.

- pseudobacillum Grun.

- radiosa $\mathrm{Ktz}$.

- streptoraphe Cl.

- tabellaria Ktz. var. stauroneiformis.

- transversa A. S.

- ventricosa $\mathrm{Ktz}$.

- viridis $\mathrm{Ktz}$.

Nitzschia scolaris IV. Sm.

- sigmoidea W. Sm.

Pleurostauron acuta var. maxima.

Stauroneis amphilepta Eh.

- anceps Eh.

- - var. amphicephala.

- Baileyi Eh.

- gracilis Eh.

- Phonicenteron Eh.

Surirella cardinalis Kitt.

- elegans Eh.

- robusta Eh.

Tabeliaria fenestrata Ktz.

No 375 YAGIANA BAY - OREGON (Etats-Unis)

Amphora angusta $\mathrm{Eh}$. var.

Cocconeis scutellum Eh.

Epithemia musculus W. Sm.

Melosira Borreri Greg.

- mummuloïdes Ag.

Navicula didyma $\mathrm{Ktz}$.

- humerosa Bréb.

Nitzschla longissima Ralfs.
Nitzschia sigmatella Greg.

Pleurosigma angulatum W. Sm.

$$
\begin{array}{ll}
\text { - } & \text { balticum W. Sm. } \\
\text { - } & \text { formotum W. Sm. } \\
\text { - } & \text { latum Gl. var. } \\
\text { - } & \text { strigosum W. Sm. }
\end{array}
$$

Synedra affinis Ktz. 


\title{
Nor 376-378 MORON - PROVINCE DE SÉVILLE
}

\author{
(Espagne)
}

\section{Dépots fossiles marins}

Nous donnons ici la liste à peu près complète des espèces qui se rencontrent non seulement dans les $n^{\text {os }} 376$ à 378 qui proviennent du gisement voisin de la gare de Moron même, mais aussi dans ceux qui suivent et qui en diffèrent notamment.

Actinocyclu: Ehrenbergii Ralfs.

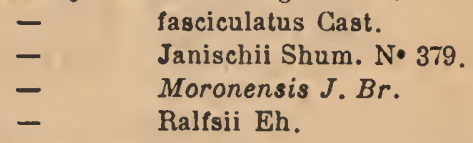

Actinoptychus amblyoceros A. S. N• 378.

- ellipticus Grun. N•378.

- interpositus J. Br.

- undulatus Eh.

Arachnoidiscus ornatus Eh.

Aetorolampra affinis.

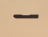

$\overline{-}$

Dallasiana Greo.

$-$ Grevilloi Wall. No 378.

\section{Grun.}

Marylandica Eh.

Aéteromphalus Moronensis Grev.

Aulacodiscus Greoilleanus Norm.

Aullecue cœlatus var. Bail.

- - - var. constricta.

- - var. major.

- - var. mergens Rett.

- incertus A. S.

- Moronensis Greo.

- Normanianus Greo.

- sublceois Grun.

Biddulphla Johnsoniana Greo.

- Tuomeyi Bail.

Calonel Kinkeriana Truan.

Cestodiscus Johnsoniana Greo. N· 382.

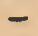

ovalis Grev. - Stokesianus Grev.

Cocconeis dirupta. Greg. var. californica $\mathrm{Cl}$.

- Grevillei W. Sm. N॰382.

- Moronensis A. S.

- oculus cati J. Br.

- pseudo-marginata Greg.N॰382.

- regalis Grer.

- oenusta A. S. N• 384.

Cosclnodiscus apiculatus $\mathrm{Eh}$.

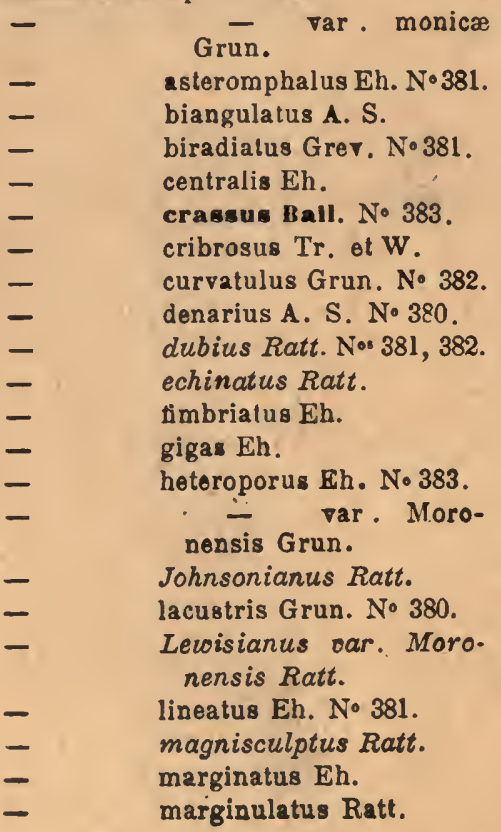


Coscinodiscus marginulatus $v$, curvatostriata Grun.

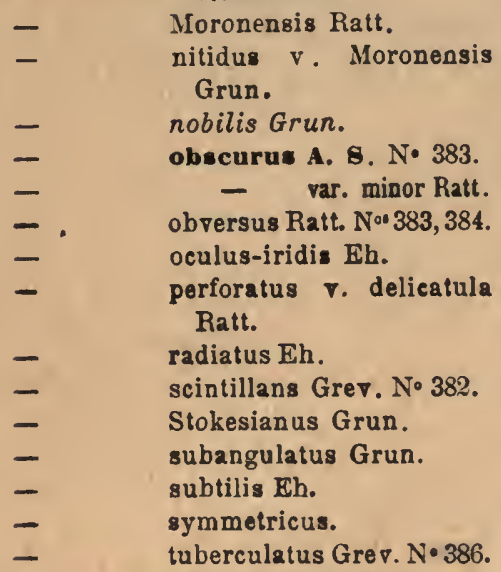

Craspedodiscus coscinodiscus Eh. N• 385 .

Entopyla australis Eh.

Euodia gibba Bail.

- - var. Moronensis Temp. et Per. Nor 378, 379.

Fragilaria antarctica Cast. o. moronensis Truan. No1 376, 378.

Gephyria media Arnott. N• 377.
Grammatophora Moronensis Greo. - robusta Dippel.

Mastogonia actinoptychus Eh. N॰ 378 .

Navicula crabro $E h$.

- elliptica W. Sm. No 379.

- expedita A.S.

- lyra Eh.

- prætexta Eh. N. 383.

Omphalopelta Moronensis Greo.

Periptera tetracladia Eh. N• 380 .

Pleurosigma affine v. fossilis Grun.

Radiopalma dichotoma J. $\mathrm{Br}$.

Raphoneis nitida. Grun.

- surirella. Eh. No 378.

Stephanopyxis corona Eh. N• 379.

stictodiacus californicus $v$. areolata.

Surirella Neumayeri Janisch.

Triceratium cinnamoneum Grev. N• 376.

- guttatum $\mathrm{Cl}$.

- lobatum Greo.

- Moronensis Greo.

- nicobaricum var. Grun.

- parallelum.

- $\quad$ quinquelobatum Greo.

- Stokesianum Greo.

\section{No 379 et 380 CORTIGO DE JALAPA - MORON (Espagne)}

N* 381 et 382 TIERRA DEL SALADO - MORON (Espagne) 
$\mathrm{N}^{\circ} 385$ et 386 TIERRA DE PASADA ALTA - MORON (Espagne)

Nor 387 et 388 ROUILHAS BAS - PUY-DE-DOME (France)

1)épot fossile d'eau douce

\section{BIBliographie. - Frère J. Héribaud. Les Diatomées d'Auvergne 1893}

Achnanthes lanceolata Grev.

Amphora affinis $\mathrm{Ktz}$.

- ovalis $\mathrm{Ktz}$.

- pediculus Grun.

Cocconeis placentula Ehr.

Cyclotella comta v. arverna M. P. et F. H. Cymatopleura solea Bréb.

Cymbella affinis $\mathrm{Ktz}$.

- amphicephala Mæg.

- 2nglica Lag.

- aspera Ehr.

- cistula Hemp.

- cuspidata $\mathrm{Ktz}$.

- cymbiformis Ehr.

- gastroides 0. minor Kt.

- lanceolata oar. Ehr.

- naviculiformis Auersw.

Encyonema ventricosum Ktz.

Epithemiagibba v. parallela Grun.

- - v. ventricosa Grun.

- turgida v. granulata Grun.

- zebra Ktz.

- - v. longissima M. P. ot F. H.

Eunotia arcus Eh.

- - ₹. bifrons Grun.

- impressa v. angusta Grun.

- gracilis V. major M. P. ot F. H.

- monodor Ehr.
Fragilarla capucina Desm. .

- construens $\nabla$. genuina Grun.

- elliptica Schum.

- parasitica Grun.

- virescens Ralfs.

Gomphonema acuminatum o. coronata Eh.

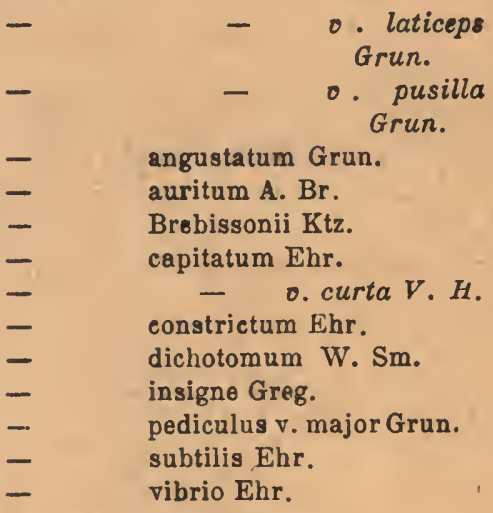

Hantzschia amphioxys Grun.

Melosira crenulata $\vee$ ambigua.

- Róseana Moor.

Meridion circulare Ag.

Navicula ambigua Ehr.

- ampliata Ehr.

- amphigomphus Ehr. 
Naricula bicapitata Lag.

- borealis $\mathrm{Ktz}$.

- columnaris Ehr.

- cuspidata Ktz.

- dactylus Ehr.

- decurrens Ehr.

- dilatata Ehr.

- firma Ktz.

- elliptica Ktz.

- esox Ehr.

- gastrum Donk.

- gentilis Donk.

- lævissima Grun.

- lata o. minor Bréb.

- legumen v. vix. undulata V. H.

- limosa Ktz.

- mijor Ktz.

- mesolepta v. stauroneiformis Grun.

- nobilis Ehr.

- pupula $\nabla$. minuta Ktz.

- radiosa Ktz.

- - - acuta Grun.

- rupestris $\mathrm{Ktz}$.
Navicula stauroptera Grun.

- termes $\nabla$. stauroneiformis Ehr.

- viridis $\mathrm{K} t z$.

- - v. commutata Grun.

Nitzschia Brebissonii $W$. Sm.

Btauroneis anceps v. hyalina $M$. P.et $\mathrm{J} . \mathrm{Br}$.

- gracilis W. Sm.

- Phœnicenteron Ehr.

- - $\quad$ . crassa M. P. et F. H.

- $\quad$ - $\quad$ v. gracilis J.

J. Br. et F. H.

v. lanceolata fa major J. $\mathrm{Br}$.

Bynedra capitata Ehr.

- ulna v. longissima W. Sm.

Burirella biseriata Bréb.

- elegans Eh.

Tabellarla fenestrata Ktz.

- flocculosa Ktz.

$N^{\circ s} 389$ et 390

ELESD (Hongrie)

Bibliographie. - J. Pantocseck. Diatomés de Hongrie, volume 1

Achnauthes brevipes $\mathbf{A g}$.

Actinocyclus circumdatus Pant.

$$
\text { - Ebreńbergii Ralfs. }
$$$$
\text { - subtilis Ralfs. }
$$

Actinoptychus kymatodes Pant.

$$
\begin{aligned}
& \text { - } \\
& \text { - } \\
& \text { splendens Ralfs. } \\
& \text { - } \\
& \text { Grun. } \\
& \text { undulatus Eh. balionyx }
\end{aligned}
$$

Amphora granulata Greg.

$$
\text { - monilifera Greg. }
$$

Anaulus mediterraneus Grun.

Arachnoldiscus Ehrenbergii Bail.
Asterolampra Marylandica Eh.

Auliscus sculptus Ralfs.

Biddulphia elegantula Grev.

- pulchella Greg.

- Regina W. Sm.

- Tuomeyi Bail.

Campylodiscus adriaticus Grun.

- clypous Eh.

- Dæmelianus Grun.

- ecclesianus Grev.

- Echeneis Eh.

- hibernicus Eh.

- striolatus Grun.

- Thuretii $\nabla .$, Baldjikiana Grun. 
Clavicula biharensis Pant.

Cocconeis pellucida Grun.

- pseudo marginata Greg.

Coscinodiscus apiculatus Eh.

$\begin{array}{ll}\text { - } & \text { asteromphalus Eh. } \\ \text { - } & \text { biharensis Pant. } \\ \text { - } & \text { magans Grev. } \\ \text { - } & \text { marginatus Eh. } \\ \text { - } & \text { Martoufli Pant. } \\ \text { - } & \text { oculus iridis Eh. } \\ \text { - } & \text { radiatus Eh. } \\ \text { - } & \text { robustus Grev. } \\ \text { - } & \quad-\quad \text { v.latemarginata }\end{array}$

Endictya minor A. S.

Epithemia biharensis Pant.

- gibberula Ktz.

Grammatophora maxima Grun.

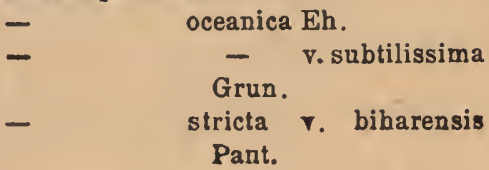

Hyalodiscus radiatus Grun.

- . $\quad$ - v. biharensis Pant - subtilis $\nabla$, australiensis.

Leudugeria epithemoides Temp.

Melosira biharensis Pant.

- clavigera Grun.

- granulata Ralfs.

- nummuloides v. Elesdiana.

- Omma Gl.

- sol Ktz.

Navicula crabro Eh.

- didyma Eh.

- Elesdiana Pant.

- ercipata Grev.

- fusca Ralfs.

- halionata Pant.

- Hennedyi W. Sm.

- humerosa Bréb.

- latissima Greg.
Navicula lyra Eh.

- - - connectens Grun.

- nebulosa Greg.

- pseudofusca Pant.

- Sandriana Grun.

- Smithii Bréb.

- yarrensis Grun.

Nitzschia antiqua Pant.

- Tryblionella Hantz $\nabla$.

- biharensis Pant.

Orthonels splendida Grun.

Paralia sulcata $\mathrm{Gl}$.

Plagiogramma biharensis Pant.

Pleurosigma balticum W. Sm.

Pyxidicula cruciata Eh.

Rhabdonema adriaticum $\mathrm{K} t z$.

Stephanodiscus Kanitzii Pant. of Grun.

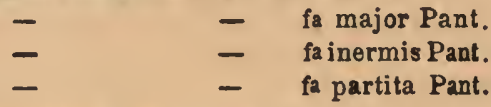

Stephanopyxis corona Grun.

- turris Eh.

stictodiscu calicornicus Grer.

Surirella antiqua Pant.

- biharensis Pant.

- rotunda Pant.

- striatula Turp.

Synedra Hennedyana Greg.

Terpsinoë americana Ralfs.

\section{- - fa trigona Pant. et Grun . \\ - intermedia Grun.}

Triceratium balearicum Cl. et Grun.

- favus Eh.

- Grovei Pant.

- lætum Pant.

- polygibbum Pant.

Xanthlopyxis cingulata Eh.

- oblonga Eh.

N॰ 391 APPLEBY - WESTMORLAND N० 2

Achnanthes minutissima $\mathrm{Ktz}$. Coratonels arcus Ktz.
Gomphonema angustatum Ktz. gracile $\mathbf{E b}$. 
Gomphonema gracile var, aurita .

- rhombicum A. S.

- subclavatum Grun.

Navicula radiosa $\mathrm{Ktz}$.
Odontidium mesodon $\mathrm{K} t \mathrm{z}$.

Surirella minuta Bréb.

Tabellaria flocculosa $\mathrm{Ktz}$.

Tetracyclus lacustris Ralfs.

No 392

\section{VALLEE DE TOLUCA (Mexique)}

\section{1)épôt fossile d'eau douce}

Achnanthes lanceolata.

Cocconois placentula Eh.

Cymbella cistula Hemp.

Fpithemia turgida $\mathrm{Ktz}$.

$$
\text { - zebra Ehr. }
$$

Eunotia eruca Grov.

$$
\text { - major Rab. }
$$

Fragilarla construens Ehr.

Comphonema affine $\mathrm{K} t z$.

$\begin{array}{ll}\text { - } & \text { imbricatum Ktz. } \\ \text { - } & \text { gracile Eh. } \\ \text { - } & \text { parvulum Ktz. } \\ \text { - vibrio Eh. }\end{array}$

Melosira crenulata $\mathrm{K} t z$.

Navicula lanceolata $\mathrm{Ktz}$.

- pupula Ktz.

Neillum iridis Eh. var.

Pinnularia dactylus Eh.

- hemiptera $\mathrm{Ktz}$.

- nobilis Eh.

- subcapitata Greg.

Stauroneis Phonicenteron Eh.

Synedra ulna Eh.

Tabellaria fenestrata Ktz.

Tetracyclus emarginatus Eh.

No 393

ILE MAURICE (Mauritius)

Lavage de coquilles

Actinocyclus Ralfsii Bail. et var.

Actinoptychus heterostrophus A. S. var.

- splendens Ralfs.

$$
\text { - undulatus Eh. }
$$

Amphora lævis Grog.

Amphiprora lepidoptera Greg.

Arachnoidiscus ornatus Eh.

Auliscus cœlatus Bail.

Biddulphia obtusa $\mathrm{Ktz}$. pulchella Graz.
Campylodiscus ambignus Grov.

$\begin{array}{ll}- & \text { Heufleri Grun. } \\ \text { - } & \text { ornatus Grev. } \\ \text { - } & \text { Ralfsii W. Sm. } \\ \text { - } & \text { samcensis Grev. }\end{array}$

Climacosphenia monilifera Eh.

Cocconeis heteroidea Hantz.

Cuscinodiscus apiculata Eh. denarius A. S. 
Coseinodiscus lineatus Eh.

$\begin{array}{ll}- & \text { nitidus Greg. } \\ - & \text { oculus-iridis Eh. } \\ \text { - } & \text { radiatus Eh. } \\ \text { - } & \text { subtilis Eh. }\end{array}$

Craspedodiscus coscinodiscus Eh.

Melosira sulcata $\mathrm{Ktz}$.

Naricula apis Donk.

- aspera Eh.

- braziliensis Grun. var.

- bomboïdes A. S.

- gemmatula Grun.

- Graeffi Grun.

- Kamorthensis Grov. var.

- lyra Eh. et var.

- multicostata Grun.

- nitescens Greg.

- notabilis Greg.

- pandura Bréb.

- splendida Greg.

Nitzschia Graeffii Grun.

- panduriformis Greg.

thoneis splendida Grun.
Pollocystis adriatica Ktz.

Podosira ambigua Grun.

Plagiodiscus Martensianus Grun.

Pleurosigma affine Grun.

$\begin{array}{ll}\text { - } & \text { decorum W.Sm.. } \\ \text { - } & \text { delicatulum W.Sm. } \\ \text { - } & \text { rigidum W. Sm. var. }\end{array}$

Rhabdonema adriaticum Eh.

- $\quad$ mirificum W. Sm.

Rhoïcosigma compactum Grun.

- oceanicum H. P.

- Weissflogii Grun.

Stephanopyxis corona Eh.

Surirella hybrida Grun.

- fastuosa Eh.

Syuedra formosa Hantz.

Triceratium arcticum Bright.

- v. pentagonalis.

favus Eh.

quinquelobatum Grev. zonulatum Grev.

\section{No 394 MACLEAN'S POND-PICTON Co (Canada)}

Amphora libyca Eh. var.

$$
\text { - ovalis } K t z \text {. }
$$

Cocconeis lineata Eh.

Cyclotella Temperei F. H. et M. Per.

Cymbella capitata M. Per. et F. H.

- cistula Kirch.

- cuspidata Ktz.

- Ehrenbergii Ktz.

- gastroides $\mathrm{Ktz}$.

- heteropleura Ktz.

- maculata Ktz.

Diploneis elliptica Ktz.

- Smithii Brẻb.

Epithemia amphicephala Grun.

- gibba Ktz.

- turgida Ktz.

- - var. granulata.
Epithemia zebra Ktz.

Eunotla lunaris Grun.

- major Rab.

- monodon Ell.

Gomphonema acuminatum Eh.

$$
\begin{aligned}
& \text { - } \quad \text { - var. pusilla. } \\
& \text { - - var. trigono- } \\
& \text { cephala. } \\
& \text { - augur Eh. } \\
& \text { - capitatum Eh. } \\
& \text { - constrictum Eh. } \\
& \text { - montanum Schum. var. }
\end{aligned}
$$

Melosira tenuis Ktz.

Navicula acrospheria Bréb. var. dilatata.

- americana Eh. o. bacillaris.

- amphirhynchus Eh. 
vavićnla amphisbæna Borz.

- bacilliformis Grun.

- Braunii Grun.

- cardinalicus Cl.

- columnaris Eh.

- cuspidata Ktz.

- dactylus Ktz.

- dicephala Eh. var.

- dilatata Eh.

- divergens W. Sm.

- firma Ktz.

- - var. subampliata.

- flexuosa Cl.

- gentilis Donk.

- gibba Ktz.

- hemiptera Ktz.

- Hitchcockii Eh.

- iridis Eh. var. ampliata.

- legumen Ktz.

- limosa Ktz.

- macilenta Eh.

- major Ktz.

- - var. asymetrica.

- mesolepta Eh.

- - var. stauroneiformis.

- mesotyla Eh.
Navicula microstauron O'Mea.

- nobilis $\mathrm{Ktz}$.

- producta W. Sm. var.

- popula $\mathrm{Ktz}$.

- radiosa $\mathrm{Ktz}$.

- stauroptera Grun.

- streptoraphe $\mathrm{Cl}$.

- tabellaria Ktz.

- transversa A. S.

- ventricosa $\mathrm{Ktz}$.

- viridis $\mathrm{Ktz}$.

- - var. fallax.

Pleurostauron acuta W. Sm.

Stauroneis amphilepta $E$.

- anceps Eh.

- - var. amphicephala.

- Baileyi Eh.

- gracilis Eh.

- phonicenteron Eh.

- $\quad$ - fa maxima.

Surirella elegans $W . S m$.

- robusta Eh.

- tenera Greg.

- - car.neroosa.

Tabellaria fenestrata Ktz.
Cyclotella Terryana Temp. et M. Per.

$$
\text { - - var. major. }
$$

Cymatopleura apiculata W. Sm.

$$
\text { - solea W. Sm. }
$$

Cymbella cistula Kirch.

- gastroïdes $\mathrm{Ktz}$.

Bncyonema Mac Kayiana Temp. et M. Per. n. sp. Semblable à la formereprésentée dans I'atlas A. S. pl. 10 fig. 49, 50, mais à partie centrale beaucoup plus développée, à stries moins radiantes et fortement granulees $2 u$ nombre de 7 au dos, 8 au ventre en $10 \mu$. Long. $60 \mu$, largeur $20 \mu$.

\section{Eunotia gracilis Rab.}

Gomphonema constrictum $\mathrm{Eh}$.

Navicula acrosphaeria Bréb.

- Braunii Grun.

- cuspidata Ktz.

- Duriana A. .

- esox Ktz.

- firma var. subampliata.

- interrupta W. Sm.

- logumen Ktz.

- limosa Ktz.

- macilenta Eh. 
Navicula major $K t z$.

- - var. asymetrica.

- Paulensis Grun.

- polyonca Bréb.

- tabellaria Ktz. var. acros. sphæriaformis n. var - Extrémités aussi larges que le milieu; area axial large jus. qu'aux extrémités qui sont semblables à Nav. acrosphoeria.

- transversa A. 8 .

- trigonocephala $\mathrm{Cl}$.

- viridis $\mathrm{Ktz}$.

Nitzschla sigmoidea $W$. Sm.

- spectabilis W. Sm.

stauroneis anceps El. var. elongata $\mathbf{n}$. var. Encore plus long et plus élancé que la var. hirostris, moins finement striè. Long. $145 \mu$, larg.

$15 \mu, 18$ stries en $10 \mu$.

Stauroneis Baileyi Eh.

- gracilis El.

- Plœenicenteron Eh. - $\quad$ var. lanceo-

- phyllodes Eh. var. elegans n. var. Plus allongé que le type, porte parfois un granule central au milieu du nodulemédian.Long. $150 \mu$, largeur $30 \mu, 13$ à 15 stries en $10 \mu$.

Surirella cardinalis Kitt.

- elegans Bh.

- Kittonii A. S. var.

- saxonica Auers.

- tenera Grey.

Synedra biceps Ktz.
Achnanthes delicatula $\mathrm{K} t z$.

- subsessilis $\mathrm{ktz}$.

Amphora acutiuscula Ktz.

- turgida Greg.

Berkeleya rutilans var. Dillwynii.

Cocconeis molesta $\mathrm{Ktz}$.

Melosira nummuloides Ag.

Navicula gregoria Donk.

- subsalina Donk. var. fuscata.
Nitzschia acuminata W. Sm. var. Per. Diat. de France 70/21.

Rhoicosphenia curvata var. marina .

Schizenema comoides Ag.

- neglectum Thw.

Synedra affinis Ktz. var, gracilis.

- var. tabulata.

- pulchella Ktz.

\section{N• 397 OLYMPIA - PUJET SOUND (États-Unis)}

Actinocyclus nebulosus $M$. Per.

Actinoptychus ternariu's Jan. (valves primaires A. undulatus).

$$
\text { - } \quad \text { undulatus Eh. }
$$

Amphiprora alata $\mathrm{Ktz}$.

$$
\text { - } \quad \text { - fa minor. }
$$

Biddulphia aurita Bréb.

- longicruris Grun var. 
Coscinodiscus excentricus Eh.

$$
\text { - } \quad \text { leptopus Grun }
$$

Diploneis didyma $E h$.

- interrupta Ktz.

Navicula cyprinus W. Sm.

- digito radiata Grun.

- directa W. Sm.

- formosa Greg.

- liber W. Sm.

- pennata A.S.

- rostellata Ktz.

Nitzschia acuminata Grun.

- bilobata W. Sm.

- - var. minor.
Nitzschia sigma $W$. Sm.

- - v. Habirshawii.

- - var. rigida.

- Tryblionella var, victoriæ.

Pleuroslgma balticum W. Sm.

- $\quad$ - var. maxima.

- elongatum oar. Balearica.

- Olympianum Terry.

- strigosum W. Sm.

Scoliopleura tumida var. adriatica.

Scoliotropis latestriata Gl.

Stauroneis Gregorii Ralfs.

Surirella gemma Eh.

Trachyneis aspera Eh.

Van lleurckia interposita Lewis.
Achnanthes breoipes $A g$. var.

maiulata n. var. Présente un area blanc à chaque extrémité de la valve supérieure et du même côtś du pseudo-raphé no 398.

Actinocyclus subtilis Ralfs.

Biddulphia pulchella Graz.

$$
\text { - reticulata Rop. }
$$

Campylodiscus ambigua Greo. no 399.

Climacosphenia moniligera Eh. no 399 .

Cocconels pellucida $\mathrm{Ktz}$. var. minor

- pseudo-marginata var. Ste-

vensci n. var. - Le raphé

s'épanouit vers le nodule central et donne sous un faiblegrossissement l'aspect du navicula hyalina $n^{\circ} 398$. - scutellum Eh.
Coscinodiscus lineatus $E h$.

Grammatophora marina Ktz.

- var. macilenta.

Licmophora Lyngbyei Grun.

Mastogloia quinquecostata Grun. n॰398.

Melosira sulcata $K t z$.

Navicula cancellata Donk. n० 399 .

$$
\text { - - var. Gregorii. }
$$

Orthoneis binotata Grun n 398 .

$$
\text { - fimbriata Grun no } 398 .
$$

Podosira Montagnei litz. nº 399

Stephanodiscus astraea Grun. var. spinulosa.

Synedra formosa Hantz. no 399.

Trachyneiw aspera $\mathrm{Cl}$.

- $\quad$ sp. A. S. atlas $48 / 30$. 


\section{N॰400 NEUSSARGUES - PUY-DE-DOME (France)}

\section{Récolte d'eau douice}

Achnanthes Hauckiana Grun. subsessilis $\mathrm{Ktz}$.

Amphiprora paludosa $W$. Sm.

Amphora pediculus fa major.

Cocconeis pediculus Eh.

- placentula Eh.

Cyclotella Kützingiana.

Diatoma tenue $\mathrm{Ag}$.

- - var. elongata.

- - var. vulgare v. minor.

Gomphonema olivaceum Ktz.

Melosira Borreri Grev, var.

- varians.
Navicula ambigua $E h$.

- menisculus Schum.

- viridula Ktz. var. hungarica.

Nitzschia fonticola Grun.

- Hungarica Grun.

- Tryblionella Hantz.

Pleurosigma acuminatum Grun.

Rhoicosphenia curvatum Grun.

Stauroneis Phcenicenteron Eh.

Surirella Brightwellii W. Sm.

- crumena Bréb.

- ovata Ktz.

- - var. suevica.

Synedra affinis Kitz. var. tabulata.

\section{$N^{\text {os }} 401$ et 402 HONOLULU - ILES SANDWICH}

\section{Lavage de coquilles}

Aotinoptychus intermedius A. S. oar. $8 p$ ? Intermédiaire entre l'A. Grunowii et l'A. hexagonus var. tenella.

Amphora arenicola Gruin. var. major.

- cymbifera Greg.

- farcimen Grun.

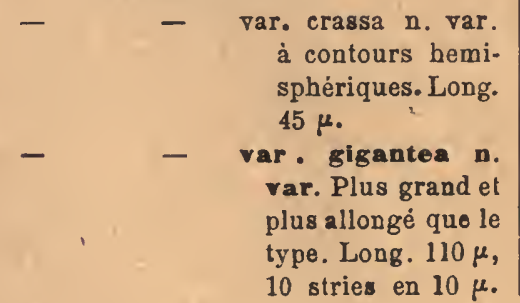

Amphora gigantea Grun o. fusca.

- inflata Grun.

- Javanica A. S.

- Schleinitzii Jan. var.

- spectabilis Greg. oar.

- obtusa Greg. var.

- turgida Greg.

- sp? A. S. atl. $27 / 61$.

Anorthoneis maculata M. Per. n. sp. Largement elliptique, stries marginales laissant un area très développé, dans lequel le raphé, droit, excentrique, à pores medians éloignés est accompagné, du côté de l'area, le plus 
grand, de deux macules. Long. $20 \mu$, larg. $14 \mu$, 16 stries en $10 \mu$.

Aulacodiscus orientalis Grev.

Biddulphia pulchelia Gray. - reticulata Roper.

Campylodiscus Kinkerii A. S. var.

$\begin{array}{ll}\text { - } & \text { Samoensis Grun. } \\ \text { - } & \text { singularis A. S. } \\ \text { - } & \text { triumphans A.S. } \\ \text { - } & \text { undulatus Grev. var. }\end{array}$

Climacosira mirifica Grun.

Climacosphoenia moniligera Eh.

Cocconeis gibbo calyx $\mathrm{J}$. Br.

- heteroidea Hantz.

Coscinodiscus curvatulus Grun.

\begin{tabular}{ll} 
- & \multicolumn{1}{c}{$\begin{array}{c}\text { var. latius- } \\
\text { triata. }\end{array}$} \\
- & $\begin{array}{l}\text { decrescens Grun. } \\
\text { bustior. }\end{array}$ \\
- & Kützingii A. $S$. \\
- & leptopus Grun. \\
- & marginatus Eh. \\
- & nitidulus Grun. \\
- & nitidus Grun. \\
- & symbolophorus Grun.
\end{tabular}

Diploneis cynthia A. S.

- crabro Eh. v. separabilis.

- fusca Ralfs.

- Mauleri J. Br. var.

- mediterranea $\mathrm{Ktz}$. var. elliptica n. var. Longuement elliptique, forme de passage au D. cynthia.

- nitescens Ralfs.

- notabilis Grun.

- - Var. expleta.

- Smithil Bréb. var.

- splendida Greg.

- vetula A. S.

- sp.? A. S. atl. 8/14.

Endictya oceanica Eh.

Epithemla gibberula Ktz.

- hirundinella J. Br.

- musculus Ktz.

Eunotia cistula var. lunaris.

- formica Eh.

- major Rab.
Grammatophora caribaea $\mathrm{Cl}$.

- oceanica Eh.

- - var. macilenta.

Hyalodiscus radiatus Grun. fa minor.

Mastogloia Portieriana Grun.

Melosira sulcata Ktz. v. strigillata.

Navicuia cancellata Donk.

$$
\begin{aligned}
& \text { - } \quad \text { var. retusa. } \\
& \text { - } \quad \text { Hennedyi W. Sm. } \\
& \text { - liochstetteri Grun. } \\
& \text { - } \quad \text { var. } \\
& \text { - liber W. Sm. } \\
& \text { - maxima Greg. var. exentrica. } \\
& \text { - Ny Cl. var. } \\
& \text { - pennata A. S. } \\
& \text { - perplexa? var. minutissima n. } \\
& \text { var. Semblable a la forme } \\
& \text { Pl. } 23 / 13 . \text { Per. Diat. marines } \\
& \text { de France, mais beaucoup } \\
& \text { plus petite et plus finement } \\
& \text { striée. Long. } 35 \mu, 10 \text { stries } \\
& \text { en } 10 \mu \text {. }
\end{aligned}
$$

Nitzschla angularis $W . S m$.

- granulata Grun.

- Jelineckii Grun.

- panduriformis Greg.

- $\quad$ - var. delicatula.

$$
\text { - - - var. paralbata. }
$$

Orthotropis lepidoptera Greg.

- $\quad$ - var. delicatula.

Plagiogramma Gregoryanum Greo.

Pleurosigma rigidum W. Sm.

Raphoneis amphiceros Eh. v. rhombica.

Rhoicosigma oceanicum Per. T. corsicana.

Skeletonema mediterraneum Grun. var.

Stephanodiscus astraea $\mathrm{Ktz}$.

Surirella fastuosa $\mathrm{Eh}$.

- - var. robusta.

Terpsinoë intermedia Greo.

Trachyneis aspera Eh.

- - var. minor.

- $\quad$ - var. pulchella.

- consors $A . S$.

- oblonga Bail. velata A. S. 
Trachyephæenia acuminata M. Per. n. sp. Semblable au Tr. australis, mais à extrémités supérieurealtẻnuée et acuminèe 8. 9 stries en $10 \mu$, peu distinctement granu- lées et non distinctoment interrompues par un pseudo-raphé. australis P. P.

Triceratium bullosum W. H. zonulatun Grev.

No 403

GOLFE JUAN (France)

Sondage

Actinocyclus Ralfsii W. Sm.

Actinoptychus undulatus Eh.

Amphiprora elegans $W$. Sm.

$$
\text { lepidoptera Greg. }
$$

Amphora mexicana A. S.

Auliscus sculptus W. Sm.

Astorolampra Grevillei v. adriatica .

$$
\text { - Marylandica Eh. }
$$

Biddulphia pulchella Gray.

$$
\text { regina W. Sm. }
$$

Campylodiscus adriaticus v. massiliensis.

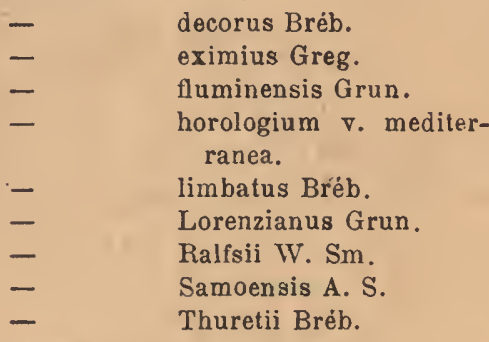

Cocconeis pseudo-marginata Greg.

Coscinodiscus devius A. S.

$$
\text { - } \quad \text { gigas } \mathrm{Eh} \text {. }
$$

Endyctia oceanica Eh.

Ifyalodiscus subtilis Bailey.

Mastoglvia reticulata Grun.

Navicula abrupta Greg.

- aspera Eh.
Navicula Beyrichiana A. S.

- bombus Eh.

- californica v.campechiana Grun.

- Hennedyi W. Sm.

- - var. manca.

- liber.

- Iyra Eh.

- _ v. elliptica.

- $\quad$ - v. subtypica.

- - v. recta.

- $\quad$ - v. A. S. atlas 2/25.

- multicostata Grun.

- prætexta Eh.

- $\quad$ - var. A. S. atlas 3/34.

- Sandriana Grun. var.

- Smithii Bréb.

- spectabilis Grev.

Nitzschia insignis IV. Sm.

- panduriformis Greg.

- sigma W. Sm.

- valida Grun.

Pleurosigma affinis Grun.

$$
\begin{aligned}
& \text { - balticum W. Sm. } \\
& \text { - decorum W. Sm. } \\
& \text { - } \quad \text { - var. adriaticum. } \\
& \text { - rigidum W. Sm. } \\
& \text { - _ - " var. giganteum. }
\end{aligned}
$$

Pyxidicula mediterranea Grun.

Rhabdonema adriaticum Kız.

Rhoïcosigma robustum Grun.

Surirelia Baldjickii Norm.

- fastuosa Eh.

- - var, abludens. 
Suricella fastuosa var. opulenta.

- fluminensis Grun.

- hybrida Grun.

- lata Eh.

- var. minor.

Synedra baculus Greg.

- fulgens W. Sm.
Synedra robusta Ralfs.

- undulata Greg.

Triceratlum arcticum Bright.

- antediluvianum Eh.

- farus Eh.

- pentacrinus WVall.

spinosum Bail.

\section{$\mathrm{N}^{\mathrm{o}_{5}} 403$ bis et 404 FAUFOUILLOUX - CANTAL (France)}

Dépôt fossile d'eau douce

\section{BIBLlOG R APHIE. - Frère J. Héribaud. Diatomées fossiles d'Auvergne 1903}

Achnanthes lanceolata Grun.

Amphora lybica Ehr.

Campylodiscus costatus TV. Sm.

Coscinodiscus dispar $\mathbf{v}$, radiata F. II.

Cyclotella iris J. Br. et F. II.

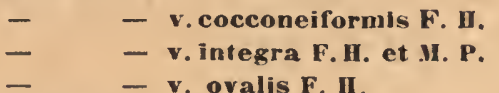

Cynıtopleara solea Bréb.

Cymbella aspera Ehr.

- Bouleana F. I.
- capitata F. H.
- cistula Hemph.
- conifera F. H.
- cymbiformis Ehr.
- lanceolata Ehr.
- leptoceros Ktz. fa curta.
- Pauli M. P. et F. H.

Epithemia Hyndmannii WV. Sm.

$$
\begin{aligned}
& \text { - } \quad \text { sorex Ktz. } \\
& \text { - } \quad \text { turgida Ktz. } \\
& \text { - } \quad \text { - } \quad \text { vebra } \mathrm{Ktz} \text {. porcellus F. H. } \\
& \text { - } \quad \text { - } . \text { proboscidea Grun. }
\end{aligned}
$$

Eunotia gracilis Rab.

- polyglyphis Grun.

Fragilaria brevistriata Grun.

Gomphonema cantalicum F, If.
Gomphonema capilatum Ehr.

$$
\text { - } \quad \text { olivaceum Ehr. }
$$

IIantzschia amphioxys Grun.

Melosira canalifera $\mathbf{F}$. II.

$$
\begin{aligned}
& \text { - } \quad \text { - } \quad \text { v. anastomosans F. H. } \\
& \text { - tenuis Grun. } \\
& \text { - Varennarun F. I. et M. P. }
\end{aligned}
$$

Navieula bicapitata Lag.

- gigas Ehr.

- major $\mathrm{Ktz}$.

- Reinhardtii Grun.

- ventricosa Donk.

Pleurosigma attenuatum $\mathrm{K} \mathrm{tz}$. IRhoïcosphenia curvata Grun.

Rhopalodia gibba Ehr.

Stauroneis phœnicenteron Ehr.

Stephanodiscus astræa $\mathrm{Ktz}$.

Surirella ovata Ktz.

$$
\text { - saxonica Auersw. }
$$

Synedra capitata Ehr.

Tetracyclus Boryanus Pant. v. minor. F. H.

- ellipticus F. H.
-
- lamarginatus W. Sm,
lamina Ehr. 
$N^{\circ} 405$ à 407

KARAND (Hongrie)

Dépot fossile marin

\section{Bibliogra PhiE. - J. Pantocseck. Diatomées de Hongrie, volume III}

Actinocyclus Bremianus Pant.

- Ehrenbergii Ralfs.

Actinoptychus Staubii Pant.

- undulatus Eh. var.

- vulgaris Schum.

Alloioneis Castracanei Pant.

Amphiprora Pethoi Pant.

Amphora acuta Greg. v. neogena Pant.

- andesitica Pant.

- cymbifera Greg.

- Nova Caledonica Grun.

Aoteromphalus Debyi Pant.

- Kinkerii Pant.

Campylodiscus crassus Pant.

- dilatata Pant.

- eximius Grev.

- Kützingii Pant.

- reticulatns Pant.

Chretoceros biharensis Pant.

- gracilis Pant.

- hispidus Eh.

- hungaricus Pant.

- Pethoi Pant.

Climacosphenia hungarica Pant.

Coceoneis scutellum Eh.

Coscinodiscus apiculatus Eh var.

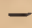

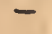

$-$

- $\quad$ Brightwelloïdes Pant.

- lineatus Eh.

- oculus iridis Eh.

- Pethoi Pant.

- pseudo-lineatus Pant.
Epithemia gibberula $\mathrm{Ktz}$. v. producta Grun.

- incisa Pant.

Grammatophora oceanica Eh.

llemiaulus rostratus Pant.

IIyalodisous radiatus Bail.

- scoticus Grun.

- subtilis Bail.

Mastogloia antiqua $\mathrm{Cl}$.

- neogena Pant.

- Pethoi Pant.

- Smithii Thw.

Melosira spinosa Pant.

- sulcata $\mathrm{Ktz}$.

Navlcula Boryana Pant.

- crabro Eh.

- elliptica Ktz. var.

- Hennedyi W. Sm.

- muscæformis Pant.

- seriosa Pant. var.

- Smithii Bréb.

Orthoneis splendida Eh.

Paralia Pethoi Pant.

Plagiogramma Boryana Pant.

Pleurosigma biharense Pant.

Podosira hormoïdes Mont.

Pseudodictyoneis hungaricus Pant.

Rhabdonema musica $J$. $B r$.

Rhizosolenia hungarica M. Per. Espèce tigurée, mais non nom. mée par M. H. Peragallo dans sa monographic des Rhizosolenia P. 21 5/19.

stictodlscas hưnganicus Pant. 
Surireila striatula Turp.

- subfastuosa Pant.

- torquata Pant.

Synedra crystallina W. Sm.

Triceratiun elatum Pant.
Triceratium spinosum Bail. - vastum Pant.

Xanthiopyxis oblonga Eh.

Zygoceros hungaricus Pant.

\section{No• 408 et 409 NEUSSARGUES - CANTAL (France)}

\section{Dépôt fossile d'eau douce}

\section{BIBLIOGR APHIE. - Fıère J. Héribaud. Les Diatomées fossiles}

d'Auvergne 1902

Amphoraaffinis $\mathrm{Ktz}$.

- ovalis $\mathrm{Ktz}$.

- podiculus Grun.

Cocconoi» pediculus Ehr.

- lineata Grun.

- piacentula Ehr.

Cyciotella comta Ktz.

Cymatoplenra solea Brẻb.

Cymbelia aspera Ehr.

- eymbiformis Ehr.

- lanceolata Eh.

- leptoceros Ktz.

- microcephala Grun.

- Pauli M. P. ot F. H.

Diatoma hyemale Heib.

- oulgare W. Sm.

Diploneis elliptica Ralfs.

Bncyonema ventricosum $\mathrm{Ktz}$.

Bpithemia Hyndmannii W. Sm.

- sorex Ktz.

- turgida $\mathrm{Ktz}$.

- zebra Ktz.

- $\quad-$ v. minor F. H.

- - - $\quad$ - proboseidea Grun.

Eunotia gracile o. capitata $M . P$. et F. $H$.

- polyglyphis Grun.

Fragilaria brevistriata Grun.

- elliptica Schum.

- Harrisonii Grun.

- mutabilis Grun.
Gomphonoma brevistriata F. H.

- capitatum v. curta V. H.

- subclavatum Grun.

Melosira distans Ehr.

- arenaria Moor.

- Boulayana M. P.

- crenulata $\mathrm{Ktz}$.

- granulata Ehr.

- tenuissima Grun.

Meridion constrictum Ralfs.

Navicula acrosphæria Brẻb.

- bacillum Ehr.

- Bonhardi F. H.

- Dariana A. S. miocenica F. H. et $M$. $P$.

- elliptica $\nabla$. minutissima Grun.

- limosa Ktz.

- legumen v. undulata V. H.

- major Kitz.

- Malinvaudi F. H.

- menisculus Schum.

- peregrina Herb. v. Lossilis F. H.

- placentula Ehr.

- radiosa $\mathrm{Ktz}$.

-. Reinbardtii Grun.

- rupestris $\mathrm{Ktz}$.

- sculpta Ehr.

- slesoicensis Grun.

- tenella Bréb.

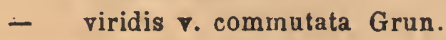

Nitzschia sigmoidea W. Sm.

Opephora Martyi $F$. $H$. 
Pleurosigma acuminatum Grun.

Rhoicosphenia curvata Grun.

Rhopaiodia gibba v. ventricosa Grun.

Surirella biseriata Bréb.

- crumena Bréb.

- robusta Ehr.

- splendida Elur.

- tenera Greg.

- $\quad$ - v. splendidula Greg.
Stauronels acuta W. Sm.

- phœnicenteron Ehr.

Synedra ulna Ehr.

- - amphirynchus.

- $\quad$ - v. danica.

- Ramesi F. H.

Tetracyclus ellipticus. F. H.

- emarginatus. W. Sm.
$\mathrm{N} \cdot 410$

No 411

$\mathrm{N}^{\circ} 412$

No 413

LE HAVRE (France)

Rhabdonema arcuatum Ktz.

BOIS DE BOULOGNE - PARIS

Gomphonema olivaceum Eh.

BOIS DE BOULOGNE - PARIS

Diatomy tenue Ktz.
LE HAVRE (France)

Surirella gemma Eh. 
Vavicula nobilis Ku.

Surirella elegans Eh.

- robusta Eh.
Surirella tenera Greg.

Tetracyclus lacustris var. = (Biblarium glacis Eh.)

\section{$\mathrm{N}^{\text {os }} 415$ et 416 CORMACK'S TOP - OAMARU}

\section{(Nouvelle-Zélande)}

\section{Dépot fossile marin}

Actinodiscus Barbadensis Grev.

Actinoptychus glabratus Grun.

- $\quad$ vulgaris.

Arachnoidiscus Ehrenbergii Ralfs.

Aulacodiscus amœnus Grev.

$\begin{array}{ll}\text { - } & \quad \text { - var. } \\ \text { - } & \text { angulatus Grev. } \\ \text { - } & \text { cellulolus Gr. et St. } \\ \text { - } & \text { crux Eh. } \\ \text { - } & \text { Huttonii Gr. et St. } \\ \text { - } & \text { Janischii Gr. et St. } \\ \text { - } & \text { margaritaceus v. Debyi. } \\ \text { - } & \text { Nova Zelandica A. S. } \\ \text { - } & \text { Rattrayi Gr. et St. } \\ & \quad \text { var. avec } 2 \text { pro- } \\ & \text { cossus. }\end{array}$

Uidululphia Johnsoniana Greo.

$$
\begin{aligned}
& \text { - } \quad \text { punctata Grev. } \\
& \text { - } \quad \text { rigida A. S. } \\
& \text { - Tuomeyi Bail. }
\end{aligned}
$$

Brightwellia pulchra Grun.

Coscinodiscus oculus iridis Eh. var.

$\begin{array}{ll}\text { - } & \text { angulatus Greo. } \\ \text { - } & \text { argus Eh. } \\ \text { - } & \quad \text { - } \quad \text { var. minor. } \\ \text { - } & \text { radiatus El. } \\ \text { - } & \text { spiniferus Gr. et St. } \\ \text { - } & \text { symbolophorus Grun. } \\ \text { Ruthii Grun. }\end{array}$

Eunotogramma productum Gr. et St.
Goniothecium odontella Eh.

Hemiaulus includens Grun. var.

Hyalodiscus permaculatus Grove.

- radiatus Grun.

- - var. maxima.

Isthmia enervis Eh.

Kittonia elaborata Gr. et St.

Melosira clavata Eh.

- clavigera Grun.

- Oamaruensis Gr. et St.

- sol Eh.

- sulcata Eh. var.

- truncata Grove.

Pyxidicula cruciata Eh.

rodosira argus Grun.

- hormoides Mont.

Stephanogonia Nova Zelandica Grun.

Stephanopyxis Grunowil Gr. et $\mathbf{S t}$.

$$
\text { - } \quad \text { turris Eh. }
$$

Stictodiscus californicus $\nabla$. areolata.

- Hardmannianus $\nabla$. mega-

$$
\text { pora. }
$$

- Nova Zelandica Grun.

- parallelus $f_{a}$ trigona.

- $\quad$ - fa gibbosa.

Triceratium americanum Ralfs.

- arcticum Bright.

- castellatum Grer.

- castelliferum Grun.

- $\quad$ coscinoides Gr. et St.

- crenulatum Gr. et St. 
Triceratium favus Eh.

- - - fa quadrata.

- fractum Wal. ot Cl.

- glandarium A.S.

- glandiferum Grun.

- grande Bright.

- majus Gr. et St.

- Morlandii Gr. et St.

- Nova Zelandicum Gr. et St.
Triceratiun Oamaruense Gr. et St. pulvinar A. S.

- pseudo-nervatum Gr. et St.

- rugosum Gr. et St.

- secedens A. S.

- undatum Grun.

- unguiculatum Grev.

- Weissflogii Gr. et St.

Trinacria ventricosa Gr. et St.

HANOVRE (Allemagne)

Terres fossiles d'eau douce

La terre fossile blanche $\left(\mathrm{N}^{\circ} 417\right)$ ne diffère de la grise (No 418 ) que par la prédominance du Synedra ulna. Ce sont deux parties d'une même couche diatomifère.

Achnanthes lanceolata Bréb.

Amphora ovalis Ǩtz.

Cymbella cistula Ktz.

- cuspidata Ktz.

Encyonema cœspitosum Ktz.

Epithemia sorex $\mathrm{Ktz}$.

- zebra Ktz.

Fragilaria capucina v. lanceolata. - mutabilis W. Sm.

Gomphonema capitatum Eh. clavatum Eh.
Gomphonema montanum Shum. tenellum Ktz.

Melosira tenuis $\mathrm{Ktz}$.

Navicula bacillum Eh.

- limosa Ktz.

- placentula Eh.

- pupula Ktz.

Stephanodiscus astraea v. spinulosa.

- - v. minutula.

Synedra ulna Ktz.

Tetracyclus marginatus W. Sm.
Achnanthidium flexellum Brób.

Amphora ovalis Ktz.

Cyclotella comta Eh var.
Cymbella æqualis W. Sm.

- cesati Grun.

- cuspidata Ktz.

- cymbiformis Eh. 
Cymbella gastroides $\mathrm{Ktz}$.

- maculata Ktz.

- norvegica Grun.

Denticula tenuis $\mathrm{Ktz}$.

Epithemia gibba Ktz.

$$
\text { - } \quad \text { sorex Ktz. }
$$

Encyonema cœspitosum Ktz.

$$
\text { - gracile Rab. }
$$

Eunotia arcus Eh.

$$
\begin{aligned}
& \text { - depressa Eh. } \\
& \text { - incisa Greg. } \\
& \text { - pectinalis Ktz. } \\
& \text { - gracilis Eh. }
\end{aligned}
$$

Mastoglola lacustris Grun.

Gomphonema acuminatum $\mathrm{Ktz}$.

$$
\text { - intricatum } \mathrm{Ktz} \text {. }
$$$$
\text { vibrio Eh. }
$$

Navicula amphirynchus Eh.

- biceps Eh.

- Brebissonii var.

- cuspidata Ktz.
Navicula dactylus Eh.

- firma Ktz.

- gibba Eh.

- gigas Eh.

- Hilseana Jan.

- limosa Ktz.

- major $\mathrm{Ktz}$.

- mesolepta $\nabla$. stauroneiformis.

- platycephala.

- radiosa.

- - var. acuta.

- rostellata Ktz.

- serians $\mathrm{Ktz}$.

- viridis var. commutata.

- viridula Ktz.

- tuscula Eh.

Nitzschla communis Rab.

Stauroneis anceps Eh.

- phœnicenteron Eh.

Surirella elegans Eh.

- robusta Eh.

Tabellaria fenestrata.

Van lleurckia rhomboides Bréb.
Achnanthes longipes Ag.

Actinocyclus subtilis Bail.

Amphitetras antediluviana $\mathrm{Eh}$.

Biddulphia pulchella Gray.

Campyloneis Grevillei W. Sm.

Cocconeis costata Greg.

- dirupta v. flexella.

- pseudo-marginata.

- scutellum fa parva.

Grammatophora macilenta W. Sm.

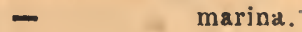

Hyalodiscus scoticus Grun.

Licmophora Lynghyoi Ktz.

Plenrosigma delicatulum $\mathrm{W}$. sm. decorum W. Sm.

Podosira hormoides Mont.

Rhabdonema adriaticum Ktz .

Synedra affinis $\mathrm{Ktz}$.

- Gaillonii Klz.

- dalmatica Ktz.

- undulata Greg.

Triceratiun arcticum Bright. 


\section{No 421 SAINT-LUNAIRE. - ILLE-ET-VILAINE (France)}

Sur la vase

Navicula veneta et vars. Ktz.

$\mathrm{N}^{\text {os }} 422$ à 425 BERGONZANO. - REGGIO I''EMILIO

(Italie)

Dépôt fossile marin miocène

Bibliogra Phie. - Dr Áchille Forti. Primo elenco della Diatomee fossili contenute nei depositi miocenici di Bergonzano ( $L a$ Nuova ${ }^{\circ}$ Notarisia Serie XIX 1908.) L'analyge suivante nous a été communiquée avec les matériaux par le Dr Forti, de Vérone.

Actinocyclus Ralfsii Eh.

- $\quad$ - var. samœensis Gr.

Actinoptychus decumanus $A . S$.

- hexagonus Gr. var.

- sculptilis A. S.

- undulatus Eh.

Actinosphtenia splendens Ralfs.

Arachnoidiscus ornatus Eh. var.

Asterolampra affinis Grev.

$$
\begin{gathered}
\text { - Dallasiana Greo. } \\
\text { - } \quad \text { Marylandica Eh. } \\
\text { - } \quad \text { var. fossilis } \\
\text { Pant. }
\end{gathered}
$$

Asteromphalus Grevillei Wall.

Aulacodiscus amoenus Greo.

- $\quad$ - var . hungarica Pant.

- Schmidtii O. W.

Biddulphia Tuomeyi Bail.

Campyloneis Grevillei Wall.

Cerataulus Smithii Bréb.

Cestodiscus ovalis Grev.
Cladogramma conicum Grev.

$$
\text { - var. campanula- }
$$
tum Forti.

Cocconeis præcellens Pant. v. ellipticostriata Forti.

- - $\quad$ var paucistriata Pant.

- pseudo-marginata Greo. oar.

- Lanzii n. sp. Forti.

Coscinodiscus crassus Bail.

- decrescens Grun.

- diorama A. S. var.

- Gazellae Jan. (en fragmerits).

griseus Greo. Nec. A. S. intumescens Pant.

lentiginosus Jan.

Lewisianus Grev. v. minor Temp.

- $\quad$ lineatus Eh.

- $\quad$ - $\quad$ v. intermedia Ratt.

- $f_{a}$ irregularis n. fa. 
Coscinodiscus nodulifer A. S.

- oculus iridis.

- proteus Ratt.

- radiatus Eh.

- - $\quad$ G. irregularis

- rhombicus Grun. v. italicus Forti.

- $\quad$ Rothii Grun.

- superbus Hardm.

Craspedodiscus coscinodiscus Eh.

$$
\begin{aligned}
& \text { - elegans Eh. } \\
& \text { - insignis } A . S \text {. }
\end{aligned}
$$

Endictya oceanica Eh.

Gephyria gigantea Greo.

Goniothecium danicum Grun.

$$
\text { - odontella Eh. }
$$

Grammatophora marina $K t z$.

Gyrodiscus vortex $\mathrm{O}$. W.

Ilemianius polymorphus Grun. oar.

- Weissii Grun.

Hyalodiscus radiatus var. arcticus Grun.

Isthmia Squinaboli Forti n. sp. (=Pyxilla Squinaboli Forti, olim.)

- Szaboi Pant.

Licmophora angustata Grun.

Melosira clavigera Grun.

$$
\begin{aligned}
& \text { - } \quad \text { A.S. } \\
& \text { - expectata A.S. } \\
& \text { - subornata A.S. } \\
& \text { sulcata var. separanda A.S. }
\end{aligned}
$$

Navicula circumsecta Grun.

- gemmata $\nabla$. pristiophora Jan.
Navicula protexta Eh. fa minor.

- nitescens Greg.

- polysticta Greo.

- - o.circumsecta Grun.

Orthoneis splendida Grun.

Periptera tetracladia Eh.

Pseudopyxilla vasta (Pant.) Forti.

$$
\begin{array}{ll}
\text { - } & \text { dubia (Grun.) Forti. } \\
\text { - } & \text { rossica(Pant.) Forti. } \\
& \text { Tempereana Forti n. sp. }
\end{array}
$$

Septroneis caduceus Eh. oar.

Stephanogonia actinoptychus Grun.

$$
\text { - } \quad \text { cincta Pant. }
$$

Stephanopyxis Broschii fa aculeis obtusis $\mathrm{Gr}$.

- Grunowii Gr. et St.

- turris Ralfs. $\quad$. cylindrus fa paucispina Grun.

Stictodiscus confusus $T r$. et $W$.

- Grunowii Tr. et W.

- Kinkerianus Tr. et W.

Temperea miocenica Form. n. g. (=Aulacodiscus miocenicus Forti, olim).

Triceratium antediluvianum Gr. et V. II.

- Pantocsekii Grun.

- grande Bright. o. quadrangularis.

- nancoorense Grun.

Xanthiopyxis globosaEh.

- oblonga Eh.

panduræformis Pant.

Les numéros 426 et 427 proviennent d'un lavage d'estomacs de conserves d'Huîtres du Japon, exposées à Paris en 1889; lo numóro 428 d'un lavage des conserves d'Huîtres exposées en 1900 au pavillon Japouais.

Achnanthes angusta.

- Jaoanica Grun. var.

- mammalis Gl. var.

Actinocyclus Ehrenbergii Ralfs.

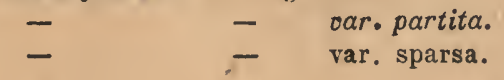


Actinocyclus moniliformis Ralfs. var. nebulosus M. Per.

Actinoptychus areolatus Eh.

- undulatus Eh.

- $\quad$ - fa minor.

Alloioneis antillarum Cl.

- Debyi Leud. Fort.

Amphiprora alata $\mathrm{Ktz}$.

Amphora angusta Greg.

- costata W. Sm.

- crassa Greg.

- cymbifera Greg.

- obtusa Greg.

- ostrearia Bréb.

- proteus Greg.

- Pusio Gl.

- salina W. Sm.

Bacillaria paradoxa Gmel.

Bacteriastrum carians Laud.

Biddulphia chinensis Gror. - pulchella Graz.

Campylodifcus Pfitzeri $A$. S.

Cerataulus Petiti Loud. Fort.

Chretoceros Kelleri J. Br.

Climacosphoenia elongata Bail.

Cocconeis psoudo marginata Greg.

- scutellum Eh.

Coseinodiscus decipions Grun.

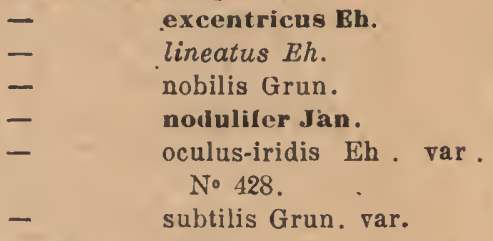

Cyclotella striata Ralfs. v. sinensis.

Diploneis bombus Eh.

- diplosticta Grun.
- $\quad$ interrupta Gl.
- Smithii Cl.

Dytilium Brightwellii West.

Donkinia carinata Ralfs.

Epithemia constricta Bréb.

$$
\text { - } \quad \text { gibberula Ktz. }
$$

Grammatophora oceanica Eh. Ilantzschia marina Grun.
Ilantzschia virgata Grun .

Hyalodiscus stelliger Bail.

Liemophora flabellata $\mathrm{Ag}$.

Mastogloia Braunii Grun. fa anomala. La ligne des logettes est à l'intérieur de la valve et non contre le bord.

- Jelineckii Cl. o. marina.

- Macdonaldii Greo.

- quinquecostata Grun.

Melosira sulcata $\mathrm{Ktz}$. v. coronata.

- - - o. radiata.

Navicula Baileyana Grun.

- brevis Greg.

- Brunii Pant.

- cancellata Donk.

- clacata Greg. o. impressa.

- crucifera Grun.

- cyprinus Ktz.

- forcipata Grev. v. densestriata.

- granulata Bréb.

- - var. javanica.

- Hochstetteri Eh.

- jejuna A. S.

- Karmorthensis Grun.

- luxuriosa Grev.

- lyra Eh.

- - v. elliptica.

- - o. subtypica.

- pygmea Ktz.

- scopuloruin Bréb. var. perlonga.

Nitzschia bilobata W. Sm. var.

- Jelineckii Grun.

- panduriformis Greg.

- punctata Grun.

- sigma Grun. No 428.

Plagiotropis elegans Grun.

Pleurosigma angulatum IV. Sm.

- Japonieum Cast.

- naviculaceum Bréb.

- $\quad$ - v. æquatoriale.

Rhizosolenia amputata Est.

Scoliopleura latestriata Grun. No 428.

Skeletonema costatum Cl.

Stauroneis salina W. Sm.

stictodiscus Grorei A. S. 
Surirella curoifacies J. Br. car. conspicua 0 . $n$. Stries facilement visibles, granulées formées de points croisés à angles droits."

- fastuosa Eh.

- - var. recedens.

- gemma Eh.

- patens A. S. var. laevis M. P. n. var. Forme extérieure du S. patens (A. S. atl. 4/10) la bande des stries du milieu fermée en demi-cerclo aux extrémités; côtes nulles repré- sentées seulement par de petites parties striées. Long. $120 \mu$, largeur $60 \mu$.

Synedra formosa Hantz. - superba Ktz.

Toxarium Hennedyanum Gr.

Tracheneis aspera Cl.

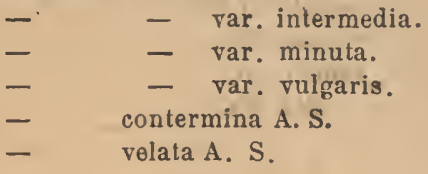

No 429

\section{ORCAS ISLAND (Etatś-Unis)}

\section{Dépôt fossile d'eau douce}

Achnanthes lanceolata Bréb. var. Haynaldii.

Amphora libyca $E$.

- pediculus $K t z$.

Cocconeis placentula Eh.

Cyclotella comta $\mathrm{Ktz}$.

Cymbella cistula Kirch.

$$
\begin{aligned}
& \text { - } \quad \text { cuspidata Ktz. } \\
& \text { - } \quad \text { ghrenbergii Ktz. } \\
& \text { - }
\end{aligned}
$$

Diatoma tenue Ag. cum valvis internis.

Encyonema turgidum Grun.'

$$
\text { - ventricosum Grun. }
$$

Epithemia musculus $\mathrm{Ktz}$.

$$
\begin{aligned}
& \text { - } \quad \text { turgida Ktz. } \\
& \text { - } \quad \text { zebra Ktz. } \\
& \text { - } \quad \text { - var. amphicephala. }
\end{aligned}
$$

\section{Eunotia gracilis Rab.}

- impressa Eh. var. ventricosa. n. v. Grande forme infléchio du côté ventral et à extrémités atténuées récurvées.

- incisa Greg.

- minor lkal.

- lunaris Grun.

- - var. attenuata.

- - var. undulata.
Eunotia major Rab.

- - var. ventricosa.

- minor V. II.

- prarupta Eh. v. bidens.

- quaternaria Eh.

- tridentata Eh.

Fragilaria æqualis Heib.

- capucina Desm.

- - - oar. mesolepta.

- virescens Ralfs.

Gomphonema acuminatum $\nabla$. pusilla .

- angustatum kitz.

- - v. obtusatum.

- $\quad$ - v. producta.

- apicatum $\mathrm{Cl}$.

- auritum Braun.

- constrictum Eh. fa elongata $60 \mu$.

- $\quad$ intricatum $\mathrm{Ktz}$.

- montanuh $v$. suecica.

- vibrio Eh.

IIantzschia amphioxys Grun.

Melosira crenulata Kitz.

- lineolata Grun.

Meridion constrictum Ralfs.

Navicula acrosphæria Ktz.

- bacilliformis Grun. 
Navicula brevicostata Cl.

- cuspidata Ktz.

- dactylus Ktz.

- elliptica Ktz.

- gibba Ktz.

- gigas Ktz.

- limosa $\mathrm{Ktz}$.

- major $\mathrm{Ktz}$.

- mesolepta Eh.

- radiosa Ktz.

- semen Eh.

- viridis Ktz.
Nitzschia sigmoidea $W$. Sm.

Stauroneis anceps $E h$.

- Baileyi Eh.

- gracilis Eh.

- phœnicenteron Eh.

8tephanodiscus astraea Grun.

$$
\text { - _ - v. spinulosa. }
$$

Synedra ulna Eh.

- - var, danica.

Tabellaria fenestrata Ktz.

- flocculosa Ktz.

Nor 430 et 431 TROUVILLE (Pélagique) (France)

La préparation No 430 est dans le médium et le 431 à sec

Loptocylindrus danicus Cleve

N· 432

ILE SAINT-PAUL (Mers Australes)

Sondage .

Achnanthes longipes $A$. S.

Arachnoldiscus indicus Eh. var.

Biddulphla aurita Bréb. pulchella Graz.

Campyloneis Grevillei W. Sm.

Cocconeis pellucida $\mathrm{Ktz}$.

Coscinodiscus radiatus $\mathrm{Eh}$.

Entopyla australis Eh.
Hyalodisous ambiguus $=$ (Podosira ambigua).

- lævis Eh.

- subtilis Eh.

Grammatophora arctica Cl.

- serpentina Eh.

Navicula aspera $\mathrm{Eh}$. fa minuta

- crabro Eh. var.

Pleurosigma validum Shadb.

Surirella fluminensis Grun.

$N^{\circ} 433$

ILE D'AMSTERDAM

Dépôt fossile d'eau douce

Achnanthes lanceolata Eh.

Navlcula subcapitata var. paucistriata.
Melosira Roseana Eh.

\begin{tabular}{|ccc} 
Melosira Roeseana & Lh. \\
- & - & var. dendroteres.
\end{tabular} 
Amphiprora alata Ktz.

Aetínocyclus Ralfsii. IV. Sm. - elegans W. Sm.

Amphora ovalis Kız.

Campylodiscus Echeneis Eh.

Coscinodiscus excentricus Eh.

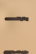

-

-

Cerataulus polymorphus $\mathrm{Ktz}$.

Cymatopleura elliptica Ktz.

Cymbella lanceolata Eh.

Epithemia musculus Ktz.

- zebra Ǩtz.

Melosira Borreri Grev.

- - var. hispida Cast.

Mastogloia apiculata W. Sm.
Navicula amphisbæna Bory.

- bombus Ktz.

- cyprinus W. Sm.

- elliptica Ktz.

- forcipata Grev.

- lyra Eh.

- marina Donk.

Nitzschia acuminata Grun.

- marginata Grun.

- punctata Grun.

- rigida W. Sm.

- sigma W. Sm.

- - var. intercedens Grun

- - var. sigmatella Grun.

- tryblionella Grun.

Plenrosigma æestuarii W. Sm.

- balticum W. Sm.

- - var. diminutum.

- formosum W. Sm.

Surirella gemma Eh.

- striatula Turp.

$N^{\circ} 435$ et 436 SANTA-MARIA (Californie)

Dépot fossile marin

Actinocyclus Ehrenbergii Ralfs.

Actinoptychus splendens Ralis.

- undulatus Eh.

Amphitetras antediluviana Eh.

Arachnoidiscus Ehrenbergii Ralfs.

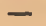

- var. californica Grun.

ornatus Eh.

Asteromphalus moronensis Grev.
Aulacodiscus affinis Grun.

$$
\text { - } \quad \text { compactus Ratt. }
$$

- - var sparso-radiata.

Auliscus Hardmanianus Grev.

- mirabilis Greo.

- Stöckardtij Jan.

Campylodiscus coronilla J. Br. 
Campylodiscus ecclesianus Greo. toniatus A. S.

Cestodiscus superbus Hardm.

Cocconeis Montereyi Grun.

- pellucida Hantz.

- regalis Grev. var.

Coscinodiscus argus var. A. S.

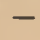

$-$

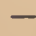

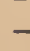

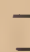

ב

$-$

\section{ב}$$
\bar{z}
$$$$
\bar{z}
$$

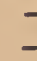$$
\bar{z}
$$$$
\overline{-}
$$$$
-
$$$$
\overline{-}
$$$$
=
$$$$
\overline{-}
$$$$
\overline{-}
$$$$
\text { apiculatus Eh. }
$$$$
\text { centralis } f \text { maxina. }
$$$$
\text { crassus Bail. }
$$$$
\text { curvatulus Grun. var. }
$$$$
\text { decrescens Grun. }
$$$$
\text { elegans Grev. }
$$$$
\text { - fimbriatus Eh. }
$$$$
\text { floridulus } A \text {. S. }
$$$$
\text { gigas Eh. }
$$$$
\text { herculus J. Br. }
$$$$
\text { implicatus Ratt. }
$$$$
\text { - lineatus Eh. }
$$$$
\text { marginatus Eh. }
$$$$
\text { micans Grun. }
$$$$
\text { obscurus A. S. }
$$

Coscinodiscus relatus A. S. var.

Entopyla australis var. gigantea Grev.

Grammatophora robusta Eh.

Hyalodiscus laevis Eh.

$$
\text { - maximus Grun. }
$$

Isthmia neroosa $K t z$.

Melosira clavigera Grun.

- sol Eh.

- sulcata Eh.

Navicula angelorum $\mathrm{Cl}$.

- aspera Eh.

- Iyra Eh.

- pedalis J. Br.

- prætexta Eh.

- - var. abundans A. S.

- ornata. A. S.

Orthoneis splendida Grun.

Porpeia quadriceps Grev.

Pseudauliscus peruvianus Ratt.

Rhablonema biquadratum $J, B r$.

Rntilaria hexagona Grun.

Stephanopyxis corona Eh.

$$
\begin{array}{ll}
\text { - } & \text { spinosissima Grun. } \\
\text { - } & \text { superba Grev. } \\
\text { - } & \text { turris Eh. }
\end{array}
$$

Stictodiscus californicus.

Triceratium arcticum var. californica Grun. elegans Grev.

- parallelum Grov.

- - fa trigona.

Xanthiopyxis umbonatus Grev. 
Amphiprora decussata Grun.

Amphora crassa Greg.

- - var. punctata.

- spectabilis Grun.

Aulacodiscus Johnstonii Arnold.

Biddulphia pulchella Graz.

Campylodiscus decorus Bréb.

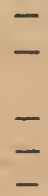
eximius Greg. horologium $\nabla$. mediterranea.

limbatus Bréb. samcensis Grun. Thuretii Bréb.

Cerataulus Smithii Ralfs:

Coscinodiscus excentricus Eh.

$\begin{array}{ll}\text { - } & \text { lineatus Eh. } \\ \text { - } & \text { marginatus Eh. } \\ \text { - } & \text { nitidus Greg. } \\ \text { - } & \text { oculus-iridis Eh. } \\ & \text { scintillans Grev. }\end{array}$

Grammatophora serpentina Eh.
Melosira sulcata Eh.

Navicula apis Donk.

- aspera Eh.

- bombus Eh.

- californica Grev. var.

- crabro Eh.

- excavata Grov.

- longa Greg.

- lyra Eh.

- pandura Bréb.

- prætexta Eh.

- pristiophora Jan.

- Smithii Bréb.

Nitzschia distans Greg. var.

- panduriformis Greg.

- valida GI. et Grun.

Orthoneis splendida.

Pleurosigma balticum W. Sm.

- decorum W. Sm.

- formosum W. Sm.

- rigidum W. Sm.

Rhablonema adriaticum Ktz.

\section{$\mathrm{N}^{\text {os }} 439$ à 441 WILLIAM'S BLUFF - OAMARU}

(Nouvelle-Zélande)

Dépot fossile marin

Aetinoptychus vulgaris v. maculata Gr. et St.

splendens o. fusca.

Anthodiscus floreatus $\mathrm{Gr}$. et St.

Arachnoidiscus Ehrenbergii Bail.

Asterolampra decora Grev.

$$
\text { - } \quad \text { marylandica, Eh. }
$$

Aulacodiscus amonus Grov.

$$
\text { - angulatus Grev. }
$$

$\begin{array}{ll}\text { - } & \text { cellulosus Gr. et St. } \\ \text { - } & \text { crux v. subsquamosa Grun. } \\ \text { - } & \text { Janischii Gr. et St. }\end{array}$

Aulacodiscus margaritaceus $\nabla$. Debyi. Ratt.

$$
\begin{aligned}
& \text { - - V. undosa } \\
& \text { Gr. et St. } \\
& \text { - nova zelandica Gr. ot St. } \\
& \text { - patens Ratt. } \\
& \text { - Rattrayi Gr. et St. }
\end{aligned}
$$

Auliscus Hardmannianus Grev.

- - $\quad$ v. bifurcata Ratt.

- oamaruensis Gr. et St.

- ovalis Grov.

- Stöckhardtii Jan.

Biddulphia oamaruensis $\mathrm{Gr}$. et $\mathrm{St}$. - punctata Grer. 
Biddulphia rigida $\mathrm{A}$. $\mathrm{S}$.

$$
\text { - Tuomeyi Bail. }
$$

Ceraṭalus subangulatus Gr. et St.

Coscinodiscus argus Eh.

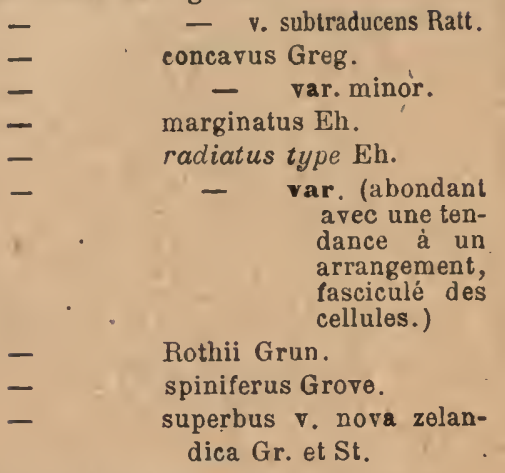

Eunotogramma productum Gr. et St.

Ilemiaulus includens Grun var.

IIyalodiscus radiatus Grun:

- var. arctica Grun.

Isthmia enervis Eh.

Melosira clavigera Grun.

- oamaruensis Gr. et St.

- sulcata Eh. var.

- truncata Gr. et St.

Podosira hormoides oar.

Porodiscus hirsutus Gr. et St.
Psendauliscus diffusus Ratt.

Pyxidicula cruciata Eh.

Stephanopyxis Grunowii Gr. et $S t$.

Stictodiscus californicus Grev. var.

- Hardmannianus Grev.

$\begin{array}{ll}\text { - } & \text { v. megapora } \\ \text { Gr. et St. }\end{array}$

Triceratium anastomosans Grove.

$\begin{array}{ll}\text { - } & \text { castellatum Grun. } \\ \text { - } & \text { crenulatum Gr. et St. } \\ \text { - } & \text { fallaciosum Grun. } \\ \text { - } & \text { favus Eh. } \\ \text { - } & \text { fractum Guadrata. } \\ \text { - } & \text { glandarium A. S. } \\ \text { - } & \text { glandiferum Grun. } \\ \text { - } & \text { lineatum Grev. } \\ \text { - } & \text { majus Gr. et St. } \\ \text { - } & \text { nova zelandicum Gr. et St. } \\ \text { - } & \text { oamaruense Gr. et St. } \\ \text { - } & \text { planoconcaoum J. Br. } \\ \text { - } & \text { pseudo-nervatum Gr. et St. } \\ \text { - } & \text { rugosum Gr. ot St. } \\ \text { - } & \text { trisulcum v. cuneata Grove. }\end{array}$

\section{Bibliogra Phie. - J. Pantocseck. Diatomées de Hongrie, volume I}

Achnanthes brevipes Ag.

$$
\begin{aligned}
& \text { - } \quad \text { - } \quad \text { v. contracta Grun. } \\
& \text { - } \quad \text { subica Grun. }
\end{aligned}
$$

Actinocyclus circumdatus Pant.

$$
\text { - subcrassus Ratt. }
$$

Actinoptychus leptomitos Pant."
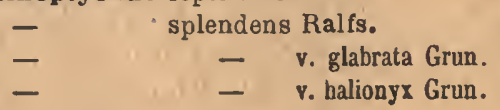

Actinoptychus undulatus Eh.

$$
\text { - vulgaris } \nabla \text {. doljensis Pant. }
$$

Amphora crassa $v$. punctata Grun.

- moniligera Greg.

- intersecta A. S. v. striata.

- - - - sarmatica Pant.

Asterolanpra marylandica Eh.

Aulíscus cœlatus Bail.

- sculptus Ralfs. 
BIddulphia elegantula Grev.

Campylodiscus Dæmelianus Grun.

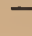

$-$
Ralfsii $\nabla$. fossilis Grun. Thuretii $\nabla$. baldjickiana Grun.

Chretoceros affin $\theta$ Laud.

Climacosphenia moniligera $\mathrm{Eh}$.

Cocconeis pellucida Grun.

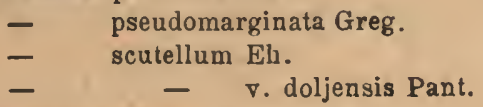

Coscinodiscns asperulus Grun.

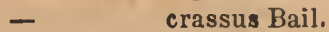

- decrescens Grun.

- doljensis Pant.

- excentricus Eh.

- elegans Grev.

- lineatus Eh.

- marginatus Eh.

- nitidulas Grun.

- obscurus A.S.

- oculus-iridis Eh.

- perforatus Eh. จ. cellulosa.

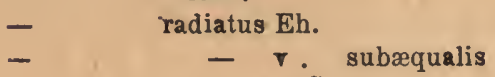

Grun.

- robustus Grev.

- sarmaticus Pant.

- 'Stokesianus $\nabla$. baldjikiana Grun.

tumidus Jan.

Dimeregramma nanum Greg.

Epithemia gibbarula Ktz.

Grammatophora insignis Grun.

$\begin{array}{cl}- & -\quad \text { vadoljensis Grun } \\ - & \text { maxima Grun. } \\ - & \text { oceanica Eh. } \\ - & \text { v. subtilissi- } \\ \text { ma Grun. } \\ \text { - } & \begin{array}{l}\text { robusta Dippel. } \\ \text { stricta Eh. }\end{array} \\ & \text { Grun. fossilis }\end{array}$

Ilenidiscus cuneiformis Wall.

Uyalodiscus lævis Eh.

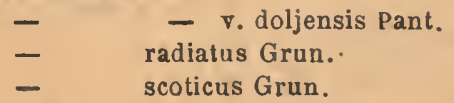

Hyalodiscus subtilis Bail. var. - australiensis Grun.

Leudugeria $\theta$ pithemioides Temp.

Mastogloia doljensis Pant.

Melosira clavigera Grun.

Navicula cancellata Donk.

- caribea Cl.

- coarctata A. S.

- directa W. Sm.

- doljensis Pant.

- fusca Ralfs.

- Gorjanovicii Pant.

- Hennedyi W. Sm.

- humerosa Brèb.

- interrupta Ktz.

- latissima Greg.

- - v. karmothensis Grun.

- lineata Donk.

- lyra Eh.

- $\quad$ - . dilatata A.S.

- - - olliptica.

- scutellum O'Meara.

- Smithii Bréb.

- suborbicularis Greg.

- Vakotinovicii Pant.

- Yarrensis Grun.

Nitzschia doljensis Pant.

Orthoneis binotata Grun.

- splendida Grun.

Paralia sulcata Cl.

Plagiogramma Gregorianum Grev.

Pyxidicula cruciata Eh.

Raphoneis amphiceros Eh.

- rhombus Eh.

- subtilissima Pant.

Rhabdonema adriaticum Ktz.

- hamuliferum Kitton.

Stephanopyxis corona Grun.

- turris Eh.

Stictodiscus californicus Grer.

Surirella fastuosa $\mathrm{Eh}$.

- rotunda Pant.

Synedra crystallina Ktz.

- $\quad$ - fa gibba Pant.

Thalassfonema Frauenfeldii $\nabla$. doljensis Pant.

Triceratiun favus Eh.

spinosum Bail. 
Actinoptychus hexagonus Grun.

Aulacodíscus Elirenbergii Jan. - margaritaceus Ralfs.

Biddulphia balaena Bright.
Biddulphia pulchella Gray. Cerataulus turgidus Eh. Navicula aspera Eh. Triceratium Robertianum Grev.

\section{$N^{\circ} 445$ à 447 ILE MAURICE (Océan Indien)}

\section{Lavage d'algues}

Achnanthes anguatata Grev.

Actínocyclus Ehrenbergii Ralfs.

$$
\begin{gathered}
\text { - Ralfsii v. australiensis } \\
\text { Grun. }
\end{gathered}
$$

Arachnoidiscus ornatus Eh.

Auliscus cœlatus Bailey.

Biddulphia obtusa Grun.

$$
\text { pulchella Gray. }
$$

Campylodiscus ambiguus Grun.

$$
\begin{array}{cc}
\text { - } & \text { intermedius Grun. } \\
\text { - } & \text { ornatus Grun. } \\
- & \text { - var. amphileia } \\
\text { - } & \text { Grun. } \\
- & \text { samoensis Grun. } \\
\text { tæuiatus A. S. }
\end{array}
$$

Climacosphenia moniligera Eh.

Cocconels gibbocalyx $J$. $\mathrm{Br}$.

$$
\text { - } \quad \text { peteroidea Hantz. }
$$

Cosclnodiscus concavus Grev.

$$
\text { - marginatus Eh.. }
$$

Epithemia musculus Ktz.

$$
\text { - succincta Bréb. }
$$

Gephyria media Arnott.
Grammatophora marina W. Sm. Hyalodiscus subtilis Bailey.

Iydrosera Boryana Pant.

- mauritiana Bergon.

Ifydrosilicon rimosa $\mathrm{J}$. $\mathrm{Br}$.

Isthmia capensis Grun.

Mastoglvia quinquecostata var.?

Melosira nummuloides Ag.

Navicula apis Donk.

- aspera Eh.

- clavata Greg. var.

- fluitans J. Br.

- gracilis $\mathrm{K} \imath$. var.

- lyra Eh.

- polita J. Br.

- prisca A. S.

- velata A. S.

Nitzschia decora Kitton.

- ventricosa Kittun.

- Danduriformis Greo.

- - var. minor.

Orthoneis fimbriata.

Plagiodiscus Martensianus Grun.

Podocystis adriatica Ktz. 
Pleurosigma delicatulum W. Sm.

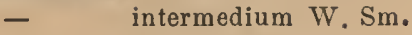

- rigidum W. Sm.

- $\quad$ speciosum W. Sm.

- strigosum W. Sm.

Pyxidicula mediterranea Grun.

Rhablonema adriaticum. Eh. - mirificum. W. Sm.

Surirella fastuosa Eh.

Synedra Gaillonii Eh.
Synedra dalmatica Grun.

- Frauenfeldii Grun.

- Hennedyana IV. Sm.

Triceratium arcticum Bright.

- compar A. S.

- grande var. 7 gona.

- pentacrinus Wall.

- quinquelobatum Grun.

- Shadboldtianum Grev.

- Wallichianuin Ralfs. zonulatum Grev.

$N^{*} 448$ et 449

ABASHIRI (Japon)

Dépôt fossile marin

BIbliographie. - Dr J. Pantocsek. Beitrage zur Kenntniss der fossilen Bacillarien Ungarns. Part. III.

Actinoptychus areolatus Eh. simbirskianus $A$. S. undulatus Eh.

Arachnoidiscus Ehrenbergii Bail. var. californica.

$$
\text { - indicus Eh. var. }
$$

Chretoceros pliocenum J. Br.

Clavicula arenosa $J . B r$.

- Jimboi Pant.

Cuscinodisous asteromphalus Eh.

$$
\overline{-}
$$
elegans Grev. fulguralis J. Br. griseus Grev. var. heteroporus Eh. v. moronensis Ratt.

- megaloma A. B.

- micans A. S.

- oculus-iridis Eh.

- radiatus Eh.

- $\quad$ robustus Grev.

- suspectus Jan.

- symbolophorus Grun. var.
Coscinodiscus transyloanicus Pant.

Dicladia capreolus Eh.

Goniotheclum odontella Eh.

Grammatophora robusta Dipp.

Isthmia nerrosa Ktz.

Melosira sol $\mathrm{Ktz}$.

Navicula Sieboldii Pant.

- spectabilis Greo.

Pterotheca subulata Grun.

Pyxilla americana Eh.

Rhabdonema mikado Pant.

$$
\text { - musica J. Br. }
$$

Rutilarla longicornis Temp, et Brun.

Bkeletonema ventriculosa $J . B r$.

Stephanopyxis Grunowii Gr, ot St.

- turris o. cylindrus.

furris v, intermedia.

Surirella gemma Eh.

Triceratium interjectum $A$. $S$.

Xantiopyxis umbonatus Grev. 
No 450

MONKSTEAD SKYE (Ecosse)

1)épôt fossile d'eau douce

Cocconeis placentula Eh.

Cymbella cymbiformis $\mathrm{Eh}$.

Encyonema ventricosum Ktz.

Epithemia gibba Ktz.

- Hyndmannii.

- sorex $\mathrm{Ktz}$.

- turgida Ktz.

- zebra Ktz.

Eunotia arcus Eh.

- bigibba Grun.

- gracilis Rab.

Uantzschia elongata Grun.

Gomphonema acuminatum Ktz.
Gomphonema capitatum. Eh.

Navicula elliptica $\mathrm{Ktz}$.

- - - v. oblongella.

- gibba Ktz.

- gigas Eh.

- divergens W. Sm.

- major Ktz.

- radiosa $\mathrm{Ktz}$.

- serians $\mathrm{Ktz}$.

- viridis Ktz.

Surlrella linearis W. Sm.

- Mölleriana Grun.

- robusta Eh.

Synedra ulna Ktz.

BIBliOgR APHIE. - C. Janisch. Zur Charakteristik des Guano's von verschiedenen Fundorten. - C. Mereschkowsky

Note on Diatoms from Chincha Guano

Les préparations proviennent des dépôts suivanțs : Chincha Island,

Chipana. Lobos de Afuera, Pabellon da Pica

Actinocyclus moniliforme Ralfs.

Actinoptychus areolatus Bright.

$-$ capensis Grun.

glabratus Grun.
Actinoptychus Janischii Grun.

- pellucidus Grun.

- splendens Grun. 
Actinoptychus undulatus Eh.

Amphora affinis $\mathrm{Ktz}$.

Anaulus scalaris $E h$.

Arachnoidiscus Ehrenbergii Ralfs.

$$
\text { - ornatus Eh. }
$$

Asteromphalus arachne Bréb.

$\begin{array}{ll}\text { - } & \text { elegans Greo. } \\ \text { - } & \text { flabellatus Grev. } \\ \text { - } & \text { heptactes Ralfs. } \\ & \text { Ralfsianus Grev. }\end{array}$

Aulacodiscus Brightwelli Jan.

$\begin{array}{ll}- & \text { Comberi Arnott. } \\ - & -\quad \text { var. irregularis. } \\ - & \text { crux Eh. } \\ - & - \text { var. tenera O. Witt. } \\ - & \text { formosus Arnott. } \\ \text { - } & \text { Kittonii Arnott. } \\ \text { - } & \text { margaritaceus Ralfs. } \\ - & \text { scaber Ralfs. } \\ \text { - } & \text { - var. Jonesiana. }\end{array}$

Auliscus cœlatus Bail.

$\begin{array}{ll}\text { - } & \quad \text { - var. strigillata. } \\ \text { - } & \text { ovalillei Jan. } \\ \text { - } & \text { prolatus Ratt. } \\ \text { - } & \text { rugosus Ratt. } \\ \text { - } & \text { sculptus Ralfs. } \\ \text { - } & \text { Stöckhardtii Jan. }\end{array}$

Biddulphia aurita Bréb.

- $\quad$ peruviana Grun.
- $\quad$ reticulata.
rhombus Eh.

Campylodiscus clypeus Eh.

$$
\text { - Hodgsonii var. }
$$

Campyloneis Grevillei Grun.

Chactoceros diadema Gran.

$$
\begin{aligned}
& \text { - } \quad \text { dicladia Cast. } \\
& \text { - } \quad \text { didyma Eh. } \\
& \text { - } \quad \text { porenziana Grun. }
\end{aligned}
$$

Cyclotella striata Grun.

Cocconeis costata Greg.

$$
\begin{aligned}
& \text { - } \quad \text { - var. pacifica Grun. } \\
& \text { - } \quad \text { peruviana Eh. } \\
& \text { scutellum. }
\end{aligned}
$$

Coscinodiscun apiculatus Eh.

asteromphalus v. pabelanıca Grun.

v. parallela Grun.

boliviensis Grun.

- bisculptus Ratt.

- centralis Eh.

- concavus Greg.

$-$ concinnus W. Sm.

- v. Jonesiana Ratt.

cristatus Ratt. curvatulus Grun.

- v.densius striata devius A. S. diorama Grun. divisus Grun. excentricus Eh. fasciculatus A. S. flexilis. Ratt. gigas Eh. - var.californica Ratt. Janischii A. S. labyrinthus Roper. lineatus Eh. Macræanus Grev. marginatus Eh. minor Eh. modestus Ratt. oculus-iridis.

- v. subspinosa Grun. odontodiscus Grun. omphalanthus Eh. perforatus Eh.

- : v. cellulosa Grun. Peruanus Grun. radiatus $\mathrm{Eh}$.

- var.media Grun. - var. minor.

subtilis Eh. superba Janish. symbolophorus Eh. umbonatus Greg. Woodwardii Eul.

Endyctia oceanica Eh.

Eucampia zodiacus Eh.

Gephyria media Arnott.

Grammatophora angulosa Eh.
- marina Eh. maxima Grun. 
Grammatophora serpentina Eh.

Hyalodiscus subtilis Bail.

Melosira sol Eh.

- sulcata. Eh.

Navicula aspera Eh.

- didyma Ktz.

- Hennedyi W. Sm.

- - var. subrostrata Meresk.

- liber W. Sm.

- lyra Eh.

- Smithil Bréb.

Pleurosigma balticum IV. Sm.

- formosum IV. Sm.

- nicobaricum Grun.

Pseudauliseus peruoianus Ratt. radiatus Ratt.
Raphoneis lumen $J, B r$.

$$
\text { - superba Jan. }
$$

Rhabdonema adriaticum Eh.

Rhizosolenia styliformis Bright.

Septroneis caduceus $E h$.

- marinum Grun.

Skeletonema costatum. Grev.
- $\quad$ - var. spiralis Me. resk.

Spermatogonia antiqua Leud. Fort.

Stephanopyxis turris Grev.

Synedra affinis $\mathrm{Ktz}$.

Triceratium alternans Eh.

- $\quad$ var . variabilis
- $\quad$ favight.
- $\quad$ pentacrinus Wall.

\section{$N^{\star 8} 457$ et 458 CHERRYFIELD - MAINE (États-Unis) \\ - Dépôt fossile d'eau douce}

Cymbella Ehrenbergii Ktz.

Eunotia bactriana Eh.

- camelus Eh.

- hemicyclus Eh

- diadema Eh.

- incisa Greg.

- triodon Eh.

- tetraodon Eh.

- robusta Ralfs.

Gomphonema acuminatum $\mathrm{Ktz}$.

Navicula acrosphæria W. Sm.

- amphigomphus Eh.

- bacillaris Greg.

- bacillum Eh.

- dilatata Eh.

- dactylus Eh.

- divergens W. Sm.

- elliptica Kítz.

- firma Ktz.

- follis Eh.
Navicula gigas Eh.

- iridis Eh.

- macilenta Eh.

- nobilis Eh.

- polyonca Bréb.

- rliomboides Eh.

- - var. diaphana Eh.

- serians. Bréb.

- stauroneiformis W. Sm.

- tabellaria Eh.

- trochus Eh.

- tumescens Eh.

Stauroneis anceps Eh.

- gracilis Eh.

- leguinen Eh.

- phcenicenteron Eh.

stonopterobia anceps Bréb.

Surirella intermedia IV. Sm.

- linearis WV. Sm.

Tabellaria fenestrata. Ktz. 


\section{No 459 ORDIE - ABERDEEN (Écosse)}

\section{Dépôt tourbeux}

Achnantidium flexellum $\mathrm{Ktz}$.

Cymatopleura hibernica IV. Sm.

Cymbella cistula Hemp.

- cymbiformis Ehr.

- Ehrenbergii Ktz.

- lanceolata Eh.

Bncyonema gracile Rab.

- ventricosum Ktz.

- turgidum Grun.

Epithemia argus Ktz.

- gibba $\mathrm{Ktz}$.

- sorex Ktz.

- turgida v. granulata.

- zebra Ktz.

- $\quad$ - var. proboscidea.

Bunotia argus Eh.

- gracilis Eh.

- impressa Greg.

Gomphonema acuminatum $\mathrm{Ktz}$.

- $\quad$ capitatum $\mathrm{Ktz}$.

- constrictum Ktz.
Gomphonema intricatum $\mathrm{K} \mathrm{lz}$.

Il antzschia elongata Grun.

Mastogloia lacustris Grun.

Melosira crenulata $\mathrm{Ktz}$.

Navicula Brebissonii $\mathrm{Ktz}$. var.

- cardinalis Eh.

- Hebes Ralfs.

- hemiptera Ktz.

-. legumen Eh. var.

- limosa Ktz.

- major $\mathrm{K} t z$.

- nobilis $\mathrm{Ktz}$.

- oblonga Ktz.

- parva Greg. var.

- radiosa $\mathrm{Ktz}$.

- - sphærophora Eh.

Stauroneis phcenicenteron $\mathrm{Eh}$.

Synedra capitata Ktz .

- ulna $\mathrm{Ktz}$.

Tabellaria fenestrata. Ktz.

Nos 460 et 461 . TOOME BRIDGE (Islande)

\section{Dépôt fossile d'eau douce}

Amphora exilis $\mathrm{Ktz}$.

- ovalis Ktz.

- pediculus $\mathbf{K} \mathbf{z}$.

Campylodiscus hibernicus Eh.

Cocconei placentula Eh.

Cymatopleura elliptica Eh.

-

hibernica Rh.

Cyclotella antiqua W. Sm.
Cymbella cymbiformis Eh.

- Ehrenbergii Ktz.

Encyonema gracile Rab.

Epithemia gibba $\mathrm{Ktz}$.

- Hyndmanni W. Sm.

- sorex Ktz.

- turgida $\mathrm{Ktz}$.

- zebra Ktz. 
Gomphonema acuminatum $\mathrm{Ktz}$.

$$
\begin{array}{ll}
\text { - } & \text { constrictum Eh. } \\
\text { - } & \text { geminatum Ag. } \\
\text { subtile Eh. }
\end{array}
$$

Mastogloia lacustris W. Sm.

Melosira arenaria Moore.

- crenulata Ktz.

- Normanni Arnott.

Navicula anglica Ralfs.

- dactylus Eh.

- dicephala Eh.

- elliptica Ktz.

- gentilis Donk.

- gigas Eh.
Navicula major Etz.

- radiosa $\mathrm{Ktz}$.

- rhomboides Eh.

- tenella Ktz.

Pleurosigma attenuatum W. Sm. Stephanodiacus astraea Grun.

Surirella biseriata Bréb.

$$
\text { - - - var. minor. }
$$

- elegans W. Sm.

- robusta Eh.

- turgida W. Sm.

Synedra capitata Ktz.

- ulna Eh.

Tabellarla fenestrata. Ktz.
Actinoptychus adriaticus o. balearica.

$$
\text { - } \quad \text { undulatus Eh. }
$$

Amphora acuta Greg.

- crassa var. A. S. atl. 28/18.

- obtusa Greg.

- spectabilis Greg.

Auliscus cœlatus Bail.

- sculptus Pritch.

Biddulphia pulchella Graz.

$$
\text { - regina W. Sm. }
$$

\section{Campylodiscus adriaticus Grun.}

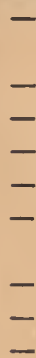

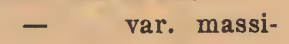

liensis.

biangulatus Greg.

decorus Bréb.

eximiun Greg.

fluminensis Grun.

horologium $\nabla$ : mediterranea.

limbatus Bréb.

samoensis Grun.

Thuretii var. simulans W. Sm.
ClJmacospheniá elongata Bail.

Cocconeis pseudo-marginata.

Coscinodiscu centralis Greg.

- concavus Greg.

- leptopus Grun.

- radiatus Eh.

Navicula aspera Eh.

- Beyrichiana A. E.

- bomboïdes A. S.

- bomibus Eh.

- crabro Eh.

- Hennedyi var. niceaensis H. P.

- - var. manca.

- longa Greg.

- lyra Eh. var. dilatata.

- - var. elliptica.

- - var. recta.

- - var. subtypica.

- multicostata Bréb.

- prætexta Eh.

- separabilis A. S.

- Smithii Bréb.

$$
\text { - var. A. S. atl. 7/19. }
$$

Nitzschia hybrida Grun. var. 
Nitzschia insignis Grog.

- macilenta W. Sm.

- panduriformis Greg.

- valida $\mathrm{Cl}$.

Pleurosigma affine Grun.

$\begin{array}{ll}- & \text { balticum W. Sm. } \\ \text { - } & \text { decorum W. Sm. } \\ \text { - } & \text { formosum W. Sm. } \\ \text { - } & \text { rigidum W. Sm. } \\ \text { - } & \text { robustum Grun. } \\ \text { - } & \text { speciosum W. Sm. }\end{array}$

Rhoicósigma oceanicum H. P. Cette curieuse espéce, qui se retrouve dans la préparation de La Rochelle, a été rattachée par $M$. Petit dans les Diatomées de France au Pl. Wans. beckii, espèce dont elle differe totalement a mon point de vué. (Voir la Monographie des Plourosigma.)

Surirella Baldjickii Eh.

- rastuosa Eh.

- - var.opulenta Grun.

- . - var. collare.

- Guinardii H. P.

- lata W. Sm.

- - var. constricta.

Synedra baculus Greg.

- Gaillonii var. macilenta Eh.

- robusta Pritch.

- undulata W. Sm.

Trlceratium antediluvianum:

- $\quad$ - var. $\beta$.

- $\quad$ arcticum Bright.

- favus Eh.

spinosum Bail.

Nos 463 et 464 SZURDOK-PUSPÖKI (Hongrie)

Dépôt fossile saumâtre

Bibliogra PHiE. - Dr J. Pantocsek. Diatomées de Hongrie, volume II

Amphora acutiuscula $\mathrm{K} \mathrm{tz}$.

- $\quad$ - $\quad \mathrm{v}$. fossilis.

- bituminosa Pant.

- coffeæformis $\mathrm{Ktz}$.

- $\quad$ - $\quad$ v. fossilis.

- - $\quad$ - salinarum.

- fossilis Pant.

- hevesensis Pant.

- minuta Pant.

- protracta Pant.

- salina W. Sm. $\nabla$. fossilis.

- striolata Pant.

- Szaboi Pant.

- Wiesneri Pant.

Cocconeis Galifornica Grun. var. meneletica Pant.

- pediculus Eh.
Cymbella hevesensis Pant.

- hungarica Pant.

- salina Pant.

Epithemia crucæformis Pant.

- salina Pant.

Fragilaria bituminosa Pant.

$\begin{array}{lll}\text { - } & - & \text { v. curta. } \\ - & - & \text { v. elongata. } \\ \text { - } & - & \text { v. minor. }\end{array}$

- brevistriata Grun. v. fossilis.

- microcephala Pant.

- minuta Pant.

Gomphonema olivaceum Eh. $\vee$. fossilis.

Melosira bituminosa Pant.

- Dickeii Ktz. 
Navicula Beckii Pant.

- debilis Pant.

- grata Pant.

- halionata Pant.

- - $\quad$ v. directa.

- heteroflexa Pant.

- - v. constricta.

- - v. minor.

- insignis Pant.

- interposita Lev.

- interrupta Ktz.

- - v. fossilis.

- Kochii Pant.

- Makreana Pant.

- meniletica Pant.

- microrynchus Grun.

- ovalis Hilse.

- _ v. fossilis Pant.

- procera Pant.

- tenella Brẻb. v. fossilis Pant.

- yarrensis Grun.

- $\quad$ - $\quad$. bituminosa Pant.

- - - v. valida Pant.
Nitzochia bituminosa Pant.

- - $\quad$ - . tenuior.

- frustulum Grun.

- - v. acuta Pant.

- - v. curvata Pant.

- - v. minuta Pant.

- , - v. obtusa Pant.

- $\quad$ - $\quad$. producta Pant.

- hevesensis Pant.

- neogena Grun.

- spectabilis Grun.

- Szaboi Pant.

- Tabyana Grun.

Staurosira mormonorum Grun.

- venter v. fossilis Grun.

Surirella costata Neup.

- Neupauerii Pant.

- patella Eh.

- rotunda Pant.

- - v. minor.

Synedra salinarum Pant.

$\mathrm{N}^{03} 465$ et 466 TUF DE LA, MONNE. - PUY-DE-DOME (France)

Bibliographie. - F. Héribaud. Les Diatomées d'Auvergne.

Achnanthes subsessilis Ktz .

$$
\text { - ventricosa Eh. }
$$

Amphora ooalis $K t z$.

- pediculus Ktz. fa major.

Asterionella formosa Hass. var. gracillima.

Campylodiscus hibernicus Eh.

Cocconeis californica Gr. $\nabla$. subcontinua.

- lineata $G r$.

- - var. euglypta.

Cymatopleura apiculata W. Sm.

- $\quad$ elliptica W. Sm.

- hibernica W. Sm.

- $\quad$ - $\quad$ - major.

- solea Bréb.
Cymbella affinis $K t z$.

- - var.

- amphicephala Naeg.

- arcus Eh.

- cistula Hemp.

- - var. maculata.

- cuspidata Ktz.

- cymbiformis Bréb.

- Ehrenbergii Ktz.

- gastroïdes $\mathrm{Ktz}$.

- - var. minor.

- helvetica Ktz.

- lanceolata Eh.

- parva V.H.

- turgidula Grun.

Diatoma anceps $E h$. 
Diatoma hiemale Ktz.

- - var. mesodon.

Encyonema lunula Grun.

Epithemia argus Ktz. v. amplicephala. gibba Ktz.

- - var. ventricosa.

- gibberula Ktz. $\nabla$. producta.

- sorex Ktz.

- turgida Ktz.

- - var. granulata.

- $\quad$ - var. vertagus.

- zebra Ktz.

- - var. proboscidea.

Eunotia bicapitata Grun.

- gracilis Rab.

- minor Rab.

- pectinalis Rab. $f_{\mathbf{a}}$ elongata.

- Soleirolii Ktz.

Iragilaria bidens Heib.

- brevistriata Grun. v. pusilla.

- $\quad$ - v. subacuta.

- construens Grun. v. binodis.

- - v. venter.

- elliptica Schum.

- intermedia Grun.

Comphonema acuminatum Ell.
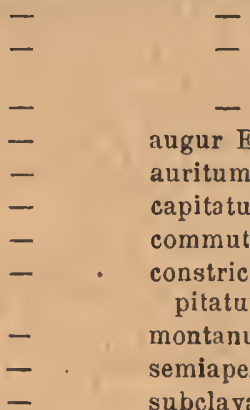

v. laticeps.

$\nabla$. trigonocephala.

$$
\text { - var. }
$$

augur Eh, var.

auritum Braun.

capitatum Eh.

commutatum Grun.

- constrictum Eh. v. subcapitatum.

montanum Schum,

- semiapertum $\nabla$. tergestina.

- subclavatum Grun:

Melosira crenulata $\mathrm{Ktz}$.

- - var. ambigua.

- distans Ktz.

- laevis Grun.

- Tonuigsima Grun.

- varians Ag.

Meridion circulare Ag.

Navicula acrosphæria Brẻb.

- americana Eh. $\nabla$. bacillaris.

- amphirynchus Eh.
Navicula ampliata Eh.

- aponina Ktz.

- divergens $W . S m$.

- - var. undulata.

- elliptica var. oblongella.

- firma Ktz.

- gastrum Donk.

- gibba Ktz.

- gracillima Ralfs.

- interrupta W. Sm.

- legumen Eh.

- limosa Grun.

- - - gibberula.

- major Ktz.

- mesolepta Eh.

- - - v. stauroneiformis.

- mesostyla Eh.

- minuscula Grun.

- nobilis Kitz.

- producta W. Sm.

- pupula Ktz. fa minuta.

- radiósa $\mathrm{K} t z$.

- stauroptera Grun.

- viridis $\mathrm{K} t z$.

- - var. commutata.

Nitzschia sigmoidea $W . S m$.

Pleurosigna attenuatum W. Sm.

- Spencerii W. Sm.

IR holcosphenia curvata Grun.

Stauroneis acuta W. Sm.

- ancops Eh.

- gracilis Eh.

- phœnicenteron Eh.

- platystoma Eh.

Stephanodiscus astraea Grun.

- - - var. minutula.

Surirella apiculata W. Sm.

- bifrons $\mathrm{Ktz}$.

- biseriata Bréb.

- linearis W.Sm.

- minuta Bréb.

- norvegica Eh.

- saxonica Auers.

- turgida W. Sm.

Synedra acus Grun.

- capitata Eh.

- delicatissima W. Sm.

- ulna Eh. 
No $46 \dot{7}$ LE HAVRE (France)

Pleurosigma lecoriun W. Sn. var. Dalmaticuin

Nos 468 et 469 SAN-PEDRO. - CALIFORNIE (États-Únis)

Dépôt fossile marin

Actinocyclus incertus Grun.

Actinoptychus biformis $\mathrm{J}$. Br.

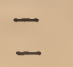
glabratus $\nabla$. incisa. halionyx $v$. dilatata $n$. var. Double structure marginale seulement, aréas développées, cel- les des compartiments épineux dilatées, mais non cruciformes.

- intermedius Gr. var.

- nitidus Grev.

- - - var.

- perisetosus J. Br. var. inermis n. var. Diffère du type par l'absence d'épines dans les compartiments secondaires et par la forme de ses appendices évasés et non tubulaires.

undulatus Eh.

Amphora crassa Greg.

- proteus Greg.

Anaulus mediterraneus Grun. var.?

Aulacodiscus amonus Greo.

- Kittonii Arn.

Auliscus Biddulphia Kïtt. var.

- prominens.

- elegans Ratt.

- intestinalis $A$. S.

- pruinosus Bail.

- - v. subreticulata.

- punctatus Bail. var.

Biddulphia aurita Bréb.

- granulata Rop. oar.

- Tuomeyi Rop.

Campyloneis regalis 'Grun.

Cerataulus turgidus Eh.

Chatoceros californicus Grun. (spores).

- diadema (spores).

Cladogramma californicum Eh.

Cocconeis dirupta Eh.

- praestans A. S.

- scutellum Eh. v. Baldjickiana.

- superba Jan.

Coscinodiscus apiculatus Eh.

$-$

$-$

$-$

$-$

$-$

$-$

$-$

-

一

-

-

$-$

$-$

=.

$-$

$\overline{-}$ argus Eh.?

diorama Grun.

dubius Ratfs.

exentricus Eh.

heteroporus Eh. v. moroniensis.

leptopus Grun.

marginatus Eh.

- var. inter. media.

var. submarginata.

monicæ Ratt.

nitidus Greg.

obscurus A. S.

oculus-iridis Eh.

radiatus Eh.

robustus Greo.

strigillatus Witt.

subtilis Eh.

symbolophorus Grun. 
Dimeregranma minus Ralfs.

Diploneis apis Eh.

- bombus Eh.

- crabro Eb.

- didyma Eh.

- Smithii CI.

Entopyla australis Eh. v. incurvata.

$$
\text { - - o. ocellata. }
$$

Epithelion curvatum Pant.

Bunotogramma laeois Grun.

Grammatophora robusta Dip.

Hemiaulus ambiguus Jan.

- polycistinorum Eh.

- $\quad$ sp.? (spore).

Ltholesmium californicum Grun.

$$
\text { - minusculum Grun. }
$$

Melosira clavigera Grun.

$$
\begin{aligned}
& \text { - sol Ktz. } \\
& \text { - sulcata Ktz. } \\
& \text { - - } \quad \text {. coronata. } \\
& \text { - - o.biseriata. } \\
& \text { - - v. radiata. }
\end{aligned}
$$

Mülleriella limbata V. H.

Navicula clavata Greg.

- excacata Greg.

- lyraEh.

- $\quad$ - var. elliptica.

- nebulosa Greg.

- nummularia Greo.

- prætexta Eh.

- spectabilis Greo.

vitzsehia sigma W. Sm. v. rigida.
Opephora Schivartzii P. P.

Orthoneis splendida Grun.

Plagiogramma Gregorianum Greo.

Rhabdonema adriaticum $K t$.

Raphoneis amphiceros Eh. v. elongata.

llouxia Californica M. P., n. sp. Diffëre du R. Peragalli en ce que toute la surface est couverte de stries indistinctementponctuées. Long. $40 \mu$, largeur $7 \mu, 14$ stries en $10 \mu$.

- Californica car. elongata plus étroite et plus allongéo que le type. Long. $60 \mu$, larg. $5 \mu$.

Stephanopyxis turris Ralfs.

$$
\text { - } \quad \text { - } \quad \text { - } \quad \text { ४. crassispina. }
$$

Stictodiscus Buryanus Grev. var.

- Californicus Grun.

- - var nankoorense.

Trachyneis aspera $C l$.

- oblonga Bail.

Triceratium nicobaricum $G r$.

- $\quad$ paralellum o. coloniensis.

Xanthiopyxis alata Eh.

- aristata J. Br.

- cingulata Eh.?

- oblonga Eh.

- umbonata Greo.

N॰ 470 LAC DE GENEVE (Suisse)

Amphora affinis $\mathrm{Ktz}$.

- gracilis Eh.

- ovalis $\mathrm{Ktz}$.

- pediculus Ktz.

Cocconeis pediculus Eh.

- placentula Eh.

Cymatopleura apiculata W. Sm.

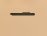
elliptica WV. Sm.

$$
\text { - solea. }
$$

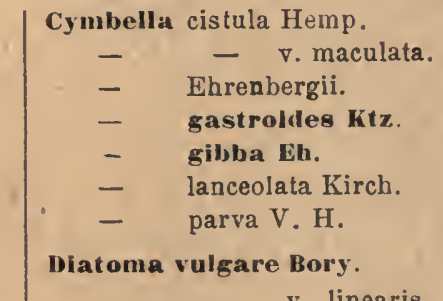

Cymbella cistula Hemp.

- $\quad$ - v. maculata.
- $\quad$ Ehrenbergii.
- gastroldes Ktz.
- gibba Eh.
- lanceolata Kirch.
- parva V. H.

Diatoma vulgare Bory. 
Encyonema cospitosum Ktz.

- prostratum Ralfs.

- ventricosum Grun.

Epithemia gibba $K t z$.

- sorex Ktz.

Fragilaria brevistriata Grun.

- capucina Desm.

- $\quad$ - v. mesolepta.

- construens $\mathrm{v}$. binodis.

Gomphonema acuminatum Eh.

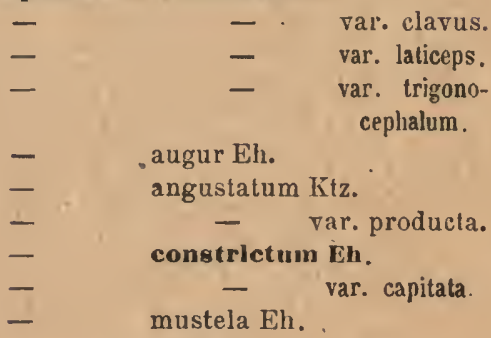

Hantzschia amphioxys Grun.

Melosira varians Ag.

Navicula ambigua Eh.

- amphirhynchus Eh.

- amphishoena Bory.

- avenacea Bréb.

- Brebissonii Ktz.

- cuspidata Ktz.

- dubia Eh.

- firma Ktz.

- - var. subampliata.

- gracilis Eh.

- humilis Donk.

- iridis Eh.
Navicula limosa hitz.

- - var. gibberula.

- - var. undulata.

- menisculus Schum.

- radiosa $\mathrm{Ktz}$.

- - var. acuta.

- rhyncocephala var. amphiceros.

- ventricosa Ktz. var. trunculata.

- viridula Kítz.

vitzschia angustata W. Sm.

- dissipata Grun.

- - - v. media.

- linearis W. Sm.

- sigmoidea W: Sm.

- tenuis W. Sm.

- Tryblionella Hantz.

- vermicularis llantz.

Pleurosigua acuminatum Grun.

Rhoicosphenia curvata Grun.'

Surirella angusta Grun.

- linearis $\mathrm{Ktz}$.

- . - var. amphioxys.

- minuta Bréb.

- $\quad$ fa longior.

- ovata Ktz.

- splendida Eh.

- tenera Greg.

Synedra capitata Eh.

- ulna Eh.

- - v. aqualis.

- $\quad-$ v. amphirhynchus.

- $\quad-$, v. danica.

- - v. oxyrhynchus.

- - v. vitrea.

Van lleurckia vulgaris V. II.

$N^{\text {s }} 471$ à 473 LOUBET. - CALIFORNIE (Etats-Unis)

Dépot fossile marin

Actinocyclus moniliformis Ralfs.

$$
\text { - } \quad \text { sparsus Ratt. }
$$

Actinoptychus areolatus Eh.

- Clevei A. S. oar.

- undulatus Eh.
Araclinoidiscus ornatus $\nabla$. Montereyana.

Asteromphalus Darwinii Eh.

senectus Temp. et $\mathrm{Br}$. Wallichianus Ralfs.

Biddulphia longicruris Gran. 
Campyloneis Grevillei Grun.

$$
\text { - var. regalis. }
$$

Chatoceros californicum Grun.

$$
\text { - monicæ Grun. }
$$

- spinosum Loud Fort.

- Wighamii Bright.(Endocyst)

Cladogramma Californicum Eh.

Coscinodiscus Eginensis A. S. var curvatula. - Présente, en partie, l'arrangement des cellules du Cosc. curvatulus.

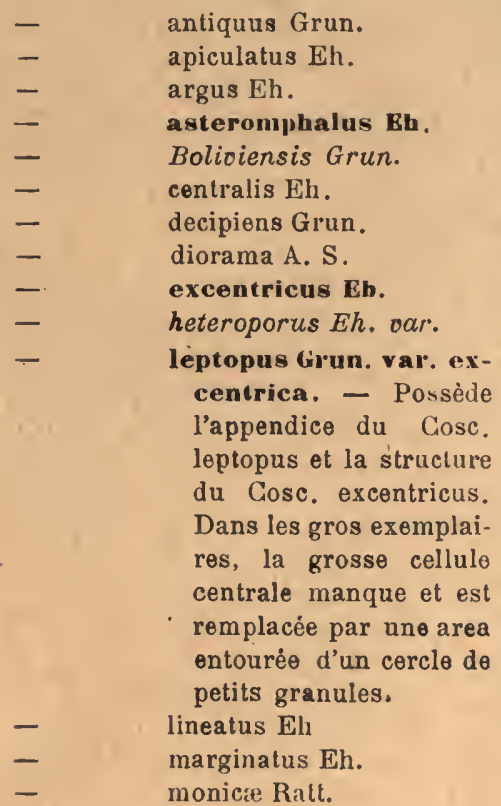

Coscinodiscus pobile Grun.

$$
\begin{aligned}
& \text { - obscurus A.S. } \\
& \text { - oculus iridis El. } \\
& \text { - } \quad \text { var. Mor- } \\
& \text { siana. } \\
& \text { - } \quad \text { radiatus Eh. } \\
& \text { - Symbolophorus Grun. } \\
& \text { - tuberculatus var, monicæ. }
\end{aligned}
$$

Dicladia capreolus $E$.

Eunotia gibba Bail.

Ilemiaulus ambignus Grun.

Lithodesmium californicum Grun.

$$
\text { - minutissimum Grun. }
$$

Melosira sulcata var. coronata.

Navicula lyra Eh.

- pennata A.S.

Nitzschia gracilis Hantz.

Opephora Schwartzii P. P.

Raphoneis amphiceros $\mathrm{v}$. californica.

- - var. elongata.

Stephanopyxis aristata T'emp. et $\mathrm{Br}$.

$\begin{array}{ll}\text { - } & \text { corona Grun. } \\ \text { - } & \text { turris Ralfs. } \\ & \text { sp. A. S. atlas 123/11. }\end{array}$

Stictodiscus californicus Grev.

Thalangiothrix nitzchioicles Grun.

- Frauenfeldii Grun. var.

- sinuata. - En fragments plus ou moins sinueux, 11 points en $10 \mu$.

Triceratium quadrangulare Greo.

Van Ieurckiella admirabilis Pant.
Actinoptychus glabratus Grun. undulatus Eh. sp. A. S. atlas $153 / 6$.
Arachnoidiscus indicus Eh.

$$
\text { - ornatus Eh. }
$$

Aulacouliseus scaber Ralfs. 
Coscinodiscus asteromphalus Eh. var. hybrida.

$\begin{array}{ll}\text { - } & \text { leptopus Grun. } \\ \text { - } & \text { oculus-iridis Eh. }\end{array}$

Craspedodiscus insignis A. S. (Par son bord rugueux, cette espèce appartient aux Endictya.)

Endictya oceanica Eh.

Isthmia enervis Eh.
Isthmia neroos $\alpha$ Ktz.

Navicula formosa Greg.

Pleurosigma balticum W. Sm.

Podosira argus Grun.

stictocliscus californicus var. aggregata. - Granules gros, seriés en rayons intersulqués jusqu'à moitié du rayon, le ceńtre restant a vec quel. ques granules èpars.

Surirella sp. ? A: S. atlas 24/25.
No 476

Biddulphia aurita Bréb.

$$
\text { - mobiliensis Bail. }
$$

Chætoceros curvisetum Cl.

- didymum Eh.

- laciniosum Schütt.

- scolopendra Cl.

Coscinodiscus oculus-iridis Eh.

Ditylium Brightwellii West.

Eucampia zodiacus Eh.

Guinardia flaccida $\mathrm{H}$. Per .

Nitzschia socialis var. baltica.

Pleurosigma angulatum W. Sm. - naviculaceum Bre̊b.

Rhizosolenia imbricata Bright.

- Shrubsolii Cl.
TROUVILLE (France)

\section{Pélagique}

Triceratium Crouanii $M$. Per. - De forme analogue à celle du Tric. Archangelskianum, mais à granules formant des 'lignes rayonnantes bien marquées, extrémités oculiformes, sans épines. Longueur du rayon, $55 \mu$, côté $90 \mu$ de long. papillatum var. acuta. Diffère de A. S. atlas $167 / 6$ par ses angles plus aigus et I son centre brusquement surèlevé. Longueur du rayon $40 \mu$, côté $60 \mu$. Roberstianum Grev.

No 477 CAP DE BONNE-ESPÉRANCE (Afrique)

\section{Pélagique}

Bacteriastrum varians Laud. Chretoceros coarctatum Laud.
Chratoceros compactum Schatt. furca $\mathrm{Gl}$. 
Chatoceros Lorenzianum Grun.

Melosira sol Eh.

Rhizosolenia alata Jright. . rabusta Norm.
Rhlzosolenia setigera Bright. styliformis Bright.

Temperei H. Per. var. acuminata.

\section{Nos 478 et 479 COTES DE LA NOUVELLE-ZÉLANDE}

\section{Lavage d'Haliotis bleus}

Ces matériaux et leur analyse nous ont été communiqués par M. Paul Bergon

Actinocyclus curvatulus Janisch.

- nebulosus M. Per.

$$
\text { - Ralfsii W. Sm. }
$$

Actinoptychus adriaticus Grun. $\mathbf{v}$. balearica?

- hexagonus var.

- splendens Shad. et var.

$$
\text { - trilingulatus. }
$$

Alloinoeis antillarum et var. Cl. et Grun.

Amphora crassa Greg. var.

- mexicana A. S.

- porcellus Kitton. var.

Amphitetras antediluoiana $\mathrm{Ehr}$. oar.

- tesselatus Shadb.

Arachnoidiscus ornatus $E h$.

Asteromphalus arachne Bréb.

Aulacodiseus crucifer Schadb.

$$
\text { - } \quad \text { Kittonii Arnott. et var. }
$$

Auliscus colatus Bail v. strigillata A.S. - ocalis Arnott.

Biddulphia Mac Donaldii Pritch.

- Grundleri A. S.

- pulchella Gray. et var.

- reticulata Roper. et var.

- rhombus Eh.

- Tuomeyi Bail.

Campylodiscu biangulatus Greo.

- circumactus A. S.

- crebrecostatus Greo.o.n.

- Domelianus Grun. var.
Campylodiscus decorus Bréb. o. eudecora.

echeneis Eh.

fluminensis Grun.

Kinkeri A. S. et var. nova Kittonianus Greo. car.? latus Shadb. Pfitzeri A. S. car. Thuretii Bréb.

Samoensis Grun. et o.n. Wallischianus(fragments)

Campyloneis Grevillei (W. Sm.) Grun. var.

- microsticta Grun.

Cerataulus Smithii Ralfs.

Climacosphenia elongata Bailey. var.

Cocconeis australis $P . P$.

- calata Arnott.

- curoirotunda Temp. et Br. var.

- bifrons Grun.

- imperatrix o. pactica Grun.

- pseudo-marginata Greg.

- Schleinitzii Jan. var. (Forme à côtes perlées beaucoup plus nombreuses et rapprochées.)

- scutellum Eh, v. dilatata A.S.

Coscinodiscus curoatulus Grun. denarius Eh. var. Janischii A. S. lineatus Eh. fa latestriata. 
Coscinodiscus nitidus Greg. oar.

$\begin{array}{ll}\text { - } & \text { nodulifer A. S. } \\ \text { - } & \text { oculus-iridis Eh. var. } \\ \text { - } & \text { robustus Greo. }\end{array}$

Cosmiodiscus elegans Grev. var.

Craspedodiscus coscinodiscus Ehr.

Cyclotella stylorum Bright.

Eurlyctia oceanica Eh.

Entopyla australis $E h$.

- ocellata var. pulchella Grun.

Euphyllorlium spathulatum Shadb.

Eupodiscus Jonesianus Grev, var.

Glyphodiscus stellatus Greo.

Hyalodiscus maximus Grun.

$$
\text { - stelliger Bail. }
$$

Isthmia nervosa Kütz.

Leudugeria Janischii Temp.

Mnstoglola Craveni Leud. Fort. oar. (A placer entre Craveni et cru- ciata. 1

- quinquecostata Grun. oar. elongata Leud. Fort.

Melosira sol Eh.

- sulcata Eh. v. coronata.

- teres $\mathrm{Br}$. var.

Navicula abrupta Greg.

- brasiliensis Grun oar.

- bullata Greo.

- clacata Greg. var.

- crabro Eh. v. multicostata.

- - var. separabilis A.S.

- Johnsoniana Greo.

- indica Greo. var.

- lyra Eh. typica.

- - oar. noo.

- - var.subcarinata Grun.

- maxima Donkin. var.?

- notabilis Greo.

- permagna Bail.

- proetesta Eh.
Navicula Smithii Bréb.

- ispectabilis oar.?

- suborbicularis Greg. var.

Nitzschia panduriformis Greg et var.

Pleurosigma formosum W. Sm. oar. balearica Grun.

rigidum.

Podosira hormoides $\mathrm{Ktz}$. var. Montereyi?

Ihabdonema hamuliferum Kitton.

- mirificum W.Sm.

scoliopleura tumida Rab.

Scoliotropis latestriata Bréb.

Stephanopyxis Grunowii Grove et St. var.?

Surirella contorta Kitt.

- eximia Grev. var.

- fastuosa Eh. et var.

- - oar. noo.

- incuroata A. S.

- Macræana Grev. var.

- pandura Per. var.

Synedra robusta Ralfs.

- undulata Greg.

Terpsinoë americana Greg. . .

- musica Eh.

- inflata $J . B r$.

Tracheneis aspera Eh. et var.

Tricoratium affine Grun.

- arcticum Bright.

- attenuatum Grev. var.

- bicorne Gl.

- formosum fa trigona.

- _ fa tetragona

- fimbriatum Wall.

- incisum A. S.?

- latum var. dissimula?

- Montereyi Bright.

- pentacrinum fa tetragona.

- - fa pentagona.

- scitulum fà trigona.

- . - fa tetragona. sculptum Shadb. 
Nos 480 et 481 COLLINE DE SODDO (Ethiopie)

])épôt fossile d'eau douce

BIBLIOgRaPHiE. - Dott. Achille Forti. Diatomacee quaternarie subfossili d'acqua dolce raccolte in Etiopia dal Dott. Giovanni Negri (Contribuzioni Diatomologiche Venezia 1910).

Amphora lybica Eh.

- pediculus Grun.

Cocconeis lineata Eh.

- pediculus Eh.

- placentula Eh.

Cymbella Abyssinjca Grun.

- Australica Cl.

- lanceolata Kirch.

- maculata Ktr.

Camplodiscus clypeus Eh.

$$
\text { - contortus Pant. }
$$

Cerataulus orbicularis Forti. n. sp.

Cymatopleura elliptica var. constricta Grun.

$$
\begin{array}{cc}
- & -\quad \text { fa subconstric: } \\
\text { - } & \text { ta V.H. } \\
- & \text { solea } W . \text { Sm. } \\
\text { - } & \text { - oar. claoata O. M. } \\
\text { - } & \text { - var. laticeps O.M. } \\
\text { - } & \text { - var. rugosa O. M. } \\
& \text { - var. subconstricta } \\
& \text { O. Mull. }
\end{array}
$$

Diatoma oulgare Bory.

Encyonema Grandi F. H.

- intermedium F. H.

- turgidum Grun.

Epithemia argus $\mathrm{Ktz}$. ventricosum Grun.

- - la minor.

- cistula Ralfs.

- Hyndmannii W. Sm.

- sorex Ktz.

- turgida Ktz.

- Westermanni Ktz.

- zebra Ktz.

Eunotia pectinalis Rab.
Fragilaria capucina Desm.

- construens Grun.

- $\quad$ - var. venter Grun.

- virescens Ralfs. var. exigua Gr.

Gomphocymbella Beccarii (Grun.) Forti.

Gomphonema intricatum Ktz.

$$
\begin{array}{ll}
\text { - } & \text { clavatum Eh. } \\
\text { - } & \text { commutatum Grun. } \\
\text { - } & \text { subclavatum Grun. } \\
\text { turris Eh. }
\end{array}
$$

IIantzschia Abyssinica Grun.

Melosira arenaria Moore.

- Bruni M. Per. et F. H.

- canalifera J. Br. et F. H.

- crenulata Ktz.

- distans Ktz.

- granulata Ralfs.

- varians Ag.

Navicula ambigua Eh.

- - fa craticula.

- amphisbcena Bory.

- amphirhynchus Eh.

- dubia Eh.

- elliptica $\nabla$. minutissima Grun.

- Elsae Thum, et Pant.

- gibberula Ktz.

- mutica Ktz. var. Cohnia (Hilse) Gr.

- placentula Ktz.

- pupula Ktz.

- sculpta Eb.

- sphærophora Ktz.

Nitzschia commutata Grun.

- intermedia Hantx.

- palea W. Sm. 
Pinnularia cardinalis $W . S m$.

- divergens W. Sm.

- gigas Eh.

- major Eh.

- molaris Grun.

- viridis Eh.

Rhopalodia ascoidea O. Mull.

- gibba O. Mull.

- gracilis O. Mull.

- hirudiniformis O. Mull.

Stauroneis acuta W. Sm.

- Heufleriana Grun.

- Javanica Cl. var. minor Hi.
Stauroneis phœnicenteron Eh.

Surlrolla biseriáta Bréb.

- constricta Eh.

- ooalis Bréb.

- ocata Ktz.

- spiralis Ktz.

- splendida Ktz.

Synedra oxyrynchus Ktz.

- - var. medioconstricta Forti.

- ulna var. lanceolata Grun.

- - var. longissima J. Br.

- - var. vitrea V. H.

$N^{\circ 3} 482$ et 483

LAC ZIJAY (Ethiopie)

Dépôt fossile d'eau douce

BIBllographiE. - Dott. Achille Forti. Diatomacee quaternarie subfossili d'acqua dolce raccolte in Etiopia dal Dott. Giovanni Negri (Contribuzioni Diatomologiche Venesia 1910).

Amphora lybica Eh.

- ovalis Ktz.

Cocconeis pediculus Eh.

$$
\text { - placentula Eh. }
$$

Cymbella Abyssiniea Grun.

Campylodiscus clypeus Eh.

$$
\text { - echeneis Eh. }
$$

$$
\text { - noricus Eh. }
$$

Cymatopleura elliptica W. Sm.

$$
\begin{array}{cc}
\text { - } & \text { subconstricta.Witt. } \\
\text { - } & \text { solea W. Sm. } \\
\text { - var. subsconstricta } & \text { O. Mull. }
\end{array}
$$

Cerataulus orbicularis Forti. n. sp. Cyolotella comta $\mathrm{Ktz}$. var. trinotata F. H.

Encyonema Grandi F. H.

Epithemia argus Ktz.

$\begin{array}{ll}\text { - } & \text { cistula Ralfs } \\ \text { - } & \text { sorex Ktz. } \\ \text { turgida Ktz.' }\end{array}$

Fragilarla construens Grun.

$$
\text { - - var. venter Grun. }
$$

Gomphocymbella Beccarii (Grun.) Forti.

Gomphonema intricatum $\mathrm{Ktz}$.

- subclavatum Grun.

Melosira crenulata $K t z$.

- italica Ktz.

- $\quad$ - var. tenuissima (Grun.)

$$
\text { O. Mull. }
$$

- Nyassensis 0. Mull.

Navicula amphisbœna Bory.

- bacillum Eh.

- dubia Eh.

- elliptica Ktz.

- gastrum Eh.

- oblonga Ktz.

- Perrotettii Grun.

- placentula fa minor Grun.

- pupula Ktz.

- radiosa $K t z$.

- - var. acuta Grun. 
Navicula silicula Eh.

- sphærophora Ktz.

- tuscula Eh.

Nitzschia acuminata Grun. - sinuata Grun.

Pinnularin viridis (N.) Eh.

Rhoicosphenia curvata Grun.

Rhopalodia clavata (Dickie) Forti. - fa perlonga Friche.

$\begin{array}{ll}\text { - } & \text { gibba O. Mull. } \\ \text { - } & \text { gracilis O. Mull. } \\ & \text { rentricosa O. Mull. }\end{array}$

Stauroneis Heidenii Forti. $(=S$. inflata Heid. nec. Ktz.)

- legumen Ehr.

Surirella biseriata Bréb.

- Fullebornii O. Mull. var. constricta O. Mull.

- $\quad$ - var.elliptica 0.M.

- Mallombae O. Mull.

- Mülleri Forti.

Synedra familiaris Ktz.

- ulna var. lanceolata Grun. fa. longior $\mathrm{Gr}$. et $\mathrm{V} . \mathrm{H}$.

- - var. longissima J. Br.

Terpsinoe musica Eh.

\section{LLYN ARENIG BACH (Pays de Galles - Angleterre) \\ Dépôt fossile d'eau douce}

Encyonema gracile Rab.

Epithemia turgida Ktz.

Eunotia arcus Eh.

$$
\begin{aligned}
& \text { - incisa Greg. } \\
& \text { - major Rab. } \\
& \text { - } \quad \text { var. bidens. } \\
& \text { - pectinale Rab. } \\
& \text { - } \quad \text { - var, undulata Ktz. } \\
& \text { - vodon Eh. }
\end{aligned}
$$

Gomphonema acuminatum Eh.

$$
\text { - } \quad \text { intricatum Ktz. }
$$

Navicula alpina W. Sm.

- cardinalis Eh.

- divergens W. Sm.
Navicula gibba Eh. var.

- gigas Eh.

- major Ktz.

- mesolepta Ktz.

- serians $\mathrm{Ktz}$.

- transversa A. S.

Melosira distans Ktz.

- sp.?

Stauroneis gracilis Eh.

Surirella linearis. W. Sm.

- - var. constricta.

- robusta Eh.

- saxonica Auers.

Tahellaria fenestrata Kitz.

Van lleurchia rhomboïdes Bréb. 


\section{N० 486}

No 487
LE HAVRE (France)

Synedra ulna var. Danica.

PYRENÉES (France)

Odontidium hyemale var. Lyngh.

Nos 488 et 489 BLANKENBERGHE (Belgique)

Actinocyclus subcrassus Ratt.

Actinoptychus splendens Ralfs. - undulatus $\mathrm{Eh}$.

Biddulphia aurita Bréb. - rhombus Eh.

Campylosira cymbelliformis Grun.

Cerataulus Smithii Ralfs.

Coscinodiscus denarius A.S.

- excentricus Eh.

- lineatus Eh.

- radiatus $\mathrm{Eh}$.

Ditylium sol V. H.

Hyalodiscus stelliger Bailey.

Melosira sulcata $\mathrm{Ktz}$.

- Westii W. Sm.

Navicula maxima Greg.
Navicula maxima var. umbilicata Grun.

Nitzschia panduriformis var. minor. - sigma.

Pleurosigma restuaril W. Sm .

- affine Grun.

- angulatum W. Sm.

- balticum W. Sm.

- hippocampus W. Sm.

- iquadratum W. Sm.

- Spencerii W. Sm.

- strigosum W. Sm.

Raphonois amphiceros Eh.

$$
\text { - surirella Eh. }
$$

Scoliopleura tumida Grun.

Surirella gemma Eh.

Triceratium favus Eh.
Actlnocyclus Ralfsii Pritch.

Actinoptychus undulatus Eh.

Cerataulus Smithii Ralfs.
Coscinodiscus concinnus $W$. Sm.

- gigas Eh.

- oculus iridis Eh. 
Coscinodiscus radiatus Eh.

- symmetricus Grev. var. (= Cosc. denariús A.S.)

Cyclotella striata Ktz.
Cyclotelia stylorum Bright.

Eupodiscus radiatus Bail.

Polymyxus coronalis Bail.

Triceratium favus Eh.

Nos $49 \mathrm{l}$ et 492 LAMIOUZE-ROCHEFORT - PUY-DE-DOME.

\section{(France)}

Aohnanthes lanceolata Bréb.

Anphora gracilis Eh.

Cocconels lineata Eh.

- molesta Ktz.

- Placentula Eh.

Cymatopleura solea W. Sm.

Cymbella affinis Ktr.

- cistula Kirch.

- var. fusidium.

- cuspidata Ktz.

- Ehrenbergii Ktz.

- Janceolata Kirch.

- leptoceros Rab.

- maculata Ktz.

- parva V. I.

Diatoma anceps Kirch.

Epithemia gibba kítz.

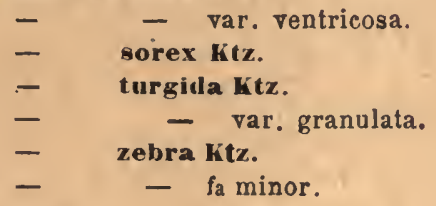

Eunotia linaris Grun.

Fragllarla capucina var. mesolepta.

- construens Grun.

- - var. binodis.

- - var.excisa v. $\mathrm{n}$.

Le bord présente une forte encoche médiane ot unilatéralo.

- construens var. pumila.

- - var. venter.

- elliptica Grun.

- intermedia Grun.
Gomphonema acuminatum Eh:

$$
\begin{aligned}
& \text { - } \quad \text { - } \quad \text { - var. clavus. } \\
& \text { - - var. pusilla. } \\
& \text { - angustatum Ktz. } \\
& \text { - } \quad \text { - var. producta. } \\
& \text { - augur Eh. } \\
& \text { - auritum Braun. } \\
& \text { - constrictum Eh. } \\
& \text { - - } \quad \text { var.capitatum } \\
& \text { - - var. subcapi- }
\end{aligned}
$$

- intricatum $\mathrm{Ktz}$.

- , turris Eh.

Melosira crenulata $K t$.

- lævis Eh

- varians $\mathrm{Ag}$.

Meridion constrictum Ralfs.

Navicula cuspidata $K t z$.

- elliptica Ktz:

- firma Ktz. car. subampliata.

- iridis Eh.

- mesolepta Ktz. o. stauroneiformis.

- pupula Ktz.

- radiosa kitz.

- - - var. acuta.

- rupestris Hantz.

- viridis $\mathrm{Ktz}$.

Vitzschia neutiuscula Grun.

- fossilis Grun.

- intermedia Grun.

- minuta Bleisch.

- sigmoïdea W. Sm.

Rhoicosphenia curvata Grun. 
Synodra acus Ktz.

- - var. fossilis.

- capitata Eh.

- delicatissima W. Sm.

- $\quad$ - var. mesolepta.

- ulna Eh.
Synedra ulna var. æqualis.

- - $\quad$ - va.. danica.

- - var. lanceolata.

- - - fa brevis.

- - var. oxyrhynchus.

- - var. vitrea.
Campylodiscus clypeus Eh.

Cymbella lanceolata Eh.

Nitzchia spectabilis Eh.

Navicula bohemica.
Navicula biceps $E h$.

- major Ktz.

- silicula Eh.

- sculpta Eh.

- viridis Eh.

No 494

SOURCE DE CHATEL-GUYON

PUY-DE-DOME (France)

\section{BIBLIOGRAPHiE. - Frère J. Héribaud. Les Diatomées d'Auvergne}

Achnanthes lanceolata $\mathbf{v}$. elongata.

Amphora ooalis $K t z$.

Cocconeis placentula Eh.

Diatoma hyemale $\nabla$. mesodon.

Encyonema ventricosum Grun.

Eunotia minor $R a b$.

Fragilaria construens Grun.

$$
\begin{array}{lll}
\text { - } & \text { - var. binodis. } \\
\text { - } & \text { - var. excisa. } \\
\text { - } & \text { - var. venter. }
\end{array}
$$

Gomphonema constrictum $E h$.
Melosira crenulata Ktz.

- laevis Eh.

- laeoissima Grun.

- varians $\mathrm{Ktz}$.

Navicula atomoïdes Grun.

- elliptica Ktz.

- pseudo-bacillum Grun.

Nitzschia minuta Bleisch.

Synedra ulna Eh.

- - car. amphyrynchus.

- - var. æqualis.

- - oar. danica. 
$N^{\bullet} 495$ VAL D'ENFER - PUY-DE-DOME (France)

\section{Bibliographie. - Frère J. Héribaud. Les Diatomées d'Auvergne}

Achnanthes Biasolettiana Grun.

- minutissima var. cryptocephala Ktz.

Cymbellı maculata Ktz.

Denticula valida Ped.

Epithemia gibberula Ktz.
Eunotia minor $R a b$.

Navicula cincta Eh.

- var. Heufleri.

Nitzschia vitrea var. Gallica.

Ithoicosphenia curvata Grun.

Surirella ovalis Brób.

$N^{\circ} 496$

HELSJON - HOLLANI) (Suède)

Anomoeonei follis Ehr.

Cymbeila amphicephala Nægeli.

- naviculacea Grun.

Encyonema gracile Rab.

- hebridicum Greg.

Eunotia arcus Ehr.

- major var. bidens.

- robusta Ralfs.

Frustulia crassinervia Bréb.

Navicula radians Ktz.

Neldium afûne Ehr.
Pinnularia biceps Greg.

- dactylus Ehr.

- gentilis Donk.

- major Ktz.

- microstauron Eh.

- stauroptera Grun.

- stomatophora Grun.

- undulata Greg.

- viridis Eh.

stauroveis phcenicenteron Eh.

- gracilis Eh.

\section{No 497 WORCESTER - MASSACHUSSETS (Etats-Unis)}

\section{Dépôt fossile d'eau douce}

Cymbella cuspidata Ktz.

- Ehrenbergii Ktz.

- hoteropleura Ralfs.
Encyonema gracile Rab.

Eunotia major Rab.

- pectinalis Dillw. 
Eunotia polyglyphis Eh.

- - polyodon Eh.

Fragilaria capucina Desm.

$$
\text { - undulata TV. Sm. }
$$

Gomphonema acuminatum Eh.

$$
\text { - capitatum Eh. }
$$$$
\text { - intricatum } \mathrm{K} t z \text {. }
$$

Melosira distans Eh.

Navicula amphigomphus El.

- amphirynchus Iih.

- ampliata Eb.

- biceps Greg.

- dilatata E.h.

- divergens W. Sm. var. minor.
Navicula gibba Eb.

- gigas Eh.

- major Ktz.

- mesolepta Kiz.

- nobilis Eh.

- Peisonis Grun

Stauronsis amphilepta Eh.

- gracilis Eh.

- phonicenteron El.

Surirella tenera Greg. var. splendidula forma major A. S.

Tabellaria fenestrata $\mathrm{Ktz}$.

Van Ileurckia rliomboides Bréb.

BIBLiOgRaPhiE. - H. Peragallo. Note sur quelques Diatomées saumâtres du Médoc

Ampliprora paludosa $\mathrm{WV}$. Sm.

Chetuceros Whighamii Bright

Cocconcis placentula Eh.

- $\quad$ pediculus $\mathrm{Ktz}$.

Mastogloia Braunii Grun.

- Dansei Thur.

- Smithii v, anphicephala W. Sm:
Mastogloia exigua Levis.

Vavicula ambigua $\mathrm{Ktz}$.

- elliptica Ktz.

- limosa Ktz.

- viridula $\mathrm{Ktz}$.

Ithoïcosigma curvata $\mathrm{Kiz}$.

Stauroneis spicula Dick.
Achnanthes longipes Ag.

$$
\text { - subsessilis Ag. }
$$

Actinocyclus subtilis Eh.

Cocconeis scutellum El.

$$
\text { - - var. stauroneiformis }
$$

Grammatophora macilenta $\mathrm{K} t z$. .

$$
\text { - marina Ktz. }
$$

Hyalodiscus scoticus $\mathrm{K} t z$.

Navicula aspera Eh.

- Smithii Bréb. var.

Orthoneis binotata Roper.

- fimbriata Bright.

Rhabdonema adriaticum $\mathrm{Ktz}$. 
$\mathrm{N} \cdot 500$ LUSSAT - PUY-DE-DOME (France)

Bibliographie. - Nrère J. Héribaud. Les Diatomées d'Auvergne

Achnanthes Americana CI.

$$
\text { - }
$$

lanceoláta Bréb.

Amphora oeneta $\mathrm{Ktz}$.

Coccoueis intermedia M. Per.

Diatoma hiemale Heib.

$$
\text { - - var. inesodon. }
$$

Encyonema ventricosum Grun.

Epithenia gibberula $K t z$.

$$
\text { - Lurgida Ktz. . }
$$

$$
\text { - zebra Ktz. }
$$

Eunotla lunaris Grun.

Fragilaria construens Grún. v. venter.

$$
\text { - elliptica Grun. }
$$

Comphonema acuminatum $\mathrm{Eh}$.

$$
\begin{array}{ll}
\text { - } & \text { angustatum Ktz. } \\
\text { - } & \text { parvalum Ktz. } \\
\text { - } & \text { subclaoatum Grun. }
\end{array}
$$

IIantzschia amphioxys Grun.

Melosira varians Ag.

Navicula appendiculata vur. irrorait.
Navicula arenacea Bréb.

- borealis $\mathrm{Ktz}$.

- Brebissonii Ktz.

- cryptoccphala Ktz.

- elliptica $\mathrm{Ktz}$.

- gracilis Eh.

- menisculus Schum.

- silicula Grun.

- viridis Ktz.

Nitzselia amphibia Grun.

- commutata Grun.

- Crustulum Grun.

- - - var, tenella.

- Hantzschiana Rab.

- linearis W. Sm.

Surirella augusta Ktz.

Synedra splendens Kiz.

- ulna Eh.

- - var. æqualis.

- - var. amphirynchus.

Van IIeurckia vulgare Rab.

Bibliographie. - Dr J. Pantocsek. Diatomées de Hougrie, volume II

Amphiprora decussata Grun. - duplex Grun.

Amphora acutiuscula Ktz.
Amphora coffeæformis Ktz.

$$
\begin{array}{lll}
- & - & \text { จ. fossilis Pant. } \\
\text { - } & \text { fossilis Pant. }
\end{array}
$$


Amphora libyca Eh. T. interrupta Pant.

- lineata Greg.

- permagna Pant.

- striata Pant.

Cocconeis californica Grun. pediculus Eh.

Cymbella hevesensis Pant.

Epithemia crucæeormis Pant.

- subsalsa Pant.

- succinta Bréb.

Fragilaria bituminosa Pant.

- $\quad$ - v. minor.

- brevistriata Grun. v. fossilis

Mastogloia lanceolata Thw.

Melosira bituminosa Pant

$$
\text { - } \quad \text {. dilatata. }
$$

Navicula ammoplila Grun.

- - - v. degenerans.

- $\quad$ - v. intermedia.

- arenaria Donk.

- bituminosa Pant.

- halionata Pant.

- Heerii Pant.

- heteroflexa Pant.

- interposita Lev.

- interrupta $\mathrm{Ktz}$.
Navicula interrupta $\mathbf{r}$. fossilis.

- - - v. Talyana.

- microrynchus Grun.

- ovalis Hilse.

- - - v. fossilis Pant.

Nitaschia bicuspidata Pant.

- bituminosa Pant.

- frustulum Grun.

- $\quad$ - v. acuta Pant.

- $\quad$ - v. curvata Pant.

- $\quad$ - v. constricta Pant.

- $\quad$ - $\quad$. hungarica Pant.

- $\quad$ - v. minuta Pant.

- $\quad$ - v. obtusa Pant.

- - - v. producta Pant.

- fusiformis Grun.

- neogena Grun. .

- spectabilis Grun.

- Talyana Grun.

Staurosira mormonorum Grun.

- venter Grun. v. fossilis.

Surirella minuta Bréb.

- subsalsa W. Sm.

Synedra Demerarae Grun.

- lævissima Grun. v. fossilis.

- salinarum Pant.

- tenella Grun. v. brevis.

\section{Nos 503 à 505 ILE DE LA GUADELOUPE}

Actinocyclus Ehrenbergii Ralfs.

$$
\text { - subtilis Eh. }
$$

Amphiprora pusilla Greg.

Arachnoïliscus Ehrenbergii Bail.

Biddulphia aurita Bréb.

- pulchella Greg.

- - var., n०505.

- Zanziluarica A. S., n*503.

Campylodiscus ecclesianus Grev. Fortii Temp n, sp.

Climacosphenia elongata Bail.

Cocconeis scutellum var. parva.

Coscinodiscus nitidus Greg.

Glyphodesmis eximia Greg.
Grammatophora oceanica var.

llomoeocladia Vidowichii Grun.

Mastogloia capitata Greg.

Navlcula apis Donk.

- aspera Eh.

- Braziliensis var. A. S.

- elliptica Ktz.

- elongata var.

- excentrica Grun.

- longa Greg.

- separabilis A. S.

Nitzsehia angularis Greg.

- constricta Greg.

- , longissima Ktz.

- panduriformis. 
Nitzschia scalaris Greg.

$$
\text { - sigma var. }
$$

Orthoneis fimbriata Bright.

$$
\text { - splendida Grun. }
$$

Plagiogramma obesum Gree.

Pleurosigma affine fa major.

-

- antillarum II. P., n 503.

balticum v. diminuturn.

validum var.
Podosira ambigua Grun, $n^{\circ} 503$.

- Vontagnei Kít., n 505.

Ihahlonenı allriaticum Klz, n* 503.

Rhoicosigma compactum Greg.

Striatella unipunctata.

Synedra formosa $\mathrm{K} \mathrm{z}$.

- - var. lrevigata, no 505.

Triceratium pentacrinus Wall., n 503.

\section{No 506 FONTAINE DE THEIX - PUY-DE-DOME (France)}

\section{BIbliogra Phie. - Fı̀̀̀e J. Héribaud. Les Diatomées} d'Auvergne ainsi que pour les deux numéros suivants

Ceratoneis arcus $K t_{z}$.

Cymbella cistula Kirsch.

Epithemia zebra hitz.

Fragilaria construens var. binodis.

- - - v var. eximiæ.

Gomphonema constrictum Eh.

- var. capitatum
Gomphonema montanum Schum.

$$
\text { - subclaoatum Grun. }
$$

Hantzschia amphioxys Grun.

Velosira varians $\mathrm{Ag}$.

Navicula major litz.

- - oiridis kitz.

Nitzschia intermedia Hantz.

Synedra ulna Eh, oar. cequalis.

\section{No 507 FONTAINE DE VERNEUGES - PUY-DE-DOME (France)}

Achnanthes lanceolata Bréb.

Encyonema ventricosum fa minuta.

Fragilaria construens $v$. binodis.

Gomphonema parvulum $\nabla$. subcapitata.

IIantzschia amplioxys Grun. v. minuta.
Melosira varians Ag. 1

Navicula atomus Grun.

Nitzschia communis Grun. $v$ abbreviata.

Gurirella minuta $B r e ́ b$.

Syuedra ulna Eh. 


\section{No 508 FONTAINE DE ROYAT - PUY-DE-DOME (France) \\ Achnantues lanceolata Bréb: \\ Diatoma hyemalo $\nabla$. mesodon. \\ Encyonena ventricosum Grun. - $\quad-\quad$ fa minuta. \\ Fragilaria construens Grun. - - var. binodis. \\ Gomphonema parvulum $\nabla$. subcapitata. Meiosira carians $\mathrm{Ag}$. \\ Nitzschia fonticola Grun. paiea var. debilis. \\ Synedira ulna var. æequalis. \\ Van Heurckia vulgare Bréb:}

No 509

LE HAVRE (France)

Pleurosigma strigosum W. Bn.

$N^{\circ} 510$

TROUVILLE (France)

Pleurosigma aestuarii $W$. Sn.

No 511 TOULOUSE (France)

Nitzschia vitrea Norm.

N• 512

TOULOUSE (France)

Nitxschia lamprocampa Ilantz.

N• 513 PALAVAS - MARAIS SAUMATRES (France) Striatella unipunctata Ag. 


\section{N* $う 14$ et $51 う$ FELMÉNES - HONGRIE}

\section{Dépôt fossile saumâtre}

\section{BIBLIOGRA PHIE. - J. Pantocsek. Diatomées de Hongrie, volume II}

Amphora acutiuscula Ktz.

- $\quad$ - $\quad$ v. fossilis.

- coffeæformis Ktz.

- Eulensteinii Grun. v. fossilis.

- libyca Eh. v. interrupta Pant.

- lineata Greg.

Cocconeis Californica Grun.

- pediculus Eh.

Cymbella hevesensis Pant.

Epithemia crucæformis Pant.

$$
\text { - subsalsa Pant. }
$$

Fragilaria bituminosa Pant.

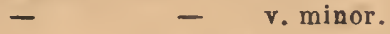

- brevistriata Grun. v. fossilis.

- pinnata Eh.

Gomphonema intricatum Ktz. v. fossilis. Vavicula anomoplila Grun.

- - - v. degenerans Grun

- arenaria Donk.

- arenicola Grun.

- debilis Pant.

- hordeiformis Pant.

- interrupta Ktz.

- microrynchus Grun.
Navicula ovalis Hilse.

- _ v. fossilis.

- sculpta Eh.

Nitzschia frustulum Grun.

$\begin{array}{lll}- & \text { - } & \text { v. acuta Pant. } \\ \text { - } & \text { - } & \text { v. constricta Pant. } \\ \text { - } & \text { - } & \text { v. hungarica Pant. }\end{array}$

- fusiformis Grun.

- Kittlii Grun.

- neogena Grun.

- spectabilis Grun.

- Talyana Grun.

*atarosira Kavnensis Pant.

- mormonorum Grun.

- venter $\nabla$. fossilis Grun.

Stephanodiseus biharensis Pant.

Surirella minuta'Bréb: .

- patella Eh.

- striatula Eh.

Synellra Demeraræ Grnn.

- fasciculata K'tz. v. obtusa.

- lævissima.v. fossilis Grun.

- salinarum Pant.

- tenella v. brevis Grun.

\section{$\mathrm{N}^{\circ} 516$ LAC DES ESCLUSES - PUY-DE-DOME (France)}

\section{BIBliggraphie. - Frère J. Héribaud. Les Diatomées d'Auvergne}

Amphora gracilis Eh.

Cocroneis placentula Eh.
Cyubella cistula Kirch.

- cuspidata Ktz. 
Cymbella cymbiformis Kt\%。

- maculata Ktz.

Encyonema ventricosum Grun.

Epithemia amphicephala Grun. gibba Ktz.

- - var. parallela.

- gibberula Ktz.

- $\quad$ turgida Ktz.

- zebra Kiz.

Eunotia lunaris Grun.

Fragilaria construens Grun.

- intermedia Grun

- virescens Ralfs.

Gomphonema acuminatum Eh.

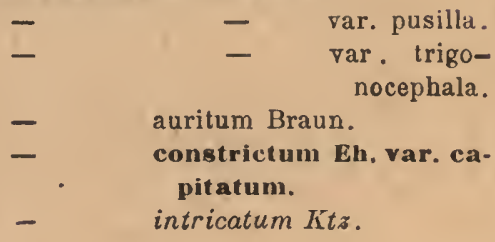

Gomphonema parvulum var.

Mastoglola Dansei Eh.

Melosira crenulata Irzz.

- varians $A g$.

Navicula bacilliformis Grun.

- elliptica Ktz.

- hemiptera Ktz.

- iridis Eh.

- - var. ampliata.

- $\quad l i m o s a k t z$.

- major Ktz.

- radiosa $k i z$.

- - var. acuta.

- - var. tenella.

Stauroneis phcenicenteron $E h$.

Surirella robusta $\mathrm{Eh}$.

- splendida Eh.

Synedra ulna Elr.

- - var. danica.

Tabellaria fenestrata $\mathrm{Ktz}$. floceulosa $\mathrm{Ktz}$.

\section{Jépot fossile d'eau douce}

Amphora enoculata M. Per. et F. H.

- libyca Eh.

- ovalis Ktz.

Campylodiscus costatus W. Sm. Cymatopleura apiculata $\dot{W} . S m$. - elliptica W. Sm.

Cymbella cistula Kirch.

- cuspilata Kitz.

- cymbiformis Bréb.

- Ehrenbergii Ktz.

- gastroides $\mathrm{K} t z$.

- lanceolata Kirch.

- mdeulata $\mathrm{Ktz}$.

Eplthemia amphicephala Grun.

- orex Kítz.
Fpithemia turgida Ktz.

$$
\begin{aligned}
& \text { - } \quad \text { - fa crassa. } \\
& \text { - } \quad \text { zebra Ktz. } \\
& \text { - } \quad \text { - fa minor. } \\
& \text { - } \quad \text { var. proboscidea. }
\end{aligned}
$$

\section{Gomphonema clavatum Eh.}

Melosira arenaria Moore.

- - var. laevis.

- crenulata Ktz.

- granulata Ralfs.

Navicula amphigomphus Eh.

- bacilliformis Grun.

- bisulcata Lag.

- brasiliensis Grun. จ. fossilis.

- cardinalis O'Meara. 
Navieula commutata Ktz.

- elliptica Ktz.

- firma Ktz.

- gastrum Eh.

- - var. major.

- Gendrei var. intermedia.

- gigas Ktz.

- limosa $\mathrm{K} t \mathrm{z}$.

- - var. gibberula.

- major Ktz.

- meniscus Schum.
Navicala peregrina $K t z$.

- radiosa $\mathrm{Ktz}$.

- - var. acuta.

- ventricosa Eh.

Nitzschia hungarica Grun.

Stephanodiscus astraea Girun.

Surirella bifrons $\mathrm{K} t z$.

synedra delicatissima Ktz. $\nabla$. mesoleia. ulna var. amphirynchus.

Bibliográphie. - J. Pantocsek. Diatomées fossiles de Hongrie, $3^{\circ}$ partie

Achnanthes lanceolata Grun.

Cocconeis boryana Pant.

- californica v. hungarica Pant.

- lineata Grun.

Cymbella austriaca $v$ excisa Grun.

- cistula $\nabla$. hungarica Pant.

- cymbiformis $\nabla$. producta. Pant.

- gastroides $\mathrm{Ktz}$.

- - var. crassa Grun.

- lanceolata $\nabla$. fossilis Pant.

- latestriata Pant.

- leptoceros Grun.

- obtusa Pant.

- plutonica Pant.

- Staubii Pant.

- Sturii Grun.

- turgida Greg.

Himantidium boryanum Pant.

Epithemia cistula v. Iunaris Grun.

- gibba v. boryana Pant.

- gibberula v. perlonga Pant.

- Pethoi Pant.

Eunotia flexuosa v. trachychita Pant.

- incisa Greg.
Eunotia minor Grun.

- pectinalis Rab.

- polyglyphis Eh.

Melosira arenaria $\nabla$. hungarica Pant.

- crenulata Ktz.

- granulata Ralfs.

- - v. boryana Pant.

- lœois Grun.

- Rœseana Rab.

- scala Grun.

- undulata $\mathrm{Klz}$.

- - v. minor Pant.

Navicula arata Grun.

- bacillum Eh.

- Clementis Grun.

- dactylus Eh.

- dicephala W. Sm.

- Dubravicensis Grun.

- elliptica v. grandis.

- gastrum $\nabla$. boryana Pant.

- Ilauerii Grun.

- macilenta Eh.

- major Kitz.

- mesolepta Ktz.

- Neumayeriana Pant.

- nobilis Ktz. 
Navicula radiosa $\mathrm{Ktz}$.

- sculpta Eh.

- styriaca Grun.

- subsolaris Grun.

- viridis. . fossilis Pant.

Nitzschia Kittlii Grun.

- scalaris W. Sm.

- tryblionella v. maxima Grun.

Peronia boryana Pant.

Balacia boryana Pant.

Stauronels boryana Pant.

- Szontaghii Pant
Staurosira construens Eh.

- Grunowii Pant.

- Harrisonii Grun.

- - v. amphitetras Grun.

- intermedia Grun.

- venter Grnn.

Surirella Clementis Grun.

- subsalsa W. Sm.

- tenera o. fossilis Pant.

Terraryclus larustris o fossilis Pant

\section{No 520 ALMOLOYA DE ALQUESIRAS (Mexique)}

Cymhella cymbiforme Ktz. parva W. Sm.

Cocconeis placentula W. Sm.

Encyonema turgidum Greg.

Epithemia gibberula Ehr.

- turgida Ktz.

- zebra Ktz.
Eunotia eruica Eh. var.

Fragilarla construens Eh.

Gomphonema subclavatum Grun.

Navicula cuspidata Ktz.

Melosira crenulata lítz. var.

stauroneis phonicenteron Eh.

Stephanodiscus astraed Grun.

No 521

BEZZIERS (France)

Achnanthe minutissima Ktz.

Cymbella affinis litz.

Diatoma oulgare $\mathrm{Ag}$.

Encyonema ventricosum Grun. fa minuta. '
Gomphonema angustatum Grun var. producta.

Meridion circulare Ag.

Synedra ulna Eh. var, æequalis.
Achnanthes brevipes $\mathrm{Ag}$.

Melosira Borreri Grev.

- - var. hispida.
Striatella unipunctata $A g$.

Synedra affinis rar. delicatula. 
N० 523

NICE (l'rance)

Aohnanthes brevipes Ag.

- $\quad$ - var. intermedia.

- longipes Ag.

Achnanthes subsessilis $K t \boldsymbol{x}$.

Melosira nummuloides Ag.

BROYTORP - POJO (Finlande)

BIBLIOgRAPHIE. - Cleve. Diatomées de Finlande

Cymbella cuspidata Ktz.

- gastroides $\mathrm{K} t z$.

D iploneis Finnica Cl.

Eunotia robusta Ralfs.

Neidlum tirmum Ktz.

Pinnularia brevicostata var. leptostanron $\mathrm{Gl}$.

\section{- dactylus Eh.}

- episcopalis Cl.
Pinnularia legumen Eh.

- macilenta (Eh.) Cl.

- major Ktz.

- nobilis Eh.

- platycephala Eh.

- stomatophora Grun.

- stomatoptera Eh.

- viridis Eh.

Surirella robusta Ralis.

Tetracyclus lacustris Ralfs.

No 525

RADE DE YOKOHAMA (Japon)

Plankton récolté par l'expédition de la Vega

Asterionella Bleakeleyi W. Sm. Bacteriastrum varians Laud.

Biddulphia aurita Bréb.

- chinensis Grev.

Chretoceros distans Cl.

$\begin{array}{ll}\text { - Javanieun Cl. } \\ \text { - } & \text { paradoxum Cl. } \\ \text { - } & \text { peruvianum Bright. } \\ & \text { Ralfsii Cl. }\end{array}$

Coscinodiscus abyssalis Cart.

- fragilissimus Grun.

- lineatus Eh.

- $\quad$ punctiger (Cast.) H. P.

Cresswellia palmeriana Grev.

Dactylioselen $\mathrm{sp.}$ ?

Ditylium sol Grun.

Eurampia cornuta Cl.

- zodiacus Eh, 
nemianlus Heibergii. Cl.

Pleurosigma acutum Norm. var.

Rhizosolenia arafurensis Cast.

- alata Bright. var. polydactyla Cast.
RhIzosolenia setigera Bright.

- Stolterfothii H. P.

- styliformis.

Skeletonema costatum Grev.

Thalamsiothrix Frauenfeldii Grun.

\section{$\mathrm{N}^{*} 526$ et 527 LONG ISLAND SOUND (Etats-Unis)}

Amphiprora elegans W. Sm.

- lepidoptera Greg.

- pulchra Bail.

- pusilla Greg.

- vitrea W. Sm.

Amphora angusta Eh.

- cymbifera Greg.

- obtusa Greg.

Coscinodiscus excentricus Eh.

Eplthemia musculus Ktz.

Mastogloia apiculata Grun.

Melosira sulcata $\mathrm{K} \mathbf{z}$.

Nivicula apis Donk.

- cancellata Donk.
Navicula cyprinus Eh.

- formosa Greg.

- fusca Greg.

- Grundleri A. S.

- lyra Eh.

- marina Ralfs.

- peregrina Eh.

- quadriseriata Gl. var.

- yarrensis Grun.

Pleurosigma balticum W. Sm.

- elongatum W. Sm.

- Wansbeckii Donk.

Rhabdonema adriaticum Ktz.

Scoliopleura latestriata Bréb.

- incisa Greg.

$\mathrm{No}^{5} 528$ et 529

ERDÖBENYE (Hongrie)

Dépôt fossile saumâtre

Bibliographie. - J. Pantocsek. Diatomées de Hongrie, volume II

Amphora coffeaeformis Krtz.

- libyca Eh. v. interrupta Pant.

- lineata Greg. .

Cocconeis californica Grun.

- pediculus Elı.

Cymbella Chyzerii Pant.

- Erdöbẻnyana Pant.
Cymbella hevesensis $\mathrm{P}$ ant.

- hungarica Pant.

- Neupauerii Pant.

- salina Pant.

Epithemia erucaeformis Pant.

- - - v. validior Pant. 
Fragilaria bituminosa Pant.

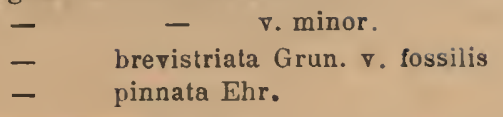

Gomphonema intricatum Ktz. $\nabla$. fossilis.

$-$

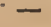

- salinarum Pant.

- salsa Pant.

Navicula bituminosa Pant.

- cincta Pant.

- debilis Pant.

- halionata Pant.

- Hazslinszkyi Pant.

- interrupta Ktz.

- microrynchus Grun.

- ovalis Hilse.

- $\quad$ - v. fossilis Pant. troglodytes Pant.
Nitzschla bituminosa Pant.

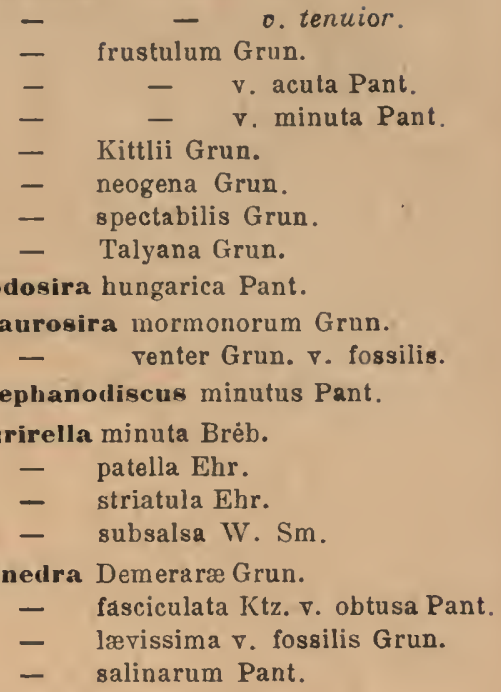

\section{Bibliographie. - J. Pantocsek. Diatomées de Hongrie, volume II}

Amphora euprepes Pant.

- suavis Pant.

- transylvanica Pant.

Campylodiscus Euleinsteinii Pant. hibernicus Eh.

- noricus Eh. $\nabla$. fossilis Pant.

Coscinodiscus biradiatus Grev, var.

- . subtilis Eh.

$$
\text { - } \quad \text { transylvanicus Pant. }
$$

Cymatopleura elliptica W. Sm. var.

- solea W. Sm.

- subconstricta Pant.

Cýmbella aspera Pant.

- capitata Pant.

- gigantea Pant.
Cymbella Kochii Punt.

- lanceolata Eh.

Epithemia transylvanica Pant.

- turgida Ktz. var.

- zebra v. parallela.

Fragilaria pinnata El.

- sepulta Pant.

- transylvanica Pant.

Gomphonema transylvanica Pant.

- vibrio Eh. var.

Melosira granulata Ralfs.

- Varennarum M. Per.

Navicula bacillum Eh. var.

- basilica Pant.

- bituminosa Pant. var. Bodosensis Pant. 
Vavicula Brebissonii Ktz, v. fossilis.

- Budayana Pant.

- elliptica Pant.

- Gutvinskii Panz.

- Hyrthii Pant.

- latevittata Pant.

- lucida Pant.

- major Ktz.

- Maulerii J. Br.

- paludinarum Pant.

- - v. gracilior.

- paripinnata Pant.

- pressa Pant.

- Proserpinæ Pant.
Navicula transylvanica r. producta Pant. - yarrensis Grun.

Nitzschia antiqua Pant.

I'leurosigma hippocampus IV.Sm, var.

Surirella amoena Pant.

- Kochii Pant.

- lanceolata cuspidata Pant.

- rubusta Eh. var.

- transylvanica Pant.

- turgida W.Sm.

- verrucosa Pant

Tetracyclus emarginatus Eh.

\section{No' 532 et 533 PORT-LOIJIS (Ile-de-France)}

\section{(Sur les Corallines)}

Actinocyclus Ehrenbergii Ralfs.

$$
\text { - Ralfsii W. Sm. }
$$

Amphora bigibba Grun.

- crassa var. junctata.

- cymbelloides Grun.

- lineata Greg.

- robusta Greg.

Arachnoidiscus ornatus Eh.

Bidululpia pulchella Gray.

- Tuomeyi Bail.

Campylodiscus undulatus Grun.

Climacosphenia elongata Bail.

Cocconeis pellucida $\mathrm{K} t z$.

$$
\begin{aligned}
& \text { - } \quad \text { - var. flexella Ktz. } \\
& \text { - scutellum Eh. } \\
& \text { - - var. ampliata Grun. }
\end{aligned}
$$

Coscinodiscus nitidis Grev.

$$
\text { - } \quad \text { - fadiatus } E h \text {. }
$$

Epithemia musculus Ktz.

Enodia gibba Bail.

Grammatophora caribcea $\mathrm{Cl}$.

- gallopagensis Grun.
var.

Isthmie caponsis.
Lichmophora Ehrenbergii Eh.

$$
\text { - Jurgensii Ag. }
$$

Mastogloia ovata Grun.

$$
\text { - undulata Giun. }
$$

Melosira sulcata Eh.

$$
\text { - - - var. coronata. }
$$

Navicula aspera El.

- clavata Greg.

- consors A.S.

- crabro Eh.

- - var. intermedia.

- didyma Eh.

- diplosticta Grun.

- gemmatula Grun.

- liber W. Sm.

- lyra Eh.

- papula A. S.

- velata A. S.

- Weissflogii A. S.

Nitzschia angularis Greg.

- fluminensis Grun.

- panduriformis Greg.

- - var. minor.

- salinarum Grun.

Orthoneis fimbriata Bright. - splendida Grun. 
Pagiolliscas Martensianus Grun. Pleurosigma formosum W. Sm. Podocystis adriatica Ǩlz.

Podosira ambigua Gron.

- - argus Grun.

- hormoides Mont.

Raphoneis amphiceros Eh.

- - - var. cruciata.

Rhablonema mirificum WV. Snı. - adriaticum Kítz.

Schizonema obtusum WV. Sm.

- Smithii Ag.
Stictodiscus californicus var. Grev.

Surirella fastuosa Eh.

Synodra lievigata var, hyalina Grun.

- formosa kitx.

- pulcherima $\mathrm{K} \mathrm{z}$.

- undulata Grev.

Triceratium arcticum Shadb.

- bulbosum Witt.

- latum Greo.

- pentacrinus IVall.

- quinquelobatuin IVall.

- Shadboldtianum. zonulatum Grev.

No 534

CULTURE DU D ${ }^{r}$ P. MIQUEL

Nitzschia palea Kız, avec auxospores

No 535

MER NOIRE

Vase

Amphitetras antediluviana Eh. var.cruciformis.

Campylodiscus Thuretii Bréb.

Coseinodistus concavus Greg.

$$
\text { - - radiatus Eh. }
$$

Melosira Borrerii Grev.

Navicula lyra Eh.

Nitzschia circumsuta Bail.
Pleurosigma balticum $W$. Sm.

thablonema adriaticum $\mathrm{Fit}$.

Scoliopleura tumida Grun.

Surirella gemma Eh

- fastuosa Eh.

Synedra fulgens $\mathrm{IV}$. Sm. et var.

- undulata Greg.

Triceratium favus Eh.

$\mathrm{N}^{\circ 5} 536$ et 537 LE MONASTIER - IIAUTE-I_OIRE

(France)

BIBliographie. - Frère J. Héribaud. Diatomées fossiles d'Auvergne 1903

Achnanthes Joursacensis F. H. et M. P. Coscinodiscus pygmæus M. P. et F. H. Coscinodiscus Boulei II. P. Cymbella affinis Ktz. 
Cymbella Ehrenbergii Greg.

$$
\begin{aligned}
& \text { - } \quad-\quad \text { v. conica F. H. } \\
& \text { - } \quad \text { lanceolata Ehr. } \\
& \text { - } \quad \text { meniscus F. H. et M. P. } \\
& \text { - tumidula Grun. }
\end{aligned}
$$

Encyonema ventricosum $\mathrm{Ktz}$.

Epithemia cistula Ralfs.

- Hyndmannii W. Sm.

- turgida $\mathrm{Ktz}$.

- $\quad$ - v. granulata Grun.

Eunotia polyglyphis Grun.

Fragilaria æqualis Lag. v. capitata F. H.

- brevistriata Grun.

- $\quad$ - v. mormonorum Grun.

- $\quad$ - v. pusilla Grun.
Fragilaria construens Grun.

$$
\text { - } \quad \text { - } \quad \text { - } \quad \text { v. binodis Grun. }
$$

\section{- Zellieri F. u.}

Melosira lineolata Grun.

- undulata Ktz. v. producta A.S.

- tenuis cirun.

Navicuia amphibola Cl. $\nabla$. Perrieri F.H. et M. P.

- Berriati F. H.

- 'v. minor F. H.

- Chaberti F. H.

- major Ktz.

- placentula Ehr.

Opephora Martyi F. H.

ithopalodia gibba Ehr.

Stauroneis phœnicenteron Eh.

No 538

LAC LEMAN (Suisse)

Cyclotella boranica Eulenst

$N \cdot 539$

TOULOUSE (France)

Nitzsehia frustulum ktz.

N०540

LE HAVRE (France)

Lichmophora tincta $\Delta \mathbf{g}$.

N०54l

MER CASPIENNE

Sondage

BIBLIOGRAPHIE. - Grunow. Algen und Diatomeen aus dem Karpischem Meere. Isis 1878

Actinocyclus Ehrenbergii Ralfs. var. - subtilis Ralfs.

Campylodiscus Daemelianus Grun.

-
Echeneis Eh.
Coscinodiscus centralis Eh.

- radiatus var. Grun.

subtilis Eb.

I Navieula formosa Greg. 
Navicula Schneideri Grun.

Nitzschia circumquta Bril.

- longissima Ralfs.

- sigma W. Sm. var.

Pleurosigma balticum WV. Sm.
Rbaludonema adriaticum $K t$.

Surirella striatula Turpin.

Synedra affinis oar. hybrida Grun.

- robusita Ralfs.

$N^{\circ} 542$ et 543 PALAZZOLO ACREIDE (Italie)

Achnanthes inflata Grun.

Amphora gracilis Eh.

Campylodiscus costatus $W$. Sm.

Cymbella cistula Kirch.

$$
\text { - gastroïdes Ktz. }
$$

Denticula valida. Ped.

Epthemia gilbba Ktz.

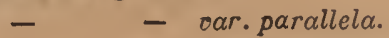

- - val. ventricosa.

- gibberula KIz.

- turgida Ktz.

Gouphonenia clavatum Eh.

- constrictum Eh.

- subtile Eh.

- intricatum $K t z$.

Melosira crenulata $K t z$.

- varians Ag.
Navicula ambigua $E h$.

- borealis $K t z$.

- gibba Ktz.

- interrupta var. stauroneiformis.

- ladiosa Ktz. var. tenella.

- sculpta Eh.

- Slesvicensis Grun.

- sphærophora Ktz.

- viridis Ktz.

Nitzschia Tryblionella Hantz.

$$
\text { - - var. Levidensis. }
$$

Pleurosigma attenuatum W. Sm.

Stephanodiscus astraea Grun.

Surirella elegans. Eh.

- ooata Ktz.

- tenera var, nervosa.

Bynedra ulna Eh.
Cocconeis placontula Eh.

Cymbella affinis $K t z$.

- cistula Kirch.

- gastroïdes $\mathrm{Ktz}$.

Diploneis elliptica $C l$.

Epithemia cistula Eh.

\footnotetext{
- var. lunaris.
}

Epithemia gibberula Rab.

$$
\text { - } \quad \text { - } \quad \text { var. directa. }
$$

Eunotia major Rab.

Faagilarla brevistriata Grun.

- construens Grun.

- elliptica Grun. 
Fragilaria Harrisonii Grun.

- - var. major.

- nitida M. Per. et F. H.

Iantzschia amphioxys Grun.

Melosira arenaria Moore.

- crenulata Ktz.
Navicula gastrum Eh.

- - var. maxima.

- Gendrei Herib. v. intermedia.

- major Ktz.

Synedra ulna Eh. fa undulata.

Tabellaria fenestrata $K t_{\text {z }}$.

N० 546

PILA (Hongrie)

Dépot fossile d'eau douce

Cocconeis placentula Ek.

Cyubella cymbiformis Bréb.

- gastroides Ktz.

- leptoceros Rab.

- parva V. H.

Eunotia gracilis Rab.

- Junaris Grun.

- major Rab.

- minor Rab.

Fragilaria Jrevistriata Grun.

- construens Grun.

- endocystifera M. Per, At F. H.

Comphonema angustatum Kiz. - intricatum Ktz.

Velosira crenulata $\mathrm{Ktz}$.
Velosira crenulata var. tenuis.

- - var. tenuissima.

- laevis Grun.

Navicula amphibola $\mathrm{Cl}$.

- commutata Grun.

- gastrum.Eh.

- gibba Ktz.

- gibbula Cil. var. cantalina.

- hemiptera $\mathrm{Ktz}$.

- major Ktz.

- rupestris Grun.

Stauroneis anceps Eh.

- antediluviana M. Per. et F. $H$.

- gracilis Eh.

Stephanodiscus astreae Grun.

No 547 BIBARCZFALVA (Hongrie)

Dépôt fossile d'eau douce

Bibliographie. - J. Pantocsek. Diatomées de Hongrie, partie III

Amphora suavis Pant.

- transylvanica Pant.

Campylodiacus hibernicus Eh. var. fossilis. 
Cocconeis lineata Eh. var. fossilis. Coscinodiscus transylvanicus Pant.

Cyclotella pygmæa Pant. (Ne me parait être qu'une petite forme du Cos. transylvanicus.)

- transyloanica Pant.

Cymatopleura gigantea Pant.

Cymbella Budayana Pant.

- - var. gracilior.

- Kochii Pant.

Epithemia argus $K t z$. var. fossilis.

- gibba Ktz. var. Buryana.

- perlonga Pant.

- transylvanica Pant.

Eunotia polyglyphis Eh.

Fragilitria sepulta Pant.

- transylvanica Pant.

Gomphonema Kinkerii Pant.

- transylvanicus Pant.

Melosira crenulata $K t z$. var. hungarica Pant.
Navlenla amphigomphus Eh.

- Bodosensis Pant.

- Budayana Pant.

- Gutwinskii Pant.

- hasta Pant.

- hilarula Pant.

- Hitcheockii Eh.

- Hyrthii Pant.

- inculta Pant.

- Mauleri J. Br.

- ostracodarum Pant.

- paludinarum Pant.

- Proserpinæ Pant.

- Smithii Bréb.

- Toular Pant.

Nitzsehia Kohleri Lauby.

Pleurosigma Kochii Pant.

Pleurostanron Smithii Grun.

Staurosira Harrisonii Grun. var. fossilis.

Stephanodiscus transylvanicus Pant.

Surirella lanceolatocuspidata Pant.

- vorrucosa Pant.

\section{Nor 548 à 550 DOLGEVILLE - CALIFORNIE (Etats-Unis)}

\section{Dépôt fossile marin}

Actinoptychuः glabratus Grun.

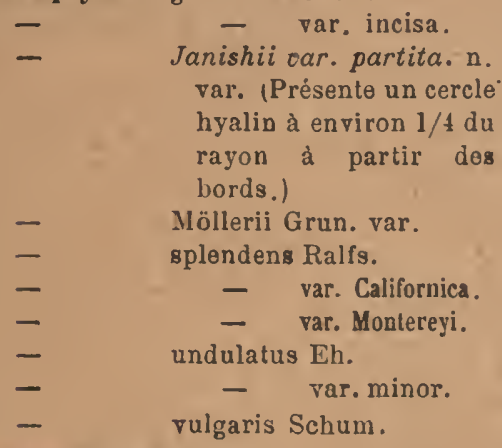

Amphora crassa Greg.

- quadrata Bréb.
Anaulus mediterraneus oar. intermedia

Arachnoïliscus Ehrenbergii Bail.

$$
\text { - ornatus Eh. }
$$

Aulacodiseus Kittonii Arn.

- margaritaceus Ralfs.

Auliscus Biddulphia var. prominens.

- colatus Bail.

- Grunowii var. californica.

- Hardmanianus Greo.

- Moronensis fa bioculata.

- pruinosus Bail.

- sculptus Eh.

- Stöckhardtii Jan.

Biddulphia Tuomeyi Rop.

Campylodiscus Montereyanu Grev. 
Campyloneis Grevillei Grun.

Cerataulus turgidus Eh.

Chretoceros incurvum Bail.

Cocconeis dirupta Greg.

- pseudomarginata Greg.

Coscinouliscus apiculatus Eh.

$\begin{array}{ll}\text { - } & \text { asteromphalus Eh. } \\ \text { - } & \text { cocconeiformis } A . S . \\ \text { - } & \text { curcatulus Grun. } \\ \text { - } & \text { meatus Eh. } \\ \text { - } & \text { monicæ Ratt. } \\ \text { - } & \text { oculus iridis Eh. } \\ \text { - } & \text { radiatus Eh. } \\ \text { - } & \text { robustus Grec. } \\ \text { - } & \text { subtilis Eh. } \\ \text { - } & \text { symbolophorus Grun. }\end{array}$

vineregramna marinum Ralf.

Diploneis Lombus Eh.

- crabro Eh.

- nitescens $\mathrm{Cl}$.

- Smithii Cl.

Endictya nceanica Eh.

Encopyla australis Eh.

Euodia gibla Bail.

Glyphodiscus stellatus Gret.

Gramnatophora robusta Dipp.

Iy alodiscus reticulatus $A$. $S$.

Isthmia nervosa Ktz.
Melosira clavigera Grun.

- sol Eh.

- sulcata Ḱtz. var. coronata.

Navicula $a b r u p t a$ Greg. excavata Greo.

- Fischeri A. S.

- lyra Eh. et var.

- prætexta Eh.

- spectabilis Greg.

Orthoneis splendida Grun.

Plagiogramma Gregorianum Greo.

- oalidum var. tumidu. lum.

Porpeia quadriceps Bail.

Stephanogonia actinoptychus Eh.

Stephanopyxin Grunowii Grove et S.

- turris Ralfs.

- - var. crassispina.

Stictoulisens californicus Grev.

Surirella fastuosa El.

Syndenurium diadema Eh.

Trachyneis aspera $\mathrm{Cl}$.

$$
\text { - oblonga Bail. }
$$

Triceratium biquadratum Jan .

Ianthiopyxis alata Eh.

- cingulata Eh.

- oblonga Eh.

- umbonatus Grev.

$N^{0} 551$

NOUMEA - CAYENNE

Sur les algues

Actinocyclus complanatus Cast. var.

- Ehrenbergii var. sparsa.

- incertus Grun.

- nebulosus M. Per.

- rotula J. $\mathrm{Br}$.

Actinoptychus hexagonus Grun.

Amphiprora lepidoptera Greg.

Amphora arcus Greg.

- Grundlerii Grun.
Amphora proteus Greg.

Aulacodiscus Kittonii Arn.

Auliscus pruinosus var. Zansibarica.

Bacteriastrum spirillum Cast.

Biddulphia aurita Bréb.

- $\quad$ - pulchella Gray.


Campylodiscus ambiguus Grun.

$$
\begin{aligned}
& = \\
& \overline{-} \\
& \overline{-}
\end{aligned}
$$
biangulatus Grev. intermedius Grun. Kitlonianus Grev. Robertsianus Greo undulatus tirev. Wallischianus Grov.

Chetoceros spinosum Leud. Fort.

Climacosphenia elongata Bail. var.

Cocconeis pseudo marginata Greg.

Coscinodiscus Janischii A. S.

oboersus Ratt.

Diploneis crabro Eh.

Diploneis splendida Greg.

Euphyllodium spathulatum Shad.

IIyalouliscus stelliger Bail.

Isthnia minima Bail.

Lampriseus circulare Grun.

\section{- Schadboltianum Ġreo.}

Mastogloia quinquecostata Grun.

Navicula bullata Nor.

- claoata Greg.

- lyra Eh.

- - var. intermedia.
Navicula lyra var, recta.

- maxima var. excentrica.

Nilzschla panduriformis Greg.

Orthoneis cribrosa Grun.

$$
\text { - fimbriata Grun. }
$$

Plagiodiscu* Martensianus Grun.

Pleurosigma formosum IV. Sm.

- rigidum W. Sm.

Rhablonema alriaticum Kitz. - mirificum W. Sm.

Surirella eximia Greo.

- fastuosa Eh.

Synedra formosa Iantz.

Synedrosphenia cuneata Grun.

Terpsinoë intermedia oar.

Toxarium undulatum.

Trachyneis aspera $\mathrm{Cl}$.

$$
\text { - _ - var, vulgaris. }
$$

Triceratium arcticum var. californica.

- bullosum $\mathrm{O}$. W.

- formosum oar. pentagona-

$$
\text { lis. }
$$

- punctatum Br.

- sculptum Shadb. scitulum fa quadrata.
Actinocyelus Ehrenbergii Ralfs.

$$
\text { - incertus Grun. }
$$

Actinoptychus areolatus Eh.

$$
\text { - } \quad \text { spinifer Grun. }
$$

Actinoptychns undulatus Eh.

$$
\text { - } \quad \text { - valgaris schum. }
$$

Arachnoüdiacus Ehrenhergii Bail.

- ornatus El. 
Asteromphalus Brookei Bail.

$$
\text { - Darwinii Eh. }
$$

Aulacodiscus argus A. $S$.

Auliscus pruinosus Bail.

- sculptus Eh.

Biddulphia Tuomeyi Rop.

Cerataulus turgidus $E h$.

Chatoceros californicus Grun.

$$
\begin{array}{ll}
\text { - } & \text { didymum Eh. } \\
\text { - } & \text { incurvum Bail. } \\
\text { monicce Grun. }
\end{array}
$$

Cladogramma Californicum El.

Cocconeis dirupta Greg.

$$
\text { - scutellum Eh. }
$$

Coscinodiscus apiculatus Eh.

$\begin{array}{ll}\text { - } & \text { asteromphalus Eh. } \\ \text { - decipiens Grun. } \\ \text { - } & \text { denarius A. S. } \\ \text { - } & \text { diorama A. S. } \\ \text { - } & \text { exasperens Ratt. } \\ \text { - } & \text { excentricus Eh. } \\ \text { - } & \text { lineatus Eh. } \\ \text { - } & \text { marginatus Eh. } \\ \text { - } & \text { nitidulus Grun. } \\ \text { - } & \text { oculus iridis Eh. } \\ \text { - } & \text { radiatus Eh. } \\ \text { - } & \text { robustus Greo. } \\ \text { - } & \text { subaulacodiscoidalis Ratt. } \\ \text { - } & \text { symbolilis Eh. }\end{array}$

Cosmiolliscuss elegans Grev. (Actino-

Denticula lauta Bail. cyclus?)

Dimeregramma Ischaboensis Grun.

Diploneis splendida Greg.

Eudyctia oceanica Greg.

Eunotograuma debilis Grun.

Gephyria media Arn.

Goniothecium crenatum Eh.

Graya argonauta Grooe et $S t$.

IIemiaulus ambiguus Grun.
Ifercotheca mamillaris Eh. var.

liyaloniscus scoticus Grun. subtilis Bail.

Istbuia enervis Eh.

$$
\text { - nervosa } \mathrm{K} \text { iz. }
$$

Leudugeria epithemioides Temp.

Lithodesmium minusculum Grun. undulatum Eh.

Melosira sulcata $\mathrm{K} t \mathrm{z}$.

$$
\text { - - var. coronata. }
$$

Molleriella limbata V. H.

Navicula lyra Eh.

- - var. atlantica.

- peregrina Ktz.

Ploaria petasiformis Pant.

Pyxilla carinifera Grun.

Raphoneis amphiceros var. Californica.

- $\quad$ - var. elongata.

Rhizosolenia pileolus Eh.

- styliformis Bright.

Septroneis geminata Grun.

- nitzschioïdes Grun.

Stephanogonia actinoptychus.

Steplanopyxis appendiculata Eh.

$\begin{array}{ll}\text { - } & \text { corona Grun. } \\ \text { - } & \text { cruciata. Temp. et Per. } \\ \text { - } & \text { diadema Eh. } \\ \text { - } & \text { Grunowii Grov. et St. } \\ \text { - } & \text { spinosissima Grun. } \\ \text { - } & \text { turris Ralfs. } \\ & - \text { var. crassispina. }\end{array}$

Stictodiscus Californicus Grev.

- Kittonianus Grun.

Syndentrium diadema Eh.

Tualassiothrix Frauenfeldii Grun.

- marina Grun.

Triceratium condecorum Eh.

- interpunctatum Grun.

ranthiopyxis oblonga Eh.

- umbonatus Grov.

zygoceros quadricornis Grun. 
N* 556 MOISSAC - TARN-ET-GARONNE (France)

Vitzschla Brebissonii $w$. Sm.

1. Stauroneis phoenlcenteron Eh.

No $55 \overline{7}$

\author{
ESTUAIRE IDE LA TAMISE \\ SHEERNESS ON SEA (Angleterre)
}

Rhizosolenia Shrubsolei Cleve

$\underline{-}$

No 558 à 560 LOsT SPRING RANCH - CALIFORNIE

(Etats-Unis)

Jépôt fossile d'eau douce

Amphora gracilis Eh.

- ovalis $\mathrm{Ktz}$. fa minor.

- pediculus Grun.

Campylodiscus hibernicus Eh. var.

Coceonels placentula Eh.

- - var. lineata.

Cymatopleura apiculata W. Sm.

- olliptica W. Sm.

Cymbella cistula Kirch.

- cuspidata $K t$.

- delecta A. S.

- Ehrenbergii Ítz.

- - var, minor.

- gastroïdes $\mathrm{Klz}$.

- heteropleura Ktz.

- - var. minor.

- Kamtschatica Grun.

Encyonema crespitosum lítz.

- triangulum $\mathrm{K} t z$.

- ventricosum Grun.
Epithemia amphicephala Grun.

- cistula Ralfs.

- gibberula Ktz.

- sorex $K t z$.

- turgida Ktz.

- - var. granulata.

- - oar.porcellus.

- zebra Ktz.

- $\quad$ - var. proboscidea.

- $\quad$ - fa minor.

Eunotia arcus var. tenella.

- Tormica Eh.

- impressa Eh.

- minor V. H.

- pectinalis Rab.

- - var. stricta.

- tridentata Eh.

Fragilaria construens Grun.

Gonıhonema acuminatum $\mathrm{Eh}$.

- apiculatum $\mathrm{Cl}$. 
Gomphonema commutatum Grun .

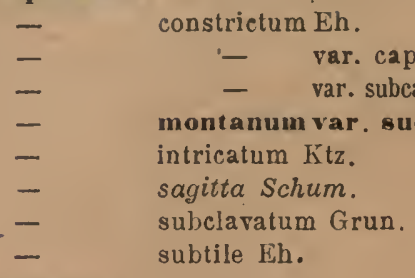

llantzschia amphioxys Grun.

- elongata Grun.

Melosira crenulata $\mathrm{Ktz}$.

- - var. tenuis.

Navicula acrosphoria oar. parca.

- americana Eh.

- - var minor.

- amphigomphus Eh.

- amphirynchus Eh.

- bacilliformis Grun.

- bicapitata Lag.

- - var. hybrida.

- borealis Brèb.

- Brebissonii Ktz.

- - var. diminuta.

- commutata Grun.

- cuspidata Ktz.

- - var. craticula.

- dicephala var. Eh.

- dubia Eh.

- elliptica Ḱlz.

- esox Eh. var. recta.

- gentilis Kítz.

- - var, hyalina.

- hemiptera $\mathrm{K} t z$.

- iridis Eh.

- - var. ampliata.

- isostauron Pant.

- lata Bréb.

- limosa Ktz.

- - var.gibberula.

- macilenta Eh.

- major Ktz.

- mesolepta Eh.

- - var. stauroneiformis.

- mesostyla Eh.

- mutica Ktz.

- - var. subundulata.
Naricula nobilis $\mathrm{Ktz}$.

- nodosa Ktz.

- pachyptera Eh.

- parva En.

- polygramma Schum. var.

- radiosa $\mathrm{Ktz}$.

- - var. acuta.

- - var. tenella.

- rhynchocephala var. amphiceros.

- rupestris Hantz.

- transversa A. S.

- trinodis Lewis.

- ventricosa Ktz.

- viridis $\mathrm{Ktz}$.

Vitzschia sigmoïdea W. Sm.

Pieurostauron acuta Rab.

Rhopalodia gibba Mũll.

$$
\text { - } \quad \text { - var. paralella. }
$$

gtauronels anceps $\mathrm{Eh}$.

- Baileyi Eh.

- mesopachya Eh.

- gracilis Eh.

- phœenicenteron Eh.

Stephanodiscus astræa Grun.

- - var: spinulosa.

Surirella bifrons Ktz. fa minor.

- elegans Eh.

- - var. producta n. var. ( $A$ extrémité inférieure atténuée, produite et pointue.)

- linearis W. Sm.

- - var. constricta.

- Mölleriana Grun.

- robusta Eh.

- splendida Eh.

- tenera Greg.

- - var. nervosa.

- - var. splendidula.

- - var. torta $n$. var. (Valve légèrement tordue autour de l'axe longitudinal.)

- sp. A. S. atlas 23/26.

Synedra ulna Eh. 


\section{Nos 561 et 562 THURSDAY-ISLAND (Nouvelle-Guinée)}

\section{Sur les algues}

Actinocyclus incertus Grun.

Actinoptychus intermedius Grun.

- hexagonus Grun.

- splendens Rialfs.

- undulatus Eh.

- $\quad$ - var. erosa n. var. (Les marques des compartiments se. condaires sont effacés à l'exception de quelques-unes éparses et très visibles.)

vulgaris Schum.

Aulacodiscus argus A. $S$. - Kittonii Arn.

Auliseus colatus Bail.

- - var. latecostata.

- reticulatus Greo.

- sculptus Eh.

Biddulphia anrita Bréb.

- pulchella Greg.

- rhombus W. Sm.

- Tuomeyi Rop.

Campylodiscus concinus Grun.

- ecclesianus Grov.

- Kittonianus Grev.

- undulatus Grev.

- Wallichianus Grev.

Cerataulus turgidus Eh.

Climacosphoenia elongata Bail.

Cocconeis pseudo-marginata Greg.

Coscinodiscus apiculatus Eh.

- denarius A. S.

- Janischii A.S.

- lineatus Eh.

- nitidus Grun.

- oculus iridis Eh.

- $\quad$ radiatus Eh.
Coscinodiscus symbolophorus Grun . symmetricus Grev.

Cyclotella stylorum Bright.

Diploneis Weissflogii $\mathrm{Cl}$.

Eunotogramma Frauenfeldii Gru?.

Ilyalodiscus stelliger Bail.

Loulugeria epithemioïdes Temp.

Melosira sulcata $K t_{\text {.z. }}$.

- - var. coronata.

- - var. radiata.

Navicula arabica Grun.

- brasiliensis Grun.

- bullata var. Mölleriana.

- clavata Greg.

- - var. elongata.

- Iyra Eh.

- - var. elliptica.

- - var. intermedia.

- - var, recta.

- maxima Greg.

- robusta Grun.

- spectabilis Greg.

Nitzsebia Jelineckii Grun.

- panduriformis Greg.

Omphalopelta hispida Eh.?

Plagiogramma Gregoryanum Greo.

Pleurosigma formosum IV.Sm.

IRhabdonema adriaticum $\mathrm{Ktz}$.

Surirella Ceylanensis Leud. Jort.

-- fastuosa Hantz.

Trachyneis aspera $\mathrm{Cl}$.

Triceratium bicorne $\mathrm{Cl}$.

- favus Eh.

- pentacrinus Eh.

- scitulum fa quadrata.

- sculptum Shadb. 
No 563

PORT DE SUE\%

Sur les algues

Cocconeis scutellum Eh. fa parva.

Homoeocladia Vidovichii Grun.

Grammatophora marina Grun.

Synedra acus hitz, var, intermedia.

\section{$\mathrm{N}^{\text {os }} 564$ et 565 BILIN - BOHÈME (Autriche)}

\section{Terre fossile d'eau douce}

Amphora ovalis $\mathrm{K} t \mathrm{z}$.

Cocconeis placentula Eh.

Cymatopleura solea Eh.

Cymbella cistula Hemp.

- cuspidata Ktz.

- cymbiformis Eh.

- Ehrenbergii Ktz.

Epithenia gibba $\mathrm{Ktz}$.

- sorex Ktz.

- turgida Kitz.

- - var. granulata.

- zebra Ktz.

Fragilaria construens Grun. n• 565.

- $\quad$ - var. binodis n 565 .

- $\quad$ - var. venter n० 565 .

- mutabilis Grun n० 565 .

- _ $\quad$ - var.elliptica n० 565 .

- - var. minutissima n० 565 .
Gomphonema constrictum Ktz.

Melosira granuluta Eh.

Navicula ambigua $\mathrm{Ktz}$.

- amphigomphus Eh.

- elliptica Ktz.

- major hitz.

- mesolepta Ktz.

- nobilis Eh.

- serians Ktz.

- viridis Eh.

Vitzschia sigmoïdea Eh.

- palea Ktz.

Stauroneis plicenicenteron Elı.

Surirella elegans Eh.

- robusta Eh.

Synedra acus K'tz.

- capitata $\mathrm{E}$.

- ulna Eh.

$\mathrm{N}^{\circ} 566$

CANNES (France)

Sondage

Actinocycius Ralfsii W. Sm.

- subtilis Ralfs.
Actinoptychus undulatus Eh.

Asterolanıpa Marylandica Eb. 
Amphora crassa Greg.

Auliscus cœlatus Eh.

- sculptus Eh.

Biddulphia pulchella Gray.

Campylodiscus adriaticus var. massiliensis.

$\begin{array}{ll}- & \text { decorus Bréb. } \\ \text { - } & \text { eximius Greg. } \\ \text { - } & \text { fluminensis Grun } \\ \text { - } & \text { limbatus Bréb. } \\ \text { - } & \text { Lorenzianus Grun } \\ \text { - } & \text { Horologium o. m } \\ & \text { ranea A. S. } \\ \text { - } & \text { Ralfsii W. Sm. } \\ - & \text { Samoensis Grun. } \\ \text { - } & \text { Thuretii Bréb. }\end{array}$

Cocconeis pseudo-marginata Greg.

Coscinodiscus lineatus $E$.

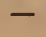

nodulifer A. S.

$-$ radiatus Eh.

Endyctia Oceanica Eh.

Mastogloia reticulata Grun.

Navicula aspera Eh.

- Beyrichiana A. S.

- bombus Wh.

- carinifera Grun.

- crabro Eh:

- exemta A. S.

- gemmata Greo.

- Hennedyi.

- $\quad$ - var. niceænsis H. P.

- longa Greg.

- lyra Eh.

- _ var. elliptica.
Navicula lyra var. subtypica.

- multicostata Bréb.

- nebulosa Greg.

- Powelli Lewis.

- prætexta Eh.

- Smithii fa maxima.

- Sandriana Grun.

vitzschia insignis Grun.

- notabilis Grun.

- panduriformis Greg.

- sigma W. Sm.

- valida Gl. et Grun.

Opephora Schoartzii var.? P. P.

Orthoneis splendida Grun.

Pleurosigma affine Grun.

$\begin{array}{ll}\text { - } & \text { balticum W. Sm. } \\ \text { - } & \text { decorum var. dalmaticum. } \\ \text { - } & \text { formosum W. Sin. } \\ \text { - } & \text { littorale W.Sm. } \\ \text { - } & \text { speciosum W.Sm. }\end{array}$

Podlosira ambigua Grun.

Surirella collare A. S.

- fastuosa Eh.

- - var. abludens.

- - var. opulenta.

- Guinàrdii H. P.

- intercedens Grun.

- lata W. Sm.

Synedra baculus Greg.

- fulgens W. Sm.

- Hennedyana Greg.

- robusta W. Sm.

Triceratium antediluvianum Eh.

- favus Eh.

- $\quad$ spinosum Bail.

No 567

CANNES (France)

Sur les Corallines

Actinocyclus Ehrenbergii Ralfs.

Bidululphia pulchella Gray. balaena.
Campylodiscus decorus Bréb.

Thuretii Bréb.

Coscinodiscus concarus Greg. 
Grammatophora macilenta Grun. serpentina Eh.

Orthoneis splendida Grun.

Plenrosigma decorunı W. Sm. - rigidum IV. Sm.

Podocystis adriatica Ktz.
Rhabionema adriaticum $\mathrm{Ktz}$.

Synedra formosa Hantz.

- Galllonii W. Sn.

- undulata W. Sm.

Triceratium Shalboltianun Grun.

- antediluvianum et var.

No 568

LE HAVRE (France)

Grammatophora marina litz.

Nos 569 et 570 OTAGO - OAMARU (Nouvelle-Kélande)

Actinoptychus vulgaris var. maculata Gr. et St.

Arachnoidiscus Ehrenbergij Bail.

Asterolampra decora Grev.

$$
\text { - vulgaris Grev. }
$$

Aulacodiscus amcenus Grev.

- cellulosus Gr. et St.

- crux var. subsquamosa Grun.

- Janischii Gr. ot St.

- Rattrayi Gr. et St.

- Nova Zelandiæ Gr. et St.

Aullecus inflatus Gr. et St.

Birlulphla Oamaruensis Gr. et St.

$$
\text { - _ Tuomeyi Bail. }
$$

Brightwellia coronata Ralfs.

Coseinodiscus angulatus Grev.

-

$\longrightarrow$

-

-

$-$

$-$

- Rothii Grun.

- $\quad$ spiniferus Gr. et St.

- symbolophorus Grun.

- vetustissimus Pant.

Eunotogramma productum Gr. et St,

Goniothecium odontella Eh.

Iemianlus ornithocephalus Grev. - polymorphus Grove.

Hyalodiscus radiatus Grun.

- $\quad$ - $\quad$ var. arctica Grun.

Isthuia enervis Ehr.

Kittonia elaborata Grr. et St.

Melosira Oamaruensis Gr. et $\mathrm{St}$.

- sol El.

- sulcata Grun.

- - var. biseriata.

- truncata Grove.

Podlosira corolla A. S.

- hormoïdes Mont.

Pyxidicula cruciata Eh.

Pyxilla dubia Grun.

- reticulata Gr. et St.

Stephanopyxis Grunowii Gr. et St.

Stictodiscus Californicus Grev. rar.

- Hardmanianus var. megapora Gr. ot St.

- nitidus Gr. et St.

- Nova Zelandica Grun.

- paralellus fatrigona Gr. et St.

- - var. gibbosa Gr. et St. 
Triceratium castellatum var. fracta Grun.

$\begin{array}{ll}\text { - } & \text { castelliferum Grun. } \\ \text { - } & \text { crenulatum Gr. et St. } \\ \text { - } & \text { fallaciosum Grun. } \\ \text { - } & \text { - fa quadrata. } \\ \text { - } & \text { glandarum A. S. } \\ \text { - } & \text { glandiferum Grun. } \\ \text { - } & \text { grande Bright. }\end{array}$

Triceratium majus Gr. et St.

- Morlandii Gr. et St.

- - var. aperta.

- $\quad$ - var. subaperta.

- Nova Zelandicum Gr. et St.

- pseudo marginatumGr. et St.

- rugosum Gr. et St.

- undatum Grun.

Trinacria ventricosa Gr. et St.

\section{No 571 CANCALE $N^{n} 1$ - ILLE-ET-VILAINE (l'rance)}

Amphiprora ornata Bailey. Amphora sulcala Bréb. fa parva.

Lichmophora Ehrenbergii El. Navicula Gregoria Donk.

Nitzschia hyalina Greg.
Plagiotropis Van Heurckii Grun.

Pleurosigma æstuarii W. Sm.

- Brebissonii Donk.

- fasciola W. Sm.

\section{No 572 CANCALE No 2 - ILLE-ET-VILAINE (France)}

Amphiprora elegans A. S.

Nitxschia acuminata W. Sm.

- sigma W. Sm.

Pleuroaigma restuarii W. Sm.

- balticum W. Sm.

- littorale W. Sm.
Pleurosigma fasciola W. $S m$.

- strigosum W. Sm.

- Parkerii Har.

- Wansbeckii Donk. faminor.

Scoliopleura latestriata Grun.

Surirolla gemma Eh.

No 573 CHAVILLE - SEINE-ET.OISE (France)

Navicula cuspidata Ktz. 


\section{SAINT-LÉGER - OISE (France)}

Sur les Sphagnums

Van Heurckia rhomboides, var. Saxonica Rab.

$\mathrm{N}^{\circ} 575$

LAC LADOGA (Finlande)

A la surface du lac

Asterionella formosa Hass.

Cyclotella Kützingiana Cl.

Cymatopleura solea W. Sm.

Cymbella cistula Hemp. vo maculata.
Eunotia Clevei Grun.

Gomphonema geminatum $\mathrm{Ag}$.

Melosira granulata Ralfs.

Stephanodiscue astraea Grun.
Ceratonels arcus Ktz.

Cymbella amphicephala Næg.

Diatoma hiemale $\nabla$. mesodon.

Epithemla turgida Kty. var.

Eunotla arcus Eh. v. hybrida.

- diodon Eh.

- exigua Rab.

- gracilis Rab.

- henaglyphis Eh.

- inciara Greg.

- Iunaris Grun.

- prærupta Eh.

- robusta Rab. v. tetraodon.

- Soleirolei Rab.

Fragilaria construens Grun.

- virescens Ralfs.

Gompihonema angustatum KIz.

- parvulum Kitz.

- subclavatum Grun.

Melosira distans Ktz. v. nivalis.
Navicula aftinis Eh.

- bicapitata Lag.

- - var. hybrida.

- bisulcata Lag.

- borealis Ktz.

- Brebissonii Ktz.

- gibba Ktz.

- iridis Eh.

- lata Bréb.

- major Ktz.

- viridis $\mathrm{Ktz}$.

Stauroneis anceps Eh.

- _ - var. linearis.

- gracilis Eh.

- phœnicenteron.

Surirella linearis W. Sin.

- tenera Greg.

Tabellarie flocculosa Ktz.

Van Heurchía crassinervia Bréb. rhomboïdes Bréb. 
Cymbella amphicephala Næg.

Diatoma hyemale var. mesodon.

Encyonema gracile Rab.

$$
\text { - ventricosum Grun. }
$$

Eunotia arcus Eh. rar. hybrida.

- gracilis Rab.

- incisa Greg.

- major Rab.

- parallela Eh.

- pectinalis Rab.

- pentaglyphis Eh.

- robusta var. tetraodon.

- - var. triodon.

Fragilaria virescens lialfs.

Gomphonema commutatum Grun. subclavatum Grun.

Melotira distans Kitz, var. nivalis.
Navicula bicapitata Lag. var. hybrida.

- bisulcata Lag.

- borealis Kitz.

- Brebissonii Ktz.

- dactylus Eh.

- gibba Eh.

- gigas $\mathrm{Ktz}$.

- major.Ktz.

- viridis $\mathrm{Ktz}$.

Stauroneis anceps Eh.

- _ var. linearis.

- phœnicenteron Eh.

Surirella linearis W. Sm.

- tenera Greg.

Tabellaria flocculosa $\mathrm{Ktz}$.

Yan ffeurckia crassinervia Bréb. rhomboïdes Bréb.

\section{N.578 GOSCHENEN ALP (Suisse) $N^{\circ} 3$}

Coratoneis arcus $\mathrm{Ktz}$.

Cymbella amphicephala Næg.

Diatoma anceps Kirch.

- hyemalo var. mesodon.

Encyonema gracilis Rab.

$$
\text { - ventricosum Grun. }
$$

Eunotia arcus Eh.

- - var. hybrida.

- gracilis Rab.

- incisa Greg.

- lunaris Grun.

- major Rab.

- minor Rab.

- pectinalis Rab.

- proerupta Eh.

- robusta var. tetraodon.

- $\quad$ - var. triodon.
Fragilaria virescens Ralfs.

Gomphonema angustatum Ktz.

- intricatum $K t z$.

- parvulum Ktz.

- subelavatum Grun.

Molosira distans $\mathrm{Ktz}$. var. nivalis.

Meridion constrictum Ralfs.

Navicula affinis Eh.

- bicapitata Lag.

- - var. hybrida.

- bisulcata Lag.

- borealis $\mathrm{Ktz}$.

- Brebissonii Ktz.

- iridis Eh.

- major Ktz.

- pupula. 
Navicula subcapitata var. Stauroneiformis.

- viridis $\mathrm{Ktz}$.

Pleurosigma attenuatum W. Sm.

Stauroneis anceps Eh.

- - - var. linearis.

- gracilis Eh.
Stauroneis phoenicenteron Eh.

Surirella linearis W. Sm.

- - var. constricta.

- ooalis Ktz.

- tenera Greg.

Van Heurckia crassinervia Brẻb.

BIBLIOGRAPHIE. - Cleve. Le Diatomiste,-volume II, page 99.

Achnanthes hungarica Grun.

Amphipleura Lindheimeri Grun. var. intermedia.

Cocconeis placentula Eh.

$$
\text { - - var. lineata. }
$$

Epithemia gibberula $\mathrm{K} \downarrow$.

Eunotia monodon Eh.

Gomphonema subclavatum Grun .

Navicula anglica Ralfs.

- divergens W Sm.

- - var elliptica.

- luagerheimii Gl.

- latevittata $\mathrm{Cl}$.
Navicula elliptica $K(z$.

- major Ktz.

- viridis $\mathrm{Ktz}$.

- - var. distiguenda.

Nitzschia linearis $W$. Sm.

- spectabilis Ralfs.

Ithopalodia gibha O. M.

- $\nabla$. ventricosa.

Surirella splendida $E$.

- tenera. Greg.

- - oar. nercosa.

Synedra ulna Eh.

- - var. danica.

$\mathrm{N}^{\circ} 580$

TESALIA (Equateur)

Achnanthes hungarica Grun.

- Janceolata Bréb.

Epithemia amphicephala Grun.

- argus Ktz.

- gibberula $\mathrm{Ktz}$.

Gonphonema angustatum $v$. intermedia.

- subclavatum Grun.
Inantzchia amphioxys Grun.

Navicala americana Grun.

- lacunarum Grun.

- sculpta Eh.

Surirella crumena Bréb.

- ovalis Bréb.

- ovata Ktz. 
Achantidium lanceolatum Bréb. Encyonema ventricosum Grun.

Epithemia gibberula Litz.

Eunotia minor Rab.

- monodon Eh.

- prærupta Eh.
Gomphonemn turris Eh. intricatum $\mathrm{K} t z$.

Melosira Roeseana Rab.

Navicula laterittata var. Domingensis.

Nitzschin spectabilis Ralfs.

Rhopalodia gibba $\mathbf{O}$. M.

\section{No 582 HARBOR BAY - MAINE (Etats-Unis)}

Runotia flexuosa Ktz.

- lunaris Grun.

- minor Rab.

- monodon Eh.

- pectinalis Rab.

Gomphonensa intricatum $K t z$

Navicula amphigomphus Eh.

- Brebissonii Ktz.

- divergens IV. Sin.

- firma Ktz.

- gibba Eh.

- gigas Ktz.

- gracillima Ralfs.

- iridis Eh.

- - var. ampliata.

- major Ktz.
Navicula microstauron Eh.

- serians Ktz.

- - var. minor.

- streptoraphe Cl.

- viridis Ktz.

Nitzechia sigmoïdea W. Sm.

Stauroneis gracilis $E h$.

- phonicenteron.

Stenopterobia anceps Bréb.

Tabellaria fenestrata Ktz. - flocculosa $\mathrm{K} t \mathrm{x}$.

Van Heurckia rbomboides Breb.

- - var.lineata.

- viridula Bréb.

Nos 583 et 584 . LAC DE SARNEN (Suirse)

Achnauthidium lanceolatum Bréb. Amphora gracilis Eh.

$$
\text { - ocalis } K t z \text {. var. }
$$

Campylodiscus noricus Eh.
Cyclotella Bodanica Eul.

Cymbella cistula Kirsch.

- cuspidata Kiz.

- Ehrenbergii Ktz. v. minor. 
Cymbella gastroïdes Kitz.

- heteropleura Ktz.

- $\quad$ maculata Ktz.

Diploneis Smithii Cl.

Encyonema gracile Rab.

- oentricosum Grun.

Epithemia turgida $K t z$.

Eunotia Arcus Eh.

- hidens Eh.

- tlexuosa Kız.

- formica Eh.

- gracilis Rab.

- hendecaodon Eh.

- lunaris Grun.

- major Rab.

- ininor Rab.

- monodon Ell.

- parallela Eh.

- pectinalis Rab.

- - var. undulata.

- - var. ventricosa.

- pentaglyphis Eh.

- polyodon Ralfs.

- prierupta Eh.

- - var. bidens.

- - var, inflata.

- robusta var. tetraodon.

- - var. triodon.

Gomphonema acuminatum Eh.

- constrictum Eh.

- - var. capitata.

- montanum var. suecica.

Irantzschla elongata Grun.

Melosira crenulata Ktz.

- undulala Ktz.

- carians Ag.

Meridion constrictum Ralfs.

Navicula acrosphæria var. 'dilatata.

- aftinis Eh.

- americana Eh.

- amphigomphus Eh.

- amphirynchus Eh.

- binodis W. Sm.

- borealis $\mathrm{Ktz}$.

- Brebissonii Ktz.

- bisulcala Lag.

- commutata Grun.

- dactylus Iitz.
Navicula divergens W. Sf.

- $\quad$ - var. elliptica.

- dubia Eh.

- Esox Eh.

- firma Ktz.

- $\quad$ - var. subundulata.

- gibba Eh.

- gigas $\mathrm{K} t z$.

- gracillima var. stauroneiformis.

- halophila Grun.

- $\quad$ - fa craticula.

- hemiptera $\mathrm{Ktz}$.

- Hitclicockii El.

- iridis Eb.

- $\quad$ - var. ampliata.

- Jata Bréb.

- latevittata Cl.

- legumen Eh.

- leptogongyla Grun.

- limosa Ktz.

- major Ktz.

- marina Ralfs.

- mesolepta Eh.

- microstauron Eh.

- nobilis $\mathrm{Kt} z$.

- nodosa Eh.

- peregrina Ktz.

- pusilla, oar. alpestris.

- radiosa $\mathrm{Ktz}$.

- rupestris Hantz.

- semen Eh.

- serians Ktz.

- stomatophora Grun.

- streptoraphe Cl.

- tabellaria Istz.

- transversa A. S.

- viridis $\mathrm{ki} z$.

- vulpina IItz.

Nitzschia spectabilis Ralfs.

Pleurostauron acula Rab.

- javanica Grun.

- ( - var.) rhombica

n. sp. à contours rhombiques anguleux, 11 stries en $10 \mu$, long. $210 \mu$.

Stauroneis amphilepta Eh.

- anceps Eh.

- _ - var. amphicephala. 
Stauronels anceps car. linearis.

$$
\begin{aligned}
& \text { - } \quad \text { Brunii M. Per. } \\
& \text { - } \quad \text { gracilis Eh. } \\
& \text { - } \quad \text { phonicenteron Eh. } \\
& \text { - } \quad \text { pteroides Eh. }
\end{aligned}
$$

Stephanodiscus astrace oar. spinulosa. Surirella elegans. Eh.

- robusta Eh.

- splendida Eh.
Surirella tenera Greg.

- - var. splendidula.

Synedra ulna Eh. var. danica.

Tabellaria fenes/rata $\mathrm{Ktz}$.

$$
\text { - flocculosa Ktz. }
$$

Tetracyclus lacustris Ralfs.

Van Ileurckia rhomboïdes Breb.

$$
\text { oar. amphi- }
$$
pleuroides.

\section{No ว̌85 GROTTE DE MALDONADO - VALPARAISO}

\section{(Chili)}

Actinoptychus glabratus Grun.

$$
\begin{array}{ll}
\text { - } & \text { splendens Shad, var. } \\
\text { - } & \text { undulatus Eh. } \\
\text { vulgaris Schum. }
\end{array}
$$

Billulphia pulchella Greg.

Cerataulus laeois Ralfs.

Coscinodiscus concinnus $W . S m$.

$\begin{array}{ll}\text { - } & \text { cribrosus T. W. } \\ \text { - } & \text { denarius A. S. } \\ \text { - } & \text { oculus-iridis Eh. } \\ & \text { radiatus Eh. }\end{array}$

llemiaulns ambiguus Grun.

IIyalodiscus stelliger Bail. subtilis Bail.
Melosira granulata Ralfs.

- sulcata Ktz.

Navicula lateoittata var. Domingensis.

Nitzschia circumsuta Grun.

Rhabdonema adriaticum $\mathrm{Ktz}$.

Surirella striatula Turp.

synedra crystallina $K t$.

Terpsince intermedia Grun.

Triceratium alternans Bail.

$$
\begin{aligned}
& \text { - } \quad \text { javus Eh. } \\
& \text { - } \quad \text { megastomum Eh. }
\end{aligned}
$$

\section{$\mathrm{N}^{\circ} 586$ à 588 CRANE POND (Etats-Unis)}

Amphora Delphinea Bail. v. minor.

- ooalis Ktz.

- Libyca Eh.

Cocconeis lineata Eh.

- placentula Eh.

cyclotella Bodanica Eul.

Cymbella americana A. S.
Cymbella cistula Kirch.

- cuspidata Ktz.

- Ehrenbergii Ktz.

- - var. minor.

- gastroïdes $\mathrm{K} t z$.

- Hauckii W. H.

- heteropleura Ktz. 
Enoyonema ventricosum Grun.

Epithemia amphicephala Grun.

$$
\begin{array}{lc}
\text { - } & \text { truncata } M . \text { Per. } \\
\text { - } & \text { turgida hlz. } \\
\text { - } & \text { - var. granulata. } \\
\text { - } & \text { - var. vertagus. }
\end{array}
$$

Funotia arcus Eh.

- bidentula W. Sm.

- formica Eh.

- gracilis Rab.

- impressa Eh.

- incisa Greg.

- lunaris Grun.

- minor W. H.

- parallela Eh.

- pectinalis Rab.

- - var. undulata.

- - - var. ventricosa.

- prcerupta Eh.

- - var. bidens.

- tetraodon Eh.

Fragilaria æqualis Heib.

$$
\text { - construens Grun. }
$$

Gomphonema acuminatum $\mathrm{Eh}$.

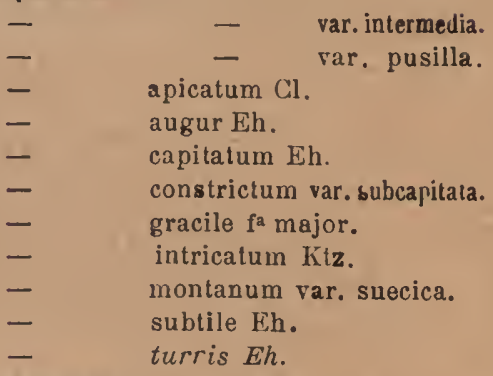

IIantzschia amphioxys Grun.

$$
\text { - elongata Grun. }
$$

Melosira crenulata $\mathrm{Ktz}$.

- tenuis $\mathrm{Ktz}$.

- tenuissima Grun.

- undulata Kítz.

Meridion constrictum Ralfs.

Navicula acrosphæria Bréb. $\nabla$. dilatata.

- rstuarii Cl.

- americana El.

- - var. bacillaris.

- - var. minor.

- amphigomphus Eh.

- amphirynchus El.
Navicula anglica Ralfs.

- bacilliformis Grun.

- bicapitata Lag.

- Bogotensis Grun. oar.

- Brebissonii Ktz.

- cardinalicus Cl.

- commutata Grun.

- cuspidata Ktz.

- dactylus Kiz.

- Dariana A.S.

- dilatata Eh.

- divergens W Sm.

- dubia El.

- - var. acuminata n. v. elliptique extrémités acuminées.

- esox Eh.

- firma Ktz.

- gastrum Ell.

- gibba Ktz.

- gigas Ktz.

- hemiptera Ktz.

- Hitchcockii Eh.

- integra Grun. var.

- iridis Eh.

- - var. ampliata.

- legumen Eh.

- limosa Ktz.

- - var. gibberula.

- major $\mathrm{Ktz}$.

- - var. asymetrica.

- mesolepta Eh.

- - var. stauroneiformis.

- mesotyla Eh.

- nobilis Ktz.

- nodosa Eh.

- polyonca Rab.

- pseudo-bacillus Hantz.

- radiosa $\mathrm{K} t z$.

- - var. acuta.

- rupestris Grun.

- sillimonorum Eh.

- Smithii Brèb.

- stomatophora Grun.

- tabellaria $\nabla$. stauroneiformis.

- transversa A. S.

- trigonocephala Cl.

- ventricosa Eh.

- viridis $\mathrm{K} t z$.

Nitzschia scalaris IV. Sm.

Odontidium tabellaria W. Sm. 
Pleurostauron acuta Rab.

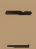
jaoanica Grun. Smithii Grun.

Rhopalodia gibba O. M.

Stauroneis anceps Eh.

- - var. elongata.

- Baileyi Eh.

- lanceolata Kiz.

- qracilis Eh.

- phrnicenteron Eh.

- Sieboldii Eh.

Surirella $\operatorname{arcta} A . S$.
Surirella cardinalis Kitt.

- linearis IV. Sin.

- $\quad$ - var. constricta.

- elegans Eh.

- splendida Eh.

- tenera Greg.

- - var. splendidula.

Synedra delicatissima W. Sm.

- amphirynchus Ktr.

- ulna Eh.

- - var. danica.

Tahellaria fenestrata $\mathrm{K} t z$.
Cymbella amphicephala Næg.

- cuspídata $\mathrm{K} \mathrm{tz}$.

- Ehrenbergii oar minor.

- gastroïdes $\mathrm{Ktz}$.

- heteropleura kitz.

- leptoceros Ktz.

Encyonema ventricosum Grun.

Eunotia gracilis Rab.

- Iunaris Grun.

- major Rab.

- minor Rab.

- monodon Eb.

- parallela Eh.

- pectinalis Rab.

- - var ventricosa.

- polyglyphis Eh. var.

- roluusta Eh, var.

- tetraodon Eh.

Gomphonema acuminatum Els.

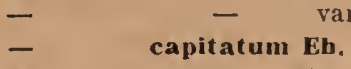

var. pusilla.

Melosira crenulata Kłz.

Navicula affinis Eh.

- ambigua Eh.

- amphigomphus Eh.

- bicapitata Lag.
Navicula bicapitata car. hybrida,

- Brebissonii $\mathrm{K} l \mathrm{z}$.

- commutata Grun.

- dactylus Kiz.

- dilatata Eh.

- firma Kitz.

- gibba kilz.

- gigas hitz.

- hemiptera Ktz.

- iridis Eh.

- - var. ampliata.

- major kitz.

- mesolepta v. Stauroneiformis.

- nobilis Ktz.

- pseudo-bacillum Grun.

- rupestris Hantz.

- semen Eh.

- viridis $\mathrm{Ktz}$.

Nitzschia sigmoldea? W. Sin.

Odontidium tabellaria W. Sm.

Stauroneis anceps Eh.

- Baileyi Eh.

- gracilis Eh.

Surirella tenera Greg.

Tabellaria fenestrata Kítz.

Van Ifeurckia rhomboïdes Bréb.

- var. lineolata. 


\section{Nos 590 et 591 THURSDAY ISLAND N॰2}

\section{(Nouvelle-Guinée)}

Achnanthes javanica Grun. car. rhombica.

Actinocyclua confluens Grun.

$$
\text { - incertus Grun. }
$$

Actinoptychus intermedius $A . S$.

$$
\text { - oulgaris var. australis. }
$$

Aulacodiseus Johnsonii Arn.

Auliscus colatus Bail.

Bacteriastrum oarians Laud.

Billdulphia aurita Bréb.

$$
\begin{array}{lc}
\text { - } & \text { pulchella var. Gray. } \\
\text { - } & \text { reticulata Rop. } \\
\text { - } & -\quad \text { var. trigona. } \\
\text { - Tuomeyi Rop. }
\end{array}
$$

Campylodiscus biangulatus Grev.

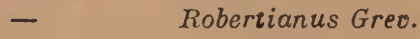

- undulatus Grev.

- sp. A. Sch. atlas 207/13.

Corataulus Petitii Loud. Fort.

Climacosphenia elongata Bail.

Cocconeis heteroïdea var. sigmoidea.

$$
\text { - pellucïda Hantz. }
$$

Coscinodiscus blandus A. S.

$$
\begin{array}{ll}
\text { - } & \text { concinnus W. Sm. oar. } \\
\text { - } & \text { excentricus Eh. } \\
\text { - } & \text { subtilis Eh. }
\end{array}
$$

Diploneis splendida Greg.

Endictya oceanica $E h$.

Eplthemia hirundinella J. Br.

Euphyllodium spathulatum Shadb.

Grammatophora oceanica Eh. oar. $m a$ cilenta.
IIyalodiscus radiatus Grun.

- stelliger Bail.

Isthmia capensis Grun.

Melosira sulcata $K t z$. oar. coronata.

Navicula arabica Grun.

- liber W. Sm. var umbilicata.

- lyra et var.

- maxima Greg. var. bicuneata.

- notabilis Grev.

- nummularia Grev.

- robusta Grun.

- scapulorum Brẻb.

- $\quad$ - var. craticula.

Nitzschia alata Leud. Fort.

- Jelineckii Grun.

- - longissima Ralfs.

- panduriformis Greg.

- sigma W. Sm.

Orthoneis fimbriata Grun.

Pleurosigma angulatum W. Sm.

$$
\begin{aligned}
& \text { - } \quad \text { intermedium W. Sm. } \\
& \text { - } \quad \text { rigidum W. Sm. }
\end{aligned}
$$

Hhalodonema adriaticum $I \bar{t} z$.

Surirella fastuosa Eh.

Synedra crystallina var. conspicua.

- robusta Ralfs.

Toxarium Hennedyanum Grun.

Trachyneis aspera $\mathrm{Cl}$.

$$
\text { - - var. vulgaris. }
$$

Triceratium bicorne Cl.

- gibbosum.

- scitulum var. quadrata.

Tropidoneis lepidoptera $\mathrm{Cl}$. 
$\mathrm{N}^{\circ} 592$ et $593 \quad$ THURSDAY ISLAND No 3

\section{(Nouvelle-Guinée)}

Achnanthes longipes Ag.

Actinocyclus Ehrenbergii Ralfs. subtilis Ralfs.

Actinoptychus hexagonus Grun. var.

$$
\begin{aligned}
& \text { - splendens Ralfs. } \\
& \text { - vulgaris Schum. } \\
& \text { - - var. australis. }
\end{aligned}
$$

Allö̀neis antillorum $\mathrm{Cl}$.

Amphora aurita Bréb.

- granulata Rop.

- pulchella Greg.

- Tuomeyi Rop.

Campylodiscus biangulatus Grev.

$\begin{array}{ll}\text { - } & \text { Kittonianus var. Zanzi- } \\ \text { barica. } & \text { Samoensis Grun. } \\ \text { - } & \text { toenioüdes Deby. } \\ \text { - } & \text { undulatus Grev. }\end{array}$

Cerataulus Smithii Ralss.

Cocconeis pellucida Hantz.

Coscinodiscus excentricus Elh.

$\begin{array}{ll}- & \text { lineatus Eh. oar. } \\ \text { - } & \text { nitidulus Grun. } \\ \text { - } & \text { nodulifer var. apiculata. } \\ \text { - } & \text { radiatus Eh. } \\ \text { - } & \text { Rothii Grun. } \\ & \text { symmetricus Grun. }\end{array}$

Cyclotella striata Grun.

Dictyoneis marginata Cl.

Diploneis apis Eh.

- bombus Eh.

- crabro Eh. var.

- nitescens Cl.

- Smithii Cl.

- splendida Greg.

Eudictya oceanica Eh.

Glyphodesmis margaritacea Cast. llyalodiscus maximus Eul.

- stelliger Bail.

- subtilis Bail.

Isthmia nervosa Ktz.

Mastogloia quinqueco:tata Grun.

Melogira sol Eh.

- sulcata Kítz.

- - var. coronata.

- - var. radiata.

Nivicula abrupta Donk.

- arabica Grun.

- bullata Norm.

- clavata Greg.

- lyra Eh.

- - var. intermedia.

- - var. subcarinala.

- Perrottetti Grun.

- robusta Grun.

Nitzsehia marginulata Grun.

- panduriformis Greg.

Orthoneis aspera $\mathrm{Per}$.

- fimbriata Grun.

Podosira ambigua Grun.

Stephanopyxis turris Eh.

Surirella fastuosa Eh.

- incurvata A. S.

Synedra robusta Ralfs.

Trachyneis aspera $\mathrm{Cl}$.

- clepsydra Donk.

Triceratium areticum Bright.

- favus Eh.

- formosum Bright.

- muricatum Bright.

- pentacrinus Wall.

- scitulum fa quadrata.

- sculptum Heib.

Weissnogia Macdonaldii Jän. 
No 594

PORT DE DUNKERQUE (France)

Actinocyclus Ehrenbergii Ralfs.

Actinoptychus splendens Ralfs.

$$
\text { - undulatus Rh. }
$$

Aulacodiscus argus A. S.

$$
\text { - Rogersii A. S. }
$$

Auliscus sculptus Ralfs.

Biddulphia aurita Bréb.

- heteroceros Cirun.

- rhombus W. Sm.

Campylosira cymbelliformis Grun.

Cerataulus Smithii Ralfs.

Coscinodiscus excentricus Eh.

- nitidulus Grun.

- radiatus Eh.

Cyclotella striata Grun.

Cymatosira Belgica Grun.

Diploneis bombus Eh.

- didyma Eh.

- interrupta $\mathrm{Cl}$.

- Smithii Cl.

IIantzechia amphioxys Grun.

- virgata Grun.

Hyalodiecus stelliger Bail.

- subtilis Bail.
Melosíra nummuloïdes Ay.

- sulcata Ktz.

- Westii W. Sm.

Navicula distans Ralfs.

$$
\begin{aligned}
& \text { - formosa Greg. } \\
& \text { - Hennedyi W. Sm. } \\
& \text { - humerosa Bréb. } \\
& \text { - Lyra Eh. } \\
& \text { - palpebralis Bréb. } \\
& \text { - } \quad \text { subsalina Donk. }
\end{aligned}
$$

Nitzochia granulata Grun.

- navicularis Brèb.

- panduriformis Greg.

Plagiogramma Gregoryanum Greo.

Raphoneis amphiceros Eh.

$$
\begin{array}{lcc}
- & - & \text { var. belgica. } \\
\text { - } & - & \text { var. rhombica. } \\
\text { - } & \text { nitida var. liburnica. } \\
\text { - } & \text { surirella Grun. } \\
\text { - } & - & \text { var. australis. }
\end{array}
$$

Scoliopleura tumida Bréb.

- - var. adriatica.

Surirella fastuosa Eh.

Triceratium alternans Bright.

- faous Eh.

\section{No 595 WIMEREUX - PAS-DE-CALAIS (France)}

Amphora cymbifera Greg.

Anorthoneis excentrica Grun. Drurigea geminata Donk.
Navicula forcipata Grer. var.

Plagiogramma adriaticum Grun.

$N^{\circ} 596$ SAN-LUIS (Buenos-Aires)

Achnanthes lanceolata Bréb. var. Donticula oalida Per.
Gomphonema lagenula kitx. parvulum $\mathrm{K} \mathbf{z}$. 
Hentzochia amphioxys Eh.

Navicula a mbigua Eh.

- anglica Ralfs.

- halophila Grun.

- microstauron Eh.

- viridis $\mathrm{Ktz}$.

vitzschia linearis W. Sm.
Rhopalodia gibba Mul.

Surirella ovalis Bréb.

- tenera Greg.

$$
\text { - - var. nervosa. }
$$

Synedra ulna Eh. var. æqualis.

- - var. amphirynchus.

- - var. splendens.
No 597 LE HAVRE (France)

Melosira dubla Ktz.

\section{No 598 AMBLETEUSE - PAS-DE-CAI_AIS (France)}

Actinocyclus Roperii Kitt.

Amphora cymbifera Grog.

Anorthoweis excentrica Grun.

Biddulphia aurita Bréb.

Donkinia recta Grun.

Druridgea geminata Donk.

IIantzschin amphioxys Grun. - marina Grun.
Navicula forcipata Grev, var.

- hyalosira $\mathrm{Cl}$.

- lanceolata Ktz.

- palpebralis Bréb.

Striatella unipunctata $\mathrm{Ag}$.

Trachyneis aspera $\mathrm{Cl}$.

Tropidoneis lepidoptera Cl.
Cocconeis lineata $E h$.

Cymatopleura apiculata W. Sm. - solea W. Sm.

Cymbella cistula Kirch.

Eunotia lunaris Grun.

- minor Rab.

Gomphonenı acuminatum Eh. var. laticeps. var. clava.
Navicula ambigua Eh.

- amplirynchus Eh.

- amphisbcena Bory.

- Brebissonii Ktz.

- cuspidata Ktz.

- limosa var. gibberula.

- oblonga KIz.

- rafiosa Ktz.

- rhynchocephala $\mathrm{K} / \mathrm{z}$.

- subsalina Donk.

- viridula var. minor. 
Nitzechla dubia W. Sm.

- hungarica Grun.

- linearis W. Sm.

- sigmoidea W. Sm.

Rhopalodia gibba O. Mull.

Pleurosigma acuminatum Grun.
Stauroneis Smithii Grun.

Synodra acus Ktz.

- capitata Eh.

- ulna Eh.

- _ var equalis.
Bidlulphia aurita Bréb.

Cocconeis Britannica Næz.

Coscinodiscus apiculatus Eh.

- excentricus Eh.

firammatophora marina $\mathrm{Ktz}$.

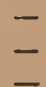
oceanica Eh. serpentina Eh.

- fa minor.
Licmophora Ehrenhergii var. augustata.

$$
\text { Jurgensii Ag. }
$$

Melosira sulcata $\mathrm{K} t z$. fa minor.

Orthoneis binotata Grun.

- splendida Grun.

Pollosira hormoïdes Ktz.

Rhahdonema arcuatum Ktz. .

Synedra Gaillonii Eh.
Achnanthes lanceolata Bréb.

Cocconeis placentula Eh.

$$
\text { - - var. lineata. }
$$

Cyclotella antiqua $W$. Sm. Cymbella cymbiformis Grun.

Encyonema ventricosum Grun.

Epithemia amphicephala Grun.

- cistula Ralfs.

- ocellata Ktz.

- sorex Ktz.

- zebra Ktz.

Eunotia arcus Eh.

- - var. plicata.

- bidentula.

- gracilis Rab.

- incisa Greg.

- lunaris Grun.

- monodon Eh.
Eunotia minor W. H.

- prcerupta oar. bidens.

Fragilaria virescens Ralfs.

Gomphonema acuminatum Eh.

$\begin{array}{ll}\text { - } & \text { augustatum Ktz. } \\ \text { - } & \text { constrictum Eh. } \\ \text { - } & \text { intricalum Ktz. }\end{array}$

Navicula commutala Grun.

- divergens W: Sm.

- Gendrei Her, et M. Per.

- gibba Ktz.

- gigas Ktz.

- major Ktz.

- microstauron Eh.". *

- pupula Kt.

- radiosa ktz.

- Reinhardtii Grun.

- serians Ktz. car. minor. 
Navicula stomatophora Grun.

- ventricosa Eh.

- viridis $\mathrm{K} t z$.

Nitzschia sigmoïdea W. Sm.

Rhopalodia gibba O. Mull.

Stauroneis anceps Eh.

- gracilis Eh.
Stauronels phonicenteron Eh.

Surirella gracilis car.

- ocalis Bréb.

- robusta Eh.

- tenera Greg.

Tabellaria fenestrata $\mathrm{Ktz}$.

- flocculosa $\mathrm{Ktz}$.
Achnanthes minutissima $\mathrm{K} t z$.

Amphora ooalis $K \boldsymbol{t}$.

Ceratoneis arcus $\mathrm{K} t z$.

Cymatopleura apiculata W. Sm.

Cymbella amphicephala Nceg.

- subrqualis Grun.

Encyonema ventricosum Grun.

Gomphonema angustatum $\mathrm{Ktz}$.

var. producta. parvulum var. subcapitala. subclaoatum Grun.

Hantzschia amphioxys Grun.

Meridion circulare $\mathrm{Ag}$.

Navicula Brebissonii $\mathrm{Ktz}$.

- - - var. minor.

- cryptocephala Klz.
Navicula cryptocephala var. exilis.

- lancettula Grun.

- limosa Ktz.

- mesolepta v. stauroneiformis.

- microstauron Eh.

- rhyncocephala Kitz.

- $\quad$ - var. amphicephala.

- viridis $K t z$.

Nitzschia acicularis W. Sm.

-. linearis W. Sm.

- minuta Bleisch.

- minutissima W. Sm.

Pleurosigma scalproitles $R a b$.

Surirella minuta Brèb.

Synedra ulna Eh.

- - var æqualis.

Van Heurckla vulgare Bréb.

No 603

LAC DE LUCERNE (Suisse)

Cyclotella Bodanica Eul, var.

| Fragilaria crotonensis, Kitt.

-

\section{No 604}

ILE DE MALTE

Dépôt fossile d'eau douce

Amphora pediculus Grun.

Cocconeis placentula $\mathrm{K} t z$.

Cymbella cistula Hemp.

- cuspidata $\mathrm{Ktz}$. 
Cymbella helvetica $\mathrm{K}$ z.

- lanceolata Eh.

- turgidula Grun.

Encyonema cæspitosum W. Sm. var.

Epithemia turgida Ktz.

- sorex Ktz.

- zebra Ktz.

Fragilaria construens var. pumila Grun. - mutabilis Grun.

Gomphonemx angustatum Grun.

- Brebissonii Ktz.

- capitatum fa curta.

Gomphonema clavatum Eh.

- montanum Eh.
Melosira creuulata var tenuis kitz.

- $\quad$ - var. procera.

Navicula bacillum Eh.

- elliptica $\mathrm{K}^{\prime} \mathrm{z}$.

- exigua Grey.

- gastrum El.

- placentula Eh.

- pupula $\mathrm{K} \mathbf{z}$.

- veneta Kitz.

Stephanodiscus astraea Grun.

- - var. minutula.

Synedra ulna Kiz.

- - var. longissima.

Tabellaria flocculosa $\mathrm{K} t \mathrm{z}$.
Amphora libyca Eh.

- lineata var. euglypta.

Cymbella cistula Hemp.

- cuspidata Ktz.

- Ehrenbergii Ktz.

- gastroides Ktz.

Cymbella heteropleura $\mathrm{K} t z$.

$$
\text { - lanceolata } \mathrm{Klz} \text {. }
$$

Encyonena gracile Rab.

- lunula Grun.

Epithemia gibba $\mathrm{Ktz}$.

- turgida Ktz.

- - var. grauulata Ktz.

- zebia Kitz.

Eunotia arcus var. uncinata Eh.

Comphonema acuminatum Eh.

-

$$
\text { constrictum Eh. }
$$

Hantzschia amphioxys Grun v. elongata.

Melosira arenaria Monre.

- crenulata var Jonensis.

Navicula amphirhynchus Eh.

- ampliata Eh

- cardinalis oar. $\beta$. Kitt.
Navicula cuspidata $K t$ z.

- - _ var. gracilis M. Per.

(On trouve assez communément une forme naviculaire présentant tous les caractères de $N$. cuspidata mais plus grêle et à stries plus fortes $(13$ en $10 \mu)$; on trouve également cette même espèce a vec la forme craticulaire, ce qui me confirme dans cette detormination.

- cuspidatá lítz. fa craticula var gracilis.

- elliptica Ḱtz.

- firma $\mathrm{Ktz}$.

- fusca Pritch.

- gibba Eh.

- gigas $\mathrm{Eh}$.

- Hitchcockii Eh.

- limosa $\mathrm{Ktz}$.

- var. gibberula. 
Navicula mesolepta Kíz.

- - - var. stauroneiformis.

- peregrina Eh.

- pseudobacillum Grun.

- pupula Kitz oar.

- radiosa Ktz.

- transversa A. S.

- ventricosa Donk.

- viridula $\mathrm{K} t z$.

- - var. major.
Navicula oiridis $K^{\prime} t$ s car commutata.

Stauroneis acuta W. Sm.

- anceps Eh. var.

- gracilis W. Sm.

- phønicenteron Eh.

- producta Grun.

Surirella elegans Eh.

- lunáris W. Sm. var. commutata.

- robusta Eh.

\section{N 606 BLACK MOSS - ABERDEEN (Écosse)}

\section{Dépôt fossile d'eau douce}

Amphora affinis Ktz.

Cymbella cuspidata $\mathrm{K} \mathbf{z}$,

Cymbella cymbiformis Eh.

$$
\text { - lanceolata Eh. }
$$

Encyonema cœspitosum $\mathrm{Ktz}$.

Epitheruia gibba $\mathrm{Ktz}$.

$$
\text { - sorex } \mathrm{KIz} \text {. }
$$$$
\text { - zebra Eh. }
$$

Eunotia arcus Eh.

$$
\text { - diodon Eh. }
$$

Fragilaria brevistriata Grun.

- construens Grun

- $\quad$ - var. venter.

- _ - var. binodis.

- - virescens Ralfs. var.

Comphonema acuminatum Eh.

-. commutatum Grun.

- constrictum Eh.

- geminatum Ag.

- montanum Eh.

Melosíra crenulata $\mathrm{K} t z$.
Navicula ámphirhynchus Eh.

- dactylus Eh.

Navicula divergens W., Sm.

- firma $\mathrm{Ktz}$.

- gigas Eh.

- Hobes Ralfs.

- hemiptera Ktz, var.

- legumen Elr.

- major Eh.

- nobilis Ktz.

- stomatophora Ktz.

- viridis $\mathrm{Ktz}$.

- - var. commutata.

Stauronels gracilis Eh.

- phœnicenteron Eh.

Surirella robusta Eh.

synedra capitata Eh.

- ulna Eh.

- - var. longissima.

Tabellaria fenestrata $\mathrm{Ktz}$.

- flocculosa: Ag. 
$N \cdot 608$

VILLEFRANCHE-SUR-MER (France)

Synedra undulata Greg.

$\Lambda \cdot 609$

DIEPPE (France)

Navicula scopulorum Bréb.

No 610 SAINT-SERVAN - ILLE-ET-VILAINE

(France)

Biddulphia rhombus $W$. sm.

No 611

PREMNAY PEAT (Angleterre)

BIBliographIE. - Prof. Dickie, Ann. of nat. Hist. Août 1818, Type Van Heurck, $n^{\circ} 51$. - Ce dépot de tourbe d'eau douce est un de ceux qui ont servi à W. Smith pour sa Synopsis.

Epithemla gibba Ktz.

$$
\text { - turgida Ktz. }
$$

Eunotia formica Eh.

- major Eh.

- minor Rab.

- tetraodon Eb.

Navicula alpina $w$. Sm.

- bacillum Eh.

- Brebissonii $\mathrm{K} t z$.

- $\quad$ - var. commutata Grun.
Navicula divergens $\mathrm{TW}$. Sm.

- dactylus Eh.

- major Ktz.

- nodosa Ktz. var.

- stauroptera Grun.

- viridis $\mathrm{Eh}$.

Surirella biseriata Bréb. fa major.

- robusta Eh. var.

Van Ileurckia viridula Bréb. 


\section{No612 LA DARSE - VILLEFRANCHE-SUR-MER}

\section{(France)}

Achuanthes longipes Ag.

Actinocyclus subtilis Ralfs.

Amphora sulcata Greg.

Biddulphla pulchella Greg.

Canpylodiscus Ralfsii W. Sm.

Cerataulus turgidus Eh.

Cocconeis pseudo-marginata Greg.

Grammatophora serpentina Eh.

Homoecladia Vidovichii Grun.

Melosira sulcata Kitz.
Navicula aspera Eh.

Pleurosigma decorum IV. Sm.

- delicatulum W. Sm.

formosum W. Sm.

Striatella delicatula IV. Sm.

- unipunctata. Ag.

Synedra dalmatica K'tz.

- Hennedyana Greg.

- undulata Greg.

Triceratium antediluvianum Eh. arcticum Bright.
Actinoptychus undulatus Eh.

Biddulphia aurita Breg.

- rliombus IV. Sm.

Campylosira cymbelliformis Grun.

Coscinodiscus excentricus Eh.

$$
\text { - radiatus Eh. }
$$

Epithemia musculus $\mathrm{Ktz}$

Navicula arenaria Donk.

- cruciformis Donk.

- rhombica Grev.

- Trevelyana Donk.

Nitzschla sigma IV. Sm.

- virgata Roper.
Pleurosigma aestuarii W. Su.

$$
\begin{array}{ll}
\text { - } & \text { balticum W. Sm. } \\
\text { - } & \text { delicatulum W. Sm. } \\
\text { - } & \text { lippocampus W. Sm. } \\
\text { - } & \text { marinum Donk. }
\end{array}
$$

Podosira maculata. W. Sm.

Raphoneis amphiceros Eh.

- - var. rhombica.

Scoliopleura latestriata Bréb.

- tumida Bréb.

Surirella gemma Eh. 


\section{Estomacs d'Holothuries}

Actinoptychus undulatus Eh.

Amphora elegans W. Sm.

$$
\begin{aligned}
& \text { - lepidoptera Greg. } \\
& \text { - } \quad \text { maxima Greg. } \\
& \text { - }
\end{aligned}
$$

Auliscus sculptus $\mathrm{Ktz}$.

- coelatus Bail.

Biddulphia pulchella Greg.

Campylodiscus adriaticus Grun.

- decorus Greg.

- $\quad$ - var. pinnata H.P.

- limbatus Bréb.

- Samoensis A. S.

Eudictya oceanica Eh.

Navicula aspera Elı.

- crabro et var. Eh.

- lyra et var. Eh.

vitzschia macilenta W. Sm. - scalaris Greg.
Nitzenchia sigma W. Sin.

$$
\text { - valida Cl. }
$$

Pleurosigma balticum $\mathrm{W} . \mathrm{Sm}$.

- decorum W. Sm.

- $\quad$ elongatum W.Sm.

- formosum var. balticum Cl.

- obscurum v. mediterranea.

- $\quad$ rigidum $\mathrm{W}$. $\mathrm{Sm}$.

- speciosum W. Sm.

Rhabdonema adriaticum Klz.

Surirella Comis A. S.

- fastuosa Eh.

- - var. abludens.

- - var. collare.

Synedra formosa Ktz.

- fulgens W. Sin.

- robusla Eh.

- undulata Greg.

Triceratium antediluvianum $\mathrm{Eb}$. Shadboltianum Eh.
Actinoptychus splendens Shad.

$$
\text { - } \quad \text { undulatus Eh. }
$$

Amphora obtusa Greg.

Biddulphia pulchella Greg.

$$
\text { - Tuomeyi Bail. }
$$

Campylodiscus adriaticus Grun.

$$
\begin{array}{ll}
\text { - } & \text { decorus Bréb. } \\
\text { - } & \text { limbatus Bréb. }
\end{array}
$$

Cocconeis pellucida Eh.

Coscinodiscus radiatus Eh.

Dictyoneis rugosa $\mathrm{Cl}$.
Endictya oceanica Eh.

Iyalodiscus subtilis Bail.

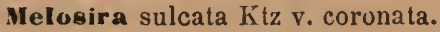

Navìcula aspera Eh.

- crabro Eh.

- pennatula Grun.

- maxima Greg.

- Lyra El.

- - v. abnormis.

- - o. recta.

- prætexta Eh.

- Zanardiana Grun.

Nitzschid panduriformis Grun. 
Orthoneis splendida Eh.

Pleurosigma affine Grun.

$$
\begin{aligned}
& \text { - balticum W. } \mathrm{Sm} \text {. } \\
& \text { - } \quad \text { formosum } \mathrm{v} . \text { Balearicum. }
\end{aligned}
$$

Podocystis Adriatica $K t$.

Scoliopleura tumida Bréb.

Surirella fastuosa Eh.
Surirella gemma Eh.

- recedens $\mathbf{A}$. S.

Synedra baculus o. minor.

- robusta Ralfs.

Triceratiun favus Eh.

spinosum Bail.
Actinocyclus Ehrenbergii Ralfs. var,

$$
\begin{array}{ll}
\text { - } & \text { moniliformis Ralfs. } \\
\text { - } & \text { nebulosus M. Per. }
\end{array}
$$

Actinoptychus undulatus Eh.

Ampliora acuta Greg.

- arcus Greg.

- obtusa Greg oar.

- proteus Greg. et var.

Auliscus coelatus Bail.

- sculptus Ralfs.

Biddulphia aurita Bróh.

Campylodiscus Thuretii Bréb.

Cerataulus Smithii Ralfs.

Cocconeis scutellum Eh.

Coscinodiscus concinnus $W$. Sm.

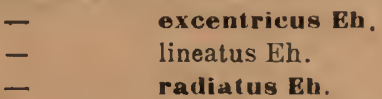

Dineregramma marinum Greg.

Diploneis apis Donk.

- bombus Eh.

- dilyma Eh.

- - var. major.

- fusca Cl.

- - var. delicatuli.

- _ - var. norvegica.

- interrupta $\mathrm{Ktz}$.

- Smithii Cl.

- splendida Bréb. var.

Epithemia gibberula Ktx.
Granimatophora oceanica var. macilenta. serpentina Eh.

llyalodiscus scoticus Grun.

- stelliger Bail.

- subtilis Bail.

Mastogloia apiculata W. Sin.

Melosira sulcata $\mathrm{Ktz}$. var. coronata.

Vavicula abrupta Donk.

- blanda A. S.

- Baileyana A. S.

- brevis Greg.

- digitoradiata Greg.

- forcipata Grev.

- Hennedyi IV. Sm.

- - var. nebulosa.

- humerosa Bréb.

- liber W. Sm.

- - var. elongata.

- lyra Eh.

- - var. atlantica.

- - var. elliptica.

- - var. intermedia.

- - - $\quad$ - fa dilatata. n. $\nabla$. à partie médiane fortement dilatee et extrèmités coniques. Long. $150 \mu$; larg. $60 \mu, 5,5$ stries en $10 \mu$.

- - var. recta.

- maxima Greg.

- - $\quad$ - var. bicuneata. 
Nitzschił acuminata Grun.

- punctata Grun.

- _ - var. elongata.

- sigma W. Sm. oar. rigida.

- - var. sigmatella.

orthoneis aspera H. Per.

Pleurosigm ^ formosum W. Sm.

Pyxilla baltica Grun.

Rhablonema areuatum ktz.

\section{- minutum ktz.}

Ithoïcosphenia curvata Grun. var. marina

Surirellu fastuosa Eh.

- $\quad$ - var. cuneata.
Surirella fastuosa var. opulenta.

- fluminensis Grun.

Syndendrium diadema Eh.

Synedra affinis $\mathrm{K} t z$

- buculus Greg.

- crystallina Kiz.

- Gaillonii Elı.

Toxarium undulatum Bail.

Trachyneis aspera Cl.

$$
\begin{aligned}
& \text { - - oar. minor. } \\
& \text { - - oar. pulchella. } \\
& \text { - - var. vulgaris. }
\end{aligned}
$$

Triceratium antediluvianum.

\section{No 618 GROTTE DE LERNO - BUENOS-AIRES}

\section{(Rép. Argentine)}

Achnauthes ventricosa Eh.

Amphora affinis Eh.

Cerataulus levis Ralfs.

Denticula valida Ped.

Gomphonema angustatum $v$, intermedia.

- commutatum Grun.

- constrictum Eh.

- intricatum Ktz.

Melosira tenuis Ktz.

- varians $\mathrm{Ag}$.

Navicula ambigua Eh.

- avenacea Bréb.

- cuspidata $\mathrm{K} \mathrm{z}$.
Navicula parva V. $H$.

- sculpta El.

- sphcerophora Eh.

- tenella Bréb.

- ventricosa Kt.

Nitzschia hungarica Grun.

Rhopalodia gibba 0 . M.

- - var. ventricosa.

- - gibberula O. M. var.

Surirella tenera o. splendida.

synedra tenera $W$. Sm.

- ulna Eh.

" - - var. danica.

- - var. splendens. 


\section{No 619 GROT'TE DE PALERMO - BUENOS-AIRES}

\section{(Rép. Argentine)}

Achnanthes inflata Eh.

Amphora aftinis Eh.

Cerataulus lævis Ralfs.

Denticula valida Ped.

Gomphonema angustatum Grun.

$$
\text { - constrictum Eh. }
$$$$
\text { - intricatum } K t z \text {. }
$$

Navicula ambigua Eh.

- $\quad$ - fa craticula.

- acenacea Bréb.

- cuspidata $\mathrm{Ktz}$.

- gibba Eh.
Navicula parva $V$. $H$.
- sculpta Eh.
- sphcerophora Eh.
- viridis $\mathrm{Ktz}$.
Ifhopalodia gibha O. 1 .

$$
\begin{aligned}
& -\quad \text { - var. parallela. } \\
& \text { - } \quad \text { gar. ventricosa. }
\end{aligned}
$$
Surirella tenera Greg.
Synedra ulna Eh.
- _ var. splendens.
Terpsinee̋ intermedia Grun.

$\left.N^{\circ} 621\right)$

ILES AÇORES (Océan Atlantique)

\section{Sondage}

Biddulphia pulchella Gray.

$$
\text { - regina W. Sm. }
$$

Brightwellia coronata oar" radians.

Cerataulus turgidus Eh.

Coscinodiscis apiculatus oar.

$$
\begin{array}{ll}
\text { - } & \text { concinnus W. Sm. } \\
\text { - } & \text { nitidulus Grun. car. } \\
\text { - } & \text { oblongus Greo. } \\
\text { - } & \text { radiatus Eh. } \\
\text { - } & \text { radiosus Grun. }
\end{array}
$$

Craspedodiscus oblongus Grun.

Endictya oceanica Eh.

Eunotogramma Weissii $E h$.

Euodia gibba var. atlantica.
Ilemiaulus alatus Grev.

- polycistinorum Eh.

Melosira clavigera Grun.

Vavicula protexta $E h$.

Pleurosigna balticum W. Sm.

$$
\text { - rigidum W. Sm. }
$$

Porodiscus sp.?

Stephanopyxis Grunowii Grov, et St. Stictodiscus hexagonus Cast.

Surirella lata W. Sm. var.

$$
\text { - striatula Turp. }
$$

Trachyneis aspera $\mathrm{Cl}$.

Triceratium etlanticum Cast.

$$
\begin{aligned}
& \text { - arcticum Bright. } \\
& \text { spinosum fa quadrata. }
\end{aligned}
$$


Actinocyclue curvatulus Jan.

- denticulatus Cast.

Actinoptychus splendens Shadb. car. undulatus Eh.

Amphora Clara A. S.

$$
\text { - egregia Eh. }
$$

Asterolampra Marylandica Eh.

Asteromphalus elegans Grev.

BIduluiphia reticulata Rop.

Brightwellia pulchra Grun.

Cestodiscus pulchellus Greo.

$$
\text { - gemmifer Cast. }
$$

Cocconeis heteroïdea Hantz.

Coscinodiscus africanus Jan.

- bulliens A. S.

- curvatulus Grun.

- $\quad$ - var. latius striata.

- anguste lineatus A.S.

- concinnus IV. Sm.

- $\quad$ _ var. Jonesianus

- excentricus El.

- elegans var. spinifera.

- fimbriatus Eh.?

- gigas Eh. var.

- heteroporus Grun.

- leptopus Grun.

- lineatus Eh.

- marginatus Eh.

- var. intermedia
Coscinodiscus nodnlifer Jan.

- oculus iridis Eh.

- radiatus $\mathrm{Eh}$.

- symbolophorus Grun.

Diploneis crabro $E h$.

Endictya minor $A$. $S$.

Ethmodiscus giga Cast.

Euodia cuneiformis Wall.

$$
\begin{aligned}
& \text { - gibba Bail. } \\
& \text { - }- \text { var. atlantica. } \\
& \text { - } \text { - var. ventricosa. }
\end{aligned}
$$

Ilemiaulus alatus Grev.

- polycistinorum Eh.

- polymorphus Grun.

Iyalodiscus subtilis Bail.

Nitzschia Tryblionella Hantz.

Pleurosignia balticum W. Sm.

Rhabdonema adriaticum Ktz.

Roperia tessellata Grun .

- - oar.? (Cosc. Kryophila Ratt.)

Stephanopyxis Grunowii Gr. et St.

Stictodiscus radiatus Cast.

Triceratium antediluoianum $E h$.

- cuspidatum Jan.

- faous Eh.

- oar. quadrata.

\section{Nos 622, 623 DÉTROIT DE MALACCA (Mer de Chine)}

Achnanthes Bengalensis Grun.

Actinocyclus Ehrenbergii Ralfs. confluens Grun. incertus Grun.
Actinocyclus nebulosus $M$. Per.

- Ralfsii var. samoensis.

- rotula $J \mathrm{Br}$.

Actinoptychus hexagonus Grun. var. 
Actinoptychus intermedius A. S.

$\begin{array}{ll}\text { - } & \text { splendens Shadh. } \\ \text { - } & \text { trilingulatus Bright. } \\ \text { - } & \text { undulatus Eh. } \\ \text { - } & \text { vulgaris Schum. }\end{array}$

Alloioneis antillarum $\mathrm{Cl}$.

Anıphiprora lepidoptera Greg.

- ear. proboscidea.

Amphora crassa var. spuria.

$$
\text { - egregia Eh. }
$$

Arachnoilliseus ornatus $E h$. var.

Astoromphalus elegans Greo.

Asterolampra Marylandica Eh.

Aulacoulisens orientalis Greo.

Auliscus colatus Bail.

- Grunowii A. S. var.

Bacterlastrum hyalinum Laud.

$$
\text { - varians Laud. }
$$

Bidlulphia aurita $B r \cdot e b$.

$\begin{array}{ll}\text { - } & \text { grañulata Roper. } \\ \text { - } & \text { pulchella Grog. } \\ \text { - } & \text { reticulata Rop. } \\ \text { - } & \text { - f trigona } \\ \text { - } & \text { Tuomeyi Rop. }\end{array}$

Campylodiscus ambiguus Grev.

$\begin{array}{ll}\text { - } & \text { biangulatus Grev. } \\ \text { - } & \text { bimarginatus A. S. } \\ \text { - } & \text { contiguus A. S. } \\ \text { - } & \text { ecebrecostatus Grev. } \\ \text { - } & \text { intermedius Grun. } \\ \text { - } & \text { Kittonianus var. Zanzi- } \\ & \text { barica. } \\ \text { - } & \text { Normanianus Grev. } \\ \text { - } & \text { Wndulatus Grev. } \\ & \text { Wallichianus Grun. }\end{array}$

Caupyloneis Greoillei Grun.

Cerataulue levis Ralfs. var.

Climacosphenia elongata Bail.

Cocconeis heteroidea Hantz. pseudo marginata Greg.

Coscinodiscus centralis Eh.

$\begin{array}{ll}\text { - } & \text { concinnus var. Jonesiana. } \\ \text { - } & \text { debilis Grove? } \\ \text { - } & \text { excentricus Eh. } \\ \text { - } & \text { Janischii A. S. }\end{array}$

Coscinotiscus marginatus Eh.

$\begin{array}{ll}\text { - } & \text { nitidus Greg. } \\ \text { - } & \text { nodulifer Jan. } \\ \text { - } & \text { oeulus iridis Eh. } \\ \text { - } & \text { ralliatus Eh. } \\ \text { - } & \text { Rothii Grun. } \\ \text { - } & \text { subtilis Eh. }\end{array}$

Crasperlodiscus coscinodiscus Eh.

koorense. var. Nan-

Cyclotella striata Grun.

Diploneis apis Eh.

- crabro Eh.

- gemmata Eh.

- nitescens $\mathrm{Cl}$.

- notabilis Cl. var. expleta.

- Smithii Cl

- splendida Greg.

Endictya oceanica Eh.

Euodia gibba Bail.

- - var. atlantica.

Euphyllodium spathulatum Shadb.

Ilemiaulus alatus Greve.

- polymorphus Grun.

Iy alodiscus stelliger Bail.

- subtilis Bail.

Isthmia capensis Grun.

Mastogloia angulata Laeois.

- Howathiana Grun.

Melosira sulcata $\mathrm{Ktz}$

- - var. coronata.

Vavlcula abrupta Donk.

- approximata v. substauronelformis.

- Bleishii Jan.

- Brasiliensis Grun.

- bullata Norn.

- clavata Greg.

- formosa Greg.

- liber IV. Sm.

- lyra Eh.

- - var. intermedia.

- - - r recta.

- - var. recta fa elliptica.

- scopulorum fa craticula.

- spectabilis Greg.

- tumescens Grun.

Nitzschia alata Leud. Fort.

- Campechiana Grun. 
NItzschia circumsuta Grun.

$$
\begin{aligned}
& \text { - Jelineckii Grun. } \\
& \text { - panduriformis Greg. } \\
& \text { - - var. lata. } \\
& \text { - } \quad \text { - var. minor. } \\
& \text { - sigma W. Sm. } \\
& \text { - - oar sigmatella. }
\end{aligned}
$$

Orthoneis cocconeiformis Grun.

Pleurosigna decorum var. dalmaticum.

$$
\text { - } \quad \text { formosum W. Sm. }
$$

Rhabdonema adriaticum Kt\%.

$$
\text { - mirifeum } w \text {, su. }
$$

Rhoicosigma antillarum Cil.

Roperia tenella Grun.

Stephanopyxis Grunowii Grov. et St

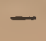
Joysonii A. S. var. turris Eh.

Stictodiscus Truanii Witt. car.

Surirella fastuosa Eh.

- gemma Eh.

- Lorenziana Grun.

- striatula Turp.
Synedra cuneata Eh.

- formosa Hantz.

- fulgens IV. Sm.

- robusta Ralfs.

Trachyneis aspera $\mathrm{Cl}$.

$$
\begin{array}{ll}
- & \text { - var. intermedia. } \\
\text { - } & \text { - var. minor. } \\
\text { - } & \text { - var. vulgaris. }
\end{array}
$$

Triceratium antediluvianum Eh.

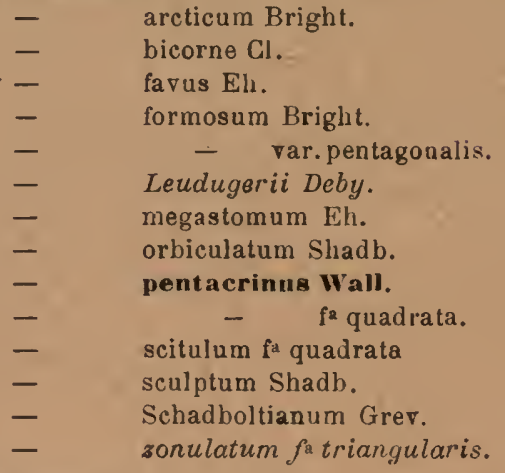

Xanthiopyxis oblonga Eh.

\section{Nos 624, 625 CARSON CITY-NEVADA (Etats-Unis)}

Cymbella Charetoni F. H. et M. Per.

- cistula Kirch.

- cuspidata Kitz.

- Ehrenbergii Ktz:

- maculata Ktz.

Cunotia gracilis W. Sm.

Fragilaria virescens Ralfs.

Melosira crenulata $\mathrm{Ktz}$.

- Jyrata Grun.

- scalaris tirun.

- tenuis Ktz.

- undulata $\mathrm{Ktz}$.

- $\quad$ - var. debilis M. Per.

- $\quad$ - var. producta.

Navicula amphibola $\mathrm{Cl}$.

- - - var. stauroneiformis

- commutala Grun.

- dactylus Ktz.
Navicula Dariana Elı.

- dicephala Eh.

- episcopalis Eh.

- gastrum Eh.

- gentilis Donk.

- gigas Ktz.

- major Kiz.

- - var, asymetrica.

- ventricosa Eh.

- viridis Ktz.

Stauroneis phoenicenteron Eh.

stephanodiscus Carconensis Grun. v. inermis.

Tetracyclus constrictus M. Per. n sp. panduriforme à extrèmités arrondies, long. 60 à $70 \mu$. 
Tetracyclus glans Eh.

- lamina Eh.

- Peragalli F. H. et M. Per.

- stella Eh.
Tetracyclus stella var. dilatata $n$. var., de largeur égalerou supér. à la longueur. tripartitus J. Br.

No 626

CALTANISETTA - SICILE (Italie)

Dépôt fossile marin

Actinocyclus moniliformis Ralfs.

Coscinodiscus argus $E$.

$\begin{array}{ll}\text { - } & \text { centralis Eh. } \\ \text { - } & \text { curvatulus Grun. } \\ \text { - } & \text { fimbriatus } E \text {. } \\ \text { - } & \text { lineatus } E \text {. minor. }\end{array}$

Coscinodiscus minor Eh. patina Eh. radiatus $\mathrm{Eh}$. radiolatus Eh. subtilis Eh.

Euodia gibba $E h$.

- minor Eh.

\section{$\mathrm{N}^{\circ}$ 627, 628 WASHINGTON COUNTY (Etats-Unis)}

Amphora libyca Eh.

- ovalis $K \boldsymbol{t}$.

- pediculus fa major.

Cocconeis lineata El.

- placentula Eh.

Cocconema australicum A. S.

- Janischii A. S.

- mexicanum A. S.

Cymbella cuspidata $\mathrm{Ktz}$. var.

Epithemia gibba Kitz. var. ventricosa.

- turgida $\mathrm{KIz}$. var. granulata.

- Westermanij Ktz.

- zebra Ktz.

Eunotia major Rab.

Fragilaria rhombus Elh.

- virescens Ralfs.

Gomphoneis elegans $\mathrm{Cl}$.

$$
\begin{gathered}
\text { - } \\
\text { - } \\
\text { - } \\
\text { mainilla (Eh.) Cl. }
\end{gathered}
$$

(iomphonema constrictum o. capitata $C l$.
Gomphonema Grovei $M$. Sch.

$$
\begin{array}{ll}
\text { - } & \text { intricatum Ktz. } \\
\text { - } & \text { lanceolatum Eh. } \\
\text { - } & \text { occidentale M. Sch. } \\
\text { - } & \text { - }
\end{array}
$$

Melosira solida Eul.

Navicula ampliata Elı.

- bacillaris var. inconstantissima.

- major Ktz.

- mesolepta Kiz. v. stauroneiformis.

- radiosa $\mathrm{Ktz}$.

- Reinlıardii Grun.

- ventricosa Donk.

Nitzschia sigma W. Sm.

stauroneis amphilepta Eh.

stephanoliscus astræa Grun. var. minutula.

Surirella saxonica Auersw.

moicosphenia curvaty la minor. 
No 629

PICHINCHA (Equateur)

(Région des neiges éternelles)

Cymbella spuria $\mathrm{Cl}$.

- turgida Ktz. (Encyonema).

- ventricosa Grun.

Veidium iridis Eh.

- - var. amphigompha.

Pinnularia borealis El.

- dactylus Eh.
Pinnularia divergens W. Sm .

- major Kiz.

- stauroptera Ktz.

- viridis $\mathrm{Ktz}$.

Stauroneis anceps $\nabla$. linearis Eh.

Surirella tenera Grun.

\section{$\mathrm{N}^{\text {os }} 630,631$ PITT RIVER - OREGON (Etats-Unis)}

Amphora pediculus Ktz.

Cocconeis placentula Eh.

Cyclotella Meneghiniana.

Cymbella Janischiana. mexicana Eh.

Encyonema triangulum Eh.

Epithemia sorex $\mathrm{Ktz}$.

- turgida Ktz.

- zebra Ktz.

Fragilaria construens Eh.

- mutabilis W. Sm.

Gomphonema Eriense Grun o. rostrata.

- intricatum Ktz. $\mathbf{\nabla}$. vibrio.
Gomphonema Grovei M. Sch. Oregonicum Eh.

Melosira granulata Ralfs.

- solida Eul.

Navicula elliptica Kítz.

- Ludlowiana A. S.

- major Ktz.

- viridula Ktz.

Pinnularia viridis $\mathrm{Kt} z$.

Rhoicosphenia curvata v. gracilis.

Stephanodiscus astræa Eh.

carconensis var. minor.

$\mathrm{N}^{\circ 1} 632,633$

DUBRAVICA (Hongrie)

Dépôt fossile saumâtre

BIBLIÔGRAPHIE. - Grunow. Beitrage zur Paleontologie Osterreich Ungarn et J. Pantocsek, Diatomées de Hougrie. Vol. III.

Cocconeis Dubravicensis Pant.

- lineata Grun.
Cymatopleura solea Bréb.

Cymbella abnormis Grun. v. fossilis Pant. 
Cymbella austriaca var. prisca Grun.

- Clementis Pant.

- cymbiformis Eh.

- - var.producta Pant.

- Dubravicensis Pant.

- gastroïdes var. crussa Grun.

- Grunówi Pant.

- lanceolata Eh.

- leptoceros Ktz.

- pachyptera Pant.

- simplex Pant.

- Sturii Grun.

- vegeta Pant.

Encyonema ventricosum $K t z$.

Epithemia cistula Eh. var. Iunaris Grun.

Eunotia Ehrenbergii Grun. v. quaternaria - gracilis Rab. o. fossilis Pant. - polyglyphis Grun.

Comphonenra Dubravicense Pant.

- intricatum $\mathrm{Ktz}$.

- $\quad$ - v. fossilis Pant.

- - var. pumila.

- montanum Grun.

Melosira arenaria Moore.
Meloeira bituminosa var. dilatata pant. - crenulata Eh. rar: ambigua.

- - var. debilis Pant.

- tenuissima Grun.

Navicula arata Grun.

- Carpathorum Pant.

- Dubravicensis Grun.

- elliptica Ktz. var. minor.

- Haueri Grun.

- major Ktz.

- microrynchus Grun.

- neogena Pant.

- pachyptera Kiz.

- Scythica Pant.

- subsolaris Grun.

Scoliopleura tumida Rab.

Staurosira Harrisonii Grun.

- venter Grun.

Surirella Glementis Grun.

- salsa W. Sm. var. hungarica Pant.

Syntolra delicatissima W. Sm.

- Dubravicensis Pant.

- - ulna Eh. var. aqualis $\mathrm{Ktz}$.

- - var. fossilis Pant.

No 634

ILE MAC-DONALD (Océan Austral)

Sondage

Achnanthes inflata Grun.

Actinocycius curvatulus Jan.

-

Oliverianus O. Mea.

-

Actinoptychus undulatus Eh.

Amphora angusta var. ventricosa.

Asteromphalus antarcticus Cast.

$$
\text { - Humboldtii Eh. }
$$

Biddulphia pulchella Graz.

$$
\text { - Weissflogii Grun. }
$$

Chretoceros californicum? Grun.

$$
\text { - radiculum Eh. }
$$

Coscinodiscu concinnus̄ W. Sm.

- elegantulus Grev.

- Gallopagensis Grev.

- lentiginosus Jan.

- marginatus Eh. fa minor. Diam. $50 \mu$ nodulifer. A. S. oar.

- nodulifer. A. S.

- pectinatus Ratt.

- radiatus Eh.

- tumidus Jan, oar.

Dicladia capreolus Eh.

Diploneis expedita A. S. car. 
viplor Subcincta A. S.

Entopỳla ocellata v. pulchella.

Fragiiaria antarctica Cast.

Grammatophora arcuata $\mathrm{E} h$.

- - maxima Grun.

Ifemiaulus ambiguus Grun.

Hyalouliecus maximus P. P.

$$
\text { - radiatus Grun. }
$$

Mastogloia quinquecostata Grun. var.

Melosira sulcata var. coronata.

$$
\text { - - var. radiata. }
$$

Vavícula pennata A. S, var.

\section{$-314-$.}

Nitzschia angularis $\nabla$. boreatis.

- panfuriformis Greg.

- $\quad$ - $\quad$ var. peralbata.

Orthotropis maxima var.

Pleurosigma longissimum $\mathrm{Cl}$.

Pyxilla americana oar. Eh.

Rhizosolenia hastata Grun.

Synedra fulgens H. Sin.

- robusta Ralfs.

Trachyneís aspera Cl.

- - var. intermedia.

oblonga Bail.
$N^{\circ} 635$

$\mathrm{N} \circ 636$

a
LE HAVRE (France)

Synedra tabulata litz.
LE HAVRE (France)

Melosira Jurgensii Ag.

N 637 BRANFORD - CONNECTICUT (Etats.Unis)

Sondage

Actinocyclus Ehrenbergii Ralfs. var. intermedia.

Actinoptychus areolatus Eh.

- undulatus Eh.

Amphiprora conspicua Grev.

Biddulphia pulchella Greg.

Campylodiscus Echeneis Eh.

Coscinodiscus asteromphalus Eh.

- excentricus Eh.

- radiatus Eh.
Navicula formosa Greg.

- Kamortensis Grun.

Nitzschia sigma IV. Sm.

Pleurosigma affine Grun.

- balticum W. Sm.

- strigosum W. Sm.

Rhablonema adriaticum W. Sm.

Scoliopleura tumida Bréb.

Surirella Febigerii Lewis.'

Tropidonels seriata $\mathrm{Cl}$. 
Cymbella affinis kitz.

Comphonema angustatnm Ḱtz. var.

Navicula tenella.

\section{producta.}

Vitzschia linearis $W$. Sm. tubicola (irun.

Synedra acus Grun.

- oxyrhynchus Kitz.

\section{$\mathrm{N}^{\text {ss }} 639,640$ BEATTIES POND - CONNECTICUT (E.-Unis)}

\section{Dépôt saumâtre}

Achnanthos brevipes Ag.

$$
\text { - parvula Ktz. }
$$

Actinoptychus undulatus Eh.

$$
\text { - - rar. minor. }
$$

Amphiprora pulchra var. pulchella.

Amphora libyca Eh.

- ovalis $\mathrm{Ktz}$. var. minor. .

- proteus Greg.

Brebissonia Boeckii Grun.

Cocconeis scutellum Eh.

Coscinodiscus excentricus Eh.

$$
\text { - } \quad \text { lineatus Eh. }
$$

Cyclotella striata Grun

Cymbella gastroìdes $\mathrm{Ktz}$

Diploneis coffeaeformis Cl.

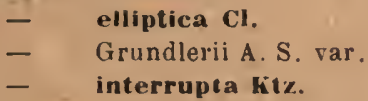

Encyonema prostratum Ralfs. var.

$$
\text { - ventricosum Grun. }
$$

Epithemia gibberula Ktz.

$$
\text { - zebra oar. proboscidea. }
$$

Eunotia arcus Eh.

$$
\text { - - var plicata. }
$$

- flexuosa Grun.

- gracilis W. Sm.

- impressa Eh
Eunotia lunaris Grun.

- major Bab.

- minor W. H.

- pectinalis Rab.

- - var. ventricosa.

- prcerupta var. bidens.

- robusta var. tetraodon.

Fragilaria virescens Ralfs.

Gomphonema acuminatum Eh.

- - var, pusilla.

- Brebissonii Klz.

- constrictum var. capitata.

- gracilo Eh.

- $\quad$ - famajor.

- intricatum $\mathrm{Klz}$.

- montanum val. succisa.

IIyalodiscus maximus $E u l$.

- scoticus Grun.

Melosira sulcata var. coronata.

Navicula acrosphæria Brèb.

- zstuarii Ci,

- amphirynchus Eh.

- borealis Ktz.

- Brasiliensis Grun.

- commutata Grun.

- cruciformis Donk.

- cryptocephala Kitz.

- divergens oar. elliptica.

- elegans var. cuspidata.

- firma Ktz. 
Navicula formosa Greg.

- gastrum fa minor.

- gentilis Donk.

- gibba Ktz.

- hemiptera Ktz.

- Hennedyiana W. Sm.

- icostauron Grun.

- limosa Ktz.

- inajor Kiz.

- - var. asymetrica.

- microstauron Eh.

- nodosa Ktz.

- peregrina ktx.

- peripunctata J. Br.

- puella W. Sm.

- semen Eh.

- ventricosa $\mathrm{K}$ tz.

- viridis $\mathrm{K} I \mathrm{z}$.

- vulpina Ktz.

Nitzschia bilobata W.Sm.

- circumsuta Grun.

- granulata Grun.

- littoralis Grun.

- obtusa W. Sn.

- plana W. Sin.

- punctata Grun.

- scalaris W. Su.

- sigma W. Sm.

- spectabilis Ralfs.

- Tryblionella Hantz.

- - var.

Plagiotropis Van Heurckii Grun.

Pleurosigma balticum W. Sm.

Pyxilla baltica Grun.

Raphoneis amphiceros Eh.
Raphonels amphiceros var. belgica. - var rhombicá.

Stauroneis anceps Eh.

- - var amphicephala.

- gracilis Eh.

- phonicenteron Eh.

- $\quad \operatorname{salin\alpha } W . S m$.

Stenoneis? amphipleuroïdes M. Per. n. sp. a la forme extérieure du Van Heurckia viridula ; les bourrelets arqués partaut du nodule central finissent en pointe avant d'arriver à un nodule terminal, fort, allongé, ne touchant pas l'extrémité de la valve.

Surirella Mülleriana Grun.

- - var constricta. $n$. var. à milieu rétréci et à extrémités atténuées acuminées.

- ovata Ktz.

- saxonica Auerswo.

- splendida Eh.

- tenera Greg

- - oar. splendidula.

Synedra pulchella Ktz.

- ulna car. danica.

Tabellaria fenestrata $\mathrm{Ktz}$.

Triceratium alternans Bail.

Tropidoneis lepidoptera var proboscidea.

Van Hourckia vulgaris V. H.
Achnanthes brevipes $\mathrm{Ag}$.

$$
\text { - - var. galinarum. }
$$

Amphora commutata Grun.

Cocconeis scutellum Eh.

Epithemia gibberula Ktz.

Navicula lyra Eh.
Pleurosigna balticum W. Sm.

striatella unipunctata $\mathrm{Ag}$.

Surirella fastuosa $\mathrm{E} h$.

- striatula 'Turp.

synedra affinis litz.

1 - pulchella Kitz. 
Asteriouella formosa var. gracillima. Cyclotella comta $\mathrm{K} t \mathrm{z}$.
Cyclotella Kützingiana Chauv.

Fragilaria crotonenais kitton.
Achnanthes lirevipes $\mathrm{Ag}$.

Biddulphia mobiliensis Bail.

Cynatopleura elliptica 0 , rhomboides.

$$
\text { - solea W. Sm. }
$$

Diploneis bombus var. densestriata.

$$
\text { - interrupta Ktz. }
$$

\section{Melosira Borreri Grev.}

- nummuloides Ag.

Navicula anglica Ralfs.

.. - Brasiliensis Grun.

- $\quad$ granulata Bréb.

- humerosa Bréb.
Navicula palpebralis $B r e b$

- peregrina Ktz.

- subsalina Donk.

Nitzsehia navicularis Bréb.

- rigida $v$. rigidula.

Pleurosigua elongatum W. Sm.

- strigosum W. Su.

Surirella gemma $E h$.

- medulica H. Por.

- tenera oar. nervosa.

Synedra aftinis $\mathrm{Ktz}$.

- pulchella Kiz.

- ulna Eh. et var.

N• 644 BRIANÇON - HAUTES-ALPES (France)

\section{Récolte faite à $1600 \mathrm{~m}$ d'altitude}

Cocconeis placentula Eh.

Cymbella affinis $\mathrm{Ktz}$.

- cymbiforınis Bréb.

- helvetica $\mathrm{Ktz}$.

- lavis Naeg.

- maculata $\mathrm{Ktz}$.

- parva V.H.

biatoma hyemalo var. nesoidon.

Bncyonema ventricosum Grun.

Eunotia arcus Eh.
Comphonenıa angustatum Grun.

tissinua.

var. angus-

Navicula elliptica $K \mathrm{tz}$.

- iridis Eh.

- $\operatorname{limosa}$ Ktz.

- tenella Bréb.

Nitzschia linearis $W$. Sm.

Synedra delicatissima W. Sm

- ulna var. Uanica. 


\section{Nos 645, 646 SAN BERNARDINO - CALIFORNIE (E -Unis)}

\section{Dépot fossile marin}

Aetinocy clus Eh́renbergii Ralfs. Actinoptychus glahratus A. S.

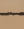

- splendens Ralfs.

- $\quad$ - var. californica

- - var. halionvx.

Asteromphalus Dallasianus Éh.

- Darwinii Eh.

Brunia mirabilis Temp. oar.

Coscinodiscus apiculatus Eh.

- - var.
Coscinouliscus crassus Eh.

$\begin{array}{ll}- & \text { excentricus Eh. } \\ \text { - } & \text { heteroporus Eh. } \\ \text { - } & \text { marginatus Eh. } \\ \text { - } & \text { nelus iridis Eh. } \\ & \text { radiatıs Eh. }\end{array}$

Melosira sulcata $\mathrm{Ktz}$.

Navicula lyra Eh.

- Smithii Brẻb. -

Stephanopyxis turris Eh.

Triceratium elegans Grev.

$\mathrm{N}^{\text {os }} 647,648$ ILE SAMOA (Océan Pacifique)

Achnanthes Bengalensis? Grun.

- brevipes $\mathrm{Ag}$.

- indica $J \mathrm{Br}$.

- $\quad$ - var. sulcata M. Per. Le bord de la valve supérieure est marqué par un sillon.

Ce sillon peut être observé dans la photographie de J. Brun (fig. 13 d.), j'ai observé des valves.ne possédant pas cé sillon; je les considere cornme typiques.

- longipes Ag.

- subsessilis oar. cuneata Öst.

Actínocyclus incertus Grun.

- nebulosus M. Per.

- Ralfsii var. australiensis Grun.

- _ - major.

- - var. samoensis forma inermis.

- tenuissimus Cl.
Aetinoptychus campanulifer A. S. guttatus Öst. n. sp. M. Per. (Syn. A. moronensis var. guttata Öst:) Cette formene peut être considérée comme une variété de l'A. moro$\mathrm{n}+\mathrm{nsis}$.

- hexagonus var. tenella.

- undulatus Eh.

- rulgaris Schum.

Amphora angusta Greg.

- costata W. Sm.

- crassa Greg.

- cymbifera Greg

- dubia Greg. var.

- farcimen var. fusca A. S.

- fusca fa la M. Per.

- Javanica var. oculata M. Per. Semblable à la forme reprèsentée dans A, S., atlas, Pl. 27, fig. 31 , mais.avec un area lisse au nodule central. 
Amphora ostrearia Bréb. obtusa Grnn.

- pusio Cl.

- Samoensis (capensis var. ?) M. Per. Semblable à l'A. capensis A. S. Atlas $25 / 49$, 50 ; en diffère principalement par une côte stauroneiforme au nodule central du côté dorsal. Long. 50 à $60 \mu, 8$ à 9 côtes faiblement granulées en $10 \mu$. - spectabilis freg.

- subalata n. sp. M. Per. Face ventralo semblable à celle de A. robusta (A. S. atlas $2: / 40$ ). mais arec une aile plate comme celle de l'A, alata H. Per. (Diat. de France 43/46) Long $100 \mu, 12$ stries en $10 \mu$.

- turgida Greg.

- $\quad$ - forma minor

- separanda M. Per. (Amphora sp. A. S. atl. 25/57). Ciett 2 forme qui ne peut pas étre réunie à l'A. contracta, mérite un nom particulier.

- sp. A. S. atlas $27 / 57$.

- sp. A. S. atlas 28/58.

- sp. Per. Diat. de France $40^{\circ} / 21$.

- farciminosa Per De forme semblable à celle do A. farcimen, mais s'en distingue par sa striation et son nodule central. Le sillon est placé au sommet d'un angle très prononcé, et prèsente l'apparence de la bando transparente des Caloneis Long. $93 \mu$ larg. $19 \mu, 11$ à 12 stries en $10 \mu$

- granulata var. lineata Per. Diffère de la figure de A. S. atl. 27/44 par sa structure faite de gros granules allongès, disposés en lignes longitudinales dont les deux premières, du eôté du raphé, sont interrompues devant le nodule central. Long. $75 \mu, 9$ stries en $10 \mu$ et 4 à 5 lignes longitudinales.
Asteromphalus Hoolieri $\mathrm{E} h$.

Aulacodiscus orientalis Grev.

Bicldulphia aurita Bréb.

$$
\begin{aligned}
& \text { - } \quad \text { pulchella ciray. } \\
& \text { - } \quad \text { reticula Rop. } \\
& \text { IRoperiana Grev. }
\end{aligned}
$$

\section{Campylodiscum ambiguus firev}

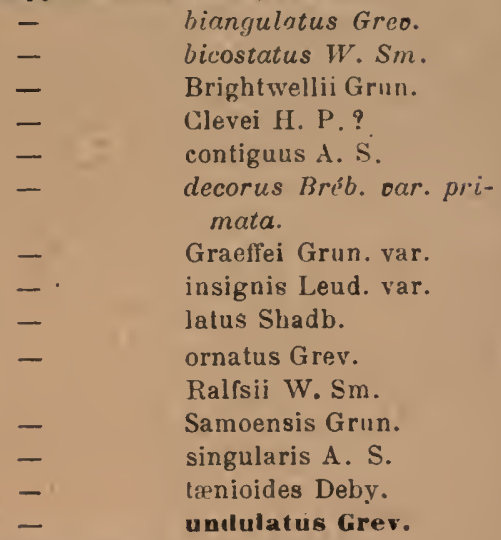

Climacosphenia moniligera Eh.

Cocconeis Emmericii Jan.

- heteroidea Ktz.

- gibbocalix J. Br.

Coscinodiscus excentricus El.

- marginatus Eh.

- nitidulus Grun.

- _ - Grun.var. scintillans M. Per. Granules alignés en rayons alternativement courts et longs. Diam. $58 \mu$.

- nitidus Greo.

Cymatoneis sulcata $\mathrm{Cl}$.

Dlmeregramma fulvum.

Diploneis Beyrichiana A. S.

- cynthia $A . s$.

- - var. intermedia M. Per. Intermédiaire entro le type (A. S. atlas $8 / 41$ ) et la forme $A$. S. atlas $3 / 45$.

- didyma Kiz.

- fusca Cl.

- interrupta var. Noræ Zelandiæ Per. Voisin de la variété 
Samoensis A. S. atl. 12/12, mais s'en distingue l'après la forme des sillons.

biploneis mediterranea $\mathrm{Cl}$.

- multicostata Grun.

- nitescens var. rhomboïdes $M$.

Per. Largement rhomboïdal, à angles longement arrondis.

Long. $45 \mu$, larg. $27 \mu$

- notabilis Grev.

- Schmidtii Cl.

- Smithii var. recta M. Per. Petit variétẻ à sillons étroits et non dilatés autour du no. dule central.

- splendida Greg.

Endictya oceanica Cl.

Epithemia hirundinella J. Br.

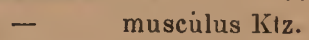

Euphyllodium spathulatum Shadb.

Graumatophora marina $\mathrm{K} t \mathrm{z}$.

$$
\text { - } \quad \text { maxima Grun. }
$$

IIomoeoclaclia Vidovichii Grun.

fyalodiscus ambiguus Grun.

$\begin{array}{ll}\text { - } & \text { laevis Eh. } \\ \text { - } & \text { radiatus Grun. } \\ \text { - } & \text { scoticus Grun. } \\ & \text { stelliger Bail. }\end{array}$

Isthmia capensis Grun.

Mastogloia Portierana Grun. - quinquecostata Grun.

Melosira coronaria Mann. A. S. atl. 158/9 $=$ Porodiscus calyciflos var. Porodiscus calyciflos n'appartient pas au genre Porodiscus.

- nummuloildes Ag.

- sulcata Ktz.

- - var. coronata.

- - var. radiata.

Navicula clavata Greg.

- Fischeri A. S.

- formosa Ġreg.

- Hochstetteri Ehr.

- lyra Eh.

- Ny Cl.

- pinnata A. S.

- Reicharti Grun. var. intermedia M. Per. Intermędiaire entre le type et la Nav. Ny Cl. Les nodules terminant en faucille. le raphé est accompagné d'une ligne de granules allongès en de trẻs courtes séries. A. Schmidt représente dans son atlas 70/26, 29 des formes analogues.

Navicula robusta Grun.

- sp. A. S. atl. 8/15.

Nitzechia angularis W. Sm.

- distans Greg. var.

- Jelineckii Grun.

- majuscula Grun.

- panduriformis Greg.

- - var. delicatula Grun.

- _ var. peralbata Per.

- scalaris W. Sm.

- sigma W. Sm.

Orthoneis fimbriata Grun.

Periptera sp.?

Plagiodiseus nervatus Grun.

Plagiogramma pulchellum Grec.

Plenrosigma validum Shadi).

Potiocystis spathulata Shadb.

Pollosira hormoïles Mont.

Ihabdonema adriatum Ktz.

$$
\text { - ' mirificum W. Sm. }
$$

Raphoneis amphiceros var. gemmifera.

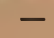
var.obesa M. Per. Contour absolument circulaire avec deux petites pointes; lignes degranules médians alternativement courtes et longues. Long. $45 \mu$. Diam. $35 \mu$

Rhoïconeis Gorkeana var. N'a que 16 stries en $10 \mu$ seulement.

Rhoicosigma compactum Grun.

Surirella fastuosa Eh.

- - var. opulenta.

- Ceylanensis Leud.

- gemma Eh.

Synedra formosa Hantz.

- Hennedyana Grun.

- undulata Grun.

Synedrosphenia cuneata (Grun.) H..Per. 
Terpsinoe intermedia Grun.

- fa musica M. Per. A la forme extérieure de $\mathrm{T}$. musica, mais la structure est celle de $\mathrm{T}$. intermedia.

Tracheneis aspera $\mathrm{Cl}$.

- var. vulgaris $\mathrm{Cl}$.

- consors A.S.

- velata $\mathbf{C l}$.

Triceratium antediluvianum Eh.

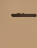

- var.trigona M. Per.

Triangulaire à côtés convexes presque circulaire, long. $55 \mu$.

- bullosum 0 . Witt.
Triceratium areticum Bright $\nabla$. Japonica.

$\begin{array}{ll}\text { - } & \text { folmosum Bright. } \\ \text { - } & \text { pentacrinus Wall. } \\ \text { - } & \text { Sculptum Shadb. } \\ \text { - } & \text { versicolor J. Br. v. trigona. } \\ \text { - } & \text { zonulatum Grev. } \\ \text { - } \quad \text { ? fa trigona. } \\ \text { (Lampriscus) Leudugerii. } \\ \text { var. Samoensis Per. } \\ \text { Pas tout á fait circulaire, } \\ \text { angles proéminents. Long. } \\ \text { du côté } 55 \mu, 6 \text { lignes do } \\ \text { granules en } 10 \mu \text { au bord. }\end{array}$

Tropidoneis lepidoptera Cl.

$N^{* s} 649,650$

LIMBOURG (Autriche)

Dépôt fossile marin

Actinocyelus crassus $\mathrm{V}$. H. moniliformis Ralfs.

Actinoptychus splendens Ralfs.

- stella var. Thumii.

- undulatus Eh.

- - var. minor.

Anorthoueis striata $n s p$. M. Per. Valve presque circulaire, raphè fort rectiligne très excentrique à pores centraux ècartés; area très développé; stries courtes un peu plus longues que le $1 / 3$ de la distance lu bord ou raphè, divisées en deux parties, l'une marginale où elles sont robustes, l'autre intérieure où elles sont plus faibles. Long. $38 \mu$, larg. $35 \mu, 10$ stries en $10 \mu$

Aracbnoidiscus Ehrenbergii o. Oamaruensis.

Asteromphalus Dallasianus Ralfs.

Aulucodincus affinis Grun.

Cerataulas turgidus Eh.
Chatoceros hispidum Bright.

Climacomphenia moniliger $\alpha \mathrm{Eh}$.

Cosinodiscus asteromphalus Eh.

- curvatulus Grun.

- dubius Ralfs.

- lineatus Eh.

- marginatus Eh.

- minor Eh.

- neogradensis Pant. rar.

- nitidulus Greo.

- nitidus Greg.

- obscurus A.s.

- oculus-iridis. El.

- perforatus $\nabla$. ambigua.

- radiatus Eh.

- spiraliterpunctatus Pant.

- stellaris? (Rop. A. S. Atl. $164 / 4)$.

La partie centrale de la valve parait bien être identique à celle de la fig. citèe, mais les nombreux fragments paraissant se rapporter à cette partie centrale font présumer un diamètre 
beaucoup plus considérable, diaprès moi, la fig. de Schmidt ne peut se rapporter au C. stellaris Roper.

Coscinodiscus subtilis Eh.

$$
\text { - tuberculatus Grev. }
$$

Craspedodiscus Mölleri A. S.

Cyclotella striata Grun.

Dic-ladia capreolus Eh.

$$
\text { - mitra Eh. }
$$

Dlploneis interrupta $\mathrm{Cl}$.

$$
\text { - Smitlij Cl. }
$$

Endictya oceanica Eh.

Goniothecium odontella Eh.

Grammatophora robusta Dipp.

Heniaulus bipons Grun.

- polymorphus Grun.

Ifercotieca mamillaris Eh.

Hyalodiscus scoticus Grun. subtilis Bail.

Melosira cincla Pant.

- clavigera Grun.

- crenulata Ktz.

- interjecta Jan.
Melosira ounina Cl.

- sol Eh.

- sulcata $\mathrm{v}$. radiata.

Navicula abrupta Donk.

- Hennedeyi W. Sm.

- Hachstetteri Grun.

- lyra Eh.

- pretexta Eh. var.

Nitzchia circumsuta Grun.

Orthoneis splendida Grun.

Periptera saxogallica $\mathrm{J}$. $\mathrm{Br}$.

- tetracladia Eh.

Ploaria petasiformis Pant.

Pyxilla americana Eh.

- dubia Grun.

Rhaphidodiscus microtatos Pant.

Raphoneis amphiceros Eh.

$$
\text { - - gemmifera Eh. }
$$

Stephanogonia actinoplychus Eh.

Aynedra salinarum Pant.

Terpaino ë intermedia car. musica.

Xanthiopyxis cingulata Eh.

- oblonga Eh. umbonata Greo.

$\mathrm{N}^{\circ s} 651,652$ ROYAL SOUND (Kerguelen)

\section{Actinocyclus curvatulus Jan.}

- Olverianus O'Mea.

- umbonatus clast.

Amphora angusta var. ventricosa.

- rubusta Greg.

Auliacus coelalus Bail.

- reticulatus Greo.

Bidifulphia aurita Bréb.

- punctala Grev.

- Roperiana Grev.

- Wrissfloyii Grun var.

Cerataulus Smithii Ralfs.

Cegturlincus gemmifer? Cast. var. ?

Chretoceros gastridium Eh.
Cocconeis costata Greg.

- dehiscens A.S. var.

- imperatrix A.S.

- pseado-marginala Grun.

- scutellum Eh. v. dilatata.

Coscinodiscus asteromohalus Eh.

$\begin{array}{ll}- & \text { concinnus W. Sm. } \\ \text { - } & \text { dubiosus Grove. } \\ \text { - } & \text { elogantulus Grev. } \\ \text { - } & \text { griseus Greo. } \\ \text { - } & \text { lentiginosus Jan. } \\ \text { - } & \text { aculus-iridis Eb. } \\ \text { - } & \text { pacificus Ratt. } \\ \text { - } & \text { pectinatus Ratt. }\end{array}$


Coscinodiscus radiosus Grun. tuberculatus Grev.

Dicladia capreolus Eh.

Diploneis sideralis $J . B r$. oar. - oagabunda J.Br. oar.

Endictya oceanica $E h$.

Eutopyla australis var. gigantea.

- - var. incurvata.

- ocellata var. calaritana.

- - var. pulchella.

Grammatophora arcuata Eh.

- maxima Grun.

IIemiavlus ambiguus Grun.

Hyaloulscus pustulatus A.S.

- maxionus $\mathrm{P}$. P.

- radiatus Grun.

- subtilis Bail.
Melosira interjecta Jan.

- recedens $A . S$. oar.

- sol. Eh.

- sulcata v. radiata.

Navicula pennata A,S. var.

Nitzschia angularis $W$. Sm.

- panduriformis Greg.

- - var. paralbata.

Rhizosolenia hastata Grun. Synedra fulgens W. Sm.

Trachyneis aspera $\mathrm{Cl}$.

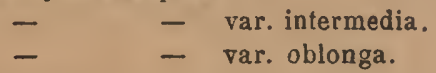

Trlceratium antarcticum Jan.

- interjectum f $^{2}$ quadrata.

- formosum var. (Jan Gaz. $\mathrm{XI} / \mathrm{L3)}$.

Nos 653,654

JAEN (Espagne)

Jaen, Moutemayor, Osuna et Moron paraissent appartenir à un méme dépót de grande étendue, et en être les différents effleurements le long du versant de la Sierra Nevada; dans tous les cas, ce sont les mêmes espèces avec une répartition différente.

Actínocyclus ellipticus Eh.

- $\quad$ rasciculatus Cast.
- $\quad$ moniliformis Ralfs.
- $\quad$ moronensis Deby.

Actinoptychus areolatus Eh.

$\begin{array}{ll}\text { - } & \text { splendens Shadb. } \\ \text { - } & \text { unilulaius Eh. } \\ \text { - oulgaris oar. maculata. }\end{array}$

Amphora excisa Greg.

- robusta Greg.

Arachnoilliscus ornatus Eh.

Asterolampra Grovillei Grev. var. - Marylandica Eh.

Asteromphalus Moronensis Ratt.

Aulacoliscus radiatus Greo.

Aullscus cœlatus Bail.

- incertus A. S.
Auliscus Moronensis o. quadriocellata.

Bidulphia pulchella Gray.

- Tuomeyi Rop.

Campylodiscus decorus Bréb.

$$
\text { - Tluuretii Bréb. }
$$

Campylonels Grevillei $\nabla$. regalis.

Chetoceros hispidum Bright.

- incurvum Bail.

Cocconels dirupta o. californica.

- Misronensis A.S.

- pseudo marginata Greg.

Coscinodiscu a apiculatus Eh.

- asteromphalus Eh.

- birarliatus Greo.

- concinnus W. Sm.

- crassus Bail.

- curvatulus Grun.

- $\quad$ v. latiustriata. 
Coscinodiscus diversus M. Per. N. Sp.
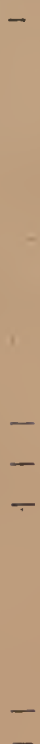

$-$

Craspedodiscus coscinodiscus var. hispanicus n. var. semblable à la variété Nankoorensis, mais -les cellules de l'an. neau extérieur sont plus grosses 13 à $3 \mathrm{l} / \mathrm{k}$ en $10 \mu$ ) et disposées en lignes rayonnantes plus régulières: centre très enfoncé.
Dimeregramma? Achnanthes M. Per n. sp. semblable à $D$. marinum, mais la valve est genouillée comme dans les Achnanthes, long, $130 \mu ; 5$ i 6 granules en $10 \mu$.

Diplonei Beyrichiana oar. - Smithii Cl.

Endictya oceanica Eh.

Entopyla australis Eh.

Euodia gibba var. Moronensis.

Gephyria media Arn.

Goniothecium odontella Eh.

Grammatophora Moronensis Grev. robuista Dipp.

Hemiaulus ambiguus Grun.

Ilyalodiscus scoticus Grun. - subtilis Bail.

Isthmia enervis Eh.

Melosira sol Eh.

- sulcata var. radiosa.

Navicula abrupta var.

- Hennedyi W. Sm.

- prætexta Eh.

- spectabilis Greg.

Orthoneis splendida Grun.

Pleurosigma affine var. fossilis.

Pyxilla baltica Grun.

Raphoneis amphiceros Eh.

Rhabdonema adriaticum $K t z$.

Stephanopyxis corona Eh.

- Grunowii Groo. et St.

- turris Eh.

Stictodiscus littonianus var.

synedra salina Pant.

- superba Ktz.

Triceratium antediluvianum Eh.

- $\quad \operatorname{arcticum} \mathbf{v}$. Californica.

- balearicum Cl. et Grun.

- interpunctatum Grun.

Xenthiopyxis alata Eh. oblonga Eh. 


\section{N*65็-657 MONTEMAYOR - CORDOUE (Espagne)}

Actinocyclus fasciculatus Cast.

- incertus Grun.

- moniliformis Ralfs.

- Moronensis Deby.

Actinoptychus areolatus Eh.

$\begin{array}{ll}- & \text { Clevei A. S. } \\ \text { - } & \text { undulatus Eh. } \\ \text { - } & \text { oulgaris Shum. }\end{array}$

Arachnoïdiacus ornatus Eh.

Asterolampra Dallasiana Grev.

- Grevillei Grev.

- Marylandica Eh.

Asteromphalus Brookei Bail.

- $\quad$ Moronensis Ratt.

Aulacodiscus angulatus Grev. oar.

- Grunowii Cl.

- - oar. divergens.

- $\quad$ radiatus Grev. var.

Auliscus Normanianus Greo.

Biddulphla Johnsoniana Greo.

- Tuomeyi Bréb.

Brunia mirabilis Temp.

Campyloneis Grevillei v. regalis.

Campylodiseus Graeffii Grev.

Cestodiscus gemmifer Cast.

- Moronensis Grev.

Cocconeis dirupta o californica.

Coscinodiscus apiculatus Eh.

- asteromphalus Eh.

- biangulatus A. S.

- biradiatus Giev.

- concinnus W. Sm.

- crassus Bail.

- curvatulus Grun.

- $\quad$ - var. latius striata.

- devius A. S.

- diorama A. S.

- 1 dubius Ratt.

- flexuosus $\nabla$. hispanica.
Coscinodiscus heteroporus Eh.

- leptopus Grun.

- Lewisianus Greo.

- $\quad$ - v. Moronensis.

- luctuosus Grove.

- narginatue Eh.

- Monicæ Ratt.

- olsacurus A. $\mathbf{S}$.

- obversus Ratt.

- oculus-iridis Eh.

- radiatus Eh.

- stellaris A. S.

- subtilis Eh.

- tuberculatus Grev.

Craspedodiscus coscinodiscus $v$, hispanica.

coscinodiscus c. Nankoorensis.

Diploneis crabro Eh.

- Beyrichiana ear.

- futilis d. $S$.

- gemmatula o. spectabilis.

- splendida Greg.

Eudictya oceanica Eh.

Goniothecium odontella $\mathrm{Eh}$.

Grammatophora robusta Dipp.

Isthuia enerois $\mathrm{Eh}$.

Melosira sulcata v. radiata.

Orthoneis splendida Grun.

Pleurosigma affine $\nabla$. fossilis.

Pyxilla baltica Grun.

Stephanopyxin turris Eh.

Stictodiscus Jeremianus fa hexagona.

Surirella fastuosa Eh. var.

Tracheneis clepsydra Cl.

Triceratiun antediluvianum Eh.

- rellulosume. Simbirskiana.

- Pantocsekii A. S.

- Sarmaticum? Pant.

- Stokesianum Greo. 


\section{No 658660 OSUNA - SÉVILLE (Espagnej}

Actinocyclus fasciculatus Cast.

$\begin{array}{ll}- & \text { elliptícus Eh. } \\ \text { - } & \text { incertus Grun. } \\ \text { - } & \text { Moniliformis Ralifs }\end{array}$

Actinoptychus amblynceros Eh.

$$
\begin{array}{ll}
\text { - } & \text { areolatus Eh. } \\
\text { - } & \text { stella A. S. } \\
\text { - } & \text { undulatus Eh. }
\end{array}
$$

Arachnoïliscus ornatus Eh.

Axpeitia Temperej M. Per n. g. - N.Sp. Triangulaire, à côtés presque droits el à angles arrondis; structure cellulense ne s'étendant pas jusqu'au bout de la valve. Azpeitia Temperei ; cellules hexagonales en lignes rayonnantes courles et décussées $(5$ en $10 \mu$ au milieu du rayon, plus petites au centre et sur les bord-1. Marge lisse, étroite, bordée le long des còtés et des angtes d'une ligne de perles fines ( 10 en $10 \mu$ ) divísée en parties à peu près ègales par des perles plus grosses. Long. du coté 80 à $90 \mu$. Le Triceratum antiquum Pant. rentre dans ce genre.

Asterolampra Dallasiana Grev.

$$
\text { - } \quad \text { Grevillei Grev. }
$$

Asteromphalus Moronensis Ratt.

Auliscus coelatus v. latecostata.

- Normanianus Grev.

Biddulphia pulchella Gray.

$$
\text { - Tuomeyi Bail. }
$$

Campyloneis Greoillei Grun.

Cestodiseus hispanicus M. Per. N. Sp. (Cest. convexus var.?) A la partie centrale, les granules serrés donnent l'aspect de l'Actinocyclus Eh. renbergii, à la partie mosenne, ils sont plus lâches et dècussés en zigzag; les lubrrcules beaucoup moins nombreux que dans le Cest. convexus Cast.

Cestodiscus Moronensis Grev.

Climacospheuia moniligera? Eh.

Coscinodiscus apiculatus Eh.

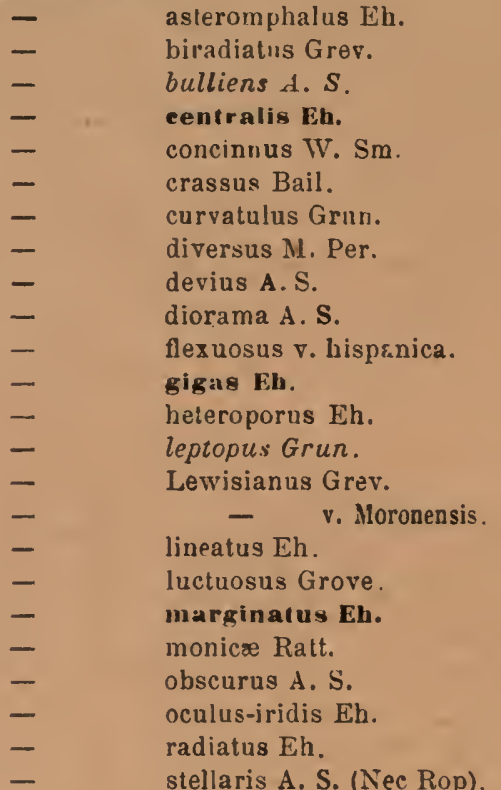

- stellaris A. S. (Nec Rop).

- _ - Roper.

- subtilis Eh.

- tumidus Jan.

Craspedodiscus coscinodiscus Eh.

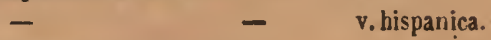

Diplonels bombus Eh.

Grammatuphora Moronensis Greo. robusta Dipp.

Ilemiaulus ambiguus Ġrun.

Isthmia enerois $E h$. 
Matogonia Actinoptychus Eh.

Melosira sulcata o. coronata.

Navicula protexta $E h$.

Pleurosizma affine $\nabla$. fossilis.

Pseudotriceratium cinnamoneum.
Stephanopyxis corona Eh.

- turris Eh.

Synedra salina Pant.

Triceratium antelliluvianum $\mathrm{Eh}$.

(Azpestia) antıquum Pant.

\section{No 661 ETANG DE VILLEBON - MEUDON (France).}

\section{Fragilaria vlrescens IRalfs}

\section{No 662 COLCHESTER - ESSEX (Angleterre)}

Campylodiscus bicostatus W. Sm. Nitzschia hungarica Grun.
Nitzschia vitræa Norm.

Surirella ovalis Bréb.

No 663

COLCHESTER - ESSEX (Angleterre)

Saumâtre

Cocconels pediculus Eh.

- placentula Eh.

Cymbella Ehrenbergii Ktz.

Epithemia sorex kitz.

- turgida Ktz.
Epithemia turgida v. granulata.

- $\quad$ - v. purcellus.

- zebra Ktz.

- _ fa minor.

- - var. proboscidea.

Navicula peregrina Eh.

No 664 HUMBOLDT - NEVADA (Etats-Unis)

Cymbella cistnla Kirch.

- cymbiformis Bréb.

- maculata $\mathrm{Ktz}$.
Melosira arenaria Moore var.

- crenulata $\mathrm{KIz}$.

- - var. tenuis. 
Melosira distans $\mathrm{Ktz}$.

\section{- granulata Ralis.}

Navicula commutata Grun.

- legumen $\mathbf{v}$. undulata.
Navicula major $\mathrm{Ktz}$.

Tetracyclus Peragalli $\nabla$. gracilis $n$. var. - strumosum Eh.

Van Ileurckia rhomboïdes Bréb.
Achnanthes Flahauti Her. et Per. rar.

Cymbella americana A.S.

- cistnla Kircl. .

- gibba Brèb.

Fragilaria virescens Ralfs.

- undulata W. Sm.

\section{Velosira crenulata fitz.}

- - rar. tenuis.

- decipiens Grun var.

- undulata oar. producta.

Navicula Bohemica Eh.

- Brebissonii Ktz.

- Dariana Eh.
Navicula gibba Ktz.

- gigas $\mathrm{ktz}$.

- lata Bréb.

- major Ktz.

- nobilis Ktz.

- peregrina $K t z$.

- pseudo bacillum Grun.

- viridis $\mathrm{Ktz}$.

- - var. commutata.

Tabellaria fenestrata $\mathrm{Ktz}$.

Tetracyclus lacustris Grun.

- Peragalli Her. et Per.

- stella Eh.
Achnanthos hungarica var.

Amphora pediculus Grun.

Cocconeis placentula Eh.

- líneata Eh.

Diatoma anceps Kirch.

Gomphonema acuminatum Eh.
Gomphonema acuminatum v. clavus.

- constrictum o. capitatum.

- micropus Kiz.

- mustela Eh.

- parvulum litz.

IIantzsehia amphioxys v. minor. Synedra delicatissima W. Sm.
Achnanthes coarctata Bréb.

- brevipcs Ag.
Achnanthes longipes $A g$. - subsessilis $\mathbf{K h}$. 
Homoeocladia Vidovichii Grun.

Licmophora Dalmatica K,

Navicula gracilis Grun.
Navicula rhyncocephala Eh. var.

Nitzschia lanceolala W. Sm.

Synedra aftinis Ktz.

No 668

PYRÉNEES (France)

Meridion circulare Ktz.

No 669 IVASHINGTON - SUSSEX (Angleterre)

Melosira variuns Ktz.

No 670

LUCHON - PYRÉNÉES (France)

Melosira (Orthosira) arenaria Moore

No 671 VILLEFRANCHE-SUR-MER (France)

IIonococladia Vidovichii Grun.

No 672 GLEN FESHIE - INVERNESS (Ecosse)

Eunotia diodon El.

- major Rah.

- monodon El.

- pectinalis Ktz.

Fragilara virescens Rirla.

Melosira distans $\mathrm{Kt} z$.

Navicula alpina W. Sm.

- borealis Eh.

- Brebissoni Ktz.
Navicula dactylus Eh. var.

- gentilis Ktz.

- major Ktz.

- pachyptera Ktz.

- rhomboïdes Eh.

- viridis Eh.

Surirella biseriata Bréb.

Tabellaria fenestrata $\mathrm{K} / \mathrm{z}$.

- flocculosa Ktz. 


\title{
N673-675 MONTE GIBBIO-SASSUOLO-EMILIA (Italie)
}

\author{
Dépôt fossile tertiaire marin
}

BIBLIOGRAPHIE. - Dr Achille Forti, Primo elenco delle Diatomee fossili cuntenute nei calcari marnosi biancastri di Monte Gibbio (Nuova Notarisia 1912).

Actinocyclus Ehrenhergii Ralfs $r$. intermodia Grun.

- Janischii Schum.

Actinoptychus glabratus Grun.

$\begin{array}{cc}\text { - } & \text { kymatodes Pant o. te- } \\ \text { tramera n. v. } \\ \text { minutus Grev. } \\ \text { - } & \text { Moronensis (Greo) Grun. } \\ \text { - } & \text { Mpinifer Grun. } \\ \text { - } & \text { undulatus El. } \\ \text { - } & \text { - fa maximaA.S. } \\ \text { - } & \text { vulgaris Sclium. } \\ \text { - } & -\quad \text { v. neogradensis }\end{array}$

Antelminellia gigas (Cast. Schütt).

Arachnoidiscus Ehrenbergii Bail.

- Greolleanus Hardm.

- indicus Eh. fa minor H.et B.

- orualus Eh. v. Muntejeyana A. S.

Asterolampra affinis Grev. v. cellulosa A. S.

- Marylandica Eh.

- rotula Greo.

Asteromphalus Grevillei Wall.

Aulacodiscus amcenus Grev. v. hungarica Pant.

- italicus Forti.

- margaritaceus Ralfs.

- Petersii Eh.o.trimera n.v.

Auliscus colatus Bail.

- $\quad$ - var. rhipis A. S.

- - var. strigillata A.S.

- pruinosus Bail.

- sculptus (W. Sm.) Ralfs.

Biddulphia Fortiana Temp. 1. sp.

- pulchella Gray.

- Tabellariceformis Forti n. sp.
Biddulphia Tuomeyi Bail.

$$
\text { - } \quad \text { - } \quad \text { - va hungarica Pant. }
$$

Campylodiscus Kidstonii Pant.

Cladogramma conicum v. campanulata Forti n. v.

Cocconeis Lanaii Forti n. sp.

- præcellens Pant. v. paucistriata.

- splendida Greg.

Coscinodiscus asteroïdes $\mathrm{Tr}$. et W.

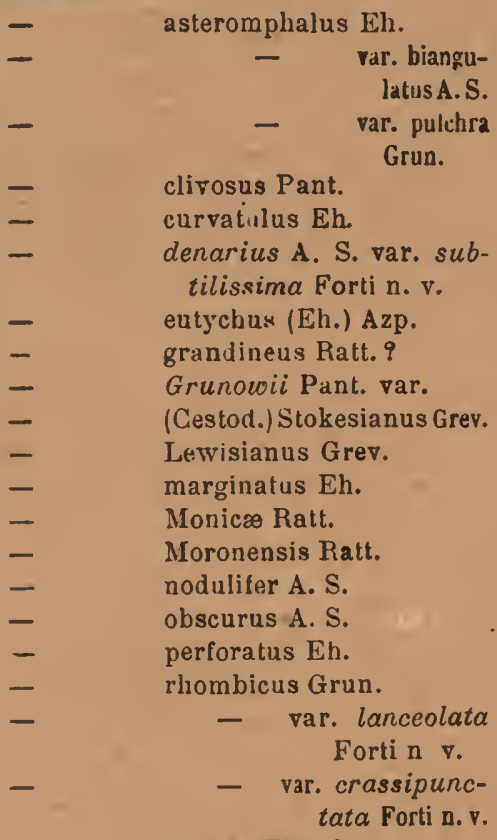

Rothii (Eh.) Grun. 
Craspedoporu Pantocsekii J. Br.

Bnilyctia oceanica Eh.

Gephyria Rinnböckii (Pant.) Forti.

Goniotuecium odontella Eh.

- $\quad$ - var.danica Grun.

Grammatophora robusta Dipp. $\nabla$. gracilis Pant.

Hyalodiscus arcticus Grun.

$$
\text { - stelliger Bail. }
$$

Isthmia nervosa $\mathrm{Ktz}$.

\section{- Squinaboli Forli.}

- $\quad$ - v.crassior Forlin.v.

Lendugeria epithemioides Temp. v. subarcuata Temp.

Lithodesmium Ehrenbergii (Grnn).

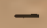

fa dicenlrica quadragous.

Melosira ornata Grun. v. reducta n. v.

Palmeria capillaris (J. Br.) Forti.

Paralia suicata (Ktz.) C.l.

- var. Siberica Grun.

Nuvicula lyra Eh. $v$. intermedia Per.

- $\quad$ - fa ambigua Per.

Podosira argus Grun.

- ambigua Grun.

Pseudopyxilla Tempereana Forti.

Rhablonema adriaticum Ktz.

Stephanozonia cincla Pant.

Stephanopyxis aculeata Ktz.

$\begin{array}{ll}\text { - } & \text { crassispina Grun. } \\ \text { - } & \text { grosse cellulata Pant. } \\ \text { - } & \text { Grunowii Gr. et St. } \\ & \text { lineata Eh. }\end{array}$

Stephanopyxi polaris Grun.

$\begin{array}{ll}- & - \\ \text { - } & \text { var. } \\ \text { - } & \text { turris Eh. vantiqua Pant. cylindrus Grun. }\end{array}$

Stictodiscus parallelus Grev. $\nabla$. Kinkeriana Tr. et W.

- parallelus $1^{2}$ minor.

- $\quad$ - $\quad f^{2}$ trigona.

- confusus Tr. et WV.

Surirella striatula Turp. var. Azpeiliæ Forti.

- recedens $A . S$.

Temp rea miocena Forti.

Triceratium antediluvianum Eh.

- biquadratum Jan.

- condecorum (Eh.) Bright.

- cucullatum v. lat or. Pant.

- formosum Bright.

- granle Bright fa quadrata.

- Grovei Pant.

- Nancoorense Grun.

- Pantocsekii A. S.

- $\quad$ - var. convexa Pant.

- $\quad$ - var. reciangularis

Forti n. v.

- radiata punctatum $A$. S.

- radians Temp. et $\mathrm{Br}$. var. italica $n$. $v$.

subrotundatum A. S.

- tridactylum Bail. v. valida Grun.

- Wittii Jan, fatalica quadrata, $n$. v.

Xanthiophyxis panduræformis Pant.

- globosa Eh.

acrolopha Forti n. sp.

No $676^{\circ}$ SHEERNESS ON SEA (Angleterre)

Biddulphia aurita Bréb.

No 677 LÜNEBERG - HANOVRE (Allemagne)

Amphora ovalis Ktz.

Cocconeis placentula Eh.
Cymbella cymbiforme Eh.

- cuspidata $\mathrm{K} \mathrm{tz}$. 
Epithemia zebra $\mathrm{Ktz}$.

Fragilaria construens Eh.

- mutabilis W. Sm.

Gomphonema capitatum Eh.

Gouphoneua clavatum Eh.

- mustela Eh.
Melogira crenulata $K t z$.

Navicula limosa $\mathrm{K} t z$.

- placentula Eh.

- radiosa Eh.

- cuspidata Ktr.

Stephanodigcus astrea $\mathrm{Eh}$.

No 678 HOPKINTON - NEWF-HAMPSHIRE (E.-Unis)

Dépôt fossile d'eau douce

Encyonema gracile Rab.

Eunotia polyglyphis Eh.

- robusta th

Fragilaria undulata W. Sm.

Melosira granulata $\mathrm{Ktz}$.

Navicula affinis $K t z$.

- amphirynchus El.

- appendiculata Ḱtz.

- bicapitata Lag.

- commutata Ktz.

- dactylus Eh.

- dilatata Eh.

- dubia Greg.
Navicula gigas Eh.

- iridis Eh.

- major Kz.

- mesolepta Eh.

- - var.stauroneiformis

- nobilis Ktz.

- rhomboïdes Eh.

- serians Ktz.

- seinen Eh.

- viridis Eh.

Stauroneis gracilis $\mathrm{K} \mathrm{z}$.

- phœnicenteron Eh.

Tabellaria fenestrata $\mathrm{Ktz}$.

No 679 DRAKESVILLE - NOVA-SCOTIA (Canada)

Dépôt fossile d'eau douce

Cocconeis lineata Eh.

- placentula EH.

Cymbella arcus $E h$.

- cistula Hempr.

- cymbiformis Eh.

Encyonema turgidum Greg.

Epithemia gibba Ktz.

- turgida kitz.

- Westermannii Kitz.

- zebra El.

- - var. proboscidea.

Fragilaria capucina var. mesoleia.

- construens $\mathrm{v}$. binodis.
Fragilaria construens var. genuina.

Gouphonema acuminatum $\mathrm{Eh}$.

$\begin{array}{ll}\text { - } & \text { capitatum Eh. } \\ \text { - } & \text { constrictum Eh. } \\ \text { - } & \text { intricatum Ktz. } \\ \text { turris Eh. }\end{array}$

Navicula amphigomphus Eh.

- cuspidata Kta.

- limosa Ktz.

- peregrina Kitz.

- tadiosa Kíz.

Synedra capitata Eh.

- ulna Eh. 
No 680

KLIEKEN - HANOVRE (Allemagne)

Dépôt fossile d'eall douce

Amphora affinis $K$ tz.

$$
\text { - ovalis Kiz. }
$$

Cyclotulla comta $\mathrm{Ktz}$.

$$
\text { - } \quad \text { - var. radiosa. }
$$

Cynatopleura solea Eh.

Cymbella amphicephala Næg,

- cistula Hemp.

- cuspidata Ktz.

- cymbiformis Eh.

- Ehrenbergii Ktz.

- lanceolata Eh.

Encyonema cuespitosum $\mathrm{Ktz}$.

- - var. lata.

- ventricosum Ktz.

Epithenia gibba $\mathrm{Ktz}$.

- zebra Kiz.

Fragilaria capucina Desm.
Fragilaria intermedia Eh.

Gomphonema clavatum $\mathrm{Eh}$.

- montanum Eh. var.

Melosira crenulata $\mathrm{K} t z$.

Navicula cuspidata Iŕtz.

- bacillum Eir.

- firma Eh.

- gigas Eh.

- gastrum Eh.

- limosa $\mathrm{Ktz}$.

- major Ktz.

- oblonga Ktz.

- placentula Elı.

- radiosa Kitz.

Stephanodiceus astræa Eh.

Surirella biseriata Brẻb.

- linearis IV. Sm.

Synedra ulna var. subitqualis.

$N^{*} 681$

\section{PORT D'OAMARU (Nouvelle-Zélande)}

Biddulphia obtusa Ralfs.

$N^{\circ} 682$

INVERNESS (Écosse)

Amphora affinis $\mathrm{Ktz}$.

Campylodiscus costatus W. Sn.

Cymatopleura ellıptica IV. Sm.

$$
\text { - solea Eh. }
$$

Cymbella gastroives Ktz.

- subrqualis Grun.

Diploneis elliptica $\mathrm{Cl}$.

- oblongella Cl.
Navicula limosa $K t$.

- viridis Ktz.

Ntzschia marina Grun.

- sigmoïdea W. Sm.

Surirella bifrons $K t z$.

- linearis W. Sm.

Synedra ulna $\nabla$. amphirynchus.

Tetracyclus lacustris $\mathrm{Ktz}$. 
No 683

Chatoceros danicum Cl.

I.eptocylindru danicus $\mathrm{Cl}$.

\section{EEBELO (Danemark)}

Rhizosolenia gracillima $\mathrm{Cl}$.

N० 684

DÉTROIT DE DAVIS No ${ }^{\circ}$

\section{Pélagique}

Chætoceros boreale var. Bright.

- decipiens CI.

- Peruviana Bright.
Coscinodiscus polyachanthus var. Rhizosolenia alata Bright.

- setigera Bright.

No 685

\section{DÉTROIT DE DAVIS No 2}

Fragilaria striatuia Lyng.

Licuophora tincta Ag.
Vavicula complanate Grun.

- constricta Eh. fa minuta.

N• 686 HUECORIO - LAGO DI PEZENARO (Mexique)

Dépôt fossile

Epithomin turgida v. granulata

Stephanodiscus Niagarge Eh.

Melosira crenuiata Kít.

(5)

\section{No 687 SAN'TIAGO PAPASQUIANO (Mexique) \\ Dépôt fossile}

Amphora veneta Ǩtz.

Cocconeis placentula Eh.
Cymbelia cistula Hemp.

- leptoceros Rab. 
Rpithemia turgida Eh.

Encyonema turgidum Greg.

Fragilaria Harrisonii Grun.

Gomphonema vibrio $\mathrm{Eh}$.

vavicula oblonga $\mathrm{Ktz}$.
Navicula radiosa $\mathrm{Ktz}$.

Nitzschia denticula Grun.

Pinuularia subcapitata Greg.

Synedra ulna Eh.

- capitata Eh.

Nos 688-690 PATUXENT RIVER (Etats-Unis)

\section{Dépot fossile marin}

Actlnocyclus Ehrenbergii Ralfs. moniliformis Ralfs.

Actinoptychus heliopelta Grun.

$\begin{array}{ll}\text { - } & \text { - o. versicolor J. Br. } \\ \text { - } & \text { excelsus Schum. } \\ \text { - } & \text { praetor A.S. } \\ \text { - } & \text { triooloa J. Br. } \\ \text { - } & \text { undulalus Eh. }\end{array}$

Anaulus mediterraneus Grun.

Asterolampra Marylandica Eh.

Asteromphalus Dallasianus Greo.

Aulacodiscus $\operatorname{crux} E h$.

$$
\begin{array}{lcl}
- & \text { margaritaceus Ralf. } \\
\text { - } & - & \text { v. distans. } \\
\text { - } & \text { Rogersii. A. S. }
\end{array}
$$

\begin{tabular}{|c|c|}
\hline - & - var. ambigua \\
\hline & - var. monicæ. \\
\hline & argus Eh. \\
\hline- & asteroides $T$. et $W$. \\
\hline - & asteromphalus Eh. \\
\hline 一 & - v. ambigua. \\
\hline- & biangulatus. A. S. \\
\hline- & bulliens A. S. \\
\hline- & borealis Eh. \\
\hline- & duriusculus Ratt. \\
\hline- & excentricus Eh. \\
\hline- & Levoistanus Greo. \\
\hline- & nobilis (irun. \\
\hline- & oculus-iridis Fh. \\
\hline 一 & omphalinthus Eh. \\
\hline - & $\begin{array}{l}\text { pertoratus Eh. } \\
\text { radiatus Eh. }\end{array}$ \\
\hline - & subtilis Eh. \\
\hline
\end{tabular}

Biddulphla Tuomeyi Bailey.

Cerataulus Smithii Ralf.

Coscinodiscus apiculatus Eh.
Coscinodiscus symbolophorus Eh.

Crasperlodiscus coscinodiscus $\mathrm{Eh}$.

$$
\text { - elegans Eh. }
$$

Eupodiscus inconspicuus Ratt.

Goniothecium decoratum J. Br.

$$
\text { - odontella Eh. }
$$

Liracliscus minutus Greo.

Melosira sulcata $\mathrm{Ktz}$.

Navicula Hennedeyi W. Sm.

- lyra Eh.

- prisca A. S.

- Witti A.S.

Periptera tetracladia Eb.

Pleurosigma Marylandicum Grun.

Pseullauliscus radiatus Ratt.

Pyxilla americana Grun.

Rhizosolenia sp.

Raphoneis amphiceros Eh.

- gemmifera $\mathrm{Eh}$.

- scalaris Eh.

septronels caducens Eh.

Stephanogonia actinoplychus Eh.

Stephanopyxis corona lih.

$$
\text { - } \quad \text { limbata Eh. }
$$

Stictolliscus Kitlonianus Grev.

Triceratium americanum Ralf.

- $\quad$ arcticum Bright.

- Brightwollıi Witt.

- Fischeri A S.

- Montereyi Bright.

- multifrons $J . B r$.

- solenuceros Eh.

- undulatum Eh. 
Nos $691-693$

ILE DE ZANTE (Grèce)

Dépôt fossile marin

Actinocyclus Ehrenborgii Ralf.

Actinoptychus undulatus Eh.

Biddulphia tridens $\mathrm{Eh}$.

Coscinodiscus dubius Ratt.

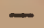

-

elegans Grev. var. Cette à fait, moins les épines marginales, à la variété spinifera (A. S. atlas $138 / 16$ offre la particularité de présenter des variétés et anomalies produites par l'ẻtablissement des granules de la partie centrale accom. pagnée do resserrement des lignes de granules rayonnantes qui se transforment en stries perlées et tinissent par donner à cette forme l'aspect d'un Hyalodiscus radiatus à structure très grossière.

Coscinodiscus markinatus Eh.

$\begin{array}{ll}\text { - } & \text { limbatus Eh. } \\ \text { - } & \text { oculus iridis Eh. } \\ \text { - } & \text { punctatus Eh. } \\ \text { - } & \text { radiatus Eh. } \\ \text { - } & \text { robustus Greg var. } \\ \text { - } & \text { turgidulus Ralfs. }\end{array}$

Hyalodiscus radiatus Bail. var.

Molosira sulcata $\mathrm{Ktz}$ var. radiata.

Pleurosigma sp?

Raphoneis gemmifera Eh.

Stephanopyxis turris Grun var.

- $\quad$ sp?(Cioscinodiscus cruciatus Eh)

N०694

NAGY-TAPOLCSANY (Hongrie)

Récent

Achnanthes lanceolata Grun.

Gomphonema obtusatum kitz.

Meridion constrictum Ag.

Navicula Brebissonii $K t z$.

Vitzschia frustulum Grun.
Nitzechia vermicularis Grun.

Surirella angusta $\mathrm{Ktz}$.

- minuta Bréb.

Synedra radians $W$. Snı.

- ulna var. aequalis.

N॰695 LONG LAKE WINSTED - CONNECTICUT (E.-U.)

Amphora àffinis $\mathrm{Ktz}$.

Cocconeis placentula Eh.

Cyclotella comta Eh.
Cymbella cuspidata Ktz.

- maculara W. Sm.

- lanceolata Eh. 
Epithemia turgida $\mathrm{Ktz}$.

Eunotia major Rab.

- undulata Els.

Navicula cardinalis Eh. var.

- dactylus Eh.

- dubia Greg.

- divergens W. Sm.

- fusca Greg var.

- Hitchcockii Eh.

- legumen Eh.

- limosa Eh.
Navicula radiosa Eh.

- stauroptera Ralis.

- tabellaria Eh.

Pleurosigma Spencerii var.

Stauroneis gracilis Eh.

- anceps Eh.

- phœnicenteron Eh.

Surirella splendida Eh.

- linearis Eh.

- robusta Eh.

Nos 696698

LICATA - SICILE (Italie)

Dépôt tertiaire marin

(L'analyse suivante nous a été fournie par M. le docteur ACHILLE FORT'I)

Actinocyclus Ehreniergii Ralf.

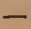

- moniliformis Ralfs.

- Ralfsii W. Sm.

- subcrassus Ratt.

Actinoptychus kymatodes Pant. var. radiolata Forti.

- undulatus Eh.

- $\quad$ - var paralleli . striata Forti.

- - var. labyrinthyformis Forti.

- vulgaris var. neogradensis Pant.

Asterolampra acutiloba Forti n. sp. (A.

Marylandica var.?)

$$
\text { - rotula Grev. }
$$

Auliscus colatus Bail.

Biddulphia Tuomeyi Bail. var.

Coscinodiscus appendiculatus Eh. var. æginensis A. S.

argus Eh.

- crassus Eh. var.
Coscinodiscus curvatulus Eh. var.

$\begin{array}{ll}- & \text { Eutychus (Eh.) Azp. } \\ \text { - } & \text { excentricus Eh. } \\ \text { - } & \text { leptopus Grun. } \\ \text { - } & \text { marginatus Eh. } \\ \text { - } & \text { minor Eh. } \\ \text { - } & \text { monicre Ratt. } \\ \text { - } & \text { neogradensis Pant. } \\ \text { - } & \text { radiatus Eh. var. } \\ \text { - } & \text { subtilis Eh. } \\ & \text { symbolophorus Grun. }\end{array}$

Euodia gibba v. moronensis.

- minor.

Ilyalodiscus arcticus O'Mea.

Navicula circumsecta Grun.

- crabro Eh. var.

- mujor Cl. $\nabla$. permagna Pant.

Rhabdonema adriaticum v. fossilis Pant.

IRhizosolenia robusta? fragments.

Stephanopyxis lineata (Eh.) Forti.

Synedra affinis Ktz.

Triceratium biquadratum Jan. 
$N^{\text {os }} 699700$

\section{JASTRABA (Hongrie)}

\section{Dépôt fossile d'eau douce}

BIBLIOGRAPHIE. - Ehrenberg. Mikrogeologie, T. VIII. I. (Ėchantillon notablement différent de celui étudié par Ehrenberg).

Amphora protracta Pant.

Cocconeis placentula Eh.

Cymbella cymbiformis Bréb.

- Ebrenbergii r. minor.

- gastroides Ktz.

- hungarica Grun.

- leptoceros v. obtusa.

Encyonema ventricosum Grun.

Epithemia cistula Ralfs.

- - - var. lunaris.

- gibberula Ktz.

- - - var. producta.

- turgida Ktz.

- zebra Ktz.

Eunotia flexuosa Ktz.

- formica $E h$.

- gracilis Rab.

- major Rab.

- minor V. H.

- monodon Eh.

- parallela Eh.

- pectinalis 0 . ventricosa.

Fragilaria capucina Desm.

- construens Grun.

- - var. venter.

- elliptica A. \&.

- endocystifera M. P. et F. H.

\section{Fragilaria nutabilis Grun.}

- nitida M. P. et F. H.

- virescens v, exigua.

Gomphonema angustatum v. producta.

- $\quad$ intricatum Ktz.

- subclaratum Grun.

IIantzschia amphioxys v. elongata?

Melosira arenaria Moore.

- crenulata Ktz.

- tenuis $\mathrm{Ktz}$.

- tenuissima Grun.

- undulata Ktz.

Navicula acrosphceria Bréb.

- amphibola $\mathrm{Cl}$.

- - - var. Perrieri.

- borealis Ktz.

- esox Ktz.

- gastrum Eh.

- hemiptera Ktz.

- major Ktz.

- protracta fa minor Grun.

- semen Eh. var.

Nitzgchia Kittlii Grun.

- vitrea Norm.

Van Ileurckia rhomboïdes $c$. amphiploroides.

\section{No8 701-726 ILES BARBADES (Antilles)}

Le dépôt des Barbades est l'un des pluz intéressants qui soient, aussi bien par la richesse et la rareté des formes qu'il contient que par leur ancienneté géologique. Ces magnifiques formes, dont la découverte a illustré 
Greville, sont cependant très rares dans ces dépóts composés presque exclusivement de Polycistines et ce n'est gu户̀re qu'en examinant un nom ${ }^{h} r e$ considèrable de Slides, le poil du triage à la màin, qu'on peut espérer en faire moisson. On sera toujours récompensé de sa peine par la richesse du résultat.

Découvert par Sir Robert Shomburg en 1846, ce dépôt fut envoyé à Ehrenberg qui le publia par des notes parues dans les Comptes Rendus de l'Académie des Sciences de Berlin en 1846, 1847 et 1850. En 1854, dans sa Microgéologie, il le mélange sur la même planche avec le dépôt des îles Nicobares; dans le dessin d'eusemble con-acré aux Barbades (fig. B), il ne représente aucune Diatomée, tandis qu'il figure un Arachnoïdiscus pour les Nicobares (fig. C). Dans les dessins de détail, consacrés presque exclusivement aux Polycistines, on peut se rendre compte facilement en se reportant à son mémoire ultérieur de 1875 que sur les 10 " polygastriques " qu'il figure et dont 8 seulement sont des Diatomées, l'Hemiaulus Polysticinorum et les plaques internes d'Asterolampra sont tout ce qu'il a pu voir dans ce riche dépôt en tant que Diatomées.

En 1875, dans ses "Mikrogeologischen Studien » sur les 3û magnifiques planches consacrées aux Polycistines, 10 figures de la planche 1 sont consacrées aux Diatomées; on y retrouve purement et simplement les plaques internes d'Asterolampra, l'Hemiaulus Polycistinorum déjà figurées en 1854 et comme nouveauté le modeste Triceratium microporum.

Dans ses tableaux syuoptyques, Ehrenberg cite Ehrenberg et ne cite qu'Ehrenberg. Toutes les découvertes de Greville lui restent incounues. Mais pour masquer la pauvreté de sa liste des Barbades, il y ajoute les Bermudes. Or, le soi-disant dépôt des Bermudes u’est probablement pas autre chose qu'un tripoli du Maryland, un "Nottingham " qui a été vendu en Amérique sous le nom de "Bermuda Tripoli ». En tout cas, si ce dépôt existe en réalité, il se rattache aux dépôts américains et non aux dépôts des Barbades.

Et cependant à cette époque Ehrenberg n'ignorait pas les travaux de Greville, et d'après son mémoire il possédait presque tous les dépôts des Barbades plus mème que nous n'en possédons aujourd'hui.

Il cite : Mount Hillaby, Haggats Plantage(Saint-Andrew), Icerves ou Boscobelle, Springfield, Burnt Hill, Scotland Ricer, Bissex Hill, Tank Smith House et Chalky Mount. 11 est vrai qu'il ne cite pas Cambridge où Greville a fait tant de belles découvertes.

C'est effectivement Greville qui a découvert les Diatomées des Barbades. 
Il les a décrites et figurées dans une série de remarquables mémoires pu bliés de 1861 à 1863, dans le "Quaterly journal of Microscopical Science " et les transactions de la Société de microscopie de Londres alors annexées à ce journal. Il semble qu'il ait eu ses matériaux de seconde main de Norman Johnson et d'autres diatomistes anglais. Jusqu'en 1863, il ne distingue pas les dépóts, il dit simplement "Barbados ») et cite cependant Cam. bridge en 1861 et Chimborazo en 1862, deux dépôts qui ne figurent pas sur la liste d'Ehrenberg. M. Brun croit que le " Burbadoes "primitif de Greville est Chimborazo. Ce n'est effectivement qu'au commencement de 1863 que Greville recoit de Johnson le dépôt de Cambridge et à partir de cette époque il cite toujours les dépôts partiels, Cambridge en général et quelquefois Springfield.

Greville était loin d'avoir épuisé le sujet. Bien d'autres explorèrent cette mine après lui et y firent de belles découvertes.

Walker et Chase dans les mêmes matériaux Springfield, Cambridge et dans Newcastle dont le nom apparaît pour la première fois à ma connaissance, découvrent de nouvelles espèces bien mal figurées par eux en 1887 .

Schmidt dans son atlas ea donne au contraire de magnifiques figures; de nouveaux dépôts sont découverts : College Hill, Chalky Cliff (Chalky mount?), Colonial et Clealand et de nouvelles espèces sont triées.

Sur les triages de Tempère et surtout de Barbó, Brun, décrit et figure dans les mémoires de la Société d'histoire naturelle de Genòve et dans le Diatomiste (1891-92), toute une série de magnifiques formes tirées de ces dépôts nouveaux.

Comme âge du dépôt, Ehrenberg et Brun le donnent comme .Miocène: Pantosseck, dans l'étude si intéressante qu'il a publiée en 1894 sous le titre de Die Bacilarien als Gesteinsbilder und Altersbestimmer, considérant à juste raison les Diatomées comme des fossiles, au même titre que toute autre coquille et même peut-être à mon avis à un titre supérieur vula minutie de détermination et d'identification qu'elles présentent, s'en sert pour la détermination de l'âge des couches géologiques (à défaut de renseignements stratigraphiques) suivant les mêmes règles qui sont admises en géologie pour les autres fossiles.

Ainsi interrogés, par leurs espèces et genres en général, éteints aujourd'hui, les dépôts des Barbades revendiquent leur place parmi les anciennes formations crétacées de la période secondaire.

H. P. 


\section{Principaux dépòts des Harbades}

Nos 701-703 MOUNT HILLABY.

Nos 704.707 COLLEGE HILL.

Nos 708-710 CHIMBORAZO.

Nos 711-714 SPRINGFIELD.

Nos 715,716 HOPEWELL.
Nos 717,718 PIC TENERIFF.

Nos 719-722 CHALKY CLIFF.

Nos 723,724 CHALKY MOUNT.

Nos 725,7:26 CAMBRIDGE ESTATE.

(Nos 92,93(publlé p. 50) NEWCASTLE.)
Actinella scala $\mathrm{J}$. $\mathrm{Br}$.

Actinuliseus Barbadensis Greo.

$$
\text { - } \quad \text { gloria J. Br. }
$$

Actinocyclus tenuissimus $\mathrm{Cl}$.

Actinogonium septenarium Eh.

Actinoptychns adamans Temp. et $\mathrm{Br}$.

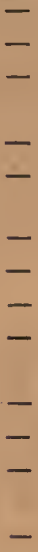

$\overline{-}$
-
$\overline{-}$
-
$\overline{-}$
-
-
-
baccatus $J$. Br. constellatus $J$. $B r$. heliopelta car. cersicolor J. Br.

Hillabyanus $J$. Br.

- $\quad$ oar. coralli-
lobatus J. Br. Br.
madreporus J. Br.
paradoxus Greo.
pericatatus o. tropicalis
J. Br.
ratiolatus Grun.
trivaloa J. Br.
undulatus oar. Barba-
densis A. S.
Wittii Jan. o. scutifor-
mis W. et Ch.

Amphora obtusa Greg. oar. miocena J. Br.

Anaulus primordialis $J$. $B r$.

Arachnoinliscus Barbadensis A. $S$.

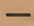

Ehrenbergii o. splendens $J . B r$.

- indicus.
Asterolampra cemulans Grec.

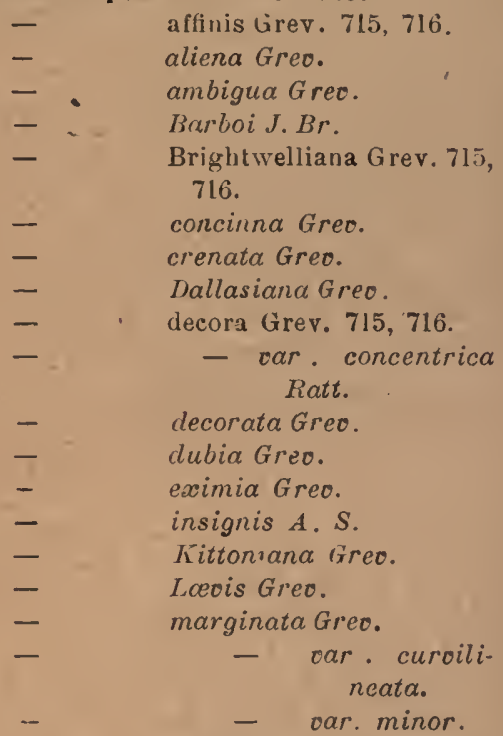

- Marylandica Eh. var.

Barbadensis.

- pulchraGreo.

- punctata Greo.

- Ralfsiana Grev. 721, 722.

- Rylandsiana Greo.

- scutula Greo.

- simulans Greo.

- splendida Greo.

- stellulata frreo.

- traducens Rait. 
Astcrolampra vulgaris Grev.

$$
\begin{array}{cc}
- & \text { oar. cellulosa } \\
\text { - } & \text { Greo. } \\
\text { - } & \text { War.planiorGreo. } \\
\text { - Wallishiana Greo. } & \text { Weisflogii Grun. }
\end{array}
$$

\begin{tabular}{|c|c|}
\hline- & - subdecora Ratt. \\
\hline - & angulatus Gireo. \\
\hline - & anthoïdes A. S. \\
\hline - & attenuatus Ratt. \\
\hline - & Barbadensis Ralfs. \\
\hline - & $\begin{array}{l}\text { Carruthesianus Kitt. et } \\
\text { Grove. }\end{array}$ \\
\hline - & cinctus Grev. \\
\hline - & compactus Ratt. \\
\hline - & decorus Greo. \\
\hline - & - oar. Stoschii Jan. \\
\hline- & extans Greo \\
\hline - & gigas Grev. \\
\hline- & $\begin{array}{l}\text { grandis W. et Ch. } \\
\text { gracilis Ratt. }\end{array}$ \\
\hline - & $\begin{array}{l}\text { Huttonii oar. dichotoma } \\
\text { J. Br. }\end{array}$ \\
\hline- & inflatus Greo. \\
\hline & - var. minor Ratt. \\
\hline - & $\begin{array}{c}\text { - oar. spinifer } \\
\text { J.Br. }\end{array}$ \\
\hline - & - var.stellata Ratt. \\
\hline - & Johnsonii Arnott. \\
\hline - & Kilkelianus Grieo. \\
\hline - & - $\quad$ car.minor. \\
\hline - & lucidus Greo. \\
\hline - & mammosus Greo \\
\hline- & notatus $G r$. et $S t$. \\
\hline- & pallidus Greo. \\
\hline - & $\begin{array}{l}\text { paradoxus Greo. } \\
\text { pellucinus Greo. }\end{array}$ \\
\hline - & Petersii Eh. \\
\hline - & - var.expansa Ratt. \\
\hline - & $\begin{array}{l}\text { oar. rara Ratt. } \\
\text { probabilis A. S. }\end{array}$ \\
\hline - & radiatus Greo. \\
\hline- & robustus Ratt. \\
\hline - & $\begin{array}{l}\text { rotulus Ratt. } \\
\text { sparsus Greo. }\end{array}$ \\
\hline - & $\begin{array}{l}\text { sparsus Greo. } \\
\text { spectabilis Greo. }\end{array}$ \\
\hline- & - oar depressa. \\
\hline & $\begin{array}{l}\text { Stoschii Jan. ( }=A \text { deco- } \\
\text { rus var.) }\end{array}$ \\
\hline - & $\begin{array}{l}\text { superbus Kitt. } \\
\text { tabernaculum } J . B r .\end{array}$ \\
\hline
\end{tabular}

Aulacodiscus amonus Greo.
Aulacodiscus tetrapurgon $\mathrm{J}$. Br. - umbonatus Greo.

Auliscus Barbadensis Greo.

- Bourgognei Bergon.

- colatus Bail.

- - var. latecostata A.S.

- craterifer J. Br.

- curoatoradiatus $J . B r$.

- elegans Greo.

- - car. Grunowii Ratt.

- Grunowii A. S.

- incertus A. $S$.

- nanus A: S.

- nitidus Ratt.

- paroulus Greo.

- - punctatus Bail.

- Ratrayi Cl.

- squamosus J. Br.

- Stōckardtii Jan.

Baxteria Brunii V. $H$.

- formosa J. Br.

Bergonia Barbadensis Temp.

- tetrapora J. Br.

Bidldulphia aurita Bréb.

- Barbadensis Greo.

- bifenestrata J. Br.

- chinensis Greo.

- - corpulenta Greo.

- decorata Greo.

- elegantula isreo.

- flmbriata Greo.

- giganteaGrec.

- inflata Grev.

- mammosa Greo.

- nitida Grev.

- podagrosa líreo.

- punctata Freo.

- rhombus o. fossilis $\mathrm{J} . \mathrm{Br}$.

- sinuata Greo.

- tenuicornis Greo.

- Tuomeyi Bailey.

- sp. A. S. atlas 118/11, 12 .

- sp. A. S. atlas 119/19.

Brightwellia coronata Ralfs.

$-$

- var. radians Ratt.

- elaborata Greo.

- excellens Ratt.

- Johnsonii Ralfs 715.

- $\quad$ pulchra "Grun. 702, 703

- splendida Ratt. 
Bruniella coronalis $V . H$.

Campyloneis notabilis var. oculata J. Br.

Capsula Barboi J. $\mathrm{Br}$.

- biformis J. Br.

Cerataulus scaber (Breo.) Ralfs.

Cestodiscus interruptus M. Per. n. sp. Diam. environ $50 \mu-d u$ bord au centre : une zone marginale striée (9 stries en $10 \mu$ ). un cercle de granules $(5$ en $10 \mu)$ interrompu par de petites épines ou tubercules; une area lisse circulaire (environ 2 $\boldsymbol{\mu}$ de large); des lignes ra: diantes de granules ( 5 en $10 \mu)$ de longueur inègale et n'atteignant pas le centre qui est lisse, nos 701703.

- $\quad$ pulchellus Grev, 713. Giun. superbus Hardm.

Chretoceros californicum Grun. 702.

Cladogramma conicum Greo.

Clavularia Barbadensis Greo.

$$
\text { - catenata J. Br. }
$$

Cocconeis armata Greo.

$$
\text { - nodulifer Groce. }
$$

Coscinodiscus africanus Jan.

$\begin{array}{ll}\text { - } & \text { angulatus Greo. } \\ \text { - } & \text { apages Ratt. } \\ \text { - } & \text { apiculatus Eh. } 715 . \\ \text { - } & \text { apollonis Eh. } \\ \text { - } & \text { argus Eh. } \\ \text { - } & \text { armatus Greo. } \\ \text { - } & \text { Barbadensis Greo. } \\ \text { - } & \text { biradiatus Grev. } \\ \text { - } & \text { boliviensis Grun. } \\ \text { - } & \text { borealis Bail. } \\ \text { - } & \text { bractea J. Br. } \\ \text { - } & \text { bulliens A. S. } \\ \text { - } & \text { concinnus W. Sm. } \\ \text { - } & \text { convexus A. S. } \\ \text { - } & \text { crassus Bail. var. } \\ \text { curvatulus v. latius striata } & \quad \text { Ratt. } \\ \text { - } & \quad \text { v. recta Ratt. }\end{array}$

Coscinodiscus decrescens Grun.

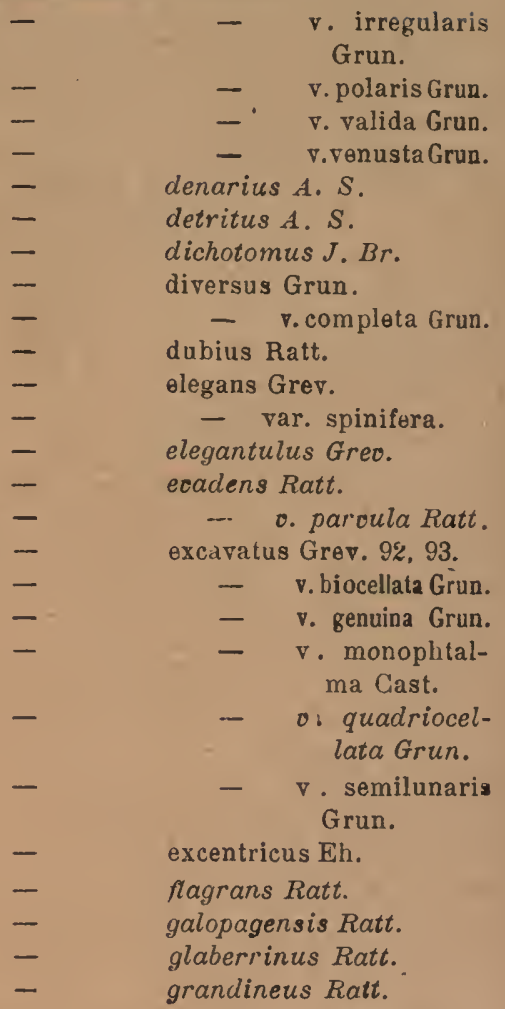

$-$

o. dentata Ratt.

- griseus Grev.

- $\quad$ - $\quad$. apiculata Ratt.

- Groveanus Ratt.

- heteroporus Eh.

- interlineatus Ratt.

- Janischii A. S.

- lineatus Eh.

- Macrceanus var.

- Marginatus Eh.

- minor Eh.

- Morsianus Greo.

- nitidus Greo.

- nitidulus Greo.

- Normannianus Greo.

- Oamaruensis Br. et St.

- ooum Temp. et M. Per

n. sp. Contouren forme d'œuf; zone marginale 
très étroite, strièe (16 stries en $10 \mu$ )garnie de petites épines; lignes rayonnantes élégam inent courbes; alternativement courtes et longues de granules isolés apiculés ( 5 en $10 \mu$ au milieu du rayou où îls sont le plus gros). Rayons respectivement $14,18,21 \mu$ environ. Nos $702,703$.

Coscinodiscus oblongus Grev.

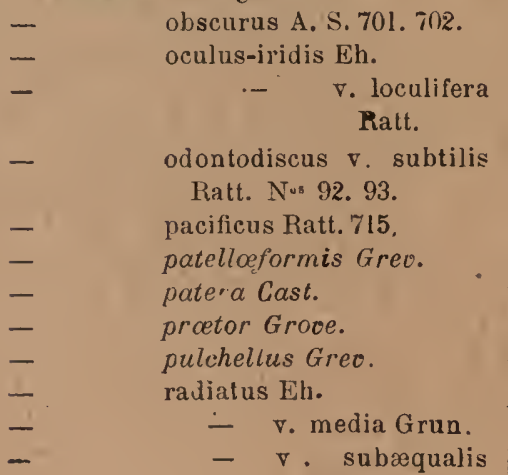
Grun.

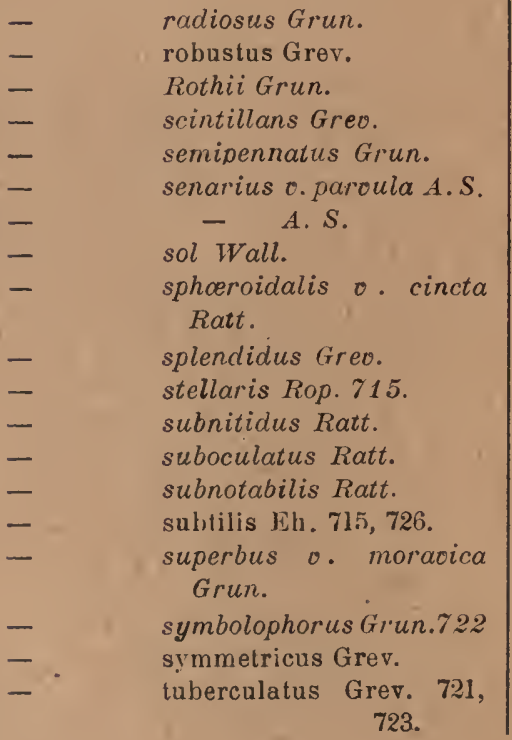

Coscinodiscus tuberculatus oar. monicoe Grun.

$$
\text { - } \quad \text { turgidus Ratt. 725, } 726 .
$$

Cotyledon coronalis $J . \mathrm{Br}$

Craspedodiscus coronatus Bright.

- coscinodicus v. apicu-

latus n. v. Diam. $50 \mu$.

- Partie périphérique a vec des granules isolès, apiculés, décussés $(1 \dot{a} 5$ en $10 \mu)$ Nos 715,716 .

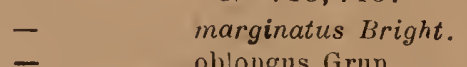
oblongus Grun.

- - v. acuminata n. v. - Variété allongèe à extrémités pointues mélangé au type.
- semiplanus Bright.
- umbonalus Greo.

Craspedoporus Johnsonianus Greo.

$$
\text { - Ralfsianus Greo. }
$$

Cresswellia Barbadensis Greo.

$$
\text { - } \quad \text { minuta Grev. }
$$

Dicladia Barbadensis Greo.

- robusta Greo.

Entogonia Abercrombieana Greo.

-

-

- amabilis Greo.

- approximata Greo.

- Bergonii J. Br.

- Brunii Bergon.

- Davyana Greo.

- - var. distans Bergon.

- - var. - fabian- gulata Bergon.

- $\quad$ - var.intermedia Bergon.

- - oar. conspicua (Greo.) Bergon.

- $\quad$ - oar. propincua Bergon.

- $\quad$ - o. trigemma J. Br. et Bergon.

divergens Bergon. 
Entogonia elegains Grec.

- furcata J. $\mathrm{Br}$.

- gratiosa Greo.

- $\quad$ - oar.intermedia Bergon.

- inopinata Greo.

- $\quad$ - fa linearis Bergon.

- Letourneurii Bergon.

- pulcherima Greo.

$-$
- - o. marginata (Grev.) Ber- gon.

- - v. punctulata.

- reticulata Greo.

- Temperii Bergon.

- $\quad$ - o. intermedia Bergon.

- tripodiformis Bergon.

- oariegata Greo.

- venulosa Greo.

Eunotia castalunga $J$. $B r$.

Enpodiscus Barbadensis líreo.

- Hardmanianus Greo.

- minutus Greo.

- paroulus Greo.

- - o. concentrica.

- punctulatus Greo.

- scaber Greo.

- simplex Greo.

Fenestrella Barbalensis freo.

Gephyria media Arnodt. $f^{2}$ miocena.

Goniothecium prolongatum Greo.

$-$

Ilemiaulus alatus Ǵrev.

- allar J. Br.

- angustus Greo.

- Barbadensie Grun.

- capitatus Greo.

- crenatus Greo.

- diversus Grun.

- dubius Grun.

- exiguus Gree.

- hastatus Greo.

- hostilis Heib.

- lobatus Greo.

- longicornis Grev. 709, 710.

- lyriformis Grev.

Hemiaulus lyriformis oar. rostrata $\mathbf{n}$. Rostre médian trés développé plus long et plus robusle que les cornes latérales. $N^{\text {os }} 715,716$.

- minutus Greo.

- mucronatus Grec.

- ornithocephalus Grun.

- pileolus var. baccata J. Br.

- podagrosus (Greo.) Grun.

- polycistinorum Eh.

-

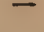

var. mesolepta Grun.

- proteus Heib.

- puloinatus Greo.

- punctatus Greo.

- reticulatus Greo.

- robustus Greo.

- subacitus Grun.

- symmetricus Grun.

- tenuicornis Grev. 721, 722.

- tubuloous J. Br.

- Wittii Grun.

Heterodyction Rylandsianum Greo. - $\quad$ splendidum Greo. 703.

Isouliscus coronalis $J$. Br.

Isthmia enerois Eh.

- Johnsonii A. S.

- minima Bail.

- ? reticularis J. Br.

Kittonia Barboi $J$. $B r$.

- gigantea cireo.

- Hyllabyana J.Br.

Liostephania comta $E h$.

$$
\begin{aligned}
& \text { - magnifica Eh. } \\
& \text { - } \quad \text { rotula Eh. } \\
& \text { - } \quad \text { car. A. S. atlas } \\
&
\end{aligned}
$$

Liradiscus Barbadensis Grun.

$$
\begin{aligned}
& \text { - } \quad \text { ellipticus Greo. } \\
& \text { - } \quad \text { minutus Greo. } \\
& \text { - oodis Greo. }
\end{aligned}
$$

Litbodesmium Ehrenbergii Greo.

- minusculum Greo.

- undulatum Eh.

Mastogloia Grovei $C l$.

- Hillabyana J. Br.

- rutilans $J . \mathrm{Br}$.

- sagitta cupidinis J. Br.

- splendens J. Br. 
Molosira sol Eh. 717, 718.

- sulcata $\mathrm{Ktz}$.

- - var. coronata.

Navicula camplanata $J . B r$.

- cuneoquadrata J. Br.

- Fischeri fa major n. f. Longueur $190 \mu, 5$ à 6 lignes de granules en $10 \mu \dot{x}$ la partie médiane. No 709, 710.

- fluitans var. biconvexa J.Br.

- inelegans Br. et St.

- sp. A. S. atlas 6/35, 36 .

Orthoneis Barbadensis (Greo.) Cl. 706.

- Grovei Cl.

- naviculoides (Greo.) Cl.

- $\quad$ oar. miocena

J. $B r$.

Peponia angulata (Greo.) H. Per.

- Barbadensis Grev.

PIagiogrunima angulatum Greo.

- Bardadensis Greo.

\section{- - oar. acti-}

Podosira sphcerica $J . B r$. nellas. Br.

Porodiscu calyciflos Temp. et $\mathrm{Br}$.

- conicus Greo.

- corniger J. Br.

- elegans Greo.

- major oar. densa Rati.

- nitidus Greo.

- - oar. armata Ratt.

- oblongus Grev.

- $\quad$ spiniferus Riatt.

- $\quad$ splendidus Grev. 702, 703.

- - var. marginata

Porpela inflexa $A . S$. Ratt.

- ornata Greo.

- quadrata Grev.

- quadriceps Greo

Pseudo-Auliscus ambiguus(Greo.)Ratt.

- - oar. major.

Ratt.

- elaboratus ( $R$ alfs)

Ratt.

- $\quad$ - var.tetra.

pod i a

J. $B r$.
Pseudo-Aullecue

Johnsonianus oar.craterifer $J . B r$.

Johnsonianus oar. eludens Ratt.

nebulosus Leud. Fort. notatus (Greo.) Ratt. ornatus (Greo.) Ratt. Ralfsianus (Greo.) Ratt.

rotatus Ratt.

- $\quad$ spinosus Christion.

- tetraphtlhalmus $\mathrm{Cl}$.

Pseudo-Stictodiscus angulatus Grun.

Pseudo-Triceratium araneosum (Greo.) Grun.

- labyrinthicum (Greo.) Grun.

Pyxilla Baltica Grun.

- Barbadensis Greo.

- caput-avis J. Br.

- - car. gracilis Temp. et Forti.

- gracilis Temp. et Forti.

- - var.buccinalis Temp. et Forti.

- Johnsoniana Greo.

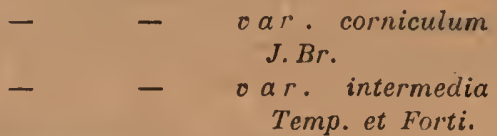

Radiopalma Brunii $V . H$.

inutilaria elliptica Greo.

- superba Grec.

- ventricosa Greo.

- - oar. rostellatus $\mathrm{J} . \mathrm{Br}$.

Rylandsia biradiata Greo.

Skeletonema Barbadensis Greo.

- - oar.argus J. $B r$.

Solium princeps $M$. Per. n. sp. Forme à 4 cornes du Trinacria princeps Witt. Nor $702,703$.

Stophanogonia Hillabyana Temp. et M. Per. Rayons très peu marqués; valves couvertes de granules épars (10 à 12 en $10 \mu$ onviron) sans vermiculurè ; centre hélicoïdal. Nor 702, 703. 
Stephanogonia Hillabyana oar. tenera Temp. et $M$. Per. Valve moins robuste à granules presque punctiformes. Nor 702, 703 .

Stephanopyxis appendiculata o. intermedia Grun.

Barbadensis (Greo.) Grun.

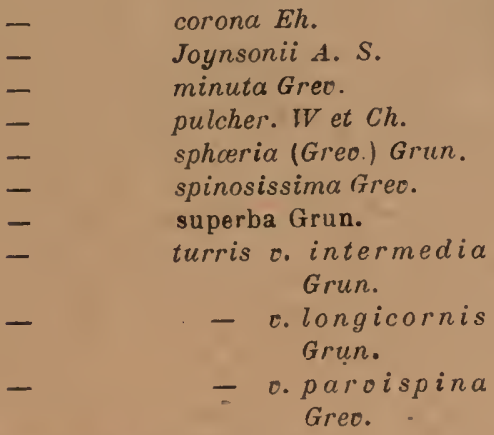

Stictodiscus Greoilleanus $W$. et $\mathrm{Ch}$. insignis Greo. reticulo-cellulosus Bergon.

Strangulonema Barbadense Greo.

syndetocystis Greoilleana Grec.

Synedra costalunga J. Br .

- fibula J. Br.

Synedrosphenia clavata (Gireo.) H. Per: Syringidium eximium Grun.

Wittii Grun.

Thaumatonema Bardadense Greo. - . complanata J. Br. - costatum Greo.

Terebraria Barbadensis Greo.

Triceratium acceptum Greo.

$\begin{array}{cl}\text { - } & \text { aculeatum Grun. } \\ \text { - } & \text { acutangulatum Greo. } \\ \text { - } & \text { americanum Ralfs. } \\ \text { - } & \text { amplexum A. S. } \\ \text { - } & \text { areolatum Greo. } \\ \text { - } & \text { - car. venosaGrun. } \\ \text { - } & \text { atomus Greo. } \\ \text { - } & \text { attennatum Grev. } \\ \text { - } & \text { car. (Le Dia- } \\ \text { - } & \text { tom iste I } \\ \text { - } & \text { Barbadense Greo. } \\ \text { - } & \text { Barboi J. Br. }\end{array}$

Triceratium biformis $J . B r$. - blanditum Greo.

- breoineroum Greo.

- cancellatum Greo.

$-$ oar. minor $W$. et $\mathrm{Ch}$.

capitatum Greo.

- cariboum W. et Ch.

- cariboum W. et C

$-$

ar. major West.

- cellulosum Greo.

- concinnum Greo.

- claoatum Cl.

- condecorum Bright.

- constans Greo.

- cornutum Greo.

coscinoides Grove et St.

$-$ cruciforme A. S. curoicostatum J. Br. curvilimbum J. $\mathrm{Br}$. definitum Greo. delicatulum trieo. denticulatum Greo. digitale J. Br. disciforme Greo. dioisum Grun. dulce Greo. Ehrenbergii Grun. exornatum Grec. Febigerii Walker. ferox A. S. firmum Greo. fooeatum Greo.

- car. minor A.S. fractum W. et Eh. Frauenfeldii Grun. giganteum Greo.$$
-
$$

$-$

(a) 
Triceratium inelegans Greo. o. tubulosa J. $\mathrm{Rr}$.

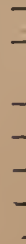

inconspicuum Greo.

inflatum Greo.

oar. (A.s. atlas $7 \pi / 19,21,28$.

insigne Gree.

- car. minor A.S. irregulare Greo.

Jansenianus Grun.

latum Greo.

lautum Grev.

ligulatum Greo.

lineatum Greo.

lineolatum Greo.

lobatogemmatum $\mathrm{J}$. Br.

lobatum Greo.

mammosum Greo.

marginatum Bright.

microcephalum (j)eo.

microporum Eh.

microstictum Greo

microstigma Eh.

microstriatum Greo.

minutum $W$. et $C h$.

mirificum $J . B r$.

modestum ireo.

nebulosum Greo.

neglectum Greo.

nitescens Greo.

nitidum Greo.

Normanianum Greo.

notabile Greo.

obesum Greo.

obtusum Eh.

oculatum Greo.

ornatum Greo.

pallidum Greo.

partitum Greo.

panperculum Greo.

perminutum Greo.

perpusillum Greo.

picturatum firreo.

plicatum Grun.

plumosum Greo.

protenue Greo

iroductum Greo.

prominens Greo.

quadrangulare Greo.

quadratum Greo.

radiatum Greo.
Triceratium radioso reticulatum Grun. repletum is ireo.

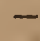

reticulatum Grec.

reticulum Eh.

rotundatum Greo.

Rylandsianum Greo.

semicirculare Bright.

Smithianum Greo.

solenoceros Eh.

squamatum Pant o. radiata J. Br'.

subcapitatum Gree.

tridactylum Bright.

trifolium A. S.

trilineatum Greo.

tripartitum Grun c. miocena $J . B r^{\circ}$.

tripecten $J . B r$.

- tripos J. Br.

- $\quad$ triplex J. Br.

- truncatum Bright

Tulkii Temp.

tumidum Greo.

- var. costulata A.S.

turgidum A. S.

umbilicatum Ralss.

undulatum Eh.

unguiculatum Grev.

uoiferum $A$. $S$.

venosum Bright.

- faparca.

cenulosum Greo.

Westianum Greo.

-

$-$

Weissflogii W. et Ch.

wonulatum Greo.

- sp. A. S. atlas $76 / 4$.

- $\rightarrow \quad$ sp. A. S. atlas $77 / 42$.

- $\quad$ sp. A..S. atlas 88/9.

- sp. A. S. atlas 94/10.

- $\quad$ sp.A.S atlas 150/8,17,24.

- sp. A. S. atlas 151/42.

Trinacria conifera (J. Br.) Grun.

- insipiens $v$. intermedia.

- ligulata (Greo.) Br. et St.

- pileolus o. baccata $\mathrm{J} . \mathrm{Br}$.

- prcetenuis (Gree.) Grun.

- - oar. perminuta.

- subcapitata (Greo.) Grun.

Xanthiopyxis cingulata $E h$.

- constricta Eh. oblonga Eh. 


\section{No 727 FRANZENSBAD - BOHÈME (Autriche)}

Dépôt fossile d'eau douce

Eunotia lunaris Eh.

Gomphouema montanum Schum. var. subelavatum Grun.

Melosira crenulata v. ambigua.

Navicula atomoides Grun.

- bacillaris v. inconstantissima Grun.

- cincta Eh.

- leptogongyla Eh.

- limosa $\mathrm{Ktz}$.
Navicula major Ktz.

- peregrina $\mathrm{K} t z$.

- ventricosa v. truncatula Grun.

- viridis Ktz.

Nitzschia frustulum.

- spectabilis.

Synedra affinis v. delicatula Grun.

- minuscula Grun.

- pulchella v. Smithii Ralfs.

\section{$\mathrm{N}^{\text {s }} 728,729$ TODD HOLLOW - PLYMOUTH CONN.}

\section{(Etats-Unis)}

Amphora ovalis var. minor.

$$
\text { - pediculus Grun. }
$$

Cocconeis placentula $\mathrm{Eh}$.

Cymbella americana A. S.

- amphicephala Næg.

- anglica Lag.

- austriaca Giun. var.

- cuspidata Kítz.

- Ehrenbergii var. minor.

- gastroides $\mathrm{Ktz}$.

- gibba Eh.

- Laubyi M. Per. et F. H

- maculata Ktz.

Diatoma anceps var. constricta $n$. var. à extrémités capitées et partie médiane rétrécie.

Encyomema gracile Rab.

- ventricosuın Grun.

Eunotia bidens Eh.

- formica Eh.

- flexuosa Kt.
Eunotia incisa Greg.

- lunaris Grun.

- major Bréb.

- - var. ventricosa.

- minor V. H.

- monodon Eh.

- perpusilla Grun.

- prærupta var. bidens.

- robusta var. tetraodon.

Fragilaria construens Grun.

- elliptica Sclium.

- virescens Ralfs.

Gomphonema acuminalum Eh.

$\begin{array}{ccc}- & - & \text { var. clavus. } \\ - & - & \text { var. trigono- } \\ \text { cephalus. } \\ \text { - } & \text { angustatum Ktz. } \\ \text { - } & -\quad \text { var. producta. } \\ \text { - } & \text { capitatum Eh. } \\ \text { - } & \text { dichotomum Ktz. } \\ & \text { elongatum W. Sm. }\end{array}$


Gomphonoma intricatum $\mathrm{Ktz}$.

- parvulum Ktz.

- subclavatum Grun.

Hantzschia amphioxys.Grun.

- $\quad$ - var. elongata.

Molosira crenulata $\mathrm{K} \mathrm{I}$.

- - - var. tenuis.

- scalaris Grun.

- tenuissima Grun.

Meridion constrictum Ralfs.

- Zinkenii $\mathrm{K} / \mathrm{z}$.

Navicula amphigomphus Eh.

- amphirynchus Eh.

- bacilliformis Grun.

- biceps Eh.

- bisulcata Lag.

- Brannii Grun.

- Brebissonii var.

- cardinalicus Cl.

- columnaris Eh.

- cuspidata Ktz.

- dactylus Ktz.

- decurrens Eh.

- divergens Eh.

- dubia Eh.

- elegantissima M. Per.

- firma $\mathrm{Ktz}$.

- - var. subampliata.

- gibba Ktz.

- gigas $\mathrm{Ktz}$.

- guttata J. Br.

- Hilseana Jan.

- Hitcbcockii Eh.

- interrupla var. stauroneiformis.

- iridis Eh.

- - var. ampliata

- legumen Eh.

- limosa var. gibberula.

- inajor $\mathrm{Ktz}$.

- - - var. asymetrica.

- mesolepta var. stauroneiformis.
Navieula mutica $K_{t z}$.

- nobilis Ktz.

- pachyptera Eh.

- parva Eh.

- producta W. Sm.

- pseudo-bacillum Grun.

- raliosa var..

- rhyncocephala Ktz.

- rostellata Ktz.

- subcapitata var. stauroneiformis.

- Termes A. S.

- transversa A.S.

- binodu Lewis.

- centricosa Eh.

- sp. 9 A. S. atlas $49 / 13$.

Nitzschia brevissima var.

Stauroneis anceps Eh.

- _ - var. amphicephala.

- $\quad$ - var. fossilis.

- Baileyi kh.

- gracilis Eh.

- lineolata Eh.

- Sieboldii Eh.

- Smithii Grun.

Surirella angusta Ḱtz.

- cardinalis Kitt.

- elegans Eh. var.

- linearis W. Sm.

- robusta Eh.

- saxonica Auers.

- tenera Greg.

- _ var. splendidula.

Synedra ulna var. danica.

Tabellaria fenestrata $\mathrm{Ktz}$.

. - flocculosa $\mathrm{Ktz}$.

Van Ileurckia crassinervia var.

- rhomboides Bréb.

- $\quad$ var . amphipleuroides. vulgare $\mathrm{V} . \mathrm{H}$.

Nos 730, 731 POND’S POINT - CONNECTICUT (Etats-Unis)

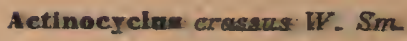

Actinoptychus undulatus Eh.
Amphiprora pulchra Bail.

Anphora carenaria Donk. 
Amphora Eulensteinii Grun.

- proteus Greg. et var.

Auliscus cœlatus Bail.

- fenestratus Gr. et St.

- pruinosus Bail.

- - var. Zanzibarica.

BIddulphia pulchella Greg.

- rhombus W. Sm.

Cerataulus turgidus Eh.

Coscinodiscus excentricus Eh.

$\begin{array}{ll}\text { - } & \text { nitidus Greg. } \\ \text { - } & \text { oculus-iridis Eh. } \\ & \text { radiatus Eh. }\end{array}$

Cyclotella striata Grun.

Diploneis bombus Eh.

- interrupta $C l$.

- littoralis $\mathrm{Cl}$.

Eunotogramma loevis Grun.

Hyalodiscus radiatus Grun.

Mastogioia angulata Lew.

Melosira sulcata oar. coronata.

- - var. radiata.

Navicula Brasiliensis var.

- clavata Greg

- Dariana A. S.

- formosa lireg.
Navicula gigas Ktz.

- Hennedyi W. Sm.

- humerosa Bréb.

- lyra Eh.

- - var. elliptica.

- - oar. insignis.

- - var. intermedia fa recta.

- major Ktz.

- permugna Bail.

- Powellii Lewis.

- Wrightii 0. Mea.

- Yarrensis var.? A. S. atlas $45 / 17,18$.

Nitzschia plana W. Sm.

- scalaris W.Sm.

Plagiogramma Gregorianum Grev.

Pleurosigma angulatum W. Sm.

- Terryanum II. Per.

Raphoneis amphiceros Eh.

Rhablonema adriaticum W. Sm.

Rhopalodia musculns $\mathrm{H}$. Per.

Scolispleura tumida Bréb.

Stauroneis Gregoryi Ralfs.

Surirella recedens $A$. S.

- striatula Tur.

Triceratium antediluvianum Eh.

No 732 STONY CREEK - CONNECTICU'T (Etats-Unis)

Actinoptychus splendens Shadb.

$$
\text { - undulatus Eh. }
$$

Aniphora ooalis $K t z$.

Asteromphalus Hookerii $\mathrm{Eh}$.

Aulacodiscus argus A. $S$.

Aullacus colatus Bail. var. - pruinosus Bail.

Biddulphia aurita Bréb.

- pulchella Graz.

- rhombus W.Sm.

Breblssonia Boeckii Grun.

Campyloriscus echeneis Eh.

Cerataulus turgidus Eh.
Cerataulus lævis Ralfs.

Cocconeis scutellum Eh.

Coscinodiscus excentricus Eh.

$$
\begin{array}{ll}
\text { - } & \text { gigas Eh. } \\
\text { - } & \text { oculus-iridis Elı. } \\
\text { - } & \text { radiatus Eh. }
\end{array}
$$

Cyclotella striata Grun.

Cymbella gastroides $\mathrm{Ktz}$.

- maculata Kirsch.

Diploneis elliptica $C l$.

- interrupla Cl.

- littoralis CI.

- Smithii Cl. 
Eunotia arcus Eh.

- bidens Eh.

- formica Eh.

Hyalodiscus radiatus Grun.

$$
\text { - stelliger Bail. }
$$

Lithodesmium undulatum Eh.

Melosira Borreri Orev.

- sulcata var. coronata.

- - var. radiata.

Navicula Braziliensis Grun.

- dactylus Ktz.

- divergens W. Sm.

- formosa Greg.

- lyra Eh.

- - var. elliptica.

- major Ktz.

- pachyptera Eh.

- Yarrensis var.? A. S. atlas $46 / 17,18$.

Nitzschia acuminata Grun.

- circumsuta Grun.

- scalaris W. Sm.

- $\quad$ sigma W. Sm.
Nitzschia sigma var, intercedens.

- - var. aigmatella.

- tryblionella Hantz.

- . - var. maxima.

Pleurosigma Terryanum $H$. P.

Podocystis americana Bail.

Pyxilla baltica Grun.

Raphoneis amphiceros Eh.

Rhablonema adriaticum IV. Sm.

Rhopalodia gibba $\mathrm{O}$. M.

Stauroneis Gregoryi Ralfs. phcenicenteron Eh. var.

Surirella Febigerii Lewis.

- incuroata A.S.

- recedens $\mathrm{A}$. $\mathrm{S}$.

- striatula Temp.

Synedra affinis var. tabulata.

Tabellaria fenestrata Kiz.

Triceratium favus Eh.

Tropidoneis lepidoptera $\mathrm{Cl}$. vitrea $\mathrm{Cl}$.

\section{N*733 BESANCOON’S LAKE - PICTON C` (Nova Scotia)}

Cymbella amphicephala Noeg.

Encyonema gracile Rab

- ventricosum Grun.

Eunotia arcus Eh.

- bidentula W. Sm.

- gracilis WV. Sin.

- incisa Greg.

- - var. oblusiuscula.

- minor V. H.

- monodon Eh.

- pectinalis Rab.

- - var. undulata.

- - var. ventricosa.

- robusta var. hexaodon.

- - var. tetraodon.

- - polyodon Eh.

Fragilaria virescens Ralfs.

- undata W. Sm
Melosira crenulata Ktz.

- tenuis $\mathrm{Ktz}$.

Navicula affinis Eh.

- - var. amphirhynchus Eh.

- amphigomphus Eh.

- bisulcata Lag.

- biceps v. stauroneiformis.

- Bogotensis Grun.

- Brebissonii Ktz.

- decurrens Ktz.

- firma Ktz.

- gihba Kítz.

- gigas Ktz.

- hemiptera $\mathrm{Ktz}$.

- iridis Eh. .

- - var. ampliata."

- lineolata Eh.

- macilenta Grun. 
Navicula mesolepta $\nabla$. stauroneiformis.

- major Ktz.

- nobilis Ktz.

- rupestris Grun.

- serians Eb.

- tabellaria Ktz.

- transversa A. S.

- viridis Eh.

Pleurostauron sagitta Grun.

Pseudo-Eunotia hemicyclus Grun.

stauroneis anceps $\mathrm{Eh}$.

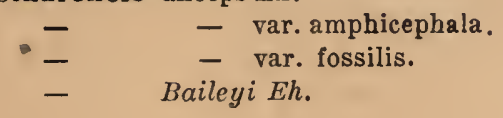

Stauroneis gracilis Eh.

- Phonicenteron Eh.

Stenopterobia anceps Bréb.

Surirella bifrons Eh.

- linearis W.Sm.

- - var. constricta.

- robusta Eh.

- saxonica Auers.

- splendida Eh.

- tenera Greg.

Tabellaria fenestrata $K t z$.

- flocculosa Ktz.

Van Ileurckia rhomboides Bréb.

\section{No 734, 735 LA GARDE - CANTAL (France)}

\section{Dépôt fossile d'eau douce}

BIBLIOGRApHiE. - Frère Héribaud. Les diatoméeś d'Auvergne, $3^{e}$ mémoire 1908.

Cocconeis lineata Grun.

$$
\begin{array}{lll}
- & - & \text { var. euglypta Grun. } \\
\text { - } & \text { - var. rotunda. }
\end{array}
$$

Coscinodiscus pygmæus v. micropunctata. Cymatopleura solea Bréb. $\nabla$. apiculata.

Cymbella cantalense sp.nov. M.P. et F. H.

- cymbiformis Eh.

- Foucaudi F. H.

- gastroïdes Ktz.

- lævis Næg.

- lanceolata Eh.

- Laubyi F. H.

- leptoceros $\mathrm{K} t z$.

- parva W. Sm.

- pseudo turgidula n. sp. M. P. et F. $\mathrm{H}$.

Epithemia cistula (Eh.) Pant.

$$
\text { - - v. lunaris Pant. }
$$

Eucyonema cospitosum Rab.

Eunotia pectinata Rab.

- - v. minor Rab.

- pendens sp. n. M. P. et F. H.
Fragilaria brevistriata $\nabla$. Mormorum Grun. - construens Grun.

- - - var. pumila.

- - var. venter.

$$
\text { - Zeilleri F. H. }
$$

Gomphonema acuminatum Eh.

$$
\begin{array}{cc}
- & -\quad \text { var. coronata Eh. } \\
- & \text { var. intermedia } \\
\text { Grun. }
\end{array}
$$

Melosira Mauryana M.P. et F. H. sp. n.

- . undulata $\mathrm{v}$. debilis.

- - - v. producta D, S.

Navicula amphibola var. stauroneiformis M. P. et F. H.

- acrosphæria v. badeana. 
Navicula Braunii v. moissacensis.

- Corbieri F.H.

- gastrum Donk.

- major Ktz.

- $\quad$ - v. convergentissima.

- peregrina $\nabla$. fossilis.

- placentula Eh.

- pseudo-bacillum Grun.

- pupula v. major F. H.

- radiosa v. acuta Grun.

- viridula $\mathrm{Ktz}$.
Opephora cantalense var. obtusa.

- Glangeaudi sp. n. M.P. et F.H. Martyi F. H.

Stauroneis gracilis W. Sm.

- phœnicenteron Eh.

Synedra capitata Eh.

- delicatissima W. Sm.

- ulna Eh.

Tabellaria fenestrata.

- flocculosa Ktz.

Van Heurckia rhomboïdes Bréb.

\section{$\mathrm{N}^{\circ}$ 736, 737 LUGARI)E - CANTAL (France)}

\section{Dépôt fossile d'eau douce}

BIBLIOGRAPHIE. - Frère Héribaud. Les diatomées d'Auvergne, $3^{\ominus}$ mémoire 1908.

Achnanthes joursacence F. H. Anorthoneis Peragalli F. $\mathrm{H}$.

Cocconeis lineata Grun.

$$
\text { - - var euglypta Grun. }
$$

Cymbella aspera Eh.

- cymbiformis Eh.

- gastroïdes Ktz.

- - var. minor.

- lanceolata Eh.

- leptoceros $\mathrm{Ktz}$.

Epithemia cistula (Eh.) Pant.

- $\quad$ - v. lunaris Pant.

- gibba Ktz.

- $\quad$ - v. paralella.

- - $\quad$ - ventricosa.

- gibberula Eh. v. producta.

- Hyndmannii W. Sm. v. perlonga.

- sorex $\mathrm{Ktz}$.

Eunotia gracilis Eh.

- paralella Eh.

- pectinalis $\mathrm{Kt} \mathrm{z}$.

- - v. minor.

- prærupta Eh.

Fragllaria Harrisonii v. major.

- nitida F. H.
Fragilarla nitida $\nabla$. curta.

Gomphonema commutatum Grun. intricatum Kitz.

Melosira granulata Eh. . australiensis V. H.

- undulata $\mathrm{Ktz}$.

- - - v. debilis.

- $\quad$ - $\quad$. producta.

- Rieufii F. H.

- varennarum.

Navicula amphibola Gl. v. stauroneiformis.

- Berriati F. H.

- - var. minor.

- $\quad$ - $\quad$. splendida F. H.

- borealis $\mathrm{Ktz}$.

- Costei F. H.

- divergens W. Sm.

- gastrum Donk.

- limosa $\mathrm{Ktz}$.

- major Ktz.

- - var. convergentissima.

- nobilis Eh.

- ventricosa Donk.

Opephora cantalense $\nabla$. obesa.

staurosira Grunowii Pant. 
Staurosira (irunowii var. biangulata.

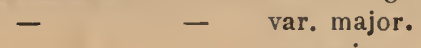

Staurosira Grunowii var. triangulata. 'Synedra ulna Eh. .

Tetracyclus glans var. paucicostata.

\section{Nos 738, 739 ALLANCHE - CANTAL (France)}

Amphora Borneti F. H.

Cocconeis lineata $\nabla$. euglypta.

Cyclotella perforata $\mathrm{F}$ : $\mathrm{H}$.

Cymbella cuspidata Ktz. v. minor.

- cymbiformis Eh.

Cymatopleura Martyi F. H.

Encyonema cuespitosum v. Auerswaldii Eh.

Epithemia cistula (Eh.) Pant.

- gibba Ktz.

- $\quad$ - . ventricosa.

- turgida Ktz.

Fragilaria Gustavei F. H.
Fragilaria minutissima Grun.

- mutabilis Grun.

Gomphonema parvum F. H.

Melosira crenulata v. ambigua Grun.

- strangulata M. P. et F. H.

- tenuissima Grun.

- undulata Ktz. v. producta.

Navicula Berriati v. minor.

- pseudo-bacillum Grun.

- radiosa Ktz.

- - triangulifera F. H.

Opephora cantalense F. H.

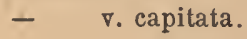

\section{$\mathrm{N}^{\text {os }} 740,741$ ARAULES - HAUTE-LOIRE (France)}

Achnanthes lanceolata Bréb.

Amphora araulensis M. P. et F. H.

- protracta Pant. $\nabla$. gallica M. P. et F. H.

Cymbella cymbiformis Eh.

- leptoceros Ktz.

- miocenica M. P. et F. H.

Encyonema ventricosum Ktz.

Eunotia gracilis Rab.

- lunaris Grun.

Fragilaria araulensis M. P. et F. H.

- brevistriata Grun.

- construens Grun.
Fragilaria elliptica Schum.

- endocystifera M. P. et F. H.

Gomphonema Gallandi M. P. et F. $\mathrm{H}$ :

- insigna Greg.

- intricatum $\nabla$. dichotoma.

Melosira crenulata Ktz. v. armata.

- distans $\nabla$. scalaris.

- undulata $\mathrm{Ktz}$.

Navicula aquitaniæ $F$. $H$.

- bohemica Eh.

- Dubravicensis Pant. $\nabla$. gallica.

- rupestris $\mathrm{Ktz}$.

- sculpta Eh.

vitzschla sigmoidea W. Sm. 
LE HAVRE (France)

Licmophora Lynebyei kitz.

No 743

LE HAVRE (France)

Sur les algues

\begin{abstract}
Cocconeis scutellum Eh. v. stauroneiformis.

Grammatophora marina Eh.

- serpentina Eh.

Rhablonema arcuatum $K t$. .

Rhabdonema minutum Ktz.

Schizonema Grevillei Ag.

Synedra Gaillonii Eh.

Triceratium antediluoianum Eh.
\end{abstract}

No 744

ENGHIEN (France)

Navicula amphisboena Rovy

\title{
Dépôts fossiles marins du Dapon
}

Jusqu'en 1888, il n'avait jamais été question de matériaux fossiles marins japonais, et c'est tout à fait par hasard que le premier dépôt de ce genre fut trouvé sóus la forme d'un calcaire bitumineux rapporté par M. l'abbé Faurie et remis par lui au Laboratoire de Botanique de $\mathbf{M}$. Bureau au Museum d'Histoire Naturelle qui, n'y découvrant rien qui puisse l'intéresser ou lui être utile, le fit porter au Laboratoire de Paléontologie du même établissement où $M$. Fischer y reconnut la présence de nombreux reste de poissons, malheureusement indéterminables, de quelques Gastéropodes et Pélécypodes. 
Ce calcaire, originaire de Sendaï (Nord du Japon) se présentait sous deux formes assez différentes; la plus remarquable avait l'aspect de cailloux roulés, sorte de galets de grosseurs variables d'uu gris brunâtre, très durs, à cassure fine et presque cristalline; l'autre, était représentée par des débris de couleur ochracée, très irréguliers et paraissant provenir de masses perforées plus fragiles.

Un de nos amis, M. Ch. Schlumberger qui travaillait au laboratoire de M. Fischer et à qui M. Gaudry àvait remis quelques échantillons de ce calcaire y remarqua, en en examinant la cassure à la loupe, des points brillants qui, détachés et placés sous le microscope, se trouvèrent être de beaux Arachnoidiscus.

Il parait certain que sans celte observation fortuite ces matériaux, n'offrant aucun autre intérêt, eussent été rejetés pour toujours.

Dans la journée, M. Schlumberger m'apporta un morceau de ce dépôt en me faisant part de sa découverte et le soir même, après un traitement à l'acide, nous nous trouvâmes en présence d'une florule toute nouvelle et d'un grand intérêt. Je priai alors M. Schlumberger de me faire envoyer tout ce qu'il pourrait réunir de ce superbe dépôt qui fit l'objet d'une étude toute spéciale qui parut en avril 1889.

La caractéristique du dépót de Sendaĩ, à part les nombreuses espèces nouvelles qu'il renferme, est la dimension considérable, pour des Diatomées, de certaines formes (Coscinodiscus, Arachnoidiscus, Aulacodiscus, Brunia) qui atteignent jusqu'à 1 mill. de diamètre, et, chose assez curieuse, c'est surtout en traitant les débris dont j'ai fait'mention que ces formes, et des plus belles, furent trouvées.

Je crois que malgré toutes les recherches faites depuis, il a été jusqu'ici impossible de retrouver ce dépôt, elles en ont toutefois fait découvrir d'autres dans l'île de Yeso, à Tukuro, Abashiri, Satanaigori, Hakodate et Wembets qui ont été étudiés par le docteur J. Pantocsek dans la $3^{\mathrm{me}}$ partie de ses Diatomées de Hongrie. Ces dépôts, dans lesquels on retrouve un certain nombre d'espèces de Sendaï, ne renferment pas de grands exemplaires. On trouvera la description et les figures des espèces nouvelles de ces dépôts dans :

Tempère et Brun, Diatomées du Japon, Genève 1889; J. Brun, Espèces Nouvelles. Le Diatomiste, vol. II. - Doct. J.Pantocsek, Beitrage zur Kennstniss fossilen Bacillarien Hungarns. Part. III. (Pozsony 1905 ) et de très beaux dessins dans l'atlas des Diatomées de Ad. Schmidt. 


\section{Principaux dépòts fossiles marins du Japon}

\begin{tabular}{ll|ll}
$745-747$ & HAKODATE YESO. & SENDAI, publié sous les Nos 22-25. \\
$748-749$ & SE'TANAIGORI YESO. & TUKURO, & - \\
750 & WEMBETS YESO & ABASHIRI, & - \\
\end{tabular}

\section{Liste COMPLÈTE DES ESPÈCES}

Achnanthes Leudugeri Temp. et $\mathrm{Br}$.

Actinocyclus calyx Temp. et $B r$.
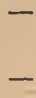

$-$

$-$ ellipticus Gr. v. Sendaïana Temp. et $\mathrm{Br}$. elongatus Grun. flos $J . B r$. Ralfsii o. australiensis V. $H$.

rotula $\mathrm{J} . \mathrm{Br}$.

Actinodiscus Barbadensis Greo.

Actiniscis pennatus Grun.

Actinoptychus adamans Temp. et $\mathrm{Br}$. anemone $J . B r$. asiaticus Temp. et Br. aster $J . B r$. baccatus J. Br.

\section{Barboi J. Br.} biformis $\mathrm{J} . \mathrm{Br}$. clavatus $\mathrm{J}$. Br. decorans v. japonica A.S. erinaceus Temp. et $\mathrm{Br}$. glabratus Grun.

- var. incisa.

- var. subangulata. interpositus $\mathrm{J}$. Br. lucidus $\mathrm{J}$. Br. marmoreus J. Br. nitidus Greo. papilio J. Br. pericavatus $J . B r$. perisetosus $\mathrm{J} . \mathrm{Br}$. summissus A. S. Temperei A. S.
Actinoptychus trifolium Temp. et $\mathrm{Br}$.

$\begin{array}{cc}- & \text { trifurcatus Temp. et Br. } \\ - & \text { trivoloa J. Br. } \\ - & \text { turgidus Temp. et Br. } \\ - & \text { undulatus Eh. } \\ - & -\quad \text { var. A. S. atlas } \\ - & \text { 155/3. } \\ - & \text { var. A. S. Nord } \\ - & \text { Diat. 3/29. } \\ - & \text { velatinum A. S. Montereyi. }\end{array}$

Alloioneis antillarum Cl. et Grun.

Amphiprora coarctata $J . B r$. - fragilis Temp. et $B r$.

Amphitetras Groffeana Wit.

Amphora arcus Greg.

- crassa Gireg.

- exisa Greg.

- fallax Temp. et Br.

- labuensis Cl.

- lanceolata var. incurvata J. Br.

- Petiti Temp. et Br.

- Pleurosigma Temp. et Br.

- proteus Greg.

- Sandaïana J. Br.

- Solsoigensis P. P.

- spectabilis Greg.

T - oar.A.S.atlas 40/20. 23.

- sebrata Temp. et Br.

Anaulus latecavatus $J$. Br.

Aruchnoidiscus Ehrenbergii Bail.

- var. californica. 
Arachnoidiscus indicus $E h$.

Asterolampra decora Greo. oar. japonica J. Br.

stellaris Temp. et $\mathrm{Br}$.

Asteromphalus Brockei Wall.

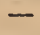
senectus 'T'emp. et $\mathrm{Bl}$. Wallischianus Greo.

Aulnoodiscus adonis Temp. et $B r$.

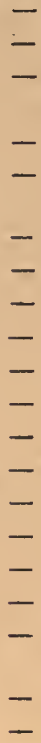
amcenus Greo. angulatus Greo.

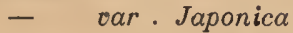

Temp. et $\mathrm{Br}$.

asiaticus J. Br. - var. vallonea J. Br.

Brunii $A$. S. cornutus $J . B r$. crater J. Br. giganteus Temp. et $\mathrm{Br}$. Jimboi Pant. multispadix Temp. et Br nigricans Temp. et $\mathrm{Br}$.

- var. spinifera. pallidus Greo.

Temperii A. S. tripartitus Temp. et Br. tubulo-crenatus Temp. et $B r^{*}$.

tumulifer $J . B r$. ooluta-coeli J. Br.

Auliscus ambiguus $J . B r$.

- asiaticus $\mathrm{J} \mathrm{Br}$.

- crystallinus $J . B r$.

- curoatoradiosus J. Br.

- Grunowi A.S. o flammula

Temp. et $B r$.

- gigas Eh.

- Haradce Pant.

- Jimboi Pant.

- luminosum J. Br.

- pressus Leud. Fort.

- Stöckardtii Jan.

- tricorona J. Br.

- trigemmis J. Br.

- trilunaris J. Br.

Auricula complexa Greg.

- Japonica J. Br.

- ostrea Temp. et Br.
Biddulphia aurita Bréb.

- birostrum J. Br.

- calamus Temp. et Br.

- capucina A. S.

- cornu-caprae J. Br.

- decumana A.S.

- Edwardsii Feb.

- indica Roper.

- Japonica Pant.

- nobilis J. Br.

- obtusa Ralfs.

- - oar. spinosa Temp. et $B r$.

- polyacanthos $J . B r$.

- primordialis J. Br.

- - var. inermis.

- pustulata J.Br.

- reticulata Roper.

- rhombus W. Sm.

- rigida $\mathrm{A} . \mathrm{S}$.

- Roperiana freo.

- tubulosa J. Br.

- Tuomeyi Bail.

- vitrea J. Br.

Brunia mirabilis Temp.

Campylodiscus altar $J . B r$.

$\begin{array}{ll}\text { - } & \text { angularis ireg. } \\ \text { - } & \text { canalisatus Temp.et Br. } \\ \text { - } & \text { chrysanthemum J. Br. } \\ \text { - } & \text { clioosus J. Br. } \\ \text { - } & \text { hypodesianus A. S. } \\ \text { - } & \text { Jimboi Pant. } \\ \text { - } & \text { notatus oar. oitiensis } \\ \text { - Grun. } \\ \text { - } & \text { Ralfsii W. Sm. } \\ \text { - } & \text { rioulosus Temp. et Br. } \\ \text { - } & \text { scalaris Temp. et Br. } \\ \text { - } & \text { simplex Temp. et Br. } \\ & \text { toeniatus oar. radiosa } \\ \text { - } & \text { Temp. et Br. }\end{array}$

Cerataulus Japonicus Pant.

- turgidus Eh.

Cestodiscus Japonicus Cl:

- Johnsonianus Grev.

- pulchellus Grev.

- - oar. gemmifera Cast. 
Chatoceros clavigerum Grun.

$\begin{array}{ll}\text { - } & \text { didymum Eh. } \\ \text { - } & \text { distans Cl. } 0 . \text { subsecunda. } \\ \text { - } & \text { incuroum Bail. } \\ \text { - } & \text { Javanicum Cl. } \\ \text { - } & \text { plicatorugosum J. Br. } \\ \text { - } & \text { pliocenum J. Br. } \\ \text { - } \quad \text { Ralfsii Cl. } \\ \text { - } \quad \text { sigmocalamus Temp. et Br. }\end{array}$

Cladogranma conicum Greo.

Clavicula arenosa $J . \mathrm{Br}$.

- delicatav. radiata Temp.et Br.

- filiformis J. Br.

- Jimboi Pant.

- robusta J. Br.

Cocconeis antiqua Temp. et $\mathrm{Br}$.

- costata Greg.

- curvirotunda Temp. et Br.

- dirupta Grev.

- formosa J. Br.

- Harada Pant.

- Japonica Pant.

- Jimboi Pant.

- Kinkerii Pant.

- nitida Greo.

- notabibis Pant.

- oculus-catis J. Br.

- pellucida Grun.

- scutellum Eh.

- - - v. ornata.

- sigma Pant.

- - o. sparcipunctata Temp. et Br.

- sigmo-radians Temp. et Br.

- splendida Greg.

$\begin{array}{ll}- & -\quad \text { o. crucifera } \\ \text { - } & \quad \text { et Br. } \\ - & \text { o. lurida. } \\ - & \text { sparsipunctata J. Br. } \\ \text { - } & \text { verrucosa J. Br. } \\ \text { - } & \text { versicolor J. Br. } \\ \text { - } & \text { vitrea J. Br. }\end{array}$

Corethron cometa J. Br.

Coscinodiscus asonumce Pant.

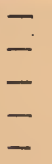
asteroides $\mathrm{Tr}$. et $\mathrm{W}$. asteromphalus Eh. borealis Bail. crasisus Bail.

- cum placenta J. Br.
Coscinodiscus elegans Greo. entoleion Grun. Euryoma A. S. excentricus Eh.

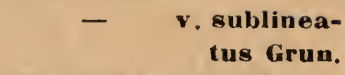

fimbrio-limbatus Eh.

flexuosus J. Br. fulguralis $\mathrm{J}, \mathrm{Br}$. gigas Eh.

- var. stellifera

Temp. et $\mathrm{Br}$.

herculus J. Br.

heteroporus Eh.

var. Moronensis.

intersectus J. Br. Japonicus Pant.

Jimboi Pant.

lineatus Eh.

marginatus Jan.

megaloma A. S.

obscurus A. S.

- v. borealis.

oculus-iridis Eh.

Peragalloi Pant. .

præclarus A. S.

radiatus Eh.

robustus Grun.

v. amoena

Temp. et $\mathbf{B r}$.

stellaris Rop.

subconcavus Grun, fa major.

- $\quad$ subtilis Eh.

- symmetricus Greo.

- tabulatus Temp. et Br.

- Temperei J. Br.

- tubiformis Temp. et $\mathrm{Br}$.

- tumulus J. Br.

- vesiculosus $\mathrm{J} . \mathrm{Br}$.

- vigilans A. S.

- Woodwardii Eul.

Cotyledon circularis $J . B r$.

- toronalis J. Br.

Craspedoporus corolla J. Br.

$$
\text { - Pantocsekii J. Br. }
$$

Cyclotella asiatica J. Br.

- Castracanei J. Br.

Cymatosira Debyi Temp. et Br. 
Cymatosira Japonica Temp. et $\mathrm{Br}$.

Cymbella gastroides Ktz.

- marina Pant.

- yarrense A.S.

Dicladia capreolus Eh

- Japonica Pant.

Dictyoneis marginata $C l$.

- - o. Janischii Cl. - rugosa Temp. et Br.

Ditylium segmentale $J . B r$.

Entogonia conspicua Greo. o. trigemma

$$
\text { J. Br. }
$$

Epithemia argentina $J . B r$.

Eunotia formica $E$.

- monodon Eh.

Euodia gigontea.

- inornata Cast.

- $\quad$ - v. curvirotunda Temp. ot $\mathrm{Br}$.

- margaritacea J. Br.

- ventricosa Cast.

Eupodiscus scaber 0 . heliodiscus J. Br. Ethmodiscus oitrifacies Temp. et $\mathrm{Kr}$.

Fenestrella gloriosa $J$. $B r$.

Fragilaria pliocena J. $\mathrm{Br}$.

$$
\text { - - o acutiforme J. Br. }
$$

Gephyria gigantea líreo.

$$
\text { - - o. minor Greo. }
$$

Gomphonenia curvirostrum Temp. el $\mathrm{Br}$.

Goniothecium tenue J. $B r$.

Gramuatophora arcus $J . B r$.

$\begin{array}{cc}\text { - } & \text { flexuosa o. japonica } \\ \text { Temp. et Br. } \\ \text { - } & \text { Japonica Pant. } \\ \text { - } & \text { lyrata Grun. v. Japonica. } \\ \text { - } & \text { monilifera Temp.et Br. } \\ \text { - } & \text { o. linearis } \\ & \text { J. Br. } \\ \text { - } & \text { Moronensis Greo. O.Ja- } \\ & \text { robusta Dippel. } \\ \text { - } & \text { striata Eh. } \\ \text { - } & \text { tabellaris J. Br. }\end{array}$

nydrosera triquetra Wall.

$$
\text { - } \quad \text { o. tetragona Temp. }
$$

Hemiaulus applanatus $J$. Br.

$$
\text { - simplex J. Br. }
$$

Iemiaulus verrucosus $J . B r$.

Isthmia enerois $E h$.

- nereosa Ktz.

I.endugeria Janischii Temp.

Liostephania Japonica J. Br.

Liradiscus lucidus J. Br.

Lithodesuium californicum Grun.

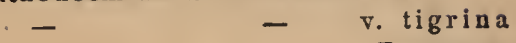

Temp.et $\mathrm{Br}$.

Mastogloia albifrons $J . B r$.

$$
\begin{array}{ll}
\text { - } & \text { angulata Lewis. } \\
\text { - } & \text { Cleoci J. Br. } \\
\text { - } & \text { ooata Grun. } \\
\text { - } & \text { sinuata A. S. } \\
\text { - } & \text { reticulata Grun. } \\
\text { - } & \text { Temperei Cl. }
\end{array}
$$

Melosira arcuata Pant.

$$
\begin{aligned}
& \text { - clypeus J. Br. } \\
& \text { - cornuta Temp. et Br. } \\
& \text { - mediterranea Grun. } \\
& \text { - mirabilis J. Br. } \\
& \text { - } \quad \text { sol Eh. } \\
& \text { - } \\
& \text { sulcata v. lurida J. Br. }
\end{aligned}
$$

Navicula adonis $J . B r$.

- - o. gibbosa J Br.

- anthracis Cl. et Br.

- arabica Grun.

- baccata J. Br.

- basilica J. Br.

- bomboides Eh. o. media Cl. et Grun.

- bombus Ktz.

- Californica o. Campechiana.

- circumnodosa J. Br.

- compressicauda A. S.

- crabro o. Japonica A.S.

- $\quad$ - fa A. S atlas.49/1.

- crucifix Temp. et Br.

- cubitus Temp. et Br.

- debilis Pant.

- distans W. Sm.

- excavata o. angelorum Cl.

- - Greo. o. A. S. atlas $3 / 23$.

- foliala Temp. et Br.

- fusca Greg.

- Grunowii Rab.

- Guinardiana J.Br.

- Harada Pant.

- Hennedyi o. manca. 
Navicula index Temp. et $\mathrm{Br}$.

- Johnsoniana Greo.

- libellus Greg.

- maxima Greg.

- - o. asiatica Temp. et $\mathrm{Br}$.

- mikado Pant.

- monodon J. Br.

- oscitans A.S.

- polygona J. Br.

- polysticta A.S.

- prætexta Eh. et var.

- reticulo-radiata Temp. et Br.

- Reusii Pant.

- scalarifer J. Br.

- scintillans Temp. et Br.

- Sieboldii Pant.

- simiceoultus J. Br.

- Smithii Bréb.

- spathulata J. Br.

- spectabilis Greo.

- splendida Greg.

- subcincta A. S.

- subtilis Greg.

- superimposita A.S.

- Temperei J. Br.

- vitriscala J. Br.

- sp. A. S. atlas 48/12, 13.

Nitzschia angularis $W . S m$.

- asiatica Temp. et Br.

- longissima 0. fossilis J. Br.

- pennata Temp. et Br.

- protuberans J. Br.

- vitrea Norm.

Paralia Hokkaideana Pant.

Plagiogramma fenestra $J . B r$.

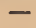

Gregorianum o. robusta J. Br.

- polygibbum A.S.

Pleurosigma candidum Norm.

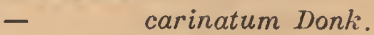

- Clevei Grun. o. fossilis.

- decorum W. Sm.

- dircctum Grun.

- hamuliferum $J . B r{ }^{\prime}$

- hungaricum Cl. et Br.

- lanceolatum Donk.

- Peragalli J.Br.

- sagitta Temp. et Br.

Podosira maculata W. Sm.

- maxima Er.

- spino-radiata $J . B r$.

Porodiscus calyciflos Temp. et $\mathrm{Br}$.

Pcerotheca spada Temp. et Br.

Pyxilla americana $\mathrm{Cl}$.

- BarboiJ.Br.

Raphoneis asiatica $\mathrm{J}$. Br.

- lumen J. Br.

- pinnularia Temp. et Br.

Rhabdonema biquadratum $J$. Br.

$$
\begin{array}{cc}
- & \text { elegans Temp. et Br. } \\
- & \text { formosum. } \mathrm{Br} . \\
- & \text { Japonicum Temp. et Br. } \\
- & \text { var. recta Temp. } \\
\text { - } & \text { et Br. } \\
& \text { var. sparsicos } \\
\text { tata Temp. et } & \text { Br. } \\
\text { - } & \text { mikado Pant. }
\end{array}
$$

Rhizosolemia inermis Cast.

- robusta Norm.

- styliformis Bright.

Rutilaria capitata Temp. et Br.

- hexagona v. cornuta Temp. et $\mathrm{Br}$.

- Kernerii Pant.

- longicornis Temp. et Br.

Septroneis coluber $J . B r$.

Schizonenı Japonicum $J$. $B r$.

Skeletonema Mediterranea 0 . punctife$\mathrm{raJ} . \mathrm{Br}$.

- utriculosa J. B.

Staurosigma asiaticum Temp. et $B r$.

Siauroptera oblonga Bail.

Stephanodiscus elegans $J . B r$.

-

$-$

Stephanopyxis appendiculata o. interaristata Temp. et Br. Brunii A. S.. media.

- corona Eh.

- $\quad$ - var. A. S. atlas 123/10, 17.

:- $\quad$ Grunowii Gr. et St.

- limbata v. cristagalli Temp. et $\mathrm{Br}$. 
Stephanopyxis nidulus Temp. et $\mathrm{Br}$.

- Peragalli Temp. et Br.

- $\quad$ turris v. aretica Grun.

- Weissflogii A. S.

Stictodlscus Hardmannianus Greo,

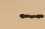

$$
\text { o. Japo- }
$$

Kittonianus o. Greo.

Surirella fastuosa $E h$.

Synedra aftinis $\nabla$. hybrida Grun.

- cristallina o. bacillaris Grun.

- nitzschioïdes Grun.

- parva $f^{\alpha}$ major Gl. et Grun.

- pulchella Kti.

- tibialis Temp. et Br.

- Van Heurckii J. Br.

Tabulina testudo J. $\mathrm{Br}$.

Terpsinoë americana Ralfs. v. trigona.

- Brunii Pant.

- inflata J. Br.

Thalasiothrix Frauenfeldii $\nabla$. javanica Grun.

longissima $\mathrm{Cl}$.

Tracheneis aspera v. intermedia Grun.

Triceratium albifrons $\mathrm{J} . \mathrm{Br}$.

\section{- $\quad$ arcticum Bright.}

_ _ _ v.lucida Temp.et Br.

- v. vulcanica Temp. et $\mathrm{Br}$.

balaniferum Temp. et $\mathrm{Br}$.

- $\quad$ Couturianum Temp. et Br.

- coerulescens Pant.

- cellulosum o. Japonica

J. $\mathrm{Br}$.

- $\quad$ Constellatum Temp. et Br.

- cultum A. S.

- curoilimbum J. Br.

- cyclamen J. Br.
Triceratium dulce Greo. o. japonica Temp. et Br.

elegans Greo. o. japonica Temp. et $\mathrm{Br}$.

elegans 0 . pusilla.

Forresteri Tomp.

interjectum A. S.

- Jimboi Pant.

- Kinkerianum J. Br.

- luminosum Temp. et Br.

- Montereyi Bright.

- $\quad$ - o. primordialis J. Br.

- multifrons J. Br.

- Peragalli J. B'r.

- $\quad$ - $\quad$ r. subrotunda J.Br.

- plano-concavum J. Br.

- punctatum Bright.

- quadrangulare Grev.

- $\quad$ radians Temp. et Br.

- $\quad$ - v. quadrata J. Br.

- radiatopunctatum o. calcarea Temp. et $\mathrm{Br}$.

- Sendaïense A. S.

$$
\begin{array}{cc}
\text { - } & \text { Sendaiense A. S. } \\
\text { - } & \text { Schlumbergeri Temp.et Br. } \\
\text { - } & \text { simplex J. Br. } \\
\text { - } & \text { Stokesianum o. moravica } \\
& \text { Grun. } \\
\text { - } & \text { trinitas J. Br. } \\
\text { - } & \text { - v. minima J. Br. } \\
\text { - } & \text { tripolaris Temp. et Br. } \\
\text { - } & \text { truncatum J. Br. } \\
\text { - } & \text { venulosum o. japonica } \\
& \text { Temp. et Br. } \\
\text { - } & \text { versicolor J. Br. } \\
\text { - } \quad \text { v. cuneata J. Br. }
\end{array}
$$

Trinacria pileolis $O$. W.

Xanthopyxis umbonatus Grev.

Zygoceros circinus v. trapezol̈dalis Temp. et $\mathrm{Br}$. 
$\mathrm{N}^{*} 751$

CHIMBORAZO (Equateur)

(Collection P. T. Cleve)

Achnanthes inflata Grun.

Amphipleura Lindheimeri Grun.

Diadesmis peregrina W. Sm.

Eunotia pectinalis fa maxima Rab.

Frustulia vulgaris de Toni.

Gomphonema affine Ktz.

turris Eh.
Navicula anglica Rab.

- dicephata Ktz.

- radiosa Ktz.

- pupula Ktz.

vitzschia linearis $\mathrm{Ag}$.

Pinnularia latevittata Cl.

Synedra ulna Eh.
Amphora libyca Eh.

Campylodiscus hibernicus Eh.

$$
\text { - noricus Eh. }
$$

Cymatopleura apiculata W. Sm.

$$
\begin{aligned}
& \text { - } \\
& \text { - }
\end{aligned} \quad \text { - } \quad \text { v. minor. }
$$

Cymbella cuspidata Ktz.

- Ehrenbergii Ktz.$$
\text { - lanceolata Eh. }
$$

- cymbiformis Eh.

- perfecta Pant.

Encyonema paradoxum Ktz.

Epithemia argus $\mathrm{Ktz}$.

- directa Pant.
Epithemia gibba $K t z$.

- perlonga Pant.

- turgida litz.

- zebra Ktz.

Melosira arenaria Moor.

Navicula cuspidata o. craticula.

- hemiptera Eh. oar.

- limosa Eh.

- mijor Ktz.

- oblonga Ktz.

- sphorophora Ktz.

- viridis $\mathrm{Ktz}$.

\section{Pleurosigma liochii Pant.}

Surirella bifrons Kítz.

- splendida Kı.

Synedra ulna Eh. var.

$N^{0} 753$

HAMMAN LIF - TUNIS (Afrique)

Amphora cymbifera Greg.

Navicula salinarum Grun.

Navicula cincta $k$ itz. 


\section{No 754 RIVER SHANNON (Estuaire) (Ecosse)}

Achnanthes longipes $\mathrm{Ag}$.

Actinocyclus Ralfsii W. Sm.

$$
\text { - sparsus Greg. }
$$

Actinoptychus undulatus Eh.

Amphora marina W. Sm.

Auliscus cœlatus Eh.

Campylodiscus Thuretii Bréb.

Cerataulus turgidus Eh.

Coscinodiscus excentricus Eh.

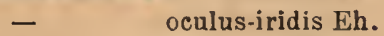

- radiatus Eh.

Epithemia musculus Ktz.

Grammatophora marina $\mathrm{Ktz}$.

- serpentina $\mathrm{Ktz}$

Melosira sulcata $\mathrm{K} t z$.

Navicula abrupta Greg.

- apis Donk.

- aspera Eh.

- brevis Greg.

- cyprinus W. Sm.
Navicula didyma Ktz.

- formosa Greg.

- liber WV. Sm.

- lyra Eh.

- - var. elliptica.

- - var. subtypica.

- humerosa Bréb.

- maxima Greg.

- multicostata Bréb.

- notabilis var. explita Grev.

- polysticta Grev.

- Smithii Bréb.

- Trevelyana Donk.

Pleurosigma balticum Eh.

- formosum W. Sm.

Rhabdonema arcuatum Ktz.

$$
\text { - minutum } \mathrm{K} \mathbf{z} \text {. }
$$

Surirella fastuosa Eh.

Synedra baculus Greg.

- undulata Greg.

Triceratium antediluvianum Eh.
IIantzschia amphioxys $\nabla$. minor Q run.

Navicula Brebissonii v. diminuta Grun.

- cincta var. Heufleri Grun.

- subcapitata Greg.
Navicula viridis var. commutata Grun :

Surirella angusta $\mathrm{Ktz}$.

- miruta Bréb.

- pinnata W. Sm.

$N^{0} 756$

IZSOPALLAGA SERGES (Hongrie)

BIBliographie. - J. Pantocsek. - Beitrage fur Kenntniss der fossilen Bacillarien Ungarns. Part. III.

Actinocyclus disseminatus Pant.

- Loczyi Pant.
Actinoptychus notabilis Pant.

- Semseyi Pant. 
Actinoptychus Staubyi Pant.

- vulgaris Schum.

Amphora acuta o. neogena Pant.

- inoidenda Pant.

- juoenalis Pant.

- Nooa Caledonica Pant.

Aurlcula Szontaghii Pant.

Campylodiscus neogradensis Pant. - Szontaghii Pant.

Coscinodiscus apiculatus Eh.

Dimeregramma Boryanum Pant.

Endyctia Lunyacsekii Pant.

Epithemia gibberula v. producta Grun.

Grammatophora hiharensis Pant.

IIyalodiscus radiatus Bail. subtilis Bail.

Mastogloia Szontaghii Pant.

Melosira sulcata $\mathrm{Ktz}$ v. radiata.

Navicula bacillifera Pant.

- bimaculata Pant.

- Boryana Pant.

- Brazilensis Grun oar.

- didyma Ktz. var.

- difficilis Pant.

- Flattei Pant.

- illustrá Pant.

- includens Pant.
Navicula Kolleri Pant.

$$
\begin{aligned}
& \text { - lyra v. connectens Grun. } \\
& \text { - } \quad \text { - v. elliptica. } \\
& \text { - } \quad \text { - } \text {. hungargaritifora Pant. } \\
& \text { - maxima Eh. var. } \\
& \text { - Orphei Pant. } \\
& \text { - perlonga Pant. } \\
& \text { - peroasta Pant. } \\
& \text { - Smithii Bréb. var. } \\
& \text { - } \quad \text { subfusca Pant. } \\
& \text { - fusca Ralfs. }
\end{aligned}
$$

Nitzschia panduriformis Grun.

Orthoneis splendida Grun.

Podosira hormoides Mont.

Pseudodictyoneis hungarica CI. Maphoneis Kinkeri Pant.

Rhabclonema minutum Ktz.

Stictodiscus Szontaghii Pant.

Surirella fastuosa Eh.

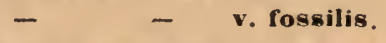

Synedra crystallina vossilis.

- Juranyi Pant.

Terpsinoë polygibba Pant.

- triquetra Pant.

Triceratium spinosum Bright. oastum Pant.

\section{N 757 WEEQUACHICK LAKE - NEW JERSEY \\ (Etats-Unis)}

Amphora ovalis $\mathrm{Ktz}$.

Cocconeis lineata Eh.

Cyclotella striata Grun. v. mesoleia.

Cymatopleura solea Bréb.

Cymbel]a anglica Lag.

- aspera Eh.

- clstula Hemp. var.

- cymbiformis Eh.

- Ehrenbergii Ktz.

- lanceolata Eh.

- muculata Grun.
Cymbella parva w. Sm.

Encyonema gracile Rab.

- turgidum.

- v - var. ventricosa.

Epithemia gibba Ktz.

$$
\begin{aligned}
& \text { - } \quad \text { - var. ventricosa. } \\
& \text { - turgida Ktz. } \\
& \text { - } \quad \text { - var. granulata. } \\
& \text { - } \quad \text { zehra kitz. }
\end{aligned}
$$

Euuotia diodon Eh. 
Fragilaria brevistriata Grun.

- - - var. construens.

- Harrisonii Grun.

Gomphonema acuminatum Eh.

\begin{tabular}{ll} 
- & \multicolumn{1}{c}{ o. pusilla. } \\
- & capitatum Eh. \\
- & intricatum Ktz. \\
- & sphærophorum Eh. \\
- & subtilis Eh. forma augusta. \\
- & turris Eh.
\end{tabular}

Mastogloia exigua $\mathrm{Ktz}$.

Melosira distans $\mathrm{Ktz}$.

Navicula bacilliformis Grun.

- biceps Greg.

- Brebissonii Ktz.

- commutata Grun.

- cuspidata Ktz.
Navicula dicephala Eh.

- limosa Ktz.

- major Ktz.

- oblonga Ktz.

- peregrina $\mathrm{Ktz}$.

- radiosa $\mathrm{Ktz}$.

- Reinhardtii Grun.

- sphcerophora Ktz.

- tuscula Kız.

- viridis $K t z$.

Stauroneis acuta W. Sm.

- gracilis Eh.

- Phœnicenteron Eh.

- phyllodes Eh.

Surirella linearis $W$. Sm.

- - o. constricta.

Synedra capitata Eh.

- ulna Eh.

\section{No 758 DEGERNAS - WESTERBOTTEN (Suède)}

\section{Achnantidium fexellum Bréb.}

Anomoeoneis exilis Grun.

$$
\text { - serians Bréb. }
$$

- Zellensis Grun.

Cymbella aqualis W. Sm.

$$
\begin{array}{ll}
\text { - } & \text { arctica Lag. } \\
\text { - } & \text { Cesati Ral. } \\
\text { - } & \text { cuspidata Ktz. } \\
\text { - } & \text { helvetica Ktz. } \\
\text { subæualis Grun. }
\end{array}
$$

Encyonema norvegica Grun.

Epithemia argus $\mathrm{K} t z$.

$$
\text { - gibba Ktz. }
$$

Eunotia arcus Eh.

$$
\text { - prærupta Eh. }
$$

fiomphonema acuminatum Eh.
Gomphonema constriclum Eh.

$$
\begin{array}{ll}
\text { - } & \text { gracile Eh. } \\
\text { montanum Schum. }
\end{array}
$$

Navicula hebes Ralfs.

- - radiosa $\mathrm{Ktz}$.

$$
\text { - subtilissima Cl. }
$$

Neidium iridis Eh.

Pinnularia biceps Greg.

- borealis Eh.

- commutata Eh.

- gracillima Greg.

- legumen Eh.

- mesogongyla Cl.

- stomatophora Grun.

- viridis Eh.

- undulata Greg.

Tabellaria fenestrata Kiz. 


\section{No 759 PAUTRASK - STENSELE LAPPMARK (Suède)}

Achnanthes fexellum Bréb.

A nomoeoneis serians Bréb, var.

Cymbella aqualis $W$. Sm.

$$
\begin{array}{ll}
\text { - } & \text { arctica Lag. } \\
\text { - } & \text { cesati Rab. } \\
\text { - } & \text { cuspidata } \mathrm{K} t z \\
\text { - } & \text { navictica } \mathrm{Ktz} . \\
\text { - } & \text { subrequalis Grun. }
\end{array}
$$

Encyonema norvegica Grun.

Epithemia argus Ktz.

Eunotia arcus Eh.

Frustulia crassinervia Bréb.

Gomphonema constrictum Eh.

- $\quad$ sagitta Schum.

Navicula hebes Ralfs.

- radiosa $\mathrm{Ktz}$.

Neidium iridis Eh.

Nitzschia angustata Grun.

Pinnularia biceps Greg.

$$
\begin{array}{ll}
\text { - } & \text { gentilis Donk. } \\
\text { - } & \text { gracillima Greg. } \\
\text { - } & \text { microstauron Eh, } \\
\text { - } & \text { stauroptera Grun. } \\
\text { - } & \text { stomatophora Grun. } \\
\text { - } & \text { viridis Eh. }
\end{array}
$$

Stauroneis anceps Bréb.

$$
\text { - gracilis Eh. }
$$

Tabellaria fenestrata $\mathrm{Ktz}$.

\section{No 760 TROUVILLE - CALVADOS (France)}

Amphora cymbifera Greg.

- excisa Greg.

- fasciata Greg.

- lævis Greg.

- obtusa Greg.

- pellucida Greg.

Amphiprora lepidoptera Greg.

$$
\text { - pusilla Greg. }
$$

IIantzschia marina Grun.

Navicula arenaria Donk.

- fortis Greg.
Navicula granulata Bréb.

- humerosa Bréb.

- littoralis Donk.

- minor Greg.

- northumbrica Donk.

- palpebralis Bréb.

- pygmœa Eh.

- retusa Bréb.

- Treveleyana Donk.

Nitzschia salinarum Grun.

- spathulata Bréb.

No 761

CETTE - HÉRAULT (France)

Estomac d'Ascidies

Achnanthes longipes Ag.

Actinoptychus vulgaris Schum.
1 Actinoptychus undulatus Eh.

I Amphiprora lepidoptera Greg. 
Biddulphia pulchella Greg.

$$
\text { - Tuomeyi Bail. }
$$

Campylodiscus decorus Bréb.

$\begin{array}{ll}\text { - } & \text { eximius Grun. } \\ \text { - } & \text { limbatus Bréb. var. } \\ \text { - } & \text { Thuretii Bréb. }\end{array}$

Cocconeis pellucida $E h$.

- scutellum Eh.

Cosclnodiscus excentricus Eh.

Cymatopleura elliptica W. Sm.

Bndictya oceanica Eh.

Epithemia musculus $\mathrm{K} t z$.

Gramniatophora marina o. major.

$-$ serpentina Eh.

Hy alodiscus stelliger Bail.

Melosiru nummuloïdes Ag.
Melosira sulcata $K t z$.

Vavicula aspera Eh.

- crabro Eh.

- Hennedyi IV. Sm.

- lyra Eh.

- maxima Greg.

- Smithii Bréb.

Orthoneis splendida Eh.

Pleurosigma decorum W. Sm.

- rigidum W. Sm.

Rhablonema adriaticum W. Sm.

Surirella fastuosa Eh.

Synedra basulus var. minor Grev.

- robusta Ralfs.

- undulata Greg.

Triceratiun antediluvianum Eh.

- pentacrinus Wall.

Nos 762, 763 ANDREUGEOLET près de SAUGUES HAUTE-LOIRE (France)

BIBLIOGRA PHIE. - Frère J. Héribaud. Diatomées fossiles d'Auvergne, $3^{\ominus}$ mémoire 1908.

Achnanthes lanceolata Bréb.

Amphora enoculata M. Per. ot F. H.

- ovalis Ktz.

- pediculus Grun.

Cocconeis lineata Grun.

- placentula Eh.

Cyclotella comta var. quadrinotata.

- Temperei F. H.

Cymatopleura elliptica $\nabla$. rhomboides.

- solea W. Sm.

Cymbella cistula v. fusidium.

- gallica M. P. et F: $\dot{H}$.

- gaştroides $\mathrm{Ktz}$.

- $\quad$ - v. prolongata M. P.

- helvetica $\mathrm{Ktz}$.

- lanceolata Eh.

- leptoceros Eh.
Cymbella maculata $\mathrm{Ktz}$.

Encyonema Auerswaldii Rab.

- ccespitosum $\mathrm{Ktz}$.

- $\quad$ turgidum Greg.

Epithemia gibba Eh.

$$
\begin{array}{cc}
- & -\quad \text { v. ventricosa. } \\
\text { - } & \text { Hyndmannii W. Sm. } \\
\text { - } & -\quad \text { v. perlonga. } \\
\text { - } & \text { turex Ktz. } \\
\text { - } & \text { - var. granulata. } \\
\text { - } & \text { - var. vertagus. } \\
\text { - } & \text { zebra Ktz. } \\
\text { - } & \text { - var. proboscidea. }
\end{array}
$$

Eunotia pectinalis $\nabla$. stricta.

Fragilaria elliptica Schum.

- parasitica Grun. 
Gomphonema acuminatum Eh.

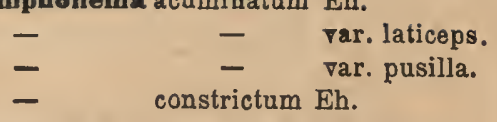

Melosira Mauryana var. granúlosa.

Navicula arenaria Donk.

- borealis Eh.

- cardinalis Ktz.

- Chaberti F. H.

- commutata Grun.

- cuspidata Ktz.

- elliptica Ktz.

- esox Eh. var. recta F. H.

- gentilis Donk.

- halophila Grun.

- - - major.

- Heribaudi M. Per.

- hemiptera Ktz.

- limosa Ktz.

- major $\mathrm{Ktz}$.

- nobilis Eh.
Navicula placentula Eh. v. minor M. P. et $\mathrm{F} . \mathrm{H}$.

- radiosa $\mathrm{Ktz}$.

- $\quad$ - - acuta.

- $\quad$ - . maxima M.P.etF.H.

- Reinhardtii Grun.

- rupestris Ktz.

- transversa A. S.

- ventricosa Donk.

- viridis Ktz.

Nitzechia sigmoidea W. Sm.

Pleurosigma Kủtzingii Grun.

Stauroneis Baileyi Eh.

- gracilis W. Sm.

- phcnicenteron Eh.

- pteroides Eb.

Surirella robusta Eh.

- - var. A. S. atl. 22/4.

- splendida Eh.

Synedra ulna Eh.

Tetracyclus omarginatus W. Sm.

\section{N' 764,765 FONTILLOU - CANTAL (France)}

\section{BIBLIOGRAPHIE. - Frère J. Héribaud. Diatomées fossiles d'Auvergne, $3^{\ominus}$ mémoire 1908.}

Anortboneis Peragalli M. P. et F. H.

Campylodiscus costatus W. Sm.

Cocconeis lineata Grun.

Coscinodiscus pygmæus.

Cymatopleura solea Bréb.

var. micropunctata.

- $\quad$ - var. apiculata.

Cymbella affinis $\mathrm{Ktz}$.

- aspera Eh.

- Brevieri F. H.

- cistula Hemp.

- $\quad$ - var. fusidium.

- conifera F. H.

- cymbiformis Eh.

- lanceolata Eh.
Cymbella parva W. Sm.

- Pauli M. Per.

Bncyonema prostratum Ralfs.

$$
\text { - ventricosum } \mathrm{Ktz} \text {. }
$$

Epithemia gibba Eh.

$$
\begin{aligned}
& \text { - - v. parallela. } \\
& \text { - gibberula v. producta. } \\
& \text { - Hyndmannii W. Sm. } \\
& \text { - } \quad \text { sorex } \overrightarrow{\mathrm{Ktz}} \text {. } \quad \text { verlonga. } \\
& \text { - turgida Ktz. } \\
& \text { - Westermannii Ktz. } \\
& \text { - . zebra Ktz. } \\
& \text { - - } \quad \text { - proboscidea. }
\end{aligned}
$$

Eunotia polydentula Eh.

Fragilaria brevistriata Grun.

- construens Grun. 
Fragilaria elliptica Schum.

- lapponica Grun.

Gomphonema cantalicum F. H.

- commutatum Grun.

intricatum $\mathrm{Ktz}$.

Meloslra varennarum M. P. et F. H.

Navienla acrosphæria var. elongata F. H. et M. P.

- elliptica Ktz.
Navicula major $\mathrm{Ktz}$.

- nobilis Eh.

- radiosa $\mathrm{Ktz}$.

- Reinhardtii Grun.

Surirella Brunhesí M. P. et F. H.

- Norvegica Eh.

- robusta Eh.

- tenera Greg.

stauroneis phœnicenteron Eh.

\section{Sur les algues}

Achnanthe brevipes Ag.

Cocconeis scutellum Eh.

Grummatophora marina Ktz.

Rhabdonema arcuatum Jtz.
Rhoïcosphenia curvata $\nabla$. marina Ktz.

Synedra affinis $\mathrm{Ktz}$.

- Gaillonii Eh.

Triceratium antediluvianum Eh.

$\mathrm{N} \times 767$

WESTERAS (Suède)

Récolte saumâtre

Amphora libyca Eh.

- ovalis $\mathrm{Ktz}$.

- - - fa minor.

Campylodiscus Balticus Cl.

$$
\text { - bicostatus W. Sm. }
$$

Cocconeis pediculus Eh. var.

Cymatopleura apiculata W. Sm.

$$
\text { - elliptica W. Sm. }
$$

Cymbella Ralatonis Grun.

- cistula Kirsh.

- cymbiformis Brèb.

- lanceolata Kirch.

- maculata Ktz.

Diplonels bombus Eh.

- interrupta $\mathrm{Cl}$.
Diploneis papula A. S.

- pelagi A. S.

- Smithii Cl.

Encyonema prostratum Ralis.

Epithemia amphicephala Grun::

- argus $\mathrm{Ktz}$.

- gibba Ktz.

- - $\quad$ v. parallela.

- Hyndmannii W. Sm.

- sorex Ktz.

- turgida Ktz.

- - var. granulata.

- Westermannii Ktz.

Gomphonema intricatum $K t z$.

Melosira arenaria Moore

- undulata var. producta. 
Navicula Baileyana Grun.

- humerosa Bréb.

- major $\mathrm{Ktz}$.

- oblonga Ktz.

- sculpta Eh.

- Schumanniana Grun.

- tuscula car.

Nitzschia sigmoidea W. Sm.

- Tryblionella Hantz.

- - oar. Levidensis.

- _ - var. marina.

Pleurosigma attenuatum W. Sm.
Pleurosigma strigilis W. Sm.

Podosira hormoides $K t$ t.

Rhoicosphenia curoata Grnn.

Scoliopleura tumida Bréb.

- - var. adriatica.

Surirella bifrons fa minor.

- crumena Bréb.

- Kittoni var.

- ovalis Bréb.

- striåtula Turpin.

Thallasiosira Baltica Grun.

\section{No 768 SAINT-LEGER-EN-YVELINES - SEINE-ET-OISE}

\section{(France)}

Eunotia impressa Eh.

- pectinalis Rab.

- $\quad$ - v. undulatus.

- - - ve ventricosa.

Navieula serians Ktz.
Navicula serians var. minor.

Stenopterobla anceps Bréb.

Tabellaria fenestrata ktz.

- flocculosa $\mathrm{Ktz}$.

Van Heurckia rhomboities Brób.

\section{No 769 SAINT-LÉGER-EN-YVELINES — SEINE-ET-OISE}

\section{(France)}

Encyonema gracile Rab.

Eunotia flexuosa var. pachycephala.

- lunaris Grun.

- major Rab.

- pectinalis Rab.

- - v. ventricosa.

- tetraodon Eh.

Navicula gibba Eh.

- gigas Eh.
Navicula major $\mathrm{Ktz}$.

- mesolepta o. stauroneiformis.

- serians Ktz.

- viridis $\mathrm{K} t z$.

Stauroneis phœnicenteron Eh.

Stenopterobia anceps Bréb.

Tabellaria fenestrata $\mathrm{Ktz}$.

- flocculosa $\mathrm{Ktz}$.

Van Hourckia rhomboides Bréb. 


\section{No 770 ETANG DE SAINT-QUENTIN — SEINE-ET-OISE}

\section{(France)}

Achnanthes minutissima rar. cryptocephala. Amphora pediculus Grun.

Cocconeis placentula Eh.

Cyclotella Kützingiana Thw.

Cymatopleura apiculata W. Sm.

Cymbella amphicephala Næeg.

- cistula Kirch.

- gibba Eh.

- lanceolata Kirch.

Fragilaria capucina Desm.

$$
\text { - - } \quad \text { v. mesolepta. }
$$

Gomphonema acuminatum Eh.

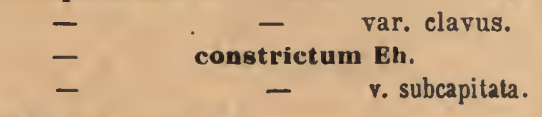

Melosira varians Ag.
Meridion circulare Ag.

Navicula cryptocephala Ktz.

- gracilis $\nabla$. neglecta.

- lancettula Schum.

- limosa Ktz.

- oblonga v. curta.

- radiosa o. acuta.

- Reinhardtii $\nabla$. elliptica.

Surirella pinnata $W$. Sm.

Synedra acus Ktz.

- biceps $\mathrm{Ktz}$.

- capitata Eh.

- radians $\mathrm{Ktz}$.

- ulna Eh.

- - var. æqualis.

- - var. rostrata.

- - var. vitræa.
Actinoptychus undulatus Eh.

Amphiprora alata Ktz.

Amphora proteus Greg.

Biddulphia aurita Bréb.

- pulchella Gray.

- rhombus W. Sm.

Cerataulus Smithii Ralfs.
Coscinodiscus leptopus Grun. Hyalodiscus stelliger Bail.

Navicula cyprinus W. Sm.

Nitzschla acuminata Grun. - sigma W. Sm.

Plourosigma acuminatum Grun. - angulatum IV. Sm. - balticum W. Sm. 
Pleurosigma balticum var. minor.

- $\quad$ quadratum W. Sm.

- - var. minor.

Scoliopleura latestriata Bréb.
Scollopleura tumida Bréb.

- var. adriatica.

Stauroneis salina W. Sm.

Surirella gemma Eh.

\section{No 773 POPE'S CREEK - MARYLAND (U. S. A.)}

Actinocyclus Ehrenbergii Ralts.

- moniliformis Ralfs.

- undulatus CI.

Actinoptychu Bismarkii A. S.

$\begin{array}{ll}- & \text { heliopelta Grun. } \\ \text { - } & \text { intermedius A.S. } \\ \text { - } & \text { nitidus Greo. } \\ \text { - } & \text { stella oar. Thumii. } \\ \text { - } & \text { undalatus Bh. } \\ \text { - } & \text { - valgaris Schum. minor. }\end{array}$

Asterolampra Marylandica Eh.

Aulaoodiscus Rogersii $A$. $S$.

Bidduiphia suborbicularis Ģ̣run.

Tươmeyi Rop.

Cerataulus antediluoianus M.Per.n.sp. Forme du Triceratium papillatum, mais avec deux appendices seulement, les deux autres remplacés par de fortes épines; structure du T. antediluvianum.

-Diam. $62 \mu$.

Cestodiscus proteus $\nabla$ ar.

Coscinodiscus aplculatus Eh. et var.

$\begin{array}{ll}\text { - } & \text { asteromphalus Eh. } \\ \text { - } & \text { Boliviensis Grun. } \\ \text { - } & \text { bulliens A. S. } \\ \text { - } & \text { curoatulus oar. } \\ \text { - } & \text { dubius var. } \\ \text { - } & \text { leptopus o. irregularis. } \\ \text { - } & \text { lineatus Eh. } \\ \text { - } & \text { marginatus Eh. } \\ \text { - } & \text { nodulifer Grun. } \\ \text { - } & \text { oculus-iridis var. } \\ \text { odontophorus Grun. } & \text { symbolophorus Grun. }\end{array}$

Craspedodiscus coscinodiscus Eh.

Cymbeila americana A. S.

- gastroides $\mathrm{Ktz}$.

Diploneis didyma $\mathrm{Eh}$.

- prisca Cl.

- succincta $\mathrm{Gl}$.

Fragilaria Ischaboensis Grun.

Goniothecium odontella Eb.

Hemiaulus bipons Grun.

- petasiformis oar.

- polymorphus Grun.

Hercotheca mamilliaris Eh.

Melosira ornata var. A. S. atlas 178/41.

- sulcata Ktx.

- - var. coronata.

- - var. radiata.

Navicuia excavata Grev.

- heroina A. S.

- lyra o. elliptica.

- Yarrensis Grun.

Pleurosigma Marylandicum Gr.

Plourostanron Javanicum Grun.

Pyxilla americana Grun.

- gracilis Forti.

- Johnsoniana ₹. intermedia.

Raphoneis gemmifer Eh.

- - fa curta.

Rhizosolenia pileolus Eh.

Septroneis caduceus Eh.

stephanogonia actinoptychus Grun.

Stephanopyxis corona Eh.

- ferox Grev.

- Joynsonii A. S.

- Palmoriana Grun. 
Stephanopyxis turris Eh.

Stictodiecue Kittonianus Grev. Trlceratium americanum Ralfs.
Triceratium nobile 0 . W.

- obscurum oar.

- Weissii A. S.

Xanthiopy $\mathbf{x}$ oblonga $\mathbf{E b}$.

\section{N•774 PORT D'OAMARU (Nourelle Zélande)}

Achnanthes brevipes Ag. var.

Actinocyclus Ralfsii Sm.

- subtilis Ralfs.

Biddulphia aurita Bréb.

- Roperiana Grev.

Campylodiscus Thuretii Bréb.

Coscinodiscus lineatus Eh. fa minor.

- marginatus Eb.
Navicula aspera Eh.

- Beyrichiana A. S.

- didyma Ktz.

vitzechia sigma W. Sm. var.

Plourosigma obscurum W. Sm. v. mediterranea.

Synedra capensis Grun.

Triceratium spinosum Bail.

No 775 NASSAU - ILES BAHAMAS (Antilles)

Actinocyclus moniliformis Ralfs.

Actinoptychus splendens var.

$$
\begin{array}{ll}
\text { - } & \text { trilingulatus Bright. } \\
\text { - } & \text { undulatus Cl. } \\
\text { - } & \text { vulgaris Schum. }
\end{array}
$$

Alloioneis antillarum $\mathrm{Cl}$.

$$
\text { - Debyi Leud. Fort. }
$$

Amphora crassa Greg.

- - oar. sloesoicensis.

- Graeffei Grun. oar.

- Grundleri Grea.

Auliscus colatus Bail.

- - var. Aucklandica.

- - var. latecostata.

- reticulatus Grev.

Biddulphia mobiliensis Bail.

- Peruviana Grun. var.

- pulchella Gray.

- reticulata Rop.

- Tuomeyi Rop.
Campylodiscu: ambiguus Grev.

-

$-$

biangulatus Grev.

contiguus A. S.

- Daemelianus o. comminuta.

Graeffei var.

intermedius Grun.

Kittonianus Grev.

Normannianue Grev. ornatus $\nabla$. amphileis.

Samoensis Grun. undulatus (irev. Wallichianus Grov.

Cerataulus Smithii Ralfs.

Cocconeis heteroidea Hantz.

Coscinodiscus concinnus $\nabla$. Jenseniana.

- excentricus Eh.

- leptoptus Grun.

- nitidulus Grun.

- nitidus Greg. 


\section{Coscinodiseus nodulifer Jan.}

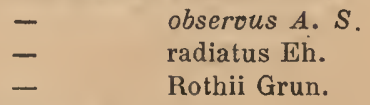

Diploneis crabro Eh.

- demta A. S.

- multicostata A. S.

- Smithii Cil.

- splendida Greg.

Endictya oceanica Eh.

Entopyla australis o. incurvata.

Euodia gibba Bail. var.

Euphyllodium spathulatum Shadb.

Hyalodiscus radiatus Grun.

- stelliger Bail.

- subtilis Bail.

Melosira sulcata $\mathrm{Ktz}$.

Navicula Barbitos A. S.

- bullata Norm.

- - var. Mölleriana.

- clavata Greg.

- formosa Greg.

- liber W. Sm.

- lyra Eh.

- - var. elliptica.

- - var. intermedia,

- maxima var. bicuneata.

- proetexta Eh.

- spectabilis Greg.

Nitzschia Campechiana Grun.

- panduriformis lireg.

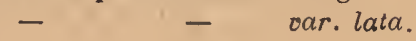

Nitzschia panduriformis var. minor.

- plana var.

Orthoneis Barbadensis o tenuipunctata.

- cribrosa var.

Orthotropis lepidoptera Grun.

Plagiogramma tessellatum Greo.

Rhoicosigma oceanicum v. corsicana.

Surirella fastuosa Eh.

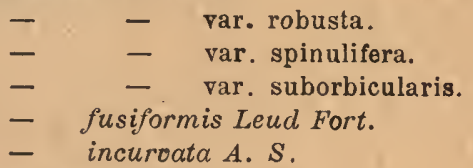

Synedra formosa Ktz.

Trachyneis aspera $\mathrm{Cl}$.

$$
\begin{array}{lll}
- & \text { - var. intermedia. } \\
\text { - } & \text { - var. pulchella. }
\end{array}
$$

Triceratium favus Eh.

$\begin{array}{ll}\text { - } & \text { fimbriatum Wall. } \\ \text { - } & \text { formosim v. pentagonalis. } \\ \text { - } & \text { latum Grev. } \\ \text { - } & \text { parallelum fa trigona. } \\ \text { - } & \text { punctatum fa pentagona. } \\ \text { - } & \text { Robertsianum v. inermis. } \\ \text { - } & \text { sculptum Shadb. } \\ \text { - } & \text { var. A. S. atlas } \\ \text { - } \quad 76 / 10,11 . \\ \text { - } \quad \text { spinosum Bail. } \\ \text { - } \quad \text { zonellarium Bright. } \\ \text { zonulatum Grev. }\end{array}$

\section{N• 776 PHILLIPS - MAINE - CONN. (U. S. A.)}

Cymbella cuspidata Ktz.

- heteropleura $K t_{z}$.

- Laubyi M. Per. et F. H.

Diatoma anceps var. capitata n. v. Etroitement bacillaire, à extrémités coniques et absolument capitées.

Encyonema ventricosum Grun.
Eunotia arcus Eh.

- - var. hybrida.

- bidentula W. Sm.

- gracilis W. Sm.

- impressa Eh.

- - var. angusta.

- lunaris Grun.

- - var. arcuata. 
Eunotia major Rab.

- minor V. H.

- pectinalis Rab.

- - var. ventricosa.

- polyglyphis Eh.

- prarupta Eh.

- - v. bidens.

- $\quad-\quad$ v. inflata.

- robusta var.

- tetraodon Eh.

Fragilaria capucina Desm.

Gomphonema acuminatum o. pusilla.

$\begin{array}{ll}- & \text { angustatum } \mathrm{Ktz} . \\ - & -\quad \mathrm{v} \text {. angusta- } \\ \text { tissima. }\end{array}$

Uantzschia amphyoxis Orun.

$$
\text { - elongata Grun. }
$$

Melosira bellicosa M. Per. et F. H.

- crenulata Ktz.

Meridion constrictum Ralfs.

Navicula acrosphæria Bréb.

- - var. laevis.

- amphigomphus Eh.

- bacilliformis Grun.

- biceps Eh.

- bisulcata Lag.

- Brebissonii Ktz.

- commutata Grun.

- Dariana A.S.

- dicophala Eh.
Navicula dicephala fa intermedia.

- divergens $f^{2}$ minor.

- $\quad$ - var. elliptica.

- dubia Eh.

- elegantissima M. Per.

- firma var. subundulata.

- gibba Ktx.

- gigas Ktz.

- Huei var. lata.

- instabilis A. S.

- iridis Eh.

- lata. var.

- latevittata var. domingensis.

- legumen fa major.

- limosa Ktz.

- major Ktz.

- mesotyla Eh.

- microstauron Eh.

- nodosa $\mathrm{Ktz}$.

- pachyptera Eh.

- rupestris Grun.

- semen Eb.

- serians Ktz.

- stomatophora Ktz.

- termes var. stauroneiformis.

- viridis $\mathrm{Ktz}$.

Nitzschia sigmoidea W. Sm.

Stauroneis acuta W. Sm.

- - var. major.

- amphilepta Eh.

- anceps Eh.

- $\quad$ - var. amphicephala.

- Brunii M. Per. et F. H.

- gracilis Eh.

- lanceolata Ktz.

- phoenicenteron Eh.

Tabellaria fenestrata Ktz.

- flocculosa Ktz.

Van Heurckia rhomboides Breb.

\section{No 777 MONTGOMERY - ALABAMA (U. S. A.)}

\section{Dépot fossile d'eau douce}

Cymbella gastroides Ktz.

Epithonia zobra' Ktz.
Eunotia major Rab.

Melosira Rosseana Rab. 
Navícula Americana Eh.

- acrosphæria Bréb.

- firma Eh.

- gigas Eh.

- iridis Eh.

- major Ktz.

- mutabilis A. S.
Navlcula nobilis Ktz.

- subacuta Eh.

- tabellaria Eh.

- viridis Eh.

- - - commutata.

Stauroneis acuta W. Sm.

- phœnicenteron Eh.

No 778

\section{ABERDEEN (Ecosse)}

Bpithemia Hyndmannil $\mathbf{W} .8 \mathrm{~m}$.

No 779

CÔTES DU JAPON

Sur les algues connues dans le commerce de la droguerie sous le nom d'Agar-Agar, et qui serventà faire les gelées végétales et à falsifier celles de fruits telle que la groseille dans lesquelles on trouve les espèces suivantes :

Arachnoidiscus ornatus Eh.

| Orthoneis splendida Grun.

No $780^{(1)}$

LAGO di LUGANO (Suisse)

Pélagique

Asterionella gracillima Heib. Cyclotella comensis Grun.

- comta var. tenuis Fricke.
Cycloteila melosiroïdes Lemm.

Fragilarla crotonen sis var. media Seh. et Vog.

Gomphonema olivaceum Ktz.

(1) Nous devons les matériaux de 780 à 788 ainsi que leur analyse à l'obligeance de M. Moister, d'Horgen (Suisse). 
Astorlonella gracillima Heil. Cyclotella melosiroides Lemm. Molosira italica Ktz.

No 782
Synedra Schroeteri Moister. - tonera W. Sm.

Fragilarla crotonensis var. curtasch. et Vog. | Tabellarla fenestrata Ktz.

No 783 VIERWALDSTATTERSEE (Suisse)

Pélagique

BIBLIOGRAPHIE. - Fr. Meister. Die Kieselalgen der Schweiz

Aaterionella gracillima Heib. Cyclotella lemanensis Lemm.
Cymatopleura elliptica var.genuina Meister. - $>$ - var. orata Grun. Fragilaria crotoneñsis var. media Sch. et Vog.

No 784 ZURICHSEE près HORGEN (Suisse)

Fragilariacrotonensis var. media Sch. et Vog. - $\quad$ - $\quad$ var. curta Sch. et V.
Tabellaria fonestrata pastorionellolde: Grun. 
BIBLIOGRA PHIE. - Fr. Meister. Die Kieselalgen der Schweiz

Achnanthidium lanceolatum Bréb.

Cocconeis pediculus Eh.

Cymbella affinis Ktz.

- delicatula Ktz.

- prostrata Cl.

- ventricosa var. Auerswaldia Meister.

- $\quad-\quad$ v. lunula Meister.

- - v. ovata Cl.

Diatoma hiemale v. genuina Grun.

- vulgare v. brevis Grun.
Diatoma vulgare $\vee$. genuinum Grun.

- - $\quad$ - v. productum Grun.

- tenue $\mathrm{v}$. normale $\mathrm{K} t z$.

Fragilaria intermedia Grun.

Gomphonema angustatum v. obtusatum Grun.

Meridion circulare $\mathrm{Ktz}$.

Microneis minutissima $\mathrm{Cl}$.

Navicula gracilis Grun.

- minima v. atomoïdes Cl.

Nitzschia dissipata Gruń.

synedra ulna var. equalis $\mathrm{Br}$.
Achnanthidium lanceolatum Bréb.

Cymbelia æqualis W. Sm.

Diatoma tenue $\nabla$. minus Grun.
Melosira varian: Ag.

Merldion circulare Ag.

synedra vitrea $\mathrm{Ktz}$.

$N^{\circ} 787$

URSERENTHAL (Suisse)

BibliographiE. - Fr. Meister. Die Kieselalgen der Schweiz

Ceratoneis arcus v. genuina ffolm.

Cymbella parva W. Sm.

- ventricosa $\nabla$. lunula Meister.

Diatoma hiemale $\nabla$. mesodon Grun.

Diatomella Ralfouriana Greo.
Gomphonema parvulum Grun.

Navicula cryptocephala Ktz.

Synedra radians Ktz.

- vitrea Ktz. 
No 788

RHIN près ERMATINGEN (Suisse)

BIBLIOGRA PHIE. - Fr. Meister. Die Kieselalgen der Schweiz

Cyelotella bodanica Eul.

- comta v. radiosa Grun.

- $\quad$ - $\quad$. tenuis Fricke.

-. Kützingiana Thw.

- stelligera Cl. et Grun.

- inelosiroídes Lemm.

Cymbelle æqualis W. Sm.

- 2 ffinis $\mathrm{Ktz}$.

- cesatii Grun.

- cistula v. typica Meister.

- helvetica $\nabla$. gracilis Meister.

- lanceolata Kirch.

- leptoceros Grun.

- maculata $\mathrm{Ktz}$.

- ventricosa $\mathrm{Ktz}$.

Denticula frigida $\mathrm{Ktz}$.

- tenuis $\mathrm{Ktz}$.
Eucocconeir flexella Cl.

Fragilaria capucina Desm.

Gomphonema angustatum $\mathrm{K} \mathbf{z}$.

$$
\begin{array}{ll}
\text { - } & \text { intricatum Ktz. } \\
\text { - } & \text { subclavatum } \mathrm{Cl} .
\end{array}
$$

Microneis microcephala Cl.

Navicula cryptocephala $\mathrm{Ktz}$.

- radiosa $\mathrm{K} t \mathrm{z}$.

- rhynchocephala Ktz.

- viridula $\mathrm{Ktz}$.

- vulpina Ktz.

Pleurosigma attenuatum W. Sm.

Synedra nana Meister.

- Schröteri Meister.

- splendens Ktz.

N• 789 ROYAN - CHARENTE-INFÉRIEURE (France)

Pélagique

Actinoptychus splendens Shad.

$$
\text { - undulatus Eh. }
$$

Asterionella Japonica Cl.

Bacteriantrum hyalinum Laud.

$$
\text { - varians Laud. }
$$

Biddulphie Baileyi WV. Sm.

$$
\text { - mobiliensis Bail. }
$$

Campylosira cymbelliformis Grun.

Cerataulus Smithii Ralfs.

Chretoceros cochlea Schütt.

- curvisetum Cl.

- deeipiens $\mathrm{Cl}$.

- densum $\mathrm{Cl}$.
Coscinodiscus asteromphalus $\mathrm{Th}$.

- excentricus Eh.

- gigas Eh.

Bucampia zodiacus Eh.

Lithodesmium undulatum Eh.

Navicula peregrina $\mathrm{Ktz}$.

Nitzschia seriata $\mathrm{Cl}$.

Pleurosigma delicatulum W. Sm.

Rhizosolenia alata Bright.

- styliformis Bright.

Stephanopyxit turgida Ralfs.

Surirella gemma Eh.

Thalasaionema nitzschioïdes Grun. 
N• 790

ARCACHON - GIRONDE (France)

Pélagique

Eacteriastrum hyalinum Laud.

- varians Laud.

Biddulphia Baileyi W. Sm.

- granulata Rop.

- mobiliensis Bail.

Chretoceros armatum Weil.

- curvisetum Cl.

- danicum $\mathrm{Cl}$.

- didymum Eh.

- Groelandicum Cl.

- $\quad$ - $\quad$ v. leptopus.
Chretoceros Lorenzianum Grun. socialis Laud.

Corethron hystrix Hensen.

Coscinodiscus concinnus W. Sm. - excentricus Eh.

Ditylium Brightwellii' West.

Pleurowisma balticum W. Sm. - quadratum W. Sm.

Stephanopyxis turgida Ralfs.

Surirella gemma Eh.

N० 791

ARCIACHON - GIRONI)E (France)

Pélagique

Bacteriastrum varians Laud.

Bellerocher mallous V. $\mathrm{H}$.

Biddulphia Baileyi W. Sm.

Chrtoceros cochlea Schūtt.

Cocconeis scutellum Eh.

Eucampia zodiacus Eh.
Guinardia flaccida $\mathrm{M}$. Per. Rhizosolenia alata Bright.

- $\quad$ - $\quad$. corpulenta - v. gracillima. setigera Bright.

Striatella unipunctata Ag.

N० 792

ARCACHON - GIRONDE (France)

Pélagique

Chretoceres cochloa schütt.

- curvisetum $\mathrm{Cl}$.

- decipiens $\mathrm{Cl}$.

- didymnm Eh.
Rhizosolenia alata Bright.

- $\quad$ - $\quad$ - $\quad$. corpulenta.

Surirella gemma Eh. 


\section{Pélagique}

\section{Bacteriastrum varians Leud.}

Bidduiphla longicruris Grev.

Chatoceros cochlea Schütt.

$\begin{array}{ll}\text { - } & \text { curvisetum Cl. } \\ \text { - } & \text { danicum Cl. } \\ \text { - } & \text { decipiens Cl. } \\ \text { - } & \text { didymum Eh. } \\ \text { - } & \text { diversum Cl. } \\ \text { - } & \text { sorenzianum Grun. } \\ \text { socialis Leud. }\end{array}$

Coscinodiscus asteromphalus Eh. gigas Eh.
Diploneis Smithii Cl.

Ditylium Brightwellii West.

Guinardia flaccida $\mathrm{H}$. Per.

Navicula cuspidata $K t z$.

- formosa Greg.

Nitzechia seriata $\mathrm{Cl}$.

$$
\text { - - } \quad \text {. fraudulenta. }
$$

Rhizosolenia alata Bright.

- robusta Nor.

- styliformis Bright.

Temperei H. Por.

Thalassionoma nitzschioides Grun.
Achnanthes hexagona $\mathrm{Cl}$. et $\mathrm{Br}$.

$$
\text { - longipes? Ag. }
$$

Actínocyclus Ehrenbergii Ralfs.

$$
\begin{array}{ll}
\text { - } & \multicolumn{1}{c}{\text { v. sparsa. }} \\
\text { - } & \text { noniliformis Ralfs. } \\
\text { - } & \text { nalfsii v. samoensis. } \\
\text { - } & \text { sparsus Ratt. } \\
\text { - } & \text { subtilis Ralfs. }
\end{array}
$$

Actinoptychus areolatus Eh. unduletus Eh.

Alloioneis antillarum $\mathrm{Cl}$.

Amphipror lepidoptera Greg.

Amphora acuta Greg.

- alata H. Per.

- contracta Grun.

- costata W. Sm.
Amphora crassa Greg.

- excisa Greg.

- gigantea Grun.

- - oar.

- Grevilleana Greg.

- - $\quad$ v. Campechiana.

- laevis Greg.

- mexicana $A . S$.

- obtusa Greg.

- oculus A. S.

- ostrearia 0. vitrea.

- proteus Greg.

- sp. A. S. 2tl. $27 / 51$.

Arachnoidiscus Ehrenbergii r. californica.

$$
\text { ornatus Eh. }
$$

Auricuia intermedia $\mathrm{Cl}$, var.

Bacteriastrum varians Laud. 
BiddnIphia anrita Bréb.

$\begin{array}{ll}\text { - } & \text { chinensis Grev. } \\ \text { - } & \text { granulata Rop. } \\ \text { - } & \text { pulchella Gray. } \\ \text { - } & \text { Tucula Rop. }\end{array}$

Campylodiscus ambiguus Greo.

-
-
$=$
$=$
$=$
$=$
$=$
$=$
$=$
angularis Greg. biangulatus Grev. circumactus A. S. contiguus A. S. ecclesianus Grev. emarginatus Deby. incertus $A$. $S$. latus Shabd.

- Ralfsil W. Sm.

- stellatus Greo.

$\overline{-}$ Sumbawanus $A . S$. tæniatus A. S. Thuretii Bréb. undulatus Grov.

Campyloneis Grevillei W. Sm. Climacosphenia elongata Bail.

Cocconels contermina A. S.

- curvirotunda J. Br.

- $\quad$ - $\quad$ v. Grovei.

- cyclophora Grun.

- divisa A.S.

- heteroidea Hantz.

- insignis Jan.

- japonica A. S.

- scutellum Eh.

- $\quad$ sigmoradians Temp. ot $\mathrm{Br}$.

- transversa A. S.

- voluta J. Br.

Coscinodiscus biconus Ost.

- blandus A.S

- concinnus $\nabla$. Jonesiana.

- dubiosus Jan.

- $\quad$ excentricus Eh.

- gigas Eh.

- lineatus Eh.

- marginatus Eh.

- $\quad$ nitidus Greg.

- $\quad$ radiatus Eh.

- Woodwardii Eh.

Cyclotolla striata Grun.

Diplonels crabro Eh.

- - v. multicostata.

- Debyi oar.
DipIoneis didymus Eh.

- fusca Cl.

- geminata Eh.

- nitescens Cl.

- Schmidtii GI.

- Smithii Cl.

- splendida Grey.

- suborbicularis $\mathrm{Cl}$.

Endictya oceanica Eh.

Epithemia sorex $\mathrm{Ktz}$.

$$
\text { - } \quad \text { turgida Ktz. }
$$

Ethmodiscus Japonicus Cast. var.

Euodia gibba Bail.

Euphyllodium spathulatum Shadb.

- $\quad$ $\quad$. Ostrupi $n$.

o. Les marque sont formées de 4 granules ou de 2 assemblès transversalement. = E. spath. Ostrup. Fl. of Kch Chang $1 / 3$.

Gephyria media Arn.

Glyphodesmis Williamsonii Grun.

Goniothecium decoratum $\mathrm{J} . \mathrm{Br}$.

Grammatophora marina Ktz.

$$
\begin{array}{ll}
\text { - } & \text { maxima Grun. } \\
\text { - } & \text { serpentina Eh. } \\
\text { - } & \text { undulata Eh. }
\end{array}
$$

Hyalodiscus maxima Eul.

$$
\text { - } \quad \text { radiatus Grun. }
$$

Hydrosilicon mitra $J . B r$.

Mastogloia quinquecostata Grun.

Melosira sulcata Ktz.

Navicula brasiliensis Grun, oar.

- cancellata Donk

- clacata Greg.

- Hennedyi W. Sm.

- $\quad$ - $\quad$. furcata.

- jejuna A. S.

- liber W. Sm.

- lyraEh.

- - $\nabla$. intermedia.

- pratexta Eh.

- spectabilis Greg.

Nitzschia Jelineckii Grun. 
Nitzschia panduriformis Greg.

$$
\text { - superba Leud Fort. }
$$

Orthoneis aspera H. Per.

\section{- fimbriata Grun.}

- splendida Grun.

Pleurosigma affine $\mathrm{v}$. moronensis .

decorum W. Sm.

ellipticum M. Per. n. sp. (rigidum var. ?). Contour elliptique, à extrémités très légèrement produites, $105 / 25 \mu, 21$ stries obliques et transversales en $10 \mu$

- naviculaceum Bréb.

- rigidum IV. Sm.

Pseudo Amphiprora cruciata J. Br. elliptica M. Per. $n$. sp. (jugata var.?). Longuement elliptique, $100 / 21 \mu$; 12 stries en $10 \mu$. difficilement visibles sur la partie marginale.

Rhabdonema adriaticum $\mathrm{K} z$.
Rhabdonema mirificum W. Sm.

Rhoïconeis Gorkeana Grun.

Ihoicosigma antillarum ( $\mathrm{Al}$. - oceanicum H. Per.

Stophanopyxis cruciata Eh.

- Grunowii Gr. et St.

Surirella fastuosa $\mathrm{Eh}$. et var.

- interrupta Leucl. Fort.

- lata IV.Sm.

- - v. Macraeana.

- $\quad$ - . robusta.

- recedens $\mathrm{A}$. S.

Synedra crystallina K'tz.

Trachyneis aspera $\mathrm{Cl}$.

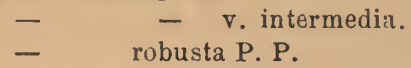

Triceratium antediluoianum Eh.

- formosum Bright.

- $\quad$ - v. pentagonalis.

- gibbosum Bail.

- parallelum v. sparsa.

- pentacrinus Wall.

- - fa quadrata.

Xanthiopyxis umbonatus Grev. var.

No 798

\section{YOKOSKY - VERNY (Japon) \\ Lavages d'algues}

Actinocyclus Ehrenbergii v. sparsa.

$$
\text { - rotula } \mathrm{J}, \mathrm{Br} \text {. }
$$

Actinoptychus splendens Shad. var.

$$
\text { - } \quad \text { undulatus E. fusca. }
$$

Alloioneis antillarum Cl.

Amphiprora lepidoptera Greg.

Amphóra cingulata $\mathrm{Cl}$.

$$
\text { v. proboscidea. }
$$

- obtusa lireg.

$$
\text { - proteus Greg. }
$$

Arachnoidiscus Ehrenbergii $\nabla$. californica. ornatus Eh.

Aulacodiscus affinis Grun.
Aulacodiscus amonus Greo. car.

- Macraeanus Grev. var.

Auliscus cœlatus Bail.

- $\quad$ - fa triocellata.

- _ - var. latecostata

- - - var. rhipis.

- pruinosus v. zanzibarica.

- reticulatus Grev.

Biddulphia aurita Bréb.

$$
\begin{aligned}
& \text { - } \quad \text { pulchella Gray. } \\
& \text { - } \quad \text { Teticulata Rop. } \\
& \text { - Tuomeyi Rop. }
\end{aligned}
$$

Campylodiscus ambiguus Grev.

- angularis Greg. bicinctus Cast. 
Campylodiseus Daemelianus Grun. ecclesianus Grev.

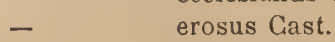

\section{- Greenleafianus Greo.}

- ornatus o. amphileia.

- Pfitzeri A. S.

- Tialfsii W. Sm.

- $\quad$ stellatus Grev.

- undulatus Grev.

Campyloneis Grevilloi Grun.

Cerataulus turgidus Eh.

Climacosphenia elongata Bail.

Cocconeis curvirotunda v, bifrons.

- - - v. Grovei.

- heteroidea Hantz.

- japonica A. S.

Coscinodiseus curvatulus Grun.

- dubiosus Jan.

- elegans Grev.

- excentricus Eh.

- lineatus Eh.

- marginatus Eh.

- nitidulus Grun.

- nobilis Grun.

- nodulifer Jan.

- radiatus Eh.

Cyelotella striata Grun. symbolophorus Grun.

Dictyoneis Thumii GI.

Diploneis crabro Eh.

- - $\quad$ v. multicostata.

- didymus Eh.

- Smithii Cl.

- suborbicularis Cl.

Endictya oceanica Eh.

Epilhemia turgida Kítz.

Euodia gibba Bail.

- - v. atlantica.

Gephyria media Arn. ,

llyalouliscus radiatus Grun.

- stelliger Bail.

Ifyulrosilicon mitra $J$. Br.

Velosira sulcata Kítz, et var.

Navicula Brasiliensis Grun. oar.

- californica var. campechiana.

- clavala Greg.

- - rar. H. n. rar. Liensemble des aréas forme un $\dot{H}$ dont les branches non amincies, n'arrivent pas jusqu'au bord de la valve.

Navicula Fischeri A. S.

- formosa Greg.

- Hennedyi W. Sm.

- liber W. Sm.

- lyra v. dilatata.

- $\quad$ - var. elliptica.

- polysticta o. circunzuta.

- protexta Eh.

- spectabilis Greg.

Nitzsehia alata Leud. Fort.

- Jelineckii Grun.

- panduriformis Greg.

Ortloneis fimbriata Grun.

Plenrosigma decorum WV. Sm.

- naviculaceum Bréb.

- rigidum W. Sm.

Podosira maxima Grun.

- variegata A. S.

Ruablonema adriaticum Ktz.

- mirificum W. Sm.

Rhoicosigma antillarum Cl.

Stephamopyxis Grunowii Gr. et St.

Surirella eximia Greo.

- fastuosa Eh.

- Kittoniana Leud Fort.

- recedens A.S.

Synedra formosa Hantz.

- Hennedyana Greg.'

Terpsinos inflata $\mathrm{J}$. $\mathrm{Br}$. oar.

Trachyneis aspera Cl.

- $\quad$ - $\mathrm{v}$. intermedia.

- $\quad$ - $\quad$. oblonga.

Triceratium bicorne Cl.

- favus Eh.

- formosum Bright.

- $\quad$ - v. pentagonalis.

- impressum Grun.

- junctun A. S.

- Pantocsekii A.S.

- $\quad$ paquadiata.

- parallelum Grev.

- pentacrinus Wall.

- - fa quadrala.

- Shadboltianum Grev.

- sculptum Shad. 


\section{FORMIGNANO - CESENA (Italie)}

Dépôt fossile marin miocène

Actinocyclus Bremiaus Pant.

- circumdatus Pant.

- Ehrenbergii Ralfs.

- $\quad$ - oar. intermedia Grun.

Bactoriastrum sp. (fragments).

Budlulphia tridentata $\mathrm{Eh}$.

- Tuomeyi Eh.

Chictoceros sp. (fragments).

Coscinodiscus curvatulus Grun.

$\begin{array}{ll}\text { - } & \text { denarius A. S. } \\ \text { - } & \text { excentricus Eh. } \\ \text { - } & \text { leptopus Grun. } \\ \text { - } & \text { Rothii (Eh.) irun. } \\ \text { - } & \text { subtilis Eh. } \\ \text { - } & \text { symbolophorus Grun. } \\ \text { - } & \text { symmetricus Grev. }\end{array}$

Fragilaria Grunotoii Pant.

Grammatophora robusta Dip.
IIyalodisens lrevis v. doljensis Pant.

Mastugloia Smithii Thw.

Melosira clavigera o. crassiradiata Fort.

- granulata Ralfs. var.

Navicula bombus Eh.

- crabro Eh.

- IIennedyi IV. Sm. c. brachypleura Forti.

Nitzschia panduriformis o. Loczii Pant.

Orthoneis splendida Grun.

Pleurosigma Normannii Ralfs.

Bhabdanema adriaticum v. fossilis pant.

Rhaphoneis nitida oor. Debyi Pant.

$$
\text { - } \quad \text { - o. liburnica. }
$$

Stephanopyxis turris Eh.

Synedra cretae Sauoage.

Thalassionema nitzschioides Grun.

(1) BILIOGRAPHIE. - Fr. Meister. Beitrage zur Bacillariaceen flora Japans (Archiv. für Hydrobiologie. Vol. VIII, 1912/13).

Achnanthidinu lanceolatum $v_{\bullet}$. ellipticum Cl.
Acbuanthidium lanceolatum var. Haynaldii Cl.

(1) Nous devons les matériaux et leurs analyses des.Nos $\$ 01$ à 809 à l'obligeance de M. Fr. Meister, d'Horgen. 
Asterionella subtilissima Meister.

Caloneis silicula oar. cuneata Meister.

- ventricosa Meister.

Ceratoneis arcus Ktz.

Cocconeis placentula Eh.

Cyclotella Kützingiana Thw.

Cymbella australica $A$. $S$.

- cuspidata Ktz.

- microcephala Grun.

- turgidula Grun.

- ventricosa $\mathrm{Ktz}$.

Diploneis elliptica CI.

- ovalis var. oblongella $\mathrm{Cl}$.

Epithemia zebra $\nabla$. saxonica Grun.

Eunotia incisa Greg.

- lunaris Grun.

Fragilaria construens var. venter Grun.

Gomphonema augur Eh.

- capitatum Eh.

- globiferum Meister.

- olivaceum Ktz.

Gyrosigma acuminatum Rab.
Melosira granulata v, mustraliensis Grun.

- Japonica Meister.

- pusilla Meister.

- varians $\mathrm{Ag}$.

Microneis exigua $\mathrm{Cl}$.

- hungarica Cl.

- minutissima Gl.

Navicula cincta Grun.

- cryptocephala Ktz.

- hasta Pant.

- hungarica v. capitata Cl.

Rhoicosphenia curvata Grun.

Surirella minuta Bréb.

Synedra Japonica Meister.

- pulchella Ktz.

- rostrata Meister.

- tenera W. Sm.

- ulna v. æqualis Brun.

- vaucheriæ $\mathrm{Ktz}$.

Tabellaria fenestrata Ktz.

- flocculosa Ktz.

No 802

AUSEELI près HORGEN (Suịsse)

Melosira italica htz.

No 803

FONTAINE GADMENTAL (Suisse)

Cymbella niaculata kitz.

No 804

AAR près BERNE (Suisse)

Cymbella parva $w$. Sm. 


\section{No 805 PONT DU RHIN - CONSTANCE (Suisse)}

\section{BIBLIOGRAPHIE. - F. Meister. Die Kieselalgen der Schweiz}

Cyclotella Kützingiana Thw.

- melosiroides Lemm.

Cymbella cymbiformis Bréb.

- leavis Næg.

- lanceolata Kirch.

- subalpina Meister.

Denticula crassula Nag.

biatoma grande $v$. clavigerum Meister.
Eucocconeis flexella Cl.

Gomphonema angustatum $\mathrm{Ktz}$.

Microneis exilis Cl.

- microcephala CI.

Synedra longissima W. Sm.

- paludosa Meister.

Tabellaria fenestrata $\mathrm{Ktz}$.

\section{No 806. FREIBURG - BREISGAU (Allemagne)}

Achnanthidium lanceolatum Bréb. Navicula cryptocephala litz.
Surirella apiculata W. Sm.

- ovata Ktz.
Cymbella cesalii Grun .

Eunolia lunaris Grun.

- tetraodon Eh.

Frustulia torfacea $A$. Br.

Gomphonema acuminatum Eh.

- capitatum Eh.
Microneis exilis Eh.

Pinnularia viridis v. Clevei Meister.

Synedra vitrea Ktz.

Tabellaria fenestrata Ktz.

- flocculosa $\mathrm{Ktz}$. 
No 808

RICHTERSWIL (Suisse)

BIBLIOGRAPHIE. - F. Meister. Die Kieselalgen der Schweiz

Cymbella ventricosa v. Aúerswaldii Mreis-

ter.

- $\quad$ - v. lunula Meister.

Microneis exilis Cl.

Microneis minutissima (Al..

Veidium an phirhynchus v. minus Meister. Synedrie nana Meister.

- vitrea Ktz.

No 309 FONTAINE près TVETTSIVIL - ZURICH (Suisse)

Cyclotella Kützingiana Thw..

Cymbella Cesatii Grun.

- helvetica var. curta Cl.

- laevis Neg.
Incoceoneis flexella $\mathrm{Cl}$.

(iomphonema angustatum Kiz.

syuedra longissima W. Sm.

- palulosa Meister. var.

\section{Note sur les dépóts d'Damaro (Nouvelle-Zélande)}

Le prototype de ces nombreux dépôts, Cave Valley, a été communiqué par M. Morland à MM. Grove et Sturt. Ces messieurs le placent à un étage géologique inférieur aux couches oligocènes d'Oamaru, il serait donc Eocene superieur.

Les auteurs précités font ressortir ses affinités avec le dépôt de Cambridge (Barbades); tout cela est fort plausible. Le Dr Pantocsek, suivi par MíM. Woolman et David, vieillissent un peu ce dépôt et le placent, soit dans le Crétacé supérieur, soit dans l'Éocène inférieur.

Mes recherches personnelles, sur l'application de la loi de Lyell, aux significations paléontologiques de la proportion des formes récentes et fossiles dans les dépôts diatomifères, me permettent de confirmer ces données. D'après la courbe que j'ai établie, le \% des récentes, à la séparation des terrains secondaire et tertiaire serait d'environ $20 \%$; or, les dépôts anciens se classent ainsi d'après leur \% en Diatomées actuellement vivantes. 


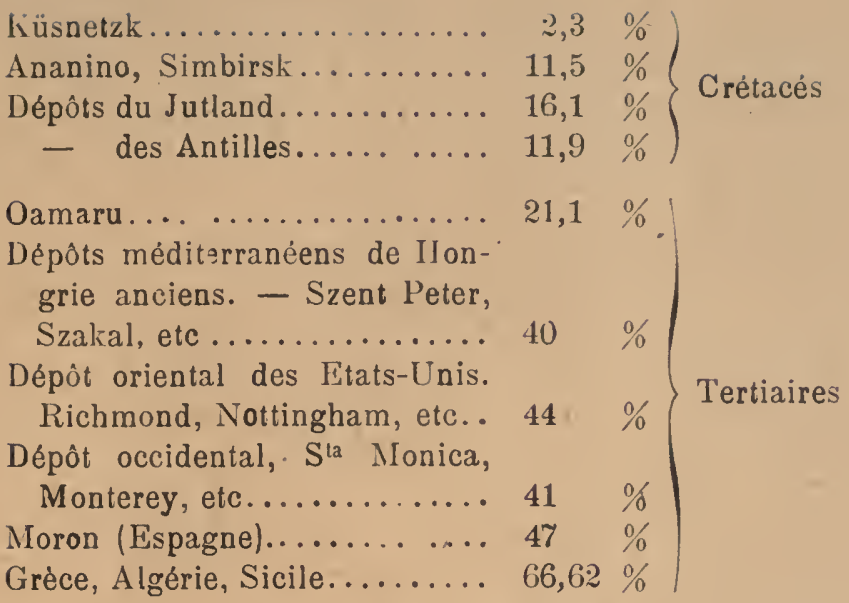

D'après cela, les dépôts d'Oamaru se placent bien à la limite du Crétacé et de l'Eocène, plutôt au début de l'Eocène.

D’après la courbs que j'ai établie. les dépôts des Antilles (I3arbades, Trinité, Jémémie) se placeraient au milieu de la période crétacée et seraient par suite beaucoup plus anciens que ceux d'Oamaru.

Les genres et espèces qui les composent sont d'ailleurs fort différents. Il n'y a à Oamaru que des traces des genres Entogonia et Asterolampra si caractéristiques des Barbades.

II. P.

\section{Principaux dépóts fossiles maring d'Damaru (Nouvelle Zélande)}

Nos 810-813 TROUBLESOMEGULLY.

- 814.817 TOTARA.

- 818.820 ALLAN'S FARM.

- 821,822 BORRIES.

JACKSON'S PADDOCK, publié sous les $N^{\text {os } 1,2 .}$
BAIN'S FARM, publié sous' les Nos 139-141.

FORRESTER'S ROCK, publié sous le No 250.

CORMACK'S TOP, publié sous les Nos 415-416.

WILLIAMS BLUFF, publié sous les Nos 439-441.

OTAGO, publiésous les Nos 569-570. 
BIBLIOGRAPHIE. - Grove et Sturt : On a fossil marin Diatomaceous Deposit from Oamaru Otago (New Zealand) (1886). J. Brun : Espèces nouvelles (Diatomiste, vol. II, 1893-1896). Ad. Schmidt : Atlas. Dr J. Pantocselk : Diatomées de Hongrie, vol. III.

Actinodiscus Barbadensis Grev.

$$
\text { - Grayii Grooe. }
$$

Actinoptychus constrictus Gr. et St.

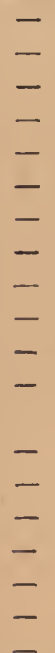

\section{decorans $A$. $S$.}

elegantulus Gr. et St.

fuscus Gr. et St.

glabratus Giun.

Grooei Thoimas. interpositus J. Br. maculatus Gr. et St. maculosus Pant. Mölleri Grun. nitidus Grun. Damaruensis Grun. sis. I. $\mathrm{Br}$. splendens Ralfs. tener Gr. et St. trilunatus J. Br. undulatus Eh. Wittii Jan. pericacaus o. Ocmarue"pulchellus o.tencra Grun. vulgaris Schum.

Amphiprora cornuta $\mathrm{W}$. et Gl.

Amphora cingulata $C l$.

- contracta Grun.

- crassa Greg.

- furcata Leud. Fort.

- interlineata Gr. et St.

- labuensis $\mathrm{Cl}$

- obtusá Greg.

- subpunctataGr. et St.

- tessellata Gr. et St.

A.naulus birostratus Grun.

- subconstrictus Gr. et St.

Anthodiscus floreatus Gr. et St.

Aporodiscus Oamaruensis Ratt.

Arachnoidiscus Ehrenbergii Bail.

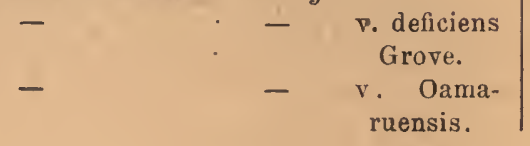

Arachnoidiscus indicus $\mathrm{Eh}$.

Asterolampra affine v. punctifera Grun. decora Grev. insigne A. S.' marylandica Eh. uraster Gr. et St. vulgaris Grev.

Aulaconliscus amæenus Grev.

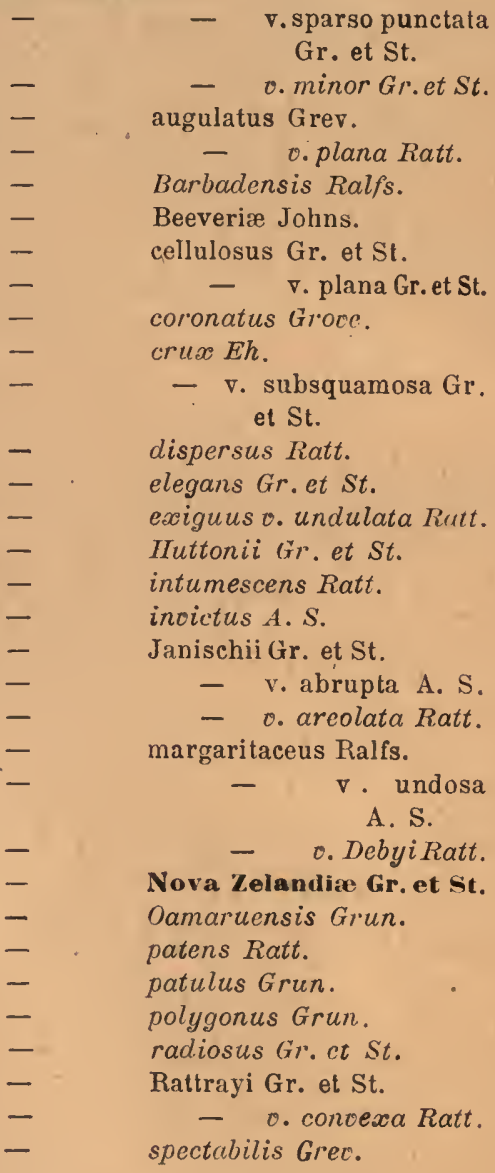


Inlacodiscus Sollitianus v. Novæ Zelandise Gr. et St.

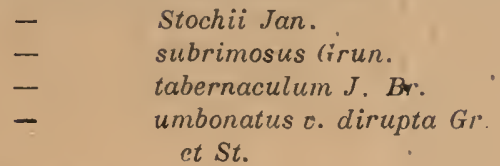

Auliscus accedens Ratt.

- Barbadensis Greo. .

- coelatus o. delicatula Ratt.

- coincidens A.S.

- confluens Grun.

- conoolutus Groce.

- dubius Temp. (A. S. atlas 125/9).

- ellipticus Arnott.

- fenestratus Gr. et St.

- fulcratus A.S.

- Grevillei Jan.

- Hardmannianus Greo.

- inflatus Gr. et St.

- intermedius Gr. et St.

- interruptus o. sparsa Ratt.

- lacunosus Gr. et St.

- Macroanus Grco.

- microleion A. S.

- Moronensis Greo.

- notatus Greo.

- Oamaruensis.

- ooalis Arnott.

- pectinatus Ratt.

- polyphemus $A$. $S$.

- propincuus Gr. et St.

- pruinosus Bail.

- - o. robusta.

- punctatus Grev.

- Raeanus Ratt.

- racemosus Ralf.

- Stöckardtii Jan.

- - o. aspera Grove.

- - - o. inconspicua Ratt.

- - o. subpunctata Ratt.

- subccelatus Ratt.

- transpennatus. $T . B r$.

Bıddulphia chinensis Greo.

- cornuta J. Br.

- dissipatd Gr. et St.

- elegantula Grev.

- $\quad$ - v. polygibba Pant.

- fossa Gr. et St.

- Grocei Pant.

Biddulphia lata $G r$. et $S t$.

- miraoulosa J. Br.

- Oamaruensis Gr. et St.

- podagrosa Greo.

- punctata Grev.

- reticulata fa trigona.

- reversa Gr. et St.

- rhombus o. fossilis fa trigona.

- rigida $A . S$.

- tenera Gr. et st.

- Tuomeyi Bail.

- virgata Gr. et St.

- vittata Gr. et St.

Brightwellia pulchra Grun.

Campyloneis argus Grun.

Cerataulus Johnsonianus Grun.

- marginatus Gr.et St.

- polymorphus fa minor,

- rotundus Temp. et Br.

- subangulatus Gr. et St.

Chretoceros gastridium Grun.

Clavicula apicephala Pant.

Cocconeis antiqua Temp. et $\mathrm{Bl}$.

- Barbadensis Grun.

- costata Greg.

- cyclophora Grun.

- naviculoides Grun.

- nodulifer Gr. et St.

- pellucida Grun.

- pseudo-marginata v. intermedia Grun.

- Totarce J. Br.

\section{Coscinodiscus angulatus Grev.}
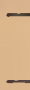

$-$

$-$

$-$ apiculatus o. Woodicardii Riatt. apollonis Eh. argus $\nabla$. subtraducens. argus $E h$. biradiatus Greo. bulliens A. S. centralis Greg. concavus Greg. - v. punctata. conclusus Grove. cureatulus Grun. debilis Groce. decrescens o. repleta. decussatus Gr. ct St. densus $G r$. et St. dubiosus Groce. 
Coscinodiscus dubiosus o.curoans Ratt.

$\begin{array}{ll}- & \text { elegans Grev. } \\ - & \quad \text { v. spinifera. } \\ - & \text { excentricus Eh. } \\ - & \text { Gallopagensis Ratt. } \\ - & \text { inæqualis Gr. et St. } \\ - & \text { Kützingii A. S. } \\ - & \text { lacunosus Grove. } \\ - & \text { lentiginosus Jan. } \\ - & \text { luctuosus Grove. } \\ - & \text { marginatus Eh. } \\ \text { - } & \text { megacentrum Grooe. } \\ \text { - } & \text { micans A. S. } \\ - & \text { minor Eh. } \\ \text { - } & \text { nitidus Greg. }\end{array}$

- Oamaruensis Gr. et St.

- oblongus Grev.

- oculus-iridis EH,

Cosmiodiscus Normanianus Greo.

Cotyledon circularis $J, B r$.

Craspedoporus elegans Gr. et St.

Cyclotella Castracanei J. Br.

Dehya Oamaruensis Riatt.

Dictadia capreolus Eh.

Dictyoneis marginata $\mathrm{Cl}$.

Dimeregramma fulvum Ralfs.

o. Janischii $\mathrm{Cl}$.

Ditylium Grovei J. Br.
Donkinia antiqua $G r$. et St.

Eunotogramma bivittata Grove et Pant.

Weissci Eh. o. elongata.

$-$ - v. producta Gr. et St.

Euodia striata Gr. et St.

Fenestrella convexa J. $\mathrm{Br}$. - gloriosa J. Br.

Gephyria incuroata $v$. Oamaruensis.

Glyphodesmis marginata $\mathrm{Gr}$. ct St. - interpositus $J . \mathrm{Br}$.

Glyphodiscus bipunctatus $A$. $S$.

- Grunowii A. S. o. lacuno. sa Temp.

- Oamaruensis Grun.

- scintillans A. S.

- $\quad$ stellatus Greo.

- strigillatus A.S.

Goniothecium decoratum $J . B r$.

Grovea pedalis Gr. et St.

Gyroptychus contabulatus $A$. S.

llemiaulus angustus Grev.

- amplectans Gr. et St.

- - o. major.

- Barbadensis Grun.

- claviger A. S.

- dissimilis Gr. et St.

- includens Grun.

- lyriformis Grev.

- unicornutus J.Br.

- ornithocephalus Grev.

- polymorphus Grun.

- tenuicornis Grev.

Inttonia alternans $G r$. et $S t$.

- virgata Gr. et St.

Iyalodiscus arcticus Grun.

- punctatus A. S.

- radiatus Grun.

- subtilis Bail.

- valens A. S.

Isodiscus coronalis $J . \mathrm{Br}$.

- Debyi Ratt.

- mirificus Ratt.

Isthmia neroosa $E h$.

Kittonia elaborata Gr. et St.

- Greoilleana Gr. et St.

- virgata Gr. et St. 
Lamella oculata $J . B r$.

Lampriscus Debyi Gr. et $S t$.

Lepirlodiscus imperialis $J . B r$.

I.endugeria epithemioides Temp.

Liraliseus ovalis Grev.

Melosira architecturalis J. Br.

- Borreri IV.Sm.

- clavigera Grun.

- cospectata A. S.

- Oamaruensis Gr. et St.

- major Grove.

- pontificalis $J . B r$.

- prceclara A. S.

- saturnalis J. Br.

- sol kïitz.

- sulcata Kûtz.

- - $\quad$ v. biseriata fa coronata.

- - - - fadiata.

- - $\quad$. crenulata fa Barbadensis.

- truncata Grove.

- Westii IV. Sm.

Monopsia mammosa Gr. et St.

Navicula apis $E$ a.

- biconstricta Gr. et St.

- braziliensis Grun.

- decora Gr. ei St.

- definita Gr. et St.

- dispersa Gr. et St.

- dlubia M. S.

- geminata Greo.

- Grundleri A. S.

- inelegans Gr. et St.

- interlineata Gr. et St.

- lobata Gr. et St.

- margino-lineata Gr. et St.

- margino-punctata Gr. et St.

- mediopartita Grove.

- Oamaruensis Grun.

- placida A. S.

- placita Gr.et St.

- prcelexta Eh.

- - o. A. S. atlas 258/10.

- pulchella M.S.

- Sandriana Grun.

- Smithii c. nitescens Greg.

- sparsi.punctata Gr. et St.

- spectabilis o. Oamaruensis.

- trilineata Gr. et St.

- cariolata Cl.
Nitzschia antiqua Gr. et St.

- - o. carnosa J. Br. Grundleri Grun.

Orthoneis Barbadensis Greo. o. tenuipunctata $J . B r$.

- splendida Grun.

Piunularia excellens Cl.

$$
\begin{array}{cl}
\text { Plagiogramma atomus Greo. } \\
\text { - } & \text { bilobatum } J . B r \\
- & \text { Nancoorense Grun. } \\
\text { - } & \text { neogradense Pant. } \\
\text { - } & \text { tessellatum Greo. }
\end{array}
$$

Poilosira argus Grun.

- corolla A.S.

- hirsutus Gr. et St.

- hormoides Grun.

- interruptus Gr. et St.

- maxima Kütz.

Pseuto-Auliscus anceps Ratt.

- diffusus Ratt.

Psendo-Rutilaria monile Gr. et St.

Pterotheea aculeifera Grun.

Pyxilicula cruciata Eh.

Pyxilla dubia Grun.

- Johnsoniana Grev,

- prolongata J. Br .

- reticulata Gr. et $\mathrm{St}$.

IRaphoneis nodulifera $A$. S.

liutilaria epsilon Greo.

- $\quad$ - o.tenuis.

- excacata J. Br.

- lanceolata Gr. et St.

- liudiata Gr. et St.

Stephanogonia Nova Zelandiæ.

Stephanopyxis Barbadensis Greo.

- ferox Grun.

- Grunowii Gr. et St.

- permarginata Grove.

- turris Grun.

- _ - v. brevispina Grun .

Stictollesmis australis Grev.

Stictouliscus californicus.

-
-
-
Grocei A. S.

Iardmannianus Grev. nitidus Gr. et St. 
Stictodiscus Novæ Zelandiæ Grun. parallelus Grer.

- v.gibbosa Gr.etSt.

Stochia punctata Gr. et St.

Synedra crystallina Kütz.

Terpsinoè americana Bail.

$$
\text { - o. trigona. }
$$

Triceratium alceolatum Bergon.

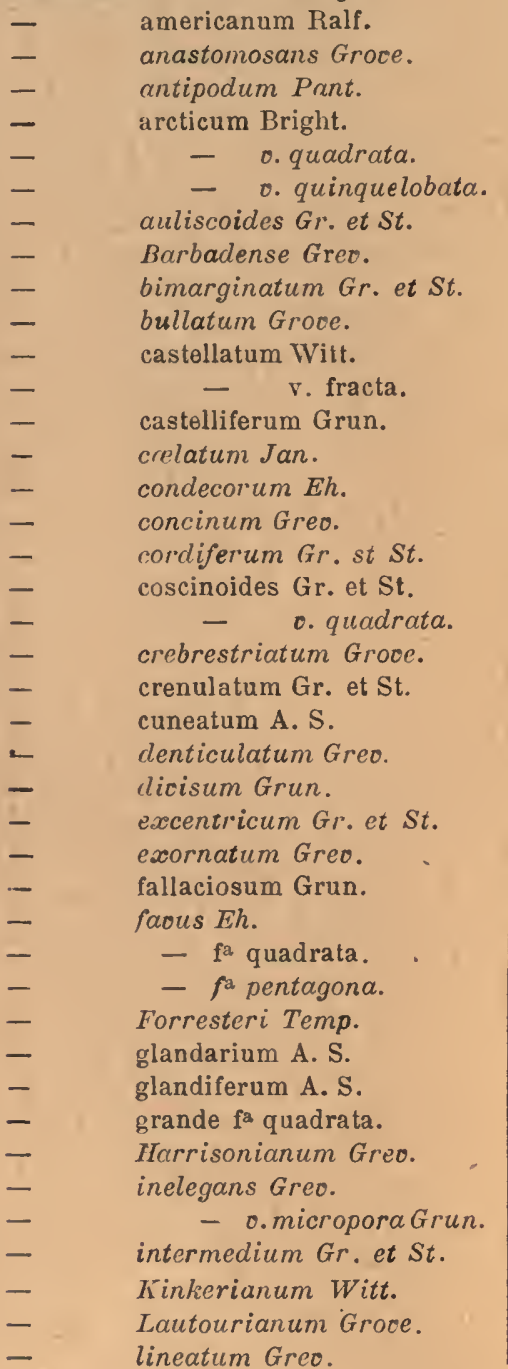

Triceratium lineatum $c$. biangulata.

lobatum Greo. majus Gr. et St.

Morlandii Gr, et St. Montereyi Pright. neglectum Greo. nitescens Greo.

Novie Zelandiæ Grun. Oamaruensis Gr. et St.

v. sparsus punctata.

- $\quad$ obesum Greo.

- parallelum Greo.

- - - fatrigona.

- partitum Greo.

- plano-concaoum $\mathrm{J} . \mathrm{Br}$.

- plenum Gr. et St.

- plumosum Greo.

- productissimum Bergon.

- pseudo-nervatum Gr. el St.

- pulvinar A. S.

- rectangulare Gr. et St.

- repletum Greo.

- rotundatum Greo:

- rugosum Gr. et St.

- secedens A. S.

- sexpartitum Gr. et St.

- simplex J.Br.

- Stokesianum Grev.

- triorbicum A. S.

- $\quad$ trisulcum Bail.

- - v. cuneata A. S

- unguiculatum Grev.

- venosum Bright.

- cenulosum Greo.

- Weissflogii Gr. et St.

- Weissei Grun.

- Websterii Temp.

Trinacria altar $J . B r$.

- fragilis Grun.

- ligulata Gr. et St.

- Mölleri Pant.

- pileolus o. jutlandica Grun.

- simulacrum Gr. et St.

- ventricosa Gr. et St.

Tubularia pistillaris $J . \mathrm{Br}$.

Van Ireurckia admirabilis Pant.

Xanthiopyxis constricta Eh.

- oblonga Eh. 


\section{$\mathrm{N}^{\text {os }} 823,824$ JARLANI) - ABERDEEN (Ecosse)}

\section{Dépôt fossile d'eau douce}

Achnantidium flexellum Bréb.

Amphora gracilis Eh.

$$
\text { - libyca Eh. }
$$$$
\text { - ovalis } \mathrm{Ktz} \text {. }
$$

Cyclotella comta $\mathrm{K} t z$.

Cymatopleura elliptica W. Sm.

$$
\text { - solea IV. Sm. }
$$

Cymbella americana A. S.

- cistula Kirsch.

- cuspidata Ktz.

- cymbiformis Bréb.

- Ehrenbergii Klz.

- gastroides Ktz.

- gracilis Ktz.

- maculata ḱtz.

- subæqualis Grun.

Diploneis elliptica $C l$.

- Smithii Cl.

Encyonema turgidum (rirun.

$$
\text { - ventricosum Grun. }
$$

Epithemia amphicephala Grun.

$$
\begin{array}{ll}
\text { - } & \text { argus Ktz. } \\
\text { - } & \text { sorex Ktz. } \\
\text { - } & \text { turgida Ktz. } \\
\text { - } & \text { - } \quad \text { v. granulata. } \\
\text { - } & \text { zebra Eh. }
\end{array}
$$

Eunotia arcus Eh.

- - v. hybrida.

- - v. tenella.

- exigua Rab.

- formica Eh.

- gracilis Rab.

- lunaris Grun.

- parallela Eh.

Fragilaria elliptica Schum.

- Harrisonii Grun.

Comphonensa acuminatum Eh.

$$
\begin{aligned}
& \text { - - } \quad \text { v. coronata. } \\
& \text { - - } \quad \text { v. pusilla. } \\
& \text { - constrictum Eh. } \\
& \text { - intricatum Klz. }
\end{aligned}
$$

Gomphonema sagitta Schum.

$$
\text { — } \quad \text { subclavatum Grun. }
$$

Mastogloia Dansei Thw.

Melosira solida Eul.

Navicula amphirynchus Eh.

- bacilliformis Grun.

- bacillum Eh.

- Erebissonii Ktz.

- commutata Grun.

- cuspidata Ktz.

- dactylus Ktz.

- divergens W. Sm.

- . - v. elliptica.

- dubia Eh.

- gentilis Donk.

- gibba Ktz.

- gigas Ktz.

- Hebes Ralfs.

- iridis Eh.

- - v. ampliata.

- lanceolata Ktz.

- legumen Eh.

- limosa Ktz.

- major Ktz.

- mesolepta Eh.

- - $\quad$. stauroneiformis.

- microstauron Eh.

- nobilis Kitz.

- nodosa Eh.

- radiosa $\mathrm{Ktz}$.

- - var. acuta.

- rupestris Hantz.

- semen Eh.

- tenella Brẻb.

- transversa A. S.

- tuscula Eh.

- viridis Ktz.

- viridula Kitz.

Nitzschia sigmoidea $W$. Sm. Pleurostauron acuta Rab.

Rhopalodia gibba $\mathrm{O}$. $\mathrm{M}$. 
Stauroneis anceps Eh.

- $\quad$ - vracilis Eh.
- $\quad$ mesopachya Eh.
- $\quad$ phcenicenteron Eh.
orirella elegans Eh.
- robusta Eh.

surirella tenera Greg.

Synedra biceps Ktz.

- capitata Eh.

- ulna Eh.

- $\quad$ - v. requalis.

Tabellaria fenestrata $\mathrm{Kt} \mathrm{z}$. flocculosa its.

\section{No $825 \quad$ KISSTIB - CAUCASE (Russie)}

Achnanthes lanceolata $\nabla$. Haynaldii. ventricosa Eh.

Amphora caucasica $\mathbf{M}$. Per: n. sp. Elégamment arqué à extrémitẻs prolongées, capilées, aire centrale fortement dilatè des deux côtés. - 15 à 18 stries très nettes des deux côtès.

Longueur 60 à $100 \mu$.

- gigantea $\mathrm{f}^{\mathrm{a}}$ minor.

- lybica Eh.

- pediculus fa major.

Campylodiscus clypeus Eh.

Cocconeis californica o. subcontinua.

Cymbella cistula Kirsch.

- maculata Kitz.

- mexicana Eh.

Denticula thermalis Ktz.

- valida Pedic.

- - - v. minor.

Diploneis elliptica Cl.

Epithemia argentina $\mathrm{J}$. $\mathrm{Br}$.

- gibberula Ktz.

- $\quad$ - v. producta.

Fragilaria æqualis Heib.

- bituminosa Pant.

- brevistriata Grun.

- elliptica Schum.

- endocystifera M. Per. et F. H.

- transylvanica Pant.

- virescens Ralf.

Gomphonema insignis Greg.

- $\quad$ - fa minor.

- subclavatum Grun.
Ilantzschia amphioxys 5 . major.

Navicula ambigua $E h$.

- amphirhynchus Eh.

- bohemica Eh.

- Brebissonii Kitz.

- cuspidata Ktz.

- firma Iitz.

- interrupta v. stauroneiformis.

- iridis Eh.

-- - v. ampliata.

- major litz.

- peregrina kitz.

- sculpta Eh.

- - var. major.

- streptoraphe Cl.

- ventricosa v. maculata.

- viridis $\mathrm{K} t z$.

Nitzschia acutiuscula v. fossilis.

- linearis W. Sm.

- sigmoidea IV. Sm.

- vitrea W. Sm.

Rhopalodia gilhba O. M.

$$
\text { - } \quad \text { - v. parallela. }
$$

Schizostanron rhombicum M. Per. n. sp. - De forme rhombique à stauros large et èvasé dont le fond est garni d'un petit triangle de stries, lumens aux extrẻmitès. Long. $60 \mu ; 13$ stries èn $10 \mu$.

Surirella antiqua Reich. $f^{a}$ acuta. Synedra ulna rar. cequalis. 


\section{Nos 826, 827 NEWARK - NEW-JERSEY (États-Unis)}

Achnanthes brevipes Ag.

Actinocyclus Barkleyi Grun.

Actinoptychus areolatus $\mathrm{Eh}$.

$$
\text { - undulatus Eh. }
$$

Amphiprora alata Ktz. var.

$$
\text { - pulchra Bail. }
$$

Auliscus pruinosus $\nabla$. Zanzibarica.

Biddulphin aurita Bréb.

$$
\text { - } \quad \text { granulata Rop. }
$$

Brebissonia Boeckii Grun.

Campylodiscus echeneis Eh.

Cerataulus laevis Ralfs.

$$
\text { - turgidus IV. Sm. }
$$

Cocconeis scutellum Eh.

Coscinodiscus curvatulus v. inermis.

$\begin{array}{ll}- & \text { denarius A. S. } \\ \text { - } & \text { excentricus Eh. } \\ \text { - } & \text { radiatus Eb. } \\ \text { - } & \text { subtilis Eh. }\end{array}$

Cyclotella striata Grun.

Cymbella cistula Kirsch.

- gastroides Ktz.

- maculata $K t \tilde{z}$. var.

Diploneis bombus Eh.
- elliptica Cl.
- interrupta Gl.

\section{- Sunithii cl.}

Epithemia gibberula $\mathrm{K} \mathrm{z}$.

$$
\text { - turgida Kítz. }
$$

Vunotia monodon Eh.

Melosira mummuloides Eh.

$$
\begin{aligned}
& \text { - sulcata Kitz. } \\
& \text { - - v. coronata. } \\
& \text { - - v. radiata. }
\end{aligned}
$$

Navicula æstuarii Gl.

- braziliensis Grun.

- Delawarensis Grun.

- elegans W. Sm.

- Fischeri A. S.

- lormosa Greg.

- Hennedyi W. Sm.
Navicula latissima Greg.

- lyra Eh.

- major Ktz.

- pachyptera Eh.

- peregrina Ktz.

- permagna Edw.

- prcetexta Eh.

- spectabilis o. emarginata.

- streptoraphe Cl.

- subsalina v. major.

- vididis $\mathrm{Ktz}$.

Nitzschia circumsuta Grun.

- fasciculata Grun.

- granulata Grun.

- marginulata Grun.

- obtusa W. Sm.

- punctata Grun var.

- scalaris W. Sm.

- sigma W. Sm.

- $\quad$ - var. rigida.

- $\quad$ - var, rigidula.

- - var. sigmatella.

- Tryblionella Grun.

Plenrosigma affine var. Normanuii.

$\begin{array}{ll}\text { - } & \text { angulatum W.Sm. } \\ \text { - } & \text { attenuatum W. Sm. } \\ \text { - } & \text { hippocampus W. Sm. } \\ \text { Temperei CI. }\end{array}$

Pseudauliscus radiatus Ratt.

Pyxilla baltica Grun.

IRhablonema adriaticum Kitz.

stauroneis Baileyi $\mathrm{Eh}$.

Stephanopyxis turgida Ralfs.

Surirella fastuosa Eh.

- Febigerii Lewis.

- ovalis Bréb.

- striatula Turp.

Synedra affinis $\mathrm{K} t z$.

Terpsinoë americana Ralfs.

Triceratium alternans Baịl.

$$
\text { - } \quad \text { favus Eh. }
$$

Tropidloneis lepidoptera v. proboscidea. - seriata $\mathrm{Cl}$.

Van lleurckia Lewisiana Bréb. 


\section{No 828 ACHOLE FARM - TRANSVAAL (Afrique)}

Encyonema turgidum Grun.

Epithemia argus v. alpestris.

Navicula majol $\mathrm{K} i z$

\section{No 829 MULLBURY CUÑA - TEXAS (États-Unis)}

Achnanthes lanceolata $\nabla$. Haynaldii.

Amphora ovalis $\mathrm{Ktz}$.

$$
\text { - } \quad \text { turgida Greg. }
$$

Cocconeis lineata Eh.

$$
\text { - placentula Eh. }
$$

Cymbella cistula Kirsch.

- gracilis Rab.

- mexicana Eh.

- subæqualis Grun.

Denticula thermalis $\mathrm{K} t z$.

$$
\text { - valida Pedic. }
$$

Encyonema gracile $R a b$.

- turgidum Grun.

- ventricosum Grun.

Epithemia constricta Bréb.

- Hyndmannii W. Sm.

- gibberula Ktz.

- $\quad$ - var. producta.

- $\quad$ - var. protracta.

- turgida Ktz.

- $\quad$ - var. granulata.

Fragilaria hituminosa Pant.

- construens Grun.

- - - var. venter.

- elliptica Schum.

- nitida M. Per. et F. H.

- parasitica Grun.

Gomphonema insigne Greg.

- $\quad$ intricatum $\mathrm{Ktz}$.

- $\quad$ - var. pumila.

- parvulum Kítz.

- $\quad$ subclavatum Grun.
Mastogloia Dansei Tliw.

Melosira crenulata Ktz.

- laevis Eh.

Navicula amphiryuchus Eh.

- Bohemica Eh.

- Brebissonii Ktz.

- - - var. curta.

- cincta var. Heufleri.

- cuspidata Iitz.

- - - fa craticula.

- gigas.

- Gothlandica Grun.

- halophila Cl.

- lanceolata Ktz.

- major Kitz.

- pupula Ktz.

- sculpta Eh.

- sphærophora Ktz.

- tenella Bréb.

- viridis Ktz.

Nitzschia denticula Grun.

- sigmoidea W. Sm.

- vitrea Norm.

IR hopalodia gibbu O. M.

- _ - var, ventricosa

Seoliopleura Peisonis Grun.

Stauroneis Brunii M. Per. et F. H.

- pliœnicenteron Eh.

Surirella ovata Ktz.

Synedra acus Ktz.

- delicatissima V. Sm.

- ulna Eh.

- - var. danica. 


\section{No 830 COTES DE LA DALMATIE (Autriche)}

Auliscus sculptus Ralfs.

Bidldulphia pulchella Gray.

Canpylodiscus adriaticus Grun.

- $\quad$ decorus Bréh.

Endictya oceanica Eh.

Navicula lyra Eh. et var.

- Williamsonii O'Meara.

vitzschia panduriformis Greo.
Nitzschia sigma W. Sin.

Pleurosigma affine Grun .

$-\quad$ balticum W. Su.
$-\quad$ formosum W. Sin.

Rhabdonema adriaticum $W$. Sm.

Surirella fastuosa Eh. et var.

Synedra baculus Greg.

- formosa Hantz.

Triceratium antediluvianum Eh. favus Eh.

$\mathrm{N}^{\circ} 831$

FITZWILLIAM (États-Unis)

Dépôt fossile d'eau douce

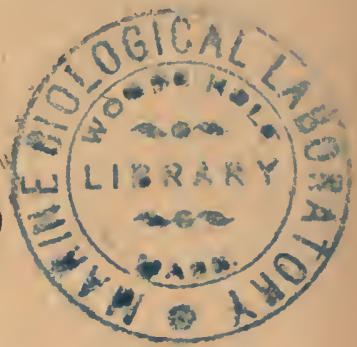

Actinella punctata Lewis.

Encyonema gracile Rab.

Eunotia pectinalis Dill.

- polyglyphis Eh.

- polyodon Eh.

- triodon Eh.

Fragilaria binodis Grun.

- undata W. Sm

Melosira distans $\mathrm{Ktz}$.

Navicula affinis Eh.

- ambigua Eh.

- amphigomphus Eh.

- conimutata Grun.

- cuspidata Ktz.

- dactylus Eh.
Navicula firma Ktz.

- gentilis Donk.

- gibba Eh.

- iridis Eh.

- major Eh.

- mesolepta hitz.

- nobilis Eh.

- nodosa Ktz.

- serians kitz.

- transversa A. S.

-- viridis Ktz.

Stauroneis amphilepta Eh.

- mesopachya Eh.

- phonicenteron Eh.

Stenopterobia anceps Lewis.

Tabellariu fenestrata Kt $\mathrm{k}$. 
BIBLIOGRAPHIE. - Doct. Achille Forti. Diatomee delle farina fossile di Castel del Piano (Contribuzioni diatomologiche II). Atti del Reale Instituto Veneto di Scienze T. LVIII. Parte seconda.

Amphora afflnis Ktz.

Cymbella lanceolata Eh.

- cuspidata Ktz.

- cymbiformis Eh.

- subæqualis Grun.

- $\quad$ - v. florentina Grnn.

Cyclotella comta Kitz.

- operculata kit..

Diatoma hyemale Heib.

Eunotia arcus $E h$.

- major Rab.

- pectinalis Rab.

Epithemia argus v. amphicephala Grun.

$$
\text { - argus } K t z \text {. }
$$

Fragilaria construens Grun.

Gomphonema acuminatum $\nabla$. coronatum Eh.

- capitatum Eh.

Navicula affinis Ell.

- cardinalis W. Sm.

- major hitz.

- mesolepta Eh.

- nobilis Eh.

- radiosa $\mathrm{Ktz}$.

- stauroptera Grun.

- undulata Grun.

- viridis $\mathrm{K} t z$.

- - v. commutata Grun.

- viridula $K t z$.

Ropalodia gibba O. M.

- parallela $O . M$.

- ventricosa $O \mathrm{M}$.

Stauroneis anceps Eh.

- phcenicenteron Eh.

Synedra capitata Eh.

- ulna Eh.

- - v. longissima W. Sm.

Tabellaria fenestrata Ktz.

- floculosa $\mathrm{Ktz}$.

\section{Lac de Cazaux. - Cironde (France)}

\section{Note sur les Diatomées de Cazaux}

Ces Diatomées proviennent de sondages faits par MM. Lalesque et Bergon dans le lac de Cazaux, au sud d'Arcachon.

Ce lac appartient à la série des lacs littoraux des Landes de Gascogne; cés lacs, sauf le bassin d'Arcachon qui communique à la mer, sont des lacs d'eau douce. 
Or, en sondant dans la fosse profonde de Cazaux, eutre 24 et 25 mètres de fond, MM. Lalesque et Bergon ont été surpris de trouver ce fond constitué par une vase à Diatomées franchement marines, mortes bien entendu.

Cette découverte jette un jour nouveau dans la question controversée jusqu'alors de la formation de ces lacs. Ce sont d'anciens bassins marins qui se sont fermés à l'èpoque quaternaire, comme le bassin d'Arcachon a tendance à le faire de nos jours.

Après leur fermeture, l'eau salée a été peu à peu remplacée par l'eau douce. Le fond de la fosse est un témoin de cet état passé; tout autour le fond est constitué par des formes d'eau douce récentes et vivantes.

Ce dépôt quaternaire récent est d'ailleurs le même que celui que l'on découvre à l'état fossile en divers endroits des Landes et qui constitue les argiles anciennes du bassin d Arcachon qui découvrent avec les basses marées. Il diffère sensiblement du dépôt marin qui se forme aujourd'hui au fond du bassin d'Arcachon.

L'étang voisin de Parentis, moins profond, n'a donné que des Diatomées d'eau douce.

H. P.

\section{No 834 LAC DE CAZAUX - GIRONDE (France)}

\section{Diatomées récentes du lac}

Achnantidium flexellum Brẻb.

Amphora libyca Eh.

- ooalis fa minor.

- pediculus Grun.

Cocconeis placentula Eh.

Cyclotella comta $\mathrm{Ktz}$.

Cymbella americana A. S.

- - - var. acuta.

- amphicephala var. acuta n. 0 . Diffère du type par ses extrémités pointues au lieu de capitées.

- cuspidata Ktz.

- _ fa minor.

- helvetica kiz.

- maculata kitz.

- subrqualis Girun.

Diplonois elliptica Cl.

- fusca Cl.
Eucyonema gracile Rab.

Epithemia argus $\mathrm{Ktz}$.

- - var. amphicephala.

- gibberula $\mathrm{Ktz}$.

- sorex $\mathrm{Ktz}$.

Funotia arcus Eh.

- - var. tenella.

- - var. uncinata.

- flexuosa Ktz.

- formica Eh.

- impressa Eh.

- incisa Greg.

- $\quad$ - var. obtusiuscula.

- lunaris Grun.

- - - var. alpina.

Fragilaria virescens Ralls.

Gomphonema acuminatum Eh.

\begin{tabular}{lll}
- & - & var. coronata. \\
\hline & - & var. pusilla.
\end{tabular}


Gomphonema intricatum Kiz.

- - - var. pumila. subtile Eh.

Mastogloia baltica Grun.

- Grevillei W. Sm.

- lanceolata Thw. var.

- Smithii Thw.

- $\quad$ - var. amphicephala.

- _ var. intermedia.

- _ var, lacustris.

Melosira granulata Ralfs.

Navicula affinis var. amphirynchus.

- amphigomphus Eh.

- bisulcala Lag.

- commutata Grun.

- dactylus Kitz.

- divergens IV.Sm.

- dubia Eh.

- follis Eh.

- Gendrei Her. et M. Per.

- gibba Ktz.

- legumen Eh.

- limosa Ktz.

- major Ktz.

- mesolepta var. stauroneifor. mis.
Vavicula producta W. Sm.

- pupula Ktz.

- radiosa Kitz.

- _ var. acuta.

- rupestris Hantz.

- stomatophora Grun.

- subcapitata o. stauronezformis.

Nitzschia angustata $\mathrm{f}^{\mathrm{a}}$ minor.

IRhopalodia gibha O. M.

- - var, ventricosa.

Stauroneis anceps Eh.

- gracilis Eh.

- phœnicenteron Eh

Surirella apiculata $W . S m$.

- linearis W. Sm.

- robusta Eh.

- Saxonica Auers.

Synedra acus Ktz.

- delicatissima W. Sm.

- ulna Eh.

Tabellaria fenestrata Eh.

- flocculosa Eh.

Van Ileurckia crassinervia Bréb.

- vulgare V.H.

\section{No 835 LAC DE CAZAUX - GIRONDE (France)}

\section{Sondage fait à 22 mètres de profondeur}

Actinoptychus splendens Ralfs. vulgaris var.

Aulisens cœlatus Pail.

- intercedens v. major n. v. -

Diam. $130 \mu$, me parait être une valve de mégafrustule do I'A. colatus.

- rhipis o. triocellata.

- sculptus Ralfs.

- Sp. A. S. atl. 30/8. Me parait être une valve de mégafrustulo de l'A. sculptus, de même que la forme $\mathrm{A}$. mediterraneus (Per. Diat. M. de France $109 / 8$ serait le mégafrustule) de $\mathrm{A}$. reticulatus.
Biddulphia obtusa Ralfs. pulchella Gray.

Campylodiscus echeneis Eh.

Cerataulus turgidus W. Sm.

Cyclotella comta oar. radiosa.

Cymbella cistula Kirch.

- cuspidata Ktz.

- heloetica Ktz.

Diploneis bombus Eh.

- fusca Cl.

- interrupta Cl.

Endictya oceanica $\mathrm{Eh}$.

Epithemia argus $\mathrm{Ktz}$.

- - var. amphicephala. sorex $\mathrm{Ktz}$. 
Eunotia arcus Eh.

- - oar. uncinato.

- gracilis Eh.

- major Rab.

- monodon Eh.

Gomphonema acuminatum Els.
- - v. coronatum.
- elongatum W. Sm.
- $\quad$ intricatum Ktz.

Mastogloia Grevillei W. Sm.

Melosira granulata Ralfs.

- sulcata var. coronata.

- - var, radiata.

- undulata var. Normanii.

Navicula abrupta Donk.

- amphigomphus Eh.

- Baileyana Grun.

- commutata Grun.

- dactylus Ktz.

- divergens W. Sm.

- $\quad$ - var. elliptica.

- formosa Greg.

- gibba Ktz.

- gigas Ktz.

- hemiptera $\mathrm{Klz}$.

- humerosa Bréb.

- iridis Eh.

- Kamorthensis Grun.
Navicula legumen Eh.

- limosa Ktz.

- lyra Eh.

- $\quad$ - v. intermedia

- - v. - f elliptica.

- - v. - fa recta.

- major Kitz.

- $\quad$ - v. asymetrica.

- mesolepta Eh.

- nobilis Ktz.

- radiosa $\nabla$. acuta.

- stomatophora Grun.

- tuscula Eh. var.

- viridis $\mathrm{K} \mathrm{z}$.

Nitzschia angusta $\mathrm{f}^{\mathrm{a}}$ minor.

- scalaris Greg. var.

IRopalodia gibba O. M .

Scoliopleura tumida Rab.

Stauroneis anceps $E h$.

- gracilis Eh.

- ph凶enicenteron Eh.

Surirella elegans Eli.

- fastunsa Eh.

- robusta Eh.

Synerlra robusta Ralfs.

Terpsinoẻ americana Ralfs.

Triceratium antediluvianum Eh.

favus Eh.

Nos 836, 837 LAC DE CAZAUX - GIRONDE (France)

Sondage fait à 24 mètres de profondeur

Bibliogra PhIE. - P. Bergon. Liste des Diatomées marines du lac de Cazaux. (Bulletin de la Société de Géographie de Bordeaux, 15 sep. 1909, n० 9.)

Actinoptychus splendens Shadb. - undulatus Eh.

Amphora cingulata Cl.

Auliscus cœlatus Bail.

- $\quad$ - var. rhipis A. S.

- mediterraneus H. P.
Bıddulphia aurita Ag.

- pulchella Gray.

- rhombus IV Sm.

Campylodiscus echeneis Eh. - Thuretii Brẻb.

Cerataulus turgidus Eh. 
Cocconeis pseudo-marginata Greg.

Endictya oceanica Eh.

Grammatophora oceanica Eh.

$$
\text { - serpentina Eh. }
$$

IIyalodiscus stelliger Bail.

Melosira sulcata Eh.

$$
\text { - - var. coronata. }
$$

Navicula abrupta Greg.

-. Baileyana A. S. var.

- boinbus Eh.

- - var. gemina A. S.

- clavata Greg.

- didyma Eh.

- formosa Greg.

- Hennedyi W. Sm.

- humerosa Bréb.

- - var. constricta.

- lyra Eh. var. recta.

- Smithii Bréb. et var.
Vavicula Trevelyana Donk.

Vitzschia circumsuta Bail.

- navicularis Bréb.

- punctata W. Sm.

Orthoneis splendida Grun.

Pleurosigma formosum W. Sm.

Scoliopleura tumida Rab.

Surirella fastuosa Eh.

- hybrirla Grun. var. balticum J. Br.

- striatula Turp.

Synedra baculus Greg.

- crystallina Lyng.

Terpsinoë americana Ralfs.

Tracheneis aspera Eil.

Triceratium alternans W. Sm.

- favus Eh.

sculptum Shadb.

$N^{0} 838$

PRAZIES (Hongrie)

Cymbella nuoiculceformis Auers.

Comphónema angustatum $K$. v, proIucta.

Hantzschia amphioxys Grun. o. major:

Navicula appendiculata $K t_{\text {z }}$.

- dicephala El.

- elliptica $\nabla$. minor.

Navicula $l i m o s a-K t z$.

- major Ktz.

- rupestris Hantz.

Vitzschia Ileulleriana Grun.

Stauroneis phonicenteron Eh.

Surirella spiralis $K t z$.

No 839

MONACO (Méditerranée)

Sondage

Achnanthes longipes Ag.

Aetinocyelus Ralfsii oar. WV. Sm.

Actinoptychus splendens Ralfs.

$$
\text { - undulatus Eh. }
$$

Amphiprora elegans W. Sm.

- lepidoptera Greg.

- pusilla Greg.
Auphora crassa Greg.

- egregia Eh.

- obtusa Greg.

- spectabilis Greg.

Asterolaupra Grevillei v. adriatica Grun.

- Marylandica Eh.

Auliscus crelatus Bail. 
Auliscus sculptus Eh.

Bildulphia pulchella $K$.

$$
\begin{array}{ll}
\text { - } & \text { regina W. Sm. } \\
\text { - } & \text { tridentata El. }
\end{array}
$$

Campylodiscus adriaticus v. massiliensis.

- decorus Bréb.

- eximius Greg.

- - var. Briocensis.

- flumimensis Grun.

- lorologium var. mediterranea.

limbatus Bréb.

- $\quad$ samoensis Grun.

- Thuretii Bréb.

\section{Cerataulus Smithii Ralfs.}

Cocconeis pseudo-marginata Greg.

Coscinodiscus concavus Greg.

$\begin{array}{ll}\text { - } & \text { elegans Greo. } \\ \text { - } & \text { excentricus Eh. } \\ \text { - } & \text { marginatus Eh. } \\ \text { radiatus Eh. }\end{array}$

Grammatophora longissima P. P.

IIyalodiscus loeois $E h$.

- stelliger Bail.

Mastogloia quinquecostata Grun.

Melosira Westii W. Sm.

Navicula aspera Eh.

- Beyrichiana A. S.

- bomboïdes A. S.

- bombus Eh.

- Californica Grev. var.

- clavata Greg.

- - var. elongata H. P.

- crabro Eh.

- didyma Eh.

- excavata Greg. var.

- fusca Greg.

- gemina $\mathrm{Ktz}$.

- Hennedyi W. Sm.

- - var. niceænsis H. P.

- incus Grun.

- lyra Eh.

- - var. elliptica.
Navicula lyra var. subcarinata.

- - var. subtypica A: S.

- maxima Greg.

- multicostata Grun.

- pandura Bréb.

- Powellii Lewis.

- protexta Eh.

- Smithii Bréb.

Nitzschia notabilis Grun.

- panduriformis Greg.

- valida Cl. et Grun.

Orthoneis fimbriata Bright.

- splendida Ḱtz.

Pleurosigna affine Grun.

- balticum W. Sm.

- decorum W. Sm.

-1 formosum WV. Sm.

- rigidum IV. Sm.

- - var . giganteum

Grun.

- speciosum W. Sm.

Podosira adriatica Grun.

- hormoïdes $\mathrm{K}$ tz.

Rhalolonema adriaticum $\mathrm{Ktz}$.

Rhoicosigma oceanicum H. P.

- var . corsica-
num H. P. robustum Grun.

Surirella Baldjickii Norm.

- fastuosa $\mathrm{Eh}$.

- - var. abludens Grun.

- - var. constricta Grun.

- - - var. Guinardii H. P.

- - var. opulenta Grun.

- fluminensis Grun.

- intercedens Grun.

- lata W. Sm.

Synedra baculus Greg.

- fulgens W. Sm.

- robusta Ralfs.

- undulata Greg.

Triceratium antediluvianum Eh.

- arcticum Bright.

- favus Eh.

- $\quad$ spinosum Bright. 


\section{No 840 DUCK POND - MAINE (États-Unis)}

Cymbella Ehrenbergii Kítz.

Eunotia biceps Eh.

- formica Eh.

- gracilis Eh.

- incisa Greg.

- polyglyphis Eh.

- robusta Eh. et var.

- undulata Ralfs. .

Gomphonema acuminatum Ktz. - capitatum Eh.

Navicula arctissima $A . S$.

- Brebissonii var.

- dactylus Eh.

- dilatata Eh.

- gigas Eh.
'Navicula iridis Ktz.

- macilenta Grun.

- major Kíz.

- nobilis Eh.

- rhomboïdes var. Eh.

- viridis Eh.

Stauroneis gracilis Eh.

- phœnicenteron Eh.

Stenopterohia anceps Bréb.

- $\quad$ interınedia Brúb.

Surirella bifrons var. A. S.

- linearis W. Sm.

- - var. constricta.

- oblonga A. S.

\section{No 841 STONY CREEK - THUMBLE ISLANI)}

\section{(États-Unis)}

Actinoptychus undulatus Eh.

Amphiprora elegans W. Sm.

Cerataulus polymorphus Eh.

Coscinodiscus oculus-iridis Eh.

Melosira nummuloïdes $\mathrm{Ag}$.

Navicula formosa Greg.

- Kamorthensis Grun.

- Smithii Brèb.
Nitzschia obtusa W. Sm.

- scalaris W. Sm.

- $\quad$ sigma W. Sm.

- Tryblionella Grun.

Pleurosigma affine Grun.

- balticum W. Sm.

- decorum IV. Sm.

- elongatum W. Sm.

Scoliopleura tumida Bréb.

Triceratium favus Eh.

\section{$N^{\text {os }} 842,843$ SANTA LUCIA - CALIFORNIE (États-Unis)}

\section{Dépot fossile marin}

Actinocyclus Ehrenbergii Ralfs.

Actinoptychus areolatus Eh.
Actinoptychus Grundleri A. S.

Janischii A.S. 
Actinoptychus spinifer Grun.

$$
\text { - vulgaris Schum. }
$$

Arachnoiliseus Ehrenbergii Bail.

$$
\begin{aligned}
& \text { - } \\
& \text { - } \\
& \text { var. califor- } \\
& \text { nica. }
\end{aligned}
$$

Asteromphalus Darwinii Eh.

$$
\text { - variabilis Grev. }
$$

Aulacoriscus amcnus Grov.

Auliseus sculptus $E h$.

Biddulphia longicornis Grun. Tuomeyi Bail.

Campylouliscus ecclesianus Grev.

Campyloneis regalis Grun.

Chretoceros didymum Eh. hispidum Eh.

\section{Cocconeis costata Greg} - pseudo-marginata Greg.

Coscinoliscus asteromphalus Eh.

$\begin{array}{ll}- & \text { biradiatus Grev. } \\ - & \text { diorama A. S. } \\ - & \text { floridulus A. S. } \\ - & \text { lineatus Eh. } \\ - & \text { margiuatus Eh. } \\ - & \text { monica Ratt. } \\ - & \text { oculus-iridis Eh. } \\ - & \text { radiatus Elı. } \\ - & \text { robustus Eh. } \\ - & \text { suboculatus Rab. } \\ - & \text { subtilis Eh. }\end{array}$

Dicladia capreolus Eh.
Gepliyria constricta Arn.
- costata Arn.

Grammatophora oalida Dipp.

IIemiaulus polymorphus Grun. llyalodiscug radiatus var. O. Meara.

Litholesmium minusculum Grun.

Melosira clavigera Grun.

$$
\text { - sol Eh. }
$$

Navicula crabro $E h$.

- prætexta Eh.

- spectabilis Grev.

Orthoneis splendida Grun.

Podosira hormoïdes Mont.

Pterotheca subulata Grun.

Rhablonema adriaticum Iitz.

Rhizosolenia setigera Bright.

$$
\text { . T styliformis Bright. }
$$

Rutilaria hexagona Grun.

Stephanopyxis corona Eh.

$$
\begin{array}{ll}
\text { - } & \text { Grunowii Gr.et St. } \\
\text { - } & \text { limbata Eh. } \\
& \text { turris Eh. }
\end{array}
$$

Stictoriscus californicus Grev.

Triceratium" Montereyi Bright.

- primordialis $J$. Br.

- quadraingulare Bright. oalidum Grun.

\section{Nos 844,845 LOS ANGELES - CALIFORNIE (États-Unis)}

\section{Dépôt fossile marin}

Actinocyclus ingens Ratt. Actinoptychus undulatus Eh.

Aulacodiscus affinis Grun. - Oregrnus Bail.

Auliscus elegans Greo.

- Hardmannianus Greo.

- mirabilis Greo.

$\rightarrow$ pruinosus Bail.
Auliscus punctatus Baii.

$$
\begin{aligned}
& \text { - - var. striolata. } \\
& \text { - subreticulatus Ratt. } \\
& \text { - - var. decipiens. } \\
& \text { - subspeciosus Ratt. }
\end{aligned}
$$

Coscinorliscus angusta lineatus A. S. - apiculatus Eh. argus Eh. 
Coscinodiscus asteromphalus Eh.

$$
-
$$$$
\text { - centralis Eh. }
$$$$
\text { - curvatulus Grun. }
$$$$
\text { - elegans Grev. }
$$$$
\text { - excentricus Eh. }
$$$$
\text { - exustus Ratt. }
$$$$
\text { - timbriatus Eh. }
$$$$
\text { - gigas Eh. }
$$$$
\text { - griseus Grev. }
$$$$
\text { - leptopus Grun. }
$$$$
\text { - Letoisianus Greo. }
$$$$
\text { - marginatus Eh. }
$$$$
\text { - nobilis Grun. }
$$

No 846
Coscinodiscus oculus-iridis Eh.

omphalanthus Eh. radiatus $\mathrm{Eh}$.

$-$

- radiosus Grun.

- subtilis Eh.

Fupodiscus oculatus Greo.

IIeliopelta nitida Greo.

Navicula angelorum $\mathrm{Cl}$.

Stephanopyxis limbata $E h$.

Stictodiscus californicus Grev.

Xanthiopyxis umbonatus Grev.
Aetinocyclns Ralfsii W. Sm. Actinoptychus undulatus Eh.

Amphora angulata $\mathrm{Cl}$.

Auliscus cœlatus Bail. - sculptus Ralfs.

Bidldulphia aurita Brèb.

Coscinodiscus excentricus Eh. - radiatus Eh.

Epithemia musculus $\mathrm{K} t z$.

Hyalouliscus stelliger Bail.

Melosira sulcata K'tz.

Navicula abrupta Greg.

$$
\begin{aligned}
& \text { - apis Donk. } \\
& \text { - aspera Eh. } \\
& \text { - bomboïdes A. S. } \\
& \text { bombus Eh. }
\end{aligned}
$$

GREBESTADT (Suède)

\section{Sondage}
Vavicula crabro Eh.
- didyma Kitz.
- entomon Eh.
- Hennedyi W. Sm.
- humerosa Bréb.
- lyra Eh.
- - var. atlantica A.S.
- _ - var. elliptica A. S.
- - var. recta A. S.
- maximia Greg.
- - var. bicuneata.
- Smithii Brèb.
- Trevelyana Donk. .
Pleurosigma balticum WV. Sm.
Surirella fastuosa Eh.
Synedra baculus Eh.
- undulata Greg.
Triceratium antediluvianum Eh.


$\mathrm{N}^{\circ} 847$ LA ROCHELLE - CHARENTE-INFÉRIEURE

\section{(France)}

\section{Lavage de Pectens}

Actinocyclus Ralfsii W. Sm.

Actinoptychus splendens Shad.

$$
\text { - - var. halionyx. }
$$

Biddulphia pulchella Gray. rhombus W. Sm.

\section{Cerataulus Smithii Ralfs.}

Coscinodiscus centralis $\mathrm{Eh}$.

$\begin{array}{ll}\text { - } & \text { concavus Greg. } \\ \text { - } & \text { eoncinnus } W . S m . \\ \text { - } & \text { oculus-iridis Eh. } \\ \text { - } & \text { radiatus Eh. }\end{array}$

Grammatophora serpentina Eh. Ayalodiscus stelliger Bail.

Navicula aspera $\mathrm{Eh}$.

- crabro Eh.

- Smithii Bréb.

Nitzschia spectabilis Ralfs

Pleurosigma rigidum W. Sm.

- balticum IV. Sm.

Rhablonema adriaticum Ktz.

Rhoicosigma oceanicum H. P.

Scoljopleura tumida Grun.

Triceratium antediluvianum V. H. - faous Eh.
Actinocyclus Ehrenbergii Ralfs.

- - var. sparsa.

- incertus Grun.

- moniliformis Ralfs.

Actinoptychus areolatus Ralfs.

- undulatus Eh.

- vulgaris Sclium.

Amphora libyca Eh.

- ooalis Ktz.

Chatoceros gastridium Eh

- . incurvum Bail.

Coscinodiscus cen!ralis Eh.

$\begin{array}{ll}- & \text { concinnus W. Sm. } \\ - & \text { curoatulus Grun. } \\ \text { - } & \text { devius A. S. } \\ \text { - } & \text { excentricus Eh. } \\ \text { - } & \text { Ianischii A. S. } \\ & \text { lineatus Eh. }\end{array}$

Coscinodiscus oculus-iridis Eh.

$$
\text { - radiatus Eh. }
$$$$
\text { - subtilis Eh. }
$$

Cymatopleura apiculata W.Sm. - $\quad$ elliptica W. Sm.

Cymbell heteropleura o. minor.

- Hantsschiatica Grun.

- lanceolata Kirch.

- maculata Ktz.

Dicladia capreolus Eh.

Diploneis elliptica $C l$.

- interrupta $C l$.

- Smithii $C l$.

Epithemia rebra $K t$.

Eunotia arcus $E h$.

- bidens Eh.

- monodon Eh.

Fragilaria oceanica Cl. 
Gomphonema constrictum Eh.

- - paroulum Kts.

- vibrio Eh.

Isthmia enerois Eh.

Lichnophora Ehrenbergii?

Melosira granulata Ralfs.

- varians $\mathrm{Ag}$.

Navicala amphirynchus Eh.

- cuspidata Ktz.

- esox Eh.

- Fischerii A. S

- iridis Eh.

- limoza Ktz.

- mesolepta o. stauroneiformis.

- microstauron Eh.

- perigrina Ktz.
Navicula radiosa $\mathrm{K} t z$.

- scandinavica Lag. var.

Pleurosignıa strigilis W. Sm.

Pleurostauron $a c u t a R a b$.

Pseuílo-eunotia doliolus Grun. var.

Rhoicosphenia curoata o. marina.

Stephanopyxis frunowii Gr. et St.

- turris El.

Surirella bifrons Eh.

Syndendrium diadema Eh.

Synedra capitata $E h$.

- erystallina Ktz.

- ulna Eh.

- $\quad$ - v. rostrata.

\section{No 849 WHitTE PLAINE - NEVAADA (États-Unis)}

Dépôt fossile marin

Coscinodiscus Rothii Grun. var.

Cette forme semble intermédiaire entre les Cosc. Rothii et polyacanthus .qui ne sont que des variétés du Cosc. subtilis Eh.

$\mathrm{N}^{\circ} 850$ MEINRADSBRUNNEN -. ETZEL (Suisse)

Achnanthes minutissima v. cryptocephala. Mericlion circulare $K t z$.

Syuedra minuscula dirun. (V. H. Syn. 39/13.)
Synedra minuscula var, undulata $\mathbf{v}, \mathbf{n}$. à face valvaire à bords ondulés. 
Nos $85 \check{l}$ à 853

\section{KERTSCH (Russie) \\ Dépô fossile saumâtre}

Bibliographie. - Dr. J. Pantocsek. Die Bacillarien des Klebschiefers von Kertsch (Verhandlungen der Kaiserlichen Russischen mineralogischen Gesellschaft zu St Petersburg. ZweiteSerie, Band XXXIX, $\mathrm{N} \cdot 2$.

Achnanthes Athenais Dant.

$\begin{array}{ll}\text { - } & \text { - } \quad \text { v. minor. } \\ \text { - } & \text { exigua Pant. } \\ \text { - } & \text { - var staurophora. } \\ \text { - } & \text { Kertschiana Pant. } \\ \text { - } & \text { longipes Ag. v. fossilis Pant. } \\ \text { - } & \text { tenuissima Pant. } \\ \text { - } & \text { vasta Pant. } \\ \text { vernalis Pant. }\end{array}$

Amphiprora hyalina Pant.

Amphora acutiuscula Ktz. v. fossilis Pant.

$$
\begin{aligned}
& \text { - } \quad \text { Granuncta Pant. } \\
& \text { - Kertschiana Pant. } \\
& \text { - mæotica Pant. } \\
& \text { - Marchesettiana Pant. } \\
& \text { - } \quad \text { revirescens Pant. } \\
& \text { - } \text { russica Pant. } \\
& \text { - Tithoniana Pant. } \\
& \text { Tomassiniana Pant. }
\end{aligned}
$$

Cocconeis hyalina Pant.

- placentula Eh. v. fossilis.

- sarmatica Pant.

Epithemia Anastasiæ Pant.

$$
\begin{aligned}
& \text { - } \quad \text { arcuata Pant. } \\
& \text { - } \quad \text { - veopatræ Pant. } \\
& \text { - } \text { geminata Pant. } \\
& \text { - mæotica Pant. } \\
& \text { - perinsignis Pant. } \\
& \text { - Schüttiana Pant. }
\end{aligned}
$$

Fragilaria acutiuscula Pant.

$$
\text { - } \quad \text { qualis Heib. v. mæotica. }
$$

Licmophora divergens Pant.

$$
\text { - Ehronbergii Grun. v. mæo- }
$$

Mastogloia Andrussowii Pant.

Melosira granulata Eh. v. mæotica.

Navicula Adami Pant.

- Andrussowii Pant.

- elliptica Ktz. v. mæotica.

- exigua Pant.

- hyalina Donk. $\quad$. fossilis.

- intacta Pant.

- ineomperta $\mathrm{v}$. fossilis.

- Josephi Pant.

- jucunda Punt.

- Kertschiana Pant

- Leonis Pant.

- mæotica Pant.

- mucronula Pant.

- Nicolai Pant.

- Olgæ Pant.

- paleacea Pant.

- Romanowii Pant.

- Sancti-Garoli Pant.

- Tithonia Pant.

- Ursina Pant.

- Zichyi Pant.

Nitzschia debilis Pant.

- granulata Grun. - mæotica.

- Lahusenii Pant.

- mæotica Pant.

- obtusa W. Sm. v. fossilis.

- Romanowiana Pant.

- Stockmayeri Pant.

- vermicularis W.Sm.v. mæotica

Pleurosigma balticum W. Sm. v. mæoticum. mæoticum Pant.

Scoliopleura mæotica Pant. 
Senıeyia mæotica Pant.

Stauroneis mæotica Pant.

- - $\quad$ - minor.
Surirella mæotica Pant. Synedra mæotica Pant.

- - $\quad$ v. minor.

№ 854

\section{SHEENE - ABERDEEN (Ecosse)}

Dépôt fossile d'eau douce

Amphora ovalis $\mathrm{Ktz}$.

Cyclotella affinis $\mathrm{Ktz}$.

$\begin{array}{ll}\text { - } & \text { cuspiclata Kitz. } \\ \text { - } & \text { eymbiformis Bréb. } \\ \text { - } & \text { Ehrenbergii Ktz. } \\ \text { - } & \text { gracilis Eh. } \\ \text { lancenlatum J. Br. }\end{array}$

Epithemia turgida Eh.

Eunotia arcus Eh.

-- gracilis Rab.

- linearis Grun.

- major Rab.

- pectinalis Rab

- pectinalis v. undulata Ralfs.

- - var. ventricosa Grun.

- serra Eh.

- tetraodon Eh.

Gomphonema acuminatum Eh.

- capitatum Eh.

Melosira lyrata Grun.

Navicula ambigua $\mathrm{Ktz}$.

- - var. craticula.

- amphigomphus var.

- amphirynchus Eh.

- bacillum Eh.
Novicula bicapitata Lag.

- brevistriata.

- cuspidata Ktz.

- gibba Ktz.

- iridis Eh.

- lata Ktz.

- limosa v. alpina.

- major Eh.

- mesolepta Eh.

- mesostyla Eh.

- molaris Grun.

- nobilis Eh.

- oblonga Eh.

- pusilla v, alpina.

- serians Bréb.

- stauroptera Grun.

- tumida W.Sm.

- viridis Eh.

- viridula Rab.

- tabellaria kitz.

Stauroneis acuta W. Sm.

- anceps Eh.

- gracilis W. Sm.

- lanceolata Ktz.

- phœnicenteron El.

- platystoma Eh.

Tabellaria fenestrata Ktz.

$\mathrm{N} \cdot 855$ JONES VALLEY - CALIFORNIE (États-Uni's) Dépôt fossile d'eau douce

Cymbella americana A. S. var. cymbiformis Eh.

Gomphonema intricatum $\mathrm{Ktz}$.
Gomphonema Heideni I. S.

- - $\quad$ v. sparsistriata.

Melosira granulata var. jonensis Grun. 
No 856 CELLE - HANOVRE (Allemagne)

\section{Dépôt fossile d'eau douce}

Amphora ovalis $\mathrm{K} \mathrm{tz}$.

Cocconeis placentula Eh.

Cymbella cuspidata Kiz.

- cymbiformis Eh.

Epithemia turgida $\mathrm{Ktz}$.

- sorex Ktz.

- zebra Eh.

Encyonema cospitosum Ktz.
Encyonema ventricosum Ktz.

Fragilaria mutabilis Grun.

Gomphonema capitatum $\mathrm{Ktz}$.

Melosira crenulata $\mathrm{K} \iota z$.

Navicula placentula Eh.

Stephanopyxis astrea Grun.

var. spinulosa.

Synedra ulna Ktz.

\section{Nos 857, 858 WINCHESTER - MASSACHUSE'T'S}

\section{(Etats-Unis)}

\section{Dépôt fossile d'eau douce}

Amphora ovalis $\mathrm{K}(\mathrm{z}$.

Cocconeis placentula Eh. Cyclotella antiqua W. Sm.

Cymatopleura solea Eh.

Cymbella amphicephala Mæg.

- cuspidata Ktz.

- cymbiformis Eh.

- Ehrenbergii Ktz.

- gastroides Ktz.

- obtusa Greg.

- parva W. Sm.

Eucyonema cospitosum $\mathrm{K} \mathrm{tz}$.

- gracile Rab.

- turgidum Grun.

Bunotia bigibba Greg.

- gracilis Eh.

Fragllaria brevistriata $\nabla$. subacuta.

- construens $\nabla$. binodis.

- $\quad$ - $\quad$ v. venter.
Gomphonema acuminatum $\mathrm{Eh}$.

$$
\begin{array}{ll}
\text { - } & \multicolumn{1}{c}{\text { var. clavus. }} \\
\text { - } & \text { constrictum Eh. } \\
\text { - } & \text { coronatum Eh. } \\
\text { - } & \text { capitatum } \mathrm{Ktz} . \\
\text { subtile Eh. }
\end{array}
$$

Epithemia argus Ktz.

- gibba Ktz.

- Hyndmannii W. Sm.

- sorex Ktz.

- turgida Ktz.

- vertagus $\mathrm{Ktz}$.

- zebra Kitz.

- - var. proboscidea.

Navicula americana Eh.

- amphirynchus Eh.

- bacilliformis Grun.

- commutata Grun.

- - v. stauroneiformis.

- cuspidata Ktz. 
Navicula dubia Greg.

- firma Kitz.

- - $\quad$ - subampliata .

- gibba Eh.

- gigas Eh.

- legumen Eh.

- limosa et var. Ktz

- major Ktz.

- mesolepta Eh.

- $\quad$ - var. stauroneiformis.

- producta W. Sn.
Navicula radiosa Ktz.

- viridis $\mathrm{K} t z$.

- vulpina Ḱrz.

Stauroneis anceps Eh.

- gracilis Eh.

- phœnicenteron Eh.

Surirella splendida Greg.

Synedra capitata Eh.

- ulna Eh.

Tabellaria fenestrata litz.

\section{(Angleterre)}

\section{Dépôt fossile d'eau douce}

Cymbella arcus'Eh.

$$
\begin{array}{ll}
\text { - } & \text { cistula Hemp. } \\
\text { - } & \text { cuspidata Ktz. } \\
\text { - } & \text { gymbiformis Eh. } \\
\text { gastroïdes lítz. }
\end{array}
$$

Epithemia gibba Kitz.

$$
\begin{array}{ll}
\text { - } & \text { sorex Ktz. } \\
\text { - } & \text { turgida Ktz. }
\end{array}
$$

Eunotia arcus Eh.

- diadema Ralfs.

- forınica Elı.

Comphonema acuminatum Eh. - geminatum Eh.
IIantzschir elongata Grun.

Navicula biceps Greg.

- divergens WV.Sm.

- gentilis Donk.

- iridis Eh.

- limosa Kiz.

- major Ktz.

- nobilis Eh.

- viridis $\mathrm{Ktz}$.

Stauroneis phœnicenteron.Eh.

Surirella Baileyi Lawis.

- biseriata Bréb.

- robusta Eh.

Synedra ulna Ktz. 
Actinoptychus dilatatus Pant.

$\begin{array}{ll}\text { - } & \text { Moronensis car. } \\ \text { - } & \text { splendens Ralfs. } \\ \text { - } & \text { stella A. S. var. Thumii. } \\ & \text { undulata Eh. }\end{array}$

Auliscus puloinatus $\mathrm{Cl}$.

Bidilulphia Tuomeyi Fail.

Coscinodiscus crassus Bail.

-
$\overline{-}$ lineatus $E h$. marginatus Eh. var. suh. marginata. Moronensis Ratt. oculus-iridis Eh. robustus Eh.
Coscinodiscus tuberculatus var. Monicæ Grev.

Grammatophora stricta Eh. IIyalouliscus radiatus O'Meara.

Meloaira separanda A. S.

Periptera tetracladia $E$.

Stephanogonia actinoptychus Eh.

Stephanopyxis Barbadensis Grer.

$\begin{array}{ll}\text { - } & \text { corona Eh. } \\ \text { - } & \text { diadema Eh. } \\ \text { turris Eh. }\end{array}$

Xanthiopyxis umbonatus Greo.

Zyguceros quadricornis Grun.
Actinoptychus splendens Ralfs. var.

uiddulphia pulchella Gray. var.

Campylodiscus limbatus $\nabla$. chilensis Gr. Eupodiscus racliatus Bailey.

Vavicula aspera Eh.

- lyra var. recta.

- - var. subcarinata.

Nitzschia circumsuta Bail.
Pleurosigma balticum W. Sm.

- decorum W. Sm.

- formosum W. Sm.

Rhablonema adriaticum $\mathrm{Ktz}$.

Surirella comis $A$. $S$.

- Febigerii Lewis.

Triceratium Brookei Laud.

- favus Eh.

N" 8633

LA MARTINIQUE (Antilles)

Sur les algues

Biddulphia aurita Bréb.

- pulchella Gray.
Biddulplia rhombus W. Sm.

Campylodiscus ecclesianus Grev. 
Cerataulus polymorphus Grun.

- - var. minor.

Climacosphenia moniligera $\mathrm{Eh}$. ·

Coscinodiscus apiculatus Eh. var.

- excaoatus Eh.

- marginatus Eh.

Eupodiscus argus Eh.

Grammatophora marina Ktz, var.

Hyalodiscus stelliger Bail.

- subtilis Bail.

Isthmia capensis Grun.
Nitzschia panduriformis Greg.

Orthoneis binotata Roper.

$$
\text { - splendida Grun. }
$$

Pleurosigma balticum W. Sm. - rigidum W. $\mathrm{Sm}$.

Podosira Montagnei Ktz.

Rhabdonema adriaticum $\mathrm{Ktz}$.

- mirificum W. Sm

Synedra crystallina Ag.

- formosa Ktz.

Triferatium antediluvianum V. H. pentacrinus Wall.

No 864

PORT D'ARICA (Pérou)

\section{Sondage}

Actinoptychus splendens Ralfs. undulatus $\mathrm{Eh}$.

Asteromphalus Ralísianus Grev.

Biddulphia rhombus W. Sm. Roperiana Grev.

Coscinodiscus apiculatus Eh. var.

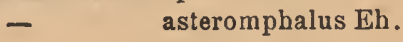

- concinnus W. Sm.

- conformis Ratt.

- excentricus Eh.

- Normanni Greg.

- obovatus Cast. v. circularis Ratt.
Coscinodiscus obscurus A. S.

- oculus-iridis Eh.

- odontodiscus Grun.

- perforatus Eh. var.

- Rothii Grun.

- $\quad$ spinuligerus Ratt. subtilis Eh.

Cresswellia turgida Grev.

Pleurosigma baiticum W. Sm.

- hippocampus W.Sm.

Surirella lata W. Sm. var.
Actinocyclus Ehrenbergii Ralfs.

Actinoptychus undulatus Eh.

Alloioneis antillarum $\mathrm{Cl}$. et $\mathrm{Gr}$.
Auliscus sculptus Eh.

Biddulphis Tuomeyi Bail.

Campylodiscus bicinctus Cast.

Daemelianus Grun.

Zebuanus Cast. 
Coscinodiscus argus Eh.

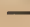

$-$

$-$

$-$

$-$

-

Cyclotella stylorum Bright.

Euodia gibúa Bail.

Iyalodiscus stelliger Bail.

Melosira sulcata Eh.

- - var coronata.

Navicula brasiliensis var (A. S 6/33).
Navicula crabro Eh.

- Kerguelensis Cast.

- lyra Eh.

- - var. elliptica.

- prætexta Eh.

- splendida Greg. var.

- Smithii Bréb.

Nitzschia cocconeiformis Grun.

- plana W. Sm.

Pleurosigua delicatulum W. Sm.

Surirella fastuosa Eh.

- - var. suborbicularis.

Triceratium favis Eh.

No 866 NORT'H TAUNTON près BRISTOL. NiASS. (États-Unis)

Dépôt fossile d'eau douce

Cocconeis placentula Eh.

Epithemia gibba Ktz.

- sorex Ktz.

- zebra Kitz.

Eunotia arcus Elı.

- Jiceps Eh.

- robusta Ralfs.

Gomphonema capitatum Eh.

Navicula crassinervia Bréb.
Vavicula gracillima Greg.

- gigas Eh.

- major Eh.

- radiosa $\mathrm{Ktz}$.

- serians Ktz.

- viridis $\mathrm{K} I \mathrm{z}$.

Stauroneis phenicenteron Eh.

Surirella robista Eh.

Tabellaria fenestrata Ktz.

\section{No 867 NEW-LONDON - IOWA (États-Unis).}

Cymbella cymbiformis Eh.

Eunotia major Rab.

- polyodon Eh.

Encyonema triangulum Eh.

Navicula ampliata $E h$.

- Brebissonii Ktz.
Navicula commutata Grun.

- firma K'tz.

- major Kitz.

- mesolepta v. stauroneiformis.

- rlıynchocephala Ktz.

- serians Ktz. 
Nitzechia linearis v, tenuis Grun.

Surirelle angusta $\mathrm{Ktz}$.

- apiculata W. Sm.

- minuta Bréb.
Stauroneis mesopachya Eh.

Synedra ulaa Eh.

Van IIeurckia vulgaris V. H.

No 868

HJERKIN DOVREFJELD

(Norvège)

Achnanthidium flexellum Brél).

Anomoeoneis serians Brẻb. $\nabla$.

Cymbella Cesati Rab.

- heteropleura Eh.

- naviculacea Grun.

Diploneis ovalis Hilse.

Encyonema hebridicum Greg.

- norvegicum Grun.

Eunotia denticula Bréb.

- gracilis Rab.

- major Rab.

- pentaglyphis Eh.

- prærupta Eh.

- triodon Eh.

Fragilaria unduta W. Sm.
Goniphonema montanum Schum. sagitta Schum.

Navicula hebes Ralrs.

- pupula Ktz.

Neidium bisulcatum $\mathrm{Cl}$.

Pinnularia divergens W. Sm .

- hemiptera $\mathrm{Ktz}$.

- mesogongyla Cl.

- stauroptera Grun.

- stomatophora Grun.

- viridis Eh.

- - var. minor.

Stauroneis anceps Eh.

- _ - var. linearis Grun.

- phœnicenteron Eh.
Achnanthe longipes Ag.

- subsessilis Ehr.

Actinocyclus Ehrenbergii Ralfs.

Campylodiscus bicostatus W. Sm.

$$
\text { - } \quad \text { clypeus Eh. }
$$

Cocconeis scutellum Eh.

Coscinodiscus asteromphalus Eh.

Diploneis Smithii Brẻb.

- didyma Eh.

Epithemia turgida v. Westermanii. Hyalodiscus scoticus Ktz.
Melosira Borreri Grev.

Navicula humerosa Brëb.

- latissima Greg.

- peregrina Elı.

Nitzschia punctata Grun.

Paralia marina $\mathrm{Cl}$.

Pleurosigma balticum Eh.

Rhabdonema arcuatum Ag. minutum $\mathrm{Ktz}$.

Surirella gemma Eh.

- striatula Turpin.

Synedra crystallina Lyngb. 
N• 870 GODFREY'S BOG - TAUNTON. MASS.

\section{(États-Unis)}

Cymbella cuspidata Ktz.

- heteroploura Ralfs.

Eanotia arcus Eh.

- biceps Eh.

- robusta v. dindema Ralfs.

- - v. hendecaodon.

Navicula affinis Eh.

- amphigomphus Ktz.

- acrosphæria Bréb. var:
Navicnla crassinervia Bréb.

- dilatata Eh.

- gentilis Donk.

- major Ktz.

- rhomboïdes $\mathrm{Ktz}$.

- viridis Eh.

Surirella robusta Eh.

Tabellaria fenestrata Ktz.

No 871 SÉVILLE - ANDALOUSIE (Espagne)

Dépôt fossile d'eau douce

BIBLIOGRAPHIE. - J. Deby. Le Diatomopelite de Séville (Journal de Micrographie, janvier 1884)

Cyclotella Sevilleana Deby. . | Cyclotella sexpunctata Deby.

$N^{\circ} 872$

SKIVE - JUTLAND (Danemark)

Dépôt fossile marin

Corinna elegans Heib.

Coscinodiscus oculus-iridis Eh.

$-$ subtilis Eh.

symbolophorus Eh.

Hemiaulus danicus Grun.

- Kittonii Grun.
Hemiaulus Weissii Grun. Odontotropis cristata Grun. Solium exsculptum Heib. Stephanopyxis turris Eh.

Trinacriu excavata Hoib. - regina Heib. 
N० 873 BLACK MOUNTAINS - BELFAST (Irlande)

Campylodiscus hibernicus Eh.

Eunotin gracilis W. Sm.

Navicula Brebissonii Ktz.

- commutata Grun.
Navicula lata W. Sm.

- major Ḱtz.

- viridis Ktz.

Surirella robusta Eh.

No 874 BELFAST - JARDIN BOTANIQUE (Irlande)

Anphora ovalis hitz.

Cynatopleura solea Eh.

hibernica $\mathbb{W} . \mathrm{Sm}$.
Nitzschia dubia IV.Sm. linearis W. Sm.

Stauroneis phonicenteron Eh.

Surirella biseriata Brèh.

\section{$\mathrm{N}^{\circ} 875$}

ILE DE MALTE (MéJiterranée)

Sur les algues

Achnanthes longipes Ag.

Actinocyclus Ehrenbergii Ralfs.

Actinoptychus splendens lialfs.

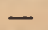
undulatus Eh.

Amphiprora lepidoptera Greg.

Aniphora arenaria Donk.

- costata W. Sm.

- crassa Greg.

- granulata Greg.

- lineata Greg.

- marina W. Sm.

- obtusa W. Sm.

Biddulphia pulchella Gray.

Bacillaria paradoxa Gmel.

Campylodiscus decorus Bréb.

$\begin{array}{ll}- & \text { Ralfsii W. Sm. } \\ - & \text { Samoensis Grun } \\ \text { - } & \text { Thuretii Bréb. }\end{array}$

Cerataulus Smithii Ralfs.

Cocconeis dirupta Greg.

$$
\begin{array}{ll}
- & -\quad \text { v. Ilexella. } \\
\text { - } & \text { Grevillei IV. Sm. } \\
\text { - } & \text { pseudo.marginata Greg. }
\end{array}
$$

Coscinodiscus concavus Greg.

$$
\begin{array}{ll}
\text { - } & \text { excentricus Eh. } \\
\text { - } & \text { nitidus Greg. } \\
& \text { radiatus Eh. }
\end{array}
$$

Epithemia musculus Kítz.

Grammatophora macilenta Eh.

$$
\text { - } \quad \text { marina Eh. }
$$

Homceocladia sigmoidea W. Sm. - Vidowichii Grun.

IIy alodiscus stelliger Bail.

Licmophora gracilis Eh. 
Mastogloia apiculata Grun.

- bisulcata $\nabla$. corsicana.

- ovata Grun.

- quinquecostata Grun.

Navicula apis Donk.

- aspera Eh.

- bombus Eh.

- crabro Eh.

- didyma Ktz.

- formosa Greg.

- fusca W. Sm.

- gemina Ktz.

- gemmatula Grun.

- Kamorthensis Grun.

- lineata Donk.

- littoralis Donk.

- lyra Eh.

- nitescens $\nabla$. stricta.

- notabilis v. expleta.

- pandura Bréb.

- pupula A.S.

- Smithii Bréb.

- splendida Greg.

- vetula A.S. var.

Nitzschia panduriformis Greg. - sigma W. Sm.
Nitzschia spathulifera Grun.

Orthoneis binotata Roper.

- splendida Grun.

Pleurosigma balticum W. Sm.

- decorum W. Sm.

rigidum W. Sm.

Podocyotis adriatica Grun.

Podosira ambigua Grun.

- hormoïdes Mont.

Pyxidicula mediterranea Grun.

Rhabdonema adriaticum Ktz.

Rhoicosigma oceanicum H.P.

Surirella fastuosa $\mathrm{Eh}$.

- gemma Eh.

Scoliopleura tumida Bréb.

Synedra affinis Ktz.

- baculus Greg. .

- formosa Ktz.

- fulgens W. Sm.

- robusta Ralfs.

- undulata Greg.

Triceratium antediluvianum Eh.

- Shadboldtianum Grev.

No 876

DUNEDIN (Nouvelle Zélande)

1)épot fossile d'eau douce

Encyonema attenuatum M. P. n. sp. Válve obèse à extrémités longuement atténuẻes, aiguës, aire centrale petite. Long. 25 à $40 \mu, 15$ stries en $10 \mu$.

rotundatum $H . P$. n. $s p$. Complètement arrondi,sans extrémités ; raphé pres- que rectiligne sans areas, nodules terminaux petits touchant le bord dorsal. Long. 15 à $30 \mu, 15$ stries en $10 \mu$.

Encyonema triangulum Grun.

Nota.- On observe les formes de passage entre ces trois espèces. 
$N^{\prime \prime s} 877,878$

BELFAST (Irlande)

Dépôt fossile d'eau douce

Anphora ovalis $\mathrm{K} / \mathrm{z}$.

- pediculus Grun.

Campyloriscus hibernicus Eh.

Cocconeis placentula Elı.

Cymatoplenra elliptica IV. Sm.

- - $\quad$ var.rlomboïdes.

- hibernica W. Sm.

Cyubella cuspidata $\mathrm{K} t \mathrm{z}$.

- cymbiformis Bı̀eb.

- Elirenbergii Ktz.

- gastroides $\mathrm{K} \mathbf{z}$.

- helvetica Ktz.

- lanceolata Kirch.

Diploneis elliptica Cl.

- - var.grandis.

Eucyonema ventricosum Grun.

Epithemia argus Ǩtz.

- $\quad$ - v. amphicephala.

- cistula Ralfs.

- Hyndmannii W. Sm.

- sorex Ḱtz.

- turgida Ktz.

- . - var. granulata.

- - var. vertagus.

- zebra Kitz.

- _ - fa minor.

Eunotia bidentula IV. Sm.

- gracilis W. Sm.

- minor V.II.

- parallela Eh.

Gomphonema acuminatum Eh.

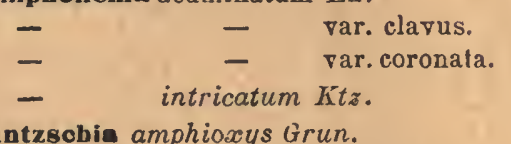

llantzscbia amphioxys Grun.
Melosira arenaria Moore.

Navicula amphirynchus $E h$.

- commutata Grun.

- cuspidala IItz.

- Fenzlii Grun.

- gigas $\mathrm{Ktz}$.

- hémiptera Kítz.

- iridis Eh.

- - var. ampliata.

- limosa lítz.

- - var. subundulata.

- major Kitz.

- mesolepta Eh.

- nobilis $\mathrm{K} t \mathrm{z}$.

- oblonga $\mathrm{Ktz}$

- peregrina $K t$.

- radiosa $\mathrm{Klz}$.

- viridis $\mathrm{K} t z$.

Pleurosigma attenuatum W. Sm. Pleurostauron acutum Rab.

IRbopalodia gibba $\mathrm{O} \mathrm{M}$.

- _ - var. parallela.

Stauroneis anceps Eh.

- Brunii M. Per.

- gracilis Eh.

- lanceolata K̇tz.

- phœnicenteron Eh.

Stephanodiscus astrae Grun.

- - $\quad$ v. spinulosa.

Surirella bifrons Eh.

- biseriata Brèb.

- elegans Eh.

- linearis W. Sm.

- - var. constricta.

- . - var. elliptica.

- tenera Greg.

- $\quad$ - var. splendidula. 


\section{N 879 MUSQUODOBOIT LAKE - NOVA SCOTIA (Canada)}

Dépôt fossile d'eau douce

Cyclotella Charetoni $\mathrm{H}$. et M. P.

Encyonema gracile Grun.

Eunotia bidentula W. Sm.

- gracilis Bréb.

- lunaris Grun.

- robusta Ralls. var.

Navicula amphigomphus Eh.

- Brebissanii Ktz

- - var, diminuta.

- commutata Grun.

- dactylus Ktz.

- gigas Kítz.

- heroina A. S.

- iridis Eh.

- macilenta Els.

- major ${ }^{-} \mathrm{Ktz}$.
Vavicula Paulensis Grun.

- rupestris Hantz.

- serians Eh.

- viridis $\mathrm{Ktz}$.

- sp. A. S. atlas 44/22.

Stauroneis anceps Eh.

- Baileyi Eh.

- lanceolata Klz.

Stenopterobia anceps Bréb.

Surirella bifrnns Eh.

- linearis W. Sm.

- - var. constricta.

- - var. elliptica.

- Rattrayi A. S.

- striatula Turp.

Van Ileurchia rhomboïdes Bréb.

No 880 DÓKUM BEDO - STAKE PLAINE. TEXAS (États-Unis)

Dépôt fossile d'eau douce

Amphora pediculus Grun.

Cocconeis placentula Eu.

Cymibella cistula Kirch.

- cymbiformis Bréb.

- obtusa Grun.

Diatoma tenie Ag.

Epithemia gibberula Kíz.

$$
\begin{array}{ll}
\text { - } & \text { Hyndmannii W. Sm. } \\
\text { - } & \text { turgilla Ktz. } \\
\text { - } & \text { zebra Ktz. } \\
\text { - } & \text { - far. granulata. } \\
\text { - } & \text { - var. proboscidea. }
\end{array}
$$

Eunotia major Rab.

Fragilaria bituminosa Pant.

$$
\begin{array}{ll}
\text { - } & \text { capucina Desm. } \\
\text { - } & \text { construens Grun. } \\
\text { - } & \text { intermedia Lirun. } \\
\text { - } & \text { mutabilis Grun. }
\end{array}
$$

Gomphonema angustatum Kiz.

$$
\begin{array}{ll}
\text { - } & \text { capitatum Eh. } \\
\text { - } & \text { intricatum Kt. } \\
\text { - } & \text { - var. pumila. }
\end{array}
$$

Melosira arenaria Moore. 
Melosira Camusi H. et M. P.

- - var. conica.

Navicula affinis $E h$.

- ambigua Eh.

- commutala Grun.

- cuspidata Ktz.

- dicephala Eh.

- divergens var.

- gastrum Eh.

- major Ktz.

- menisculus Schum.

- peregrina $\mathrm{Ktz}$.

- placentula $\mathrm{K} t z$.

- radiosa $\mathrm{Ktz}$.
Navicula rostellata $K \mathrm{tz}$.

- sculpta Eh.

- sphrerophora Ktz.

- styriaca Pant.

- viridis $\mathrm{Ktz}$.

Nitzschia sigmoïdea W. Sm.

Rhopalodia gibba $\mathrm{O}$. M.

Stauroneis amphilepta Eh.

- anceps Eh.

Syuedra biceps Ktz.

- capitata Eh.

- rostrata Eh.

- ulna Eh.

\section{N` 881 CONRAD'S RED BLUFF - CALIFORNIE (États-Unis)}

\section{Dépôt fossile d'eau douce}

Amphora libyca $\mathrm{Eh}$.

- ooalis Ktz.

Campylodiscus costatus W. Sm.

Cymbella Ehrenbergil ktz.

- gastroides $\mathrm{Ktz}$.

- obtusa Grun.

Diploneis elliptica $\mathrm{Cl}$.

Epithemia turgida v. granulata.

$$
\text { - zebra Ktz. }
$$

Bunotia gracilis W. Sm.

- impressa Eh.

Fragilaria virescens Ralfs.

Gomphonema acuminatum Eh.

$$
\begin{aligned}
& \text { - } \quad \text { apicatum Cl. } \\
& \text { - } \quad \text { constrictum v. capitata. }
\end{aligned}
$$

Hantzschia amphioxys Grun.

Melosira cienulata Ktz.

Navicula acrosphæria Bréb.

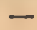

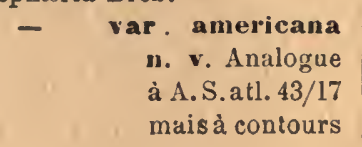

Navicula acrosphæria var. laevis.

- affinis Eh.

- amphigomphus Eh.

- bisulcata Lag.

- commutata Grun.

- dactylus K'tz.

- Dariana A.S.

- dicephala Eh. var.

- firma Ktz.

- hemiptera Ktz.

- heroina A. S.

- iridis Eh.

- - v. ampliata.

- major Ktz.

- mesolepta Eh.

- nobilis Ktz.

- rupestris Hantz.

- viridis Ktz.

Nitzschia sigmoidea $W$. Sm.

Stauroneis amphilepta Eb.

- gracilis Eh.

- phœnicenteron Eh.

Surirella bifrons $E h$. 
Surirella inducta A. S. Kittonii A. S.

- regina Jan.

- $\quad$ spiralis $K t z$.
Surirella tenera Greg.

- var. nervosa.

Synedra rostrata Eh.

- ulna var. æqualis.

No 882

\section{JONESPORT - MAINE (États-Unis)}

\section{Dépôt fossile d'eau douce}

Cymbella cuspidata Ktz.

- cymbiformis Bréb.

- delecta G. Sch.

- gastroides Ktz.

- heteropleura var. minor.

Encyonema ventricosum Grun.

Eunotia arcus Eh.

- gracilis W. Sm.

- lunaris Grun.

- major Rab.

- monodon Eh.

- pectinalis Rab.

- prærupta Eh.

- - - var. bidens.

- - var. bigibba.

- - var. influta.

- tetraodon Eh.

Gomphonema acuminatum Eh.

- $\quad$ capitatum Eh.

Navicula borealis Ktz.

- cincta var. Heutleri.

- commutata Grun.
Navicula divergens W. Sm. var.

- gibba Ktz.

- gigas Ktz.

- halophila var. craticula.

- lanceolata Ktz.

- major $\mathrm{K} \mathrm{tz}$.

- pachyptera Eh.

- rupestris Hantz.

- semen Eh.

- streptoraphe $\mathrm{Cl}$.

- viridis $\mathrm{Ktz}$.

Pleurostauron acuta Rab.

$\begin{array}{cc}\text { - } & \text { - var. major. } \\ \text { - } & \text { americana var. interme- } \\ \text { dia } \mathbf{n} . \text { var. De forme } \\ \text { intermediaire à celle du } \\ \text { Pl. Javanica. } \\ \text { - Javanica Grun. } \\ \text { - } \\ \text { J }\end{array}$

Stauroneis anceps Eh.

- mesopachya Eh.

Tabellaria fenestrata $\mathrm{K} \mathrm{tz}$.

No 883 BOWKERVILLE - NEW HAMPSHIRE

(Etats-Unis)

Dépôt fossile d'eau douce

Cymbella amphicephala Næg.

- cuspidata Ktz.
Encyonema Cesatii Rab. gracile Rab. 
Eunotia bidentula W. Sm.

- faba Grun.

- gibbosa V.H.

- gracilis Rab.

- incisa Greg.

- - var. obtusiuscula.

- major Rab.

- monodon Eh.

- pectinalis Eh.

- polyglyphis Eh.

- robusta Ralfs.

- tetraodon Eh.

- triodon Eh.

Fragilaria virescens Ralfs.

Gomphonema acurninatum v. coronata.

$\begin{array}{ll}\text { - } & \text { capitatum Eh. } \\ \text { - } & \text { intricatum Ktz. } \\ \text { - } & \text { tenellum Ktz. }\end{array}$

Melosira crenulata var. tonuis.

- distans Ktz.

- leavis Eh.

Navicula affinis $E h$.

- amphigomphus Eh.

- bicapitata var.

- commutata Grun.
Navicala cuspidata Ktz.

- esoculus Schum.

- gibba Ktz.

- heroina A. S.

- iridis Eh.

- - var. ampliata.

- lineolata Eh.

- major Ktz.

- mesolepta $\nabla$. stauroneiformis.

- microstauron Eh.

- terians Kı.

- - var. minor.

- trochus Eh.

- tumescens Grun.

Odontidium tabellaria W. Sm.

Pmeudo-Eunotia hemicyclus Grun.

Stauroneis Baileyi Eh.

- gracilis Eh.

- phonicenteron Eh.

Stephanolliscus astraea Grun.

Tabellaria fenestrata Ktz. - var. spinulosa.

- flocculosa $\mathrm{Ktz}$.

- venter Eh.

Van Ileurckia rhomboïdes Brẻb.

\section{No 884 HUDSON RIVER - NEW JERSEY (États-Unis)}

Amphora libyca Eh.

Biddulphia rhombus W. Sm.

Canpylodiscus costatus W. Sm.

\section{- $\quad$ echeneis Eh.}

Cocconeis lineata Eh.

- placentula Eh.

Coscinodiscus fasciculatus A. S. - subtilis Eh.

Cyclotella striata Grun.

Cymatopleura apiculata W. Sm.

- elliptica W. Sm.

- - var. constricta.

- - var. torta.

- $\quad$ solea W. Sm.
Cymbella Ehrenbergii Ktz.

- gastroides Ktz.

- Kamtschatica Grún.

- lanceolata Kirch.

- stomatophora Grun.

Encyonema prostratum Ralfs.

Epithemia turgida $\mathrm{Ktz}$.

- zebra Ktz.

Eunotia arcus Eh.

- - var. bidens.

- gracilis W. Sm.

- major Rab.

- - var. ventricosa.

- tetraodon Eh. 
Fragilaria crotonensis.

Gomphonema constrictum Eh.

Melosira crenulata $\mathrm{K} t \mathrm{z}$.

- varians $\mathbf{A g}$.

Navicula cuspidata $\mathrm{Ktz}$.

- Dariana A. S.

- dilatata Eh.

- firma $\mathrm{KIz}$.

- flammula A. S.

- fusca Ralfs.

- gigas Ktz.

- major $\mathrm{Ktz}$.

- nodosa Eh.

- peregrina litz.

- producta W. Sm.

- semen Eh.

- spharophora Ktz.

- viridis $\mathrm{Ktz}$.

Nitzschia apiculata Greg.

- circumsuta Grun.

- incurva Grun.

- linearis W. Sm.

- Schweinfurthii Grun.

- sigma v. sigmatella.

- sigmoidea W. Sm.
Nitzschia spectabilis Ralfs.

- Tryblionella Hantz.

Orthotropis lepidoptera Grun.

- - - var.proboscidea.

Plourosigma acuminatum Grun.

- attenuatum W. Sm.

Rhopaloulia gibba O. M.

Stauroneis anceps $E h$

- Baileyi Eh.

- phœnicenteron Eh.

Surirella bifrons Eh.

- - fa minor.

- cardinalis Kitt.

- crumena Bail.

- elegans Eh.

- Kittonii A. S.

- linearis W. Sm.

- ovalis Bréb.

- splendida Eh.

- tenera Greg.

Synedr: capilata Eh.

- ulna Eh.

Triceratlum alternans Bright.

\section{N॰ 885 WHITE LEAD LAKE - NEW JERSEY \\ (Etats-Unis)}

Dépôt fossile d'eau douce

Achnanthidium flexellum var. alpestris. Actinella punctata Grun.

Cymbella cuspidata Ktz.

- gastroides Ktz.

- heteropleura.

- - var. minor.

Bncyonema Cesatii Rab.

Eunotia arcus Eh.

- - var. plicata.

- bidentula W. Sm.

- gracilis W. Sm.

- impressa Eh.

- incisa var. obtusiuscula.
Eunotia faba Grun.

- lunaris Grun.

- - var. major.

- major Rab.

- pectinalis Rab.

- - var. undulata.

- - var. ventricosa.

- prærupta var. curta.

- robusta Ralfs.

Fragilaria binalis Eh.

Gomphonema acuminatum $\nabla$. coronata .

- capitatum Eh.

- intricatum. 
Melosira arenaria Moore.

- crenulata Ktz.

Navicula affinis $\mathrm{Eh}$.

- amphigomplus Eh.

- aponina $\mathrm{KIz}$.

- appendiculata Ktz.

- bicapitata $\nabla$. hybrida.

- Bogotensis var.

- Brebissonii Ktz.

- commutata Grun.

- divergens W. Sm.

- $\quad$ - var. elliptica.

- episcopalis $\mathrm{Cl}$.

- gibba Ktz.

- 'gigas $\mathrm{Ktz}$.

- hemiptera Ktz.

- heroina A. S.

- iridis Eh.

- legumen Eh.

- major litz.

- mesolepta $v$. stauroneiformis.

- molaris Grun.

- nobilis $\mathrm{Ktz}$.

- parva V. H.

- Paulensis Grun.

- producta W. Sm.
Navicula pseudo-bacillum Grun.

- pupula var.

- rupestris Hantz.

- serians Ktz.

- stomatophora Grun.

- sublinearis Grin.

- tabellaria Ktz.

- trochus Eh.

- tumescens Grun.

- viridis $\mathrm{Ktz}$.

Odontidium tabellaria W. Sm.

Pleurostauron productum Grun.

Pseudo-Eunotia hemicyclus Grun.

Stauroneis anceps Eh.

- Baileyi Eh.

- gracilis Eh.

- phonicenteron Eh.

Stenopterobia anceps Lowis.

Stephanodiscus astraea $v$. spinulosa.

Surirella linearis $W$. Sm.

$$
\text { - - var. constricla. }
$$

Tabellaria fenestrata Ktz.

- flocculosa Ktz.

- venter Eh.

Van Heurckia rhomboides Brél.

\section{No 886 FLORENCE - KANSAS (États-Unis)}

\section{Dépôt fossile d'eau douce}

Amphora affinis Ktz.

Cymbella cymbiformis Ktz.

- Ehrenbergii Ktz.

- lanceolata Eh.

- maculata W. Sm.

Cymatopleura solea Eh.

Epithemia gibba Eh.
Epithemia turgida Eh.

Navicula esox Eh.

- firma Ktz.

- limosa Ktz.

- major Ktz.

- radiosa $\mathrm{KI}$.

- - var. acuta.

- tuscula Grun. 


\section{No 887 ORONO RIVER - MAINE (États-Unis)}

\section{Dépôt fossile d'eau douce}

Achnanthidium flexellum Grun.

Cymbella amphicephala Næg.

- Ehrenbergii Ktz.

- maculata $\mathrm{Ktz}$.

Epithemia turgida $\mathrm{Ktz}$.

- zebra Ktz.

Eunotia arcus var. bidens.

- monodon Eh.

- pectinalis Rab.

- - var. biconstricta.

- - var. stricta.

- prærupta Grun.

- - var. bidens.

- tetraodon Eh.

Gomphonema angustatum Ktz.

- capitatum Eh.

- constrictum Eh.

- manubrium M. S.
Navicula biceps Eh.

- cuspidata Ktz.

- dactylus El.

- dilatata El.

- firma Eh.

- gentilis Donk.

- gibba Ktz.

- major Ktz.

- mesotepta Eh.

- $\quad$ - var. stauroneiformis

- pachyptera Eh.

- polyonca var. Bréb.

- termes var. stauroneiformis.

- viridis Ktz.

- - var. commutata

Nitzschia palea Ktz.

Stauroneis anceps Eh.

- phœnicenteron Eh.

Tabellaria flocculosa Ktz.
Cyclotella Meneghiniana Ktz.

Epithemia gibba Ktz.

$$
\begin{array}{ll}
\text { - } & \text { - var. ventricosa. } \\
\text { - Hyndmannii W. } \mathrm{Sm} . \\
\text { - } & \text { sorex Ktz. } \\
\text { - } & \text { turgida Ktz. }
\end{array}
$$

Navicula amphisbona Bory.

- elliptica Ktz.

- interrupta v. Tallyana Grun.
Navicula radiosa $\mathrm{Ktz}$.

- Reinhardtii Grun.

Nitzschia affinis Grun.

Rhoicosphenia curvata Grun.

Synedra affinis $\mathrm{K} t z$.

- pulchella Ktz. var.

- ulna Eh.

Tryblionella apiculata Greg. 
N• 889

Actinocyclus Ehrenbergii Ralfs.

- crassus W. Sm.

Actinoptychus undulatus Eh. splendens lialfs.

Auliscus sculptus Eh.

Bidldulphia rhombus W. Sm.

Campylodiscus echeneis El.

Cerataulus Smithii Ralfs.

Coscinodiscus centralis Eh.

fascicutatus A. S.

\section{MER DU NORD}

Sondage
Coscinodiscus oculus-iridis Eh.

radiatus Eh.

Eupodiscus argus Eh.

IIyalodiscus stelliger Bail.

Melosira sulcata Ktz.

Navicula Smithii Brèb.

Pleurosizma balticum W. Sm.

Podosira Smithiana Grun.

Scoliopleura tumida Eli.

Triceratium favus $\mathrm{Eh}$.
Amphora ovalis $\mathrm{K} t \mathrm{z}$.

Cocconeis placentula Eh.

Cyclotella striata o. ambigua Grun.

Cymatopleura elliptica Bréb.

$$
\text { - solea W. Sm. }
$$

Cymbella aspera Eh.

$$
\begin{array}{ll}
- & \text { cistula Hemp. } \\
\text { - } & \text { cuspidata Kiz. } \\
\text { - } & \text { Ehrenbergii Ktz. } \\
\text { - } & \text { Ianceolata Ḱtz. }
\end{array}
$$

Encyonema cospitosum Ktz.

$$
\text { - ventricosum Ktz. }
$$

Epithemla argus $\mathrm{Ktz}$.

$$
\begin{aligned}
& \text { - } \quad \text { gibba Ktz. } \\
& \text { - } \quad \text { turgida Ktz. } \\
& \text { - } \quad \text { zebra Eh. }
\end{aligned}
$$

Ennotia arcus Eh.

$$
\text { - incisa freg. }
$$

\section{Eunotia veltralis Eh.}

Gomphonema acuminatum Eh.

- constrictum Eh.

- $\quad$ - v. subcapitata.

- intricatum Kitz.

Melosira varians Ag.

Navicula ampliata $\mathrm{Eh}$.

- bacillum Eh.

- bisulcata Lag.

- cıspidáta Kitz.

- dnbia Greg.

- elliptica Ktz.

- gibba Eh. var.

- Hitchkockii Eh.

- limosa Grun.

- major Ktz.

- mesolepta Klz. v. slauroneifurmis.

- nobilis E.h.

- oblonga Ktz.

- peregrilla Eh. 
Navicula radiosa $\mathrm{K} 1 \mathrm{z}$.

- Smithii Brèb.

- transversa A. S.

- tuscula Eu.

- viridis Ktz.

- viridula Eh.

Nilzschia fluminensis Grun. var.

Pleurosigna attenuatum W. Sm. Spencerii W Sm.

Stauroneis acuta W. Sm

- amphilepta Éh. anceps Elh.
Stauroneis gracilis Eh.

- phœnicenteron El.

Surirella bifrons lítz.

- biseriata Bréh.

- elegans Eh.

- linearis WV. Smr.

- - rar. constricta

- robusta Brél.

- tenera Greg.

Synedra ulna Eh.

Talsellaria fenestrata Ktz.
Achnanthídium flẹxellum Bréb.

Anomoeoneis follis Eh.

- $\quad$ serians Bréb.

Cymbella cuspidata $\mathrm{kitz}$.

- gastroides Ktz.

- heteropleura Eh.

- naviculacea Grun.

Diploneis ovalis Hilse.

Eunotia arcus Eh.

- denticula Bréb.

- gracilis Eh.

- major.

- - var. bidens.

- robusta Ralfs.

- triodon Eb.
Frustulia crassinervia Bréb.

Navicula Hebes Ralfs.

Neidium bisulcatum Lag.

Pinnularia biceps Greg.

- dactylus Eh.

- divergens W. Su.

- lata Bréb.

- major Eh.

- mesogongyla Cl.

- streptoraphe Cl.

- virillis Eh.

- $\quad$ - var. intermedia Cl.

- $\quad$ - var. commutata Grun.

Staurotieis phoenicenteron Eh.

Tabellaria flocculosa $\mathrm{Ktz}$.
Achnanthes microcephala Grun.

Cymluella affinis hítz.

Diatoma tenue $\mathrm{Ag}$.

Fragilaria intermedia Grun.

- mutabilis Grun.
Gomphonema olivaceun Eh.

angustatum Ktz.

- intricatum Ktz.

Meridion circulare $\mathrm{Ag}$.

Synedra ulna Eh. 


\section{No 893 Vase entre BLANKENBERGHE et HEYST}

\section{(Belgique)}

Actinoptychus splendens Shadb.

$$
\text { - undulatus Eh. }
$$

Amphiprora pulchra Bail.

Biddulplua rhombus W. Sm.

\section{Campylodiscus clypeus Eh.}

Coscinodiscus concinnus $W$. Sm. var.

$$
\text { - } \quad \text { excentricus Eh. }
$$

Epithemia gibba $K t z$.

$$
\text { - } \quad \text { musculus Ktz. }
$$

Eupodiscus argus $E h$.

Ilantzschis virgata Grun.

Ilyalodiscus stelliger Bail.

Navicula elliptica $K t z$.

Nitzschia spectabilis Raifs.

Ruphoneis amphiceros Eh.

- Belgica o. intermedia Grun.
- $\quad$ var. rhombica.
auroneis salina W. Sm.

\section{No 894 NAARAJARVI - FINLANDE (Russie)}

\section{BIBLIOGRAPHIE. - Cleve. Diatomées de Finlande}

Cymbella cuspidata $\mathrm{Ktz}$.

Eunotia pectinalis v. biconstricta.

Fragilaria undafa W. Sm.

Hantzschia elongata Gruñ.

Melosira crenulata $\mathrm{Ktz}$.

Navicula americana Eh.

Neidium affine Eh.

- amphigomphum Eh.

- dilatatum Eh.

- Hitchcockii Eh.

Pinnularia biceps Greg.

- Brandelii Cl.

- brevicostata v. leptostauron CI.

- commutata Grun.

- dactylus Eh.
Pinnularia distinguenda $\mathrm{Cl}$.

- divergens W. Sm.

- episcopalis Cl.

- Esox Cl.

- gentilis Donk.

- isostauron Grun.

- legumen Eh.

- major Ktz.

- nobilis Cl.

- platycephala Eh.

- stauroptera Grun.

- transversa A. S.

- viridis $\nabla$. minor $\mathrm{Cl}$.

Stauroneis anceps Eh.

Surirella robusta Eh.

Tetracyclu lacustris Ralf. 


\section{No 895 VILLERS-SUR-MER - CALVADOS (France) \\ Vase saumâtre}

Amphora affinis $\mathrm{K} t \mathrm{z}$.

- ovalis KIz.

Bacillaria paradoxa Gmel.

Cyuntopleura solea Eh.

Epithenia gibba K/z.

- sorex Klz.

- zebra Elı.

Gomphonema constrictum Elı.
Navicula cuspidata $\mathrm{K} / \mathrm{z}$.

- peregrina Eu.

- sculpta Eh.

Nitzsehia acuminata IV. Sm.

- sigmoiden lih.

Pleurusigma Parkerii IIarr.

- Spencerii Arnolt.

N० 896

COTES DU MORBIHAN (France)

Lavage d'huitres

Achnanthes brevipes $\mathrm{Ag}$.

Actinopiychus undulatus Eh.

Amphiprore alata Eh.

Biddulphia pulchella Greg.

Cocconeis pellucida Eh.

$$
\text { -_ scutellum Eh. }
$$

Coscinodiscus excentricus Eh.

$$
\text { - radiatus Eh. }
$$

Epithemia musculus $K t$.

Grammatophora marina Grun.

$$
\text { - serpentina Eh. }
$$

Hyalodiscus radiatus O'Meara.

$$
\text { - } \quad \text { stelliger Bail. }
$$

ravicula apis Donk.

- aspera Eh.

- - var. minor.

- crabro Eh.

- cyprinus Eh.

- Hennedyi W. Sm.

- lyra Eh.
Navicula maxima Greg.

- sphcerophora Ktz.

Nitzschia acuminata Grun.

- insignis Grun.

- gigmoidea W. Su.

- socialis Greg.

Pleurosigma balticum W. Sm.

- formosum W. Sm.

- Spencerii W. Sm.

- strigosum W. Sm.

Rhabdonena minutun $\mathrm{Ktz}$.

Schizonema cruciferum IV. Sin.

- Greoillei Ag.

- ranosissinum AE.

Scoliopleura tumida kiél.

Stauroneis salina $\mathrm{W}$. Sm.

Surirella gemma $E h$.

- lepida A. S.

Synedra affinis $K t$. .

- baculus Greg.

- Gaillonii v. macilenta kh.

-_ undulata Grun. 
No 897 COLLIN HILL - BELFAST (Irlande)

Pinnularia lata W. 8m. = (Pinnularia pachyptera Eh.).

Achnantidium llexellum Bréb.

Anomoeoneis follis $\mathrm{Eh}$.

$$
\text { - serians Bréb. }
$$

Cymbella cistula Hemp.

$$
\text { - helvetica } \mathrm{Ktz} \text {. }
$$

Encyonena gracile Rab.

Epithemia argus $\mathrm{Ktz}$.

- gibba Kítz.

$$
\text { - gibberula Ktz. }
$$

Eunotia arcus Eh.

- bidentata W. Sm.

- gracilis Rab.

- incisa Greg.

- robusta Ralfs.

Frustulia crassinervia Bréb.

Gomphonema acuminatum Eh.

- constrictum Eh.

- geminatum Ag.

Navicula ambigua El.

- hełes Ralfs.
Navicula radiosa $\mathrm{Ktz}$.

- $\quad$ - vulpina Ktz.

Neidium affine Ell.

- bisulcatum Lag.

- iridis.

Nitzschia angustata Grun.

Pinnularia dactylus Eh.

- divergens W. Sm.

- legumen Eh.

- major Ktz.

- microstauron Eh.

- platycephala Eh.

- stomatophora Grun.

- stauroptera Grun.

- subsolaris Grun.

- undulata Greg.

Stauroneis phœnicenteron Eh.

Surirella robusta Eh.

Tabellaria fenestrata Ktz. 


\section{WARWICK (Ile de Rhode)}

\section{Dépôt fossile d'eau douce}

Cyclotella bodanica Eul.

$$
\text { - comta Eh. }
$$

Cymbella cymbiformis.

- Ehrenbergii Ktz.

Encyonema cospitosum $\mathrm{K} \mathbf{z}$.

Eunotia pectinalis.

- robusta $\mathrm{Eb}$. ventricosa.

Gomphonema acuminatum $\mathrm{Ktz}$.

Navicula dactylus.

- elliptica Ktz.
Navicula firma $\mathrm{Ktz}$.

- follis Eh.

- hebes Ralfs.

- iridis Eh.

- major Ktz.

- mesolepta Ktz.

- nobilis Eh.

- serians Ktz.

Surirella elegans Eh.

- robusta Eh.

stauroneis gracilis $\mathrm{Eh}$.

Tahellaria fenestrata $\mathrm{Ktz}$.

BIBLIOGRAPHIE. - Mitteilungen des Mikrologischen Vereins Linz-I.

Achnanthe exilis $\mathrm{Ktz}$.

- minutissima Ktz.

Achuanthidium flexellum Brèb.

Amphora enoculeata $M$. Per. ct $F$. $H$.

- gracilis Eh.

- libyca Eh.

- ovalis Ktz.

Cocconeis placentula Eh.

Cyclotella balatonis Pant.

- comta $\mathrm{Ktz}$ :

- Kützingiana Thw.

Cymatopleura elliptica W. Sm.

Cymbella affinis $\mathrm{K} t z$.

- cistula Kirch.

- cymbiformis Brél.
Cymliella delicatula Ktz.

- Ehrenbergii Ktz.

- helvetica Ktz.

- hungarica Pant.

- laevis Naeg.

- lanceolata'Kirch.

- obtusa Greg.

- parva V.H.

- pusilla Grun.

- scolica IV. Sm.

Denticula tenuis $\mathrm{K} \mathrm{z}$.

$$
\text { - } \quad \text { - var. frigida. }
$$

Diploneis elliptica Cl.

Encyonema cospitosum Kitz.

- ventricosum Grun. 
Fipithemia argus Ktz.

$$
\begin{aligned}
& \text { - } \quad \text { saxonica Kitz. } \\
& \text { - } \quad \text { sorex Ktz. } \\
& \text { zebra Ktz. }
\end{aligned}
$$

Funotia arcus Eh.

tiomphonema acuminatum v. coronatum.

$$
\begin{aligned}
& \text { - intricatum v. dichotoma. } \\
& \text { - } \quad \text { - } \quad \text { v. pulvinata. } \\
& \text { - } \quad \text { - v. pumila } \\
& \text { - subclavatum Grun. } \\
& \text { - subtile Eh. }
\end{aligned}
$$

Handmanniana austriaca M. Per

Meridion circulare Ag.

Navicula bisulcata Lag.

- Brebissonii Ktz. var.

- cryptocephala Ktz.

- Grma Kiz.

- $\quad$ - v. diminuta.

- - v. subundulata.

- Gothlandica Grun.

- iridis v. ampliata.
Vavicula limosa kitz.

- microcephala Grun.

- oblonga Ktz.

- peregrina Ktz.

- pupula Ktz.

- radiosa Ktz.

- $\quad$ - v. acuta.

- tenella Bréb.

- tuscula Eh. var.

Nitzschla angustata Grun.

- Heufteriana Grun. car.

Pleurosigma attenuatum W. Sm.

Rhopaloulia gibba o. ventricosa.

Stauroneis anceps Eh.

$$
\begin{array}{ll}
\text { - } & \text { - v. amphicephala. } \\
\text { - } & \text { - v. linearis. } \\
\text { - } & \text { gracilis Eh. } \\
\text { phcenicenteron Eh. }
\end{array}
$$

Surirella linearis $v$. constricta.

Tabellaria fenestrata Ktz.
Achnanthes lanceolata Brèb.

$$
\begin{aligned}
& \text { - } \quad \text { - v. elongata. } \\
& \text { - minutissima Kitz. } \\
& - \\
& \text { v. cryptoce- }
\end{aligned}
$$

Amphora gracilis Eh.

- Jibyea Eh.

- ovalis fa minor.

- pediculus Grun.

Coceoneis placentula Eh.

Cyclotella romta Kız.

- Kũtzingiana Thw.

- Meneghiniana Kítz.

- operculata Ktz.

Cymatopleura apiculala W. Sm. - solea W. Sm.

Cymbella affinis Kiz.

- cistula Kirch.

- gastroides Ktz.
Cymbella maculnta Ktz.

- subæqualis Grún.

Diatoma vulgare v. capitulatum.

Diploneis elliptica $\mathrm{Cl}$.

$$
\text { - } \quad \text { - } \quad \text { v. minutissima. }
$$

Encyonema ventricosum Grun.

Epithemia turgida Ktz.

Eunotia gracilis Rab.

- linearis Grun.

- - v. excisa.

Fragilaria capucina Desm.

- construens Grun.

- mutabilis Grun.

Gomphonema acuminatum Eh.

$$
\begin{array}{lcl}
- & - & \text { v. coronatum. } \\
\text { - } & - & \text { v. clavus. } \\
\text { - } & \text { angustatum Grun. } \\
\text { - } & - & \text { v. producta. }
\end{array}
$$


Gomphonema Brebissonii ktz.

-
-
-
-
-
-
commutatum Gr. constrictum Eh. molaris $\mathrm{Cl}$. mustela Eh. parvulum Grun. sarcophagus Greg. subclavatum Grun. elongatum W. Sm. car. exilissima.

Uantzschia amphioxys Grun.

Melosira distans $\nabla$. nivalis.

- varians $\mathrm{Ag}$.

Navicula bacilliformis Grun.

- Brebissonii Ktz.

- $\quad$ - v. curta.

- cincta $\nabla$. Heufleri.

- commutata lirun.

- dicephala W. Sm.

- divergens v. minor.

- lanceolata kitz.

- limosa Ktz.

- - v. signata.

- $\quad$ - v. subundulata.

- major Ktz.

- mesolepta v. stauroneiformis.

- oblonga Ktz.

- - v. curta.

Navicula pupula Ǩtz.

- radiosa Ktz.

- - - - acuta.

- rhyncocephala kitz.

- rupestris Hantz.

- sphærophora Ktz.

- ventricosa Ell.

- viridis $\mathrm{Ktz}$.

Nitzschia amphibia Grun.

- augustata Grun.

- commutata Grun.

- denticula Grun.

- frustulum Grun.

- linearis W. Sm.

- minuta Grun.

Opephora Martyi F. H.

Stauroneis amphilepta Eh.

- anceps Eh.

- $\quad$ - $\quad$. amphicephala.

- gracilis Eh.

- quadrata M. Per.

Surirella angusta Ktz.

Syneira acus $\mathrm{K} t z$.

- capitata Eh.

- tenera W. Sm.

- ulna v. danica.

- - v. rostrata.

- . - v. vitrea.

\section{No 903 HATFIELD SWAMPS - NEW-JERSEY (États-Unis)}

Achnanthes ventricosa Eh

Actinocyclus australis Grun.

\section{- Barkleyi Grun. var.}

Amphora ocalis $K t z$.

Cocconeis placentula Eh.

Cymatopleura elliptica IV. Sm.

- - $\quad$ - torta.

Cymbella cistula Kirch.

- cuspidata Ktz.

- Ehrenbergii v. minor.
Cymbella gastroiles $\mathrm{K} \mathrm{z}$.

- stomatophora Grun.

Diploneis interrupta $\mathbf{C l}$.

- Sinitlii Cl.

Epithemia amphicephala.

- turgida.

- $\quad$ - r. granulata.

- _ v. vertagus.

- zebra $\nabla$. proboscidea?

Bunotia arcus Eh.

- - P. uncinata. 
Eunotia formica Eh.

- gracilis fa major.

- major Rab.

- monodon Eh.

- pectınalis Rab.

- prærupta $\nabla$. bidens.

- tetraodon Eb.

Gomphonema apicatum $E h$.

- constrictum 0. capitatum.

- intricatum v. vibrio.

- montanum Schun.

-- $\quad$ - v. suecica.

II:utzschia amphioxys Gr. car.

Melosira crenulata $\mathrm{Ktz}$.

- semilaevis Grun.

- undulata $\mathrm{Ktz}$.

- - v. debilis.

- varians $\mathrm{Ag}$.

Navicula acrosphæria Brẻb.

- - $\quad$ v. minor.

- americana Eh.

- amphigomphus Eh.

- biceps A. S.

- cardinalicus Eh.

- commutata Grun.

- cuspidata Ktz.

- _ fa craticula.

- dactylus Kitz.

- Dariana A. S.

- divergens W. Sm. oar.

- esox var. (A. S. atlas 44/44).

- flexuosa Cl.

- gentilis Donk.

- gibba Ktz.

- - v. stauroneiformis.

- hemiptera Ktz.

- integra Grun.

- iridis Eh.

- $\quad$ - r. ampliata.
Na vicula lata Bréb.

- latevittata v. domingensis.

- legumen fa major.

- limosa Ktz.

- major Ktz.

- - v. asymetrica.

- mesolepta Ktz.

- nobilis $\mathrm{KIz}$.

- oblonga Ktz.

- polyonca Bréb.

- streptoraphe $\mathrm{Cl}$.

- viridis $\mathrm{Ktz}$.

- - v. minor.

Nitxschia circumsuta Grun.

- pJana W.Sm.

- sigmoidea W. Sm.

- tryblionella v. victoria

Plenrostauron aculum Rab.

Ifhopalodia gibba O. M.

- - $\quad$ v. ventricosa.

Stauroneis amphilepta Eh.

- Baileyi Eh.

- gracilis Eh.

- pliœnicenteron Eh.

- phyllodes Eh.

Surirella bifrons $\nabla$. minor.

- Cardinalis Kitt.

- cruciata A. S.

- elegans Eh.

- Kittonii A. S.

- linearis W.Sm.

- robusta Eh.

- splendida var.

- tenera Greg.

- - - var. splendidula.

Synedra ulna $\nabla$. danica:

Van Heurckia rhomboides $\nabla$. amphiplouroides.

\section{N॰904 SOUTH FRAMINGHAM - MADDISON C०}

(Etats-Unis)

Cymbella gastjoides Ktz.

Encyonema ventricosum Grun.
Epilliemia turgida var.

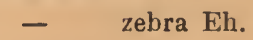


Eunotia arcus Eh.

$$
\begin{aligned}
& \text { - } \quad \text { - } \text { - uncinata. } \\
& \text { - gracilis Rab. } \\
& \text { - incisa Greg. } \\
& \text { - lunaris Grun. } \\
& \text { - major Rab. } \\
& \text { - minor V. H. } \\
& \text { - monodon Eh. } \\
& \text { - parallela var. } \\
& \text { - pectinalis Rab. } \\
& \text { - } \quad \text { - v. undulata. } \\
& \text { - prierupta Eh. } \\
& \text { - } \quad \text { - v. bidens. } \\
& \text { - } \quad \text { vetraodon Eh. }
\end{aligned}
$$

Ciouphonema acuminatum v. pusilla.

$\begin{array}{ll}\text { - } & \text { apicatum Eh. } \\ \text { - } & \text { constrictum v. capitata. } \\ \text { intricatum Ktz. } \\ \text { - } & -\quad \text { v. vibrio. } \\ \text { - } & \text { montanum Shum. } \\ \text { - } & \text { subclavatum Grun. }\end{array}$

\section{Melosira crenulate ktz.}

- $\quad$ - v. tenuis.

- undulata Ktz.

Merilton circulare Ag.
Vavicula acrosph'eria Bréb.

- americana Eh.

- bicapitata Lag.

- brevicostata v. interru: ta.

- commutata Grun.

- divergens W. Sm.

- gibba Ktz.

- hemiptera $\mathrm{Ktz}$.

- legumen Eh.

- major Ktz.

- - v. asymetrica.

- nobilis Kitz.

- Paulensis Grun.

- rupestris Hantz.

- semen Eh.

- streptoraphe Gl.

- subacuta Ralfs.

- tabellaria $\mathrm{Ktz}$.

- viridis $\mathrm{Ktz}$.

- - - . mimor.

Vituschia sigmoidea W. Sm.

Stauroneis Baileyi Eh.

- gracilis Eh.

- phœnicenteron Eh.

- phyllodes Eh.

- pteroidea Eh.

surirella splendida $E$.

Tabellaria fenestrata Kítz.

\section{No 905 BROCTON - MASSACHUSSE'TS (États-Unis)}

Cocconeis placentula Eh.

Cynlvefla acutiuscula Cl.

- americana. A. S.

- delecta A.S.

- Ehrenbergii o. minor.

- gastroides Kítz.

- heteropleura Kız.

Eunotia arcus Eh.

- - v. uncinata.

- formica Eb.

- incisa Greg.

- Iunaris Grun.

- major Rab.
Eunotia minor $\mathrm{V}$ H.

- monodon Eh.

- pectinalis Rab.

- $\quad$ - v. ventricosa.

- prærupta v. bidens.

- - v. curta.

- tetráodon Eh.

Encyonema ventricosum Grun.

Equithemia zebra var.

Gomphonema angustatum $\mathrm{K} t z$.
$-$ apicatum Eh.
- augur Eh.
- constrictum $v$. capitata. 
Gomphomemu intricatum $\mathrm{Ktz}$.

$$
\text { - } \quad \text { - } \quad \text { - v. pumila. }
$$

Navicula acrosphæria Bréb.

- americana Eh.

- amphigomphus Eh.

- amphirynchus Eh.

- bicapitata Lag.

- borealis Ktz.

- brevicostata Cl.

- commutata Gr.

- dicephala W. Sm.

- divergens W. Sm.

- dubia Eh.

- gentilis Donk.

- gibba Ktz.

- hemiptera $\mathrm{Ktz}$.

- instabilis A. S.

- integra Ralfs.
Navicula legumen Eh

- major $\mathrm{Ktz}$.

- nobilis $\mathrm{Ktz}$.

- pachyptera Eh.

- Paulensis Grun.

- rupestris Hautz.

- streptoraphe Gl

- tabellaria Ktz.

- termes A. S.

- transversa A S.

- viridis $\mathrm{Ktz}$.

Vitzschin sigmoidea W. Sm.

Stauroneis gracilis Eh.

- phœnicenteron Eh.

- phyllodes Eh.

surirella linearis W. Sm.

- splendida Eh.

\section{Nos 906-907 ESSEX - MASSACHUSSETTS (États-Unis)}

Achnanthioium minutum $\mathrm{Cl}$.

Cyclotelia comta $K t z$.

Encyonema.gracile Rab.

$$
\text { - rentricosum Grun. }
$$

Ennotia arcus Eh.

- - v. minor.

- - v. tenella.

- gracilis Rab.

- - v. major.

- impressa Greg.

- linearis Grun.

- minor V. H.

- pectinalis Rab.

- - v. undulata.

- polyodon.

- prærupta Eh.

- - $\quad$ - b. bidens.

- - $\quad$ v. curta.

- - $\mathbf{\text { v. inflata. }}$

- tetraodon Eh.

Fragilaria viresuens Ralfs.

$$
\text { - undata W. Sm. }
$$

Gomphovema intricatum Ktz.
Melosira granulata Ralfs.

- nivaiis W. Sm.

Meridion constrictum Ag.

Navicula acrosphæria Bréb.

- - v. minor.

- amphigomphus Eh.

- bisulcata Lag.

- Brebissonii $\mathrm{Ktz}$.

- brevicostata Cl.

- cincta $\nabla$. Heutleri.

- commutala Grun.

- decurrens A. S.

- dubia Eh.

- Essexii M. P. n. sp. Striation et contour de Nav. nodosa Eh. mais sans le renilement median. Long. $50 \mu, 7$ stries très courtes en $10 \mu$.

- frma Ktz.

- $\quad$ - v. subundulata.

- gentilis Klz.

- gibba Ktz.

- hemiptera Ktz. 


\section{Vavicula iridis Eh.}

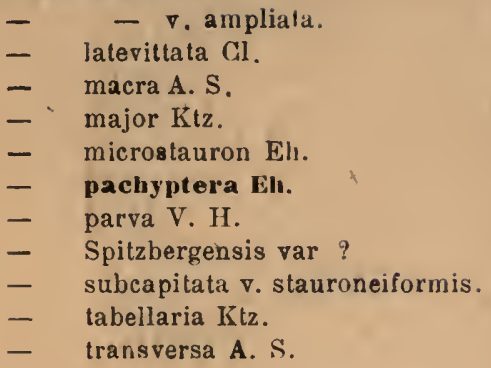

Navicula viridis $\mathrm{K} / \mathrm{z}$.

Pleurostauron acutum $v$. Ferryanum.

Stauroneis amphilepta Eh.

- Baiteyi Eh.

- gracilis Eh.

- phoenicenteron Eh.

- pteroidea Eh.

Tabellaria fenestrata Ktz.

- $\quad$ - v. intermedia.

- floceulosa Ktz.

Vau Heurckia rhomboides Bré̉.

$\mathrm{N}^{\text {os }}$ 908.909 SANTA TECLA près ACIREALE

(Sicile-Italie)

Dépôt fossile d'ean douce

\section{BIBLIOGRAPHIE. - G. Basile. L'Elefante fossile nel terreno vulcanico dell' Eina.}

Achnanthes linearis W. Sm

Amphora ovalis $\mathrm{Ktz}$.

- pediculus A.S.

Cocconeis placentula Eh.

Cyclotella operculata Ktz.

Epithemia sorex $\mathrm{Ktz}$.

- turgida Eh.

Fragilaria capucina Desm.

- construens Grun.

Gomphonema capitatum Eh.

- intricatum $\mathrm{Ktz}$.

Melosira crenulata Ḱtz.

- italica $\mathrm{Ktz}$.
Melosira varians Ag.

Navicula elliptica Krtz.

- oblonga Ktz.

- placentula Eh.

- sculpta Eh.

Nitzschia Brebissonii IV. Sm.

- denticula Rab.

- linearis W. Sm.

- sigmoidea W. Sm.

Pleurostauron Smithii Grun.

Rhoicosphenia curvata Grun.

Synedra acus Ktz.

- amphirynchus Eh. 
No 910

ORVIETO (Italie)

Dépôt fossile d'eau douce

Achnanthes lanceolata El.

Imphora affinis Ktz.

- ovalis Ktz.

- $\quad$ - v. gracilis V. H.

Campylodiscus costatus W. Sm.

Cocconeis placentula Eh.

Cymatopleura solea Bréb.

Cymbella cistula Hemp.

- cymbiformis $\mathrm{Ktz}$.

Epithemia argus Eh.

- gibba Ktz.

- $\quad$ - v. ventricosa.

- Hyndmannii W. Sm.

- ocellata Ktz.

- turgida Eh.

- $\quad$ - v. granulata.

- - v. proboscidea. $~$

- Westermannii Ktz.

Fragilaria capucina Desm.

Gomphonema constrictum v. subcapitatum.

- $\quad$ intricatum Ktz.

- $\quad$ - v. strictum.

Mastogloia Dansei Thw.

- Grevillei W. Sm.
Melosira italica Ktz.

Navicula elliptica $K t z$.

- firma Ktz.

- limosa Ktz.

- longa Ktz.

- neglecta Ktz.

- producta W. Sm.

- rliyncocephala Ktz.

- sculpta Eh.

Nitzschia Brebissonii W. Sm.

- palacea Grun.

Pinnularia oblonga Eh.

- viridis Eh.

- viridula $\mathrm{Kt}$.

Rhopalodia gibba O. M.

Rhoicosphenia curvata Giun.

Stauroneis aneeps v. linearis.

Surirella angusta $\mathrm{Ktz}$.

- ovalis Bréb.

- - v. minuta V.H.

Synedra longissima W. Sm.

- ulna Eh.

- $\quad$ - v. spathulifera Grun.

- $\quad$ - . splendens Rab.

\section{Nos 911.912 BAGNOLO - GROSSETO (Ital:}

Dépôt fossile d'eau douce

Amphora affinis Ktz.

$$
\text { ovalis } \mathrm{Ktz} \text {. }
$$

Asterionella gracillima Heib.

Cocconeis placentula Eh.

Cyclotella operculata Kı.
Cymacopleura solea W. Sm.

- _ - $\quad$ : elongata.

Cymbella cuspidata Ktz.

- cymbiformis Eh.

- gastroides $\mathrm{Ktz}$. 
Encyonema ventricosum $\mathrm{K} t \mathrm{z}$.

Epithemia argus $\mathrm{Ktz}$.

- sorex K̇z.

- tırgida Ktz.

Fragilaria capiucina $\nabla$. lanceolata.

- construens Grun.

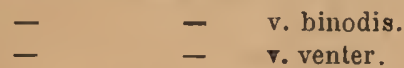

Gomphonema acuminatum Eh. - capitatum Eh.

Melosira crenulata Grun.

- varians Ag.

Meridion circulare Ag.

Varicula cuspidata v. primogena.

- firma Ktz.

- oblonga Ktz.

- radiosa v. acuta.
Pinnularia brevicostata Cl.

- major Ktz. var.

- mesolepta Eh.

- nobilis Eh.

- stauroptera Grun.

- viridis Eh.

Rhopalodia gibba O. $\mathrm{M}$.

- parallela O.M.

- ventricosa O. M.

Sauroneis constricta Eh.

- phoenicenteron El.

- robusta Eh.

Synedra capitata Eh.

- longissima W. Sm.

- $\quad$ - $\quad$. acicularis.

- ulna Eh.

- $\quad$ - v. danica.

$\mathrm{N}^{\circ 8}$ 913-914 SANTE-FIORA - GROSSETO (Italie)

Dépôt fossile d'eau douce

Amphora affinis $\mathrm{Ag}$.

- lybica Eh.

Cocconeis pediculus Eh.

- placentula Eh.

Cynatopleura solea W. Sm.

- _ - v. crassa Meister.

Cymbella affinis Ktz.

- v. elongata Moister.

- aspera $\mathrm{Eh}$.

- cuspidata Ktz.

- cymbiformis Ktz.

- Ehrenbergii Ktz.

- heteropleura fa minor.

- lanceolata Eh.

- subæqualis Grun.

Diatoma lyemale Heib.

Bunotia lunaris El.

- major Rab.

- parallela Eh.

- pectinalis Ktz.

- - $\quad$ - minor.
Epithemia argus Eh.

- sorex $\mathrm{Ktz}$.

- turgida Eh.

- - v. granulata.

- $\quad$ - v. Westermannii.

- zebra Ktz.

- - - v. proboscidea.

Fragilaria capucina Desm.

- construens Grun.

- $\quad$ - v. binodis.

- mutabilis Grun.

- virescens Ralis.

Gomphonema acuminatum Eh.

- $\quad$ augur Eh.

- Brebissonii Ktz.

- capitatum Grun.

- constrictum Eh.

- gracile Eh.

- truncatum Eh.

Melosira crenulata Ralfs.

- distans Ktz. 
Melosiru italica Ktx.

- varians Ag.

Navicula amphirynchus EL.

- amphigomphus Eh.

- bavillum Eh.

- cuspidata Ktz.

- divergens $\nabla$. elliptica.

- librile Eh.

- limosa $\mathrm{Ktz}$.

- peregrina Ktz.

- radiosa $K$ Ĺ.

- - v. acuta.

- sculpta Eh.

- tuscula Eh.

Pinnularia brevicostata $\mathrm{Cl}$.

$\begin{array}{ll}\text { - } & \text { cardinalis Eh. } \\ \text { - } & \text { commutata Grun. } \\ \text { - } & \text { dactylus Eh. } \\ \text { - } & \text { dicephala Eh. } \\ \text { - gentilis Cl. } \\ \text { - gibba Eh. } \\ \text { - } \quad \text { inæqualis Eh. }\end{array}$

Pinnularia legumen Eh.

$$
\begin{array}{ll}
\text { - major Rab. } \\
\text { - } \quad \text { mesogongyla Eh. } \\
\text { - } \quad \text { oblonilis Eh. } \\
\text { - } \quad \text { porrecta Eh. } \\
\text { - } \quad \text { viridis Eh. } \\
\text { - } \quad \text { viridula Rab. }
\end{array}
$$

Pleurosigma attenuatum W. Sm.

Rhopalodia gibba $\mathrm{O} . \mathrm{M}$.

- gibberula $\mathrm{O}, \mathrm{M}$.

Stauroneis anceps Eh.

- Baileyi Eh.

- Janceolata Ktz.

- plicenicenteron Eh.

Synedra capitata Eh.

- ulna Eh.

- - $\quad$. longissima.

- - $\quad$. oxyrynchus.

Tabellaria fenestrata $\mathrm{K} \mathrm{tz}$.

- flocculosa Ktz.
Bacterium varians Laud.

Chateceros angulatum Schûtt.

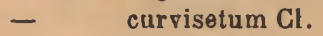

- decipiens Cl.

- pelagicus $\mathrm{Cl}$.

- perpusillum Cil.

- procerum Schütt.

- protuberans Laud.

- seiracanthum Grun.
Chretoceros teres Cil.

Dactiliosolen moditerraneus $\mathrm{H}$. Pel.

Ilemiaulus Hauckii Grun.

Rhizosolenia alata o. gracillima.

- setigera Bright.

Striatella unipunctata Ag.

Thalassionema nitzschioïdes.

Thalussiothrix Frauenfeldii. 


\section{$\mathrm{N}^{\circ} 916$}

\section{AJACCIO - CORSE (France)}

\section{Récolte pélagique}

\section{Bacterium varians Laul.}

Chretoceros angulatum Schütt.

- curvisetuen Schütt.

- decipiens Cl.

- perpusillum Cl.

- procerum Schütt.
Chretoceros protuberans Laud.

- Rulfsii Cl.

- Wollei Grun.

Dactiliosolen mediterraneus $H$. Yer.

Hemiaulus Hauckii Grun.

Rhizosolenia setigera Bright

No 917

AJACCIO - CORSE (France)

Récolte pélagique

Bacteriastrum elongatum $C l$. - varians Laud.

Chretoceros angulatum Schütt .

- curvisetum Cl.

- decipiens Gl.

- Lorenzianus Grun.

- perpusillum Gl.

- peruvianum Bright.

- procerum Schūtt.

- protuberans Laud.
Chretoceros teres Cl.

$$
\text { - Wollei Grun. }
$$

Dactiliosolen mediterraneus $H$. Per

Guinardia flaccida $H$. Per.

Ilemiaulus Hauckii Grun.

Nitzschia seriata v. fraudulenta.

Rhizosulenia alata $v$. gracillima.

- setigera Bright.

- Temperei II. Per.

Thalassionema nitzschioides Grun.
Cocconeis placentula Eh.

Epithemia sorex Ktz.

Melosira granulata Bréb.

Navicula amphirynchus Eh.

- cuspidata Ktz.

- peregrina $\mathrm{Ktz}$.
Navicula placentula Kt

- pupula Ktz.

Pinnularia nobilis Eh.

$$
\text { - viridis Rab. }
$$

Stauronei phœnicenterou Eh.

Synedra ulna Eh. 
Achnanthe ventricosa Eh.

Encyonema lunula Grun. val.

Eunotia diodon Eh.

- eruca Eh.

Gomphonema intricatum kitz. et var.

Hantzschla amphioxys Giun.

Melosira crenulata Kitz.

- _ var. valicla.
Navicula gibba Eh.

- hemiptera Eh. rar.

- major Ktz.

- parva Eh.

- viridis Ktz.

Stauroneis phuenicenturon Ell.

- scapulaformis Grev.

No 920

\section{PAKARAKA (Nouvelle-Zélande)}

Dépôt fossile d'eau douce

\section{Achnanthes inflata Grun.}

- lanceolata Bréb.

Eunotia prærupta $\nabla$. bidens Gruu.

Gomphonenia parvulum Ktz.
Melosira crenulata Pritch.

Navicula mutica v. Cobnii.

Vitzechia amphibia Grun.

- amphioxys W. Sm.

No 921 BRUNSWICK - VICTORIA (Australie)

Dépôt fossile d'eau douce

Aehnanthes lanceolata Bréb.

Ceratoneis arcus $\mathrm{Ktz}$.

Cymbella affinis Ktz.

Epithemia sorex $\mathrm{Ktz}$.
Epithemla zebra Eh.

Iragilaria construens Eh.

Srnedra ulna Eh. 
Cocconeis placentula Eh.

Encyonema ventricosum Grun .

Epithemia gibberula Ktz.

Eunotia impressa Grun.

- incequalis M. Per. n. sp. Analogue comme forme à l'Eunotia lunaris var. subarcuata, mais à extrémitès arrondies; la valve est dissymètrique, l'une des moitiés plus large que l'autre. Long. 30 à $50 \mu$, 17 stries en $10 \mu$.

- lunaris Grun.

- - var. alpina.

- - - var. major.

- - var. subarcuata.

Gomphonema angustatum $\mathrm{Ktz}$.

- Bergzrennii cl.

- intricatum o. pumila.

IIantzschia amphioxys Grun. .

Melosira crenulata $\mathrm{Ktz}$.
Melosira crenulata var. tenuis.

- nummuloïdes Ag.

- varians $\mathrm{Ag}$.

Meridion constrictum Ralfs.

Navicula borealis $\mathrm{Ktz}$.

- divergens var.

- gibba var.

- hemiptera Ktz.

- parva V.H.

- radiosa Ktz.

Stauroneis anceps Eh.

- phonicenteron Eh.

Surirella linearis v. constricta.

- minuta Bréb.

- ovalis Bréb.

- - var. Kotschyana.

- ovata Ktz.

- tenera Greg.

- - var. splendidula.

Van IIeurckia crassinervia Bréb.

vulgaris $\mathrm{V}$. $\mathrm{H}$.

$\mathrm{N}^{\text {os }}$ 923.924 KORAWARA (Nouvelle-Zélande)

Cymbella cuspidata var. $\mathrm{Ktz}$.

Gomphonema Berggrennii CI.

- commutatum Grun.

Melosira crenulata v. ambigua Grun.

Navicųla cuspidata Ktz.

Neidium affine $\mathrm{Cl}$.

Nitzschia spectabilis Ralfs.
Pinnularia brevistriata Girun.

- divergens W. Sm.

- episcopalis $\mathrm{Cl}$.

- major Ktz.

- stauroptera Grun.

- viridis $\mathrm{Ktz}$.

Stauroneis Frauenfeldii Grun.

- fulmen Eh. 


\section{No 925 BEACH HAVEN - NEW-JERSEY (États-Unis) Sondage d'un puits artésien}

Actinocyclus Ehrenbergii Ralfs. Actinoptychus cathedralis $\mathrm{J} . \mathrm{Br}$.

-

$-$

$-$

$-$

Cestodiscus Juhnsonianus Grev.

Coscinodiscus apiculatus Eh.

argus Eh.

$\overline{-}$

$\overline{-}$

$\overline{-}$

$-$

- excavatus Eh.

- fasciculatus A. S.

- interlineatus Ratt.

- marginatus Eh.

- nobilis Grun. var.

- obscurus A. S.

- oculus iridis Eh.

- perforatus Eh.

- radiatus El.

- robistus Grev.

- , subtilis Eh.
Eunotia prærupta Grun. rar.

Iiuodia Brightwellii Ralfs.

Eupodiscus Rogersii Eh.

Ilyalodiscus radiatus Bail.

Melosira sulcata Ktz.

- $\quad$ - var. coronata.

- Westii W. Sm. var.

Navicula crabro Eh. var.

- didyma kitz.

- Johnsoniana Grev.

- lyra Eh.

- _ Schultzei Kain.

Pleurosigum affine var. fossilis Grun.

Pseudauliscus radiatus Bail. - $\quad$ spinosus Ghristian.

Raphoneis amphiceros Eh. - scalaris Eh.

Stephanogonia actinoptychus Eh.

Stephanopyxis corona Eh.

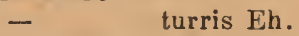

Triceratium americanum Eh. - Kainii Schultz.

\section{$\mathrm{N}^{\text {os }} 926$ à 929 GROTTE-GIRGEN'TI (Sicile-Italie) (1)}

\section{Dépôt marin tertiaire}

Actinocyclus Ehrenbergii Ralfs.

- Janischii Schum.

- moniliformis Ralfs.

- Ralfsii W. Sm. v. monicte.

Actinoptychus undulatus Eh. fa typica.

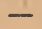

- v.parallelistria-

ta n. v. Forti.
Actinoptychus vilgaris Schum.

Amphora binodis Greg.

- crassa Greg. var. elongata Cl .

- $\quad$ - v. punctata Gr.

- proteus Greg.

- - var. oculata.

Asterolampra aculiloba Forti (6 radia).

(1) L'analyse de ce superbe dépôt a étẻ faito par le Dott. Achille Forti. 
Asterolampra Marylandica Eh.

punctifera (Grove) Forti. stellaris Temp. et $\mathrm{Br}$. var. curviradiata Forti.

Auliscus colatus Bail.

- $\quad$ - var. strigillata A. S.

Bacteriastrum varians Lauder.

Biddulphia palpebralis Forti.

- pulchella Gray.

- $\quad$ - var.moronensis (Azp.) Forti.

Tuomeyi Bail. fa margaritifera (Sliadb.) Forti.

Campylodiscus clypeus Eh. v. sicula Forti.

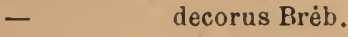

- ecclesianus Grev.

- horologium William.

- var. Plitze. rii A. S.

- punctulatus Grun.

- siculus Forti.

- $\quad$ simulans Greg. v. lemniscata Forti.

Chetoceros sp. (spora).

Cocconeis antiqua Temp. et Br.

- maxima Pant. v. septata Forti.

- Raeana Pant.

- scutellum Eh.

- - var. sicula Forti.

- vitrea J. Br.

Coscinodiscus apiculatus Eh. v. rginensis A. S.

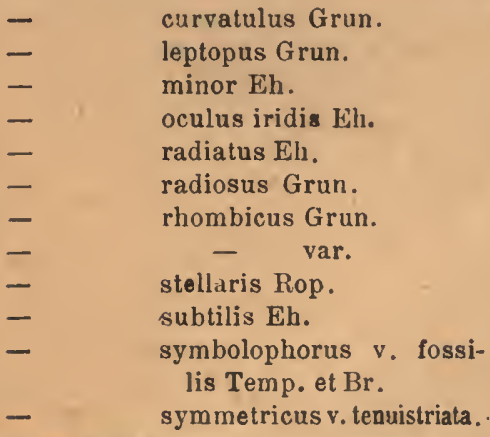

Dimeregramma marinum (Greg.) Ralfs.

Euodia gihba Bail. v. moronensis Temp. ot Per.
Eu odia gibba var. peltata Forti.

- - var. subrhombica Forti.

- minor Temp. et Per.

Grammatophora angulosa Eh.

- striata Eh.

$\begin{array}{cc}\text { - } & \text { striata Eh. } \\ - & -\quad \text { var. fossilis Pant. } \\ - & \text { undulata Eh. }\end{array}$

Ulaynaldella antiqua Pant.

Hemiaulus Hauckii Grun. var.

llyaloniscus levis Eh. v. doljensis Pant.

Vavicula approximata Grev.

- bomboides A. S.

- i var.

- bullata Grev.

- circumsuta Grun.

- crabro Eh. var. minuta.

- - var. multicostata.

- $\quad$ - var. pandura.

- - var. separabilis.

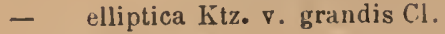

- eudoxia A.S.

- exempta A. S.

- exul A.S.

- fusca Greg.

- - var, delicata.

- - var. norvegica.

- geminata var. fussilis Pant.

- granulata Bréb. v. constricta.

- Hennedyi W. Sm.

- $\quad$ - var. fossilis Pant.

- - var. crassa Per.

- Jelineckii Grun. v. fossilis Forti.

- lyra Eh.

- - var.

- - var. intermedia.

- - var. elliptica.

- Mediterranea Br. et Cl.

- - var. fossilis forti.

- musca Greg. var.

- nigricans Pant.

- O'Swaldii Gr. v. visenda Temp.

- pennata A. S. var.

- permagna Bail. v. maxima Forti.

- perplexa Per.

- - var.

- prætexta El.

- pseudogemmata Pant.

- puella A.S.

- pulcherrima Forti.

- Sandriana Grun. v. laevis Cl. 
Navicula Smithii Bréb.

- - var. major Per.

- spectabilis Grun. v. emarginata Cl.

- splendida Greg.

- venusta Jan.

Nitzsehia punctata Grun.

Orthoneis splendida Grev.

Pleurosigma affine W.Sm. v. fossilis Grun.

- $\quad$ Eudon Pant.

Podosira ambigua Grun.

Pgeudauliscus Schmidtii Pant.

v. tetramera Forti.

Poendo-Nitzschia cretae (Eh.) Forti.

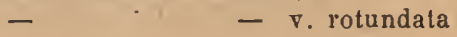
Forti.

Kaphoneis nitida Greg.

- _ - var. Debyi (Pant.) Forti.

Rhablonema adriaticum Ktz. v. fossilis Pant.

- v. diminutum

(Pant.) Forti.
Stictodiscus californicus Grev. v. areolata. Grunowii T.et W.

- parallelus (Grev) Pant.

- - var. Kinkerianus T. et WV. fa mi-

Surirella fastuosa Elı. nor Forti.

- $\quad$ - v. fossilis Pant.

Synedra baculus Greg.

Trachyneis aspera Eh.

$$
\begin{array}{lll}
\text { - } & \text { - } & \text { v. intermedia Gr. } \\
\text { - } & \text { - } & \text { v. vulgaris } \mathrm{Cl} \text {. }
\end{array}
$$

Triceratium amonum Grov.

- antediluvianum Eh. var.

- cinnamoneum Grev.

- orbiculatum v. elongatum Grun.

- spinosum Bail. T. minor fossilis A. S.

Xanthiopyxis daucispermoides Forti.

Willemoesia elegantula (Grun.) Forti.

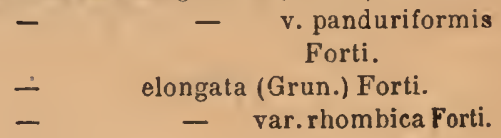

\section{No 930}

LAKE MORI (Australie)

Ejpithemia gibberula Ktz.

- sorex Ktz.

- zelira var. porcellus.

Gomphonema intricatum Eh.
Gomphonema intricatum var. tenella. Rhopalodia gibba 0 . M.

- $\quad$ - var. parallela.

N०931

BISCARROS - LANDES (France)

Dépôt saumâtre

Amphipleura Lindeimeri Grun.

Amphora libyca Eh.

- var.intermedia. Campylodiscus Echeneis Eh. 
Coccomeis placentula Eh.

Cyclotella comta Kiz.

- - var. radiosa.

Cymbella cuspidati Itz.

- cymbiformis Brèb.

- gastroides Kitz.

- parva V. H.

Diploneis elliptica Ktz.

- fusca Cl.

\section{Epithenia amphicephala Grun.}

$\begin{array}{ll}\text { - } & \text { cistula Ralfs. } \\ \text { - } & \text { gibberula Ktz. } \\ \text { - } & \text { sorex Kitz. } \\ & \text { zebra Kíz. }\end{array}$

Eunotia arcus v. uncinata.

- formica Eh.

- gracilis Bréb.

- minor V. H.

- pectinalis Rab.

- - var. undulata.

- - var. ventricosa.

Gomphonema acuminatum Eh.

$$
\begin{aligned}
& \text { - } \\
& \text { - } \\
& \text { - } \\
& \text { intricatum } \mathrm{Klz} \text {. } \\
& \text { vibrio Eh. }
\end{aligned}
$$

Mastogloia Dansei Thw.

Melosira crenulata Kiz.

- undulata $\nabla$. Normannii.

Navicula acrosphoria Bréb.

- amphigomphus Eh.

- amphirhynchus Eh.

- commutata Grun.

- divergens IV. Sm.

- dubia Flı.

- follis Eh.
Navicula Gendrei Iler, et M. Per.

- gibba Ktz.

- halophila Grun.

- Heribaudi r.elongata n. v. Plus grande que le type et $\dot{a}$ contours moins accentués. Longueur $120 \mu$.

- limosa Ktz.

- - var. subundulata.

- lyra o. intermedia.

- macilenta Eh.

- major Kítz.

- nodosa Eh.

- radiosa Ktz.

- - var. acuta.

- transversa A. S.

- sp. A. S. atlas $41 / 3 t$ var.

Nitzschia angustata Grun.

- scalaris W. Sm.

\section{Rhopalodia gihba O. M.}

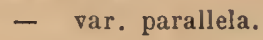

Stauroneis amphilepta Eh.

- anceps Eh.

Surirella linearis v. constricta.

- robusta Eh.

- - var. ovala n. v. Plus courte que le type et largement ovale, environ : Long. 170 u, larg. $100 \mu$.

- slesvicensis Grun.

- splendida Eh.

- tenera Greg.

- - o. nervosa.

- $\quad$ v. splendidula.

Synedra ulna Eh.

Tabellaria fenestrata $\mathrm{K} t z$.
Actinoptychus vulgaris Shum .

Amphora gigantea Grun.

$$
\text { - } \quad \text { - var. nodosa. }
$$

Biddulphla moblliensis Bail.
Cerataulus turgidus Eh.

Diploneis crabro $\mathrm{Eh}$.

Navicula clavata var. Wrightii.

- lyra v. atlantica.

Opephora Schwartzii P.P. 
Podosira hormoides Mont.

Rhabdonema adriaticum $\mathrm{K} t z$.
Tracheneis aspera $\mathrm{Cl}$.

Triceratium antediluvianum Eh.

No 933

ILE DE LA MARTINIQUE (Antilles)

Achnanthes inflata Grun.

Actinocyclus Ehrenbergii Ralfs.

- moniliformis Ralfs.

- partitus Grun.

subtilis Ralfs.

Actinoptychus amblyoceros $\mathrm{Eh}$.

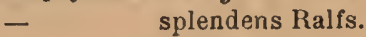

- undulatus Eh.

- vulgaris Schum.

Billulphia rhombus W. Sm.

- Tuomeyi Rop.

Campylodiscus biangulatus Grev.

$\begin{array}{ll}\text { - } & \text { crebrestriatus Grev. } \\ \text { - } & \text { singularis A. S. } \\ \text { Wallichianus Greo. }\end{array}$

Cocconeis scutellum Eh.

Coscinodiscus asteromphalus El.

$\begin{array}{ll}\text { - } & \text { centralis Eh. } \\ \text { - } & \text { concinnus W. Sm. } \\ \text { - } & \text { leptopus Grun } \\ \text { - } & \text { marginatus Eh. } \\ \text { - } & \text { nitidus Greg. } \\ \text { - } & \text { nodulifer Jan. } \\ \text { - } & \text { oculus-iridis Eh. } \\ \text { - } & \text { radiatus Eh. } \\ \text { - } & \text { robustus Greo. } \\ & \text { subtilis El. }\end{array}$

Diploneis multicostata $\mathrm{Cl}$.

- - nitescens Cl.

- notabilis $\mathrm{Cl}$.

Euodia gibba Bail.

$$
\text { - - v. atlantica. }
$$

Goniothecium odontella Elı.

IIynlouliscus stelliger Bail.

$$
\text { - subtilis Bail. }
$$

Velosira aulcata htz.

$$
\begin{aligned}
& \text { - } \quad \text { - var. coronata. } \\
& \text { - truncata Grun. } \\
& \text { - Westii W. Sm. }
\end{aligned}
$$

Navicula clavata Greg.

- liber IV. Sm.

- lyra El.

- - var. intermedia.

- - var. recta.

- macilenta Eh.

- spectabilis Greg.

Nitzschia Jelineckii Grun.

- panduriformis.

- - var. peralbata.

Orthoneis fimbriata Grun.

- splendida Grun.

Plagiotropria elegans Grun.

Pleurosigma affine Grun.

- $\quad$ balticum W. Sm.

Pyxilla baltica Grun.

Rhoicosigma compactum $\mathrm{Cl}$.

Stephanogonia actinoptychus.

Stephanopyxis corona Eh.

$$
\begin{array}{ll}
\text { - } & \text { cruciata Temp. et Per. } \\
\text { - } & \text { Grunowii Gr. et St. } \\
& \text { turris Eh. }
\end{array}
$$

surirell Ceylanensis Lend. Fort. var. a perta n. var. La partie centrale compriso entre les lignes de petites stries est élargie au inilieu et la valve a la forme rhombique.

- lepida Eh.

- fastuosa Eh.

- recedens A. S.

Tracheneis aspera $\mathrm{Cl}$.

- robusta P. P.
- $\quad$ velata Cl.
- $\quad$ sp? A. S. atlas $48 / 30$.

Triceratium sculptum Schad.

Xanthlopyxis globosa Eh. 
No 934

FLESSINGUE (Hollande)

Sondage

Actinocyclus Ehrenber hii Tialfs.

$$
\begin{array}{ll}
- & \text { Ralfsii Ralts. } \\
\text { - } & \text { sparsus Ratt. } \\
\text { - } & \text { subtilis Ralfs. }
\end{array}
$$

Actinoptyehus areolatus Eh.

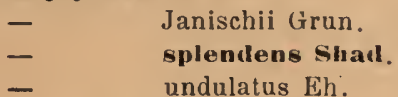

Aulacoliscus argus A. S.

$$
\text { - Roperii A. S. }
$$

Auliscur sculptus Ralfs.

Bidlulphia aurita Brẻb.

- pulchella Gray.

$$
\text { - rhombus } W \text {. Sm. }
$$

Campylodiscus clypeus Eh.

$$
\text { - echeneis Eh. }
$$

Cerataulus Smithii Ralfs.

Coscinodiscus concinnus W. Sin.

$\begin{array}{ll}\text { - } & \text { decrescens var. valida. } \\ \text { - } & \text { excentricus Eh. } \\ \text { - } & \text { marginatus Eh. } \\ \text { - } & \text { oculus-iridis Eh. } \\ \text { - } & \text { radiatus El. } \\ & \text { subtilis Eh. }\end{array}$

biploneis bombus Eh.

- didymus Eh. biploneis elliptica Cl.

Gramnatoptora robusta Dipp.

Uyalouliscus stelligel Bail.

$$
\text { - subtilis Bail. }
$$

Melosira arenaria Moore.

- sulcata Ktz.

- Westii W. Sm.

Navicula Baileyana var.

- claoata Greg.

- - v. elongata.

- forcipata Greo.

- formosa Greg.

- peregrina var.

- spectabilis Greg.

Vitzschia navicularis Bréb.

- scalaris W. Sm.

Pleurosigma balticum W. Sm.

Iraphoneis rhombus Eh.

Scoliopleura tumida Brèb.

Surirella fastuosa Eh.

$$
\text { - } \quad \text { - } \quad \text { striatula Turp. }
$$

Trachyneis aspera $\mathrm{Cl}$.

Triceratium antediluoianum Eh. facus Eh.

No 935 Expédition SCOTT au pôle Sud

Lavage d'Hydraires récoltés par $77^{\circ}, 7$ lat. Sud et $166^{\circ}$ long. Est

Actinocyclus Oliverianus 0 . Meara.

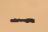

Ralfsii c. Challengerensis.

- umbonatus Cast.

Amphora Racovitzae V. $H$.
Asteromphalus Hookeri Eh.

Bidilulphia anthropomorpha V. II.

- obtusa Grun. var.

- Otto Mülleri V. H. 
Coscinodiscus australis Karst?

$\begin{array}{ll}\text { - } & \text { bifrons Cast. } \\ \text { - } & \text { chromoradiatus Karst? } \\ - & \text { dubiosus Ratt. } \\ \text { - } & \text { lentiginosus Eh. } \\ - & \text { lineatus Eh. } \\ \text { - } & \text { radiatus Eh. } \\ \text { - } & \text { radiosus Ratt. } \\ \text { - } & \text { stellaris Rop. } \\ \text { - } & \text { symbolophorus Grun. }\end{array}$

Diploneis expedita A. S. var.
Eucampia balaustium Cast.

Iyalodiscus radiatus Grun.

Melosiru omma Cil.

- sol Eh.

Plèurosigma Kerguelense Grun.

Trachyneis aspera $\mathrm{Cl}$.

$$
\text { - } \quad \text { - } \quad \text { v. intermedia. }
$$

Triceratium arcticum Bright.

Sendaiense J. Br. var.

\section{No 936 OWEN'S FERRY - SAVANNAH-GEORGIA}

\section{(Etats-Unis)}

Actinocyclus Barkleyi var.

$\begin{array}{ll}- & \text { Elirenbergii Ralfs. } \\ \text { - } & \text { moniliformis Ralfs. } \\ \text { - } & \text { sparsus Ralfs. }\end{array}$

Actinoptychus antistrophus J. Fr. oar.

$$
\begin{array}{ll}
- & \text { intermedius A. S. } \\
- & \text { splendens Shadb. } \\
- & -\quad \text { var. nicobarica. } \\
- & \text { undulatus Eh. } \\
\text { - } & \text { vulgaris Schum. } \\
\text { - } & \text { Weissflogii A. S. }
\end{array}
$$

Aulaconliscus argus A. S.

Auliscus confluens Grun.

$$
\text { - sculptus Ralfs. }
$$

Bidvilphia rhombus WV. Sm.

$$
\text { - Tuomeyi Rop. }
$$

Campylodiscus echeneis Eh.

Coscinodiscus decrescens Grun.

$\begin{array}{ll}- & \text { denarius A. S. } \\ \text { - } & \text { gigas Eh. } \\ \text { - } & \text { lineatus Eh. } \\ \text { - } & \text { nitidus Greg. } \\ \text { - } & \text { nodulifer Jan. } \\ \text { - } & \text { obversus Ratt. } \\ & \text { radiatus Eh. }\end{array}$

Coscinodiscus Rothii Grun. subtilis Eh. tuberculatus Grev.

Cyclotella striata Grun.

- stylorum Bright.

Diploneis crabro Eh.

- elliptica Cl.

- geinmata Eh.

- Grundlerii Cl.

- Smithii Cl.

Eunotia major Rab.

- monodon Eh.

- robusta o. polyodon.

- quaternaria Eh.

Eupodiscu radiatus Brél).

Ilyalodiscus stelliger Bail.

- subtilis Bail.

Melosira sulcata $\mathrm{K} t z$.

- - var. coronata.

- - var. radiata.

Navicula claoata Greg.

- formosa Greg.

- gigas $\mathrm{Ktz}$.

- Hennedyi oar. granulata

- latevittata $\nabla$. Domingensis

- lyra Eh.

- - var. recta. 
Navicula macilenta Eh.

- major Ktz.

- proetexta Eh.

Nitzechia granulata Grun.

- navicularis Brẻb.

- plana W. Sin.

- scalaris W. Sm.

- Tryblionella $\nabla$. maxima.
Plagiogramma tessellatum Grev.

Surjrella tenera v. nervosa.

Terpsinod americana Ralfs.

- intermedia Grun.

Triceratium favus Eh.

- sculptum Shadb.

sp. A. S. atlas $\mathrm{Pl}$. $84 / 9$.
Actinocyclus Ehrenbergii Ralfs.

Actinoptychus splendens Ralfs.

$$
\text { - undulatus Eh. var. }
$$

Biddulphia rhombus W. Sm.

- Tuomeyi Bail.

Coscinodiscus argus Eh.

- oculus-iridis Eh.

- subtilis Eh. var.

Cymbella lanceolata Eh.

Cyclotella striata Eh.

- stylorum Bright.

Eupodiscus radiatus Bail.

Hyalodiscus stelliger Bail.

Melosira dactylus Eh.

- sulcata Ktz.

Navicula dilatata Eh.
Navicula formosa Greg. var.

- major Iitz.

- multicostata Bréb.

- Smithii Bréb. var.

- viridis Ktz.

vitzschia plana $W$. Sm.

- Tryblionella Grun.

Raphoneis amphiceros Eh. v. rhombica.

Stauroneis phonicenteron Eh.

Surirella Capronii Bréb.

- cardinalis Kitt.

- elegans W. Sm.

- gracilis W. Sm. var.

- splendida $\mathrm{Ktz}$.

- tenera var. nervosa. .

Triceratium alternans $E h$.

- favus Eh. sculptus Shadb.

\section{No 938 FOLLY LAKE - NEW-JERSEY (États-Unis)}

Dépôt fossile d'eau douce

Achnanthidium Peragalloi Temp. n. sp. La face dorsale présente une striation tout à fait analogue à celle du navicula macula Greg.
Cyubella aspera El.

- cistula Temp.

- cuspidata Ktz.

- lanceolata Eh.

- sp. A. S. atl. 9/52. 
Encyonema gracile Rab.

Epithemia argus $\mathrm{K} \mathrm{tz}$.

$$
\begin{aligned}
& \text { - gibba Ḱtz. } \\
& \text { - - v. parallela. } \\
& \text { - turgida Kítz. }
\end{aligned}
$$

Eunotia major Rab.

- pectinalis Dilw. v. ventricosa.

- polyodon Els.

Gomphonema acuminatum Eh.

$$
\text { - } \quad \text { capitatum Eh. . }
$$

Navicula amphirynchus Eh.

- bacillum Eh.

- cuspidata K'tz. var.

- dactylus Eh.
Navicula divergens W.Sm.

- elliptica $\mathrm{Ktz}$.

- firma Ktz.

- fusca Greg.

- gibba Eh.

- major Ktz.

- nobilis Eh. var

- radiosa $\mathrm{KIz}$.

- transversa A. S.

stauroneis acula W. Sm. val.

- amplilepta Eh.

- gracilis Eh.

- phoenicenteron Eh.

Surirella linearis W. Sm.

- obtusa W. Sm.

\section{No 939 TROY - NEW-HAMPSHIRE (États-Unis)}

\section{Dépôt fossile d'eau douce}

Eucyonema gracile Eh.

Eunotia bidentula W. Sm.

- flexuosa Grun.

- incisa Greg.

- major Rab.

- pectinalis Rab. v. undulata.

- polyodon Eb.

Fragilaria undulata W. Sm. var.

Comphonema acuminatum $\mathrm{Eh}$.

$$
\text { - capitatum Eh. }
$$

Navicula affinis Eh.

- conspicua A.S.

- dicephala El.v. stauroneiformis.

- dilatata Eh.

- dubia Greg.

- firma Ktz.

— - - var tumescens.
Navicula gibba Ell.

- iridis Eh.

- major Ktz.

- nobilis Eh.

- serians ktz.

- transversa A. S.

- trochus Eh.

- viridis Ktz.

- - var. commutati.

Pseudo-Eunotia hemicyclus Grun.

Stauroneis gracilis Eh.

- phonicenteron Eh.

- - - var. crassa.

Stenopterobia intermedia Lewis.

Tahellaria fenestrata Ktz.

Van Heurckia rhomboides Bréb. 
Eunotia Iunaris var. subarcuata. - minor Rali.

Fragilaria virescens var.

Gomphonema auritum Braun.

Vavicula ampligomphus fa minor.
Navicula atomoides Grun.

- subcapitata v. stauroneiformiz.

Stauroneis anceps Eh.

Surirella linearis v. elliptica.

Van Heurckia vulgaris $v$. minor.
$N^{\circ} 941$

Fragilaria virescens Ralis.

Navicula dubia.

\section{PORTO (Portugal)}

Surirella linearis $\nabla$. elliptica.

- Rattrayi A. S.

\section{No 942 à 944 ILE DE LA TRINITÉ (Antilles)}

\section{Dépôt fossile marin}

Actinocyclus Ehrenbergii Ralfs. Actinoptychus undulatus Eh:

Asterolampra Grevillei Grow.

$$
\text { - marylandica Eb. }
$$

Cestodiscus hirtulus Grun.

\section{- peplum J. Br.}

- proteus Hartmann.

- pulchellus Grev.

- trinitatis Grun.

Coscinodiscus apiculatus var. californica Grun.

$\begin{array}{ll}- & \text { argus Eh. } \\ - & \text { asteromphalus. } \\ - & \text { decrescens Grun. } \\ - & \text { excavatus Grun. } \\ - & \text { heteroporus Grev. }\end{array}$

Coscinouliseus Lewisianus Grev.

- lineatus Eh.

- nobilis Grun. (fragments).

- nodulifer A. S.

- oculus iridis Eh.

- radiatus Eh.

- $\quad$ - var. irregularis.

- rex Wall.

- rhombicus Grev.

- subtilis Eh.

Craspedodiscus coscinodiscus Elı.

$$
\begin{aligned}
& \text { - - v. Nankoo- } \\
& \text { rensis. }
\end{aligned}
$$

Creswellia superba Grew.

Raphoneis gemmifera Eh. $\tau$. elegans.

Stephanopyxis turris Eh. 


\section{No 945 LYON'S FARM - NEW-JERSEY (États-Unis)}

\section{Dépôt fossile d'eau douce}

Cocconeis placentula Eh.

Cymbella aspera Eh.

- Ehrenbergii Ktz.

Epithenria amphicephala Grun.

$$
\begin{array}{ll}
\text { - } & \text { gibba Ktz. } \\
\text { - } & \text { turgida Eh. } \\
\text { - } & \text { - var. granulata. }
\end{array}
$$

Eunotia major Rab.

Gomphonema constrictum Eh. - montanum Schum. var.

Navicula affinis Elr.

- americana Eh.

- bacillum Eh

- cuspidata litz.

- $\quad$ fa craticula.
Navicula dilatata Eh.

- firma Ktz.

- gastrum Donk.

- gentilis Donk.

- mesolepta $\mathrm{Ktz}$.

- nobilis Eh.

- oblonga Ktz.

- rupestris Hantz.

- sphcerophora Ktz.

- viridis Ktz

Nitzschia spectabilis Rab.

Stauroneis acuta W. Sm.

- gracilis Eh.

- phcenicenteron El.

Surirella saxonica Auersi:

Synedra ulna Eh.

\section{N•946 DÉTROIT DE TORRES (Océan Pacifique) Lavage d'huîtres perlières}

Actinoptychus splendens Shad.

$$
\text { - } \quad \text { trilingulatus Bright. }
$$

Amphora crassa Greg. o. punctata.

- spectabilis Greg.

Biddulphia pulchella Greg.

$$
\text { - Tuomeyi Bail. }
$$

Cocconeis pellucida lirun.

Coscinodiscus concavus Grun. denarius A. S. excentricus Ell. lineatus $\mathrm{Eh}$. radiatus Eh. Rothii Grun. tenuis Ratt.
IIyalodiscus stelliger Bail, var. Melosira sulcata Ḱtz.

$$
\text { - - v. coronata. }
$$

Navicula aspera Eh.

- bullataGrev.

- Erythrea Grun.

- lyra et var. Eh.

- Smithii Bréb.

- splendida Greg.

- TVeisstlogii A. S.

Nitzschia panduriformis Greg.

Pleurosigna formosum var. balearica.

Triceratium favus Eh.

- pentacrinus Wall. sculptus Shadb. 


\section{No 947 WEST MELBOURNE - VICTORIA (Australie) \\ Marécages}

Achuanthes inflata Grun.

Actinocyclus Barkleyi Grun.

Amphiprora crenulata Temp.

Canıpylodiscus Diemelianus Grun.

-

Echeneis Eh.

Diploneis Smithii Bréb.

Epithemia gibberula Ktz.
Iyalodiscus subtilis Bail.

Navicula Yarronsis Grun.

- lauta Grun.

Nitzschia Tryblionella var. maxima

- circumsuta Bail

Pleurosignua balticum W. Sm.

Surirella bengalensis Gıun.

Tropidoneis mediterranea Grun.

\section{$\mathrm{N}^{\text {os }}$ 948-949 MORRIS COVE - CONNECTICUT \\ (États-Unis)}

Sondage

Achnanthes longipes Ag.

Actinocyclus Ehrenbergîi Ralfs

Actinoptychus undulatus Eh.

Amphiprora elegans W. Sm.

$\begin{array}{ll}\text { - } & \text { lepidoptera Greg. } \\ \text { - } & \text { pulchra Bail. } \\ \text { - } & \text { vitrea A. S. }\end{array}$

Amphora intersecta A. S.

- laevis Greg.

- plicata Greg.

- robusta Greg.

- sulcata Greg.

Auliscus cœlatus Bail.

- punctatus Bail.

Bicldulphia aurita Bréb.

- pulchella Gray.

- rhombus W. Sm.

Campylodiscus Echeneis Eh.

Cerataulas turgidus Elt.

Cocconeis scutellum Eh
Coseinodiscus apiculatus Eh. var.

$\begin{array}{ll}\text { - } & \text { devius A. S. } \\ \text { - } & \text { excentricus Eh. } \\ \text { - } & \text { marginatus Eh. } \\ \text { - } & \text { oculus-iridis Eh. } \\ \text { - } & \text { radiatus Eh. }\end{array}$

Cyclotella stylorum Bright. Cymatopleura elliptica $\mathrm{K} t z$.

Lithodesmium undulatum Eh.

Melosira sulcata $\mathrm{K} t z$.

- - - . coronata Kiz.

Navicula amphirynchus Eh.

- caribuea Eli. var.

- clavata var. elliptica.

- didyma Kitz.

- forcipata Grev.

- formosa Ġreg.

- fusca Greg.

- humerosa Bréb.

- irrorata Grev. 
Vavicula latissima Greg.

- lyra Eb.

- - var. elliptica.

- marina Ralfs.

- peregrina Ktz.

Nitzschia circumsuta Bail

- sigma W. Sm.

- sigmatella W. Sm.

- Tryblionella Grun.

Plagiogranuma elongatum Grev.

Pleurosigma affine $\mathrm{W}$. Sm.
Pleurosigna balticum W. Sm.

-

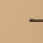
- v. diminutum.

- Wansbeckii Donk.

Pyxilla baltica Grun .

Rhabdonema adriaticum $\mathrm{k} t z$. Scoliopleura tumida Grun.

Terpsinoë musica Eh.

Triceratium alternans Bail.

- antediluvianum V. H. farus Eh.

\section{N. 950 NEW HAVEN HARBOUR - CONNECTICUT}

\section{(États-Unis)}

\section{Sondage}

Aetinocyclus crassus WV. Sm. Actinoptychus undulatus Eh.

Auliscus pruinosus Bail.

$$
\text { - } \quad \text { - var. Zanzibarica }
$$

Amphora intersecta A. S. var.

Bidolulphia aurita Brẻb.

- rhombus WV. Sm.

Campylodiscus Echeneis Eh.

Cerataulus turgidus Eh.

Coscinodiscus apiculatus Eh.

$$
\begin{array}{ll}
\text { - } & \text { excentricus Eh. } \\
\text { - } & \text { oculus iridis Eh. }
\end{array}
$$

Cyclotella striata Grun.

Melosira sulcata Ktz.

$$
\text { - _ - var. coronuta. }
$$

Vavicula approximata A. S. var.

- Baileyana Gran.

- caribca Cl.

- forcipata Grev.
Navieula formosa Greg.

- $\quad$ - var. A. S. 50/10, 14,15.

- fusca Greg.

- Hennedyi var. marina.

- humerosa Brèb.

- interrupta Ktz.

- latissima Greg.

- lyra Eh.

- - var. elliptica.

- polysticta Grev.

- prætexta Eh.

- Smithii Bréb.

Nitzschia acuminata $\mathrm{W}$. Sm.

- circumsuta Bail.

Pleurosigma aftine Grun.

Raphoneis amphiceros Eh.

Rhalsonema adriaticum Ktz.

Surirella fastuosa $\mathbf{E h}$.

Triceratium favus Ell.

Tryblionella Hantzschiana W. Sm. 
Nos 951 à 973 BASSIN D'ARCACHON (France)

Récoltes pélagiques

Les vingt-trois prépartions dont nous donnons ici une analyse d'ensemble, représentent toute uue série de récoltes faites systématiquement par notre regretté ami Paul Bergon, pendant l'année 1911, et, parmi les. quelles nous avons choisi les plus intéressantes. Elles se répartissent comme suit :

Nos 951 récolte faite le 10 févier 1911

$\begin{array}{llll}952 & - & 17 & - \\ 953 & - & 18 & - \\ 954 & - & 20 & - \\ 955 \text { à } 957 & - & 22 & - \\ 958 \text { à } 960 & - & 23 & - \\ 961 & - & 24 & - \\ 962 & - & 25 & - \\ 963 & - & 12 & \text { mass } 1911 \\ 964 & - & 15 & -\end{array}$

Nos 965 récolte faite le 2 avril 1911 966

20

$967 \quad-\quad 6$ mai 1911

968

972

7 sepl. 1911

973

Les noms d'espèces sont suivis du numéro des préparations dans lesquelles celles-ci se trourent.

Achnanthes brecipes $\mathrm{Ag} .951$.

- longipes Ag. 9:57.

Actinoptychus Janischii Grun. 954.

-

$$
\text { splendens Ralfs. } 961,965 .
$$

Amphiprora decussaia $\mathrm{Cl}$. 957, 961.

$$
\text { - paludosa W. Sm. 262. }
$$

A mphora cymbifera Greg. 962.

- ostrearia Bı́b. 954, 961,969.

Asterionella japonica CI.

Asteromphalus flabellatus Grun. 973.

Auliscus colatus Bail. 951.

Bacillaria socialis Ralfs. 965 .

Bacteriastrum hyalinum Laud. 956,96:

- varians Laud, 956, 962.
Biddulphia granulata Rop. 95:.

- mobiliensis Bail.

- pulchella Greg. 968.

- rhombus W. Sm. 964 .

- Roperiana isreo. 951.

Campylodiscus Echeneis Eh. 967, 968. - Thuretii Bréb. 970.

Campylosira cymbelliformis Gr. 958 , 959.

Cerataulus turgidus Eh. $26 \tilde{\text { J }}$

Chetoceros angulatum Sihült. 974.

- armatum West. 951, 965, 968.

- bacillaria Eh. 958, 959.

- boreale Bail. 951, $951,972$.

- cinctum irrun. $966^{\circ}$. 
Chretoceros coeblea Schitt.

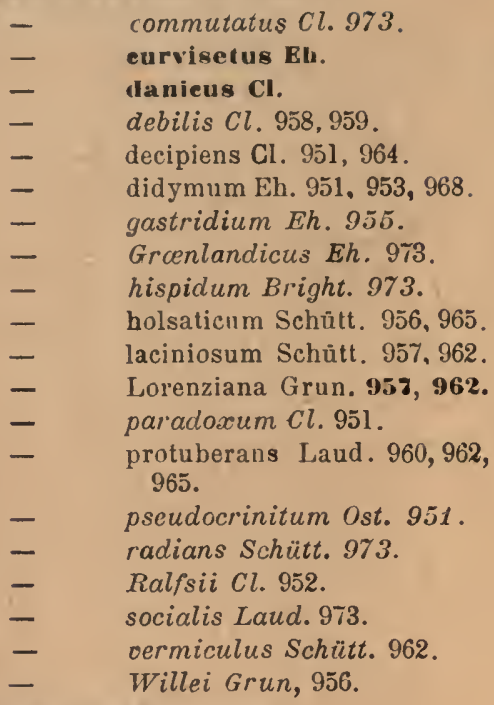

Cocconeis seutellum Eh.

Coseinodiscus asteromphalus Eh. 957.

$\begin{array}{ll}\text { - } & \text { centralis Eh. 954. } \\ \text { - } & \text { concinnus W. Sm. } 954 . \\ \text { - } & \text { excentricus Eh. } \\ \text { - } & \text { gigas Eh. 965. } \\ \text { - } & \text { leptopus Grun. 962. } \\ \text { - } & \text { oculus iridis Eh. 967,973. } \\ \text { - } & \text { papuanus Cast } 973 . \\ \text { - } & \text { radiatus Eh. } 951.960,965 .\end{array}$

Dactyliosolen antarcticus Cast. 955, 958, 959.

Diploneis bombus Eh. 951, 965 .

Ditylinm Brightwellii West.

- intricatum Grun. 953, 970.

Encyonema ccespitosum Ktz. 956, 957.

Epithemia musculus $K t z .965$.

Vueanpia Kodiaous Eh.

Grammatophora oceanica Eh. $956,957$.

Guinardia flaccida $H$. Per.

Ilemiaulus Hauchii Grun. 974, 972.

- Heibergii Cl. 95ł, 957, 972.

Ilyalodiseus subtilis Bail. 970 .

Lauderia borealis Grun. 958, 959, 962.

- delicatula H. Per. 971.

- Schroderi Bergon. 962, 965.
Lilhodesmium undulatum Eh. 973.

Melosira Borreri Grev. 962.

- crenulata v. ambigua. 952.

- nummuloïdes Ag. 951, 960.

- sulcata Ktz. 955, 960, 961.

Navieula formosa Greg. 964.

- granulata Bréb. 964 .

- lyraoar. intermedia.965,967.

- palpebralis Bail. 967.

- peregrina Ktz, 951.

- scopulorum Rréb. 958, 959, 968.

Nitzsehia acuminata Grun. 951, 965.

- circumsuta Grun. 957,960.

- granulata Grun. 962, 968.

- longissima Ralfs. 968.

- Lorenziana Grun.

- seriata Cl. 971, 968 .

- $\quad$ - var. fraudulenta. 95t;, 971.

sigma W. Sm. 958, 959, 960 .

Orthotropis lepidoptera Grun. 956, 957, 960.

Pleurosigma angulatum W. Sm. 953, 960, 96 I.

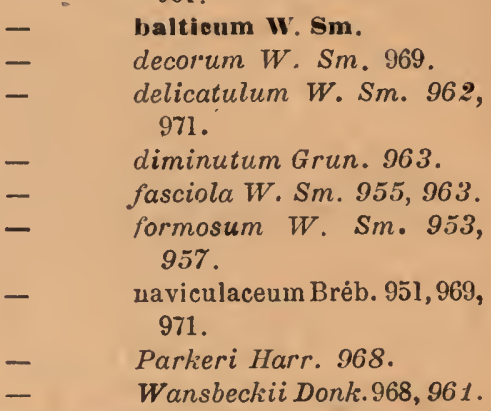

Rhizosolenia alata Bright. 966, 965 .

$$
\begin{array}{ll}
- & \text { - v. corpulenta.966,973. } \\
- & \text { formosa H. Pracillima.954,966. } 954 . \\
- & \text { robusta Norm. 973. } \\
- & \text { setigera Bright. } 973 . \\
\text { - } & \text { Shrubsolei Cl. } \\
\text { - } & \text { Stolterfathii H. Per. 954, } \\
\text { 957. } \\
\text { styliformis Bright. } 951,954, \\
\text { 972. }
\end{array}
$$

Scolioploura latestriata Grun. 969.

Skeletonema costatum Cl. 
Stephanopyxis lurgida Ralfs, 955 , $597,973$. Streptotheea Tamesis $C l 965$.

Striatella unipunctata Ag. $951.935,973$.

Surirella fastuosa Eh. 968, 971 .

- gemina Wh. 952, 906 .

Syneura fulgens o. gigantea. 960 .

Thalassionema nitzschioides Grun. 951 , 962.
Thalassiosira decipiens Grun. 960, $9 j 1$. hyaliua Grun.

Thalassiotlurix Frauenfeldii Grun. 956 .

Toxonidea Gregoryana Donk. 958, 959 , 973.

- insignis Donk. 964,971 .

Trachyneis aspera $C l .955$.

Triceratium alternans Bail. 952, 957.

\section{No 974 FUNCHAL - ILE MADERE (Portugal)}

Actinoptychus undulatus Eh. oar. Asteromphalus Roperianus Ralfs. Cerataulus laevis Ralfs.

Coscinouliscus africanus Jan.

- curvatulus Grun.

- excentricus Eh.

- lineatus Eh.

- nolulifer Jan.

- - fa ninor.

- oculus-iridis Eh.
Ditylum intricatum W'eil.

Euodia gibba Bail.

$$
\text { - - var. atlantica. }
$$

Grammatophora serpentina $E h$.

Ilemiaulus proteus Heib.

IIyalodiscus stelliger Bail.

- subtilis Bail.

Melosira granulata Ralfs.

Nitzschia angustala Grun.

Roperia tessellata Grun.

\section{No 975 SILVER LAND - BRISTOL - CONNECTICUT (Etats-Unis)}

ActInocycius Bar:kleyi Grun.

- Elarenbergii Ralfs.

- sparsus Ralfs.

Actinoptychus areolatus Eh.

- undulatus Elı.

Ampluprora conspicua Grev.

- pulchra Bail.
Auphora angusta Greg

- arenaria o. Donkinii..

- cingulata $\mathrm{Cl}$.

- Clevei Grun.

- gigantea Grun.

- obtusa Greg.

- protens Greg.

- rbombica $\mathrm{v}$. intermedia.

Auliscus cœlatus Bail. 
Auliscus proinosus $\nabla$. Zanzibarica.

- punctatus Bail.

- sculptus Eh.

BIddulphía aurita Bréb.

- pulchella Gray.

- rhombus W.Sm.

Brebissonia Boeckii Grun.

Campylodiscus costatus W. Sm. Echeneis Eh.

Cerataulus turgidus Eb.

Cocconels quarnerensis $A$. $S$.

- scutellum Eh.

Coscinodiscus centralie Eh.

- $\quad$ excentricus Eh.

Cyclotella striata Grun.

Denticula antillarum $\mathrm{Cl}$.

Diplonels bombus Eh.

$\begin{array}{ll}\text { - } & \text { didymum Eh. } \\ \text { - } & \text { elliptiea } \mathrm{Cl} . \\ \text { - } & \text { interruatula Cl. } \\ \text { - } & \text { Smithii Gl. } \\ \text { - } & \text { - var. major. } \\ \text { - } & \text { splendida Greg. }\end{array}$

Epithemia turgida o. granulata.

- zebra Ktz.

Euphyllodium spathulatum Shadb.

Grammatophora macilenta W. Sm.

- serpentina Eh.

Hyalodiscu scoticus Grun.

- stelliger Bail.

Melosira nummaloïdes Ag.

- sulcata Ktz.

- - var. coronata.

Navicula cestuarii $\mathrm{Cl}$.

- Baileyana A. S.

- cancellata Donk.

- Cardinalis Ktz.

- clavata Greg.

- formosa Greg.

- Hennedyi W. Sm.

- - var. furcata.

- humerosa Bréb.

- irrorata Grev.

- latissima Greg.

- liburnica V. H.
Naricula lyra Eh.

- - var. carinata.

- - var. cuneata.

- - var. elliptica.

- - var. insignis.

- peregrina Ktz.

- Powellii Lewis.

- Treoelyana Donk.

- viridis $\mathrm{Ktz}$.

Vitzechla acuminata Grun.

- circumsuta Grun.

- fasciculata Grun.

- granulata Grun.

- punctata Grun.

- sigma W. Sm.

- - $\quad$ var. rigida.

- Tryblionella Hantz.

Opephora pacifica P.P.

- Schwartzii P. P.

Plagiogramma antillarum $C l$.

- Gregorianum Grev.

PIasiotropis elegans Grun.

Pleurosigma affine Grun.

- naviculaceum Bréb.

Pyxilla baltica Grun.

Rhabdonema adriaticum Ktz.

Raphoneis amphiceros Eh.

- - - var. rhombus.

- surirella Eh.

Rhoicosphenia curvata $r$ marina.

Rhopalodia gibberula $\mathrm{O}$. M.

- musculus H. Per.

- var. constricta.

Scoliopleura tumida Rab.

Stauroneis Gregorii Ralfs.

Surirella fluminensis Grun.

- recedens $A$. S.

- striatula Turpin.

Syndendrium diadema Eh.

Synedra crystallina $\mathrm{Ktz}$.

- formosa Hantz.

Terpsinoẻ americana Ralfs.

Trachyneis aspera $\mathrm{Cl}$.

- - var. minor.

Triceratium alternans Bail.

- antediluvianum Eh.

- favus Eh. 
N`976 LEA'TE'S ISLAND -- CONNECTICUT' (États-Unis)

Actinoptychus undulatus Eh.

Amphiprora alata $K t z$. conspicua Grer. pulchra Bail.

Bidllulphia aurita Bréb.

$$
\text { - pulchella Greg. }
$$

Coscinodiscus centralis Eh

$$
\text { - } \quad \text { excentricus Eh. }
$$

Cyclotella striata Grun.

Cymbella gastroides $K t$ z .

Melosira sulcata Iítz.

Navicula Fischerii A. S.

- formosa Greg.

- maculata var. major.
Navicula peregrina $K$ tz.

- permagna Bail.

- rhombica Greg.

- viridis $\mathrm{Ktz}$.

Nitzschia fasciculata Grun.

- obtusa IV. Sm.

- scalaris W. Su.

- sigma W. Sm.

Orthotropis lepidoptera Grun.

Plcurosigma Terryanum II. P.

Pyxilla baltica Grun.

Rhabdouena adriaticum Ktz.

Raphoneis amphiceros Eh.

Stauroneis Gregorii Ralfs.

Surirella elegans Eh.

\section{N³ 977-978 LEATE'S CREEK - CONNECTICUT}

(États-Unis)

\section{Sondage}

Actinocyclus Ehrenbergii Ralfs.

Actinoptychus undulatus Eh.

Amphiprora elegans W. Sm.

$$
\text { - pulchra Bail. }
$$

Amphora cingulata Cl.

Bid́lulphia aurita Bréb.

$$
\begin{aligned}
& \text { - } \quad \text { pulchella Gray. } \\
& \text { - } \quad \text { rhombus WV. Sm. }
\end{aligned}
$$

Coscinodiscus excentricus Eh.

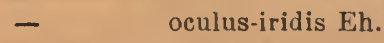

- radiatus Eh.

Epithemia musculus Ktz.

Lithodesmium undulatum Eh.

Melosirira șulcata Ǩtz.
Navicula didyma $\mathrm{K} ı$.

- distans WV. Sm.

- formosa Greg.

- fusca Greg.

- granulata Bréb.

- Hennedyi W. Sm.

- interrupta Ktz.

- Iyra Eh.

- marina Ralfs.

- peregrina Eh.

- Smithii Brẻb. var.

Nitzechia circumsuta Bail.

- scalaris W. Sm.

- sigma W. Sm.

Pleurosigma alfine Grun.

- valticum W. Sm. 
Pleurosigma strigosum W. Sm. Wandsbeckii Donk.

Pyxilla dubia Grun.

Raphoneis gemmifera Eh.

Rhabdonema adriaticum Ktz.

Scoliopleura tumida Bréb.

Stauroneis salina W. Sm.
Surirella fastuosa Eh.

- Febigerii Lewis.

- recedens $A . S$.

- triatula Turpin.

Terpsinoë musica Eh.

Triceratium antediluvianum Eh.

favus Eh.

\section{Nos 979-980 LEATE’S BAY - CONNECTICUT (États-Unis)}

Actinocyclus Ehrenbergii Ralfs.

$$
\text { - Ralfsii var. }
$$

Actinoptychus undulatus Eh.

Auliscus pruinosus Bail.

Biddulphia pulchella Gray.

$$
\text { - rhombus W. Sm. }
$$

Campylodiscus echoneis Eh.

Cerataulus turgidus Eh.

Coscinodiscue excentricus Eh.

$\begin{array}{ll}\text { - } & \text { marginatus Eh. } \\ \text { - } & \text { oculas-iridis Eh. el var. } \\ \text { - } & \text { radiatus Eh. } \\ & \text { symbolophorus Eb. }\end{array}$

Eupodiscus argus Eh.

Ilyalodiscus radiatus $\mathrm{Eh}$.

Melogira sulcata Ktz.

$$
\text { - - } \quad \text {. coronata. }
$$

Navicula distans W.Sm.

- Fischerii A. S
Navicula formosa Greg.

- Hennedyi W. Sm.

- humerosa Bréb.

- interrupta $\mathrm{Ktz}$.

- lyra Els.

- marina Donk.

Nitzschia circumsuta Bail.

- scalaris W. Sm.

- Tryblionella Grun.

Pleurosigma affine Grun.

- balticum var.

- Wandsbeckii Donk. var.

Pyxilla baltica Grun.

Rhadonema adriaticum $\mathrm{K} \mathrm{tz}$.

Scoliopleura tumida Bréb.

Stephanopyxis turris Eh.

Stictodiscus californicus Grev.

Surirella striatula Turpin.

Triceratium antediluvianum Eh.

favus Eh.

Nos 981 à 983 TALBOT - MELBOURNE (Australie)

Dépôt fossile d'eau douce

Achnanthes inflata Grun.

Amphora lybica Eh.

- pediculus Grun.
Cerataulus laevis Ralfs.

Cocconeis placentula Eh.

Cymbella delecta A. S. 
Cymbella gastroïdes Ktz.

- hercynica A.S.

- obtusiuscula Ktz.

- stomatophora Grun.

Encyonema turgidum Grun.

- ventricosum Grun.

Epithemia zebra Kız.

- - var. proboscidea.

Eunotia arcus var. tenella.

- formica Eh.

- impressa Eh.

- lunaris Grun.

- pectinalis Rab.

- - - ventricosa.

Fragilaria construens Grun.

- elliptica Schum.

- virescens var. exigua.

Gomphonema angustatum $\mathrm{Ktz}$.

- - var. producta.

- commutatum Girun.

- intricatum $\mathrm{KIz}$.

- $\quad$ - var. pumila.

- mexicana Grun.

- montanum v. succisa.

Melosira crenulata $\mathrm{Ktz}$.

- laevis Eh.

Vavicula ambigua $\mathrm{Eh}$.

- amphirynchus Eh.
Navicula Braunii var.

- Brebissonii Ktz.

- commutata Grun.

- divergens var.

- hemiptera Ktz.

- Hitchkockii Eh.

- interrupta var. stauroneiformis.

- major Eh.

- microstauron Eh.

- parra Eh.

- Paulensis Grua.

- viridis $\mathrm{Ktz}$.

Pleurostauron inflata Hoid.

Rhopalorlia gibba O. M.

- - - var. ventricosa.

Stauroneis anceps Eh.

- _ - var. amphicephala.

- fulmen Bright.

- gracilis Eh.

- phuenicenteron Eh.

Surirella arcla A. S.

- inducta A. S. v. intermedia n. v. intermédiaire entre le type et le Sur. regina. Long. $200 \mu$, 1 côté en $10 \mu$.

- tenera Greg.

Synedra ulna var. aequalis.

- _ var. splendens.

No 984 MARSH SOUTH END - CONNECTICUT

- (États-Unis)

Actinocyclus sparsus ${ }^{-}$Ratt.

Actinoptychus undulatus Eh

Amphiprora pulchra Bail.

Amphora angustata $\mathrm{Cl}$.

- Clevei Grun.

- salina W.Sm.

Bidduiphla aurita Bréb.

Brebisconia Boeckii Grun.

Coscinodiscus centralis Eh.
Coscinodiscus oxcentricus Eh.

- lineatus Eh.

- oculus-iridis Eh.

- radiatus Eh.

Cyclotella Kützingiana Thw.

- striata.Grun.

Diploneis elliptica Cl.

- fusca Cl.

- interrupta $\mathrm{Gl}$.

- Smithii CI.

- splendida Greg. 
Eunotia major Rab.

- monodon Eh.

Fragilaria virescens Ralfs.

- _ - var, subsalina.

Isthmia enerois Eh.

Lithodesnimm undulatum Eh.

Mastogloia Dansei Thw.

- - var. elliptica.

- pusilla Grun.

Melosiru nummuloïdes Ag.

- sulcata $\mathrm{Ktz}$.

- - var. coronata.

Navicula Baileyana Grun.

- brevis Greg.

- elegans W. Sm.

- 1 - var. cuspiclata."

- formosa Greg.

- granulata Bréb.

- Grevillei Ag.

- humerosa Bréb.

- Lyalina Donk.

- lyra Eh.
Navicula major $K t$.

- peregrina kitz.

- proetexta Grun.

- protiacta Grun.

- rhynchocephala $\nabla$. amphiceros.

- semen Eh.

- streptoraphe Cl.

- viridis Kiz.

- Yarrensis Grun.

Nitzschia obtusa $\mathbf{v}$. maxima.

- scalaris W. Sm.

Orthotropis lepidoptera Grun.

Pleurosigma Terryanum H. P.

Pyxilla baltica Grun.

Ruabdonema adriaticum Ktz.

Raphoneis amphiceros Eh.

Stauroneis Gregoryi Ralfs.

Surirella lluminensis Grun.

- ovata Ktz.

Synedra pulchella Ktz.

Triceratium alternans Bail.

\section{No 985 MORRIS CREEK - CONNECTICUT (Etats-Unis)}

Achnanthes brevipes $\mathrm{Ag}$.

Actinocyclus Ehrenbergii Ra:fs.

$$
\text { - sparsus Ralfs. }
$$

Actinoptychus undulatus Eh.

Amphora cingulata $\mathrm{Cl}$.

- Clevei Grun.

- gigantea Grun.

- intersecta A. S.

- laevis Greg.

- proteus Greg.

- sulcata Bréb.

Auliscus cœlatus Bail.

- pruinosus var. zanzibarica.

- punctatus Bail.

Bicldulplifa aurita Bréb.

- rhombus W. Sin.

Campylodiscus echeneis Eh.

Cerataulus turgidus Eh.
Cocconeis scutellum Eh.

Coscinodiscus excentricus Eh.

$$
\text { - } \begin{aligned}
& \text { minor Eh. } \\
& \text { - } \\
& \text { - } \\
& \text { radiatus Eh. }
\end{aligned}
$$

Cyclotella striata Grun.

Diploneis didyma El.

- fusca $\mathrm{Cl}$.

Melosira sulcata $\mathrm{K} \mathrm{tz}$.

- - var. coronata.

Navicula Baileyana Grun.

- brevis Greg.

- cyprinus $\mathrm{Ktz}$.

- forcipata Grev.

- formosa Greg.

- Hennedyi var. furcata.

- humerosa Bréb.

- irrorata Grov.

- latissima Greg. 
Navioula lyra Eh.

- - var. insignis.

- - var. subcarinata.

- marina Ralfs.

- Powellii Lewis.

- rectangulata Greg.

- Treoelyana Donk.

- Yarrensis Grun.

Nitzsehia granulata Grun.

- scalaris W. Sm.

- sigma W. Sm.

- - var. sigmatella.

- Terryana Temp. et M. Per. u. np. Frustule tordu à face connective, lègèrement sigmoïde, étroite; à face valvaire longuement lunée. Points carénaux carrés, au nombre de 7 en $10 \mu$. Stries bien visibles, 25 en $10 \mu$; longueur environ $200 \mu$.
Orthotropis lepidoptera Grun.

- - - var. pusilla.

Plagiogramma Gregoryanum Greo

Plagiotropis oitrea Grun.

Pleuresigma affine Grun.

- balticum W. Sm.

- - var. diminutum.

- rigidum W. Sm.

- Wandsbeckii Donk.

Pyxilla baltica Grun.

Rhadonema adriaticuma Ktz.

Raphoneis amphiceros Eh.

Stauroneis Gregoryi Ralfs.

Stephanopyxis turris Eh.

Surirella fluminensis Grun.

Triceratium alternans Bail.

- antediluoianum Eh.

- favus Eh.

\section{No 986 SOUTH BEACH - BRISTOL - CONNECTICUT (États-Unis)}

Actinocyclus Ehrenbergii Ralfs.

- - oar. sparsus.

Actinoptýchus undulatıs Eh.

$$
\text { - - var. minor. }
$$

Amphiprora conspicua Grev.

$$
\text { - pulchra Bail. }
$$

Amphora Eulensteinii Grun.

$$
\text { - } \quad \text { - proteus Greg. }
$$

Campylodiscus echeneis Eh.

Cerataulus turgidus Eh.

Coscinodiscus excentricus Eh.

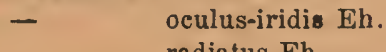

- radiatus Eh.

Cyclotella antiqua W. Sm.

- striata Grun.

Diploneis bombus Eh.

- elliptica Cl.

- tusca Cl.
Diplonels interrupta Cl.

- Smithii Cl.

Epithemia turgida $\mathrm{Ktz}$.

Eunotia major Rab.

Hyalodiscus stelliger Bail.

- scoticus Grun.

- subtilis Bail.

Melosira sulcata $\mathrm{Ktz}$.

- - var. coronata.

- - var. radiata fa minor.

Navicula Brasiliensis Grun.

- clavata Greg.

- formosa Greg.

- Hennedyi var. furcata.

- humerosa Bréb.

- lyra o. elliptica.

- perogrina Kitz.

- ventricosa Eh. 
Nitzschia granulata Grun.

Opephorá pacifica P. P.

Plourosigna balticum $W$. Sm.

Rhabdonema adriaticum Kítz.

Raphoneis amphiceros Eh.
Raphoneis amphiceros var, rhombica.

Rhopaloctia gibberula $O$. M. musculus H. Per.

Surirella fluminensis Grun.

$\mathrm{N}^{\circ} 987$.

CONSTANTINOPLE (Turquie)

Sondage

Aetinoptychus Janischii Grun, var.

- splendens Shadi.

- undulatus Eh.

Amphitetras nobilis Eh.

Auliscus colatus Bail

- sculptus Ralfs.

Biddulphia pulchelia Gray.

- rhombus W. Sin.

Campylodiscus echeneis Eh.

Cerataulus turgidus Eh.

Cocconeis pseudo-marginata Greg.

Coscinodiscus radiatus $\mathrm{E} h$.

Diploneis Beyríchiana Cl.

- Smithii var. major.

Endictya oceanica Eh.

Grammatophora serpentina oar.bacillaris.

Iyyalorliscus stelliger Bail.

$$
\text { - subtilis Bail. }
$$

Melosira sulcata Ktz.

$$
\text { - } \quad \text { - } \quad \text { v. coronata. }
$$

Navicula abrupta Donk.

- forcipata Grev.

- formosa Greg.

- humerosa Bréb.

- Bamorthensis Grun.

- latissima Greg.

- lyra Eh.

- - var. elliptica.

- $\quad$ - . intermedia.

- - $\quad$ - fa elliptica.

- - - - fa recta.

- - var. subelliptica.

- Trevelyana Donk.

Orthoneis ovata Grun.

- splendida Grun.

Pleurosigma balticum W.Sm.

Scoliopleura tumida Rab.

Surirella fastuosa Eh.

Synedra baculus Greg.

- fulgens W. Sm.

Terpsinoë americana Ralfs.

Triceratium antediluvianum Eh.

- favus Eh.

- $\quad$ spinosum Bail.

\section{N 988 WEST HARBOR - BRISTOL - CONNECTICUT (États-Unis)}

detinocyclus sparsus Ratt.

Actinoptychus areolatus Eh.
Actinoptychus undulatus Eh. Amphiprora alata $K t$. 
Amphiprora conspicua Grev.

Amphora angusta Greg.

$$
\begin{aligned}
& \text { - Clevei Grun. } \\
& \text { - giganiea Grun. } \\
& \text { - ocellata Cl. var. } \\
& \text { - proteus Greg. }
\end{aligned}
$$

Auliscus colatus oar. rhipis.

Brebissonia Boeckii Grun.

Cocconeis scutellum Eh.

Coscinodiscus blandus $\mathrm{A}$. S

$\begin{array}{ll}\text { - } & \text { centralis Eh. } \\ \text { - } & \text { decipiens Grun. } \\ \text { - } & \text { excenarius A. S. } \\ \text { - } & \text { nitidus Greg. } \\ \text { - } & \text { oculus-iridis Eh. } \\ \text { - } & \text { radiatus Eh. } \\ \text { - } & \text { sol Wall. }\end{array}$

Cyclotella striatu Grun.

Denticula antillarum $\mathrm{Cl}$.

Dictyoneis marginata $C l$.

Diploneis bombus Eh.

- elliptica Cl.

- fusca $\mathrm{Cl}$.

- Smithii Cl.

Ditylum intricatum Grun.

Epithemia turgida $K t$.

$$
\text { - zebraKtz. }
$$

Eunotogramma debilis Grun.

$$
\text { - laevis Grun. }
$$

Grammatophora macilenta IV.Sm.

$$
\text { - oceanica Eh. }
$$

IIyalodiscua scoticus Grun.

Lithodesnium undulatum Eh.

Melosira nummuloïdes $\mathrm{Ag}$.

- sulcata Ktz.

- $\quad \mathrm{f}^{2}$ minor.

- - var. coronata.

Navieula Astuarii $C l$.

- Baileyana A. S.

- breois Greg.

- cancellata Donk.

- formosa Gres.

- Heunedyi v. furcata.

- liburnica V. H.

- lyra Eh.

- - var. constricta.

- - var. cuneata.

- pennata oar. maxima.
Vavicula peregrina Ktz.

- permagna Edw.

Nitzschia acuminata Grun.

- constricta Ralfs.

- fasciculata Grun.

- granulata Grun.

- littoralis Grun.

- Lorenziana var. subtilis.

- obtusa var. maxima.

- punctata Grun.

- sigma var. major.

- tryblionella Hantz.

- - var. maxima.

Orthotropis lepidoptera Grun.

- - var.proboscidea.

- maxima var. subalata.

Plagiogramma Gregoryanum Grev.

Plagiotropis vitrea Grun.

Plenrosigna aftine Grun.

$\begin{array}{ll}\text { - } & \text { angulatum W. Sm. } \\ \text { - } & \text { - var. quadrata. } \\ \text { - } & \text { attenuatum } W . S m . \\ \text { - } & \text { balticum v. maxima fa stricta. } \\ \text { naviculaceum Bréb. }\end{array}$

Pyxilla baltica Grun.

Rhabdonema adriaticum $\mathrm{Ktz}$.

Haphoneis amphiceros Eh.

- $\quad$ surirella Eh.

Rhoïcosphenia curvata var. marina.

Rhopalodia gibba oar. ventricosa.

$$
\text { - } \quad \text { gibberula O. M. }
$$

Scoliopleura latestriata Cl.

Stauroneis Gregorii Ralfs.

Surirella crumena Bréb.

- Febigerii Lewis.

- tluminensis Grun.

- gemma Eh.

- recedens A. S.

- striatula Turp.

- - var. minor.

- tenera var. nerrosa.

Syudendrium diadema Eh.

Synedra Gaillonii v. maxima.

Terpsinoẻ americana Ralfs.

Trachyneis aspera fa minor.

Triceratium alternans Bail.

Van Heurckia Lewisiana $\mathrm{v}$. interposita. 


\section{N• 989 TIDE POND - BRISTOL - CONNECTICUT (États-Unis)}

Actinocyclus australis oar.

$$
\text { - } \quad \text { Barkleyi var. }
$$

Actinoptychus undulatus Eh.

Amphiprora pulchra Bail.

Amphora proteus Greg.

Aulacodiscu argus $A$. $S$.

$$
\text { - Rogersii A. S. }
$$

Auliscus cœlatus Bail.

- Macræanus Grev.

- pruinosus var. zanzibarica.

Biddulphla aurita Bréb.

- pulchella Gray.

- rhombus W. Sm.

- Tuomeyi Rop.

Campylodiscus echeneis Eh.

Cerataulus turgidus Eh.

Coscinodiscus blandus A. S.

- centralis Eh.

- excentricus Eh.

- marginatus Eh.

- oculus-iridis Eh.

- radiatus Eh.

Cyclotella striata Grun.

Cymbella gastroïdes $K t z$.

Diploneis bombus Eh.

- didyma Eh.

- elliptica Cl.

- interrupta Cl.
Diploneis Smithii $\mathrm{Cl}$.

Eunotia monodon $E h$.

Isthmia neroosa $K t z$.

Melosira sulcata $\mathrm{Ktz}$.

Navicula cancellata Donk.

- Fischëri A. S.

- Pormosa Greg.

- gigas Ktz.

- Hennedyi W. Sm.

- - var. furcata.

- humerosa Bréb.

- lyra Eh.

- maculata var. major.

- pachyptera Eh.

- peregrina Ktz.

- permagna Bail.

Nitzschla circumsuta Grun."

- scalaris W. Sm.

- Tryblionella $\nabla$. maxima.

Plagiotropis vitrea Grun.

Pleurosigma balticum W. Sm.

Pyxilla baltica Grun.

Rhabdonema adriaticum Ktz.

Raphoneis amphiceros Eh.

Scoliopleura tumida Rab.

Stauroneis gracilis Eb.

Darirella striatula Turp.

Triceratium antediluvianum Eh,

- favus Eh.

No 990 NEW BRITAIN - CONNECTICUT (États-Unis)

Achnantidium flexellum Brẻb. Amphora libyca Eh.
Amphora ovalis Kte.

- pediculus Grun. 
Cocconeis placentula Eh.

Cyclotella antiqua IV. Sm.

- Kǘtzingiana Thw.

- striata var. mesoleia.

Cymatopleura elliptica W. Sm

$$
\begin{array}{cc}
\text { - } & \text { - var. torta. } \\
\text { - } & \text { solea W. Sm. }
\end{array}
$$

Cymbella americana A. S.

$$
\begin{aligned}
& \text { - amphicephala Neg. } \\
& \text { - anglica Lag. } \\
& \text { - cistula Kirch. } \\
& \text { - cuspidata Ktz. } \\
& \text { - cymbiformis Bréb. } \\
& \text { - delicatula Ktz. } \\
& \text { - Ehrenbergii Ktz. } \\
& \text { - gastroïdes Ktz. } \\
& \text { - heteropleura } \nabla . \text { minor. } \\
& \text { - hybrida Grun. } \\
& \text { - parva V. H. }
\end{aligned}
$$

Denticula frigida $K t z$.

Encyonema triangulum $\mathrm{K} t z$.

$$
\text { - turgidum Grun. }
$$

\section{Epithemia argus $\mathrm{Ktz}$.}

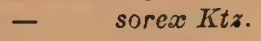

- turgida Klz.

- - var. granulata.

- - var, vertagus.

- zebra Ktz.

\section{Eunotia diodon Eh.}

Fragilaria brevistriata Grun. - construens Grun.

Gomphonema acuminatum v. coronata.

$$
\text { - constrictum Eh. }
$$

- - - var. capitata.

- intricatum $\mathrm{Ktz}$.

- - var. pumila.

- sphærophorum Eh.

- subtile Eh.

- - var. angusta.

Mastogloia lacustris Grun.

Melosira crenulata $\mathrm{Ktz}$.
Navicula affinis Eh.

$$
\begin{aligned}
& \text { - amphirynchus Eh. } \\
& \text { - amphibola Cl. } \\
& \text { - bacilliformis Grun. } \\
& \text { - bisulcata Lag. } \\
& \text { - Hrebissonii Kt. } \\
& \text { - cuspidata Ktz. } \\
& \text { - dicephala Eh. } \\
& \text { - dubia Eh. } \\
& \text { - Gendrei var. intermedia. } \\
& \text { - firma } \mathrm{Ktz} \text {. } \\
& \text { - gastrum Eh. } \\
& \text { - intorrupta } \mathrm{Ktz} \text {. } \\
& \text { - iridis Eh. } \\
& \text { - legumen Eh. } \\
& \text { - limosa Ktz. } \\
& \text { - major } \mathrm{Ktz} \text {. } \\
& \text { - mesotyla Eh. } \\
& \text { - oblonka Ktz. } \\
& \text { - peregrina Ktz. } \\
& \text { - papula Ktz. } \\
& \text { - radiosa Ktz. } \\
& \text { - - var. acuta. } \\
& \text { - sphcerophora Ktz. } \\
& \text { - termes var. stauroneiformis. } \\
& \text { - tuscula Eh. } \\
& \text { - ventricosa Eh. } \\
& \text { - viridis } \mathrm{Ktz} \text {. } \\
& \text { - viridula Klz. }
\end{aligned}
$$

Pleurosigma attenuatum W. Sm. Pleurostauron acutum Rab.

Rhopalorlia gibba O. M.

$$
\begin{array}{ll}
\text { - } & \text { - var. parallela. } \\
\text { - } & \text { gibberula O. M. } \\
\text { Stauroneis amphilepta Eh. } \\
\text { - } & \text { anceps Eh. } \\
\text { - } & \text { Baileyi Eh. } \\
\text { - } & \text { gracilis Eh. } \\
\text { - } & \text { phonicenteron Eh. }
\end{array}
$$

Surirella linearis oar. constricta.

- splendida Eh.

Synedra capitata Eh. 


\section{N`991 MILLBURY - MASSACHUSSETS (États-Unis)}

Dépôt fossile d'eau douce

Cymbella anglica Lag.

- aspera Eh.

- cuspidata Ktz.

- Ehrenbergii Ktz.

- heteropleura Ralfs.

- maculata Ktz.

Bncyonema gracile Rab.

Bunotia major Rab.

- pectinalis Dillw.

- - var. ventricosa.

- polyodon $\mathrm{Eh}$.

- prærupta Eh.

Fragilaria elliptica Grun.

- undata W. Sm.

- virescens Ralfs.

Gomphonema acuminatum Eh. var.

- capitatum Eh.

- intricatum Ktz.

Melosira lineolata Grun.

Navicula ampliata Eh.

- commutata Ktz.

- dactylus Eh.
Navicula dilutata Eh.

- divergens W. Sm.

- $\quad$ - var. minor.

- firma var. ampliata.

- gibba Eh.

- major Ktz.

- mesolepta Ktz.

- - var. stauroneiformis.

- nobilis Eh.

- Peisonis Grun.

- radiosa $\mathrm{Ktz}$.

- rupestris Hantz.

- serians Ktz. var. $\beta$.

- transversa A.S.

- viridis ktz.

Stauroneis gracilis Eh.

- phoenicenteron Bh.

Surirella linearis W. Sm.

Synodra ulna Eh.

Tabellaria fenestrata Kíx.

- flocculosa $\mathrm{Ktz}$.

Van Heurckia rhomboides Brẻb.

No 992

CORNWALLIS - NOVA SCOTIA (Canada)

Dépôt fossile d'eau douce

Cymbella aspera. Eh.

- cuspidata Ktz.

- Ehrenbergii Ktz.

Encyonema ventricosum $\mathrm{K} t z$.

Eunotia arcus Eh.

- - var. uncinata.

- prærupta El.

- $\quad$ - var. bidens.
Gomphonema Brobissonii Ktr.

- gracile Eh.

- intricatum Ktz.

- montanum Schum.

Melosira crenulata Ktz.

- lineolata Grun.

Navicula borealis Eh.

- commutata Grun. 
Navicula dilatata Eh.

- dioergens W. Sm.

- firma Ktz.

- gentilis Donk.

- gibba Eh.

- gigas Eh.

- hemiptera Klz.

- major Ktz.

- mesolepta Kt.
1. Navicula microstauron Eh.

- nobilis Eh.

- rupestris Hantz.

- seinen Eh.

- viridis Kez.

Stauroneis Baileyi Lh.

- phœnicenteron Eh.

\section{Nos 993 à 995 CONDRO - MESSINE (Sicile-Italie)}

\section{Dépôt fossile marin miocène}

Ce dépôt, ainsi que ceux de Mondaino et de Casatico, ont été étudiés et analysés par le $\mathrm{D}^{\mathrm{r}}$ Achille Forti, de Vérone, de qui nous les avons reçus.

Actinocyclus moniliformis Ralfs.

$$
\text { - stellatus Forti. }
$$

Actinoptychus Janischii Grun.

$$
\begin{aligned}
& \text { - spinifer Grun. } \\
& \text { - } \quad \text { splendens (Shadb) Ralfs. }
\end{aligned}
$$$$
\text { - vulgaris Schum. }
$$

A mphora formosa Cil.

$$
\text { - obtusa Greg. }
$$

Asterolampra acutiloba Forti.(5 rayons).

$$
\begin{array}{ll}
\text { - } & \text { marylandica Eh. } \\
\text { - } & \text { punclifera Grev. }
\end{array}
$$

Asteromphalus Grevillei Wall.

- $\quad$ variabilis (Grev.) Forti.

Auliscus colatus v. strigillata A. S.

- sculptus (Sm.) Ralfs.

Biddulphia moronensis Cl.

- permagna Pant.

- Tuomeyi Bail.

- $\quad$ - var, gibbicornis $\mathbf{n}$.

var.

Campylodiscus horologium Will. v. Pfitzerii A: S.

Cocconels Ræana Pant.

- vilrea J. Br.
Coscinodiscus curvatulus Grun.

- dubiosus Grun.

- _ - _. var. subtilissima.

- $\quad$ eutychus (Eh.) Azp.

- leptopus Grun.

- oculus-iridis Eh.

- radiolatus $\mathrm{Eh}$.

- subtilis Eh.

- $\quad$ - var.

Euodia minor Temp. et Per.

- gibba Bail. $\nabla$. moronensis Temp. et Per.

Goniothecium odontella Eh.

Grammatophora lyrata Grun.

$$
\text { - stricta Eh. }
$$

Hyalodiscus radiatus O'Meara

Navicula bomboides A. S.

- bombus Eh.

- clavata Greg.

- $\quad$ - var. caribøa Cl.

- $\quad$ - var.

- crabro Eh.

- . - var. multicostata.

- $\quad$ - var. pandura.

- _ var. separabilis. 
Navicula fusca Greg.

- geminata Grev. v. fossilis Pant.

- Henuedyi W. Sm. v. cuneata Grun.

- $\quad$ - var. manca Per.

- - var. neapolitana.

- liber W. Sm. v. robusta Grun.

- lyra Eh.

- - var. intermedia Per.

- nitescens Greg.

- O'S waldii Grev.

- $\quad$ - var. visenda Temp.

- praetexta Eh.

- perplexa Per.

- polysticta A.S. . elliptica Per.

- Sandriana Grun.

- Smithii Bréb.

- $\quad$ - var. major (Cl.) Per.

- spectabilis $\nabla$. emarginata $\mathrm{Cl}$.

- suborbicularis Greg.

- vacillans A. S.

Nitzschia insignis.

- panduriformis Greg.

Pleurosigma Eudon Pant.

- formosum W. Sm. var.

- sagitta Temp. et $\mathrm{Br}$.

Podosira corolla A. S.

Pseudonitzschia cretae (Eh.) Forti.

-

fungairiñoi (Azp.) Forti.
Raphoneis nitida Greg. fa major Per.

Rhablonema adriaticum Ktz. v. fossilis Paut.

Stephanopyxis turris v. intermedia Grun.

Synedra haculus Greg. fa reducfa nooa.

- superba kitz.

Stictodiscus californicus v. areolata Grun.

$$
\begin{array}{ll}
- & \multicolumn{1}{c}{\mathrm{f}^{\mathrm{a}} \text { major nooa. }} \\
- & \text { Hardmannianus Grev. } \\
- & \text { Jeremianus T. et WV. } \\
\text { - } & \text { parallelus Pant. fa quadrala. }
\end{array}
$$

Surirella Baldjickii Norm.

- - var.

- fastuosa Eb.

- $\quad$ - v. orbicularis Per.

Tracheneis aspera Cl.

- $\quad$ - var.intermedia.

Triceratium antediluvianum Eh.

- - var.

- cinnamomeum Grev.? var.

- formosum Bright. fa pentagona.

spinosum $\nabla$. minor fossilis

A. S.

Willemoesia elongata Forti.

- _ - v. rhombica n.var.

Nos 996 à 998 CASATICO - PARMA (Italie).

Dépôt tertiaire marin

Actinocyelus Ehrenbergii Ralfs. - Janischii Schum.

Actinoptychus crucifer $\mathrm{n}$. sp.

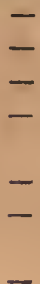
heliopelta $\nabla$. minor Br. intermedius A. S.

$-$

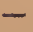
minutus fa major Forti. sculptilis A. S. v. tetramera Forti.

spinifer Grun.

splendeus $\mathrm{v}$. haliony $\mathrm{x}$ Grun.

undulatus fa maxima.

Biddulphia sp. ?
Amphora egregía Eh.

Antelminellia gigas Schüett.

Arachnoidiscus ornatus El. var.

Asterolampra aftinis Grev. v. cellulosa Forti.

marylandica Eh. v. fossilis Pant.

rotula Grev. variabilis Grev.

Aulacodiscus affinis Grun.

- amonus Grev. italicus Forti.

Cladogramma conicum Grev, 
Cocconeis Lanzii Forti.

- præcellens Pant. v. paucistriata Forti.

- splendida Grun.

- vitrea J. Br.

Coscinodiscus apiculatus $\vee$. æinensis Eh.

- asteromphalus Eh.

- centralis Ratt.

- decrescens.

- Lewisianus.

- marginatus Eh.

- moronensis Ratt.

$-$

- $\quad$. latilimba Forti.

- $\quad$ - v. major Forti.

- oculus-iridis Eh.

- - var.

- odontodiscus Grun.

- radiatus $\mathrm{Eb}$.

- $\quad$ - var.

- rhombicus Grun.

- Rothii Gr. fa spinulis densioribus.

- Stokesianus Grun.

- subnitidus Ratt.

- subtilis Eb.

- superbus Hard. v. moravíca.

Craspedodiscus elegans Eh.

-

.

$-$

Buodia gibba Bail.
Goniothecium odontella Eh.

Grammotophora robusta Dipp.

$$
\text { - } \quad \text { stricta } \nabla \text {. fossilis Grun. }
$$

Hemiaulus Weissii Grun.

Melosira clavigera Grun.

- ornata Gr. v. reducta Forti.

- Pethöi Pant.

- sulcala $\nabla$. radiata Grun.

Navicula Hennedyi WV. Sm.

- lyra v. intermedia Per.

- prætexta Eh.

- - var.

- Sandriana Grun.

Stephanogonia actinoptychus Grun.

Stephanopyxis corona Grun.

- eutycha Forti.

- grossecellulala Pant.

- intermedia Grun.

- lineala Eh.

- pediastriformis Forti.

- polaris Grun

Stictodiscus confusus T. et W.

Thalassiesira fossilis Forti.

Triceratium antediluvianum Eh.

- Nancoorensi Grun.

- $\quad$ - var. italica Forti.'

- Pantocseckii A. S.

Xanthiopyxis acrolopha Forti.

- globosa Eh.

- hystrix Forti.

- lacera Forti.

- oblonga El.

- panduriformis Pant.

N8 999-1.000 MONDAINO - F́RLI (Iıalie)

Dépôt fossile marin tertiaire miocène

BIBLIOGRAPHIE. - Castracane Fr. Degli Antelminelli. Diatomées fossiles de Mondaino ( Monografia statist economica amministrativa delle provincia di Forli 1880 ).

Actinocyclus Ehrenbergii Ralfs. Actinocyclus moniliformis Ralfs. 
Actinoptychus amblyoceros (Eh.) A. S.

- biformis J. Br.
splendens $\nabla$. Hulionyx
Grun.
- undulatus Eh.
- $\quad$ var. paralleli.
- vulgaris Schum. striata Forti.

Asterolampra acutiloba Forti.

$$
\text { - vulgaris Grev. }
$$

Bacteriastrum varians Laud.

Biddulphia Tuomeyi Bail. var. margaritifera Shad.

Cocconeis Raeana Pant.

$$
\text { - vitrea J. Br. }
$$

Coscinodiscus asteromphalus Eh.

- biradiatus Grev. var.

- curvatulus Grun.

- $\quad$ var. latestriata Grun.

- denarius var subtilissima Forti.

- $\quad$ elegans Grev. v. spinifera.

- excentricus Eh.

- minor Eh.

- oculus-iridis Eh.

- radiatus Eh.

- symbolophorus Grun.

Craspedodiscus Nankoorensis Grun.

Euodia gibba Bail.

Goniothecium odontella Eh.
Hemiaulus polvmorphus Grun.

Hyalodiscus levis Eh

- punctatus A. S.

- radiatus OMeara.

Liradiucus minutus a. sp. Forti.

Melosira Forolivensis n. sp. Forti.

Navicula crabro El. $\nabla$. mullicostata.

- Hennedyi W. Sm. var. cuneata.

- nitescens Greg

- prætexta Eh.

- Sinithii Bréb.

Parafia sulcata.

Pleurosigma incertum n. sp. Forti.

$$
\text { - } \quad \text { sagitta Temp. et } \mathrm{Br} \text {. }
$$

Pseudonitzschia cretae (Eh.) Forti,

Rhablonema adriaticum $\mathrm{Ktz} \cdot{ }^{-v a r}$. fossilis.

Stephanodiscus Pantocseckii Fricke.

Stephanopyxis corona (Eh.) Grun.

$$
\text { - } \quad \text { Grunowii Gr. et St. }
$$

Thalassionema nitzschioïdes Grun.

Trachyneis aspera Eh.

Triceratium radioso-reticulatum (irun.

- Wiltii Jan.

Willemoesia elongata (Grun) Forti. var . rhombica.

Xanthiopyxis hystrix Forti. 

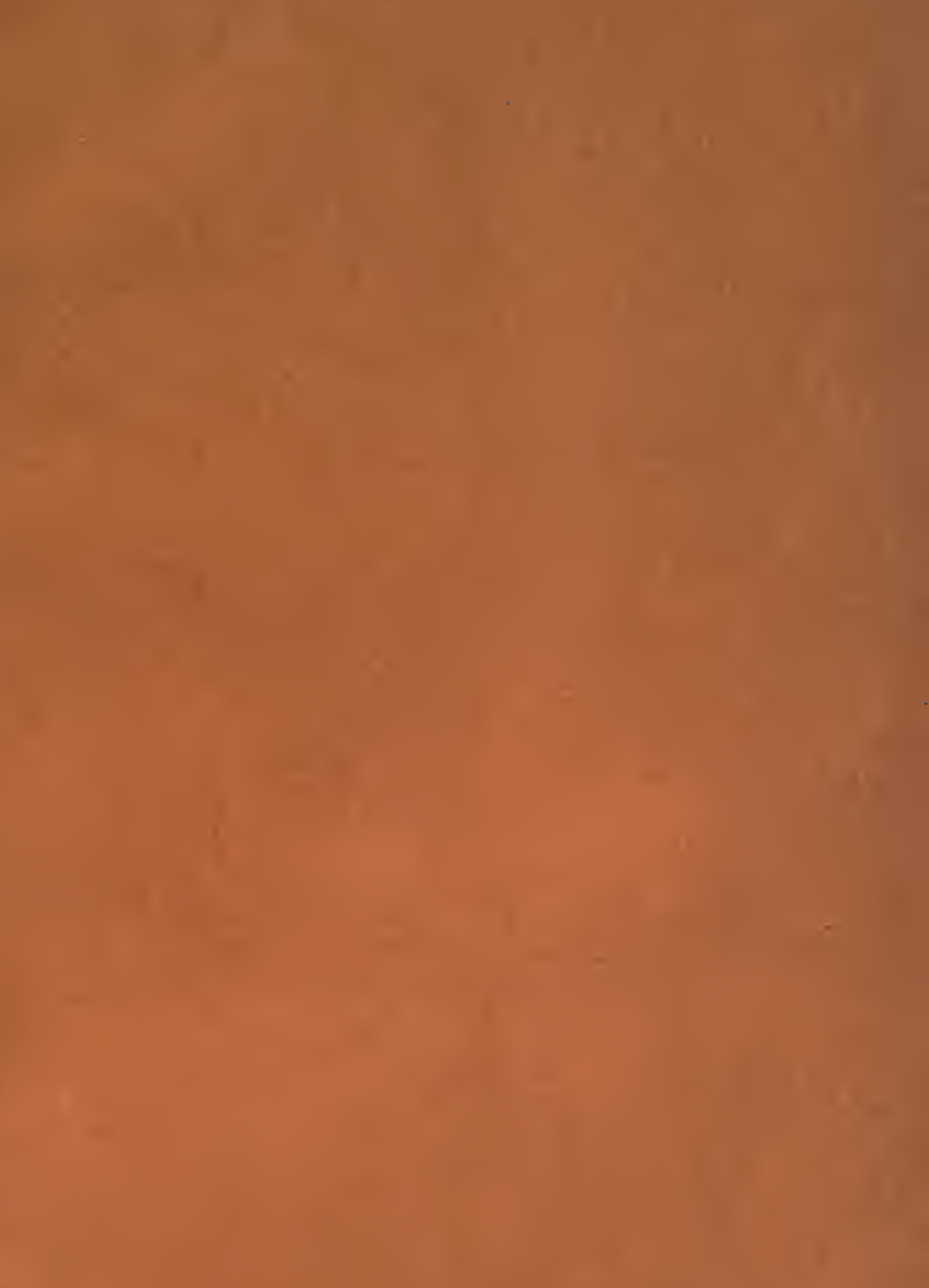


\title{
LES DIATUVEES MARINES
}

DE FRANOE

\section{et des districts maritimes voisins}

\author{
PAR
}

MM. H, et M. PERAGALLO

Ce superbe travail se compose de Irois parties :

Raphidées avec 50 planches et 1.133 figures.

Pseudoraphidées avec 39 planches et 633 figures.

Anaraphidées avec 50 planches et 421 figures.

formant un atlas de 139 planches dont une triple et 15 coloriées comprenant un total de 2187 figures dessinées d'après nature par les auteurs et accompagnées d'un texte très documenté de 560 pages avec tableaux synoptiques, analytiques et tables.

\section{Prix de l'ouvrage complet : 150 franes}

NOTA. - Tout ordre direct sera expédié franco de port contre mandal postal on chèque sur Arcachon.

Chez J. TEMPÈRE, Villa Andrée-Lucie à Arcachon (Gironde) 


\section{DIATOMEES DU. MONDR ENTIER}

\section{TABLES}

\section{COLLECTION}

Tempere et Peragallo

(2e EDITION)

Chez J. TEMPÉRE, villa Andrée-Lucie à Arcachon (Gironde) 



\section{TABLE DES PROVENANCES}

Les erreurs ou omissions dans le texte ont été corrigées ici.

Le premier chiffre indique la page du texte; les suirants, les numéros correspondant aux préparations.

D. R. Diatomées d'eau douce récentes.

M. F. Diatomées marines fossiles.

D. F. Diatomées d'eau douce fossiles.

S. R. Diatomées saumàtres récentes.

M. li. Diatomées marines récentes.

S. F. Diatomées saumâtres fossiles.

- PEL, Diatomées pélagiques ou Plankitons.

\section{田无○卫耳}

\section{ALLEMAGNE}

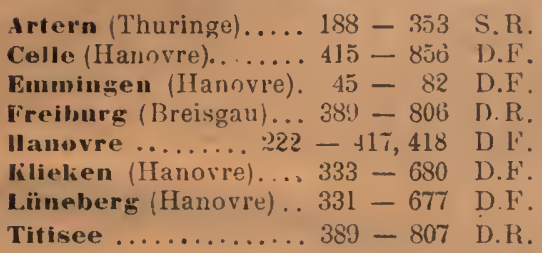

\section{ANGLETERRE}

Appleby (Westmorland). $172-320$ D.R.

$$
\text { - }-208-391 \text { D.R. }
$$

Colchester (Essex) $327-662,66^{\circ}$ S.R.

Cwm Bychau (Wales). 416 - 860 D.F.

Llyn Arinig Bach( Wa-

les).............. $253-484$ D.F.

Premnay Peal........ $312-611$ D.F.

Sheerness on Sea... $331-676$ M.R

- $\quad \ldots 279-557$

Waslingtun (Sussex). $329-663$ D.R.

\section{ECOSSE (Angleterre)}

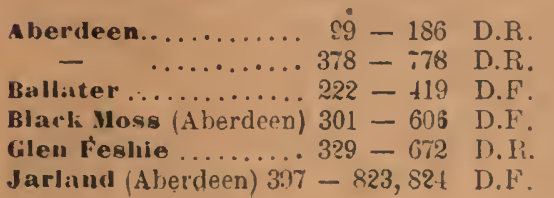

Loch Cuithais....... 298 - 601

Loch Kinuurd ... $135-248,249$

Luch Maree ....... 122 - 222

Luch Shin.......... 15 - 26

Monkstead Sliye .... 230 -450

Ordie (A berdeen) ..... 239 - 459

liver Shannon....... $365-754$

Sheene (Aberdeen)... 414-854

Sound of Null....... $91-167$

D.R.

D.F.

D.R.

D. R.

D.F.

D.F.

M.R.

D.F.

PEL .

\section{IRLANDE (Angleterre)}

Belrant....... 422 - 873, 874 D.R.

Belfiat........ 424-877, 878 D.F.

Belfast (Collin IIill).. 436 - 897 D.R.

Toume Bridge... $239-460,461$ D.F.

\section{AUTRICHE}

Alursee $\ldots \ldots \ldots \ldots \ldots 4437-901$ D.R. Bilin.......... 582 - 564.565 D.F.

Dalmatie (Yotes de)... $401-830$ II.R.

Franzenslual (Botême) 256 - 493 S.F. - $\quad$ - $\quad 349-727$ D.F.

Lissa (Dalmatie) ..... 304 - 616 M.R.

Limbourg...... $321-649,650$ M.F.

Saint-Peter (Rei Linz). 438 - 902 D.R.

Soow (Bolième)...... 13 - 21 S.F.

Styrie (Tuf de)......200 - 371 I. F. 


\section{BELGIQUE}

Blankenberghe... $254-488,489$ ...... $434-893$

Heyst............444 4893

Le Zwyn........303-613, 614

\section{DANEMARK}

Für (Ile de)...... $169-310,311$

Great Belt.......... $11-16$

Kjerteminde ....... $55-102$

Kundshoved........... $11-17$

Mors (1lo de) ...... $16-27.29$

(Ebelo.............. 33t - 683

Odense Fjord....... 54 - 101

Skive (Jutland)...... 421 - 872

\section{ESPAGNE}

\section{Cerro del Pintado}

(Moron) ....... 205 - 383,384 M. F.

Cortijo de Jalapa

(Moron)....... 205 - 379, 380

Gijon (Oviedo) .......2 264 - 517

Jaen........... $323-653,654$

Montemayor..... 325 - 655-657

Moron ......... 201 - 376-378

Osuna (Séville)... 326 - 658-660

Saint-Sébastien ...... $298-600$

Séville............421 - 871

Tierra del Pasada

alta (Moron) ... 236 - 385, 386

Tierra del Salado(Mo-

ron)......... $205-381,382$

\section{FRANCE}

Aigues-Mortes ...... 266 - 522

Aix (Provence)....... $48-89$

Ajaccio (Corse)... 446 - 915-917

Allanche (Cantal). $355-738,739$

imbleteuse ... . . . 297 - 598

Andreugcolet (H-Loire) $369-762,763$

Araules (H $H^{\text {te }}$ Loire) $355-740,741$

Arcaclion (Gironde). $38-71,72$

$$
\text { - } \quad-\quad \ldots \ldots 181-336
$$

- $\quad$ - .463-951-973

A urillac(Cantal). . $73-133,137$

Banyuls-sur-Mer.... $43-80$

$$
\begin{aligned}
& - \\
& -\quad .157-285-287 \\
& -\quad \ldots .383-793
\end{aligned}
$$

Barre de la Bidassoa. $113-210$ Béxlers........... 266 - 521

Biscarros (Landes)...452-931
M.R.

I. $R$.

It. $R$.

M. R.

II. F.

PEL.

S.R.

PEL .

II. $F$.

PEL.

S.R.

I. F.

II. F.

D.F.

I. F.

II. F.

II. F.

II. $F$.

II. $R$.

D.F .

II. $\mathrm{F}$.

II. $F$.

S. R.

D.R.

PEL.

D.F.

M. $R$.

D.F.

D. F.

II. $R$.

II. $\mathrm{R}$.

II. $R$.

PEL.

D.E.

I. R.

II. $R$.

II. $R$.

PEL.

II. $R$.

D.R.

S. R.
Boulogne-sur-Mer . . $32-58$

Briancon........... 317 - 614

Cabours........... $88-166$

Cancale........285 - 571, 572

Cannes ........... 282 - 566

- $\quad \ldots \ldots \ldots \ldots .283-567$

- $\quad \ldots \ldots \ldots \ldots . .304-615$

- (La Bocca)... 431 - 888

Cap Breton ......... 317 - 643

Ceq (Auvergne)....... 365 - 755

Celles (Ciantal)....... $67-122$

Cette.............3 $368-761$

- (Port de).......2 230 - 437

Ceyssac(Hto-Loire) $168-307,308$

Ceyssat (Puy-de-D) $189-3555.356$

Charay (Ardèche) ..... 105 - 192

Chatel-(iuyou (Pus-de-D.) $256-494$

Chaville (Seine-et-Oise) $285-573$

- $\quad 301-607$

Corge (Mousse de). $11-18,19$

Cotes du Languedoc. $229-434$

Cotes du Morbihan .. 435 - 896

Crenx Mortier (P-d-D) 153 - 278, 279

Dieppe ........... 302 - 609

Dunkerque (Port de) . $296-594$

Enghien ........... $356-744$

Etang de Saint-Quen-

tin........... $373-770$ D.R.

Etang de Saint-Lonp

(Puy-de-Dôme) ..... 10 - 13 D.R.

Erretat............ $371-766$ II.R.

Faufouilloux (Cantal) $217-403,404$ D.F.

Fontaine Delille (P-d-D) $106-196$ D.R.

Fontillou (Cantal). $370-764,765$ D.F.

Golfo Juan ........2 216 - 403 M.R.

IIontleur........... $69-125$ M. R.

He de lé $\ldots \ldots \ldots \ldots .416-x_{59}$ M. R.

Joursac (Cantal) ... $29-51-53$ D.F.

La Bade (Cantal)..... $92-169$ D F.

La Bourboule (Puy-de-D.) $63-114$ D.R.

Lac d'Aydat (Puj-de-Dóme) $162-296$ D.R.

Lac do Cazaux..... $403-834$ D.R. ...401-835-837 M. E.

Lac des Escluses (P-d D) $263-516$ D.R.

Lac d'Enghien ....... $349-727$ D.R.

Lac Leman......... 272 - 538 D.R.

Lac d'Oo............ $170-312$ D.R.

La Cassière (Poy-de-Dòme) $173-321 \cdot$ D.R.

I.a Darse (Villefranche)...303-612 M.R.

La Garde (Cantal). $353-734,735$ D.F.

Lamiouze lkochefort

Puy-de-Dômę) ... 255-491, 492 D.F.

La.Monne (Tuf de) $242-465,466$ D F.

La I'etite Sole (Nancho). $146-269$ M.R.

La Rochelle........ 23 - 43 S.R.

La Roche-Lambert $411-847$ M.R

(Haute-Loire).... $176-326,327$ D.F. 
Le Havre........, $72-132$ - $\ldots \ldots \ldots \ldots \ldots 7 \%$ 74 -138 $-\quad \cdots \cdots \cdots 77-143,144$ - $\quad$...........113 - 209 - $\quad \ldots \ldots \ldots \ldots 106-195$

- $\quad \ldots \ldots \ldots 134-244-217$

- $\quad$...........162-295

- $\quad$.............1 $181-337$

- $\quad . . \ldots \ldots \ldots 212-396$

- $\quad \ldots \ldots .220-410-413$

- $\quad . . .2 . . .2244-467$

- $\quad \ldots \ldots \ldots .254-486$

$-\quad \ldots \ldots \ldots 26262-509$

$-\quad \ldots \ldots \ldots \ldots 2272-540$

- $\ldots \ldots \ldots \ldots 284-568$

- .....2 297-59i-599

- $\quad \ldots \ldots .314-635,636$

- $\quad \ldots \ldots \ldots 356-742,743$

Le Monastier (Hite-Lo) $271-536,537$

Les Queralles (P-de-D) 89 - 162-164

Le Tréport ......... $181-338$ - $\quad \ldots \ldots \ldots 236-451$

Luchon (Pyrénèes) ... $329-670$

Lugarile (Cantal). $354-736,737$

Lussat (Duy-de-Dome). $259-500$

Menton...........240 - 46:

Meuilon (Seine-t-0ise) . . . 121 - 225

$$
\text { - } \quad \text { - } 170-313,314
$$$$
\text { - } \quad-\quad \ldots 327-661
$$

Moissac (Tarn-et-Garonne). $140-25$ '

$$
\text { - } \quad \text { - . } 170-315
$$

$$
\text { - } \quad-\quad .170-315
$$

Moissac (Cantal). . $142-262-26-$

Monaco ..........406 - 838

Montcourt (Seine-et-Ilarne). $37-70$

Neussargues (Cantal)... 214 - 400

- $\quad-219-408,409$

Niee.............267 - 523

- (Port de)........ 328 - 667

Olhy (Puy-de-Dôme) $177-328,329$

Palavas............ 269-513

- $\ldots \ldots \ldots \ldots . . . .6316-611$

Paris............. $10^{\circ} 2-294$

- $\ldots \ldots \ldots \ldots 220-411,412$

- .............271 - 534

Ponteix (Puy-de-Dôme). $41-76,77$

Pourchères (Ardéthe) $104-190,191$

Pré-Cohondy (P-de-D) $37-68,69$

Puy-de-Mur (Puy-de-D) $17-30.32$

Pyrénées........... 151 - 275

- $\quad \ldots \ldots \ldots . .254-487$

- $\quad$.............3 329-668

Randanne (Pay-de. D) $112-207,208$

Roanne (Loire)........ 156 - 284

Roull has Bas (P-d-D) $206-387,388$

Roya.........22 2239,40

- $\ldots \ldots \ldots \ldots . . .381-789$

Royat (Puy-de-Dòmo).. 262 - 508
II. $R$.

S.R.

I. R.

II. $R$.

II. $R$.

II. $R$.

M. $R$.

M. R.

S.R.

II. $\mathrm{R}$.

M. $R$.

S.R.

M. $R$.

II $R$.

M. R.

M. R.

M. $R$.

I. $R$.

D.F.

D.F.

M. R.

I. $R$.

D.R.

D.F.

D.R.

M. $R$.

D.R.

D.R.

D.R.

D.R.

D.R.

D.R.

D.F.

II. R.

D. 1 .

D. R.

D.F.

S.R.

I. $R$.

D. $F$.

S.R.

I. $R$ :

D.R.

D.R.

D. R

D. $F$.

D.F

D.F.

S.F.

D.R.

D.R.

D.R.

D.F.

D.R.

D.F.

PEL.

PEL .

D.R.
Saint-Cloud (S-et-0). $328-666$

Saint-Floret (Pay-de-D) $187-359$

Saint-Léger (Oise).... $286-574$ - $\quad$ - $372-768,769$

Saint-Lunaire....... $99-185$ - $\quad \ldots \ldots .224-421$

Saint-Malo ......... $68-123$

sainte - Marguerite

(Puy-de-Dôme) .. 127 - 231, 232

Suint-Vazaire....... $17-33$ - (Le Brivet) 18 - 34 (Port de).. $20-35$

Saint-Nectaire $\langle P$-de-D $) 62-113$

Saint-Saturnin ( $P-d-D) 124-226-228$

Saint-Servan........ 302 - 610 ........ $373-772$

Saint-Seurin (Médoc). $184-343$

- $\quad-.258-498$

Theix (Fontaine) (P-d-D, $261-506$

Toulouse.......262 - 511, 512

- $\quad \ldots \ldots \ldots \ldots 272-539$

Trouvillo........ 228 - 430,431

- $\quad \ldots \ldots \ldots .248-476$

- $\quad \ldots \ldots \ldots \ldots 262620.510$

- $\quad$............... $368-760$

Val d'En fer(Puy-de-Dome). 257 - 495

Var (Embouchure du).. $174-322$

Varennes (Pay-de-Dóme) . $161-293$

Vassivières (Puy-de-Dome) $91-168$

Verneuge(P.-de-D.) $80-149,150$

- (Fontainede) $261-507$

Villefranche-sur-Mer. $71-130$

$\begin{array}{ll}- & .193-364 \\ - & .302-608 \\ - & .329-671 \\ & .436-898\end{array}$

Villers-sur-Uer...... $99-184$

D.R.

D.R.

D.R.

D.R.

II. $R$.

M. $R$.

M.R.

D.R.

M. R.

S.R.

S.R.

D.R.

D.F.

M. $R$.

M. R.

S.R.

M.R.

D.R.

D.R.

D.R.

PEL.

PEL.

M. R.

I. $R$.

D.R.

M. $R$.

D.F.

D.F.

D.F.

D.R.

M. R.

II. $R$.

I. $R$.

I. $R$.

M. R.

II. $R$.

- $\quad$......4 $435-895$ S.R.

W'imereux......... 7 - 4 PEL. - $\quad$.........2. $296-594$ M.R.

\section{GRËCE}

Ife de Zante..... $336-691.693$ M. F.

\section{HOLLANDE}

Flessingue........455-934 M. R.

\section{ITALIE}

Bagnolo....... 444 - 911,912 D.F.

Bergonzano...... 224 - 422-425 MI.F.

Caltanisetta (Sicile). $311-626$ M. F.

Casatico....... $478-996-998$ M. F.

Castel del Piano. $402-832,833$ D.F. 
Catania (Sicile)..... $223-420$ Condro.... . . . . $477-993-995$ Formignвио........ $387-800$ Groste (Sicile).... 45ัก- $926-929$ I.icata (Sicile) ... 337 - 696.69S Marmorito....... 19خ - 367-369 Iodena........... 17.; -323 Vondaino . . . . $479-999,1.000$ Monte suiata....... $46-85$ Nonte Gibbio.... 331) - 673-675 Vaples (Golfe de).. $39-73-75$ Orvieto............ $414-910$ Palazzolo Acreide $273-512,513$ Sardaigne (Ile de)... 258 - 1:9 Santa Fiora.....445 - 913,914 Santa Tecla (Sieile) $443-908,909$

\section{HONGRIE}

Bibarczfalva ....... 27t -517 Bodlos.........269 - 530,531 Borostelek...... $171-318,319$ Bory.......... $51-94.95$ - ............. $265-518,519$

Bremia . . . . . . . $185-346-349$ Dolje.......... 232 - 442,443 Dubravica. . . . . $312-632,633$ Blesil......... 207 - 389, 390 Errlobenya...... 268 - 528,529 Felmenes.......263-514,515 Felso Estergaly ..... 49 — Gyongyos Pata .. $166-304,305$ Izsopallaga Serges... 365 - 756 Jastraba . . . . . . 338 - 699, 700 Karami ........218 - 405-407 Kavna.........185 - 346-349 Kekko......... 1.18 - 270-272 Kis Rippeny........ $193-363$ Koggik............ $181-339$ Kopecz.........110-203-205 Lac Ballaton. . . . . . . 364 - 752 Nágy-Curtos.... $129-236.238$ Nagy-Tapolesany . . . 336 - 694 Nyermegy ....... 94-173-175 Palogla..........4417 - 661 Pila.............. $274^{\circ}-516^{\circ}$ Praries........... 406 4068 Szakal ........6. $69-126-129$ Szent Peter ..... $\succ-8,9$ Szurdok Puspoki $241-463,461$ Talya..........259-501, 502 Tavarnok ......... $315-638$ Thrais ........ $273-544,515$

D.F. S. F. M. F. II. F. D.F. I. F. II. F S. F. II $\mathrm{F}$. S. F $S \mathrm{~F}$. II. $\mathrm{F}$. D. $F$. II F D. $F$. II $\mathrm{F}$. II. $\mathrm{F}$. II. $\mathrm{F}$. D. R. II. $\mathrm{H}^{\mathrm{S}}$. D. $\mathrm{F}$. D. $R$. I. $F$. D. $R$. S. F. S. F. D. F l.) $R$, II. $F$. M. $F$. S.F. S.F. D. $R$ D.F.

\section{NORVEGE}

Cìté onest ......... $23-42$ Bdsfjord.......... $22-41$

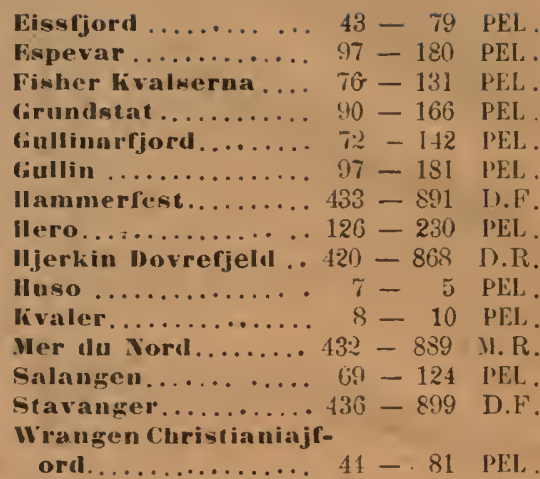

\section{PORTUGAL}

A cores $($ Iles) . . . . . $307-620$ M. $R$. Porto............ 459 - 941 D.R. Raiva Aveiro....... $459-940$ D.R. Salo (Einbouchure du) $27-50$ S.R.

\section{RUSSIE}

Ananino........ $9-\mathrm{II}, 12$ M. F. Broptorp-Pojo (Finlande) $267-524$ D R. Kertscil.........4 $413-851-853$ S. F. Kisstil) (Caucase).... $398 \div 825$ D.R. Kusnetzk ....... $140-258-261$ M. F. Larloga (Finlande)... 2800 - 575 D.R. Mer Caspienne...... 2i2 - 541 y R. Mer Nuire ........271 - 535 M.R. Naarajarvi.......... 434 - 894 D.R. Pulasjarvi........ 46 - 86 D.F.

\section{SUISSE}

Aabach............ $380-785$ D.R. Aar (Berne)........ $388-801 \cdot D . R$. A rulla (Alpes) ... I 39 - 254, 255 D.R. Auseeli (Horgen) .... 388 - 80 ? D.R. lierne............ 299 - 602 D.R. Galmental (Fontajne de). $38 \mathrm{~s}-893$ D.1i. Goschenen Alp.....2286 - 576 D.R. - $\quad-\ldots 287-577,578$ D.R. Greifensee ......... 370 - 781 PEL. Lac de Geneve ...... 245 - 470 D.R. Iac des cantons $31-56,57$ D.R. Lac de Lueverne...... 299 - 603 D.R. Lac de Sarnen... $289-583,584$ D. $R$. I.ac tle Sempach.... 317 - 612 D.R. I,ago di Lusano ..... 378 - 780 PEL . MeinradstunnenVitzol.......... $412-850$ D.R. pout du Rhin (Constance)........... $389-805$ D.R. 
Richterswil ........ $390-808$ Rhin bei Ermalengen 381 - 788 Sulzenbach......... $380-786$ Urserenthal......... $380-787$ Vierwaldstattersee. . $379-783$ Wettswil (Zurich) . . . 390 - 809 Zurichsee....... $379-782-784$

\section{SUEDE}

Antalt........... $171-316$ Areskutan (Jamtland). $150-274$ Bohuslow ........... 126 - 229 Christianstad ........ $47-88$ Còte............. 124 - 224 Dejeruas.......... $367-758$ Droblak (Christiania).. $7-6$ Grebestad.......... $410-846$ IIelisjon............257 - 496 Lyskitt........... $305-617$
D.R. D.R. D.R. D.R. PEL. D.R. PEL .

PEL . D.R. PEL. S.R. I. R. D.R. PEL . II. F. D.R. M. $R$.
Mer Batique.... $105-193,194$ M. R. North Atlantic...... 171 - 317 M.R. Pautrask.......... 368 - 759 D.R. Rysshy Calnar...... 111 - 206 D.F. Upsala........... 433 - 892 D.R. Wernamo (Smaland). $192-361,362$ D.R. Wesleo Calmar ..... 420 - 869 D.R. Westeras............ $371-767$ S.R.

\section{TURQUIE}

Constantinople ..... $472-987$ II.R. Le Bosphore...... 33 - 59, 60 M.R. Warwick (lle de Rhodes) .. $437-900$ D.R.

\section{MÉDITERRANÉE}

Malte (Ile de)........ $299-604$ D.F. - $\quad-\ldots \ldots .4422-875$ II. R.

\section{ASIF}

GEYLAN
Colombo.......... $79-148$ M.R.

\section{JAPON}

Abashiri....... $23 \check{\jmath}-448,449$ Cinte............ $378-779$ Hakorlate....... $358-745-747$ lluitres (Estomae d'). . $225-426-428$ Kobe............4 $418-865$ Lac Suwa ........ $387-801$ .ıji.............144 - 267 Nagasaki...... $383-791-797$ II. F. II. $R$. II. F. I. $R$. II. $R$. D.R. II. F. II. $R$.
Sava River......... $156-283$ Sendaii......... $14-22-25$ -- (Lignites) .. $184-314,345$ Sentenai........... $387-799$ Setanaigori..... $358-748,749$ Tukuro...........4 48 - 90 Wembets........... $358-750$ Yeso............. $164-301$ Yokohama (Rade)..... 267 - 525 (Sondage) . $164-300$ Yokosky Verny ..... $385-798$ M. R. Suez (Port de) ...... $282-563$ I. R.

\section{AFRIQU耳}

\section{ALGERIE}

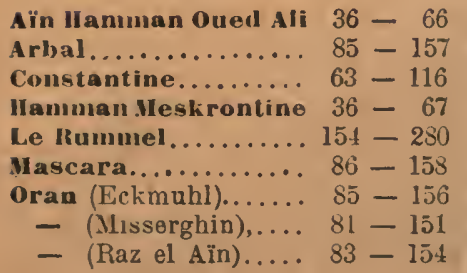

Oran (Saint-Eugène)... $84-155$ M.F. - (Village nègre).. 82 - 152 M.F. Oned Berdi........ $160-290$ D.R. Oued ben Amor..... 107 - 197 D.P. Port u'alger........ $230-438$ I. R.

\section{ETHIOPIE}

Colline de Sodo.. $251-480,481$ D.F. Lac Zuay........252 - 482,483 D.F. 


\section{MADAGASCAR}

Nossi-Bé ....... 175 - 324, 325

Tanatave...... $52-96-100$

II. $R$.

M. R.

\section{TRANSVAL}

Achole Farm

\section{TUNISIE}

Иаmman Lif......... $364-753$ D.R.

\section{AIHEIQUH}

Baffin's Bay........ $35-64$ Détroit de Davis.... . 334 - 684 - $\quad-\ldots 334-685$ spitzleerg .......... $36-65$

\section{GANADA}

Besancon's Lake (No-

va Scotia).......... $352-733$

Cornwallis (Nova Sco-

tia)...........4 4;6- 992

Drakeswille........ $332-679$

Earl Town Lake. . ...300-605

Grant Lake (Picton Co) $203-374$

II

Mackintosh Lake. $10-14,15$

Maclean's Pond (Pic-

ton $\left.\mathrm{C}^{\circ}\right) . \ldots \ldots \ldots \ldots 210-394$

Mill's Pond (Morris-

River)...........211 - 395

Musquodoboit Lake

(N. S.)...........4 $425-879$

Swamps Pond (Pic-

ton $\left.\mathrm{C}^{\circ}\right) \ldots \ldots \ldots \ldots .201-373$

\section{GTATS-UNIS}

Atkin' Pond (Conn.). $118-216$ Atlantic City (N. J.) $77-145-147$ Beach Haven (N. J.). $450-925$ Beat ties Pond(Conn.) 315 - 639, 640 Bedlington (Mlaine). $187-351,352$ Big Láke Arlington

(Wash)............ $179-334$ Birge Pond (Conn.) $20-36,37$ Boldpatte Pond (.last.). $86-159$ Bowerville (N. Hamp.) $427-883$ Boxford (Mass.)... $93-171,172$ Branford (Conn.) ..... 314 - 637 Brocton (Mass.) ......44 $411-405$ Bunnel's Pond (Conn.) $57-105,106$ Calvert Co...... 163 - 297-299 Carson City (Nerada). 310 - 624,625
PEL . PEL II. $R$. PEL .

D.F. D.F. D.F D.F D.F. D.F. D.F. D.F. D.F. D.F. L.R.

D.R. M. F. ii. $\mathrm{F}$. M. R. D. F

D.R. D.R. D.R. D.F. D.F. $\mathrm{M}-\mathrm{R}$. D.F. D.R. I. E. D.F.

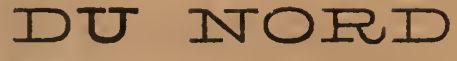

Cherryfield(Mass.) $238-457,458$ D.F. Climacum Valley

(Orcas)....... $105-302,303$ D.R.

Conradl's Red Bluf

(Cal.) ........... $426-881$ D.F.

Crane Pond ...... $291-586-588$ D.R.

Dokum Berlo (Texas).. $425-880$ D.R.

Dolgeville (Cal.) .. 275 - 548-550 M.F.

Dúck Pond (Maine)... 408 - 840 D.R.

Essex (Mass.)....442-906,907 D.F.

FallMountain (Cona.) $56-103,104$ D.R.

Fitzwilliam.......44 $401 \rightarrow 831$ D.F.

Florence (Kansas).... $430-886$ D.F.

Folly Lake (N. J.)... 457-933 D.F.

Godfrey's Bog (Mass.). 421 - 870 D.F.

Golden Gite (Cal.) ... $159-288$ D.R.

Ilaliotis (Lavage d')(Cal.) 26 - 48,49 M.R.

Uamlin's Pond

(Gonn.)........ $182-340,341$ D.R.

flarhor Bay (Maine)... 289 - 582 D.R.

IIatfield's Swamps

(N.J.)......... $439-903$ D.R.

Haughton (Mich.).... $432-890$ D.F.

llopkinton (N. H.)... $332-678$ D.F.

IIoquiam (Pujet Sound) $120-217,218$ M. R.

II ullson River (N. J.). $428-884$ S.R.

Ilumboldt (Nevada)... 327 - 664 D.F.

lce Pond (N. B. . .... $42-78$ D.R.

Jonesport (Maine) ... 427 - 882 D.F.

Jones Valley (Cal.)... 414 - 855 D.F.

Leate's Bay (Conn.). 468 - 979, 980 II. R.

Leate's Creek

(Conn.) ......4 $467-977,978$ M.R.

Leate's Island (Conn.) $467-976$ M. R.

Leat c's Pond (Conn.). $132-240$ S.R.

Long Isla ud Sound $268-526,527$ M.R.

Long Lake Winsted

(Conn.) ............ $336-695$ D.R.

Los Angeles (Cal.) $409-844,815$ I. F.

Lost Spring Ranch (Cal) ........ $279-558-560$ D.F.

Loubet (Cal.) ....2 246-471-473 M.F.

Lyon Farm (N. J.)... $460-945$ D.F. 
Marsh South End (Cono.) $469-984$ Millbury (Mass )..... $476-991$ Mobile (Ala.) ........ $47-87$ Montgonery (Ala.)... $377-777$ Monterey (Cal.)...... $63-115$ - $\quad$ - . $191-358-360$ Morris Cove (Coup.) 461 - 948,949 Morris Creek (Conn.). $470-985$ Mullbury Cuna (Texas). $400-829$ Newark (N. J.). . $399-826,827$

New Britain (Conn.).. $474-990$

New Ilaven Ilarbor

(Conn.)...........462-950

New London (Iowa). . $419-867$

Nottingham (Maryl.) 127-233 - 235

North Taunton (Mass.) $419-866$

Oakfam (Mass. ' . .....293 - 589

Olympia (Pujet Sound). $212-397$

Orcas Islanil. . . . . . . 227 - 429

órono lRiver (Maine). . 431 - 887

Owen's Ferry (Savan-

nah,............4 456-936

Patuxent River. . . $335-688-690$

Pensacola (Bay.). $178-330,331$

Phillips (Maine)...... 376 - 776

Pitt IRiver (Oregon) $312-630,631$

Ponal's Point (Conn) $350-730,731$

Pope's Creok (Mary-

land).............. 374 - 773

Port Towsend(Wash.) $61-117-121$

Puget Sound( Wash.) $179-332,333$

Quinipiac Itiver. 100 - 187-189

Kedondo Bench (Cal.) $24-45.47$

IReno (Nevada).... . . 328 - 665

IRichmond (Virginie) $98-182,183$

San bernariliuo

(Cal.)........318 - 645, 646

San Diego (Cal.).. $160-291,292$
II. $R$.

D.F.

S.P.

D. $\mathrm{F}$.

II. $\mathrm{R}$.

II $\mathrm{F}$.

S.R.

II. R.

D.R.

II. $\mathrm{R}$.

I. $R$.

I. $R$.

i). $F$.

II.F.

D.F.

D.F.

M. B.

D. $F$.

D.F.

M. $\mathrm{R}$. I. F. II. R. I). F . D.R. II, 1 .

II. $\mathrm{E}$. II $\mathrm{R}$. M. R. II. $\mathrm{R}$. II. F. D. $[4$ II. $\mathrm{H}^{3}$.

M. F M. $F$

\section{San Luiz Obispo}

((Aal.)........277-553-555 M.F.

San Pellro (Cal.). $244-468,469$ I. F.

Santa Cruz (Colo.

rado) ......... $115-213,214$ M.R.

Santa-Lucia (Cal.).. $408-842,843$ M. F.

Santa-Varia (CaJ.). $229-435,436$ M.F.

Santa-Monica (Cal.). $60-109-112$ II F.

Saco (Maine) ..... $95-176,177$ D.F.

Salinas (Cal.)........ $180-335$ M. R.

Salt See (Mansfield).. 132 - 239 S.R.

Savannah (Georgie)...457-937 S R.

Sholl Beach (Conn.) . $198-370$ M. R.

Shiloh (N.J.).... $96-178,179$ M. F.

Sing-Sing (Hudson River).. $45-84$ D.F.

South Beach (Corn.).. 471 - 986 M. R.

South Framingham

$\left(\mathrm{I} 2 \mathrm{~d} . \mathrm{C}^{0}\right)$.........440 - 904 D.R.

Stony Creck (Conn.).. $351-732$ II. R.

- $\quad$ - .4 408-841 M.R.

Swan Lake (0regor) $193-365,366$ D.R.

Tacoma (Wash.).. $58-107,108$ D.R.

Tamarack (Conn.) , $113-211,212$ D.R.

Ticle Pond (Conn.)... $474-989$ M. R.

Todul Hollow (Con.) $341-728,729$ D.R.

Tontin Lako (N. B.). $118-215$ D.R.

$\operatorname{Troy}(\mathrm{N} . \mathrm{H}.) . \ldots \ldots . .458-939$ D.F.

Viscaino IBay (Cal.)... $93-170$ M. R.

Washington $\mathrm{C} \ldots 311-627,628$ D.F.

Weequachick (N.J.) $366-757$ D.R.

West IIarbor (Conn.). $472-988$ M. R.

West River (Conno.). 154-281, 282 M. R.

White Leall Lake (N. J.) $429-885$ D.F.

White Plain (Nevada). 412 - 849 M. $\mathrm{k}^{\prime}$.

Winchester (Nass.). $415--857,858$ D.F.

Worcester (Mass.)... 257 - 497 D.1.

Yayiana Bay (Oregon). 141 - 266 M.R.

$$
\text { - _ } \quad-\quad 203-375
$$

M. $R$.

\section{AMEEIQUE DU SUD}

\begin{abstract}
Almoloya de Alquesi-
ras (Mexique).....266 - 520

Arica (Port d') (Pérou). $418-864$

Bainos (Equateur) .... 167 - 306

Buenos Aires (Rép. Arg.) $138-351$

$\begin{array}{rrr}- & - & 306-618 \\ - & - & 307-619 \\ - & - & 21-38\end{array}$

Cayenne (Guyane) ... $276-551$

Challenger Lxp (Mer

Australe) .......... 18t - 342

Chimhorazo (Equateur). . 364 - 7.51

Demerara (Guyane)... 7 - 7
\end{abstract}

D.F. II. 1 . D.R. D.R. D.R. D.R D.R II. R. II. $\mathrm{R}$. D.R. II. $\mathrm{R}$.
Demerara River $\{\mathrm{Gu}$ -

yane)......... $138-251-253$ S.R. Givatemala......... $140-257$ D.F. Honolulu (liet Harai). $214-401,402$ II. R. Inecorio.(Mexique) ... $334-686$ D.F. Mejillones(Bolivie) $121-219 \cdot 221$ M. F. Pallaranga (Equateur) $288-579$ D.R. Para Itiver (Brésil)... 25t - 490 M. R. Pérou (Guanos du). $236-452-456$ M. F. Pichincha (Equateur) . $312-629$ I.R. Pitruamo Grande

(Mexique) ........ $169-309$ D.F. Potonico (Salvador).... $13-20$ D.F. 
San Luis (Rép. Arg.). 296 - 596 Saint-Vicolas (Equa-

teur)............ $289-581$ D.R.

Santiago Papastyuiano (Mexique)....... $334-687$

Tesalia (Equateur) ... $288-580$

Valléede'Toluca (Mexique)..............209-392

Valparaiso (Chili) ...2291 - 585

\section{ANTILLES}

Cambridge Estate (Bar-

bade)......... $341-725,726$

Carenage (Trinité) ... $107-198$

Chalky Cliff (Barb.). $311-719-722$

Chalky Mount(Barb.) $341-723,724$

Chimborazo (Barb.). 341 - 708-710

College Hill (Barb.). $341-704-707$
D.R.

D.F.

D.R.

D.F. II.R.

I.F. II.R. II. F. II. F. II. F. II. F.
Hopewell (Barb.). $341-715,716$ Guacleloupe (lle de la) $260-503-505$ Jérémie (Haïti)..." $108-201,202$ lingstun Ilarbour (Ja-

maïque)........44 417862

La Brea (Trinite) . . . $107-199$

L.a . Martinique... . . $417-863$ $\ldots \ldots+454-9.38$

Mount IIIllaby (Barb) $311-701.703$ Nassau (Bahama). ... 144 - 268 - $\quad-\ldots 375-775$ Newcastle (Barb.) $50 \rightarrow 92,93$ Pic Tenerilr (Barb) $341-717,718$ Pointe Pierre (Trioité).. 159 - 289 Port Lou is (Guadel.) $250-532,533$ Porto Rico.........45 - 932 Port of Spain (Trisité). . 103 - 200 Springfieltl (Barb.).. $341-711-714$ Trinitè (lle de la) . $459-942-944$
II. F. I. $R$. Y. F. II. R. II. $R$. II $R$. II. R. M. F. II. $R$. II. $R$. I.F. II. F. D.R. II. $\mathrm{R}$. il. $R$. I. $\mathrm{P}$. il. $\mathrm{F}$. II. F.

\section{OOHAIIE}

Allan's Farm(0amaru) 391 - 818-820 Auckland (Nourelle-Zélaude) 418 - 919 Bain's Farm (0amaru) $7 \tilde{5}-139,141$ Borries (Oamaru). $391-821,822$ Brunswick (Victoria).. $448-921$ Cabbagetree Swamps

(Nouvelle-Zélande)... 123 - 223 Côte de la Nouvelle-

Zélande.......210 - 478, 479

Cormack's Top (0am) $221-\$ 15,416$

Détroit de Malacea $308-622,623$

Détroit de Torres.... $460-916$

Forrester's Rock (0am) $137-250$

King Georges Sound

(Ausiralie).......... $87-160$

King Georges Sount (Australie)..... 151-276, 277 M.P.

Korawara (Nouvelle-

Zèlande) ......449-923. 924

Ile Camplsell......... 190 - 357

Iles Carolines ........ 308 - 621

lles Marquises....... $45-83$

lle Samoa...... $318-617,648$

Iles Sandwich..... 34 - 61-63

Jackson's Padidock (0am.) $5-1,2$

Lac Rotoreia (Nou-

velle-Zélande)...... $150-273$

Lake Mori (Australie). 452-930

Pakaraka (Nouv.-Zél.) $448-920$

Port d'Oamaru (Nour-Zél) $333-681$

$$
\text { - } \quad \text { - } 375-774
$$

Port Stevens (Aust.) $213-398,399$
M. F. D.F. II. F. II. $\mathrm{F}$. D. F.

D R. II. R. It. $F$. M. R. II. R. II. F. II. $R$. D.F. II. R. M. $R$. M. R. II. $R$. II. R. M. R. D.F. D.R. D.F. II. R. II. R. II. R.
Talbot (Australie).. $468-981-983$ Tamai ltiver (Tasmania).. $277-552$ Thursilay Island $(N-G$. ) $90--165$ $\begin{array}{ll}- & -281-561,562 \\ - & -294-590,591 \\ - & -295-592,593\end{array}$

D.F. S.R. II. R. II. $R$. $\mathrm{I}, \mathrm{R}$. II. $R$. Totara (Oamaru).. $391-814-817$ M.F. Troublesome-Gully

(Oamaru)....... $391-810-813$ II. F. Waitangi (Nouv.-Zèl.) $449-922$ D.F. Wangarei (Nouv.-Zèl ). 447 - 918 D.F. West Melbourne (Vict.) $461-947$ S.R. William's Bluff (0am) $231-439-441$ II. F. Yarra IRiver (Aust.) $133-241-243$ S.R.

\section{OCÉAN INDIEN}

lle d'Amsterdam.... 228 - 143 M. R. lle Kerguelen........ $6-3$ II. R. - $\quad \ldots .322-651,652 \quad$ M. R. lle Mac-Donald...... 313 - 634 M. 1 . Ile Maurice........ $209-393$ N. $\mathrm{K}$. - $\quad \ldots .234-444-147$ II. R. Ile vicobar......2 $217-474,475$ M. R. lle de la liéunion... $24-44$ S.R. lle saint-Paul...... $228-432$ II. $R$. lle Rodrigue.... $30-54,55$ M. $R$.

\section{OCÉAN ANTARCTIQUE}

\section{Expédition Scott au} pule sud ........455-935 M. H. 


\section{.. \\ TABLE DES ESPĖCES}

N. B. - Pour I'identification des espèces contenues dans cette table, nous avons désigné de une ả cinq localités oủ elles se rencontrent le plus fréquemment; les caraclères employés pour les chiffres indiquent la plus ou moins grande abondance de l'espèce dans les préparations; les caractíres très gras indiquent que l'espéce y est presque pure ou très abondante; les caractères italiques qu'elle y est rare.

Pour les espèces très rares, mais qui néanmoins peuvent se rencontrer dans les préparations de certains dépôts publiés dans celte collection, nous avons indiqué ce dépôt en italiques.

Enfin, toutes les espéces ou genres précédés d'un (*) sont noureaux et particuliers à cette collection.

\section{Acanthodiscus Pantocsek}

rugosus Pant. 258-261.

\section{Achnanthes Bory}

americana $\mathrm{Cl} .500$

angustata Grev. 445-417.

athenais Pant. 851-853.

- $\quad$. minor 851-853.

Baldjickii Grun. 173, 175, 318, 319

Bengalensis Grun. 622, 623, 617, 618.

Biasolettiana Grun. $\mathbf{4 9 5}$.

brevipes Ag. 50, 101, 193, 353, Tô6.

- $\quad \nabla$. contracta $232,349,412,143$.

- $\quad \nabla$. intermedia 523 .

* - - v. maculata 213, 398, 399.

- - $\quad$. minor 290.

- v. salinarum 611.

clavata Pant. 851853.

coarctata Grun. 38, 78, 667.

danica Flog. 348, 349, 371, 443.

- $\quad f^{2}$ maxima 152, 153.

delicatula Ktz. 301,396 .

Flahaultii F. H. 262-264, 665 .

exigua Grun. 212, 216, 309, 832 .

- v. staurophora $851,853$.

exilis Ḱtz. 228, 232, 296, 901 .

Hauckiana Grun. 166, 214, 400.

heteromorpha Grun. 330, 331 .

hexagona Cl. et J. Br. 324, 325, -91-797.

hungarica Grun. 262-264, 579, 666.

indica J. Br. 54, 55 .

\section{Achnanthes Bory}

* indica $\nabla$. sulcata 647,648 . inflata Grun. 38, 542, $\mathbf{6 1 9}, 920$.

* inopinata $\nabla$. elliptica 301 . intermedia Kitz, var. 401, 402.

Jackii Rab. 248, 249.

Jaranica Grun. 426-128.

- v. rhombica 590,591 .

Joursacence F. II. 52, 526, 537, 736, 737 .

Kertschiana Pant. \$51-\$53.

lanceolata Brél. 13, 103, 113, 500, 580 .

- $\quad$. dubia 411, 412.

-- v. elliptica $133,136$.

- v. elongata 494.

- v. Hayoaldii 104, 108, 212, 429, 829

Lapevrerei Temp. et M. Per. 152, 153.

Ledugerii Temp. et $\mathrm{Br}$. $2 \bar{j}$.

Ligeriana F. H. $30 \%, 308$.

linearis IV. Sm. 909.

Loczyi rant. $318,349$.

longipes $\mathrm{Ag} .50,71,1(1,193$.

— $\quad$. fossilis 851.853 .

* - v. minutissina 198.

maculata 11 . Per. 398, 399.

mammalis Cl. 426-428.

microcephala Grun. 362, 892 .

minutissima Ktz. 57, 223, 521, 902.

- v. cryptoceptala $222,283, \mathbf{1 9 5}$.

parvula $\mathrm{Ktz} . \$ 51,640$.

pennæformis Grev. 24l-243, 318, 349.

* Peragalli v. fossilis 211, 212. 


\section{Achnanthos Bory}

* Temperei M. Per. 187, 189. tenuissima Pant. 853. subsessilis Eh. 33, 34, 101.

$$
\text { - } \quad \text { v. cuneata } 647,648 \text {. }
$$

subquadrata Pant. $318,319$.

vasta Pant. 85̌1-853.

ventricosa Eh. 41, 618, 825, 903.

vernalis Pant. 851-853.

\section{Achnanthidium Grunow}

flexellum Bréb. 362, 758, $759,868$.

v. alpestris 885 .

lanceolatum Brèb. 583, 581, 786, 806 .

$$
\begin{aligned}
& \text { - v. ellipticum } 801 . \\
& \text { - v. Haynaldii } 801 \text {. }
\end{aligned}
$$

minutum Cl. 206, 222, 907.

* Peragaloi Temp. 938.

\section{Actinella Lewis}

Braziliensis Grun. 343, 344. Guyanensis Grun. 251, 253. pliocenica F. H. et M. Per. punctata Lewis 831,885 . scala J. Br. (Dépóts des Barbades).

\section{Actinocyclus Ebrenberg}

alienus $v$. arctica 64.

- v. californica 109-112.

* americanus M. Per. 120, 121. asiaticus Temp. et Br. 23, 24 . Barkleyi Grun. 87, 211, 212.

Boryanus Pant. 94, 95 .

Bremianus Pant. $346,349,800$. calyx Temp. et $\mathrm{Br}$. 23, 24. circumdatus Pant. 173, 316-319, 442, 800. complanatus Cast. 342.

confluens Grev. 109-112, 358, 360, 590.

crassus W. Sm. 187-189, 619, 950 . curvatulus Jan. 118, 478, 479, 651. denticulatus Cast. 621 .

disseminatus Pant. 346-349. 756.

Ehrenbergii Ralfs. 155, 219, 220, 541

$$
\begin{aligned}
& \text { - } \quad \text { v. bisenarius } 151 . \\
& \text { - } \quad \text { v. intermedia } 109-112,637 . \\
& \text { - } \quad \text { v. minuta } 126-129 . \\
& \text { - } \quad \text { v. quindita } 426-128 . \\
&
\end{aligned}
$$

elegans W. Sm. 434

ellipticus Elı. 653, 651.

$$
\text { v. Sendaiense 24, } 25 .
$$

elongatus Grun. (Dépôts du Japon). fasciculatus Cast. 108, 268, 653, 651. flos J. Br. 23, 24.

fuscus Norm. 367,368 .

incertus Grun. 46, 359, 468, 551 .

\section{Actlnocyclus Ehrenberg}

ingens Ratt. 358-360, 844, 845 .

interpunctatus Ralfs: $145-147$.

Janischii Sch. 673, 674, 926.

knemoides Pant. 339, 346-349.

labyrinthicus Pant. 346-349.

Loczyi Pant. 339, 346-349, 756.

Marylandicus Ratt. 233, 234.

moniliformis Ralfs. 111, 269, 339.472, 617.

Moronensis Deby. 653, 651, 659 .

nebulosus M. Per. 34, 71, 101, 370, 617. obscurus Ratt. 358-360.

Oliverianus O'Mea, 634, 935.

ornatus Ralfs. 61, 62 .

partitus Grun. 233, 234, 933.

polyradiatus Cast. 634 .

radians Ratt. 358-360.

radiatus Ratt. 339.

Ralfsii W. Sm. 330, 393, 403, 532.

- v. australiensis $445,447$.

- - fa minor 647 .

- v. Challengeriensis 935.

- v. monicæ 109, 112, 926, 927.

- v. Samoensis 422-425, 622, 647 .

Roperii Kitt. 58, 161.

rotula J. Br. 268, 346, 349, 551, 798.

- v. minor 276-277.

sparsus Greg. 71, 72, 101, 153, 471.

* spiralis M. Per. 99, 100.

* stellatus Forti. 993-995.

subcrassus Pant. 126-129, 442.697.

subocellatus Ratt. 220, 221 .

subtilis Ralfs. 94, 109, 123, 399, 499.

tenellus Brèb. 101, 153, 339.

tenuissimus Cl. 687.

umbonatus Cast. 342, 651, 935.

\section{Actiniseus Ehrenberg}

pennatus Grun. (Dépóts du Japon).

\section{Actinodiscus Greville}

atlanticus K. et Sclı. 147.

Barbadensis Grev.139, 250, 813, 819.

gloria J. Br. (Dépôts des Barbades).

Grayi Grove. 810,818 .

horologium (Dépóts des Barbades).

\section{Actinogonium Ehrenberg}

septenarium Eh. 713, 723.

\section{Actinoptychus Ehrenberg}

adamans Temp. et Br. 24, 25, 712, 713 . adriaticus Grun. 43,71 .

- v. balearica 43, 71, 478.

amblỳoceros A. S. 128, 129, 183, 438, 659 .

anemone J. Br. 23, 24.

antistrophus $\mathrm{J}$. Br. 936 .

aerolatus Eh $46,187,281,554,843$. 


\section{Actinoptychus Ehrenberg}

asiaticus Temp. et $\mathrm{Br}$. 23, 24. aster J. Br. (Dépóts du Japon). baccatus J. Br. 701, 703.

Bэrboi J. Br. (Dépóts du Japon). biformis J. Br. 214, 291, 468, 469. bifrons A. S. $8,91,126,127$.

Bismarkii A. S. 45, 46, 291, 292 . Boliviensis Jan. 219, 220, 237, 238. Boryanus Pant. 91, 95. campanulifer A. S. 647,618 . capensis Grun. $452-456$. cathedralis J. Br. 145, 146. clavatus J. Br. (Dépóts du Japon). Clevei A. S. 237, 238, 271. concentricus A. S. 98, 99, 100. constellatus J. Br. (Dépóts des Barbades) constrictus Gr. et St. (Dépóts d'Oamar'u). crepido A. S. 367,368 .

* crucitinus H. P. 213,214 .

* - fa senaria 213, 214.

Coloradoï H. P. 213, 214.

decorans A. S. (Dépôts d'Oamaru). - v. Japonica (Dépôts du Japon) decumanus A. S. 422-425. delectus A. S. 71, 72, 281, 282. dilatatus Pant. 237, 238, 271, 861. elegantulus Gr. et St. (Dépóts d'Oamaru). erinaceus 'lemp. et $\mathrm{Br}$. 23, 21. excellens A.S. 233, 234, 688, 689. glabratus Grun. 43, 213, 36\%, 368 .

- v. angelorum 109-112.

- $\quad$ v. incisa 217, 218, 468.

- v. maculata $213,214$.

- v. Montereyi 358-360.

- v. subangulatus

Grovei Thomas (Dépóts d'Oamaru).

Grundlerii A. S. 236, 237, 324, 842.

* guttatus M. Per. 617, 618 .

* halionyx v. dilatata 468, 469.

heliopelta Grun. 233, 231, 197, 198, 688.

- fa minor 997, 998.

- v. versicolor $145,146,689$

heterostrophus A. S. 11, 393.

hexagonus Grun. 98, 324, 478, 551 .

- var. 276, 277.

- var. decumana 98, 324.

- v. tenella 617,618 .

Hillabyanus J. Br. 701-703.

hungaricus Pant. 236-238. v. corallina $701-703$.

Huttlingerianus $\mathrm{T}$. et W. 201, 202. indicus J. Br. 54, 55.

intermedius A.S. 97-100, 233, 238 .

interpositus J. $\mathrm{Br} .23,24$. var. $401,468,561,936$.

Janischii Grun. 236, 452, 842, 934.

* - v. notata H. P. 213.

\section{Actinoptychus Ehrenberg}

* Janischii v. partita 548, 549. japonicus Grun. 97-100. Klausenii A. S. 27,28 . Kraussi A. S. $145,146$. Kusnetzkianus Pant. 258, 260. Ky'natodes Pant. 91, 126, 127.

* - v. radiolata 696,697 .

- v. tetramera 696,697.

laevigatus Grun. 358-360.

leptomitos Pant. 442, 413.

lobatus J. Br. (Dépôts des Barbades).

Iucidus J. Br. (Dépóts du Japon).

Macraei Deby. $318,319$.

madreporus J. Br. (Depóts des Barbades). maculosus Pant. (Dépóts d'Oamaru). marmoreus J. Br. (Dépots du Japon). minutus Grer. 202, 360, 675 .

- $\quad f^{a}$ major $674,675,997,998$.

Mölleri A. S. $80,548,549$.

moronensis Cl. 91, 236-238, 861 .

mosaïca J. Br, 145, 146.

neogradensis Pant. 236-238, 367, 368.

nitidus Gre 8.469.

- var. 24, 25, 469.

notabilis J. Br. 145, 14\%, 28:.

Oamaruensis Grun. (Dépóts d'Oamaru). papilio J. Br. (Dépóts du Japon). paradoxus Grev. (Dépots des Barbades). pellucidus Grun. 45?-456

pericavatus $\mathrm{J} . \mathrm{Br} .318,319$.

- v. Oamaruensis (Dépóts d'Oamaru).

— v.tropicalis (Dépóts des Barbades).

perisetosus J. Br. 23, 24.

* - $\quad \nabla$. inermis 468, 469.

Pethoï Pant. 236-238.

Petitii Pant. 94, 95, 367, 368.

Prætor A. S. 688, 689.

* pseudoantistrophus M. Per. 189. pulchella v. tenera (Dépóts d'Oamaru). racemosus A. S. 189, 281, 282. 370 . radiolatus Grun. (Dépots des Barbades). ranunculus J. Br. 145-147, 170 .

* sculptilis v. tetramera 997, 998. seductilis A. S. 11,12 . segmentatus J. Br. 145-147. Semseyi Pant. 236-238.

Simbirskianus A. S. 11, 12. socius A. S. 358-360. spinifer Grun. 45, 291, 292, 554. spiniferus Grun. 109-112.

splendens Grun. 182, 324, 325, 934.

- v. californica $109-112,548,549$.
- $\quad$ v. fusca Gr. et St. $250,798$.
- $\quad$ v. glabrata $126-128,442$.
- $\quad$ v. haliony $219,220,324$.
- $\quad$ v. nicontereyi $518,549$.




\section{Actinoptychus Ehreulierg}

splendens v partita Pant. 91. spinulosus A. S. 291, 292.

Staubii Pant. 94, 95, 405-407.

stella A.S. $8,9,120,127$.

- v. Thumii $8,9,167,169,861$.

subangulatus A. S. 276,27 .

Szontaghii Pant. 94, 95, 367, 368.

summissus A. S. 370 .

Temperei A. S. (Dépôts du Japon). tener Gr. et St. (Dépôts d'Oamar'u). ternarius Jan. 397 .

trifolium 'Temp. et $\mathrm{Br}, 23,24$.

trifurcatus T'emp. et $\mathrm{Br}, 23,24$.

triangulatus Bright. $98,148,276,321,622$

v. major 97-100.

trilunatus J. Br. (Dépôts d'Oamar'u). trivolva J. Br. 688,689 .

Truanii A. S. $120-128$.

turgidus 'Temp. et Br. 23, 24.

undulatus Eh. 109, 281, 591, $621,936$.

$$
\begin{aligned}
& \text { — } \quad \text { v. barbadensis 7 06,713,723. } \\
& \text {, - v. compressa } 46,47 \\
& \text { * - v. orosa 561, } 562 \text {. } \\
& \text { * - v. libyrinthiformis } 6.96,6.97 \text {. } \\
& \text { - v.maxima } 367,996,997 . \\
& \text { - v. minor } 380,426,469 . \\
& \text { - v. parallelistriata } 696,926 \text {. } \\
& 929 . \\
& \text { - v. parva } 111 \text {. } \\
& \text { - v. subtilis 94, } 75 \text {. }
\end{aligned}
$$

velatinum A. S. (Dépôts du Japon). vulgaris A.S. 178, 367, 561, 622, 916.

- v. australis 590,592 .

- v. Doljensis 442, 443 .

-- v. maciulata $139,199,250,569$.

- v. monicre 46.

- v. neogradensis 673,674 .

- v. virginica $1+5,146$.

Weissflogii A. S. 936.

Wittianus Jan. 201, 202.

$$
\begin{gathered}
\text { - v. hexagonus 201, } 202 . \\
\text { - v. scutiformis (Dépts des } \\
\text { Barbades). }
\end{gathered}
$$

* Wollei M. Per. 370 .

\section{Alloioneis Sehumann}

amphora J. Br. 160.

antillarum $\mathrm{Cl}$. el Grun. $61,427,478,778$.

Castracanei Pant. 95, 407.

Debyi Leud, Fort. 420-428, 775.

Grunowii Pant. 91, 95.

\section{Amphipleura littring}

Lindheimeri Grun. (31. pellucida Kítz. 315.

\section{Amphiprora Cleve}

alata W. Sm. 123, 338, 397, 772, 892.

- v. minor 397 .

biharensis Pant. 173, 174.

coarctata J. Br. (Diatomées du Japon)

complexa Greg. 287.

conspicua Grev. 251-253, 037, 975.

cornuta W. et Cl. 570.

crenulata Temp. 9.7.

decussata Grun. 50, 285, 501, 957, 961.

dilatata Pant. $304,305$.

duplex Grun. 501, 502.

elegans W. Sm. 43, 130, 527, 839, 977.

fragilis Temp. et Br. 24.

hyalina Pant. 851-853.

lepidoptera Greg. $34,338,526,760,875$.

- v. minor. 58,130 .

- v. proboscidea $622,623,798$.

maxima Grun. 18, 285, 287.

ornata Bail. 36, 242, 397, 571.

paludosa W. Sm. 188, 400, 498, 962.

- $\quad \nabla$. nereis $251,253$.

paradoxa Grev. 322.

Pethoï Pant. 405-407.

Pozewitzii Pant. 346-349.

pulchra Bail. 187, z81, 826, 893, 976.

- . pulehella $187,189, \mathbf{3 7 0}, 639,640$. punctata Pant. 173-175.

pusilla Greg. 19, 287, 505, 760, 839.

striata Pant. $173,175$.

venusta Grev. 101.

vitrea W. Sm. 526, 527.

* - v. trinitatis M. Per. 200.

\section{Amphitetras Ehrenberg}

antediluviana Eh. 18, 420 .

จ. cruciformis 535 .

elegans Grev. 358, 359.

Græffiana Witt. (Diatomées du Japon).

minuta Grev. 146, 147.

nobilis Grev. 987.

tesselatus Shadb. 478, 479.

\section{Amphora Ehrenberg}

acuta Greg. 119, 269, 617, 794, 797.

- - armata 50.

- $\quad$. neogena 756 .

acutiuscula Ktz. $84,304,305,463,464$. v. fossilis $304,463,851853$.

affinis $\mathrm{Ktz}$. 321, 470,618 .

- v. Ergadensis 113.

- $\nabla$. proteus 76,77 .

alata H. Per. 794-797.

angulala $\mathrm{Cl}$. $187,330,846$, $98 \mathbf{1}$

angusta Greg. 187, 526, 527, 975 .

v. ven!ricosa $119,631,652$.

araulensis $\mathrm{M} . \mathrm{P}$. et F. H. 740,741 . arenicola Grun : 80 . 


\section{Amphora Ehrenberg}

arenicola v. major $276,277$.

arcuata A. S. $118,119,268,370$.

arcus Greg. 80.

arenaria Donk. $19,20,80$.

- v. Donkinii 975.

* arcolata v. elegans 99, 100.

- v. maxima 276, 277.

argus Pant. 203, 205.

biconvexa Janisch. 276, 277.

bigibba Grun. 263, 533.

binodis Greg. 928, 929.

bistriata Leud Fort. 120, 121 .

bituminosa Pant. 463, 464.

Bornetti F. H. 263, 264, 738, 739.

Boryana Pant. 94, 95.

Budayana Pant. 203, 205.

* Caucasica M. Per. 825.

cingulata $\mathrm{Cl} .189,194,269$.

clara A. S. 276, 277, 621.

Clevei Grun. 189, 975, 985.

* - - s. splendida 189.

Coffeaeformis Ktz. 304, 305, 463, 464. $\checkmark$. fossilis 305,464 . v. salinarum $305,464$.

commutata Grun. 123.

complanata Grun. 166.

conjuncta Pant. 851-853.

contracta Grun. 46, 794-797.

* corpulenta $\nabla$. capitata 268 .

costata W. Sm. 20, 188, 648. 875

crassa Greg. 74, 151, 370, 438.

- $\quad \nabla$. elongata $928,929$.

- $\quad$. minor $63,95,348,349$.

- $\quad$. punctata $173,174,348,438$.

- v. Sloestvigensis.775.

- $\quad$. spuria 622, 623 .

cuneata A. S. 276, 277.

curvata Pant. 304, 305.

cymbelloides Grun, 268.

cymbifera Greg. 210, 527, 598, 753.

decussata v. robusta.

Delphinia $\nabla$. minor 588 .

distincta F. H. 51-53, 263, 264.

dubia Greg. 648.

egregia Eh. 74, 322, 370, 622, 623.

- v. interrupta 276, 277.

elongata Greg. 19,20 .

enoculata M. Per. et F. H. 517, 762, 763.

Eulensteinii Grun. 357, 986. v. fossilis 514,515 .

Eunotia Cl. 153.

euprepes Pant. 530, 531.

exsecta Gruu. 155.

excisa' Greg. $330,654,760$.

farcimen Grun. 401, 402.

- $\quad$ v. crassa $401,402$.
- v. fusca 647,648 .

\section{Anphor Ehrenberg}

* farcimen v. gigantea $401,402$.

* farciminosa H. Per. 617, 618.

fallax Temp. et Br. 24, 25.

fasciata Greg. 760 .

formosa $\mathrm{Cl}$. 99, 100, 994.

fossilis Pant. 304, 305, 463, 404 .

furcata Leud Fort. (Dépóts d'Oamaru). fusca A. S. 268 .

- $\nabla$. lata 647. 648 .

gigantea Grun. 148, 794, 797, 975, 985.

- v. fusca 281, 282.

- $\quad$. minor 8:5.

- $\quad$ v. nodosa 932.

- v. obscara 160.

gracilis Eh. 163, 207, 279, 470 .

Graeffii v. minor 120, 276, 277,

Granii Pant. 85̆l, 85.3.

granulata Greg. 19, 20, 875.

* - v. lineata 617,618 .

Grovilleana Greg. 99, 100.

- $\quad$ v. campechiana 794-797.

Grundleri (irun. 94, 95, 346, 349 .

hevesensis Pant. $304,305,463,464$.

incerta A. S. 285, 287.

inflata Grun. 210 .

interlineata Gr. et St. (Dépôts d'Oamar'u).

intersecta A. S. 442, 443, 985 .

- v. sarmatica 412, 443.

- v. striati 330, 339, 442, 949.

invidenda Pant. $\tau 56^{\circ}$.

Janischii A. S. 268.

Javanica A. S. 401, 402.

* - - $\quad$. oculata $617,618$.

Juvenalis Pant. 756.

Kertschiana Pant. 851, 853.

Kossuthii Pant. 173, 175, 339.

labuensis Cl. 285, 287.

lævis Greg. 393, 760, 949, 985.

lanceolata v. incurvata 24, 25.

* latecingulata M. Per. 120.

lineata Greg, 19, 210, 268, 502, 875.

Loczyi Pant. 316-319.

Lunyacsekii Pant. 238 .

lybica Eh. 52, 171, 366, 824, 990.

- $\quad$. interrupta 502,514, 515.

Marchesettiana Pant. 851-853.

marina W. Sm. 20, 210, 269, 754, 875 . membranacea W. Sm. 285-287.

mexicana A. S. 75, 242, 322, 403, 478. v. ininor 120,160 .

minuta Pant. $305,464$.

Mceotica Pant. 8.51-85'3.

moniligera Greg. 442, 443.

mongolica Ost. 189.

mucronata H. L. Sm. 285-287.

neogradensis Pant. 238.

nodosa J. Br. 824, 325. 


\section{Amphora Ehrenberg}

Normanii Rab. 231, 232.

Nova Caledonica Grun. 268, 339, 756. obtusa Greg. 75, 120, 268, 330, 527.

* - - v. crassa 120 .

- v. fossilis 94,95 .

- . miocena (Dépóts des Barbades)

- v. oceanica 281, 292.

- $\quad$. rectangulata 268.

ocellata Cl. 282, 988.

oculus A. S. $237,238$.

ostrearia Brẻb. 120, 426-428, 969.

- $\quad$ v. granulata 120, 121, 219 .

- $\quad$ v. vitrea 794-797.

ovalis Ktz. 70, 222, 419, 895, 990.

- v. affinis $\mathbf{2 5 1}, \mathbf{2 5 3}, 910$.

- v. minor 311,902 .

pecten J. Br. 98, 100, 324, 325.

pediculus Grun. 164, 207, 308, 321.

- $\quad$. major 164, 400, 466.

pellucida Greg. $73,74,760$.

Petilii Temp. et Br. 24, 25.

permagna Pant. 318, 319, 501, 502.

pleurosigma Temp. et Br. 24, 25.

plicata Greg. 949

porcellus Kitt. $268,318,319,478,479$.

proteus Greg. 101, 269, 252, 640, 9 ร5.

- v. contigua 200.

* - V. oculata 50, 120, 371, 929.

protracta Pant. 304, 463, 699, 700.

- v. gallica 740, 741 .

pusilla H. Per. 166.

pusio Cl. 64, 426-428.

quadrata Bréb. 549,550 .

quadrisulcum H. Per. 213, 214.

liacovitzae V. H. 935.

revirescens Pant. 851-853.

rhombica Kitt. 80, 268.

$\nabla$. intermedia 975 .

robusta Greg. $88,158,241651$.

Rossica Pant. 851-853.

salina $W^{\top}$. Sm. 113.

- v. fossilis $304,305,463,465$.

Samoensis M. Per. 647, 618.

sarniensis Grev. 371.

Schleinitzii Jan. 401, 402.

Schmidtii Grun, 46, 47.

sejuncta Pant. 203-205.

Sendaiense J. Br. 24, 25.

* separanda M. Per. 647, 618.

Scelsvigensis P. P. (Dépots du Japon). spectabilis Greg. 19, 20, 160, 322, 839.

Staubii Pant. 203-205.

striata Pant. 346-349, 501, 502.

striolata Pant. 304, 305, 463, 464.

Sturtii Gr. et St. 140, 141.

suavis Pant. 530, 531, 547.

* subalata M. Per. 647, 648.

\section{Amphora Ehrenberg}

subpunctata Gr. et St. (Dépóts d'Oamaru). sulcata Greg. 19, 80, 322, 612, 985.

- v. parva 571 .

Szaboi Pant. 463, 464.

Szontaghii Pant. 237, 238.

tertiaria Pant. 237, 238.

tessellata Gr et St. (Dépóts d'Oamaru).

Tilhoniana Pant. 851-853.

Tomassiniana Pant. 851-853.

Transylvanica Pant. 530, 531, 547.

v. minor. 203-205.

turgida Greg. 396, 401, 402, 647.

$\nabla$. minor 648 .

veneta Ktz. 306, 500, 687.

verrucosa Pant. 203-205.

vittata Pant. 346, 349.

Wresmeri Pant. 304, 305, 463, 466.

zebrata Temp. et Br. 24, 25.

\section{Anaulus Grunow}

acutus J. Br. 213, 214.

birostratus Grun. 146, 147, 415, 441 . latecavatus J. $\mathrm{Br}$. (Dépots du Japon).

Mediterraneus Grun. 390, 468, 469

v. intermedia 549,550 .

minutus Grun. 268.

primordialis J. Br. (Dep. des Barbades). scalaris Eh. 452-456.

subconstrictus Gr. et St. 569,570

\section{* Anvellus Tempère}

* Cialifornicus Temp. 112.

\section{Anisodiscus Grunow}

Pantocseckij Grun. 91.

\section{Anomoeoneis Pfitzer}

exilis Grun. 758.

follis Eh. 899.

serians Bréb. 758, 899.

Zellensis Grun. 758.

\section{Anorthoneis Cleve}

excentrica Grun. 595, 598.

* maculata M. Per. 401, 402.

Peragalli F. H. 736, 737, 764, 765.

* striata M. Per. 619, 650 .

\section{Antelminellia Schütt}

gigas Cast. 673, 675.

\section{Anthodiscus Grove et Sturt}

floreatus Gr. et St. 140, 440, 441.

\section{Aporodiscus Rattray}

Oamaruensis Ratt. 140, 146, 819,822.

Simbirskianus Ratt. 11, 12 . 


\section{Arachnoidiacus Ehrenberg}

Barbadensis Grev. 92, 93.

Ehrenbergii Ralfs. 22, 45, 332.

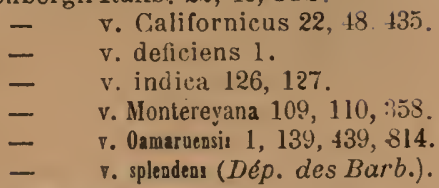

Grevilleanus Hardm. 213, 358.

indicus Eh. 291, 358, 474.

- v. minor 674 .

ornatus Eh. 291, 474, 532, ร79.

- v. Muntereyana 109, 110, 156, 358.

- $\quad$. obscura 367.

rossicus Pant. 258, 259.

Simbirskianus Pant. 11 .

Wallischianus Ralfs. 46.

\section{Asterionella flassall}

antiqua F. H. et M. Per. 12?.

Bleakeleyi Grun. 54, 55, 525.

formosa Hass. 81, 575 .

จ. gracillima $226,227,62$.

gracillima Heib. $780,781,783$.

Japonica Cl. 951, 952, 969, 973.

spathulifera Cl. 336.

subtilissima Meister 801 .

synedræformis Grun. 291, 292

\section{Asterolampra Ehrenbers}

* acutiloba Forti 926-929, 999, 1000. æmulans Grev. (Dépôts des Barbades). affinis Grev. 92,-93, 715, 716 .

- v. cellulosa 673-675, 996-998.

- v.punctifera (Dépóts d'Oamaru). aliena Grev. (Dépóts des Barbades). ambigua Grev.

Barboi J. Br.

Brebissoniana Grev. 358-360.

Brightwelliana Grev. 715, 716.

crenata Grev. (Dépôts des Barbades). concinna Grev.

* curviradiata Forti 926-929.

Dallasiana Grev. 422, 425, 655-657.

decora Grev. 140, 111, 569, 570.

- v. coneentrica (Dép des Barbades).

- v. Japonica (Dépóts du Japon). decorata Grov. (Depóts des Barbades). dubia Grev.

exımia Grev.

Grevillei Grer. 35\%, 360, 367, 655, 65i.

- v. adriatica $285,287,403,839$.

- v. eximia 285, 287.

insignis A. S. (Dépóts des Barbaules). Kittoniana Grev.

lævis Grev.

marginata Grev.

\section{Asterolampra Ehrenberg}

marginata $\nabla$. corvilineata (Dep. des Barbades). v. minor

Marylandica Eh. 92, 234, 403, 621, 830.

- v. Bárbadense ( Dép. des Barb.).

- $\quad$ r. fossilis 122, 425 .

- $\quad$ v. fragilis 367,368 .

- $\quad$ $\quad$. major 285-?87.

pulchra Grev. (Dépôts des Barbades). punctata Grev.

punctifera Forti $926-929, \overline{994}$.

Ralfsiana Grev. $721,7 \geq 2$.

rotula Grev. 360

Rylärndsiana Gr̊v. (Dépôts des Barbades) scutula Grev.

simulans Grev.

splendida Grev.

stellaris Temp. et Br. 24, 25 .

- v. curviradiata Temp. et Br. 926-928.

stellulata Grev. (Dépóts des Barbades). traducens Ratt.

uraster Gr. et St. (Dépôts d'Oamaru). variabilis Grev, 109, 112, 358, 360 .

vulgaris - Grev. 140, 569, 570, 647, 618 .

- v. cellulosæ Grev. (Dép. des Barbades).

- v. planior (Dépóts des Barbades).

Wallischiana Grev.

Weissflogii Grun.

\section{Asteromphalus Ehr.}

antarcticus Cast. 634 .

arachne Bréb. 452-456.

Brookei Wall. 46, 47, 111, 112, 454 .

Brunii Pant. 318, 319.

Debyi Pant. $405-407$.

Dallasianus Grev. 178, 179, 649, 650 .

Darwinii Eh. 111, 175, 360, 472, 473.

elegans Grev. 452-456, 621 .

flabellatus Bréb. $43,452-156$.

Grevillei Wall. 422-425, 673, 674 .

Grovei Pant. 318. 319.

Heptactis lials, 213, 214, 452, 456 .

Hookeri Eh. 213, 214.

Humboldtii Eh. 342,634 .

- Hungaricus Pant. 173, 175, 346, 349.

Kinkerii Pant. 405-407.

IIoronensis Grev. 46, 435, 651, 656.

Ralfsianus Grun. 312, 452, 456 .

robustus Cast. 285-287.

Roperianus Ralfs. 219-221, 974.

senectus Temp. et $\mathrm{Br} .24,25$.

Shadboldtianus Ralfs. 219-221.

variabilis Ratt. 291, 292.

Wallischianus Ralf. 233, 472, 473.

\section{Attheya West.}

decora West. 4. 


\section{Aulacodiscus Ehrenberg}

adonis Temp. et Br. (Dépóts du Japon). affiuis Grun . 97, 236, 300, 435, 798 Amuenus Grev. 1, 139, 415, 439, 569.

- v. hungarica 8, 236,237 .

- v. sparsoradiata 367,368 .

- v. subdecora (Dép cles Barb.). angulatus Gre $.139,140,250,415,439$.

- v. Japoniez (Dépóts du Japon).

- $\quad$ v. plana $439,440$.

anthoïdes A. S. (Dépôts des Barbades) argus (Eh.) A. S. 61, 594, 931, 936. asiaticus J. Br. (Dépots du Japon). v. rallonea (Dép. Cles Rarbades) atlanticus Temp. 445,446 . attenuatus Rall. (Dépots des Barbades). Barbadense Ralls.

Beeveriæ Johns. (Dépôts d'Oamaru).

Brightwellii Jan. 452, 456 .

Brownei Norm. 109, 112, 358, 359.

Brunii A. S. (Depots du Japon).

Carrutberianus Kitt. el Gr. (Dép. des Barbad.). catenarius WVitt. 219.

cellulosus Gr. et St. 139, 250, 415, 440, 569 .

- v. plana 139, 250, 440, 569 .

cinctus Grev. (Dépots des Barbades). Comberi Arn. 152-456.

- $\quad$. irregularis 452-456.

compactus Ratt. $435,436$.

cornutus J. Br. (Depóts du Japon). coronatus Grove (Dépóts d'Oamaru). crater J. Br. (Dépôt du Japon'. decorus Grev. 109, 110, 358, 359 .

- v. Stochii (Dép. des Barbades). dispersus Ratt. (Dépóts d'Oamaru). Ehrenbergii Jan. 182, 219, 2:20. elegans Gr. et St. (Dépóts d'Oamaru). oxcavatus A. S. 11.

$$
\text { - v. apiculata 27, } 28 .
$$

exiguus v. undulata (Depóts d'Oamaru). extans Grev. (Dépóts des Barbades). formosus Arn. 219.

giganteus Temp. el Br. (Depots du Japon).

$$
\text { v. permagna }
$$

gigas Grev. (Dépots des Barbades). gracilis Ratt.

grandis Ratt.

Grevilleanus Hardm. 673 .

Grunowii Cl. 8, 263, 270.

$$
\begin{aligned}
& \text { - v. africana } 151 . \\
& \text { - v. divergens } 655 . \\
& \text { - v. squamosa } 236,237 . \\
& \text { - v. subquamosa } 236,237,270 .
\end{aligned}
$$

Lurowii Pant. 258-260.

Habirshawii Pant. 270, 271.

Haynaldii Pant. 236.

Hungaricus Pant. 236, 237, 270, 271.

Huttonii Gr. et St. 213, 214, 415.

\section{Aulacodiscus Ehrenberg}

lluttonii r. dichotoma (Depots des Barbades). hyalinus Pant. 236, 237.

hystrix Pant. 11.

intlatus Grun. 324,3 jò.

- r. spinifer (Dépóts des Barbades).

- r. steltala

inflexus Grev. 109-111.

* italicus Forti $367,368,996$.

interruptus Pant. 258-260.

intumescens liatt. (Depots d'Oamaru). invictus A. S.

Janischii Gr. et St. 1, 139, 415, 439.

- v. abrapla (Dépots d'Oamaru).

7. areolata

Jimboi Pant. (Depots du Japon).

Johnsonii Arnott. 83, 438.

Jutlandicus Kitt. $27,28$.

Kelleri Pant. 258-26.1.

Kilkelianus Gret. (Dépóts des Bar'bades). จ. minor

Kinkerii A. S. 109, 110.

Klausenii d. S. 27, 28.

Kittonii Arnott. 115, 452, 561.

Lahusenii O. IV. 11, 36\%, 368 .

Ledebourii Pant. 258.260.

lucidus Grev. (Dépóts des Barbades).

Lunyacsekii v. maxima $236,237,270$.

Macraeanus Grev. 98-100, 148, 798.

inammosus Grer. (Depots des Barbades). margaritaceus Ralfs. 109, 178, 300, 324.

$$
\begin{aligned}
& \text { - } \quad \text { v. Debyi } 1,132,250,415,439 . \\
& \text { - } \quad \text { v. distans } 168,169 .
\end{aligned}
$$

minutus Ratt. 360 .

Molleri Grun. 232, 233.

- $\quad$. distincta Ratt. 232, 233.

multispadix Temp. et Br. (DÉp. du Japon). neogradensis Pant. 236, 237, 270.

nigrescens Pant. 258, 259.

nigricans Temp. et $\mathrm{Br}$. (Dép. du Japon).

- var. inonicæ 107-111.

notabilis Pant. $236,237$.

notatus Gr. et St. (Dépóts d'Oamaru).

Novæ Zelandiæ Gr. et St. 1, 139, 250, $415,439$.

Oregonus Bail. 48, 332, 478 .

orientalis $61,411,617$.

pallidus Grov. (Dépóts du Japon).

paradoxus Grev. (Dépóts des Barbarles). patens Ratt. $439,440$.

patulus Grun. 1.

pellucidus Grev.( Dép6ts des Barbades).

Peragalloi Pant. 258, 259.

Petersii Eh. 75, 76. 100, 324 .

- v. cruciier A. S. 96-98.

- v. expansa (Dép. des Barb.).

- v. rara (Dbpóts des Barbades). 


\section{Aulacodiscus Ehreuberg}

Petersii v. trumera Forti 673,674 . polygonus Grun. (Dépots d'Oamaru). probabilis A S. $359,359$. prominens Kitt. 11. pulcher Norm. 109, 110, 435 . - $\quad \nabla$. sparsoradiata 435. quadrans A. S. 11. radiatus Grev. 653, 655 . radiosus Gr. et St. (Dépóts d'Oamaru). Battrayi Gr. et St. 140, 415, 439, 569. - $\nabla$ convexa (Dép. d'Oamaru). reticulatus Pant. 236, 270. robustus Ratt. (Dépóts des Barbades). Kogersii A. S. 23\%, 233, 688, 689. rotulus Ratt. (Dépôts des Barbades). scaber Ralfs. 452, 456.

- v. Jonesiana 452,456 .

Schmidtii O. W. 422-425.

simplex Ratt. 358, 360 .

Sollitianus Norm. 145, 219, 232. sparsıs Gruv. (Dépôts des Barbades). spectabilis Grev.

Stoschii Jan. v. depresia

subangulatus Pant. 236, 270.

subrimosus Grun. 1, 250. superbus Kitt. (Dépôts des Barbades). suspectus A. S. 27, 28 .

tabernaculum J. Br. (Dép. d'Oamaru). Temperui A. .S. (Dépóts du Japon). tetrapurgon J. Br. 'Dép.des Barbades). Thumii A. S. 109,110 .

tripartitus Temp. es Br. 25 et dép. du Japon. T'scliestnowii Pant. 258, 259.

tubulocrenatus Temp. et Bir. (Jép. du Japon). tumulifer $\mathrm{J}$. Br.

umbonatus Grev. (Dép. des Barbades). v. dirupta (Dép d'Oamaru). volula cœeli J.Br. (Depóts du Japon). Weissflogii Pant. 11.

\section{Auliscus Bailey}

accelens Ratt. $570,813,820$.

ambiguus J. Br. 22, 25, 745-750.

- v multiclava I. Br.25, 745, 747 . antiquus Ratt. 109-112.

Asiaticus J. Br. 24, 25.

Barbadensis Grer. $92,93,701,726,820$.

Biduulphia Kitt. 45, 46, 109-112. v. prominens 548,549 .

Bourgognei Bergon (Dépôts des Barbades).

Californicus J. Br. 292.

Cielatus Bail. 43, 50, 835, 836.

- v. Aucklandica Grun. 117-119,775.

- v. constricta 220. 221, 376-378.

- v. delicatula 8:0.

- v. gigus 117-119.

\section{Aullscus Bailey}

Coelatus v. latecostata 117, 370, 561, 798.

- v. major A. S. 75.

- $\quad$ v. rhipis 50, 117-119.

- $\quad \nabla$.strigillata 452-156.

- v. triocellata 798 .

compositus A. S. $276,277$.

confluens Grun. 126, 236-233, 330, 936. convolutus Grove 116,441

craterifer J. Br. (Dépóts des Barbades). crystallinus J. Br. 24, 25, 745-750. curvato-radiosus J. Br. 24, 25, 745.750. dubius Temp. 2, 813.

elegans Ratt. 468, 469.

* - v. coloradira $213,214,814,815$.

ellipticus Arn. 2, 570, 822.

formosus Lend. Forti 148.

fulcratus A. S. $2,441,822$.

gigas Girn. 220, 221.

Grevillei Jan. 220, 221.

Grunowii A. S. $46,47.622,623$.

- v. californica 109-112,549,550

- v. flammula $22-25$.

- v. Paritocsekii 367-369.

Haradæ Pant. 448, 449, 745-747.

Hardmannianus Grev, 46, 435.

$$
\begin{aligned}
& -\quad \text { v. bifurcata. } 1,2,439,410 . \\
& -\quad \text { v. Haytianus. } 202 \text {. }
\end{aligned}
$$

Hauckii Pant. 91, 9.5.

incertus A. S. $377,653,654$.

inflàtus Gr. et St. 569. 570 .

intercedens v. major 835 .

intermedius Gr. et St. 2, 570, 820:

interruptus v. sparsa. $416,570,820$.

intestinalis A. S. 46.

Jimboi Pant. 448, 419, 745-747.

Johnsonii A. S. 291, 292.

lacunosus Gr. et St. 140, 141.

Loczyi Pant. 3.46-349.

* - v. major 367-369.

luminosus J. Br. 22-25.

Macraeanus Grev. 2, 441, 989.

Mediterraneus H. P. 836, 837.

microleion A. S. $140,414,815$.

mirabilis Grev, 213, 291,844 .

Moronensis Grev. 187, 236-238.

- . v. bioculata 549, 550 .

- $\quad$ v. quadriocellata 653,654 .

nanus A. S. 701,726 .

nitidus Ratt. 701,726 .

Normanianus Grev, 655-657, 660 .

- v. bioculata 655-65\%.

notatus Grev. $416,441,820$.

Oamaruensis Gr. et St. 1, 2, 139, 250.

ovalis Arnott. $220,221,811,812$.

parvulus Grev. 701,726 .

pectinatus Ratt. 812,813 .

polypheinus A. S. 440,812 . 


\section{Anliscus Bailey}

prælatus Ratt. 220, 237, 452-456. pressus Leud. Fort. 22, 25.745.750. prominens $468,469,732$.' propincuus Gr. et St. 570, 820, 822. pruinosus Bail. 146, 147, 300; 330 .

- v. robusta 819.821 .

- $\quad$ v. subreticulata 109-112.

- $\quad$. zanibarie 281, 282,330, 798,826

pulvinatus Cl. 91, 126-129.

- v. apiculata 237, 238.

- - $\quad$ ४. inermis 151, 237, 238.

punctatus Bail. 46, 47, 330, 570, 985.

- $\quad$. robusta 202 . v. striolata 844,845 .

racomosus Ralfs. 814,819 .

radiatus Grev. 178, 179.

Raeanus Ratt. 416, 440, 819.

Rattrayi Cl. 701, 726.

reticulatus Grov. $71,72,276,7 \subseteq 8$.

rhipis $\nabla$. triocellata 835 .

rugosus Ratt. $452-456$.

sculptus Ralfs. $33,43, \mathbf{8 2 5}, 839$.

spinosus Christian 145-147.

splendidus Ratt. 75.

- $\quad$. minor 99, 100.

squamosus J. Br. 701,726 .

Stőckardtii Jan. 140, 213, 214, 435.

$$
\begin{gathered}
\text { - } \quad \text { v. aspera } 812,813 . \\
\text { - v.inconspicua } 141,570 .
\end{gathered}
$$

subcœlatus Ratt. 816,822 .

sublaevis Grun. 377,378 .

subreticulatus Ratt. 844,845 .

$$
\text { - } \quad \text { v. decipiens } 844,815 \text {. }
$$

subspeciosus Ratt. 844,845 .

$$
\text { v. minor } 47,213,214 \text {. }
$$

textilis A. S. 109-112.

translucidus J. Br. 98, 99.

transpennatus J. Br. 2, 814 .

tricorona J. Br. 22, 25 .

trigemmis A. S. 23-25.

trilularis $\mathrm{J}, \mathrm{Br}, 22 \cdot 25$.

\section{Auricala Castracane}

amphitritis Cast. 74, 75. complexa Greg. 23, 24.

Grunowii Pant. 173-175.

insecta Grun. 80, 221. 229.

intermedia $\mathrm{Cl} .268,794-797$.

Japonica J. Br. 24, 25.

minuta $\mathrm{Cl} .268$.

ostrea Temp. et $\mathrm{Br} .23,24$.

Szontagii Pant. $318,319,339$.

\section{- Azpeltia M. Peragallo}

- Temperei M. Per. 660.

\section{Bacillaria Gmelín}

paradoxa Gmel 123, 166, 875.

socialis Ralfs. 905 .

\section{Bacteriastrum Wallisch}

elongatum Cl. 917.

furcatum Schadb. 45, 46.

Halo J. Br. (Dépots du Japon).

hyalinum Laud. 622, 789, 956.

varians Laud. 793, 916, 956, 962, 972.

- fa spirillum 160 .

\section{Baxteria Van lleurek}

formosa J. Bl. (Dépots des Barbades). Brunii V. H.

\section{Berkeley u Gregory}

hungarica Pant. 238.

rutilans v. Dillwynii 396.

- var. obtusa $\mathbf{3 3 7}$.

\section{Bergonia Tempère}

Barbadensis Temp. (Dép. des Barbades). tetrapora J. Br.

\section{Biddulphia Gray}

angustata A. S. 233-235.

anthropomorpha V. H. 925.

antiqua T. et W. 201, 202.

aurita Bréb. 6, 34, 58, 617, 6 z6.

Baileyi W. Sm. 146, 147, 789.

Barbadensis Grer. (Dép. des Barbades).

bifenestrata J. Br.

birostrum J. Br. (Dépóts du Japon).

Britloniana K. et Sch. 146, 147.

calamus Temp. et Br. 23, 24, 46 .

capucina A. S. 236-238. 347.

caraïbica T. et W. $201,202$.

chinensis Grov. 426, 794 .

Cookiana K. et Sch. $146,147$.

cornu-Capræ J. Br. (Dépbts du Japon).

corpulenta Grev. (Dép. des Barbades).

crenulata W. et Sch. 61 .

decipiens Grun. 146, 147.

decorata Grev. (Dépôts des Barbades)

- dissipata Gr. et St. (Dépôts d'Oamaru).

Edwardsii Feb. 22, 23, 288, 323.

elegantula Grev. J, 91, 237, 238, 271.

- v. polycistinea 346-349.

- v. polygibba $237,238$.

Elesdiana Pant. $346,347$.

fimbriata Grev. (Depóts des Barbades).

* Fortiana Temp. 673.

fossa Gr. et St. (Dépóts dOamaru).

gigantea Grov. (Dépots des Barbades).

granulata Rop. 58, 71, 277.

Grovei Pant. (Dépôts d'Oamaru).

Grundlerii A. S. 478,479 . 


\section{Biddulpbia Gray}

homala Pant. 8, 9, 236, 237, 347.

* hyalina H. P. 213, 214.

indica $\mathrm{J} . \mathrm{Br}$.

inflata Grev. (Dépôts des Barbaules). Japonica Pant. (Dépots du Japon).

Johnsoniana Grev.376, 377, $415,416$.

lata Gr. et St. (Dépots d'Óamaru).

Loczii Pant. 346-349.

longicruris Grun. 291, 292, 397, 471, 472.

longispina Grun. 109-112.

Mac-Donaldii Pritch. 276, 277, 479. mammosa Grev. (Dépots des Barbades).

* media H. P. 213, 214.

membranacea Cl. 80 .

miraculosa J. Br. (Dépots d'Oamaru). mobiliensis Bail. 39, 789, 790, 932.

Moronensis Cl. 993.995 .

nitida Grev. (Depots des Barbades).

nobilis J. Br. (Dépôts du Japon).

Oamaruensis Gr. et \$t. 139, 439, 440, 599, 570 .

obtusa Bail. 48, 19, 118.

- - spinosa (Dep.du Japon).

Otto Mülleri V. H 935.

$$
\nabla \text {.rotunda } 935 .
$$

* palpebralis Forti 926-929.

permagna Pant. 94, 346-319.

Peruviana Grun. 452-456, 775.

podagrosa Grev. (Dép. des Barbades). polyacanthos J. Br. (Dépt. du Jupon). primordialis J. Br. 22, 23.

$$
\nabla \text {. inermis } 22,23 \text {. }
$$

pulchella Greg. 18, 130, 401, 52:, 798.

* - v. moronensis Forti 926-929. punctata Grev. 1, 2, 439, 440 .

pustulata J. Br. 146,147 .

regina $W . S i n .48,50,130$.

reticulata Rop. 148, 590, 591 .

$$
\text { - } \quad \text {. trigona } 590,591 \text {. }
$$

reversa Gr. et St. (Dépots d'Oamaru). rhombus W. Sm. 33, 43, 58, 861, 931

- V. Consilis J. Br. (Dép. des Barb.)

$$
\text { - - lamajor (Dép.d'Oamaru) }
$$

rigida A. S. $250,415,569,821$.

Roperiana'Grev. 54, 617, 648, 774, 864.

* rotunda M. Per. $119,121$.

rutbenica 0 . W. 11,12 .

seticulosa Grun. 145-147, $18^{\circ}$.

sinuata Grev. (Dépots des Barbades). suborbicularis Grun. 233-235, 298.

* tabellariæformis Forti 673, 674.

tenera Gr. ot St. 140. 141.

tenuicornis Grev. (Dep, des Barbades).

tridentala Eh. 182, 183, 348.

- $\quad$. andesitica. 94, 95.

- v. minor $349,800,839$.

tridens Eh. 691, 692.

tubulosa J. Br. (Depots du Japon).

\section{Buddulphia Gray}

Tuomeyi Bail. 18, 34, 151, 154, 155.

- v. Boryana $94,926.929$.

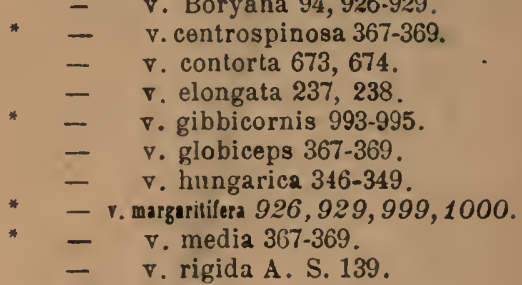

vasta Pant. 236.

virgata Gr. et St. (Dépóts d'Oamaru). vitrea J. Br. (Dépots du Japon).

vittata Gr. et St. (Depots d'Oamaru).

Weissflogil Grun. 145-147, 634.

Woolmanii K. et Sch. 145-147.

Zanzibarica A. S. 503-505.

\section{Bellerochea Van Ileurck}

malleus V. H. 791.

\section{Brebissonia Grunow}

Boeckii Grun. 187, 188, 189, 639, 610.

\section{Brightwellia Ralrs}

coronata Ralfs $140,141,250$.

- $\quad$ v. radians 620 .

elaborata Grev. (Dépôts des Barbades).

excellens Ratt.

Johnsonii Ralfs 715 .

pulchra Grun. 415, 416.

rossica Pant. 258-261.

splendida Ratt. (Dépôts des Barbades).

\section{Brunia Tempère}

mirabilis 655 .

\section{Bruniella Van Ilearck}

coronalis (Depôts des Barbades).

\section{Caloneis Cleve}

Kinkeriana Truan. 376-378.

silicula $\mathbf{v}$. cuneata Meister 801 .

ventricosa Meister 801.

\section{Campylodiscu: Ehrenberg}

adriaticus Grun. 130, $462,616$.

- r. massilieovin 71, 75, 130, 322, 403. adornatus A. S. 97, 98 .

angularis Greg. 18, 94, 346-349, 798.

- $\quad$. punctala. 94, 95.

altar J. Br. (Dépots du Japon).

a mbiguus Grev. 276, 445, 647, 648, 775.

atlanticus 324 .

balticus Cl. 767 .

biangulatus Grev. 71, 75, 324, 462, 775 . 


\section{Campy Jodiscu Ebrenberg}

bicinctus Cast. 798,865 . bicostatus W. Sm. 88. 662, 869 . binıarginatus A. S. 622,623 . Boryanus Pant. 94, 95. Bremianus Pant. 346-349. Brightwellii Grun. 61, 62, 647, 648 Californicus Duby. 46. canalisatus Temp. el Br. (Dep. du Japon). circumactus A. S. 478, 479, 794, 795. chrysanthemum J. Br. (Dép. du Japon).

* Clevei H. Per. 5ก, 617, 618. clypeus Eh. 88, 101, 239, 193, 825.

* - v. sicula.926, 927 .

clivosus J. Br. (Dépôts du Japon). cocconeiformis Grun. 252, 253. concinnus Grun. 561, 56?. contiguus A. S. $623,647,648,775$. contortus Pant. 203-205, 180.

coronilla J. Br. 110, 359, 360, 435, 436 . costatus IV. Sm. 107, 122, 682. crassus Pant. $405.40 \%$.

crebrecostatus firev. 148, 276, 622, 623, 933. Daemelianus Grun. $389,412,541,865$. v. comminuta 775 .

decorus Brèb. 75, 130, 322, 403, 566. - v. pinnuta 647,648 .

dilatatus Pant. 405-107.

ecclesiauus Grev. 276, 332, 503, 504.863. echeneis El. 87, 239, 434. $511,881$. emarginatus Deby, 791-797. erosus Cast. 798.

Eulensteinii Pant. 236, 237, 530.

eximius Grev, 70, 130, $403, \mathbf{4 6 2}, 566$. - $\quad$. briocensis $285-287,839$.

Fortii Temp. 505 .

fluminensis Grun. 50, 322, 403, 839.

Graelfei Grun. 617, 648, $655,656$.

Greenleafianus Grun. 367-369, 798.

Gregorii H. P. 50.

Grunowii Pant. $346-317$.

Heutleri Grun. 97, 98, 324.

hibernicus Eh. 34, 46. 206, 460, 558.

v. transylvanicus 203, 201, 403 .

Hogdsonii IV. Sm. 276, 452, 456.

horologium William 97, 98, 324.

$$
\text { - v. mediterranea 75, } 130 .
$$

hypodromus J. Br. (Dépôts du Japon). impressus Grun. 50, 80 .

incertus. A.S. 794-797.

insignis Leud. 617,618 .

intermedius Grev. 445-417, 551, 775.

Jimboi Pant. (Dépôts du Japon). '

Kidstonii Pant. 673675.

Kinkterii.A. S. $401,40:$.

Kiltonianus Grev. 9\%, 99, 276, 551, 775.

$$
\text { - v. zanzibarica } 622,623 \text {. }
$$

Kurtzingii Pant. $105-407$.

\section{Campylodiscus Elurenberg}

latus Grev. 276, 791-797.

- $\quad$. superba Eul. 61.

limbatus Bréb. 43, 75, 80, 566, 839.

- v. chilensis Grun. 862.

I.orenzianus Grun. 269, 285, $322,403,506$.

Montereyanus Grev. 5 18. 519.

neogradensis Pant. 127, 128 .

noricus Eh. 56, 248, 482 .

v. fossilis 530 .

Normanianus Grer. 622, 623, 775.

notatus r. vitiemis Grun. (Dép. du Japon).

obselatus Cl. 126.128, 236, 237.

ornatus Grev. 97, 98, 415.

$$
\begin{aligned}
& \text { * } \quad \text { - } \quad \text { v. a mphileia } 97,98,445,775 . \\
& \text { - v. mesolaborata } 213,214 .
\end{aligned}
$$

parvulus IV. Sm. 19, 50, 138.

Pfitzeri A. S. 426-428, 798.

punctulatus Grun. 330, 331, 925-926.

Kalfsii W. Sm. 285, 322, 325, 566, 612 .

- v. fossilis Grun. 443.

reticulatus Pant. $405-407$.

rivalis A. S. 276,277 .

rivulosus Temp. et Br. 24, 25 .

Robertianus Grev. 160, 551.

Samnensis Grun. 80, 130, 357, 566. 839. scalaris lemp. et Br. 23, 24.

Schleintzij Jan. 3.

* siculus Forti. 926-y29.

simplex Temp et Br. (Dép. du Japon).

* simulans v. lemnistica 926-929.

singularis A. S. 401, 402, 617, 648 .

stellatus Grev. 794-797, 798 .

striolatus Grun. $23,24,94,95$.

Sumbawanus A. S. 794-797.

Szakalensis Pant, 1:6-1:8.

tæniatus A. S. 61, 62, 32t, 371, 445

- r. radiosa.( Dép du Japon).

tænioides Deby 647,618 .

Thuretii Bréb. 18, 43, 123, 130, 322.

- v. Baldjickiana $346,349,390,442$.

- v. simulans $18,130,462$.

triumphans A. S. $101,402$.

undulatus Grev. 143, 551, (647.

vermicularis Temp. 325.

vitricarus Temp. et $\mathrm{Br}$. 24, 25.

Vallischianus Grev. 276, 551, 775.

zeluanus Cast. 865 .

\section{Campyloneis Grunow}

Grevillei Grun. 46, 47, 58, 160, 420

- yar. argus 160 .

microsticta Grun. 478, 479.

notabilis J. Br. 5̃ 155 .

- v. oculata Dép.des Bar.bades).

regalis Grun. $46,47,58,654$. 


\section{Cunpylosira Grunow}

cymbelliformis Grun, $489,594,614$. 789.

\section{Capsula J. Ifrun}

Barboi J. Br. (Dépóts des Barbades). biformis J. Br.

\section{Cerataulina II. Peragallo}

Bergonii H P. 142.

\section{Cerataulus lihrenberg}

*antedilurianus M. Per. 773.

Boryanus Pant 91, 95.

Japonicus Pant ?2, 23.

Johnsonianus Grev. 91, 127, 237, 300, 925.

hungaricus Pant. 236238.

Kinkerii A. S. 236-238.

Lævis Elı. 20, 34, 618, 619, 732.

* - v. robusta 188, 189.

marginatus Gr. et St. (Dép. d'Oamaru).

* orbicularis Forti 480, 481, $\mathbf{4 8 2 ,} 483$.

Peragalloi Pant. 316, 347.

Petitii Leud. 426-428. 590, 591.

polymorphus Ktz. $34,434,841,863$.

$$
\text { - v. minor } 863 .
$$

rotundus Temp. et Br. (Dép. du Japon). Smithii Ralfs $34,123,269, \mathbf{8 4 7}$.

- v. antiqua 146, 147.

subangulatus Gr. et St. 139-141, 250, 440. Thumii A. S. 367, 369.

turgiclu Eb. 71, 269, 444, 649, 932.

- $\quad \nabla$. hispidissima 94, 95.

* - $\quad$ v. major spinifer 50.

- v. minor. 47.

Weissflogii Pant. 126, 127.

\section{Coratoueis Rhrenbers}

antiqua F. H. $326,327$.

arcus $\mathrm{K} / \mathrm{z}, 222, \mathbf{3 2 0}, 603,921$.

- v. gemina Meister 787.

\section{Cestouliscus Greville}

gemuifer Cast. 621, 656.

hirtulus Grun. 943, 914.

* hispanicus M. Per. 659, 660.

intersectus J. Br. 213, 214.

* interruptus M. Per. 701, 703.

Japonicus Gl. 21, 2乞, 26 .

Johnsonianus Grev. 23, 24.

Moronensis Grev. 655-657.

ovalis Grev. 18:, 183, 377, 378, 424.

peplum $\mathrm{J}$. Br. 941.

proteus Hard. $773,912-944$.

pulchellus Grev. 24, 25, 153, 713, 943.

- v. gemmifer $24,25$.

- v. moravica (Dép. des Barb.).

superbus Hard. 90, 435, 436.

trunitatis Grun. 943, 944.

\section{Chelonioliscus Pantocreh}

Ananimensis Pant. 11, 12.

\section{Chatoceros Istrenberg}

atiline Laud. 166.

angulatum Schūtt 180, 915, 916, 917.

armatum West. 151, 790.

Atlanticum Cl. 65, 317.

$$
\text { - v. compacta Lil. } 31 \text {. }
$$

* - v spinosa 61.

bacillaria Eh. 958,959 .

balticum Ci. $6,41,89,142$.

boreale Bail. 65, 229, $951,968,973$.

- v. densa 17, 142, 180.

Californicum Grun. 15-47, 173 .

cellulosum Laud. $41,124$.

cinctum Grun. 966 .

clavigerum Grun. (Depots du Japon).

Clevei Schütt. 42.

coarctatum Land. 477.

cochlea Schütt. 131, 166, ว92,966, 973.

compactum Schütt. 65,1477 .

commutatus Cl. 973.

constrictus Grun. 6.

contortum Cast. 64, 131, 142, 166.

convolutum ciast. $6,41,64,79,317$.

criophilum Cast. 5, 65, 317.

currens Cl. 65, 79.

curvisetum Cl. 39), 952, 966, 921, 922. danicum Cl. 683. 790, 951, 956, 961.

debilis Cl. 6, 124, 958, 959.

decipiens Cl. 6, 12, 166, 683, 915.

diadema Gran. $452-456$.

dicladia Cast. 452- 156.

didymum Eh. 166, 790, 951, 95\%, 962.

- v. hyemalis 6.

distans Cl. 17, 64, 124, 131 .

- v. subsecunda (Dép du Japon).

diversus Cl. 287, 793.

fusca $\mathrm{Cl}$. 477 .

furcellatum Bail. 64, 230 .

gastridium Grun. 955.

gracile Schñtt. 6.

Grœenlandicus Gi. 316; 790.

hispidum Bright. 973.

จ. leptopus 17, 64, 316, 790, 973.

holsaticum Schūtt. 952, 956, 95z, 962.

incurvum Bail. 550, 555.

Javanicum Cl. (Dépóts du Japon).

laciniosum Cl. 124, 952, 957, 961 .

Lorenziane Grul. 316, 47ร, 955, 96', 973.

mamillanuin Cl. 180.

mitra Cl. 64.

Monice Grun.472, 473.

Ostenfeldii CI. $4 \mathrm{I}$.

paradoxum Cl. 1\%, 131, 142, 951.

pelagicus Cl. 915.

perpusillum Cl. 915, 916, 917. 


\section{Chretoceros Ehrenberg}

Peruvianum Bright. 287, 683, 917. plicatorugosum J. Br. (Dép. du Japon). pliocenum J. Br. $47,90$.

pseudocrinitum Ost. 951.

procerum Schütt. 124, 131, 915, 916, 917. protuberans Laud.131, 952, 957, 960, 972. radians Schütt. 973.

Ralfsii Cl. 916, 952.

rostratum Laud. 64.

Schütti Cl. 142.

scolopendra Cl. 142, 166, 180, 476.

seriacanthum Grun, 6, 131, 915.

vigmoralamus Temp. et Br. (Dépots du Japon). simile Cl. $6,17,142,166$.

socialis Laud. 17, 64, 166, 230, 973.

spinosum Laud. 316, 472, 473.

subtile Cl. 166.

teres Cl. 180, 915, 917.

vermiculus Sclūtt. $6,124,230,973$.

Wighamii Bright. 90, 230, 472, 473.

Willei Grun. 956.

Wollei Grun. 916, 917.

\section{Cladogramma Ehrenberg}

Californicum Eh. 112, 183, 467, 555. conicum Grev 369. 425.

* - V.campanulatum $369,425,675$.

\section{Clavicula Pantocsek}

arenosa J. Br. 448, 449.

biharensis Pant. 389,390 .

delicata $\nabla$ radiata $23,24$.

delicatula Pant. 236-238.

filiformis J. Br. (Dépots du Japon).

Jimboi Pant. 448, 449.

Kinkerii Pant. 94. 95.

polymorpha Grun. 236-238.

$$
\begin{aligned}
& \text { - } \quad \text { v. a mphilepta } 91 . \\
& \text { - } \quad \text { v. aspicephala 236-238 } \\
& \text { - } \text { - pachycephala 236-238. } \\
& \text { v. tumida 236-238. }
\end{aligned}
$$

Szakalensis Pant. 126, 127.

robusta $\mathrm{J}$. Br. 23, 24 .

\section{Ciavularia Greville}

Barbadensis Grer. (Dépots des Barbades). catenata J. Br.

\section{Climacosira Grunow}

mirifica $401,402$.

\section{Climacosponia Ehrenberg}

catena Shadb. 268.

elongata Bail. 462, 503, 532.

hungarica Pant. 91, 95, 405-407.

moniligera Eh. 61, 62, 160, 393, 398.

\section{Cocconeis Ehrenberg}

amygdalina Bréb. 365, 366 .

antiqua Temp. el Br 24, 25, 119, 921, 924 . armata Grev. (Dépóts des Barbades). australis P. P. 478, 479 .

Baldjickiana Grun. 119, 120, 346-349.

Barbadensis Grun. (Dépóts d'Oamaru). bifrons Grun. 478,479 .

biharensis Pant. 346-349.

$$
\text { - v. minor } 349 \text {. }
$$

biradiata J. Br. 330,331 .

Bonnieri F. H. 262-264.

Boryana Pant. 518, 519.

Britannica Næ. $118,119,600$

Californica Grun. 192, 502, $\mathbf{5 1 5}$.

- v. hungarica $518,519$.

- v. melinica $305,463,464$.

circumcincta A. S. 49.

v. subsontinu $192,228,279,466$.

citrina A. S. $276,277$.

cœlata Arnott. $478,479$.

contermina A. S. 160, 794-797.

costata Greg. 120, 301, 333, 420.

- v. Kerguelensis 3 .

- $\quad$. pacifica $118,119,333$,

cruciata Pant. 238.

curvirotunda Temp. et Br. 276, 478, 794-797.

$$
\begin{aligned}
& \text { - } \quad \text { v. bifrons } 276,277 . \\
& \text { - }
\end{aligned} \quad \text { v. Grovei } 794.79 i, 798 \text {. }
$$

cyclophora Grun. 794-797.

diaphana W. Sm. 18, 19.

dehiscens A. S. $3,651,652$.

dirupta Greg. 19, 34, 123.

- v. californica 119.

- v. flexella 19, 119, 210.

distans Greg. 50, 138.

divisa A. S. 794-797.

Dubravicensis Pant. 632, 633.

Eumerichii Jan. 647, 648.

eximia A. S. 61,62 .

extravagans Jan. 3 .

Febigeri J. Br. 182, 183.

formosa J. Br. (Dépots du Japon). gibbocalyx J. Br. 447. 648 .

grate A. S. 101.

Grovillei W. Sm. 382-884.

Haradæ Pant. (Depois du Japon).

helretica J. Br. 249.

heteroidea $\mathrm{Ktz} .55,62,63,160,393$.

hevosensi Pant. 463, 164.

hyalina Pant. $851-853$.

inflexa A. S. 358-360.

imperatrix A. S. $3,651,652$.

insignis Jan. 794-797.

intermedia $\mathrm{M}$. P. et F. H. 70, 77, 500 .

- v. minor 208.

interrupta Grun. 214.

Janischii A. S. 3. 


\section{Cocconeis Ehrenberg}

Japonica A. S. 794-797, 798.

Jimboi Pant. (Depóts du Japon).

Kinkerii A. S. 112.

* Lanzii Forti 367, 369, 425, 675, 998.

lineata Elh. 44, 50, 53, 210, 334.

- . euglypta $53,122,192,735,737$.

- $\nabla$. fossilis 547.

- . minor 205 .

- v. pigmea 205.

- v. rotunda $52,53,725$.

Lunyacsekii Pant. 238.

maculata Ktz. 207, 208.

- $\quad \nabla$. curta Grun 207, 208.

magnifica Jan. 3.

* maxima H. Per. 59, 60, 118, 156.

- - $\quad$. niceaensis 60 .

* - v. parva 60.

* - - v. septata 926-929.

molesta Ktz. 301, 396.

Montereyi Grun. 111, 112, 425, 426.

Moronensis A. S. 376-381, 654.

naviculoides Grun. (Dépots d'Oamaru). neogradensis Pant. 91, 238.

nitida Grev. (Dépots du Japon).

nodulifer Gr. et St. (Dépots d'Oamaru)

notabilis d. S. 358,360 .

oculus-catis J. Br. 112, 147.

ornata Greg. 50.

pediculus Ktz. 5i, 290.

- v. rotunda 137 .

pellucida Ktz. 9, 62, 63, 333, 432.

- $\quad \nabla$. fossilis 238 .

- v. minor 63.

* - $\quad$ r. Monicæ 213, 214.

- v. sigmoidea 63 .

Pensacolæ A. S. 330, 331.

perpusillus Pant. 346, 349.

Pethoi Pant. 318, 319.

placentula Eh. 192, 206, 492, 560, 607. v. minor $137,222$.

præcellens Pant. 91, 237, 238.

* - v. ellipticostriata 424, 425 .

- P. pancistriata 424, 425, 99i, 998.

praestans A. S. $111,112,469$.

prisca Cl. 358-360.

pseudonıarginata Greg. 43, 123, 287, 403,612.

Ræana Pant. 95, 318, 319 .

$$
\text { v. Stevensii } 398,399 \text {. }
$$

regalis Grev. 111,112 .

Rouxii v. euglypta $77,227$.

- v. minor 53, 77.

salina Pant. 463, 464.

sarmatica Pant. 851-853.

Schleintzii Jan. $478,479$.

scutellum Eh. 19, 123, 138.

- v. ampliata 532, 533.

- v. Baldjickiana 348,349 .

\section{Cocconeis Ebrenberg}

scutellum v. dilatata $330,331,652$.

- v. Doljensis $4+2,443$.

- v. fossilis $95,318,349$.

- $\quad$ v. major 19.

* - r. Morrissii 60 .

- $\quad$ v. obliqua 55.

- $\quad$ v. orbiculare 19.

- v. ornata 210.

- $\quad$ - parva 40,60, 420 .

* - $\quad$ . sicula 929.

- v. Mauroueiformis 138, $210,499,713$.

sigma Pant. 213, 214, 237, 238.

- т. uparsipuuctota (Dépóts du Japon). sigmoradians J. Br. $118,119,213,211$. sparsipunctata J. Br. (Dépóts du Japon). speciosa Greg. 136-138, 293.

splendida v. crucifera $24,25$.

v. lurida (Dépóts du Japon!. superba Jan. 111, 112, 40े8, 469. de Toniana Pant. 237, 238.

'Totaræ J. Br. (Dépóts d'Oamaru). transversa A. S. 794-797.

trilineatus II. Per, et F. H. 136, 137. venusta A. S. 384 .

verrucosa J. $\mathrm{Br}$, (Dépóts du Japon). versicolor $\mathrm{J} . \mathrm{Br}$. 24, 25, 114, 119. vitre2 J. Br. 24, 25, 151, 154, 928. voluta J. Br. 276, 277, 794-747.

\section{Cocconema Ehrenberg}

australicum A. S. 627,628 .

gibbum Eh. 187.

Janischii A. S. 627,628 .

mexicanum Eh. 20, 627, 628 .

ventricosum Grun. 280.

\section{Corethron J. Brun}

cometa J. Br. (Dépôts du Japon). Hystrix Hansen. 790.

\section{Corinna heiberg}

elegans Heib. 27, 28, 310, 872.

\section{Coscinodiscus Ehrenberg}

abyssalis Cast. 525.

actinocycloides Pant. 236-238.

Eginensis A. S. 4il, 472 .

* - v. curvatula 471-473.

africanus Jan. 621 .

anastomosans Grun. 109, 110, 405-407.

angusta-lineatus A. S. 201, 220, 221, 844, 815 .

annulatus Grun. 28, 29 .

antiquus Grun. 28, 29, 358-360.

apages Ralt. (Dépóts des Baibades).

apiculatus Eh. 46, 118, 233.

- $\quad$ v. æginensis 927 .

- $\quad$. ambigua 233, 234. 


\section{Coschodiscus Ehrenterg}

apiculatus $v$. californica $942,944$.

- v. maxima $182,183$.

- v. Monicse 46, 376-378. apollonis Eh. 250.

argus Eh. 2, 46, 151, 815, 865 .

- v. subtraducens 410 .

armatus Grev. 95, 182, 183, 236-235

- v. Barbadensis 36\%-369.

asonumæ Pant. (Dépôts du Japon). asperulus Grun. 182, 183.

asteroides T. et W. 22, 201, 673, 674 . asteromphalus Eb. 8. 22, 182, 187, 233.

$\begin{array}{ll}\text { - } & \text { v. Buliviensis } 219 . \\ \text { - } & \text { v. Brightwelloides } 109,110 . \\ \text { - } & \text { v. eximia } 109,120 . \\ \text { - } & \text { v. hybrida } 117,118 . \\ \text { - } & \text { v. Pabellonica } 452-456 \\ \text { - } & \text { v. parallela } 452-456 . \\ \text { v. pulceps } 219 .\end{array}$

Barbadensis Grev. 2, 301.

biangulatus A. S. 178 .

biconus Ost. 794-797.

bifrons Cast. 935.

biharensis Pant. 389, 390.

bisculptus Patt. 452-456.

biradiatus Grev. 381, 531).

blandus A. S. $110,111,187,370,590$.

Bœkii Pant. 94, 339.

Boliviensis Grun. 43, 80, 453, $214,221$. $\nabla$. spinulosa 219-221.

borealis Bail. 22, 23, 46, 47, 234.

braclea J. Br. (Dépôts des Farbades).

Bremianus Pant. 316-349.

Brightwelloides Pant. $4005-407$.

bulliens A. S. 140. 141, 178, 234, 621.

Caraïbicus T. et IV. 201, 202.

centralis Eh. $80,370, \mathbf{6 5 9}, \mathbf{9 7 5}$.

- v. maxima 435, 436.

Chambonis M. Per. et F. H. 293.

chromoradiatus Karst. 935.

cinctus Ktz. 182, 183.

clivosus Pant. 91, 236-238.

- v. Iatefasciata 91.

cocconeiformis A. S, 359, 360 .

compositus Ratt. 234

concavus Elı. 18, 535, $56 \%$.

- v. minor 250,570 .

concinnus IV. Sm. 231, 617, $521,634,818$.

- v. Jonesiana 251, 621, 775.

- $\quad$. Moseleyi 3.

conformis Ratt. 864 .

convexus A.S. (Dépóts des Barbades) crassus Bail. 22, 23, 151, 393, 861 .

- $\quad$. Morsiana. $28,29,45$

cribrosus T. et $\mathrm{W} .46,90,376,378,585$.

cristalus Ratt. 452-156.

\section{Coscinotiscus Ehrenberg}

curvatulus Grun. 74, 151, 152, 621, 626.

- v. gemina $182,183$.

- v. inermis 46,47 .

- v. latiustriata $46,47,152,621$.

- $\quad$ v. minor 183, 626.

- $\quad$ v. recta $182,359,360$.

debilis Ratt. 139, 140.

decipiens Grun. 281, 282, 312.

decrescens Grun. 213, 214.

- r. polaris (Dép. des Barbades).

- v. repleta $139,140$.

- $\quad$ v. robustior 401,402 .

- v. valida (Dép. des Barbades).

- v. renusta

decussatus fit. el St. (Dépôts d'Oamaru). denarius A. S. 119, 282, 380, 562, 585.

* - $\quad$ จ. subtilissimus 675 .

depressus Grun. 109, 110.

detritus A. S. (Dépots des Barbades).

devius A. S. $34,58,403,848$.

dichotomus J. Br. (Dépôts des Barbades).

diorama Grun. 45, 109, 117, 291.

dimorphus Cast. 61. 62.

dispar II. P. et F. H. 51-5̃3, 293.

- v. radiata $51-53,293$.

diversis Grun. 151, 654, 659.

- v. completa (Dép. des Barbades). divisus Grun, $45<-456$.

Doljensis Pant. $412,413$.

dubiosus Grun. 3, 288.

v. eurvans Ratt. (Dép. d'Oamaru).

dubius Ratt. 381, 383, 469, 773.

durimsculus Ratt. 688-699.

echinatus Ratt. 213, 214.

elegans Grev. 2, 9:, 359.

- $\quad$. parvipunctata $201,202$.

elegantulus Grev. 342, 634 .

elongatus Grun. 220, 221.

entoleion Grun. $45,4 t$.

- $\quad$. decorata 145-14\%.

epiphanes Ratt. 182, 183.

euryoma A. S. 22, 23.

eutychus Azp 696697.

evadens Ratt. (Dépôts des Barbades).

- r. parvula

exasperans Ratt. 358-360, 553-555.

excavatus Grun. 92, 93. 942-944.

- $\quad$ v. biocellata $92,93$.

- v. gemina 942-944.

- v. quadriocellata (Dép. des Barb.).

- v. monophtalma 94:-944.

- v. semilunaris 942-914.

excentricus Eh. $187,370,473,617$.

$$
\begin{aligned}
& \text { - } \quad \text { v. micropora } 158 . \\
& \text { - } \quad \text { v. minima } 58 . \\
& \text { ₹. minor } 188 \text {. }
\end{aligned}
$$

exiguus v. æqualis Ratt. 28 . 


\section{Coscinodiscus Ehrenherg}

fasciculatus A. S. 844,889 .

fimbriatus Eh. 46, 188, 435, 436. $\nabla$. californica 291, 292.

fimbrio limbatus Eh. 23, 24. flagrans Ratt. (Dépots des Barbades). flexilis Ratt. 158, 452-456.

* flexnosus v. hispanicus 6.54, 659. floridulus A. S. 110-112, 842 . fragilissinus Grun. 525. fulguralis J. Br. 448. Gallopagensis Ratt. 621. Gazellø Jan. 233. gemmifer Elı. 358-360. gigas Eh. 22, 39, 80 .

- v. duplicata 182 .

- v. laxa 358,359 . glaberrinus Ratt. (Depots des Barbades). grandineus Ratt. 3 . v. dentata (Dep. des Barbades). griseus Grev $111,112,342$

- v. apiculata (Dépóts des Barbades). v. gallopagensis 2 .

Groveanus Ratt. (Dépóts des Barbades). Grunowii Pant. 91 . $\checkmark$ minor 91 .

hispidulus Pant. 236-238.

Herculus J. Br. 22, 435 .

heteroporus Eh. 23, 157, 370, 621, 865. - v. moronensis 151 , 376-378, 468. hungaricus Pant. 91, 236-238. implicatus Ratt. 110-112, 435, 436. impressus Grun. 110-112. insequalis Gr. et St. 2. inclusus Ratt. 182, 183, 241, 242. incretus $\mathrm{A}$. S. 110-112. interlineatus Ratı. (Dep. des Barbades). interrupta Grun. $358-360$.

intersectus J. Br. 23, 24. intumescens Pant: 91, 236-238, 346. isoporus $\mathrm{Eh} .145-147$.

Kinkerianus T. et IV. 201, 202.

Kochii Pant. 236-238.

Kusnetzkianus Pant. 258, 259.

Kūtzingii A. S. 34 .

- $\quad$. glacialis 3 .

Janischii A. S. 91, 148, 182, 551, 818.

Johnsonianus Ratt. 376-378.

labyrinthicus Roper 452456.

lacustris Grun. 241-243.

- v. septentrionalis 182, 183. lentiginosus Jan. 342.

leptopus Grun. 696, 697, 772, 775, 800 .

+ v. irregularis 6,17

* - v. excentrica 472, 473.

Lewisianus Grev. 367, 369, 658, 659.

- $\quad$. minor 425 .

- $\quad$ v. monorensis 378,658,659.

\section{Coscinodiscus Ebreaberg}

lineatus Eh. 58, 182, 291, 621.

- $\quad$. tenera 201, 202.

luctuosus Grun. 658,659 .

lutescens Ratt. 201, 202.

Macræanus Grev. 452-456.

magnisculptus Ratt. 376-378.

marginatus Eh. 46,$90 ; 154,468$.

* $\quad$ - $\quad$ ₹. intermedia $151,156,468,621$.

marginulatus $\nabla$. curralostriata Grun. $376-378$.

Martoutli Pant. 389, 390.

megaloma A. S. 448.

micans A. S. $46,47,367,369$,

minor Eh. 19, 113.

modestus Ratt. 452-456.

Mölleri A. S. 213, 214.

- . macroporus irun. 11.

Monicæ Ratt. 468, 553, 555.

Moravieus Grun. 8, 236-238.

Moronensis Ratt. 376-378, 861.

$$
\begin{array}{ll}
\text { - } \quad \text { v. latilimba } 990,997 . \\
\text { - } \quad \text { v. major } 996,997 \text {. }
\end{array}
$$

Morsianus Grev. (Dépôts des Barbades). naviculoides T. et IV. 201, 202. neogradensis Pant. 91.

nitidulus Grun. 99, 100 .

* - T. scintillans 648 .

nitidus Greg. 49, 72, 148, 505, 875 .

- v. minor 533.

- v. moronensis 378 .

- v. sparsa 99, 100.

nobilis Grun. $16 \%, 182,621$.

nodulifer A. S. $50,566,621,75$.

- v. apiculata 592, 593.

* - $\quad$ v. media 50 .

- v. minor 974.

Normanii Grev. 182, 864.

notabilis A. S. $358-360$.

Nottinghamensis Grun. 233, 234.

Oamaruensis Gr. et St. 1, 2.

obliquus Ratt. $358-360$.

oblongus Grev. 201, 202.

obovatus $\nabla$. circularis 864 .

obscurus A. S. 22, 23, 383.

- v. minor 378 .

obversus Ratt. $383,384,551$.

oculus-iridis.Eh. 22, 23, 182, 310.

$$
\begin{aligned}
& \text { - v. loculifera 219. } 220 . \\
& \text { - } \quad \text { v. morsiana 27, 29, } 90 . \\
& \text { - } \quad \text { r. snbspiuora 219, 220, 452-456. }
\end{aligned}
$$

odontodiscus Grun. 92, 93, 122.

odontophorus Grnn. 773.

omphalanthus Eh. 182, 233, 234.

ovalis Roper. 173-175.

ovicentrum Grove (Depóts d'Oamaru).

* ovam Temp. et-M. Per. 702, 703. 


\section{Coscimodicus Ehrenberg}

pacificus Ratt. 358-360, 65 I, 652.

" papulosus Forti 367-369.

Papuanus Cast. 973.

partitus Gr. et St. $140,141,158$.

- v. nebulosa (Dépóts d'Oamaru).

patera Cast. (Dépôts des Barbades). patina Eh. 626.

patellæformis Grev. (Dépots des Barbades). pauper T. et W. 201, 202.

pectinatus Ratt. 634, 651, 652 .

perforatus Eh. 182, 864 .

$$
\begin{aligned}
& \text { - } \quad \text { v. ambigua } 649 . \\
& \text { - } \quad \text { v. cellulosa } 442,443,452-456 . \\
& \text { - } \quad \text { v. minor } 47 .
\end{aligned}
$$

pericompsos Ratt. 358-360.

Pethoi Pant. 405-407.

Peruanus Eh 213, 214, 452-156.

plicatus Grun. 158.

polyacanthus $\nabla$. intermedia 367-369.

preclarus A. S (Dépots du Japon).

Prietor Grove (Dépôts des Barbades). pseudolineatus Pant. 173-175, 405̄-407. pulchellus Grun. 91.

punctiger H. P. 525 .

pygmæus J. Br. et F. H. 31, 32, 293, 765 .

$$
\text { - } \quad \text {. micropunctata 53, 293, } 765 .
$$$$
\text { - } \quad \text { v. minutissima } 53 \text {. }
$$

radiatus Eh. 90, 92, 371, 61 .

$$
\begin{aligned}
& \text { - } \quad \text { v. centralis } 219,220 . \\
& \text { - } \quad \text { v. heterostriata } 236-238 \text {. media } 139,140,219,220,564 . \\
& \text { - } \quad \text { v. subrequalis } 236-238 .
\end{aligned}
$$

radiolatus Eh. 182.

radiosus $\mathbf{v}$. Kerquelensis 3 .

Rex Wall. 942-944.

rhombicus Gast. 674, 675, 928, 943.

- - v. crassipunctała 674,675.

- - $\quad$ - italicus 367-369, 423, 424 .

- - v. lanceolata 674,675.

robustus Grun. 22, 23.

- $\quad$. amœna 22, 23.

- $\quad$. latemarginata $389,390$.

Rothii Grun. 99, 674, 775, 819.

- spinuli densioribus 996, 997.

Sarmaticus Pant. 442, 443.

scintillans Grev. 342, 436.

Simbirskianus Grun. 11, 160.

secernendus A. S. 233, 234.

semipennatus Grun. (Dép. des Barbades).

senariusv. parvula (Dép.des Barbudes).

sol. Wall. 229, 324.

sphæroïdalis Ratt $358-360$.

v. cincta (Dép. des Barbades).

spiniferus Gr. et St. $140,141,250,570$.

spinuligerus Ratt. $358-360,864$

spiraliterpunctatus Pant. 236-238, 649, 650.

\section{Coscinodiscus Fhrenberg}

stellaris Rop. 23, 24.

- v. Mejıllonis 219, 220 .

Stokesianus Grun. 91, 236-238.

strigillatus Wit. 468.

symbolophorus. Grun. 155, 310, 171, 621, 773.

$$
\begin{array}{ll}
\text { - } & \text { v. fossilis } 926-928 . \\
\text { - } & \text { v. minor } 47 . \\
& \text { v. Oamaruensis } 160 .
\end{array}
$$

symmetricus Grev. 324, 561, 592.

- $\quad$ v. tenuistriata 926-928.

subaulacodiscoidalis Ratt. 553, 556.

subconcavus Grun. 11, 23, 24.

subdivisus T. et W. 201, 202.

subglobosus $\mathrm{Cl}$. ot Grun 158.

sublineatus Grun. 11.

subnitidus Ratt. (Dép. des Barbades). suboculatus Ratt. 842 .

subtilis Eh. 48, 155, 472, 473, $\mathbf{5 5 4}$.

- v. lineolata 28,29 .

subrelatus Grun. 358-360.

suspectus Jan. 418, 449.

superbus Hard. 452-456.

- $\quad$ v. moravica (Depp. des Barb.)

- $\quad$ v. trinitatis 367-369.

tabulatus J. Br. 346-349.

Temperei J. Br. 24, 25.

tenuis Ratt. 260, 261, 946.

traducens Ratt. 250.

transylvanicus Pant. 530, 531, 547.

tumulus J. Br. (Dépôts du Japon).

trochistus T. et W. 201, 202.

tuberculatus Grev. $650,652,651,657$.

จ. monicæ $47,112,473,861$.

tubiformis Temp.et Br. 24, 25 .

tumidus Jan. 342, 442, 658, 659 .

turgidus Ratt. 182, 183, 367-369.

velatus Eh. 182, 183, 231, z98.

vetustissimus Pant. 91, 569.

venulosis Cast. 3.

vigílans A. S. 12, 22, 23.

Weissflogii Pant. 236-238.

Woodwardii Eul. 182, 452, 456.

umbonatus Greg. 452-456.

undatus Grun. 91, 346-349.

undulatus Grun. 8, 9.

\section{Cosmiodiscus Greville}

elegans Grev. 358-360, 478, 479.

Normanianus Grev. (Dépóts d'Oamaru).

tenuis Grun. 358-360.

\section{Cotyledon J. Brun}

circularis J. Br. (Dépóts du Japon). coronalis J. Br. (Dér.bts des Barbades).

\section{Craspedodiscus Eirenberg}

coronatus Bright. (Dépóts des Barbades). 


\section{Craspedodicus Ehrenberg}

coscinodiscus Eh. 145, 297, 298, 638, 773. - $\quad$ P. apiculatus 715,716 .

- $\quad$ v. asterioides $996,997$.

* $\quad$ - $\quad$. hispanicus.

* - $\quad$ - Nankoorensis 146, 147, 996, 997. elegans Eh. 92, 297, 688, 998.

insignis A S. 422-425, 474, 475 .

Klawsenii Grun 27.

marginatus Bright. (Dépot des Barbades).

Mölleri A. S. 27, 619.

oblongus Grun. 620 .

* - v. acuminata 715, 716 .

rhombicus Grun. 233-235, 358.

semiplanus Bright. (Dépôts des Barbade.s). umbonatus Grer. (Dépóts des Barbades). Weissflogii Pant. 126-128.

\section{Craspedoporus Greville}

corolla J. Br. (Dépóts du Japon). elegans Gr. et St. (Dépôts d'Oámaru). Johnsonianus Bright. (Dep. des Harbades). Pantocsekii J. Br. 376-369,673,674. Ralfsianus Grev. (Dép. des karbades). Truanii Pant. 126-128, 236-238.

\section{Cresswellia Greville}

Barbadensis Grer. (Dép. des Barbades). minuta Girer (Dépôts des Barbades). Palmeriana Grev. 525. rudis Grev. 36, 358-360. sphæria Grev. (Dépôts des Barbades). superba Grev. 942-944.

\section{Cyclophora Castracane}

tenuis Cast. $26 \dot{\mathbf{5}}$.

\section{Cyclotella kützing}

antiqua W. Sm. 249, 461, 858.

- v. Vernieri 327.

asiatica $\mathrm{J} . \mathrm{Br}, 12,25$.

Balatonis Pant. 901.

Bodanica Cl. 215, 538, 603, 900.

Charetoni F. H. 169879.

- v. radiata 169 .

- v. scutiformis 169.

Castracanei J. Br. 47.

Comensis Grun. 780.

comta Eh. 192, 296, 362, 680, 900.

- v. affinis 206,215 .

- v. arverna 77, 278, 387.

- $\quad$. decressens 308.

- $\quad$. quadrinotata $326,327,762,763$.

- v. quinquenotata 326,327 .

- v. radio-a 82 .

- v. tenuis 780 .

- v. trinotata $308,483$.

\section{Cyclotella Kützing}

Guinardi F. H. 326, 327.

iris J. Br. et F. H. 53, 122, 261 .

- $\nabla$. cocconeiformis 136,404 .

- $\nabla$. integra $53,122,404$.

- v. ovalis 136, 104 .

Kützingiana Thw. 206, 575, 642, 805. v. gemmatula 263.

Lemanensis Menegh. 783.

melosiroides Menegh. 780, 781, 788.

Meneghiniana Kiz 57, 227, 341, 630, 888. $v$ rectangulata 133,134 .

operculata $\mathrm{K} \backslash \mathrm{z} 232,296,680, \mathbf{8 3 3}, 902$. - v. antiqua 296.

papillosa O'Mea. 249.

perforita F. H. 53, 264, 303, 739.

physoplea EL. 147.

pygmæa Pant. 205, 547.

radiato punctata Bail 204, 205.

sexpunctata Deby. 8 r1.

Sevilleana Deby. 8 z1.

stelligera Cl, et Grun . $163,161,273$.

striata Ktz 187, 282, 591, 936, 988.

- $\quad$. ambigua 192, 890.

- v. intermedia 34 .

- v. mesoleia 757.

* - v. radiosa 101, 308, 680.

- v. sinensis 428 .

stylorum Bright. 200, 479, 562, 855, 936.

Szakalensis Grun. 129.

Temperei M. Per. et F. H. 1।4, 372, 394, 763.

$\nabla$. inermis 326,327 .

* Terryana Temp. et M. Per. 335, 395.

* - $\quad$. major. 395.

* - v. minor 335, 366, 395.

tiansylvanica Pant. 119, 20t, 205.

$$
\text { - } \quad \nabla \text {. dissiminata punctala 204, } 205 .
$$

\section{Cymatoneis Cleve}

sulcata Cl. 160, 648 .

\section{Cymatosira Girunow}

belgica Grun. 594.

biharensis Pant. $348,349$.

Debyi Temp. et Br. 25.

japonica Temp. et Br. 25.

Lorenziana Grun. 277.

$$
\text { - } \quad \nabla . \text { lala } 263 .
$$

\section{Cymatopleura W. Smith}

apiculata IV. Sm. I49, 150, 470, 597, 767

- v. minor. 752 .

elliptica W. Sm. 56, 113, 206, 460,767.

- v. constricta 56, 122.

- v. orata 783.

- $\quad$ v. rhomboïdea $239,877,878$.

- v. subconstricta 293.

- v. torta 884. 903. 


\section{Cymatoploura W. Smith}

gigantea Pant. 587.

hibernica W.Sm. 459, 460, 461, s74.

จ. major 226, 465 .

* Mannii M. Per. 365, 366.

Martyi F. H. 262, 263, 738, 739.

solea Eh. 34, 89, 293, 824, 895.

- v. clavata 480,481 .

- v. crassa 913.

- v. elongata 85, 911, 913.

- v. laticeps 480,481 .

- v. rugosa $480,481$.

- v. subconstricta-480, 481, 482 .

- v. vulgaris 903 .

\section{Cymbella Agarilh.}

acutiuscula Cl, 249, 374, 905. æqualis W. Sm. 249, 419, 758, ซ59, 786. abnormis $\nabla$. fossilis 632,633 .

Abyssinica Grun. 480, 481, 483. affinis Ktz. 492, 621, 638, 902. alpina ₹. minor $136,137$.

Americana A. S. 159, 171, 216, 302, 855. v. acuta 834 .

amphicephala Ning $274,576,858,883,887$.

$$
\text { v. acula } 834 \text {. }
$$

* anglica Lag. 77, 150, 216, 273, 737.

angustata Grun. 249 .

arcus Eh. 679, 860 .

arctica Lag. 758, 759 .

aspera Eh. 68, 159, 293, 757, 938.

australica $\mathrm{Cl}$. 480, 481, 801 .

- $\quad \nabla$. fossilis 326,327 .

austriaca $\nabla$. excisa. $\mathbf{5 1 8 , 5 1 9 .}$

- $\quad$. prisca 632, 633.

Balatonis Grun. 767.

Bouleana F. H. et J. Br. 135, 136.

Brevieri F. H. 5l-53, 764, 765.

Bruyanti F. H. 326, 327.

Budayana Pant. 547.

v. gracilior 547 .

capitata M. Per. et F H. $263,366,374$.

catalense M. Per et F. H. 734, 735.

cesati Rab. 222, ว58, ร59, 807, 868.

Charetoni F. H. 169, 624, 625 .

Chyserii Pant. 528, 529.

cistula Hemp. 82, 222, 334. 75 ร, 859.

- - v. crassa 366, 374

- $\quad$. fusidium $341,356,763$.

- $\quad$. hungarica 518, 519 .

- v. inaculata 466, 575 .

clementis Pant. 632, 633.

conifera J. Br. et F. 'H. 134, 135, 404.

Greguti F. 'H. 51-53.

- cucumis A. S. v. delicata 366.

* cuneata M. Per. 171.

cuspidata Ktz 15, $323,429,834,854$.

- $\quad$ v.'major 374 .

\section{Cymbella Agardh}

cuspldata $\nabla$. minor 308,834 . cymbiformis Eh. 22:, 280, 296, 601, $\boldsymbol{7 5}$.

s. producta $518,5 เ 9,632,633$.

delecta A. S. 76, 77, 882, 905 .

delicatula $\mathrm{Ktz} \mathbf{2 2 2}, 255,320,501$.

- dissimilis M. Per. 365 .

Dubravicensis Pant. 632, 633 .

Ehrenbergii Ktz \$5, 169, 323, 75\%,881.

- - v. crassa 374 .

v. minor 334 .

Erdöbényana Pant. 528, 529.

Foucaudi F. H. 734, 735.

gallica M. Per. F. H 762, 763 .

gastroides $\mathrm{Ktz} 340,4$ J0, 682, 881, 903 .

$$
\begin{aligned}
& \text { - v. crassa } 518,519,632,633 \text {. } \\
& \text { - v. minor } 227,308 . \\
& \text { - v. prolongata 762, 763. }
\end{aligned}
$$

gibba Kłz 108, 216, 222, 320, 4 70.

gomphonemacea Lil. 206.

gracilis $\mathrm{K} \backslash \mathrm{z} 824$.

Grunowii Pant. 632, 633.

Hantzschiatica Grun. 848 .

Hauckii V. H. 586, 588.

$$
\text { v. fossilis } 263 \text {. }
$$

hebridicum Greg. 273.

helvetica Ktz 192 290,604, 758, 834 . $\nabla$. gracilis 788 .

Hercynina A. S. 248, 249.

heteropleura Ralls. 107, 1 76, 497, 589, 868 .

v. minor $583,587,88:, 885$.

hevesensis Pant 304, 305, 515, 527.

hungarica Grun. 197, 263, 364, 901.

incisa $\mathrm{Ktz} 352$.

Janischii A. S. 365, 366.

Kamtschatica Grun. 558, 559.

Kochii Pant. 517.

lacustris Ag 206.

Lævis Næg 222, 644, 805901.

lanceolata Eh. 57, 169, 326, 491, 890. $\nabla$. fossilis 518 .

lata Grun. 206.

latestriata Pant. 518, 519.

Laubyi F. H. 51-53, 263, כ76.

leptoceros Ktz 52, 206, 290, 589 .

$$
\text { - } \quad \text {. curta 136, 137, } 263 .
$$

v. olutusa 222 .

maculata Ktz $323,516, \mathbf{7 5 \% ,}, \mathbf{8 0 3}, 834$.

- v. curta 308 .

meniscus F. H. 51-53.

mexicana Eh. 107, 302, 334, $\mathbf{3 6 5}$.

microcephala Grun. 801.

miocenica M. Per. F. H. 740, 741.

Naviculacea Grun. $\mathbf{7 5 9}, 868,896$.

naviculiformis Aners $77,321,496$.

Neupauerii Pant. 304, 305, 528, 529.

norvegica Grun. 168, 249, 419.

obtusa Greg. 278, 578, 858, 881, 901. 


\section{Cymbella Agardh}

obtusiuscula Ktz 249.

pachyptera Pant. 632, 633.

Pagesi F. H. 51-53.

parva W. Sm. 113, 192, 757, 804, 901.

Pauli M. Per. F. H. 355, 356, 404, 764.

pisciculus Greg. 249.

Plutonica Pant. 518, 519.

- producta M. Per. 105, 106, 107. prostrata Cl. 785.

pseudoturgidula M.Per. et F.H. 734,73..

pusilla Grun. 177, 223, 350, 901 .

Rhodesi F. H. 263, 264.

salina Pant. 304, 305, 363, 529.

scotica W. Sm. 122, 249, 501.

simplex Pant. 632, 633.

spuria Cl. 629.

Staubii Pant. 518, 519.

staumatophora Grun. 280, 283, 903.

Sturii Grun. 518, 519, 632, 633 .

subæqualis Grun.602, 682, $759,829,834$.

subalpina Meister 805 .

tumida Brẻb 33.

turgida Greg. 519.

turgidula Grun. 53, 283, 604, 801 .

variabilis West. 192.

vegeta Pant. 632,633 .

ventricosa $K ı z$ 206, 629, 801 .

$$
\begin{aligned}
& \text { - } \quad \text { v. Auerswaldii 785, } 808 . \\
& \text { - } \quad \text { 7. lunula 785, } 808 \text {. } \\
& \text { - } \quad \text { ovata } 785 \text {. }
\end{aligned}
$$

\section{Dactyliosolen Castracane}

antarcticus Cast. 955, 958, 959.

mediterreneus H. P. $915,916,917$.

\section{Debya Pantocsek}

Oamaruensis Ratt. (Depots d'Oamaru).

\section{Denticula Kützing}

antlllarum Gl. 189.

crassula Næg. 805 .

elegans $\mathrm{K} / \mathrm{z} 113$.

frigida $\mathrm{Ktz} .788,901$.

inflata W. Sra. 29i, 356.

Kittoniana Grun. 253.

lauta Bail 47.

nicobarica Grun. 213, 214.

tenuis Ktz 222, 788, 901.

- v. inflata 66, 901 .

thermalis Kítz 67, 243, 825, 829 .

ralida' Ped. 495, 543, 619, 8\%5, 829.

- . minor 825 .

\section{Diadesmis Kützing}

.peregrina W. Sm. 751.

\section{Diatoma De Candolle}

anceps Grun. 13, 69, 163, 321, 575.

\section{Diatoma De Candollo}

anceps v. anomalum W. Sm. 13,150,163, 279.

- - $\quad$ - capitata 104, 776.

- $\quad$ - $\quad$. constricta 729.

- - $\quad$ v. linearis $103,104$.

Ehrenbergii v. grande 296.

elongatum $\mathrm{Ktz} 57$.

gracillima Næg. 222.

hyemale Heib. 148, 500.

v. curta 104.

mesodon Kız. 69, 500, 508, 576, 644.

pectinalis $\mathrm{K} \mathrm{z} \cdot 2 \mathrm{0} 3 \mathrm{3}, 264$.

- v. capitala 263, 264.

- $\quad \nabla$. elongata $! 63,164$.

tenue Ag. 164, 296, 412, 429, 880.

- v. minor 786 .

- v. normale 785 .

vulgaris Bory 57, 294, 4 z0.

- $\quad$. brevis 785 .

- $\quad$ v. linearís 196.

- $\quad$ v. productum 785 .

Diatomella Greville

Balfouriana Grev. 339, 787.

\section{Dicladia Ehreuberg}

Barbadensis Grev. (Dép. des Barbades).

capreolus Eh. 90, 238, 449, 634, 848 .

japonica Pant. (Dépóts du Japon).

mitra Elı. 649, 650 .

robusta Grev. 47.

\section{Dictyoneis Cleve}

marginata Cl. 268, 277, 377, 592, 593.

- $\quad \nabla$. elongata 276, 277.

- $\quad$ v. Janischii(Dép. du Japon).

naviculacea $\mathrm{Cl} .330$.

rugosa Gl. 616.

Thumii Cl. 798.

\section{Dineregramma Ralfs}

- achnanthes M. Per. 654 .

Boryanum Pant. 95.

- costatum v. rhomboides 370 .

fossile Grun. 91, 237, 238.

fulvum. Ralfs. 146, 147, 648 .

Ischaboensis Grun. 554, 555.

lanceolatum H. D. 268.

marinum Greg. 151,153, 155, 269, 929.

- $\quad$. recta Ralfs. 50.

minor Ralfs. 268.

nanum Grev. 199, 443.

nave Ciesare $K$. et Sch, $146,147$.

$$
\text { - v. obtusa } 146,147 \text {. }
$$

\section{Diploneis Ehrenberg}

apis Cl 592, 617 .

bombus Cl. 370, 594, 767 .

- $\quad$. denmestriata 370. 


\section{Diploneis Ehrenberg}

coffeæformis Cl. 610 .

crabro Eh. 469, 592, 775, 798.

- $\nabla$ separabilis 401.

cynthia A. S. 647,648 .

- - v. intermedia 647, 648.

demta CI. 775 .

didyma Cl. 370, 594, 61\%, 798, 934.

- $\quad \nabla$. major 617 .

- $\quad$ v. subconstricla 206.

Domblittensis Grun. 206.

elliptica Cl. 190, 191, 273, 371, 399.

- v. minutissima 53, 902 .

- v. oblongella 263, 264, 682, 902.

expedita A. S. 634,935 .

Finnica Cl. 362, 524.

fusca Ralfs 401, 402, 617, 834, 835 .

- $\nabla$. delicatula 617.

- - norvegica 617.

interrupta 594, 640, 767, 903, 986.

- $\quad$ マ. novæ zelandiæ 647,648 .

littoralis $\mathrm{Cl} .732$.

- $\quad$ v. arctica 64 .

Mauleri J. Br. 206, 401, 402.

mediterranea Cl. 647, 648.

- - v. elliptica 401, 402.

multicostata A. S. 775, 847, 818, 933. nitescens Cl. 549, 550, 592, 593, 933 .

- $\quad$ - $\checkmark$. rhomboides 847,848 .

notabilis Grun. 401, 402, 933.

- $\quad$. explita 401, 402.

ovalis $\mathrm{Cl} .891$.

- oblongella 801.

papula $\mathrm{Cl}$. ?67.

pelagi A. S. 767.

prisca Cl. 773.

Schmidtii Cl. 847, 848.

sideralis J. Br. $634,651,652$.

Smithii Bréb. 308, 371, 374, 394, 594.

- $\quad$. major 370 .

- $\quad$. recta 847,848 .

splendida Greg. 401, 592, 617, 847, 775 .

subcincta A. S. 634,773 .

suborbicularis Cl. 798.

vagabunda J. Br. 651, 652.

\section{Ditylum Bailey}

Brightwellii West 955, 962, 968, 972, 973.

Ehrenbergii Grun 234, 235.

Grovei J. Br. (Dépots d'Oamaru).

intricatum West. 40, 285-287, 953, 970. segmentati J. Br. (Dépóts du Japon).

sol. V. H. 488, 489, 525.

undulatum 110, 111, 234.

\section{Donkinia Raifs}

antiqua Gr. et St. (Dépóts d'Oamaru). carinata Ralfs. 18,19 .

\section{Donkinia Ralfs}

recta Grun. 598.

- $\quad$. minuta 268.

Thumii H. P. 285-287.

\section{Druridgea Donkin}

geminata Donk. 598.

\section{Eucyonema Kützing}

" attenuatum M. Per. 8z6.

cæspitosum Ktz 57, 216, 296, 856 .

$$
\begin{aligned}
& \text { - } \quad \text { v. Auerswaldii } 52,53,738,739 . \\
& \text { v. lata } 680 \text {. }
\end{aligned}
$$

hebridicum Grev. 351, 362, 496, 868. intermedium F. H. 52, 53, 480, 481 . gracile Rab $362,496,605,678,834$.

- $\nabla$. lineata 296.

- v. minor. 208

Grandi F. H. 480, 481, 482, 483.

lunula Grun. 77, 113, 222, 605, 919.

- Mackayana Temp. et Per. 395. norvegica Grun. 758, 868.

paradoxum Kt\%. 752.

- parallelum M. Per. 366.

prostratum Ralfs. 34, 113, 296, 470, 767.

- rotundatum M. Per. 8 z6.

triangulum $\mathrm{Ktz} 334,630,631,867,876$. turgidum Grun. 216, 334, 429, 828 .

- $\quad \nabla$. ventric isa 757.

ventricosum Ktz 57, 159, 283, 508, 856.

verrucosa $\mathrm{Ktz} 273$.

- $\quad$. ininuta 222, 508, 521, 602.

- $\quad$. stricła 216.

yarrense Grun. 243.

\section{Endyctia Ehrenberg}

Boryana Pant. 94.

Lunyacsekii Pant. 236, 237, 756.

minor A. S. $238,389,390$.

oceanica Eh. 151, 160, 322, 403, 987.

Schmidtii Pant. 236, 237.

\section{Entogonia Greville}

Abercrombieana Grev. (Dep. des Barb.)

amabilis Grev.

approximata Grev.

Bergoni J. Br. (Dépóts des Barbades).

biangulata Bergon (Depots d'Haiti).

Brunii Bergon (Depots des Barbades). conspicua v. Irigemma (Déots du Japon). Davyana Grev. (Depóts des Barbades).

Davyana v. distans (Dép. des Barbades).

- r. - la biangulata (Dép. des B.).

- v. intermedia (Dep. des Barbades).

- V. propineu

- $\nabla$. triganam 


\section{Entogonia Greville}

divergens Bergon (Dép. des Barbades). furcata $\mathrm{J}$. Br. elegans Grev. gratiosa Grev. v. intermedia

inopinata Grev.

$$
\text { - } \quad \text {. linearis }
$$

Jeremiana Bergon (Dépóts d'Haiti).

$$
\nabla \text {. pentagona }
$$

Letourneurii Bergon (Dép. des Barbades). pulcherrima Grev.

$$
\begin{array}{lll}
\text { - } & \text { v. intermedia } & - \\
\text { - } & \text { v. marginata } & - \\
\text { - } & \text { v. ponetnlata } & -
\end{array}
$$

punctulata Grev. (Dép. des Barbades). reticulata Grev.

Temperei Bergon

$$
\text { - } \quad \text {. intermedia }
$$

tripodiformis Bergon

variegata Grev.

venulosa Grev.

\section{Entopyla Ehrenberg}

australis Eh. 3, 48, 49, 432 .

- v. gigantea $45,110,435,436,651$.

- v. incurvata $468,469,651,652$.

hungarica Pant. 236, 237.

ocellata v. Calaritana.

- v. pulchella 474, 475, 634 .

\section{Epithelion Pantocsek}

curvatum Pant. 260, 261. rossicum Pant. 260-261.

spinifer Pant. 260, 261.

\section{Epithemia dos Brébisson}

amphicepbala Grun. 334, 516,601, 834, 931 Anastasia Pant. 851-853.

arcuata Pant. 851853.

- v. minor 853 .

argentina $\mathrm{J} . \mathrm{Br} .825$.

argus $\nabla$. alpestris $76,192, \mathbf{8 2 8}$.

- . a mphicephala. $78,122,327$.

-. $\nabla$. bidens 168.

- . fossilis 547 .

biharens.s Pant. 389, 390.

cistula Girun. 206, 544, 545.

- $\quad$ lonari Gr. $519,544,545,632,633$.

Cleopalræ Pant. 851-853.

constricta WV. Sm. 168.

crucæformis Pant. $305,463,514,515$.

- $\quad$. validior 528,529 .

Debyi Pant. 304, 305.

geminata Pant. 851.853.

gibba Ktz. 85, 465, 516, 542, 890.

- $\nabla$. Boryana 518, 519547.

- v. longissima- 76.

\section{Epithemia de Brébisson}

gibba $\nabla$. parallela $52,113,206,516,767$.

- v. ventricosa $216,334, \mathbf{3 6 6}, \mathbf{5 4 2} 888$. gibberula Eb. 44, 168, 309, 381, 61 z.

$$
\begin{array}{ll}
\text { - } & \text { v. directa } 544,545 \text {. } \\
\text { - } & \text { v. perlonga } 518,519 \\
\text { - } & \text { v. producta } 53,113,187,189,319 .
\end{array}
$$

globifera Heib. 248, 249.

hirundinella J. Br. 54, 55, 339, 400, 402.

Hyndmannii W. Sm. 50,56, 122, 190, z78.

$$
\begin{aligned}
& \text { * - } \quad \text { v. capitata } 365 \text {. } \\
& \text { - v. curta } 293 . \\
& \text { - v. interrupta } 190 .
\end{aligned}
$$

inflexa Pant. $304,305$.

maeotica F'ant $851-853$.

multicostata Pant. 304, 305.

musculus Ktr. 101, 199, 269, 375, 893. ocellata Ktz 248, 249, 327, 601, 910.

perinsignis Pant. 851-853.

perlonga Pant. 547, 762.

Pethoï Pant. 518, 519.

salina Pant. 304, 305, 463, 464.

- v. nuda 301. 305.

saxonica Kız. 961.

Schūttiana Pant. 851-853.

sorex Ktz. 34, 82, 114, 606, 663.

succincta Bréb. 168, 553.

subsalsa Pant. 501, 514, 515, 528, 529.

tertiaria Pant. 173-175.

transylvanica Pant. 530, 531, 547.

* truncata M. Per. 365.

- - $\quad$ v. debilis $\mathbf{3 6 5}, \mathbf{3 6 6}$.

turgida Ktz. 465, 6z9, ร52, ร6z..

- v. crassa 355.517.

- $\quad$ v. granulato $334,459,465,686,890$

- v. porcellus 251.252, 663 .

- $\quad$ - fa excavata 374 .

- $\quad$ v. vertagus $82,85,112,334$, ว5ร.

vittata Pant. $301,305$.

Westermannii Ktz. 113, 190, 293, 679, 767.

* - $\quad \nabla$. stricta 365, 366.

zebra Ktz. 77. 168, 216, 679, 757.

- v. curta 207.

- v. longicornis $\lceil 63,355,356,387$.

- v. minor. $216,356,374,517,663$.

- v. proboscidea $78,192,517,663,914$.

- v. saxonica 801 .

- v. undulata $355,356$.

- v. ventricosa 20 .

\section{Eucampia Ehrenberg}

balaustium Cast. 935.

cornuta Cl. 525.

Grœnlandica CI. 64.

Virginica Grun. 147.

zodiacus Eh. 229, 789, 970, 971, 973. 


\section{Eucampia Ehrenberg}

zødiacus $\mathrm{v}$. cornigera 229.

\section{Eunotia Ehrenber}

americana K. et Sch. 147. arcus Eh. 159, 374, 601, 758, 776

- v. bifrons 385.386 .

- v. hybrida $118,576,776$.

- v. minor 104.

- $\quad$. plicata 168, 211, 212, 885 .

- $\quad$. tenella 104. 559, 834.

- $\quad$. uncinata $834,903,904$

bactriana Eh. 458.

bidentula. W. Sm. $211,601,733,883,885$.

bicapitata Grun. 465, 46:i.

biceps Eh. 866.

bigibba Greg. 858 .

Burkartii Eh. 223.

camelus Eh. 458 .

castalunga J. Br. (Dép. des Barbades). Clevei Grun. 575.

compressa $v$. angusta $76,77,387,388$. denticula Bréb. $\mathbf{8 6 8}$.

depressa Eh. 419.

diodon Eh. 37, 213, 278, 274, 576.

- $\quad f^{2}$ minor. 78.

diadema v. polyodon $14,15,374$.

Ehrenbergii v. quaternaria 632, 633.

eruca Eh. 520.

exigua Rab. 576.

faba Grun 164, 883 , 885 .

flexuosa Ktz. 582.

- v. bicapitata 227 .

- v. pachycephala $176,177,769$.

formica Eli. 15, 36, 159, $\mathbf{3 4 0 , 5 5 8 . ~}$

- $\quad$ v. elongata 289.

- $\quad$ v. intermedia 289.

gibbosa V. H: 883.

gracilis Eh. $76,168,429,589,873$.

- $\quad$ v. fossilis 632,633 .

- v. major $387,903,906$.

* - v. nodosa $105,106$.

bemicyclus Eh، 458.

hexaglyplis Eh. 576 .

impressa Eh. 78, 459, 768, 776, 881.

- $\quad$ v. angusta 52, 321, 776.

* - $\quad$ - ventricosa 429 .

* inæqualis M. Per. 922.

incisa Greg. 216, 249, 481, 576, 890 .

- v. obtusiuscula $733,834,883,885$.

Junaris Grun. 13, 78, 159, 576, 854.

- $\quad$ v. alpina 834, 922 .

- $\quad$ v. arcuata 69, 776.

- v. attenuata 429 .

lunaris $\nabla$. excisa 13, 19.

- $\quad$ v. major 922.

- v. subarcuata $150,223,922$.

- v. undulata-429.

\section{Eunotia Ehrenberg}

major Rab. 13, 78, 216, 672, 776.

- v. bidens 76 .

- v. ventricosa 78, 334 .

minor Rab. 210, 356, 129, 776, 907.

monod on Eh. 36, 3i, 78, 216.

v. curta 216,374 .

obtusiuscula Grun. 374.

ophidocampa Cl. 273.

parallela Eh. $36,76,77$.

pectinalis Rab. 78, 672, 776, 854, 907.

* - $\quad$ v. arverna 172.

- v. biconstricta $887,894$.

- $\quad$ v. curta 22\%.

- $\quad$ v. elongata 465, 466 .

- v. maxima 751 .

- v. stricta 104, 122.

- v. undutata $168,211,212,484,763$.

- $\quad$ v. venter 374.

* - $\quad$ v. ventricosa 78, 768, 776, 854.

pendens M. P. et F. H. 734, 735.

pentagly phis Eh. 274.

polyglyphis Grun. 52, 497, 547, 589, 776. polyodon Eh. 484 497, 831 .

prærupta v. bidens $216, \mathbf{2 2 3}, 334,758,887$.

- v. bigibba. 882 .

- v. curta 207, 208, 885.

- $\quad \nabla$. inflata $736,852$.

quaternaria Eh. 429.

quinaria Eh. 301.

Rabenhorstii $\nabla$. monodon 207, 208.

robusta Ralfs. 15, 524.

- v. diadema $15,86,414,860,8$ ro.

- $\quad$ v. hendecaodon $\mathbf{8 7 0}$.

- v. hexaodon 733 .

- v. tetraodon $37,216,576,589$.

serra Eh. 85.

Soleirolei Ktz. 465, 466.

striata Gr. et St. 141.

ternaria 78.

tetraodon 216.

transylvanica Pant. 203, 204.

tridenta Eh. 302, 303, 429.

- $\quad$ v. bidentula 321 .

triodon Eh. 274, 577, 578, 868, 891.

ventralis Eh. 890:

ventricosa Grun. 78, 768, 776, 854, 900.

\section{Eunotogramna Weisse}

bivittata Pant. 91, 213, 214.

- v. elongata (Dép. d'Oamaru).

debilis Grun. 544, 555, 988.

Frauenfeldii Grun. 561, 562.

Laevis Grun. 730, 731, 988.

productum Gr. etSt. 139, 140, 250, 439, 440.

Weissii Eh. 12, 260, 261.

- v. producta 2, 258, 259. 


\section{Euodia Bailey}

cuneiformis Schütt. 621 .

gibba Bail. 152, 156, 292, 621, 855 .

* - $\quad$. africina 152 .

- $\quad$ v. atlantica 6:20, 623, 933, 974.

* - v. moronensis 37x, 379, 651 .

* - v. peliata 926-929.

* - v. subrhombica 926-929.

- $\quad$ v. ventricosa 621.

gigantea J. $\mathrm{Br} 22,23$.

inornata liast. $22,23,24$.

- v. curvirotunda 22, 23.

margaritacea J. Br. 621.

* minor M. Per. 251.

ventricosa Cast. 23, 24, 621.

\section{Enpodiscus Ehrenberg}

Argus WV. Sm. 34, 863, 889.

Barbadensis Grev. (Dép. des Barbades).

Calıfornicus $\nabla$. bioculata 110,111 .

Hardmanianus Grev.(Déo.tes Barbades).

inconspicuus Ratt 690.

Jonesıanus Grev. 47٪, 479.

minutus Grev. (Dépots des Barbades). oculatus Grev. 358- 60 .

punctulalus Grev. (Dép. des Barbades). parvulus Grev.

- - conceentrica radiatus Bail. $330,490,866,936$.

Rogersii-Eh. 1t5.

scaber Grev. (Dépóts des Barbades).

- p. heliodiscns J. Br. (Dépôts du Japon). simplex Grev. (Depóts des Barbades).

\section{Euphyllodium Schadbolt}

* spathulatum Schad. 551, 590,591,622, 623.

\section{Ethmodiscus Castracane}

carinatus Pant. 258, 259.

gigus Cast. 621.

Japonicus Cast. 794, 795.

rossicus Pant. 218, 219.

sellifer Pant. 248, 249.

vitrifacies Temp. et $\mathrm{Br} .22,23$.

\section{Fenestrella Greville}

Barbadensis Grev. (Dép. des Barbades). convexa J. Br. (Dépôts d'Oamaru). gloriosa J. Br. (Depôts du Japon).

\section{Fragilaria Lyngbye}

acutiuscula Pant. 852, 853.

æqualis Heib. $301,429,825$.

$$
\text { * - v. capitata } 537 \text {. } \quad \text { - mueotica } 852,853 \text {. }
$$

\section{Fragilaria Lyngbye}

æqualis $\mathbf{\nabla}$. producta $\mathbf{2 1 1}, \mathbf{2 1 2}, 301$. antarctica Cast. 342, 631.

v. moronensis 376,378 . Araulensis M. Per. el F. H. 740, 741 . bidens Heib. 465,466 . bigibua F. H. 308 .

binodis Eh. 133 137, 296, 321.

bituminosa Pant. 163. 502, 519, 825, 829.

$$
\begin{aligned}
& \text { - v. curla 304, 305. 463, } 464 . \\
& \text { - } \quad \text { v. elongala 204, 205. 463, } 464 . \\
& \text { - } \quad \text { v. minor } 305,463,502,515,529 . \\
& \text { - } \quad \text { } \quad \text {. perlonga 304, } 305 \text {. } \\
& \text { - } \quad \text { v. validior } 304,305,463,464 .
\end{aligned}
$$

brevistriata Grun. 32, 211, 212, 470, 516.

$$
\begin{array}{ll}
\text { - } & \text { v. capitata } 303 . \\
\text { - } & \text { v. fossilu } 305,464,502,515,529 . \\
\text { - } & \text { v. Laponicansis } 31,32,133-137.293,366,537,735 . \\
\text { - } \quad \text { v. pusilla } 52,53 . \\
\text { - } \quad \text { v. subacuta } 133-137,465,466,858 .
\end{array}
$$

capucina Desm. 57, 296, 470, 682, 909.

- $\quad$ v. acuta 85 .

- v. Ianceolata 418, 912.

- v. mesolepta $164,329,429,470,880$.

constricta $\nabla$. subcapitata 910.

construens Grun. $53,212,492,545.565$.

- v. binodis $34,85,472,507$.

- $\quad$ v. circulare 53.

- $\quad$ v. eximia 506.

- $\quad$ v. excisa 492, 494.

- $\quad$ v. pumila 492.

- v. minuta 85 .

- $\quad$ v. venter 53, 308, 494, 506.

crotonensis Kitt. 603, 642, 884.
- v. curta 782 .
- $\quad \nabla$. media $780,783,781$.

cylindrus Grun. 64.

elliptica Grun. 114, 321, 166, 545; 700.

- v. mincr 53.

endocystifera II. Per. et F. II. 546, 700, 741, 825.

Grunowii Pant. 800

Gustavei F. H. 52, 53, 738, 739 .

Harrissonii Grun. 106, 108, 161, 545, 687.

- v. major 546. 737.

intermedia Grun. 150. 321, 492, 516, 682. Ischaboensis Grun 773.

islandica Grun. 142.

Kochii Pant. 204, 205.

lancettula Schm. 107, 108.

Lapponica Grun. 764, 765. marina v. parva 107, 108.

microcephala Pant. 464.

minuta Pant. 305, 463, 464.

minutissima Grun. 739.

mutabilis Grun. 85, 212, 418, 565, 700.

$$
\begin{aligned}
& \text { - v. elliptica } 565 \text {. } \\
& \text { - } \quad \text { v. minutissima } 665 \text {. }
\end{aligned}
$$


Fragilaria Lyngbye.

nitida M. Per. et F. H. 545, 737, 829 . - v. curta 737.

northumbrica Grun. 222.

Oceanica Cl. 64, $\mathbf{8 1 8}$.

pacifica Grun. $31,32$.

- $\quad \nabla$. trigona 31,32 .

parasitica Grun. $164,3 \div 9,8: 9$.

pinnata Eh. 515, 529, 531 .

pliocena J. Br. 25.

v. acutifornie (Depôts du Japon).

producta Grun. 150.

rhombus Eh. 628.

sepulta Pant. 530, 531, 547.

* sinuata M Per. $365,366$.

striatula Lyng. 356,685 .

transylvanica Pant. 530, 531, 547, 825.

undata W. Sin. 831, 868, 90z, 939.

Ungeriana Grun, 20.

virescens Ralfs. 106, 296, 661, 652,941.

- $\quad$ v. elongata 69, 150.

- $\quad$. exigua 50, r00, 983.

Zeillei F. H. 52, 53, 53z, 735.

\section{Frustulia Bróbisson}

crassineria Bréb. 273, 496, 891, 899.

\section{Gephyria Arnott}

costata Arn. 882, 883.

constricta Arn. 291, 292, 359, 360 .

$$
\text { จ. costata 291, } 292 .
$$

incurvata v. Oamaruensis (Dép. d'Oamaru). gigantea Grev. 23, 24, 358-360.

- v. minor $23,24$.

media Arn. 46, 54, 156, 157, 371.

* - v. Ardissoniæformis 367-369.

- v. miocena (Dép. des Barbades).

Rimböckii (Pant.) Forti. 673-675.

\section{Glyphodesmis Greville}

eximia Grev. 268, 282, 505.

margaritacea Cast. 592, 593.

marginata Gr. et St. (Dép. d'Oamaru).

Murrayana Cast. 277.

Williamsonii W. Sm. 47.

\section{Glyphodiscus fireville}

bipunctalus A. S (Dép6ts d'Oamaru).

Grunowii v. lacunosa

Oamaruensis Grun.

scintillans A. S.

- $\quad$ v. stellatus $111, \overrightarrow{112}$.

strigillatus A. S. (Dépóts d'Oamaru).

\section{Gomphocymbella}

Beccarii Forti, 48, 481, 482, 483.

\section{Gomphoneis Cleve}

herculaneum CI. 365, 366, 627, 628 .

$$
\text { - } \quad \text { v. clavata } 265,366,62 \%, 628 \text {. }
$$

\section{Gonplioneis Cleve}

herculaneum $\nabla$. robusta 365,366 .

*

- $\quad$ v. rostrata :365, $\mathbf{3 6 6}$.

elegans Cl. $365,366,627,628$.

mamilla $\mathrm{Cl}$. 365, 366, 627, 628 .

scapha M. S 365.366.

sphærophorum Cl. 990.

subtile v. angusta 990 .

\section{Gomphonema Agardh}

abbreviatum lítz. 52, 53.

accessum F. H. 52,53 .

acuminatum Eh. $37,85,334, \mathbf{1 7 0}, 484$.

$$
\begin{array}{cc}
\text { - } & \text { v. clavus } 323,470,599,912 . \\
\text { - } & \text { v. coronala } \mathbf{2 6}, 321,615 . \\
\text { - } & \text { v. interueria } 168,374 \\
\text { - } & \text { v. laticeps } 321,470,599 . \\
\text { - } \quad \text { vusilla } 212,394,429,484.516 . \\
\hline
\end{array}
$$

affine Ktz. 38, 289, 309, 392.

- v. major $30^{\circ}, 37$.

angustatum Kız. 37, 197, 334, 470, 604 .

- $\quad \nabla$. angustissima 212, 614, 776 .

- $\quad$ v. elongata 104

- $\quad$ v. intermedia 38,618 .

- $\quad$ v. oblusatum 429, 785.

- v. producta $321,470,628,838,902$.

apicatum Cl. $334,429,776,881,904$.

arcticum Grun. 52, 53.

augur Eh. 36, 37. 159, 470, 905.

v. Gautieri 36, 37.

auritum J. Br. 168, 207, 274, 329, 429.

Berggrennii Cl 273, 922, 923.

Brazillense $v$. fossilis $320^{\circ}, 327$.

Brebıssonii Kız. 207, 249, 321. 604, 902. brevistriata M. Per. et $\mathrm{F}$. H. 51-53.

capitatum Eh. 57, 372, 329, 589, 677.

- v. curta 388,604 .

cantalicum J. Br. et F. H. 133, 404, 764, 765 .

- v. costalunga 133-137.

- v. lepida 133-137.

- $\quad$ v. major 133-137.

clavatum Eh. 604, 677, s1\%.

commutatum Girun. 44, 57.606, 618 .

constrictum Eh. 38, 274, 321, 471, ร70

- $\quad$ P. taputata 159, 334, 470, 516,905.

- $\quad$ r. elongala 429.

- $\quad$ r. subeapitatum $69,106,196,321,329$

curvirostrum Tump. el Br. 24, 25.

cygnus Eh. 207, 208, 249.

dicliotomum W. Sm. 78, 104, 321 .

Dubravicense Pant. (332, 633.

elongatum W. Sm. 36, 37 .

- v. minor 321 .

eriense Grun. 365,366 .

- v. acuminata 51-53. 


\section{Gomphonema Agardh}

eriense $\nabla$ rostrata 632,633 .

exigu!n $\mathrm{K} / 253, .127$.

exscissum F. H. 52, 53.

Gallandi M. Per F. H. 740, 741.

geminatum Ag. 186, 206, 606 .

globifprum Meisler 801.

gracile Eh. 273, 309, 372, 392.

- $\quad$. lata 41.

- $\quad$. major 639,640 .

Grovei A. S. 627,628 .

Heloridense Greg. 168, 249.

herculaneum Cil. 117, 103.

v. rohusta $107,277$.

insigne Grun. 191, 740, $741,825$.

- v. minor 53, 825.

intıicatum Kız. 37, 159, 374, 392, 679

$$
\begin{array}{ll}
\text { - } & \text { ₹. dichotoma } 740,741 . \\
\text { - } & \text { v. major } 159 . \\
\text { - } & \text { v. punila } 52,53,901 . \\
\text { - } & \text { v. stricla } 910 . \\
\text { - } & \text { v. tenella } 930 . \\
& \text { v. vibrio } 901,905 .
\end{array}
$$

Kinkerii Pant. 201, 205, 547.

lagenula Ktz. 596.

Lagerheimii Cl. 249.

lanceolatum Eh. 627, 628 .

manubrium M S. 887.

micropus $\mathrm{Itz} .52,53,106,321,666$

$$
\text { - } \quad \text { v. majur 52, 53. }
$$

molaris $\mathrm{Cl} .902$

montanum Schum. 159, 191, 604, 606, 680 .

$$
\text { - } \quad \text {. puimila } 113 \text {. }
$$

$$
\text { - V. rupciça } 334,394,429, \mathbf{5 6 0}, 881
$$

mustela Eh. $470,677,902$

- v. curvala 164, 356

obtusatum Ktz. 197, 691 .

occidentale M. S. 627,628 .

$$
\text { - v. abbreviata } 627,628 \text {. }
$$

olivaceum $\mathrm{Ktz}, \mathbf{1 0 0}, \mathbf{1 1}, \mathbf{8 9}$.

$$
\begin{aligned}
& \text { - v. fossilis } 305,463,464 . \\
& \text { - v. salinarum } 305,529 . \\
& \text { - v. staurophora } 305 \\
& \quad \text { v. vulgaris } 283,400 .
\end{aligned}
$$

Oregonicum Eh. 632, 63:3.

parvulum Ktz $350,372,516,666,787$.

- v. exilissima 902.

- $\quad$ v. subcapitala 507, 508, 516.

parvum F. H 738, 739.

pediculus v. major 388

Puiggarianum Grun. 365, 366.

sagitta A. S. $36,37,823,824,868$.

salinarum Pant. 529

saisa Pant. 529.

sarcopliaguș Grun. 70, 902.

semia pertum 305,366 .

จ. tergerstina 466 .

\section{Gomphonema Agardh}

sphærophorum Eh. 189.

subclavatum Grun. $53,191,483,520,880$.

subtile Eh. 334,901 .

- $\quad$. angusta 938 .

tenellum Ktz. 104, 249, 280, 776.

Transylvanica Pant. 530, 531, 547.

truncatum Eh. 913, 914.

turgidum Eh. 176, 17\%.

ventricosum Greg. 107, 108.

vibrio Eh. 168, 219, 392, 419, 531 .

\section{Goniothecium Ehrenterg}

crenatum Eh. $46,47,553,555$. decoratum J. Br. 90, 689, 690 . odontella Eh. y, 91, 110, 146, 298.

$$
\text { - } \quad \nabla \text {. danica 27, 28, 674, } 675 \text {. }
$$

prolongatum Grev. (Dép. des Barbad.).

Rogersii Eh. 147.

$$
\text { v. ventricosa }
$$

Szakalense Pant. 127-129.

tenue J. Br. (Dépôts du Japon).

vitripons J. Br. 90 .

\section{Grammatophora Ehrenberg}

adriatica Grun . 61-63, 285-287. africana Eh. 152, 153.

ambigua Grun. 333 .

angulosa Eh. 151, 371, 928, 929

- $\quad$. mediterranea $\mathbf{2 1 0}$

arcuata Eh. 634, 652.

al'ctica Cl. 432 .

arcus $J . B r .46,47$.

bibarensis Pant. 94, 95 .

caribca $\mathrm{Cl}$. 401, 402, 533 .

flexuosa $\nabla$. japonica 23, 24.

* fossilis H. P. 213,214.

Gallopagensis Grun. 532, 533.

gibba Cil. 18, 19.

gibberula $\mathrm{Ktz} .73,74$.

insignis Grun. 94, 95.

$$
\text { - v. doljensis } 442,413 \text {. }
$$

intermedia Grun. 198.

japonica Pant. (Dépots du Japon).

longissima P. P. 8:39.

lyrata Grun. 153, 160, 371.

- v. japonica (Dépóts du Japon).

macilenta El. 34, 269, 420, 499, 567.

$$
\text { v. subtilissima } 437 \text {. }
$$

marina Eh. 49, 398, 499, 563, 568.

- $\quad$. gibba 19.

- $\quad$ $\quad$. macilenta 398, 399.

- v. subundulata 62,63 .

- $\quad$. tropica 263, 859.

- v. undulata $62,63,153$. 


\section{Grammatophora Ehronberg}

maxima Grun. 61, 62, 389, 390652 . v. minor 169 .

monilifera Temp. et Br. 90.

$\nabla$. linearis ( $D$ epóts du Japon). moronensis lirev. $378,45 \mathrm{~s} .45 t, 60^{\circ} \mathrm{v}$.

- $\quad$ v. japonica (Dép. du Japon). nodulosa Grun. 138.

oceanica Eh. 46, 47, 94, 95, 600.

- $\quad$ v. communis 371.

- v. macilenta 671 .

- $\quad$ v. subtilissima 442, 443.

ovalariensis Grun. 268.

parallela Eh. 151-153.

robusta Dippel. 47, 110, 111, 291, 371 .

- $\quad \nabla$. gracilis 674,675 .

serpentina Eh. 34, 50, 269, 567, 847.

- - v. elongata 50 .

- v. minima 198,600 .

stricta Eh. 993-995.

- $\quad$ v. biharensis $318.319,390$.

- $\quad$. fossilis $349,442,926,929$.

subtilis Eh. 5?, 53.

subtilissima Eh. 19.

tabellaris J. Br. (Dépóts du Japon).

undulata Eh. 18, 19.

uncina Leud, $\mathbf{4 8 , 4 9 .}$

valida-Dipp. 842,843 .

\section{Grayia Grove et J. Brun}

argonauta Gr. et St. 554, 555.

\section{Grovea A: Schnidt}

pedalis Gr. et St. (Dépóts d'Oamaru).

\section{Grunowia Rabenhorst}

tabellaria Ratt. 52, 53 .

\section{Grunowiella Van Heurck}

geminata V. H. 147.

\section{Guinardia II. Peragallo}

flaccida H. P. 962,961,967,971,972.

\section{Gyrodiscus o. Witt}

hungaricus Pant. 237, 238.

vortex O. W. 260, 261.

- Handnanniana .1. Peragallo

* austriaca M. Per. 901.

\section{IIanteschia Grunow}

Abyssinica Grun. 480, 481.

amphioxys จ. major. $70,103,104$.

$$
\text { - } \quad \text {. minula } 507 .
$$

elongata Grun. 78, 605, 776, 860, 894 . marina Grun. 50, 58, 123, 598.

\section{Hantzschia Grunow}

* vivax v. granulata 103

virgata Roper. 58, 1\%0, 594, 843.

\section{II aynaldiella Pantocsel:}

antiqua Pant. 926-929.

\section{Hemiaulus Ehrenberg}

affinis Grun. 27-29, 147, 312.

alatus Grev. 620,621 .

altar J. Br. (Dépóts des Barbades).

ambiguus Jan. 469, 473, 554, 634.

amplectans Gr. et St. (Dep. d'Oamaru).

angustus Grev. v. major

applanatus J. Br.

Barbadensis Grun. 139-141.

biharensiv Pant. 318, 319.

bipons Grun. 650, 773.

capitatus Grev. (Dépôts des Barbades). caverna of. Br. 258-261.

claviger A. S. (Dépóts d'Oamaru).

crenatus Grev. (Dépóts des Barbades). danicus Grun. 27-29, 872.

diversus Grun. (Dépóts des Barbades). dubius Grev.

elegans Heib. 11, 12.

exiguns Grev. (Dépóts des Barbades).

februalus Heib. 111, 112, 310, 311.

Grunuwii Pant. 258-260.

hastutus Grev. (Dépóts des Barbades).

Hauckii Grun 915. 916. 917.

Hesbergi Cl. 525, 95t, 957, 982.

hostilis Heib. 27-29

hungaricus Pant. 128, 129, 237.

includens Grun. 12, 141 .

unicornutus J. Br. (Depóts d'Oamaru).

Kittonii Grun. 27-29, 872.

lobatus Grev. (Dép. des Barbades).

longicornis Grev. 709, 710.

lyriformis Grev. 712, 713.

$$
\text { v. rostrata } 715,716 \text {. }
$$

minutus Grev. (Dépóts des Barbades). mirus A. S. 27-29.

mucronatus Grev. (Dép. des Barbades). ornithocephalus Grev. 139, 140,509, 570. perlongus Pant. 258, 250.

petasiformis Pant. 12r, 129. 773.

pileolus v. harcata (Dép cles Barbades). podagrosus Grev.

polycistinorum th $469,620,621,712713$.

$$
\text { - v. meiolepta (Dép. des Barb.) }
$$

polymorphus Grun. $141,569,570,621,650$.

protens Heib. 311,312 .

pulvinatus Grev. (Dép. des Barbades). reticulatus Grev.

robustu: Grevं. 


\section{Hemiaulus Ehrenberg}

rostratus Pant. 405-407.

Sarutowiauns Pant. 258, 259.

simplex J. Br. (Dépóts des Barbades). subacutus Grun.

symmetricus Grun.

tenuicornis Gı・ $111,721,722$.

tubulosus J. Br. (Dep. des Barbades). verrucosus J. $\mathrm{Br}$ (Dépócs du Japon).

Weissflogii Pant. 258250.

Weissi Grun. 27-29, 369, 872.

\section{Hemidiscus Wallich}

cuneiformis Wall.442, 413.

\section{Heribaudia M. Peragallo}

ternaria M. Per. 293.

\section{Hercotheca Ehrenberg}

* armita H. P. 213-214. mamillaris Eh. 554, 555, 650.

\section{Ifeterodictyon Greville}

Rylandsianum Grev. (Dép. des Barb.). splendidum Grev. 703.

\section{Himautidiun Ehrenherg.}

Boryanum Pant. 519. pectinale Kız. 3 I3.

Soleirolel Ktz. 314.

\section{Homoeocladia Agardh}

sigmoidea W. Sm. 875 .

Widowichii Grun. $19,505,563,612,6$ z1.

\section{Iluttonia Grove et Sturt}

alternans Gr. et St. 141 . virgata Gr. et St. (Dép des Barbades).

\section{Hyalodiscus Ehrenberg}

ambiguus Grun. 432.

arcticus Grun. (Depots d'Oamaru).

* granulosus M. Per. 98-99.

læris En. 41, 49. 50, 242, 351.

— . Duljensis 44\%. 443, 800, 927.

- ४. Yarren: is 241, 242.

maculata Gl. 99. 100.

maxima P. P. $357,435,651$.

permaculata Gr. et St, $140,415,569,570$.

punctatus A. S. 2 .

radiatus Eb. 2, 33, 151, 154, 324.

- $\quad$ v. arctica $369,5 i 0$.

- $\quad$ v. bïllarensis $3 \circ 9,390$.

- $\quad$. maxima 415,416 .

- $\quad$ v. minor 251, 252, 369.

- $\quad \nabla$. radiata 3 .

reticulatus A. S. 548-550.

robustus Grev. $139,140$.

\section{Hyalodiscus Ehrenberg}

rossicus Pant. 11, 12 .

scoticus WV. Sm. 123, 269, 333, 443, 648 .

- v minor 333,357 .

stelliger Bail. 50, 113, $148,160, \mathbf{8 1 7}$.

subtilis Bail. 44, 210, 288, 333, 403.

- v. auntralienxis $270,272,389,390,403$. valens A. S. (Dépóts d'Oamaru).

\section{IIydrosera Wallish}

Boryana Pant. 94, 95, 447.

- v. hexagona $94,95,447$.

Mauritiana Bergon. 445-147.

triquetra Wall $146,147$.

- $\quad$. tetragona (Dépóts du Japon).

Wampoensis Schw. 552.

\section{Hyilrowilicon J. Brun}

mitra J. Br. 160, 794.796, 798.

rimosa $\mathrm{J}, \mathrm{Br} .445-447$.

\section{Isodiscus Rattray}

coronalis J. Br. (Depots d'Oamaru).

Debyi Ratt.

mirificus Ratt.

\section{Isthmia Agarih}

capensis Grun. 445, 53?, 622, 623, 863. enervis Ktz. 34, 5t, 185.

* gigantea M. Per. 151, 154, 156. Johusonii A. S. IDépots des Barbades). minima Bail $55 \mathrm{~L}$.

nervosa Ktz. 1 z0, 288, 322.

reticularis J. Br. (Dép. des Barbades).

* Squinabuli Forti. 367,422 .

* - v. crassior 673.

Szaboi Pant. 8, 126, $236,367$.

tenuicornis Grun. 140, 141.

\section{Janischia Grunow}

antiqua Grun. 27, 310.

\section{Keratophora Pantocsek}

nitida Pant. 260, 261.

robusta Pant. 258, 260.

\section{Kittonia Grove et Sturt}

elaborata Gr. et St. $139,140,415,416$.

Barboi J. Br. (Depots des Barbades). gigantea Grev.

Grevilleana Gr. et St. (Dep. d'Oamaru). Hillabyana J. Br. (Dep. des Barbades). viryata Gr. el st. (Dépóts d'Oamaru).

\section{Ktenodiscus Pantocsek}

hungaricus Pant. 236-238.

\section{Lamella J. Brun}

oculata J. Br. (Dépots d'Oamaru). 


\section{Lampriscus Grunow}

circulare Grun. 551 .

Debyi Gr. et St. (Dépóts d'Oamaru).

\section{Lauderia Cleve}

borealis Cl 39, 40, 958, 959, 962.

compressa H. Per. 5, 180, 229.

delicatula H. Por. 9zI.

glacialis Grun. $142,166,181$.

Schröderi Bergon. 962, 965.

\section{Lepidodiscns 0 . Witt}

elezans 0 . W. 12

imporialis J. Br.(Dépôts d'Oamaru).

\section{Leptocylindrus Cleve}

danicus Gl. 6, 10, 142, 430, 431.

\section{Lendugeria Tempère}

epithemioïdes Temp. 91, 300,324, 561, 562.

$$
\begin{aligned}
& \text { - } \quad \text { v. al'cuata 98-100, 324,325. } \\
& \text { * - v. obtusa 98-1170. } \\
& \text { * - } \quad \text { จ. subarcuata 673-675. }
\end{aligned}
$$

Janischii V. H. 478, 479.

$$
\text { * - } \quad \text { - v. brevis } 367,369 \text {. excavata } 213,214 \text {. }
$$

\section{Licmophora Agardh}

Dalmatica Ktz. 667

divergens Pant. 851-853.

Ehrenbergii $\mathrm{Ktz} 43,165,571$.

* - $\quad$ v. angustata 60,609 .

flabellata Ag. 80, 123, 361 .

gracilis Ag. 19, 875 .

Jurgensii Ag. 600 .

Lyugbyei Ktz 242.

paradoxa Ag. 80.

tincta Ag. $\mathbf{5 4 0}$.

\section{Liostephania Ehrenberg}

comta Eh, (Depots des Barbades). japonica J. Br. (Dépóts du Japon). magnifica Eh. (Depôts des Barbades). rotula $\mathrm{Eh}$.

\section{Liradiscus Greville}

Barbadensis Grev. (Depp. des Barbades). ellipticus Grev.

lucidus J. Br. (Dépots du Japon). minutus Grev. 147, 690, 1000. ovalis Grev. $1+1$.

\section{Lithodesmium Ehrenberg}

* biceps H. P. 213, 214. californicum Grun. 24, 25, 291, 292.

$$
\text { - v. tigrina 24, } 25 \text {. }
$$

\section{Lithodesmium Ehrenberg}

Ehrenbergii Grın 673-675

* - v. diceutrica quadragona 673-675. minusculus Grun 112, 292, 360. 173,8 813. undulatus En. 43, 281, 28:), 732, 789.

\section{Mastogloba Tluwites}

affinis C1. 26s, 334 .

aftirmata Cl. 268, 334.

alhifrons J. Br. (Dépóts du Japon). amiena v, turuida 268. amphibia Grun. 306.

Andrussowii Pant. 851-853.

$$
\text { จ. min } \cdot 851-853 .
$$

augulata Lewis $268,231,282,730,731$.

$$
\text { - } \quad \text { v. pusilia } 268 .
$$

antiqua Pant 40う-407.

apiculata W. Sin. $19,434,526,527,617$. asperula Girun. 26s

Bahamensis (il. 268.

baltica Cl. 834 .

bisuleata Grun. 268.

Braunii Grun. 175, 281, 282, 495.

- fa anomala 426-428.

capitata Greg. 504, 505.

Castracanei J. Br. $318,319$.

citrus $\mathrm{Cl} .61 \cdot 63,268$.

Glerei J. Br. 324, 325.

Corsicana Grun. $268,875$.

Craveni Leud. 2f $8,478,479$.

Dansei Eh. 243, 49., 516, 819, 910.

- $\nabla$. elliptica 981 .

Debyi Cl. 173-175

decussata Grun. 61-63.

Dolgensis Pant. 412, 443.

elongata Leud. 276, 277.

erythrea Grun. 18, 19, 268.

v. biocellata 268 .

euxina Cl. 268.

exigua Lewis 498.

floridea $\mathrm{Cl}, 330,331$.

Grerillei W. Sm. 249, 831, 835, 910.

Grovei Cl. (Dépóts des Barbades).

Grunnwii A. S. 160197.

Hillabiana J. Br. (Dep. des Barbades).

Howathiana Grun $522,623$.

indica $\mathrm{J} . \mathrm{Br} .54,55$

interrupta Hantz. 61-63.

Jelineckii v. marina 426-428.

Kinkerii Pant. 173-175.

lacustris Grun. 123, 419, 459, 461 .

lanceolata Thw. 18, 19, 501, 502, 834 .

lemnistica Leud. 148, 276, 277.

lineata Cil. 268.

Macdınałdii Grev. 268.

neogena Pant 173-175, 405-407.

obtusa Pant. 346-349.

ovata Grun. 19, 533, 875. 


\section{Mastogloia Thwaites}

pauduriformis Cl 98-100.

paradoxa Grun. 268.

Peragalli J $\mathrm{Br} 263$.

* -

Pethoi Pant. 40.5-407.

pisciculus Ci. 268, 330, 331 .

Portieriana Grun. 401, 402.

pusilla Grun. 210.

quinquecostata $54,398,446,634,839$. v. eling:ta 478,479 .

reticulata Grun. 73, 74, 130, 322.

rhomboidalis Pant. 173-175.

rhombus P. P. 268, 3:4, 3:5.

rimosa Cl. 268 .

rostellata Grun 268.

rutilans J. Br. (Dépots des Earbades). sagitta cupidinis $\mathrm{J} \mathrm{Br}$.

sinuata A. S. (Dépóts du Japon).

Smithii Thw. 206, 26's', 834.

$$
\begin{aligned}
& \text { - } \quad \text { v. abnormis } 268 . \\
& \text { - } \quad \text { v. amphicerbala } 50,243,498.834 . \\
& \text { - } \quad \text { v. lacusteris } \mathbf{2 1 3}, 268 . \\
& \text { - v. lanceolala } 208 .
\end{aligned}
$$

splendens $\mathrm{J}$. $\mathrm{Br}$. (Dép. des Barbades). squamosa CI. 54, 55.

Szontaghii Pant 95, 318, 319, 334, 756 .

Temperei Cl. (Dépóts du Japon).

de Toni J. Br'. 54, 55 .

tortilis $\mathrm{J} . \mathrm{Br} .54,55$.

undulata Grun. 53?, 533.

\section{Mastogonia Ehreuberg}

actinoptychus Eli $147.183,378$. crux Eh. $\% 3+, 237,298$.

\section{Melosira Agardu}

architecturalis J. Br. (Dép. d'Oamaru) arcuatí Pant. (Dépóts du Japon). a renaria 11 oore 114, 326. 630.

- $\quad$ v. hungariea 518, 519.

- v. laevis 190, 191, 301, 517.

bellicosa F. H. 307, 308, 326. :322 776.

biharensis Pant. 9, 128, 129, 272.

bituminosa Pant. ' $05,-463.464,632,633$.

- v. dilatala $305,348,4501,632$.

Borreri Grev, 19, 101, 132, 400, (\$1:3.

- v. hispida 434.

- v. ignimontana 30-32.

- v. minor 39, 198.

- v. oclogona 54, 55 .

Boulăyana MI. Yer. 32(;, 32z, 408, 409. Brunii MI. Per. et F. II. 293, 450, 481. Camusi F. H. 51-53, 880.

v. conica $51 \cdot 53,880$.

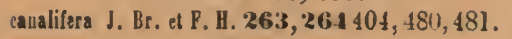

\section{Melosira Azardh}

eansliftra $\vee$. anastomosans 403 bis, $\mathbf{4 0 4}$. caput medusæ Pant. 91, 272.

cincta Pant. 1:8, 129, 644, 650.

clavigera Grun 141, $416,811,817$.

* - v. crassiradiuta $368,369,800$.

- - v. trinotata 363, 369.

clypeus J. Br. (Dépóts du Japon).

concentrica A. S. 27-29.

cornuta Temp et $\mathrm{Br} .25$.

coronarsa Mann, 647, 648 .

crenulata Kitz. $82,303,606,665,651$.

$$
\begin{aligned}
& \text { - } \quad \text { v. ambigua } 633,735,757,924 . \\
& \text { - } \quad \text { v. armata } 740,741 . \\
& \text { - } \quad \text { v. hungarica } 632,633,735,736,737 . \\
& \text { - } \quad \text { v. Jonensis } 605 \mathbf{8 5 5}, \\
& \text { - } \quad \text { v. procera } 604 . \\
& \text { - } \quad \text { v tenuis } 546,604,904 . \\
& \text { - } \quad \text { v. tenuissima } 546 . \\
& \text { - } \quad \text { v. strangulata } 931 . \\
& \text { v. valida y19. }
\end{aligned}
$$

curvatula Jan 3.

dactylus Eh. 937.

Davidsonii A S. 330, 331 .

decipiens Grun. 6 \$5.

Dickei Ktz. 463, 464 .

distans Ktz. 20, 212, 216.

- $\quad$. alpıgena 37, 113.

- v. nivalis $576,5 i 7,578$.

- v. scalaris $308,740,741$.

expectata A. S. 422-425.

fausta A. S. 145-147.

* Furlirensis Forti 1000.

fungiformis Pant. 174, 175.

granulata Eh. 122, 135, 257, 309, 661.

- v. arcuala 122.

- v. australiensis $53, \mathbf{3 0 1}, 737,801$.

- $\quad$ v. Buryana 519.

- v. curvata 301.

- $\quad$. hemicjelus 301.

- $\quad$ v. Jeremiæ 202.

- $\quad$. maeotica 855 .

Heribaudi J. Br. 30-32.

imperfecta F. H. 133 .

interjecta Jan $619,650,651,652$.

irregularis Pant. 26 $1,261$.

italica $\mathrm{K} \mathbf{z} .483,781,802,910,914$.

- v. tenuissima 483.

japonica Meister 80I.

Jurgensii Ag. 34.

Kochii Pant. 204, 205.

laevis Grun. 159, 172, 223, 546.

laevissima Grun. 494.

lineolata Grun. $307,308,326,327, \mathbf{3 3 2}$.

- v. robuita 263,20̀4.

Locyii Pant. $348,349$.

lyrata Grun: 621, 625, 854 . 


\section{Melosira gardh}

major Grove 140, 141, 416, 410, 570 .

Mauryana M. Per. et F. H. 734, 735. $\nabla$. granulata $\mathbf{3 6 2}, \boldsymbol{\gamma 6 3 3}$.

mediterranea Gıun. (Dépóts du Japon). minuta F. H. 53.

mirabilis J Br. (Dépôts du Japon). monililica Pant. 301, 3115.

nivalis W. Sm. 163, 164. 168, 90z.

nummuloides $43.123, \mathbf{1 8 8}, \mathbf{6 1 8}$

v. Elesdıana 318, 349, 390.

Nyassensis O. II. 483.

Oamaruensis Gr. tt \$1. $141,250,415,440,570$. Omına El. 238, 390, 650, 935.

orichalcea Meist. 163, 164, 355, 356.

ornata Grun. 145-147, 773.

* - v. reducta 997, 998.

Pensacolæ A. S. 330, 331 .

Peragalloi Pant. 128, 129.

polaris Grun. 47.

pontificalis J. Br. (Dépôts d'Oamaru). præclara A. S.

radiata var. minor 946.

recedens A. S. 27-29, 651,652 .

Rieufii F. H. 736, 757 .

Roeseana Ráb. 44, 113, 433, 581, 777.

$\begin{array}{ll}\text { - } & \text { v. dentroteres } 433 \text {. } \\ \text { - } \quad \text {. epidendron } 223 . \\ \text { - v. setosa } 433 \text {. }\end{array}$

* Sancta Crucis H. Per. 213, 214.

Saratoviana Pant. 260. 261.

sarmatica Pant. 2tio, 261.

saturnalis J. Br. (Dépôts d'Oamaru).

scala Pant. 518, 519.

scalaris Grun. 624, 625.

semilaevis Grun 901.

setosa Grun. 188.

Sol. Eh. 90, 288, 342, 477, 935.

* - $\nabla$. breviradiata 367-369.

solida Eul. 627, 628, 823, 824 .

- $\quad$. Haïtiensis 202.

sphærica F. H. 307, 308. 326, 327.

spinosa Pant 405-407.

spiralis $\mathrm{K} \mathrm{tz} .122$.

- v. hemisphæria 122.

- v. sphærica. 122.

strangulata M. Per et F. H. 738, 739. sulcata Ktz. 50, 27z, 773, 925, 950.

- $\quad$ coronata $160,282,835,936,950$.

- v. lurida J Br. (Dép. du Japon).

- r. minor 138, 133, 600

- v. radiala $50,102.835$.

- v. separanda. 213, 359, 360, 861 .

- v. strigillata 401, 402 .

subornata A. S. 422-427.

Temperei Pant. 204, 205.

tenuis Grun. 113, 327, 394, 537.

tenuissima Grun. 238, 409, 466, 729.

\section{Melosira Agarib}

teres J. Br. 478, 479.

Thumii Pant. 11, 12.

transylvanica Pant. 204, 205.

truncata Grove $140,141,416,440,570$.

undulata kítz. 20, 187, 191, 253.

- v. debilis $264.624,625,737,903$.

- v. Normannii 931.

- v. producla $169,264,624,737,739$.

Varennarum II. Per et P. H. 293, 366, 404, 765.

virıans Ag. 290, 450, 669, 720.

Westii W. Sm. 58. 269, 371, 839, 925.

- $\quad$. pustulata 58 .

\section{Meridion Agarlh}

circulare Ag. 13, 290 296, 602. 668

constrictum Ralfs. $13,216,303, \mathbf{4 2 9}, 922$,

* - $\quad \nabla$. elongata 104.

- v. Zinkerii 104, 728, 729.

\section{Molleria Cleve}

cornuta Grun. 167.

limbata V. H. 46, 47, 468, 469.

\section{Monopaia Grove et Sturt}

mammosa Gr. et St. (Dép. d'Oamaru).

\section{Microneis Cleve}

exilis Cl. 805, 807, 808.

microcephala $\mathrm{Cl}$. 788,805 .

minutissima $\mathrm{Cl}$. '785, 808.

\section{Navicula Bory}

abrupta Greg. 71, 96, 371, 622, 835.

- v. allantica 187.

- - $\quad$ - compressa 96-100.

- v. Iata 285-287.

- $\quad$ v. Rattrayi 281, 282, 339.

acephala M. Per et F. H. 262-264.

acrosphæria Bréb. 78, 22:3, 429, 639, 903.

* - $\quad \checkmark$ americana 881.

- $\quad$ v. Badeana 169, 734, 735.

- $\quad$. brevis 365,366 .

- $\quad$. elongata $26 \%-264,764,765$.

* - $\quad$ - dilatala $36,106,394,532,587$.

- $\quad$. leavis 108, 26:3, 355,356,776.

- $\quad$ r. minor 104, 163,172,903,909.

- v. parva. 107,108, 558-560.

acuta Ktz. 248, 249.

Adami Pant. 851, 853.

Adonis J. Br. ¿81, 282, 745-750.

- v. gibhosa 745-750.

advena A.'S. 61-03.

æstiva Douk. var. 281, 282.

æstuarii Cl. 189, 587, 639, 827, 988.

affinis $\mathrm{K} ı \mathrm{z} .78,248,372,733,870$.

- v. amphiryachus $115,106,374,738,834$.

- - maxima 36, 37, 105, 106. 


\section{Navicula Bory}

affirmata Leud. 276, $27 \%$.

alpina Ralfs 107, 108, 484, 611, 672.

- - $\quad$. elongata 107, 108.

a mbigua $\mathrm{Ktz} .58,35 \mathrm{l}, 481,589,880$.

v. craticula 70, $365,480,844$.

americana Eh. 159, 216, 777, 894, 945.

v. baeiltaru $159,216,374,586-588$.

* - v. minor 172, 321,334,558,588.

* - $\quad$ - moesta 159, 171, 172

ammophila Grun. 501, 502, 514, 515.

- $\quad$ r. degenerans $501,502,512,515$. $\nabla \cdot$ intermedia $501,502$.

amplibola Cl. 262-264, 302, 366, 5-19.

- v. Perrieri 536, 537, 699, 700.

- v. stanroneiformis 262-261, 366, 624, 736 .

amphigomphus Eh. $85,159,323,733,811$. v. major. 68,69 .

amphirynchus Eh. 78, 334, 605, 829, 938.

- v. curta 374.

v. minol 946 .

amphisbœna Bor'y 89, 123, 394, 4z0, 599. v. obtusa 254,255 .

ampliata Eh. 216, 2i8, 321, 497, 705. v. minor 149,150 .

andesitica Pant. 94, 95.

Andrussowii Pant. 851-853.

angelorum Cl. 213, 358, 435, 844, 845 .

anglica Rab. 108, 461, 579, 643, 751.

v. subsalsa 36,37 .

angustata WV. Sm. 248, 249.

anthracis $\mathrm{J} . \mathrm{Br}$. 745-750.

* antinitescens M. Per. 107, 10 S.

Apis Donk 91, 339, 438, 754, 856 .

aponina Ktz. 226-228, 465, 466, 885.

appendiculata $\mathrm{Ktz} .113,176,356,678,885$,

v. irrorata Kítz. 113, 500.

approximata Grev.189, 281, 282, 926-929. v. stauroneiformil 622,623

Aquitaniæ J. Br. 30-32, 740, 741.

- $\quad \nabla$. undulata 30,32 .

arábica Grun. 189, 561, 562, 592, 7.16.

aradina Pant. 346-319.

arata Grun. 518, 519, ५32, 633.

arctissima A S. 840 .

arenaria Donk. 50, 501, 613, 760, 762.

arenlcola Grun. 304, 305, 514, 515.

arverna M. Per. 293, 365, 366.

- $\quad$. stauroneiformis $149,150$.

aspera Eh. 80, 332, 403, 462, 566, 839.

- v. liungarica 236-238, 346-349.

- $\quad$ v. intermedia 276, 277.

- v. minor 896.

- $\quad$. pulchella 71, 72 .

atlantica A. S. 50, 71, 72 .

atomoides Grun. 350, 494, 727, 940 .

atomus Girun. 113, 507.

- Attwoodii Mr. Per. 187, 189.

\section{Navicula Bory}

avenacea Bréb. 470, 500, 618, 619 .

baccala J. Br. 745-750.

baciltaris Greg. 457, 458.

- v. inconstantissima $627,628,727$.

bacillifera Pant. $346-349,756$.

bacilli formis Grun. 159,'216, 429, 757, 902 .

bacillum Eh. 31, 323. 604, 854, 938.

- v. major $307,308$.

- v. minor 51-53,149, 150 .

Baileyana A. S. 50, 120, 370, 617, 985. Barbitos A. S. 775 .

basaltæproxima J. Br. 30-32.

v. longistriata $30-32$.

basilica Pant, 203-205, 530, 745-750.

- Bastianii M. Per, 310. 311.

Baumberii Pant. 23b-238, 316-349.

Beckii Pant. 301, 305, 463, 464.

Berriati F. H. 51-53, 262-264, 736.

- $\quad$. minor 26:-264, 537, 737, 739.

- v. splendida 736, 757.

Beyrichiana A. S. 50, 130, 157, 322, 566 .

bicapitata Lag. 321, 57i, 589, 85t, 904.

- $\quad$ v. hybrida $78,321,570,885$.

- v. sulcata 262-264.

biceps Greg. 216, 419, 497, 757, 887.

- $\quad$. stauroneiformis 733 .

biconstricta Gr. et St. 810-822.

bicuneata Grun. 61-63.

bimaculata Pant. 94, 95, 318, 319, 329.

binodis W. Sm. 583, 584 .

bipunctata Grun. 216 .

bisulcata Lag. 216, 373, 733, 834, .01.

- v. inajor 68, 69.

bituminosa Pant. 301, 305, 501, 528, 529.

- v. Bodosensis 530, 531 .

- $\quad$ v. latecapitata 304,305 .

- $\quad$ v. robusta 528, 529.

- v. signata 301,305 .

- v. staurophora $304,305$.

bivittata Pant. 304, 305.

blanda A. S. 269, 617.

Bleischiana Jan. 73-75, 622, 623.

$$
\text { - var. } 238 .
$$

Bodosensis Pant. 203-205, 530, 531.

Bogotensis Grun. 105, 106, 216, 886.

- - v. interrupla 103, 104.

* - v. undulata 103, 104.

bohemica Eh. 21, 117, 365, 740, 825.

bomboides A. S. 30-32, 151, 462, 846 .

$$
\begin{aligned}
& \text { - v. limanense } 30-32 \text {. } \\
& \text { - v. media } 30-32,745-750 \text {. } \\
& \text { v. minor } 30-32 \text {. }
\end{aligned}
$$

bombus El. 50, 130, 269, 462, 89.

- $\quad \nabla$. densestriata 80, 251, 252,268.

- $\quad$ r. egena 101. 152, 153.

- $\quad$ r.gemina 102, 251, 282, 836, 837

borealis Ktz. 216, 279, 500, 578, 882. 


\section{Navicula Bory}

borealis $\nabla$. fossilis 203-205.

$$
\text { - v. major } 76,77 \text {. }
$$

Boryana Pant. 94, 95, 318, 405, 756. Bottnica Grun. 138.

Bouhardi F. H. 408, 409.

Brasiliensis Grun. 187, 393, 517, 643, 864.

Braunii Grun. 371, 394, 728, 729, 981.

- v. interrupta 78.

- v. Moissacensis 262-264, 734, 735 .

Brebissonii Ktz. 70, 215, 372, 602, 825.

$$
\begin{aligned}
& \text { - } \quad \text { v. cominutata } 611 . \\
& \text { - } \quad \text { v. curta } 829,902 \text {. } \\
& \text { - } \quad \text { v. diminuta } 558-560,755,879 . \\
& \text { - } \quad \text { v. elongata } 168 \text {. } \\
& \text { - } \quad \text { v. minor } 602 .
\end{aligned}
$$

brevicostata Eh. 105, 216, 429, 905, 907. $\nabla$. interrupta 223,904 .

brevis Greg, 121, 187, 269, 370, 427.

- $\quad \nabla$. elliptica 117-119.

brevistriata Grun. 854 .

Lrunii Cl. 324, 325, 426-428.

Budayana Pant. 203-205, 530, 531, 547. bullata Norm. 96-100, 592, 775, 929.

- v. Mölleriana 561, 562, 775 .

Californica Grev. 96-100, 438, 839.

- v. Campechiana 403, 745-750, 798.

Campbellii M. Per. 187, 188 .

campylodiscus Grun. 268, 276, 277.

cancellata Donk 58, 160, 236, $426,974$.

- $\quad$ v. apiculata 199.

- $\quad$ v. Gregoryi 187, 251-253.

- $\quad$ v. minor 59, 60 .

- v. retusa 401, 402 .

cardinalicus $\mathrm{Cl} .216,394,587,728,903$. cardinalis $\mathrm{Ktz} .76, \mathbf{1 6} 2,248,371,695$. cari Eh. 216.

- $\nabla$. angusta 103-104.

caribaea CI. 148, 442, 918, 950 .

earinata A. S. 148.

carinifera A. S. $73-75,322,566$.

Carphatorum Pant. 632, 633.

Cellensis M. Per. 122.

Cesatii Rab. 176, $17 \%$.

Chaberti F. H. 262-264, 356, 762, 763.

Chersonensis Grun. 73.75, 276-277. $\nabla$. aniformis 160.

cincla Eh. 113, 495, 727, $\mathbf{9 5 3}, 801$.

- $\quad$. Heufleri 495, 755, 829, @02, 907.

circumnodosa J. Br. 745-750.

circumsecta Grun. 189, 696-698, 926-929

cistella Grev. $2 \times 5 \cdot 28 \%$.

clavata Greg. 34, 370, 468, 662, 974 .

- $\quad$. caribaea 285-287, 993-995.

- $\quad$ v. elliptica $130,948.949$.

\section{Navicula Bory}

* clavata $\nabla$. ellongata 50, 322. 561, 839, 934.

- - v. H. 798 .

- v. impressa 426.428.

* - v. subacuta $213,214$.

- v. Wrightii 281, 282, 932.

claviculus Greg. 101, 346-3ł9.

Clementis Grun. 518, 519.

coarcta A. S. 61-63.

coarctata Eh. 96-100, 442, 443

cocconeiformis Greg. 248-249.

coffeaeform is A. S. 268.

collumnaris, Eh. $36,37,373,394,728$.

commutata Grun. 159, 334, 601, 835, 931.

- stauroneiformis 857,858 .

complanata Eh. 685.

compressicauda A. S. 745-750.

confæderata Pant. 182, 183.

confervacea Grun. 289.

Consors A. S. $61-63,532,533$.

conspersa Eh. 203-206.

conspicua A. S. 171, 172, 373, 939.

constricta v, minuta 685 .

copiosa A. S. 219-221.

Corbieri F. H. 5l-53, 734, 735.

costata Eh. 207, 302, 303, 334, 374.

Costei F. H. 51, 53, 736, 737.

- v. bacillaris 51.53 .

crassinervia Bréb. 114, 168, 866, 8 z0.

Craveni Leud. 148.

Creguti F. H. 162-164, 207, 355, 356. cruciata Leud. 148, 2\%6, 277, 330, 331. crucicula Donk. 88.

crabro Eh. $91,160,438,761,865$.

- v. intermedia 532, 533.

- . japonica 22-25, 745-750.

- $\nabla$. limitanea 80.

- 7. minor 123, 251-253.

- v. minuta 926-929.

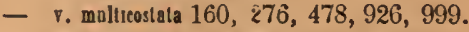

- v. pandura 96-100, 926-929, 993.

- $\nabla$. separabilis 478, 479, 926, 993.

crucifera Grun. $426,428$.

crucifix Temp. et Br. 745-750.

cruciformis Donk. 269, 613, 614, 639, 640.

crucitina M. Per. 213, 214.

cryptocephala Ktz. 89, 280,602, 770, 806.

- viexilis $89,196,222,500,602$.

cubitus Temp. et $\mathrm{Br}$. 745-750.

cuneoquadrata $\mathrm{J}$. Br. 701-706.

curtestriata Pant.203-205, 258, 304, 305.

cuspidata $\mathrm{K} t z .98,573,831,854,9)$.

* - l. eraticula 216,30:, 75\%, 8:29, 945 .

- v. gracilis 605 .

- v. Heribaudi 262-261, 278, 279.

- v. primogena 911, 912. 


\section{Navicula Bory}

cymbula Donk. 70.

cyprinus Kı. 101, 269, 397, 43t, 896.

dactylus Kitz. 14, 216, 340,518 834 .

v. curla 105, 106.

dalmatica Grun. 80 , 285-287.

Dariana A. S. 187, 216, 395, 884, 903.

debilis Pant. 463, 464, 528, 529.

Debyi Pant. v. elliptica 367-369.

decora Gr. et St. (Dépóts d'Oamaru). decrescens F. H. 262-26t.

decumana Pant. 203-205.

decurrens Eh. 103, 104, 388, 728, 733.

- v. curtecostata 51-53, 262-261. definita Gr. et St. (Dépôts d'Oamaru). Delawarensis Grum.189, 826, 827.

demta A. S. 268.

v. robusta $187,188,189$.

depressa Cl. $361,362$.

dicephala Eh. 162, 216, 321, 751, 902.

- $\quad$ v. intermedia 776 .

* - v. lata 103, 104.

- v. minor 162-164, 321 .

- $\quad$ r. slauroneiformis $248,249,939$.

didyma Eh. 50, 269, 375. 816, 925. v. Iusitanica 50 .

difficilis Pant. 173.175 756.

digitoradiata $50,189, \mathbf{3 7 0}, \mathbf{3 9 7}$.

digrediens A. S. $346-319,357$.

dilatata Eh. 86, 394, $870,915,991$.

diplosticta Grun. 148, 236-238, 276-277.

directa W. Sm. 268, 370, 397, 422, 443.

- v. cuneata 316 .

dispersa Gr. et St. (Dépóts d'Oamaru). distans W. Sm. 182, 594, 977, 979, 980.

divergens IV. Sm. 168, 223, 484, 695, 885 .

* - v. bacillaris 105, 106, 107, 108.

* $\quad$ - $\quad$. constricta 105, 106.

- $\quad$ v. elliptiea $583,584,776,835,885$.

- $\quad$ v. minor 499, 776, 902, 941 .

- v. Mullensis 248, 249.

- v. prolongata 168 .

- $\quad$ v. undulata 226-228, 465, 466.

- v. undulatocuneata 262-264.

Döcyi Print. 346-349.

Donkinii A. S. 50.

Doljensis Pant. 412, 443.

dubia Eh. 78, 215, 678, 834, 931.

* - v. acuminata 586-588.

dubitata M. Per. 5l-53.

Dubravicensis Grun.518, 519, 632,633.

Dusenii Cl. 251-253.

$$
\text { v. gallica } 740,741 \text {. }
$$

Egeria Pant. 346-349.

elegans WV. Sin. 34, 187 189, 240, 984.

- v. cuspidata 365-366, 981.

- elegantissima M. Per. 103, 104, 216, 729, 776.

Elesdiana Pant. 346-349, 389, 390.

\section{Navicula Bory}

elliptica Ktz. 82, 216, 334, 500, 910.

- $\quad$ v. grandis $218,519,9200^{\circ}-929$.

- $\quad$ v. maeotica 851.853.

- v. major 162-161.

- v. minor $632,633,838$.

- $\quad$. minutissima $164,216,222,409,481$.

- v. oblongella $133-137,240,450,465$.

elongata Greg. 503, 505.

elongatula Pant. 304, 305.

Elsae Thum. et Pant. 480, 481

cntomon Eh. 145-147, 213, 214, 846.

episcopalis Eh. 624, 525, 885.

Erythrea Grun. 187, 324, 325, 946. esoculus Schum. 883.

esox Eh. 51-53, 374, 395, 586, 700.

- v. recta 558-560, 762, 763 .

* Essexii M. Per. 906, 907.

Eudoxia A. S. 50, 109-112, 926-929. v. elongata 50.

excavata Greg. 109-112, 236-238, 438.

- v. angelorum 745-750.

excentrica Grun, 503-505.

exemta A. S. $80,566,926-929$,

exigua Greg. 604, 851-853.

exilis Grun. 168, 222.

eximia Cl. 373.

expedita A. S. 376-378.

expleta A. S. 96-100.

exul A. S. 926-929.

Falaisiensis Grun. 20, 22.

fasciata Lag. 103, 104, 105, 106.

Fenylii Grun. 877, 878.

filiformis Pant. 173-175.

Finnica Cl. 211, 212.

firma Ktz. 323, 470, 606, 777, 938.

- v. ampliata 991.

- $\nabla$. diminuta 901 .

- v. subampliata $70,470,491,729,857$.

- v. subundulata $81,372,776,901$.

- v. tumescens 939.

Fischeri A. S. 240, 339, 816, 979, 989.

* - - $\quad$. gigas 189.

* - - major 701-706.

flammula A. S. 159. 171. 172, 884.

flamatica Grun. 36, 37.

Flattei Pant. 756.

flexuosa Eh. 374, 394, 903.

* - v. cuneata 302,303 .

fluitans J. Br. $145-417$.

- v. biconvexa 701-726.

foliola Temp. et $\mathrm{Br}$. 745-750.

follis Eh. 352, 374, 458, 834, 931.

fonticola Grun. 306.

forcipata Greg. 185, 188, 639, 731,974.

fortis Greg. 187, 370, 760.

Fromentari Cil. 370 var. 


\section{Navicula Bory}

fusca Ralfs 50, 148, 187, 188, 189.

— $\quad$. delicata 50, 926, 929.

- v. major 151, 346-349.

- v. Norvegica $925,929$.

- v. permagna 94, 95.

Fuschii Pant. 94, 95, 318, 346, 349. fusiformis Cil. 17 var.

gastrum Eh. 206, 517, 604, 700, 945.

- v. elliptica 293.

* - v. maxima $365,366,544,545$.

- $\quad$. minor 639,610 .

- v. Boryana 516-519.

- $\quad$ v. major 577.

gelida $\nabla$. perpusilla 64 .

gemina Eh. 839, 875.

geminata Grev. 810-82?.

v. fossilis 9?6-929, 993-995.

gemmata Grev. 152, 153, 236-238, 566.

- v. fossilis 8,9 .

- v. pristiophora 422-425.

- v. spectabilis 73-75, 151-153, 156. gemmatula A.S. 213, 324, 393, 875.

Gendrei F. Il. et II Per. 51, 53, 601, 834,931.

- $\quad v$. intermedia $517,544,545$.

gentilis Donk. 13, 81, 672, 870, 915.

- $\quad$. hyalina 558-560.

gibba Eh. 13, 223, 374, 583, 584.

- v. hyalina 13, 162-164, 171, 172.

- v. major. 314, 345.

- v. stauroneiformis 903.

gibberula Ktz. 480,481 .

gibbula v. cantalica 546 .

gigas Eh. 351, 352, 373, 450, 589.

- v. gracilis 149,150 .

globiceps Greg. 76, 77.

gloriosa J. Br. 219-22l.

Gomontiana F. H. 122.

Gorganowicii Pant. 339, 442, 443.

Gothlandica Grun. 829, 901.

gracilis Ktz. 89, 113, 470, 667, 785.

- v. neglecta $307,308,770$.

gracillima Greg. 59, 163, 321, 582, 866 .

- v. stauroneiformis 583,584 .

Graeffii Grun. 393.

granulata Bréb. 91, 427, 760, 976, 984.

$\nabla$. constricta $926-929$.

grata Pant. 463, 464 .

gregaria Donk. 166, 396, 571.

Grevillei A.S. 984.

* Griffithii M. Per. 370.

Grundleri A.S. 189, 526, 527, 810-822.

Grunowii Ralfs. 45-47, 157, 745-750.

Guinardiana J. Br. 745-750.

Gurowii Pant, 203-205.

guttata J. Br. 340, 341, 728, 729.

Gutvinskii Pant. 530-531, 547.

Haïtiana T. et W. 201, 20\%.

\section{Navicula Bory}

halionata Pant. 94, 389, 501, 528, 529.

- $\quad$ v. directa 463, 464 .

- v. minor. $316,319$.

halophila Grun. 326, 327, 576, 762, 931 . v. craticula $583,584,882$.

v. major $307,308,762,763$.

Hantkenịi Pant. 346-349.

Haradie Pant. 745-750.

hasta Pant. 203-205, 547, 801.

Haveri Grun. 518, 519, 632, 633.

Haynaldii Pant. 91, 95.

Hayslinskyi Pant. 528, 529.

hebes Ralfs. 206, 459, $\mathbf{7 5 8 , 8 6 8 , ~ 8 9 1 , ~} 900$.

Heerii Pant. 304, 305, 501, 502.

hemiptera lítz. 113, 216, 394, 835, 885 . จ. Bielawski 162-164.

Hennedyi W. Sm. 43, 91, 617, 761, 846.

* - v. africana 1 - 1 .

* - v. brachylopleura 367-369, 800 .

- $\quad$ v. calilornica 45-47, 117-119, 213.

- $\quad$ v. coloradica 213, 214.

- $\quad$ v. crassa 926-929.

- $\quad$ r. cuueata $51,155,324,325,993-995$.

- v. fossilis $94,95,318,319,988$.

- $\quad$ v. furcata $187,974,985,988,989$.

- ve granulala $50,73,152,371$.

- v. manca 403,462, 950, 993-995.

- $\quad$ v. minuta 50 .

- V. neapolitaua 153, 155, 367-369, 993.

- v. nebulusa 617 .

- $\quad$ v. Niceaensis 58,73-75,462, 839.

- $\quad$ v. rostrata 370 .

- v. subrostrata 452-456.

Heribaudii M. Per. 203-205, 293, 762, 763.

* - v. elongata 931 .

heroïna A.S. 36, 103, 172, 881, 883.

heteroflexa Pant. 304,463, 464, 501, 502.

- v. constricta 463, 464.

- v. minor 463,464 .

Heufleuriana Grun. 113, 133, 137, 363.

Hevensis Pant. 304, 305.

hilarula Pant. 203-205, 517.

Hilseana Jan. 419, 728, 729.

Hitchcockii Eh.105, 106, 3 รม, 605, 729.

Hochstetteri Grun. 401, 402, 617, 648, 650 .

Hoffmannii Pant. 346-349.

Holstii Cl. 222.

hordeiformis Pant. 514, 515.

Huei $\nabla$ lata 776.

humerosa Bréb. 58,91, 189, 617, 974.

- $\quad$ v. constricta 836,837 .

- $\quad$ v. elongata $94,95,236-238,347$.

humilis Donk. 107,108, 231, 232, 470.

hungarica Grun. 306 var.

- v. capitata. 801.

hyalina Donk. 984.

จ. fossilis. $851-853$. 


\section{Navlcula Bory}

hyalosira Cl. 597.

hybrida M. Per. 149, 150, 162-164.

Hyrthii Pant. 530, 531, 517.

icostauron Grun. 187, 189, 230, 639-640.

ignobilis Pant. 346,349 .

imperfecta Cl. var. 6I-o3.

impressa Lag. 61-63.

includens Pant. 756.

incomperta $\mathrm{r}$. fossilis $851-853$.

inculta Pant. 203-205, 547.

incurvata Greg. 109-113, 189.

incus Grun. 73-75, 839.

- indefinita M. Per. 96-100.

index Temp. et $\mathrm{Br} .745,750$.

indica Grev. 148, 478, 479.

inelegans Gr. et St. (Dépots d'Oamaru).

inflata Greg. 211, 212.

- $\quad \nabla$. biharensis 346-349.

inhalata A. S. 236- $238,346-319$.

- v. biharensis 346-349.

insignis Pant. 463, 464.

instabilis A.S. $159,171,311,776,905$.

intacla Pant 851-853.

integra Grun. 586-588, 903-905.

interlineata Gr. et St. (Dép. d'Oamaru). interposita Lewis 463, 464, 501, 502.

interrupta $\mathrm{Ktz}$. 187, 188, 189, 354, 950 .

- $\quad$ v. lossilis $304,305,463,501,502$.

- $\quad$ v. stauroncilormis $216,365,729,825$.

Talyana 501, 502, 888 .

iridis Eh. 589, 831, 885, 906, 939.

- $\quad$. ampliata $159,373,516,825,883$.

- v. angustata 162-164.

- r. maxima 216.

irregularis Pant. 173-175.

irrorata Grun. 187, 189, 918, 974, 985.

- v. fossilis $318,319,316-349$.

isostanron Pant. 558-560.

jejuna A.S. 426-128, 791-797.

- Jelineckii v. fossilis 926-929.

Josephi Pant. 851-853.

Jucanda Pant. 851-853.

Kamortheosis Gruo. 393, 637, \$35, 841, 875.

Kefvigensis Eh. 334 var.

Kerguelensis Cast. 865.

Kertschiana Pant. 851-8:3.

Kinkerii Pant. 236-238.

Kittoniana A. S. 154, 330, 331.

Kochii Pant. 463, 464.

Koller Pant. 756.

Kossuthii Pant. 236-238.

Kützingii Grun. 80.

lacrimans A. S. 236, 238.

lacunarum Grun. 223, 580 .

lacustris Grev. 61-63, 206.

laevissima Grun. 113, 321, 387, 388.

Lagerheimii Gl. 579.

\section{Navicula Bory}

Lambertensis F. H. 326, 327.

lanceolata Kı. 59, 32!, 598, 829, 902. lancettula Schum. 770.

- Lapegrereana Temp. el Y. Per. 151, 152, 153, 156.

lata Bréb. 558-560, 685, 851, 873.

- v. minor 387,388 .

latevittata Cl. 36, 38, 339 579, 906.

v. Domingensis $581,903,936$.

latissima Greg. 50, 94, 390, 442, 974.

- $\quad$ v. capitata 94, 95.

- Kamorthensis 442, 443.

lauta Grun. 241-243, 947.

Legendrei F. H. et M. Per. 374.

legumen Eh. 159, 323, 395, 606, 885.

- $\quad$ v. major 903.

- v. undulata $408,409,664$.

- v. vis undulata $76,77,387,388$.

Leonis Pant. 851-853.

lepida Greg. 321.

leptogongyla Grun. 583, 584, ә27.

Letourneurii Pant. 94, 95.

Lewisiana Grev. 145-147.

libellus Greg. 745-750.

liber W. Sm. 34, 80, 322, 617, 933.

- $\quad$. bicuneala 80 .

- v. elongata 617 .

- . linearis 50, 130.

- v. maxima 18, 19.

- v. robusta 993-995.

- - v. tenuistriata 50.

- v. umbilicata 590, 591.

librile Eh. 913, 914.

liburnica Grun. 33, 43, 370, 974, 988.

limosa K'tz. 82, 293, 470, 835, 934.

- v. alpina 854 .

- - $\quad$. dilatata 176, 177.

- . gibbernla 122,307,466,470,599.

- v. major. $68,69,162-164$.

- v. signata, 902 .

- v.subinflata $168,216$.

- v. subundulata $877,878,902,931$.

- v. undulata 77, 207, 208, 216, 470 .

linearis Greg. 105, 106.

lineata Donk. 50, $318,319,875,883$.

- $\quad \nabla$. contracta 120.

lineolata Eh. 168, 733.

littoralis Donk. 18, 19, 58, 760, 875.

lobata Gr. et St. 819,822 .

Loczyi Pant. 340-349.

longa Greg. 61, 117, 322, 462, 504.

lucida Pant. 530, 531.

Ludlowiana A.S. 107, 108, 630, 631 .

luxuriosa Grev. 426-428.

- - v. abrupta 120.

Lyra Eh. 300, 434, 616, 775, 985.

- . abnormis 616 .

- v. acuta 236-238. 


\section{Navicula Bory}

Lyra v. atlantica $160,189,339,617,816$.

- v. - fa elliptica 213, 214.

* - v. bacilliformis 281,282 .

- v. carinata 974 .

- v. connectens $339,371,389,390,756$.

- v. constricta 71, 72, 988 .

- v. cuneata 974, 988.

- v. densestriata 120

- v. dilatata $73,75,120,187,522$.

- v. elliptica 50, 96, 130, 551, 795 .

- v. hungarica 236-238, 339, 756.

- v. insignis 189, 324,325.

- v. intermedia $71,367,617,835,945$.

- _ _ fa ambigua 15t, 155.

* - $\quad$ - $\mathrm{f}^{2}$ dilatata 617 .

- - $\quad$ fa elliptica 835, 987.

- $\quad$ - $\mathrm{f}^{\mathrm{a}}$ recta $622,730,835$.

- $\nabla$. insignis $730,731,974,985$.

* - v. lata 213, 214.

- v. producta 236-238.

* - v. pusilla 213,214 .

- v. recta $73,152,300,403,617$.

- $\quad$ - fa elliptica 621-623.

- v. subearinata 73, 478, 592, 839, 985.

- subelliptica $154,155,339$.

- subtypica 426, 428, 987.

macilenta Eh. 211, 323, 394, 733, 936. macra Grun. 150, 162-161, 906, 907. maculata Bail. 367-369.

v. major 970 .

Madagascarensis Cl. 96-100.

maeotica Pant. 851-853.

major Ktz. 84, 215, 414, 589, 885 .

- $\quad$. asymetrica $216,340,374,586$.

- v, convergentissima 51, 734, 735, 736.

* - v. dilatata 36,37 .

- v. horrida 203.

- $\quad$. interrupta 76,77 .

- v. maxima 35,37 .

- v. permagna 696-698.

- v. subacuta 373 .

Macraeuna Pant. 304, 305, 463, 464.

Malin vaudi F. H. 408, 109.

mantichora Pant. 236, 238.

margaritifera T. et W. 201, 202, 236.238, 756. marginolineata Gr.et St. (Dép. d'Oamaru). marginopunetata Gr. et St. (Dep. d'Oamaru). marina Ralfs. 18, 71, 434, 526, 948.

$$
\nabla \text {. producta } 50 \text {. }
$$

Martoufli Pant. 173, 175, 339.

Mauleri J. Br. 203-205, 530, 531, 547.

maxima Greg. 73, 193, 331, 617, 839.

- $\quad$ v. isiatica 745-750.

- v. bicuneata $43,590,591,617$.

- v. excentrica $160,401,402,551$.

- $\quad$ . umbilicata $488,489$.

mediopartita Grore (Depots d'Oamaru).

\section{Navicula Bory}

mediterranea Cl. et Br. 120, 121, 926-928.

* - $\quad \nabla$. fossilis 926,929 .

megaloptera Eh. 107, 108, 207, 208.

melinitica Pant. 304, 305, 463, 464,

menisculus Setum. $13:-137, \mathbf{4 7 0}, 500,881$.

meniscus Schum. 211, 212.

mesogongyla $\mathrm{v}$. interrupla 78 .

mesolepta Eh. 219, 321, 497, 605, 887.

- v. Alberti 307, 308.

- $\quad$. Saintignyi $149,150$.

- v. stauroneifornis $321,374,602,867$.

mesostyla Eh. 78, 216, 374, 776, 854.

microcephala Grun. 901.

microrbynehus Grun. 463, 464, 528, 529, 632.

microstatus Pant. 91, 126-129.

microstauron Eh. 374, 696, 608, 818, 981 .

$$
\text { - v. stauroneiformis } 365,366 \text {. }
$$

Mikado Pant. 745-750.

minima Grun. 125.

- v. atomoides 785 .

minor Greg. 760.

minuscula Grun. 465, 166.

moesta A. S. 339.

molaris Grun. 854,855 .

monodon J. Br. 745-750.

mucronata Pant $851-853$.

multicostata Grun. 54, 80, 300, 566, 937.

muralis Grun. 211, 212.

mnsca Greg. 213, 214, 926-929.

muscæform is Pant. 405, 407.

$$
\text { v. constricla } 80 \text {. }
$$

inutabilis A. S. 777.

mutica Ktz. 309, 353, 728, 729.

- $\quad$. Cohnii 480, 481, 920.

- v. subundulata 558-560.

navigans J. Br. 109-111.

nebulosa Greg. 36, 50, 236-238, 389.

neglecta $\mathrm{Ktz} .910$.

neogena Pant. 632, 633.

Neumayeriana Pant. 518, 519.

Nicobarica Grun. 61-63.

Nicolaï Pant. 851-853.

nigricans Pant. 926-929.

nitescens Greg. 151, 153, 236-238, 393.

- $\quad$ major $^{2}$ m4.

- var. striata 875 .

nivalis $\mathrm{v}$. interrupta 226-228.

nobilis Eh. 56, 176, 17z, 823, 824.

- $\quad$ v. gracilis 76, 77 .

nodosa Ktz. 76. 172, 611, 776, 823 .

- v. curta 78 .

northumbrica Donk. 760 .

notabilis Grev. 54, 96, 339, 398, 590.

- $\quad$. expleta $61-63,754,875$.

notata M. Per. 207, 208.

Nova Guineensis Temp. 189.

nummularia Grev, 213, 214, 590, 591. 


\section{Navicula Bory}

- nummularoides M. Per. 96-100.

Ny Cl. 401, 402, 647, 648 .

Oamaruensis Grun. 139-141, 810-822.

oblonga Ktz. 114, 599, ว5ร, 880, 990.

- $\quad$ minor 58 .

- v. curta 770, 902.

- v. directa $\boldsymbol{\gamma 0}$.

obtusa IV. Sm. 206.

occidentalis $\mathrm{Cl} .374$.

Olgae Pant. 851-853.

Olivieri F. H. $51-53$.

ornata A. S. 358-360, 435, 436. v. spinifera 109-112.

Orphei Pant. 173-175, 756.

oscitans A. S. 358-360, 744-750.

ostracodarum Pant. 203-205, 54 7.

* O'Swaldii Arn. 45-47, 236, 238, 993.

v. visenda 926-929, 993-995.

ovalis Hilse $304,463,501,511,528$.

- v. fossilis 463, 501, 514, 528, 529. pachyptera Eh. 81, 187, 885, 906, 981. Pagesi F. H. 122.

paleacea Pant. $851-853$

palpebralis Bail. 50, 189, 596, 760, 952.

paludinarum Pant. 530, 531, 517. $\nabla$. gracilior 530,531 .

pandura A. S $50,130,157,322,438$.

v. elongata 18,19 .

papula A. S. $532,533,990$.

parva Grun. 77, 164, 460, 619, 922.

patula W. Sm. 56, 206, 296.

Paulensis Grun. 273, 374, 879, 904.

pavida Pant. $339,346-349$.

pedalis J. Br. 109-112, 435, 436 .

Peisonis Grun. 497, 991.

pennata A. S. $34,120,214,634,926$.

- v. maxima 988 .

perducta Pant. 173-175, 339.

Peragalli J. Br 367-369.

peregrina Cl. $199,639,825,981,990$.

- v. fossilis $51-53,408,734,735$.

* - $\quad$. truncata 107, 108.

perfecta Pant. 236-238.

peripennata Pant. 230, 231.

peripuuctata J. Br. 36, 171, 216, 639, 640.

perlonga Pant. 318, 319, 339, 756.

permagna Bail. 240, ร30, ร.31, 989.

* - $\quad \nabla$. maxima 926, 929.

perplexa 926.929, 993-995.

* - v. minutissima 401, 402.

perpusilla Grun. 133-137.

Perrotetti Grun. 482, 483, 592, 593. pervasta Pant. 756.

* Peticolasii M. Per. 365, 366.

phalangium Pant. 346349.

pinnata Pant. 236-238, 346-349, 647.

placentula Eh. 77, 137, 600, 680, 918.

\section{Navioula Bory}

placentula $\nabla$. minor $482,483,762,763$. placita Gr. et St. (Dépóts d'Oamaru). platessa Cl. et Grove 367-369. platycephala 419 . polita J. Br. 324, 325, 445-447. polygibba Pant. 346-349. polygramma Schum. 558-560. polyonca Bréb. 457. 158, 586-588, 887. polystricta Grun. I48, 187, 754, 950.

- v. cireumseta $148,422-425,798$.

- r. elliptica 152,153, 993-995.

popola KIz. 394.

porrecta Eh. var. 293.

Powellii Lewis, 322, 566, 839, 975, 985. praeclara Pant. 203.205.

praestes A. S. 367-369.

praetexta Eh. 34, 130, 403, 839, 950.

- v. minor 422.425.

- v. abundans 358-360, 435, 436.

pressa Pant. $₹ 30,531$.

prisea A. S. $182,183,445 \cdot 447$.

pristiophora Jan. 73-75, 488.

procera Pant. 304, 305, 463, 464.

producta W. Sm. 170̄, 834, 885, 910 .

Proserpinae 984. var. A. S. atlas $49 / 40,216$.

proxima Jan. 96-100.

pseudoaspera Pant. 318, 319.

pseudobacillum Cl. et Gr. 107, 206, 374, 735 .

pseudofusca Pant. 380, 390.

pseudogemmata Pant. 926, 927.

pseudomarginata Pant. 236-238.

puella A. S. 101. 639, 640, 926-929.

pulchella M. S (Dépóts d'Oamaru).

- pulcherrima Leud. 8:6-829.

pumila Grun. 207, 208.

pupula Ktz. 85, 216, 578, 751, 918.

- v.minuta $212,216,228,466,821$.

- r. bacillarioidas 21l, 212-216, 309.

* - v. linearis 78 .

- v. minor 36,87 .

pusilla Ralfs. 101, 188, 189, 232.

- v. lanceolala 33 .

pygmaea Kitz. 187, 189, 426-428, 760.

quadriseriata Grun. $73-75,526,527$.

quadratarea A. S. 269, 370.

radians $\mathrm{F}$. H. 307, 308 .

radiosa $K t z$. 280, 308, 514, 601, 834 .

- v. acuta $36,37,89,516,834$.

- v. angustata 362 .

- v. tenella 516.

ramphoides Pánt. 304, 305.

rectangulata Greg. 269, 985.

* Reichardtii $\nabla$. intermedia $647,648$.

Reinhard tii Grun. 107, 108, 307, 628, 888. r. elliptiea 51.53, 365, 366, 770 . 


\section{Navicula Bory}

Renauldi F. H. 51-53, 133-137. reticulo-radiata Temp. et Br. 276, 277. retusa Bail. 269, 760 .

Reusii Pant. (Dépots du Japon). rhombica Greg. 120, 249, 269, 286, 976. rhomboides Eh. 351, 460, 672, 810, 870. v. major 351,352 .

rhyncocephala $\mathrm{K} t$. $.37,212,273,788,902$. v. amphiceros $216,470, \mathbf{6 0 2}$.

Robertsoniana Grev. 98-100, 330. robusta Pant. 276, 30t, 319, 339, 647. Romanoviana Pant. 339, 851, 853. rostellata $\mathrm{Ktz} .222$.

จ. minor 222, 253, 321, 397, 880 .

Rotaeana Grun. 162-164.

rugosa Jan. 98-100.

rupestris $\mathrm{Ktz} .150,168,222,776,838$.

salinarum Grun. 211, 212, 753.

v. intermedia $25 \mathrm{I}-253$.

Santa-Caroli Pant. 851-853.

Santa-Crux J. Br. 213, 214.

Sandriana Grun. 73-75, 285, 403, 566.

- $\quad$. laevis 926-929.

Sansegana Grun. 268.

Scandinavica Lag. 50, 848.

scalarifer J. Br. 213, 214.

schaarschmidtii Pant. 236-238, 270-272.

Schneiderii Grun. 541.

Schultzei Kain. 145-147, 925.

Schumanniana Grun. 206, 767.

scintillans Temp. et Br. 22-25, 15l-153.

scopulorum Bréb. 71, 72, 609 .

- v. craticula 590,591 .

- v. perlonga 426-428.

sculpta Eh. 20, 52, 240, 580, 619.

- v. major 825 .

scutelloides WV. Sm 211-213.

- $\quad$ v. Mocarensis $365,366^{\circ}$.

gcutellum O. Mea. 442, $4 \$ 3$.

Scythiae Pant. 632, 633.

sectilis A. S. 94, 95, 346-349.

- v. Boryana 94, 95, 160, 318, 319.

seductilis H.P. 73-75, 285-287.

sejuncta A. S. 346-349.

- $\quad$. Baldjichiana 316-349.

semicuciata El. 241-243.

semidecora J. Br. 213, 214.

semen Eh. 34, 171, 212, 678, эz6.

seminoïdes Eh. 306.

seminulum $v$. fragilarioides, 150, 208.

separabilis A. S. 276, 277, 324.

septentrionalis Ost. 61 .

serians Ktz. 168, 17\%, 352, 831, 939.

- . . minima 168.

- . minor 168, 17\%,

- v. Peragalli 168.

Sieboldtii Pant. 348, 349.

\section{Navicula Bory}

silicula Eh. 482, 483, 500 .

silimanorum Eh. 586-588.

simicevultus J. Br. (Depóts du Japon).

Slesvicensis Grun. 38, 542, 543.

Smithii Bréb. 33, 101, 212, 499, 756 .

- v. delicata 50 .

* - $\quad$ v. dilatata 103, 104 .

* $\quad$ - $\quad$. fossilis 213,214 .

- v. major 43, 926-929.

- v. maxima $73-75,566$.

- v. minor 95.

- $\quad$ v. nitescens (Dép. d'Oamaru).

- . permagna 50.

- v. recta 647,648 .

- v. scutellum 50 .

sparsipunctata Gr. el St.( Dép.d'Oamaru). spathulata J.-Br. (Dópôts du Japon). . sphærophora Ktz. 307,308, 459, 829, 884 . spectabilis Greg. 31, 117, 130, 775, 934.

- v. lata 98-100.

- $\quad$ v. emarginata $151,826,827$.

- -. Oamaruensis (Dep. d'Oamaru).

Spitzbergensis Cl. 906, 907.

splendida Greg. 160, 318, 865, 875, 946.

Stauntonii Grun. 251-253.

stauroptera Grun. 76, 159, 394, 611, 695 .

$$
\text { - } \quad \text { v. gracilis 162-164. }
$$

stomatophora Grun. 86, 103, 149, 215, 776. streptoraphe Cl. 189, 215, 374, 583, 825. Stuxbergii Cl. 120.

Styriaca Grun. 518. 519, 880 .

subacuta Eh. 85, 777, 904 .

subcapitata Grun. 76, 77, 177, 321, 755.

- v. paucistriata 162-161, 433 . $\nabla$. stauroneiformis 103,104 , $177,578,940$.

subfusca Pant. 756.

sublinearis Grun. 885.

sublyrata Grun. 61-63.

suborbicularis Greg. 286. 330, 331,346-349.

subsalina Donk. 33, 101, 594, 599, 643.

- v. furcata 395 .

- $\quad$ v. major $102,826,827$.

subsolaris Grun. 518, 519.

subtilis Greg. (Dépóts du Japon).

subtilissima CI. 758.

suecincta A. S. 213, 214, 669 .

superimposita A. S. (Dépbts du Japon).

Szaboi Pant. 30t, 305.

Szontaghii Pant. 91, 236238.

tabellaria Eh. 84, 168, 695, 854, 885 .

* - v. acrosphreriæformis 395 .

Talyana Grun. 5:8, 529.

Tenperei J. Br. (Dépôts du Japon). tenella Bréb. 211, 461, 638, 644, 829. - $\quad$, fossilis $304,305,463,464$. 


\section{Navicula Bory}

termes Eh. 76, 77.

- r. staur oneiformis $263,387,388,776,887$. termitiana A. S. 241-243.

Thorax J. Br. 344, 345.

Tithonia Pant. 851-853.

Toulae Pant. 203-205, 547.

transfuga Cl. 268.

transversa A. S. 14, 215, 395, 728, 831.

Transylvanica Pant. 203-205.

7. producta $530,531$.

Trevelyana Donk. 269, 613, 614, 754, 846. $\nabla$. hungarica $173-175$.

triangulifera F. H. 51-53, 738, 739 .

trigonocephala $\mathrm{Cl} .395,587,588$.

trilineata Gr. et St. (Dépsts d'Oamaru).

trinodis Lewis $78,108,216,232,560$.

trinotata Pant. 203-205.

Trochus Eh. 458, 883, 885.

Troglodytes Pant. 528, 529.

tumescens Grun. 351, 457, 883, 885.

tumida W Sm. 241-243, 851 .

tuscula Eh. 206, 242, 757, 886, 890.

undulata Greg. 241-243, 832, 833.

ursina Pant. 851-853.

vacillans A. S. 993-995.

vagabunda $\mathrm{J}$. Br. 109-112.

valida Lil. el Gr. 80.

variolata CI. (Dépóts d'Oamaru).

Vaszaryi Pant 173-175.

velata A. S. 61-63, 446, 532, 533.

veneta Ktz. 421.

Venus Pant. 236-238.

venusta Jan. 928, 9:9.

ventricosa $\mathrm{K} t z .36,37,223,30 x, 628$.

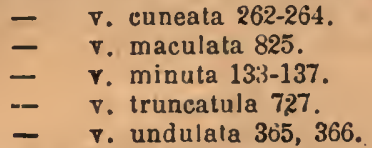

vetula A. S. $18,50,285-287,875$.

viridis $\mathrm{Ktz}$. 56, 85, 242, 625, 727 .

- v. anomala 149, 150.

- $\quad$. commulata $78,321,665,755,939$.

- $\quad$ - fa curta 350 .

- $\quad$. distiguenda 579 .

- v. fallax $36,103,104,372,394$.

viridula $\mathrm{Ktz} 82,222,498,788,890$.

- $\quad$. arenosa 211, 212, 222.

- $\quad$. hungarica 400 .

- $\quad$ v. major 241-243.

- v. minor 599.

- $\quad \nabla$. Slesticensis 34.

vitriscala J. Br. (Dépóts du Japon).

Vukotinovicii Pant. 30t, 305, 442, 443. vulgaris Heib. 2z6-228.

vulpina $\mathrm{Ktz} .206,788,857,858,899$.

Wanhöflenii Grun. 142, 166.

Weisstlogii A. S. $58,276,532,533,946$,

\section{Navicula Bory}

Williamsonii O. Mea. 830.

Wittiana K. et Sch. 145-147.

Wittii A. S. 688-690.

Wittrockii Lag. 216.

Wrightii O Mea 730, 731 .

Yarrensi3 Grun. 189, 730, ร31, 947, 984.

- v. bituminosa $304,305,463,464$.

- v. valida $304,305,317,463,464$.

Zanardiniana Grun. 285 287, 616.

Zanzibarica Grev. 98-100.

Zichyi Pant. 851-853.

zostereti Grun. 61-63, 287.

\section{Neïlium Pfitzer}

affine Eh. 274, 496.

amphirliynchus v. minor 808 .

firmum Ktz. 524.

iridis Eh. 629, 758.

- . amphigomphus 629.

sulcatum Lag. 274.

\section{Nitzchia IIassall}

acicularis W. Sm. 602.

acuminata W. Sm. 50, 187, 269, 370, 434. acuta C.l. 61-63.

acutiuscula Grun. 38, 137, 212, 356, 492.

affinis Grun. 888. $\nabla$. fos $\Delta$ ilis 825 .

alata Leud. $590,622,623,798$.

amphibia Grun. 273, 311, 500, 902, 920.

andesitica Pant. 316-319.

angularis Greg. 160, 277, 504, 532. v. affinis 58 .

angusta v. curta 255 .

angustata W.Sm. 89, 296, 362, 470, 759. v. muinor 835 .

antediluvians Pant. 237, 238.

- v. interrupta 128, 129.

antiqua Pant. $389,390,530,531$.

apiculata Greg. 884.

armoricana Grun. 34 .

asiatica Temp. et $\mathrm{Br}, 25$.

bicostata IV. Sm. 265.

bicuspidata Pant. 501, 502.

biharensis Pant. 38?, 390.

bilobata W. Sm. 72, 101, 188, 397.

- $\quad$. hybrida 350 .

- $\quad$. minor 397.

bituminosa Pant. 305, 463, 461, 501, 502.

- $\quad$ v. tenuior : $04,305,463,464$.

Brebissonii W. Sin. 84. 113, 909, 910.

brevissima Grun. 38, 229.

Campechiana Grun. 622,623.

circumsuta Bail. 87, 239, 541, 826,902. cocconeiformis Grun. 865 .

communis Rab. 350, 419.

- v. abbreviata 196, 507.

- v. obtusa 350 . 


\section{Nitzchia Hassall.}

communis $\nabla$. minor .70 .

constricta Greg. 61.63, 226, 504, 505.

$$
\text { - v. subconstricta 285-287. }
$$

costala Pant. 173-175.

closterium.W..Sm. 142, 166, 181.

curvala Dippel. 89 .

debilis Pant. 852-853.

decora Kitt. $445-447$.

delicatissima Cl. 142.

denticula Grun. 219, 306, 362, 687, 829.

dilatata H. Per. $285-287$.

dissipata Grun. 470, 785.

$$
\text { - v. nerlia } 470 \text {. }
$$

distans Greg. 285-287, 438.

- v. tumescens 160 .

Doljensis Pant. 442, 443.

dubia W. Sm. 89, 874:

fasciculata Grun. $187189,253,827$.

$$
\text { - } \quad \text {. minor } 253 .
$$

fluminensis Grun. 54, 55 .

fonticola Grun. 168, 196, 350, 400, 508 .

fossilis Grun. 133, 337, 356, 492.

Frauenfeldi $v$. major 44

frustưlum Grun. 196, 350, 500, 515, 539.

$$
\begin{aligned}
& \text { - v. acula } 305,464,502,514 . \\
& \text { - } \quad \text { v. constricta } 501,502,515 . \\
& \text { - } \quad \text { v. curvita } 305,464,502 . \\
& \text { - } \quad \text { v. hungarica } 512,515 . \\
& \text { - } \quad \text { v. minuta } 305,464,502,515 . \\
& \text { - } \quad \text { v. potusa } 304,464,502,515 . \\
& \text { - } \quad \text { v. tenella } 500 .
\end{aligned}
$$

fusiformis. Grun . 501, 502 .

gracilis Hantz, $47 \cdot 2,473$.

Graeffii Grun. 393.

granulata Leud. $148,189,206, \mathbf{3 7 0 , 9 3 6 . ~}$

$$
\text { - } \quad \text {. mceotica } 2501,853 \text {. }
$$

Hantzschiana Rab. 500.

Heufleriana Grun. 901.

hevesensis Pant. 304, 305, 463, 464.

hungarica Grun. 31; 280, 363, 599.

$$
\text { - } \quad \nabla \text {. linearis } 33 .
$$

hybrida Grun. 285.287, 462 .

ignimontana J. Br, et F. H. 133-137.

incerta Grun. 80.

incurva Grun. 188, 884 .

insignis Greg. 72, 85, 462, 566, 896.

- $\quad$. mediterranea $43,50,71,72,80$.

$$
\text { - } \quad \text {. nolabilis } 80 \text {. }
$$

intermedia Grun. 492, 506.

Jelineckii Gruı, 401, 402, 622, 623, 798. Kitlıi Grun. 301, 305̄, 350. 515, 699.

Kohleri Lauky. $5+7$.

Lahusenii Pant. 851-853.

lamprocampa Hantz. $\mathbf{5 1 2}$.

lanceolata $\mathrm{v}$. minor 25:3, 288.

* Ligeriana H. P. 11, 34 .

linearis W.Sm. 38, 290, 599, 874, 884.

\section{Nitzchia Hasgall}

linearis $v$. tenuis $\mathbf{8 6 z}$.

- lineola Cl. 41, 29.

littoralis IV. Sm. 101. 187, 188, 371 .

Loczij Pant 94, 95, 346-349.

longa Grun. 71.

longissima Ralfs $266,3 \%$

- v. fossilis (Dépots du Japon).

Lorenziana Grus. $448, \mathbf{9 5 8}, \mathbf{9 6 0}, \mathbf{9 6 5}, 968$.

- $\quad$ v. subtilis 281, 282, 988.

macilenta Greg 50, 80, 615 .

marginata Grun. 454 .

marginulata Grun. $276,592,593,826,827$. v. didyma $285-287$.

majuscula Grun. 647-648.

marina Grun. 682 .

maxima Grun. 80.

- v. minor 252, 253.

microcephala Grun. 113.

minuta Bleish. $.196,350,492,494,602$. minutissima W. Sm 602.

mæolica. Pant. 851.8533 .

navicularis Grnn. 34, 101, 594, 837, 936.

neogena Grun. 304, $361,502,515$.

notabilis Grun. 1:31), 285-287, 566, 829.

Novæ Zelandiæ Grun.-241-243.

obtusa W. Sm: 187, 240, 826, 841 .

- v. fussilis 851-853.

* - $\quad$ ৮. maxima 281, 282.

palacea Grun. 910.

palea IV. Sm. 89. 196, 350, 887.

- v. debilis 508 .

- V. tenuistriata 222.

panduriformis Greg. $80,160,462,561,651$.

- v. acuta Greg. 99, 100.

- v.delicatula 401, 402, 617, 618 .

- $\quad$. lata 622,623 .

- - . minor 393, 447, 489, 533.

- $\quad$. peralbaia 401, 647, 648, 933.

paradoxa Grun. 33.

$$
\text { จ. major 285-287. }
$$

parvula W. Sm. 249.

pennatu Temp. et Br. 118, 119.

plana W, Sm. 189, 285, 731, 865, 935.

polaris Grun. 64 .

protuberans J. Br. (Depóts du Japon).

punctata Gran. $50,101,434,617,869$.

- $\quad$. coarclata 240.

- v. constricta 276, 277 .

- $\quad$. elongata 80, 371, 617 .

- v. minor 34.

rigida $\mathrm{W} . \mathrm{Sm} .34,50,103,424$.

- - v. rigidula 34, 50,643.

Romana Grun. 283.

Romanowiana Pant. 851-853.

salinarum Grun. $533,760$.

scalaris Greg. 33, 88, 210, 639, 826.

- v. undulata 586, 587.

Schweinfurthli Grun. 884 . 


\section{Vitzschia IIassall}

seriata CI. 10, 142, 166, 316, 793.

- $\quad$ v. fraudulenta $17,64,79,142,793$.

sigma .W. Sm. 43. 269, 338, 397, 774.

- v. Habirshawii 217, 218, 39\%.

- $\quad$. intercedens 18, 71, 434, 732 .

- v. rigida $188,280,826,827$.

- $\quad$. rigidula 188 .

- $\quad$. sigmalelı 200, $370,434,617, \mathbf{7 3 2}$. sigmatella Greg. 266 . sigmoidea W. Sin. 78, 216, $170,895$. sinuata W. Sm. 155, 48\%, 483. spathulata Bréb. $58,123,760$ spatnlifera Grun. 285-287, 875. spectabilis Ralfs $105,363,493,579, \mathbf{8 9 3}$. socıalis Greg. $61,63,121$.

- v. basaltica $30-32,476,896$.

Stockmayeri Pant. \$51-853.

subsalina Donk. 194.

suparba Girun. 794.797.

Szaboi Pant. 304, 305, 363, 364.

tabellaría Grun. 122, 293.

Talyana Pant. 304,3 i3, 501, 502, 515. tenuis W. Sm. 470 .

Terryana Temp. et M. Per. 985.

thermalis $\nabla$. minor 222 .

transylvanica Pant. 203-205.

Tryblionella Hautz. 89, 187, 400, 434, 732.

$$
\begin{array}{ll}
\text { - } & \text { v. biharensis } 94,95 . \\
\text { - } & \text { v. levidensis } 34,542,513,767 . \\
\text { - } & \text { v. marina 767. } \\
\text { - } & \text { v. marima } 187,370,732,94 . \\
\text { - } & \text { v. minor } 253 . \\
\text { - } & \text { v. Victinarum } 101 . \\
\text { - } & \text { v. Yarrensis } 242,243 .
\end{array}
$$

tubicola Grun. 162-161, 293, 638.

valida Cl. et Grun. $322,403,40^{\circ} 2,566,615$.

- . minor 324, 325 .

ventricosa Kitt. 415ั-417.

vermicularis Hantz 34, 89, $\mathbf{1 7 0}, 694$.

$$
\text { - v. moeotica } 851-853 \text {. }
$$

virgata Roper. 613, 614.

vitrea Norin. 34, 113, 350, 511, 662 .

- $\mathbf{}$. gallica 113,495 .

- . major 302, 303.

vivax $W . S m .101,160$.

Wankaremæ Cl. 142.

\section{Odontella Agardh}

Heibergii Grun. 310.

Neogradensis Pant. 126-129, 236-238.

\section{Odontidinm Kützing}

anomalum $\nabla$. longissima 287.

hyemale Lynb. 487.

me-odon Ktz. 391.

tabollaria W. Sm. 584, 588, 589, 883.

\section{Odontotropis Grunow}

birostrata Pant. 259-261. carinata Grun. 27, 28. cristata Grun. 27, 28. hyalina 0 . W. I1, 12. vitrea Pant. $128,129$.

\section{omphalotheca Elirenberg}

hispida Eh. 561, 562. Jutlandica Grun. 27-29, 370.

Moronensis Grev. $376 \cdot 378$.

\section{Opephora P. Petit}

- americana M. Per. 365, 366.

Catalense F. H. 51-53.

$$
\begin{aligned}
& \text { - v. capitata } 51-53 \text {. } \\
& \text { - v. obesa } 7: 6,737 \text {. } \\
& \text { - v. oblusa } 735 \text {. }
\end{aligned}
$$

Glangeaudi M. Per, et F. H. 735 . Martyi F. H. 263, 308, 327, 537, 902.

- v. capitata $51-53,263,264$. pacifica P. P. 216.

Schwartzii P. P. 187, 370, 473.

\section{Orthoneis Grunow}

aspera $\mathrm{M}$. Per. 160.

- v. minor 160.

Barbadensis Grun. 46,47 .

- v. ienuipouctata $46,47,775$.

binotata Roper. 18, 19, 123.

- - v. Trinitatis 198.

cocconeiformis Grun. 268.

Clevei Grun. 54, 55, 268.

cribrosa Grun. 268.

fimbriata Grun. 49, 160, 276, 399, 499.

Grovei Cl. (Dépóts des Barbades).

Hillabyana J. Br.

Howatiana Grun. 268.

naviculoides Cl. (Dépôts des Barbades).

ovatá Gran. 268.

- v. intermedia 268 .

pacifica J. Br. 160, 268.

Pethoi Pant. 173-175.

splendida Grun. 71, 276, 322, 756, 863.

\section{Orthosira Thwaites}

interjecta Jan. 3.

\section{Orthotropis Grunow}

lepidoptera Grun. $268,402,775,884$, 955.

- v. delicatula 402 .

- v. pruboscidea 251-253,884.

- v. pusilla $253,268$.

maxima $\mathrm{Cl}$. 634 .

- v. subalata 988 . 


\section{Pantocsekla Grunow}

clivosa Grun. 9, 236, 237.

rossica Pant. 258-261, 271.

\section{Paralia Ileiberg}

Hokkaideana Pant. (Dépots du Japon). Pethoi Pant. 407.

polycistinea Pant. 260, 261.

sulcata $\mathrm{Cl} .238$.

- $\quad$. biseriata 348,349 .

- $\quad$ v. hungarica 358,359 .

\section{Peponia Grevilie}

angulata $\mathrm{H}$ Per. (Dép. des Barbades). Barbadensis Grev.

\section{Perlptera Ehrenberg}

clamidophora Eh. 46, 47.

saxogallica J. Br. et F. H. 30-32. tetracladia Eh. 46, 47.

\section{Peronia de Brébisson et Arnolt}

Boryana Pant. 518, 519.

Heribaudi J. Br, et M. Per. 168.

\section{Petitia M. Peragallo}

- Temperei M. Per. 268.

\section{Pinnularia Ehrenberg}

Brehissonii Ktz. 362 .

biceps Greg. 274, 36i, 496, 758, 891.

borealis Eh. 629, 758.

brevicostata $\mathrm{Cl} .309,911,912,914$.

$$
\text { - v. leptostauron } 524 \text {. }
$$

cardinalis W. Sm. 913.

commutata Grun. 274, 758, 914.

dactylus Eh. 392, 524, 629, 899, 913.

dicephala Cl. 914.

distinguenda Cl. 88.

divergens W. Sm. 361, 629, 891, 899. episcopalis Cl. 524.

exellens Cl. (Dépots d'Oamaru).

$$
\text { - } \quad \text {. interropta }
$$

gentilis Donk. 496, 759, 913.

gibba $\mathrm{Eh} 914$.

gracillima Greg. 361, 758 .

hemiptera $\mathrm{Ktz}, 392$.

inæqualis $\mathrm{Eh} 913,914$.

lata W. Sm. 897.

legumen Eh. 273, 524, 718, 899, 913.

macilenta $\mathrm{Cl} .524$.

major Ktz. 361, 521, 891, 899, 911.

- v. turgidula $\because 09$.

mesogongyla $758,891,913$.

mesolepta Eh. 274, 911, 912.

mesostyla Eh. 274.

\section{PInnularia Ehrenberg}

microstauron Eh. 496, 759, 899.

molaris Grun. 480, 481.

nobilis Eh. 274, 36l, 392, 911, 918.

oblonga Eh. 910,914.

platycephala Eh. 524, 899.

porrecta Eh. 913, 914.

sta uroptera Grun. 274, 496, 524, 629, 758.

staumatophora Grun. 496, 524. 759, 899.

streptoraphe Cl. 891 .

subcapitata Greg. 392.

subsolaris Grun. 362, 899.

undulata Greg. 362, 496, 758, 899.

viridis Eh. 274, 306, 309, $\mathbf{7 5 8 ,} \mathbf{8 9 1 .}$

- v. Clevei 807.

- $\quad$. commutata 891 .

- $\quad \nabla$. intermedia $274,891$.

- $\quad$. major 361,362 .

viridula $\mathrm{Ktz} .910,914$.

\section{Plagiodiscus Grunow et Eulenstein}

Martensianus Grun. 393, 446, 447, 533. nervatus Grun. 55, 648.

\section{Plagiogramma Greville}

adriaticum Grun. 595. angulatum Grev. (Dép. des Barbades). Antillarum Cl. 281, 282.

atomus Grev. $\mathbf{1 7}$.

Barbadensis Grer. (Dep. des Barbades).

$$
\text { - v. actinella }
$$

biharense Pant. 94, 95, 358, 359, 390.

Boryanum Pant. 94, 95, 406, 407.

Ceylanense Leud. 9y-100.

- decussatum (irev. 268.

elongatum Grev. 213, 214, 918, 919.

fenestrata J. Br. 220, 221 .

Gregoryana Eh. 19, 147, 189, 199, 443.

$$
\text { - v. robusta } 220,221 \text {. }
$$

Loczii Pant. 338, 339.

Nancoorense Grnn. 109-112.

neogradense Pant. 237, 238.

obesum Grev. 220, 221.

polygibbum A. S. (Dépots du Japon). pulchellu m Grev. 118, 119, 120, 187, 648 . salinarum Pant. 338, 339.

tessellatum Grun. 47, 330, 331, 370.

validum Grev. 213, 214.

- $\quad \nabla$. tumidulum 550.

\section{Plagintropls Pfitzer}

elegans Grun. 426, 428, 933.

seriata Cl. 189, 289, 370.

Van Heurckii Grun. 639, 640 -

vitrea W. Sm. 370 .

\section{Pleurodesmium Kützlng}

Brebissonii Ktz. ซ, 282. 


\section{Pleuroaigma W. Smith}

acuminatumW. Sm. $89,231,400,599$, эго. acutum Norm. 525 .

affine Giun. 184, 393, 403, 462, 616.

- Grun. 182, 183.

- . major 503.

- v. Marylandica 233-235.

- . Moronensis 794-797.

angulatum WV. Sm 102, 143, 772.

* Antillarum H. Per. $\mathbf{5 0 3 .}$

æstıarii Bréb. 50, 269, 434, $510,571$.

attenuatum W. Sm. $31 \mathbf{5 6}, 82,326,331$. Australe (irun. 28.5287.

balticum W. Sm. 102, 339, 526, 535, 830 .

- . diminutum 235̄-287. 431, 985.

- $\quad$. mæotica 851-853.

- $\quad$. maxima $187,189,397$.

- _ fastrieta 281,282,988.

- v. minor 772 .

- ₹. trinitatis 200.

biharense Pant. 406. 407.

* Bennetti M. Per. 217, 218.

Brebissonii Grun. 143, 144, 192, 571. Clevei ₹. fossilis (Dépôts du Japon). curvulum Grun. 31 is.

decorum W. Sm.80, 332, 420, 567, 798.

- $\quad$. adriaticum 403.

- v. dalmaticum 467, 622, 623 .

- $\quad$. inflatum 281,282 .

delicatulum W. Sm. $60.393,66^{\prime} 2,789,865$. v. salinarum 353 .

directum Grun. (Dépôts du Japon). distortum W. Sm. 43.

* ellipticum M. Per. 79 -797.

elongatum W. Sm. 43, 266, 393, 526, 643. - v. balearica 397 .

Eudon Pant. 926-929. 994.

fasciola W. Sm. 123, 571.

formosum W. \$m. 276, 357, 434, 830, 896 .

- $\quad$. adriaticum 285்-287.

- $\quad$ v. Balearicum 617. 916 .

- v. longissimum 117.

hamuliferum J. Br. (Dépots du Japon). hippocampus W. Sm. $143,013,614,864$. v. major 101 .

hungaricum Cl. et J. Br. (Dép. du Japon). incertum Forti 999, 1000.

intermedium W. Sm. 332, 590, 591.

Japonicum Cast. 426-428.

Karianum Grun. 188.

Kerguelense Grun. 935.

Kochii Pant. 547.

Kützingii Grun. 762, 763.

lacustre W. Sm. 249.

lanceolatum Donk. 230.

latum Cl. 266. 375 .

littorale W. Sm. 123, 143, 572, 613, 614 . longissimum $\mathrm{Cl} .634$.

longum $\mathrm{Cl} .166$.

\section{Pieurosigma W. Bmith}

- longum $\nabla$. lanceolata 50. marinum Donk. 193. 194, 613, f14. mæoticum Pant 851-853. Marylandicum Grın. 248. 299, 690, 773. naviculaceum Bréb. 43, 130, 426-128, 476. - $\quad$. requatoriale 426-428. neogradense Pant. 237, 238. Nicobaricum Grun. $452-456$.

Normannii Grun. $43,58,91,370$. obscurum v. mediterianea 285-287, 615 .

* Olympianum Terry 120, 217, $218,397$. O'Mearii Grun. 61.

Parkerii Harr. 895.

Peragalioi J. Br. (Dépots du Japon). prolongatum W. Sm. 285-287.

quadratum W. Sim. $123,141,338,772$. $\checkmark$. minor 772 .

* Quinipiacei M. Per. 187, 188, 189.

rigidum W. Sm. 50, 32.2, $478,839,817$. จ. gigantea $257,32: 2,403,462,829$.

robustum Grun. 462.

- Ryderii M. Per. 217, 218.

sagitta Teinp. el Br. 733, 928, 929,991. scalproides Kab. 602 .

spociosum W. Sm.51, 285-287, 615, 839,

* - $\quad$. lanceolata 160.

spectabile Grun. 251-253.

Spencerii W. Sm. 34, 123, 189, 293, 296. 7. Arnottii 280.

strigilis W. Sm. 189, 241, 242, 767.

strigosum W. Sm. 123, 509, 572, 637, 643. subrıgidum Grun. 339.

snleatum Girun. 144.

Temperei Cl. 826, 827.

Terryanum M. Per. 188, 210, 732, 976. validum Schadb. 432, 503, 617.

Wandsbeckii Dınk. 43, 1:3, 143, 144, 526 . - $\quad$. minor 572 .

\section{Pleurostauron Rahenhorst}

acutum $\mathrm{Rab} .394,583,586,877,882$.

- $\quad$. major 882 .

- v. maxima 373,374 .

- $\quad$. robusta 211,215 .

- $\quad$ v. Terryana 906.

* Americanum $\nabla$. intormedia 882.

* divisum M. Per. 251-253.

Jaranicum Grun. 228, 773, 882.

$$
\text { - } \quad \text { ₹. minor } 882 .
$$

parvulum Grun. 211.

productum Grun. 885.

- rhombicum M. Per. 583, 581.

scapulæformis Grun. 223.

Smithii Grun. 547, 588, 919.

Ploiaria Pantocsek

petasiformis Pant. 47, 238, 555, 650 . 


\section{Podocystis Kitzing}

Adriatice Kı. 19, 393, 532, 567, 875 .

Americana Bail. 370

spathulata Shadb. 668 .

\section{Podosira Ehrenbers}

ambigua Grun. 63, 393, 503, 532, 875 . argus Grun. 2, 98, 99, 250, 324

Baldjickiana Grun. $3+7,348$.

Buryana Pant. 94, 95.

corolla A. S. 5:0.

glacialis $\mathrm{Cl}$. 17, 64 .

hirsuta Grr. et St. 2.

hormoides Grun. 61, 117, 160, 420, 875.

- $\quad$ v. Montereyi 358, 359.

Hungarica Pant. 5:8, 529 .

Loczii Pant. 347,348 .

maculata W. Sm. 18, 61, 63, 339 .

maxima Grun. 61, 62, 332, 798

- $\quad$. californica 48.

- - $\quad$ - intermedia 61, 63.

Montagnei $K / z .63,123,199, \mathbf{5 0 5}, 863$.

Oliveriana Grun. 3.

robusta Pant. 304. 305.

sphæria J. Br. (Dépóts des Barbades)

spino-radıata J. Br. (Dépóts du Japon). stellulifera Grun. 73-75.

variegata A. S. 109-112, 798 .

\section{Polyuyxus Bailey}

coronalis Bail. 490.

pulchellus Grun. 109-112.

\section{Porodiscus Greville}

calyciflos Temp, et Br. 61-63.

conicus Grev. (Dépóts des barbades). corniger J. Br.

elegans Grev.

hirsutus Gr. et St. 441.

interrupta $\mathrm{Gr}$. et St 2112.

major v. densa (Depóts des Barbades).

* minulus H. P. 214 .

nitidus Grev. (Dépôts des Barbades).

- v. armat

oblongus Grev. 702, 703.

spiniferus Ratt. 'Dépóts des Barbades). splendidus Grev. 701-703.

- v. marginata (Dép. des Barbades).

\section{Porpeia Bailey}

inflexa A. S. (Dépóts des Barbades). ornata Grev.

quadrata Grev. 47, 109-112.

quadriceps Grev. 109-112, 435, 436.

robusta $\mathrm{T}$. et $\mathrm{W}, 202$.

\section{Pseudauliscus Leuduger Fortmorel} ambiguus Ratt. (Dépots des Barbades).

\section{Pgeudauliscus Leuduger Fortmorel}

anceps Ratt. 141 :

Debyi Leud. 148.

diftluens Ratt. 99;100, 141.

elaboratus Ratt. (Dép. des Barbades). - v. tetrapodia J. Br.

Johnsonianus Ratt.

$$
\text { - } \text {. craterifer }
$$

- v. cludea

nebulosus Leud. 148.

notátus Rutt. (Dépots des Barbades).

ornatus Ratt.

Peruvianus Ratt. 435, 436.

Petitii Leud. 148.

radiatus Bail. 146, 147, 233, 330, 925.

Ralfsianus Grev. 178, 179, 233-235.

rotatus Ratt. (Dep (ts des Barbades).

- Schmidiir v. trtramera 928, 929. spinosus Christian 925.

tetraphthalmus Cl. (Dep. des Barbades).

\section{Pseudo-Amphiprora Cleve}

cruciata J. Br. 791-797.

* elliptica M. Per. 794-797.

\section{Pseudo-Dictyoneis Pantocsek}

hụngarica Cl. 347-349, 405-407, 756.

\section{Pseudo-Eunotia Grunow}

doliolus Grun. 848.

hemicyclus Grun. 372, 733, 885.

\section{Pseudo-Nitzschia H. Peragallo}

* cratae Forti. 929, 995, 1000.

- - v. rotunda 929 .

- Fungairiñoi (Azp.) Forti. 993-995.

* pliocena H. P. 213, 214.

\section{Pseudo-Pyxilla Forti}

* capreolus Forti 367-369. hungarica Forti 367-369.

- obliquepileata Forti $367-369$.

- Tempereana Forti 367-3o9, 673-675.

- rossica Forti 367-369:

\section{Pseudo-Rutilaria Grove et Sturt}

monile Gr. et St. 111 .

\section{Preudo-Stictodiscus Grunow}

angulatus Grun. 29, 310, 3 [1.

\section{Pseudo-Triceratiuni Grunow}

araneosum Grev. (Dep: des Barbades). cinnamomeum Grun. 237, 238, 272.

labyrinthicum Grer. (Dep.des Barbades).

\section{Pterotheca Grunow}

spada J. Br. (Dépots du Japon). subulata Grun. 214. 


\section{Pyrgodiscus Kitton}

Kinkerii Pant. 29.

\section{Pyxidicula Ehrenberg}

Adriatica $\mathrm{K} \mathrm{z} .80$.

cruciata Eh. 1(0, 146, 160, 389, 141.

Mediterranea $445-447,875$.

\section{Pyxilla Greville}

aculeifera Grun. 311.

Americana Grua. 109, 129, 183, 299, 650.

baltica Grun. 17, 142, 189, 269, 370.

Barboi J. Br. 25.

Barbadense Grev. (Dép. des Barbades). caput avis J. Br.

$*$

carinifera Grun. 47, 311, 555 .

$$
\text { - } \quad \text {. rossica } 261 \text {. }
$$

cornuta Pant. 128, 129, 238.

directa Pant. 238.

dubia Grun. 47, 183, 235, 570, 650 .

* gracilis Temp. et Forti 773 .

* - v. buccinalis 200,261 .

- v. Saratowiana 200, 261 .

hungarica Pant. 238.

Johnsoniana Grev. 141.

- V. Corniculum (Dép. des Barb.).

* - v. intermedia 773.

Kittoniana Grun. $310,311$.

Lunyacsekii Pant. 238.

prolongata J. Br. (Dépôts d'Oamaru). rotundata Gr. et St. 141, 570.

subulata Grun. 47.

vasta Pant. 128, 129.

\section{Radiopalma J. Brun}

Brunii V. H. (Dépôts des Barbade.s). dichotoma J. Br. 376-378.

\section{Raphoneis Ehrenherg}

amphiceros $\mathrm{Eh} .5$ 5, 370, 594, 614, 925.

$$
\begin{aligned}
& \text { - } \quad \text { v. ausiralis } 276,27 \% \\
& \text { * - } \quad \text { - coloradica 213, } 214 . \\
& \text { - v. elongata } 472,473 \text {. } \\
& \text { - v. gemmifera 647, } 648 \text {. } \\
& \text { * - - v. obesa } 617,648 \text {. } \\
& \text { - v. rhombica 151, 370, 599, 614, } 937 . \\
& \text { - } \quad \text { fa elongata 213, } 214 .
\end{aligned}
$$

angustata Pant. 237, 2:38.

belgica $V$. H. 58, 146, 147, 591, 610 . - v. intermedia 893 .

Boryana Pant. 94, 95, 318, 319.

Ca,tracanei Grun. 199.

Debỳi Pant. 237, 238, 800, 928, 929.

delicatula Pant. 91.

flumineusis Grun. 146, 147.

Fuchsii Pant. 12.

\section{Raphoneis Ehrenberg}

gemmifera Eh. 146, 147. 213, 214, 273.

- $\quad$. biharensis 349 .

- v. curla 773.

- v. elegans 237, 238. 371 .

- $\quad$ v. neogradensis 237, 238.

- $\quad$ v. parcepunctata 207, 238.

- v. subtilior 319.

Gorkeana Grun. 791-797.

hungंarica Pant. 128, 129, 237, 238.

Kinkerii Pant. 756.

liburnica Grun. 50.

Moravica Grun. 237, 238.

nitida Grun. 151.

- v. Liburuca 594.

nodulifera A. S. (Dépóts des Barbades). rhombus Els. 34, 40, 58, 934.

* Santæ-Crucis H. r. 213, 214. scalaris Eh. 14\%, 689, 690. 692, 925. subtilissimus Pant. 91, 412, $4+3$. surirella Grun. 58, 72, 489, $\mathbf{5 9 4}$.

- v. australis 594.

* - v. elongata 58.

Szakalensis Pant. 128, 129.

\section{Rhabdomema liützing}

Adriaticum Ktz. 18, 152, 281, $420,551$.

* - v. diminurum 926929 .

- v. fossile 94, 95, 346, 347. arcuatum Ktz. 16, 120, 413,600 .

Atlanticum Kain. 146, 147.

biquadratum J. Br. 435, 436.

Crozieri Prit. 332, 333.

elegans Temp. At Br. (Dépóts du Japon). formosum $\mathrm{J}$. $\mathrm{Br}$.

hamuliforuın Kitt. 173-175, 442, 443, 478.

Japonicum T'emp. et Br. 23, 24, 46, 90 .

$$
\begin{aligned}
& \text { - } \quad \text {. recta 24, } 25 . \\
& \text { - v. sparso-punctata 23, } 24 .
\end{aligned}
$$

Mikado Pant. 448, 449.

minutum Kitz. 194, 61 z, 743, 754, $8 \subset 9$. mirificuin W. Sm. 62, 53:, 551, 62:2, 863 .

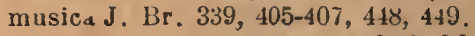
subrostratum $r$. parallola Iateralı $332,333$. valdelatum Temp. et $1 \mathrm{Br}$. 22, 23 .

\section{Rhaphidodiscus Th. Christian}

microstatus Pant. 619, 650.

\section{Rhizosolenia Ehrenberg}

alata Bright. 131, 180, 229, 689, 291.

- v. curpulenia 791, 792

- v. gracillıma 10, 16, 65, 525, 791. amputata CE॰t. 426-428.

arafurensis liast. 525.

Barbui J. Br. 47

calcar avis Schub. 180.

Gastracanei H. Per, 792, 917. 


\section{Rhizosolenia Ehrenberg}

gracillima Gl 683 .

gracilis H L. Sm. 131.

hastata Grun. 47, 634, 651, 652 .

hungarica M. Per 405-407.

imbricata Bright. 476.

inermis Cast. 81, 317.

laevis H. P. $46,47$.

obtusa Hans. 64, 166. 181.

pileolus Eh 46, 47, 773 .

polydactylus Gast. 525.

robusta Norm. 477, 792.

semispina Hens. 314.

setigera Bright. 17, 65, 168, 317, 684 .

Shrubsolei Cl. 124, 168, 1ช0, 477.

squamata Pant. 173, 175.

Stolterfothii H. Per. 30, z71.

stylifor mis Bright. 65, 131, 47z, 525, 789.

Temperei H. P. 792, 917.

- v. acuminata $47 \%$.

\section{Rhoiconeis Grunow}

Gorkeana Gr. 647, 648.

\section{Rhoiccosisma Grunow}

Antillarum Cl. 794-797, 798.

arcticum Gl. 166.

compactum Grun. 80, 393, 647, 648, 933.

lineare Grun. 285-287.

Mediterranea Cl. 285-287.

Oceanicum H. P. 322, 462, 829, 847, 875.

Reichardti Grun. 63. v. corsicana 401, 402,775, 839 .

robustum Grun. 74, 322, 403, 839.

Weissflogii $\nabla$. minor 268, 393.

\section{Rhoicosphenia Grunow}

curvata Grun. 113, 114, 280, 100, 495 .

- . gracilıs 630,631 .

- - major 630,631 .

- v. mairna 119, 138, 396, 766.

- $\quad$. minor 628 .

\section{Rhopalodia o. Müller}

ascoidea O. M. $480,481$.

clavata Forti $482,483$.

- $\nabla$. perlonga Frick. 482, 483.

gracilis O. M. 480, 481 .

gibba 579, 581, 618, 825, 931.

- . parallela $6119,825,930,931$.

- v. reatricosa $308,618,226, \mathbf{8 8 0}, 930$.

gibberula O. M. $618,619,914$.

- v Dibyi $\mathbf{3 5 4}$.

hirudiniforınis O. M. 480, 481 .

musculus H. P. 730,731 .

ventricosa O. M. $482,483$.

\section{Roperia Grunow}

tenella Grun.: 622, 623.

\section{Roperia Grunow}

tessellata Ralfs. $43,50,621$.

\section{Rouxia J. Brun et lléribaud}

* Californica M. P. 468, 469.

* - $\quad$ v. elongata 468, 469.

Peragalli J. Br. 213, 214.

- v. elongata $213,214$.

\section{Rutilarla Greviile}

capitata Temp. et Br. 22, 23.

elliptica Grev. (Dépóts des Barbades). epsilon Grev. 359, 360.

- v. longicornis $110,111$.

- $\quad$. tenuis 140,141 .

excarata J. Br. (Dépots d'Oamaru).

hexagona Grun. 4, 47, 110, 111, 843.

$$
\text { - } \nabla \text {. cornuta } 23,24 \text {. }
$$

Kernerii Pant. (Dephts du Japon). lanceolata Gr et St. (Dép d'Oamaru). longicornis Temp. et Br. 22, 23, 339, 360, 449 pulchra A. S. 110,111 .

radiata Gr. et St. $2,110,141$.

- v. uuperba Grev. (Dép. des Barbades). Szakalensis Pant. 237, $23 \%$.

ventricosa Grun. 127, 129, 237, 238.

. - v. rontellata (Dép. des Barbades).

\section{Rylandsia Gre, ille}

biradıata Grev. (Dépóts des Barbades).

\section{Salacia Pantocsek}

Boryana Pant. 91, 95.

\section{Septroneis Ehrenberg}

caduceus Eh. 147, 214, 235, 690, 773. colubert J. Br. (Dépots du Japon). cuneala Grun. 285287. genimata Grun. 354, 555. marınum Grun. 452-456.

nitzschioides Grun. 555 .

\section{Schizonema Agardh}

comoides Ag. 58, 396.

crucigerum W. Sm. 58, 896.

Grevillei Ag. 58, 123, 245.

japonicum J. Br. (Dépóts du Japon). molle W. Sm. 214.

neglectum Thw. 269, 396.

obtusum W. Sm. 533.

ramosissimum Ag. 58, 246, 896.

Sınithii Ag. 533.

\section{Schizostaurou Grunow}

* Rhombicum M. Per. 825 .

\section{Scoliopleura Grunow}

incisa Greg, 526, 527. 


\section{Scolinpleura Grunow}

latestriata Grun. 72, 121, 217, 269, 397. moeotic Bail. 851-85̄3.

Peisoni Grun. 827.

tumida Bréb. 72, 164, 337, 767, 72.

- $\quad$. adriatica $397,594,767,772$.

\section{Semseyia Pantocsek}

moeotica Pant. 851-853.

\section{Skeletonema Grevilie}

Barbadensi Grev. (Dépots des Barbades). - . argus.

costatum Cl. $142,525,964,965,971$.

v. spiralis $452-456$.

Hungaricum Pant. 129.

mediterraneum Grun. 119,187, 401, 402.

- v. punetifera (Dépóts du Japon). styliferum J. Br. 260-261.

utriculosa J. Br. (Dépots du Japon).

\section{Solium Heiberg}

exsculptum Heib. 27, 28, 310, 872.

- princeps M. Per. 702-703.

\section{Spermatogonia Leuduger Fortmorel}

antiqua Leud. $452-456$.

\section{Stauroneis Ehrenberg.}

acuta W. Sm. 82, 78, 274, 458, 776.

- v. majur 776 .

- - v. mixima 159, 176.

acutiuscula M. Per. et F. H. 278.

arnphilepta E'h 207, 497, n27, 733,831.

* amphiplearoides M. Per 69640 . anceps Eh. 78, 211, 306. 372, 605.

\footnotetext{
- $\nabla$ amplicephalu $321,372,394,776$.

- - v. cauitala 104.

- - v. elougata 395.

- v. fossilis 733 .

- v. linearis $177,187,274,629,910$.

- $\quad$. nobilis 340,341 .
}

antediluviana F. H 26\%, 263.

Bailıyi En. 215, 373, 586, 589, 885.

- Bakurii M. Per. 187.

Boryana Pant. 518, 519.

Brunii Y. Per. et P H. 159, 176, 278, 776,829.

constricla Eh. $911,912$.

Fraınnfeldii Grun. 9:3.

fulmen Eh. 923.

gallica M Per. et P. II. 162-164, 262, 263. gracilıs W. Sin. 37, 171, 176, 215, 589.

Gregorii Ralls. 50, 1є8, 189, 240, 397.

Grovei 166.

Heirleni Forli 482, 483.

Heufleriana Grun. 480,481 .

javanica $\nabla$. arvernense $262,263$.

- $\quad$. minor 480,481 .

\section{Stauroneis Ehrenberg}

Kochii Pant. 203, 204. lanceolata Ki\%. 15\%, 176, 215, 586, 776. legumen Eh. 78, 274, 458, 483.

lineolata Eh. 728, 729. mucrocephala $\mathrm{Klz}, 36$. moeutica Pant: 851-853. mesopachya Eh. 279,321,558, 559, 831 . oblonga Bail. 3 .

parvula Grun. 263, 261. phœnicenteron 159, 176, 284, 556, 885 .

$$
\begin{array}{ll}
\text { - } & \text { v. lanceolata } 395 . \\
\text { - } & \text { v. maxima } 391 .
\end{array}
$$

phyllodes Eh. 310, 757, 903, 905.

- - v. elegans 395.

platystoma Eh. 465.

producta Grun. 605 .

pteroidea Eh. 107, 762, 763, 906. quadrata M.Per. et F. H. 340,341,902. robusta Eh. 911, 912.

salina W Sm. 71, 72.

scapulæformis Grev. 919.

Sieboldti Eh. 586, 587, $728,729$.

Smithii Grun. 728, 729.

spicula Dick. 498.

- Stodderi Greenl. $\nabla$. superba 372.

Szontaghii Pant. 518, 513.

- Tokuhisai Temp. et M. Per. 301.

\section{Stauroptera Ehrenbers}

oblonga Bail (Dépot du Japon).

\section{Staurosigma Grunow}

asiaticum Temp. et Br.(Dépots du Japon).

\section{Stiurosira Ebrenberg}

construens Eh: 519.

intermedia Grun. 519.

Grunowii Pant. 519, 737.

$$
\begin{aligned}
& \text { - } \quad \text {. biangulata } 737 . \\
& \text { - } \quad \text { v. major } 737 . \\
& \text { - } \quad \text { v. minor } 737 .
\end{aligned}
$$

Harrissonii IV. Sm. 277, 519

$$
\begin{aligned}
& \text { - } \quad \text { v. amphitetras } 518,519 . \\
& \text { - } \quad \text { fossilis } 517 \text {. }
\end{aligned}
$$

Mormonorum Gran. 3u5, 463, 502, 527 . venter Grun 519.

- $\quad$. fossilis $305,463,502,527$.

\section{Stenopterobia de Brébisson}

anceps Bréb. 215, 372, 582, 769, 844. intermedia Lewis $351,352,361,840$.

\section{Stephanodiscus Ehrenherg}

Astraea Grun. 216, 308, 517, 604, 677.

- $\quad$. intermedia 366 . 


\section{Stephanodiscus Ehrenberg}

Astraea v. minutula :308. 418.

- $\quad$. spinulosa $30.3, \mathbf{3 6 6}, 418,517$.

- v. - fa IIнxima 119.

Brunii A. S. (Dépots du Japon).

Carconensis Gru॥. 366.

- $\quad$. inermis $624,625$.
- $\quad$ v. ninor $153,630,631$.
7. pusillus $\mathbf{2 5}$.

elegans.J. Br. (Dipots du Japon). Hantzschianus Grun 283.

Kanitzii Grun 8, 9, 346.

- $\quad$. inermis 389 .

- $\quad$ v. major 389.

- $\quad$. partita 389 .

Martensii Pant. 304, 305.

minutulus Grun 247.

Niagaræ Eh. 686.

Pantocsekii Fric. 999, 1000.

rotula $\mathrm{Ktz}, 249$.

transylvatica Pant. 204, 205, 547.

\section{Stephanogonia Eurenherg}

actinoptychus Eh. 112, 147,554, 861, 925.

$\Rightarrow$ aculeata Pant. 236-238.

- barbulata H. P. 213, 214. cincta Pant. 236-z38, 671, 675.

Danica Eh. 11, 12.

- Hillabyana Temp. et Br. 702, 703.

- - $\quad$ tenera 702, 703.

Nova Zelandica 140, 141, 416.

polyguna Eh. $47,146,147,234,235$.

strioliata Pant. 236-238.

Szakalensis Pant. 2:6-238.

Szontaghii Pant. 236-238.

\section{Stephanopyxis Ehrenberg}

antiqua Pant. 255-259.

apiculati Eh. 145, 146.

appendiculata Eh. 90, 310, 551, 555.

$\begin{array}{ll}\text { - } & \text { v. intermedia } 46,47 . \\ \text { - } & \text { v. parvispina } 28 . \\ & \text { v. paucispina } 28,310 .\end{array}$

aristata $\mathrm{J} . \mathrm{Br} .45,46,471,473$.

Barbadensis Grev. 92, 93, 139, 141.

Broschij ia aculeis ohtısi 422-425.

corona Eh. 91, 110, 146, 182, 183.

- $\quad$. monicæ $367-3 t^{\circ} 9$.

crassispina Grun 3578, 359, 554, 555. cruciata Temp et Br. 554, 555, 933.

diadema Eh. 554, 555.

Eutlcha Forti 99i, 997.

feros Grev. 11, 12, 141, 147, 773.

grosse cellulata Pant. $367369,996.997$. Grunowii fr et St. 140, 25i), $415,416.551$. Joysonii A. 's $36 \%-69,622,623,773$. limbata Eh. 110, 111, 146, 182 .

\section{Gtephanopyxia Ghrenberg}

limbata $\mathbf{r}$. cristagalli $22,23$.

lineata Forti 696-698.

maximu Paut. 258, 259.

ninuta Grev. (Déphis des Barbades).

nidulıs Teısp. $\mathrm{t} \mathrm{Br} 25 \overline{\text {, }} 9 \mathrm{u}$.

Palıneriana Grun. 229, 773.

Peragulli Temp. et 13r. 22, 23.

- pediastriformis Forti 367, 369, 996, 997.

permarginata Grov. (Dépots d'Oamaru). pulcher IV. et Ch. (Dép. des Barbades). rossica Pant. 208, 269.

rudis Grev. $110,111$.

spinosissima Grun 45, 46, 109, 110, 554 . superba Grev. 435.

- v. minor 90.

turris Grun. 91, 111, 141, 093, 773 .

- $\quad$. arctica $11,1 \%$.

- $\quad$ v. brevispina 141, 416.

- v. cylindrus $214,424,425$.

- v. gemina 28 .

- v. inermis 187.

- v. iutermedia $9,272,369$.

- v. nuda 272 .

- v. parvispina (Dép. des Barbades).

- $\quad$. polaris 11,12 .

tenuispina Grun. 28, 310.

turgıda Grrev. 39, 167, 2ะ9, 789, 790.

vasta Pant. 258, 259.

Weifsfloyii A. S. 22, 23.

\section{Stlctodesmis Greville}

Australis Grev, 268, 277.

\section{Stictodiscus Greville}

adspersus T. et W. (Dép.de Jérémie). Buryanus Grun. 468, 469.

- $\quad$. gracilis (Dép. de Jérémie).
- $\quad$ v. rotunda.
- v. subquadrangularis -
- v. subtriangulatus -
- v. triangularis -

Californicus Grev. 110, 236, 250, 358, 359 .

- $\quad$. areolata $415,416$.

- $\quad$ v. costata fa minur $368,369$.

- v. ecosiata 45, 46, 358, 359 .

* - v. aggregata 474,475 .

- $\quad$ $\quad$. major 993, 994.

- $\quad$ v. Nankoorense 155.

Caraïbicus T. et IV. (Dép. de Jérémie). coufusus $T$. et W.

elaboratus $\mathrm{T}$. et $\mathrm{W}$.

Ester alyensis Pant. 91.

Euleinsternii Cast.

Grovileanus IV et Ch. -

Grovei A. S. $426,428$.

Grunowi T. et W. 367, 368 .

Haïtianus T. et W. 367,368 . 


\section{Stictodiscus Greville}

Hardmanian us G:er. $2,45,109,110,358$.

- v. megripora 2, 140, 439, 440.

- minor 111, 360 .

Hungaricus Palıt. 415. 406.

Huttlingerianu- T. elW (Dip.deJérémie). insiunis Grev. (Dépóts des Barbades).

Jeremianus Cast. (Dépots de Jérémie). v. hrxagoira $\epsilon 56,657$.

Johnsonianus Grev. (Dép. de Jerémie). v. trigona 367,368 .

Kinkerianus T. et W (Dép. de Jérémie). Kittonianus Grev. 182. 183, 234, 089, 778. Kossuthii Pant 258-261.

multiplex Jan. (Dépôts de Jérémie). nitidus Gr. et St. 2 , +11 .

novæ zelandiæ Grun, $140,415,416,439,140$. Pantocsekil Temp. 258, 261. paralielus Pant. 236, 237, 238.

- v. gibbosa 2, 250, 415, 439, 410 .

- v. Kinkeriarus 928, 929.

- v. trigona $140,250,415,439,410$. radiatus Cast. 621 .

reticulo cellulosus Bergon ( $D e ́ p$. des Barbades). serpentinus T. et W. (Dép. de Jérémie). Szontaghi Pant. 756.

Truanii O. W. (Dépóts de Jêrémie). Tschestnowii Pant. 258.261.

tuberculatus Pant. 258-261.

\section{Stigmaphora Wallich}

Hungarica Pant. $316,319$.

Stoschia Janisch.

punctata Gr. et St. (Depots d'Oamaru).

\section{Strangulonema Greville}

Rarbadense Grev. (Dép. des Barbades).

\section{Streptotheca Cleve}

Támesis Cl. 965.

\section{Striatella Agardh}

deticatula W. Sm. 612 . interpunctata Heib. 81 . Girodi F. H. et J. Br. 30-31. unipunctata Ag. 81, 513, 522, 598, 641.

\section{Surirella Turpin}

alternans A. S. 182, 183.

- Americana Terry 119.

* - $\quad$. inle media 119. amcena Pant. 530, 531. anfrictuosa A. S. $6 /-63$. angusta Ktz. 232, 470, 694, 755, 867. antiqua Pant. 346-349, 390.

$$
\text { v. acuta } 825 \text {. }
$$

\section{Surirella Turpin}

apiculata WV. Sm. 834,867 . arcta $A$. S. $340,341,981,983$.

- Armoricana H. Per. 71. Baileyi k.h 372. 860 .

Baldjickii Norm. 236, 237, 403, 462. Benualensis Grun. $9+7$.

biliarensis Punt $339,389,390$.

bifruus $K$ tz. 82, 206, $215,8: 9,884$. - v. minor 884 .

biseriata Bréb. 14, 56, 107, 860 .

- v. acuminata 56.

- $\quad$. major 611.

- v. minor 56, 461 .

Brightwelli W. Sm. 34, 01, 400.

Brunhrsi M. Per. et F. H. 764, 765.

Brunii F. H. 30-32.

Ciapronii Bréb. 56, 937.

cardinalis Kitt. $\mathbf{3 6}, \mathbf{3 9 5}, \mathbf{7 2 8}, 884$.

Ceylanensis Leud. $561,562,647,648$.

*

Chiliensis Jan. 38 .

จ. aperta Leud. 933 .

Clementis Pant. 6:32.

collare A. S. 130, 462, 561, 565, 615.

comis A. S. 130,615 .

costata Neup. 304, 305, 463, 464.

cruciata A. S. $340,311,372.373$.

crumena Bréb. $34,189,408,767,884$.

curvifacies J. Br. 426428 .

Davidsonii A. S. 251.

elegans Eh. 36, 310, 558, 728, 900.

- - (anormal) 36 .

- $\quad$. elongata 56.

- $\quad$. minor 108.

- - $\quad$. producta 558, 559.

- elliptica Terry. 107, 108

eximia Grev. 61-63, 276, 478, 479.

fastuosa Eh. 80. 160, 462, 830, 934.

- v. abludens $322,403,615,839$.

- v. constricta 839 .

- $\quad$ v. cuneata 50,617 .

- v. fossilis $94,346-349,756$.

- $\quad$ v. opulenta $50,269,403,839,934$.

- $\quad$ v. panduriformis 73-75.

- $\quad$ v. spinulifera 775 .

- $\quad$ v, suborbicalarir 73-75, 80,775, 865 .

fluminensis Gruı. 72, 80.403, 432, 8?9.

Fullebornii O. M. $4 \times 2,483$

$$
\text { - } \quad \text { - v. constricta } 482,483 .
$$

fusiformis Leud. 775 .

gemina Els. 43, 143, 144, 410, 869.

gracilis Grun. 231, 232, 308.

- $\quad$. constricta 52, 53.

- v. gigantea 365.

Guatemalensis Eh, 105.

Guinardii H. Per. 73.75, 462, 566, 839.

- Guyanensis M. Per. 251. 


\section{Gurirella Turpin}

helvetica J. Br. 168, 253.

hybrida Grun 58, 71, 120, 393, 403.

- v. balliıa J. Br. 837.

incurvata A. S. 276, 502, 5118, 775.

inducta A. s. 241-243, 881, 981-983.

- - $\nabla$ intermedia 984 .

intercedens Grun. 73-75, 322, 566.

intermedia W. Sm. 457, 458.

Kelleri Pant. 203205.

Kerquelensis Grun 3.

Kinkerii Pant. 173-175.

Kittonii A. S. 107, 108, 188, 395, 881.

- - v. asperula 107, 108.

- $\quad \nabla$. elliptica 106, 798.

Kochii Pant. 530, 531.

Iævigata Eh. 241-213.

lanceolata $\mathbf{~ . ~ c u s p i d a t a ~} 530,531,547$.

lata W. Sm. 403, 566, 620, 839, 864 .

- $\nabla$. constricta 462.

- v. minor 403 .

lepida Eh. 98, 99, 324, 325.

linearis W. Sm. 106, 470, 481, 577, 879.

- $\quad \nabla$. amphioxys 470 .

- $\quad$ v. elliptica 879, 910,941.

- v. commutata 605 .

- $\quad$ จ. constricle 106,212, 484,578. 879.

Lorenziana Grun. 27h, 3:4, 325, 62:, 623 .

Macræana Girev. 478, 479.

Mallombae O if. $482,483$.

manca Jan. 268.

- medulica H. Per. 34, 643.

mexicana A. S, 268.

minuta Bréb 89, 470, 529, 694, 867.

moeotica Pant 851.853 .

Mölleriana Grun. 34. 107, 216, 450, 559 .

* - $\quad$ - constricta 639, 640.

Mülleri Forti. 482, 483.

Neumayeri Jan. 236.

Neupauerii Pant. 304, 305. 463, 464.

Norregica Eh. 465,764 .

oblonga Eh. 215, 840.

obtusa W. Sm. 938.

- Oregonica var. minor 303.

ovalis Bréb. $89,113, \mathbf{3 6 3}, 495,662$.

- $\quad$. Kostschiana 187, 188, 922.

- $\quad 7$. minuta 910 .

ovata Ktz. 33, 38, 400, 470, 596.

- $\quad$. suevica 400.

Pagesi F. H, 51, 52.

pandura H. Per. 478, 479.

patella Eh. 113, 232, 305, 529.

- patens v. lævis 426, 428.

pinnata W. Sm 755.

- pseudocruciata M. Per. 105, 106.

Rattrayi A. S. 215, 8:9, 911.

rucedens A. S. 251, 281, 330, 616, 798.

regina A. S. 107, 108, 881 .

\section{Surirella Turpin}

robusta Eh, 248, 450, 558, 723.

- - v. orata 941 .

rotunda Pant. 304, 399, 442, 463.

- $\nabla$. minor $305,464$.

salina W. Sm. 187, 188, 189.

salka Pant. 632. 633.

saxonica Auer $36,72,465,728,945$.

sentis Pant. 98, 99.

signata Pant. $318,319$.

Slesvicensis Grun. 931.

spiralis $\mathrm{K} / \mathrm{z}, 149,206,254,881$.

splendida $\mathrm{Eh}, 107,115,168,695,905$. v. minor 15.89 .

splendidula v. minura 108.

subfastuosa Pant. 405-407.

subsalsa W. Sm. 502, 5?9.

striatula Turpin 101, 102, 229, 541.

- - v. Azpeitiæ $673,674$.

- $\quad$ v. biplicata 30, 31, 102, 239.

- v. Gautieri 30, 31.

tenera Greg. 113, 256, 395. 57 596.

- $\quad$. nervosa $34.108,334,596,881$.

- $\quad$. robinsta 1117, 108.

— $\quad$. uplendidala $78,212,559,583,729$.

- - v, torta 559,550 .

* Terryi Ward 98

Thuringiaca Huntz 372.

torquata Pant. 3.39, 405-407.

Toulae Pant 339.

transylvanica Pant. 530. 531.

turgida W, sm. 190, 460, 461.

valida A. S. $105,107,302,334$.

- $\quad$. erosa 107, 108.

- $\quad$. transylvanica 107, 108.

verrucosa Pant. 530, 531, 547.

\section{Syndendrium Ehrenberg}

diadema Eh. (Dépóts des Barbades).

\section{syndetoneis Grunow}

amplectans Grun. 140, 141.

rossica Pant. 258-261.

\section{Synedra Ehrenberg}

acus Grun. 38, 280, 283, 565 .

- v. fossilis $133-137$.

- $\quad$. intermedia 563.

- $\quad$. subtilis 133-137.

- $\quad$. ventricosa 133-137.

affinis $\mathrm{Ktz}, 33,102,123,210,420$.

- $\nabla$. delicatula 522 .

- v. fasciculata $60,192,296,470,606$.

- v. gracilis 396.

- $\quad$. hybrìda $24,25,33,188$.

- $\nabla$. tabulata 33.400 , 732 .

amphicephala $\mathrm{Ktz}, 222$. 


\section{Synedra Ehrenberg}

apiculata W, Sm. 466.

armoricana $H$. Pur. 43.

baculus Greg $160,269,322,403,567$.

- v. minur iól.

- v. reducta 991.

biceps $\mathrm{K} / \mathrm{z} 341,395,770$.

biharensis Pant. 34n-349.

Bremiana Pant. 346-349.

capensis lìrun. zว4.

capituta Ktz 57.

capitella Grun 133-137.

closterinides $\nabla$. fossilis 133-13z.

costalunga J. Br. (Dép. des Barbades). cretae Sall vage 800 .

crotonensiv Grun. v. prolongata. 311 .

crystallina $\mathrm{K} \mathrm{tz} .101,269.61 \mathrm{z}$.

- $\quad$ v. bacillaris (Dep.du Japon).
- $\quad$ v. conspicua 590,591 .
- $\quad$ v. fossilica 90.
- $\quad$ v. gihba 443.

cuneata Grun. 622, 6:3.

dalmatica Kiz. 50, $1 \geq 0,446,447,612$.

delicatissima Edw. 133-13i, 296, 666.

$$
\begin{array}{ll}
\text { - } & \text { v. Mauleri 133-137. } \\
\text { - } \quad \text { v. mesoleia 212, } 517 .
\end{array}
$$

Demeraræ Grun. 501, 502, 515, 528, 529. doliolus Liast. 999, 1000.

fasciculata $\mathrm{r}$. oblusa. 51 i. 528. 529.

fibula J. Br. (Depots des Barbades).

formosa Hantz 5t, 160, 532, 551, 830 . - $\quad \checkmark$ lanvigata 501, 5U2.

Frauenfeldii Grun. $410,447$.

fulg ns W. Sm. 5ł, 2 $\$ 9,403, \mathbf{5 3 5}, 567$. Gaillonii 567, 600, ว4:3, 266 .

- - $\quad$ v. elongata 611.

- - $\quad$ v. givantea 281.

- v. macilenta $60,462$.

Goulardi Bréb. 41, 283.

- - $\quad$ - elongata 41, 283.

gracilis Grun. 113, 296.

Hennedyana Greg. 55, 80, 446, 612.

- hyalina M. Per. 199.

hyperborea Grun. 133-137.

intermedia Grun. 199.

Japonica Meister 801.

Juranyi Pant. 318, 319, 776.

lævigata $\nabla$. hyalina $532,533$.

laevissima $\nabla$ Cossilis. $501,502,515,528,529$.

lanceolata Ktz. 34 .

longissima W. $\mathrm{Sm} .805,809$.

maeotica Pant. 852, 853

- $\quad$. minor 852.853 .

minuscula Grun. 850 .

v. undulata 850 .

nana Meister 788, 808.

nitzschioides Grun. (Def.bts du Japon).

\section{Synedra Ehrenberg}

nitzschioides Grun acumináta 238, 272 . $\checkmark$ olstu-a 238 .

* oxyrunchus $\nabla$. medioconstricta 481. paludira Meister 805, 8u9. parva Ktz. v inajur 25. plincena F. H. et M. Per. 169. pulchella Ktz. $34,123,183,280,333$. pulcherrina Hantz 19, 53\%, 533 . radians $\mathrm{K} 1 \mathrm{z} .787$.

robusla Ral(s 18,41$) 3,566,835$. rostrata Eh. 283, 800, 881.

rumpens Grun 133-137, 223.

- v. familiaris 212.

- $\quad$ v. fragilarıoides 223, 283, 308, 311 .

- v. Meneghiniana 308.

- $\quad$ v. scotica 308.

salinarum Pant. 46t, 515, 528, 650.

Schröteri Me'ster $\mathbf{7 - 1 ,} 788$.

spleudens Ktz. 106, 5u0, 618, 619.

striatula Eh. v. biplicata 102.

superba KIz. 19, 26s.

tabulata Grey. 80, 511, 635 .

* - v. elongala 34 .

tenella Grun. v. brevis 502, 515.

tenera IV Sm, 781

teniata Pant. $174,175$.

tilialıs 'T'emp. et $\mathrm{Br}$ (Dépots du Japon).

trensylranica Pant. 304,305

ulna Eh. 13, 113, 470, $485,599$.

* - v. æqualis 198, $390,590^{\circ}$, (;94, 785.

- v. amibiryachus $23 \% 470,5 \wedge 10,5 \div 9,682$.

- $\nabla$. danica $212,280,486,618,644$.

- v. Ianceolata 37

- $\quad$ - 1a brevis 227, 280, 290.

- . longi-sima 57, 85, 223, 604 .

- $\nabla$. oxyrhyuchus $470,489,633$.

- $\nabla$. rosirata 210.

- v. spathulifera 910.

- v. spl-ndens 596, 910.

- v. subæqualis 279, 686.

- v. vitrea 13, 113, 308, 786 .

undulata Greg. 6\%, 1:3,32\%, 462, 608.

Van Heurckii J. Br. (Dépots du Japon).

Vaucheriæ Kız. 133-137, 800.

$$
\text { - v. parvula 133-137. }
$$

\section{Synedrosphenia II. Peragalle}

- baculæformis M. Por. 268. clavata H. Per. (Dépóts des Barbades). cuneata Grun. 551, 617, 648.

\section{Syringidium Ehrenberg}

americanum 251-253.

eximium lirun. (Depois des Barbades). Wittii Grun.

\section{Tabellaria Ehrenberg}

fenestrata $\mathrm{Ktz}$. 82, 212, 249, 232, 900. 
Tabellaria Ehrenberg

fenestrala $\vee$. asterionelloides 784 .

- v. intermerlia 907

flocculosa $K^{\prime} z .37,57,104,516$.

- v. biceps lós.

- v. ventricosa 104.

venter Eh. 883, 885 .

Tabuina J. Brun

testudo J. Br. (Dépôts du Japon).

Temperea M. Peragallo

* mephistopheles M. Per. 98-100.

\section{Temperea Forti}

* miocenica Forti 422, 423, 673.

\section{Terebraria Greville}

Barbadensis Grev. (Dép.des Barbades).

\section{Terpsinoë Ehrenberg}

americana Bail 33, 389, 836, 936, 987.

$$
\text { v. trigona } 389390 \text {. }
$$

Brunii Pant. (Dépots du Japon). inflata J. Br. $478,479,798$.

intermedia Grun. 87, 389, 619, 9.76.

- - v. musica 647, 648, 649 .

musica Eh. 116, $918,977$.

polygibba Pant. 756 .

trifoliata $\mathrm{Cl} .327$.

triquetra Pant. 756.

\section{Tetracyclus Ralfs}

Boryanus $\nabla$. minor 404.

coupressus Eh. 301.

costellatus 122 .

- constrictus M. Per. 624, 625.

decorutus J. Br. et F. H. 30-32.

elegans Eh. 122.

- v. eximia 122.

ellipticus M. l'er. 133-137, 301, 404, 409.

- v. minntissima 133-137.

emarginatus Eh. 53, 261, 329, 39:, 531 .

- - v. crassa 122 .

glans Eh. $301,624,625$.

- v. paucicustata 736, 737.

Japonicus P. P. $\mathbf{3 4 5}$.

lacustris Ralfs 86, 391, 524, 88?, 894.

- $\quad$. fossilis 519 .

lamina Eh. 404, 624, 625 .

- - v. lata 301 .

lancea Eh. 133-137.

Pagesi F. H tt II Per. 52, 53. 122.

Peragalli F. H. 53, 621, 625, 665 .

$$
\text { - v. eximia } 52,53 \text {. }
$$

rhombus Ralfs $133-137,301$.

\section{Tetracyclus Ralfs}

rhombus var maxima 366 .

- - v. producta 301.

rupextris Brann. 255.

stella M. Per. 3u8. 366. 624, 665.

- - v. dilatata 6:4, 625.

stellıre F. H. 52, 53.

tripartitus J Br. et M. Per. 133-137, 624, 625 .

\section{Thalassiogira Cleve}

baltica Grun. 166, 767.

condensata $\mathrm{Cl} 166$.

decipiens Grun. 166, 960, 961.

fossilis Forti 997, 998.

gravida Cl 167, 181 .

hyalina $958,961,969,970$.

Nordenskioldii Cil. 6, 17, 64, 167, 181.

\section{Thalassiothrix Cleve et Grunow}

elongata Grun. 6.

Frauenfeldi Grun. 525.

$$
\begin{aligned}
& -\quad \text { v. dolgensis } 442,443 . \\
& -\quad \text { v. Javanica 24, } 25 . \\
& \text { longissima G. } 24,25,312 . \\
& \text { marina Grun. } 555 . \\
& \text { nitzschioides Grun.6, 124, 166,181, } 229 . \\
& \text { - v. lanceolata } 312 .
\end{aligned}
$$

\section{Thaumatonema Greville}

Barbadense Grev. (Dép.des Barbades). complanata J. Br.

costatum Grev.

\section{Toxarium Balley}

Hennedyanum Grun. 268, 426-428. undulatum Bail. 18, 19, 268.

\section{Toxonidea Donkin}

balearica $\mathrm{Cl}$. 285-287.

Grogoriana Donk. 285-287. 958, 959, 973.

insiguis Ralfs. 285-287, $464,971$.

\section{Trachyneis Cleve}

aspera Cl. 50, 160, 297, 933, 935.

- v. iulermedia 118, 160. 226, 652, 798.

- - $\quad$. minula 50, 126, $128, \approx 68$.

- จ. pulchella 50, $118,1211,121$.

- v. vulgariı 126-128, 2ヶ8, 551, 61 \%.

clepsydra Louk. 289.

consors A. S. 401, 402, 647, 648.

contermina A. S. $1200-128$.

oblonza Bail. 468, 634. 651, 798.

robusla P. P. 7y4-797, 93:3, 935.

velata A. S. 126, 128, 401, 402, 648.

- - v. rhomboidea 160 . 


\section{Trachyephenia P. Petit}

- acuminata M. Per. 101, 102.

\section{Triceratium Ehreniserg}

Abyssorum Grun. 27 29, 258-261. acceptum Grev. (Dépots des Karbades). aculeatuin Grev

acutanqulum Grer. $91,237,238,270-272$.

acutum Eh. $182,183,235$.

affine Grun. $478,479$.

albifrons J. Br. 23, 24.

alternans En. 58, 240, 591, 975. v. variahilis $452-4 \div 6$.

alveolatum Bergon (Dephis d'Oamaru). Americ^num Ralf $141,2: 34,299,416,773$. amcenuin Grev. 926-929

amplexum A S (Depót.s cles Barbades). anastomosans Gruve $340, .341$. anlarcicum Jan. 3, 651, 65?.

antedilurianum Eb. $43,123,155,403,462$.

- - v. trigona 647,618 .

antillarum Cl. $20 \dot{8}$.

antipodum rant. (Dépots d'Oamaru). antiquum Pant. 238. 659, 660.

Archangelskianum 0 . WV. 11, 12 . arcticum Bright 23, 322, 403, 532, 612.

$$
\begin{aligned}
& \text { - } \quad \text {. Californica } 45,109,358,551 . \\
& -\quad \text { - quadragulata } 276,277 . \\
& \text { - } \quad \text { v. lucida } 22,23 . \\
& \text { - } \quad \text { v. pentagonalis } 393 . \\
& \text { - } \quad \text { v. permagna } 139,140 . \\
& \text { - } \quad \text { quadrata } 139,140,332 . \\
& \text { - } \quad \text {. quinquelobata } 139,140 .
\end{aligned}
$$

areolatum Grev. (Dép. des Barbades).

Armasewskil Pant. 258-261.

arrogans T. et W. (Dép. de Jérémie). atlanticum Cast. 620.

atomus Grev. (Depots des Barbades).

attenuatum Grev. 478, 479.

- r. quadrata (Dép. des Barb.). auliscoïdes Gr et \$ı. (Dépóts d'Oamaru). balaniferum Temp. el Br. 22, 23.

Balearicum Cl. 91, 130, 238.

Barhadense Grev. (Dep des Barbades). Barboi J. Br.

Bergonii Temp. et Br. 22.

bicorne Cl. 10n, 199. 217, 218, 479. .

biformis J Br. (Dépóts des Barbades). biliarense Paıt :18, 3:9.

bimarginatum Gr.et Si. (Dep d'Onmaru). biquadratıII Jan. 94, 95 238, 690 698. blinditum Grev (Dsp. des Barbades). blandum O W. 11, 12

brachiatum Brıght (Dép. des Barbades).

\section{Triceratium Ehrenberg}

Brandtii Pant 258.261.

breviuervum Grev. 1Dép. des Barbades). Brightwellii Witt. 6-8-690, 783.

Brunii Pant. 237, 238.

bullosum Witt. 62, 6:9, 401, 402, 535 . caducenm Pant. 258261 .

Califoruicum (irun 110-112.

canalifor J. Br. 220, 221.

cancellatuni Grer. (Dépotsdes Barbades). - v. major

castellatum West. 1, 2, 25n, 415, 416.

$$
\text { - v. fracta } 139,140 .
$$

castelliferum Grun. 1, 2, 141, 250, 415. candatım Witt. 11, 12.

cellulosum Grev. (Dép. des Barbades). v. Japonica (Dépots du Japon). cinnamnmen $m$ Grev. 376 37y-38х, 926-929. clavalum Grun. (Dépóts des Barbades). cœlatum Jan. (Dépots d'Oamaru).

cœrulescens. Paut. Dépts du Japon). Columbi O. W. 202.

compar A. S. 100, 324, 3i25, 445-447.

roncinnum Grev. (Dép des Barbades). condecorum Eh. 146, 147, 334, 335.

$$
\text { v. neogradense } 237,238 .
$$

consimile Grun. 110.112.

constans Grev. (Depóts des Barbades). constellatum Temp. et Br. 22-24.

cordiferum Gr. el St. (Dépóts d'Oamaru). cornutum Grev. 1, 2.

coscinoides Gr. et St. 2, 141, 416.

$$
\text { - v quadrata (Dép. d'Oamaru). }
$$

Contariaum Temp. et Br. (Dep. du Japon).

crenulatumı Gr. et St. 141 , $250,415,440,441$. - v. gibbosa 415,416 .

cristatum Pant. 367-369.

- Crouanii M. Per. 176. cruciforme A. S. (Dép des Barbades). cucullatum Pant. 25x-261.

$$
\begin{aligned}
& \text { - } \quad \text { v. duseminatoponctatom 258-261. } \\
& \text { - v. latior 258-261. } \\
& \text { - v. validior 258-261. }
\end{aligned}
$$

cultum A. S. 22

cuneatım A.S. (Dépôts d'Oamaru). curvibaccatın J. Br (Dép. de Jérémie): curvicosta Inm J. Br. (Dép des Barbades). curvilimbum J Br. (Dépóts du Japon). cuspidatum Jan. 621 .

cyclumen J. $\mathrm{Br}, 2 \cdot 2,23$.

Davidsonianum T. W. (Dép.de Jérémie). Debesii Punt. $258 \cdot 61$.

deductum Pant. 258-261

definitum Grev. (Dép. des Barbades). derormatum Pant. 25*-261.

delectabile Pant 258-261

delıcatulum Grev. (Dep.des Barbades). denticulatum Grev. 


\section{Triceratium Ebrenberg}

digitale J Br. (Dépôts des Barbades). disciforme Grev.

- distinctum v. qua Irata Jan. 367-369.

distinguendum I ant. 11, 12.

divisun Grev. (Dépis d'Oamaru).

dubium Bright 54,55, 270, 277.

- $\quad$. irrégularis $99,100$.

dulce Grev. (Dépóts des Barbades).

- v. Japonica 23.25.

duplicatum A. S. 11,12 .

Dutertrei Temp. 258-261.

Ehrenbergii Grun. 297, 298.

elaboratum T. et W. ( Dép. de Jérémie). elaturn Pant. 405-407.

eleyans Grev. 46, 47, 318, 319, 436.

- v. Japonica 23-25.

- v. major $110,111$.

- v. pusilla 112 .

elevatum Pant. 237, 238.

elongatum Grun. 61, 62 .

Endlicherii P:'nt. 258-261.

excentricis (ir. et St. ( Dép. d'Oamaru). exol'n.tum Grev. (Dép des Barbades). fallasciosum Gr. et St. $110,141,569,570$. fasciatum Pant, 258:61.

farus Eh. $33,148,403,616,936$.

- v. pentagona 277.

- v. quadruta 134, 140, 276, 415.

Febigeri W. etCh (Dep.drs Barbades). fenestratum 0 . W. 11, 12.

ferox A. S. (Dépôts des Barbades).

fimbriatum Wall. 13(1), 478, 479, 7\%5.

firmum Grev. (Dép'ts des Barbades).

Fischerii A. S. $146,147,689,690$.

flos Eh: 11, 12

- $\nabla$. interinedia $11,12$.

formosum Bright 18, 48,49

$\checkmark$ pentagunidis $622,623,775$.

Forresteri Temp. 22-24.

foveatum Grev. (Dépots des Barbades).

fractirm $W$. et Ch. $1,415,416,440$.

fravile Pant. 258-261.

Frauenfeldij Grun. (Dép. des Rarbades). gibbosum Bail. 590. 591

- v. crenulata 61-63

- $\nabla$. excisa 61-63.

giganteum Grev. (Dep des Barbades). - $\quad$. minor

glandarium A. \$. $39,150,415,416,440$. glandiforum Grun. 2, $1411,150,410^{\circ}, 411$. globuliferum J. $\mathrm{Br} .258261$.

Gudefroyi (irun. (Déı,̂ts de Jérémie) grande Briglıl 1, 1:39, 1:i), 3:4, 415.

- v. peistagona $\% 70$.

- v. qradiangulata 300, 673. granulatum W. et Ch. (Dép.des Barbades).

\section{Triceratium Fhrenberg}

gratum A. S. (Dépôts des Barbades). grave A. S.

Grovei Pant. 126-129, 237, 271,674.

Grundleri A. S· (Dép.des Barbades).

Grunowii Jan 96-98.

Gurowii Pant. 258-261.

guttalum Cl. $376 \cdot 378,379-388$.

Hardmanianum Grev. (Dép. des Barb.).

r. solida

Harrisonianum Grev. (Dép. d'Oamaru).

Helbergii Grun. 11, 12 .

Heilprinianım K. et Sch. 146, 147.

hilaratum Pant. 258-261.

horridum Pant. 94, 95.

hyalinum Grev. (Dép. des Barbades).

idoneum Pant. 258-261.

illustrum Pant. 258-261.

implıcitum Grev. (Dép.des Barbades).

imperator T. et W. ' Dép. de Jérémie).

inæquale Grev. (Dépóts des Barbades).

invisum A. S 160, $178,479$.

incon-picum Grer. IDép. des Barbades). indentatum $\mathrm{K}$. et $\mathrm{S} \cdot \mathrm{h}, 1+16,147$.

inelegans Grev. 359, $\approx 60$.

- v. micropora 141.

- v. tubulosa (Dép des Barbades).

inflatum Grev.

insigue Grev.

- v. minor.

insuave T. et W. (Depôts de Jérémie).

interjectum A S. 22-?4,411, 4i8.

v. quadrata 651,652 .

interpunctatum Grun. 234, $2: 35,553-555$. irregularo Grev. (Dép. des Brorbades). v. hebelat: $182,18.3$

Janischii T. et IV. (Dép. de Jérémie). Jansenianum Gran. (Dep.des Isarbad.). Jimboi Pant. (Dépóts du Japon).

Jordani T. et W. (Dép de Jérémie). jucundum Pant, 258-261.

Kainii Seh. 140, 147.

- $\quad \nabla$. constrictum 146, 147.

Kinkerianum J. Br. 22, 23.

Kiltonianum Grev. 234.235.

Kusnetzkianum Pant. 258, 259.

lætum Pant. 94, 95, 346-349.

Linusenii Paut. 258-260

lanceolatum Pant. $258-261$.

latum Grev. 98-100, 276, 325, 533.

- - v plana 154 .

Lautourianum Grove (Dép. d'Onmnru).

laulum (irev. (Depôts des Barbades).

Leudugerii Debr $6 \% 2,623$.

- - v. Simoensis 647, 64S.

ligulatum Grev. (Lép. des Barbades).

lineatum Grev. 1, 139; 1t0,439, 410.

- v. biangnlata (Dép. des Barb:). 


\section{Triceratium Ehrenberg}

lobatum Grev. 376-378, 379-388.

lobatugemunatım J. Br.1Dép. des Barb.). Loczyı Pant. 94, 9.5, 3ł6-319.

lucidum l'ant. 237, 238.

luminosim 'lemp. el Br. 2?-24.

maculitum kitt. $27-29$.

Madagascarense Grun. 96-98, 236, 237. majus Gr. et Sı. 1, 139, 150, 415, 139. mammiferum Grun. $27,: 8$. mammosum Gruv. (Dép.des Barbades). margaritacemu lialfs $234,235$. margin Htu ui Bright. (Dép. des IBarbades) Marylandicum Brighı 145-147, 2:3', 235 . megastomum Eh. 98, 324, 622,623. membianaceum Bright 182, 183.

Mereskuw.ki Pant. 258.

micrutis Grun. 237, 238.

microcephıluin Geer. (Dep. des Barb.). microporum Eh.

micr.stictum Girev.

microstigma lil.

miriticum $\mathrm{J} . \mathrm{Br}$.

mod-stum Grev.

Mölleri Pant. 237, 238.

Montereyi Brigtt 15, 109, 233, 276, 688. - v. primordialis 22, 23.

Morlandii Gr. et St. 1, 139, 140, 415.

- v. aperta 139, 140, 569, 570 . v. subaverta 56 \%). 571)

Moronense (irev 376-378, 379-388. multifrons J. Br. 23, 24, 1:9, 6s9, 690. muricatum Brinht 592, 593. v. fossiii-237, 2.38.

Nankoorense Gı un. 36736 ‘ . 773,996998 . - $\quad$ v. italica 367-36:4, 996-94:8.

nebulosun Grev. (Dép. des Barbades). neglectum Girev.

Nicobaricum Grun. 376-378, 380,468, 469. nitescens Grev. (Dépóts d Oamaru). nitidum Grev. (Dép des Barbades). nobile O. W. 11, 12, 773.

Normanianum Grer. (Dép. des Barb.). notabile Grev.

notatum Prnt. 258-261.

Nora Z handia Gr. Ut St. 1. 139, 150, 415, 439.

Oamaruense Gr. et St. $1,415,116,439,440$.

- V. sparsipnetata (Dép d'Oamaru).

obesum Grev. (Drpóts des Burbades). obliquim Grun. 110.112.

obscuruin Grev. 7\%3.

obtusum Eh 14', 147, 182, 183, 299. oculatum Grev. (Dép. des Barbades). orbicu atum Shatsd. 6:2, 6:3.

v. elong ttu un $9: 6929$.

ornatum Grev. (Dépóts des Barbades). pallidum Grev. 98110.

Pantocsekii A. S. 91, 236, 270, 271.

\section{Triceratium Ehrenber}

Pantocsekii v. convexa 236, 270, 271.

- v. pelitagonia 20

- v. reclangulari-673, 675.

papillatum Kitt (Depots d'Oamaru).

- $\quad>$ acula $+7 b^{\circ}$.

parallelum Grev. 98, 151, 376-378, 416.

- $\quad$ v. coloneusis $468,469$.

- v. spar-a 791-797.

- v. trigu.n. 436.

parlitum (irev (Dépôts des Barbades). $\mathrm{Pa}$ agonicum A. S. 22(), 2:1.

panpercul. m Girr. (Dép des liarbades). pentacrinus Wall 55, 10 (i) $325, \mathbf{5 0 3}, \mathbf{6 2 8}$. v. qualralя $160,478,479$.

Peragalli J. Br. 22, 23.

$\checkmark$ subrotunia 22,23

Peragalloi Pant. et 'Te" p. 258-261. perninutum Giev (Dep des Barbades). Perryanum T et W idép de Jéremie).

Petlioi l'ant. 23ri, "237.

Petitii Pant. 238-261.

picturalum Grev (Dćp. des Barbades). pileus Eڤ. 94. 95. 340 349.

planocuncavum Temp. $\mathrm{t}$ Br 21,25, 440. plenum Gr. et St. (Dep, d'Onmaru).

plicatum Grun. (Dé ôts des Barbades). plumosum Grer.

præerox !aut 258-?61.

præten

purp"sillum Grev.

primordial!s J. $\mathrm{Br} .842,843$.

productissimum Bergon. Dép.d'Oamaru). productum Grev. (Dép. des Burbades). prominens (irev.

proprium Pant. 258-261.

prosiractum 'rant. 258-261.

pseudo-areticnm P'ant 236, 237.

pseudo-neeratum Gr. el St. 2, 139 140, 439, 440.

pulvinar A. S $1,2,415,416$.

punctatum Wall, $71,7 ?, 2 b 9,551$.

$$
\text { - v. quarirata } 47 .
$$

quadrangulare (irev. 2), 10!. 110, 468. quadratum (irev. (DCp. dfs Barbades). quinquelobatum Grer. $370-378,394,445 \cdot 447$.

radiaus Temp. et $\mathrm{Br}^{*}, 22,23,674,675$.

$*$

- v. ilalica 177,675 .

- v. quadrata $23,21$.

radiato-punctatum A. S 8,9 126-129.

- $\quad$ - . calcaren 23, 24.

radiatum Grev. (Depóts des Barbades).

- v. minoe

radisoretieulatum Grun.

Ka trayi Pant. 258-261.

recepıum A.S. $110,111$.

rectanusulure Gr. et St. (Dép. d'Oamaru).

renunciatum Pant. 25๊, 2631. 


\section{Triceratium Ehrenterg}

repletum Grev. (Dép. des Barbades). reliculatım Eh. $182,183$.

reticulım Eh. (Dépôts des Barbades). rivale A. S. 300 .

Roberstianum Grer. 300, 444. v. inermis 775 .

robustum Grev. $146,147$.

rotundafum Grev. ( Dép des Barbades). rugosum Gr et St. $150,415,416,439,440$. ruslanilicum Temp. 258-261.

Rylandsianum Ger (Dep. des Barb.).

Rzehakii Pant. 236, 237.

Saratıvianım Pant. 258, 259.

Sarmalicum Pant. 258, 259.

Schlumbergeri T'emp. et Br. 22-24.

scitulunı Bright 2, 98, 9!, 148, 479. v. quadragulala $276,277,479,551$. sculptum Shabd 55, 100, 148, 5̄51, 936.

- v. petrupolitana $182,183$.

secedens A. S. 1,415 .

- - v. quarrala $267-369$.

secernendum A. S. $231,235$.

semicirculare Bright 146, $231,235,217,299$.

Sehdaiense A. S. 22, 035 .

$$
\text { - v. quadra'a } 22 .
$$

septnm Pant. 258, 259.

sexpartitunı Gr. et St. (Dép d'Oamaru).

Seychellense Grun. 367-369.

Shadbultian um Grev. 54, 532, „6 67, 615, 675 .

simplex J. Br (Dépôts d'Oamaru).

Smithianum Grer. (Dép. des Barbades).

Sokuluwil Pant. 268261 .

solenoceros Rab. 146, 234, 235, 689, 690.

speciosum rint. 258-261.

spinosum Bail. 51, 130,322, 462, 616.

- v. minor fossilis $928,929,994,995$.

- v. quadrata 620 .

- v. telragona 151.

squamatum Pant. 258, 259.

- v. radiata (Dép. des Barb.).

Staubii Pant. 126129.

stigmalicum Pant. 258-261.

Stokesianum Grev. 8, 9, 270, 271.

$$
\text { - } \quad \text {. moravica } 22,23 .
$$

- $\quad$ - parvula $368,369$. Stolterfothii T. et W. (Dép. de Jérémie). strabo A. S. 109.

Sturtii Pant. 94, 95, 247, 248.

subcapitatum Pant. 260, 261.

succinctum T. et W. (Dép. de Jérémie). sublime A. S. 96-98.

suborbiculare Pant. 367-369.

$$
\text { - } \quad \text { - v. latilimba 367-369. }
$$

subrotundatim A. S. $234,235,673-675$. Szakalense Pant. 126-129.

tabellarium Bright 775 .

\section{Triceratium Bhrenberg}

teclum Pant. 258-261

Temperei J. Br. 06-98.

tertiarum Pant. 258-251.

tesselatum Grev. 234, 2:35, 299.

Thumii A. S. 8, 9, 1ะ6. 246, 270.

v. quadrata 151.

triasicum Pant. 258-261.

tridactylum Bright 182, 183.

$$
\text { - } \quad \text { v. valida } 673-675
$$

trifolium A.S (Depbts des Barbarles). trilineatum Grev.

trinitas J. Br. (Dépôts du Japon).

- $\quad \nabla$ minima

tripartitum $\nabla$. miocepa (Depp. des Barb.).

tripecten $\mathrm{J}$. Br.

triplex $\mathrm{J}$. Br.

tripolaris Temp. et $\mathrm{Br} .22,23$.

tripos J Br. (Dépôts des Barbades). triorbicum A. S (Dépóts d'Oamaru). trisulcum.Bail. 128, 129, 271, 272.

$$
\begin{aligned}
& \text { - v. aculloba 367-369. } \\
& \text { - } \quad \text {. cuneala } 1,2,34 n, 341 \text {. } \\
& \text { - v. llaîtian (Dép. de Jérémie). } \\
& \text { - v. hungarica 271, } 272 . \\
& \text { - v. moravica 237, } 238 . \\
& \text { - v. proilucti } 202 \text {. }
\end{aligned}
$$

Truanii Pant 258, 259.

truncatum Bright (Dép. des Barbades).

Tulk.i Temp.

tumidum Grev.

turgidum A. S.

v. costulata -

turriferum T. et W. (Dép. de Jérémie). umbilicatum Ralfs (Dép. des I3arbades). undatum Pant. 258-261, 298, 415, 416. undosum Pant. 258-261.

undulatum Eh. 237, 238, 687, 690.

unguiculatum Grev. $415,416$.

uviferum A S. (Dépôts des Barbades).

validum Grun. 1:0, 111, 2:3, 234.

vastum Pant. 318, 319, 389, 405, 756 .

vater Pant. 253-261.

venosum Bright (Dép. des Barbades).

$$
\text { - v. parva }
$$

ventriculosum A. S. 258-261.

venulosum Grev. (Dépóts d'Oamaru). venustum $\mathrm{O}$. W. 258-261.

versicolor $\mathrm{J}, \mathrm{Br} .22,23,9 \mathrm{)}$.

- v. cuneata 22, 23.

- $\quad$. trigona 617,648 .

Wallischianum Ralfs 415-447.

Websterii Temp. (Dépóts d'Oamaru).

Weissflogii Gr. ot St. 1, 2, 415, 416.

Weissii Grun. 11, 12.

- - v. spinosa 367-369.

Westianum Grun. (Dép. des Barbades). Wittianum Truan (Dép. de Jérémie). 


\section{Trlceratium Ehernberg}

Wittii Jan. 237, 238, 773.

- - hexagona 237, 238.

* - v. italica quadrata 674,675 .

zonulatum Grev. $393,417,64 \gamma, 648,775$.

- $\quad$.triangularis $61-63,622,623$.

\section{Trinacria Ileiberg}

altar J. Br. (Dépots d'Oamaru). antiqua Pant. 258-265.

conifera J. Br. 712, 716, 720.

coronata $\mathrm{O}$. W. 11 .

excavata Heib. 27, 28, 310, 872.

v. producta 11 .

fragilis Grun. (Depots d'Oamaru).

Heibergii líitt. 27, 28, 310, 872.

- v. sparsi punctata 27, 28.

hystrix Pant. 258-261.

insipiens $\mathrm{O}$. W 11,12 .

- v. intermedia (Dép des Barb.).

Jeremiæ T. et W. (Dép. de Jérémie).

Jordanii A S. 27, 28 .

ligulata Gr. et St. 141.

Müllerii Pant. (Depóts d'Oamaru).

palmipes J. Br. 258-261.

pileolus Grun. 11, 12 .

- v. baccala (Dép.des Barbades).

- v. Jutlandica (Dép. d'Oamaru).

præcellens l'ant. 258-261.

prætenuis Grun. (Dép. des Barbades).

- r. perminata

regina Heih 2z, 28, 310, 8z2.

- v. oblusa 11 .

- $\nabla$. tetragona 27, 28.

rossica Pant. 258-261.

Semseyi Pant. 258-261.

simulacroldes Pant. 258-261.

simulacrum Gr. et St. 2 .

sparsa Pant. 258-261.

Tschestnowii Pant. 258-261.

vetustissima Pant. 258-261.

Weif-flogii O. W. 11.

ventricosa Gr. etSt. 140, 415, 416, 569, 570.

Wittii A. S. 27, 28.

\section{Trochosira Kitton}

mirabilis Kitt. 29, 311.

spinosa Kitt. 29, 311.

\section{Tropidoneis Cleve}

lepidoptera Greg. 101, 121, 598, 732.

* - v. intermedia 160.

Mediterranea Grun. 947.

seriata $\mathrm{Cl} .637$.

vitrea Cl. 732.

\section{Tryblionella W. Smith}

apiculata Greg. 888.

Hantzschii Grun. 147, 950.

punctata W. Sm. 18.

scutellum W. Sm. 147.

\section{Tabularia J. Brun}

pistillaris J. Br. (Depots d'Oamaru).

\section{Van Ileurckla de Brébisson}

crassinervia Bréb. 117, 249, 577, 578, 922.

Martoufli Pant $3: 1$.

Lewisiana Breb 217, 251, 25\%, 826, 927.

$$
\text { - } \quad \text { v. incomperta 217, } 218 .
$$

rhomboides Bréb. 481, $497, \mathbf{5} 82.268,929$.

- v. amphipleuroides 3 ri, $37,78,105,903$.

- $\quad$ v. Lineata 582 .

- $\quad$ v. major $3 i 2$.

- v. oregonica 241-243.

viridula Brèb. 37, 314. 582, 611.

- - v. lineolata 177.

vulgaris Thw $116,470,508,602,022$.

- v. minor 940 .

\section{Van Ilenrckiella Pantocsek}

admirabilis Pant. 472, 473.

\section{Weissflogia Janisch}

Macdonaldii Jan. 160.

\section{Willemoesia Castracane}

- elegantula Forti 926-929.

- - - panduriformis 926-929.

- elongata Forti 926-929, 994, 995, 1000.

- - r. rhombiea 929, 994, 995, 999, 1000.

\section{Xanthiopyxis Ehrenberg}

* acrolopha Forti 674, 675, 997, 998. aculeata Eh. 370, 371 . alata Eh. 46, 47, 370, 469, 550. - $\nabla$. armata 46, 47.

angulata Eh. 238.

aristata J. Br. 469.

cingulata Eh. 47, 369, 390, 469, 550. constricta Eh. 140, 141.

- daucispermoides Forti 928, 929.

- diaphana Forti 369. globisa Eh. 47, 424, 674, 933. hirsuta Eh. 46, 47, 120.

- Hystrix Forli 997, 998, 999, 1000.

* lacera Forti 369. oblonga Eh. 47, 91, 237, 390, 550. 


\section{Xanthiopyxis Ehrenberg}

panduriform is Pant. $328,369,424,674,675$. umbonatus Grev. 23, 24, 90, 448, 511 .

\section{Zygoceros Ehrenberg}

circinus Bail. 110, 111, 182, 183, 238.

\section{Zygoceros Bhrenberg}

circinus $v$. trapezoïdalis 23,24 . hungaricus Pant. 405, 406.

quadricornis Grun. 237, 238, 554, 861 . Weissflogii Pant. 128, 129. 


s.

$$
\begin{aligned}
& =2=37.9
\end{aligned}
$$

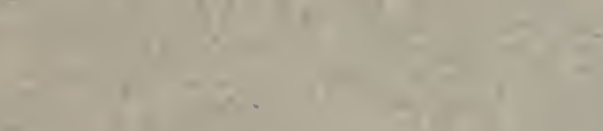$$
5 x=1-\pi, 2 \pi
$$

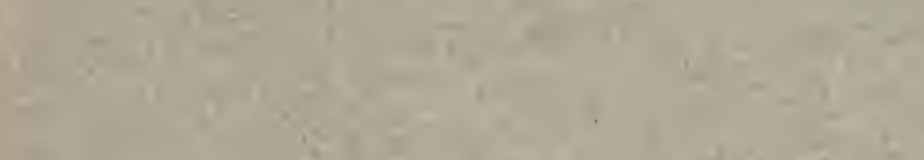

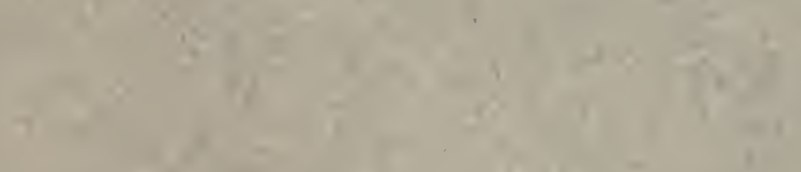

10

)

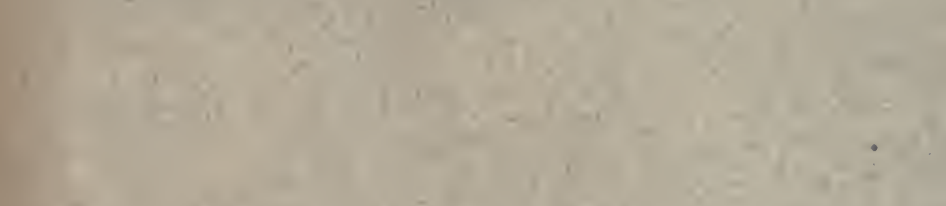

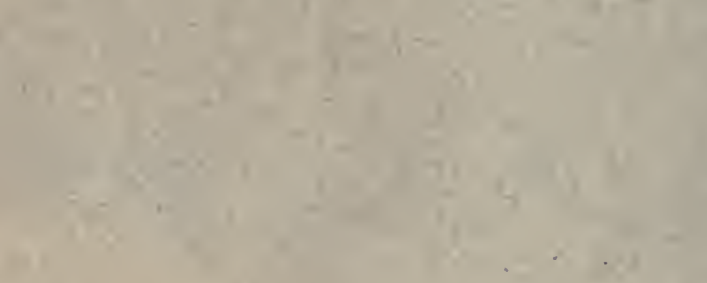

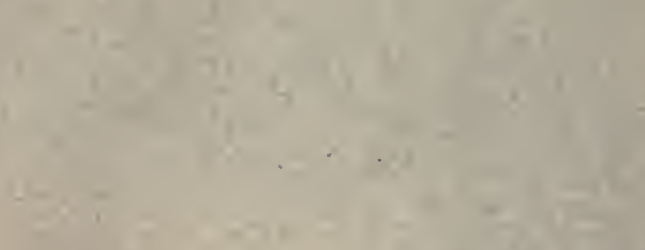

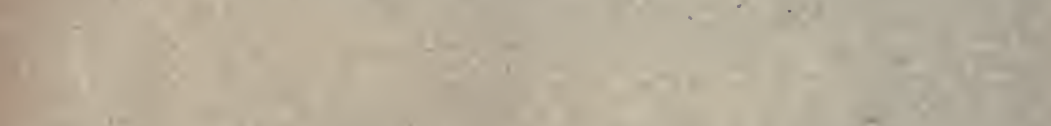

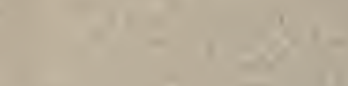

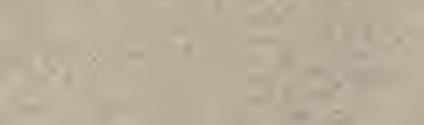

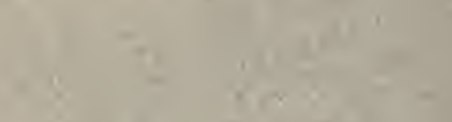

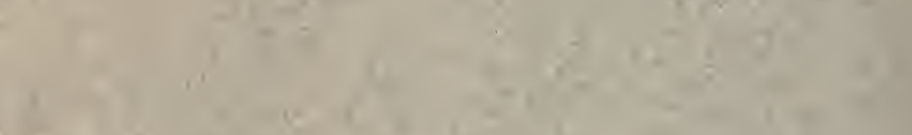

1 10

$$
\text { 1. }
$$




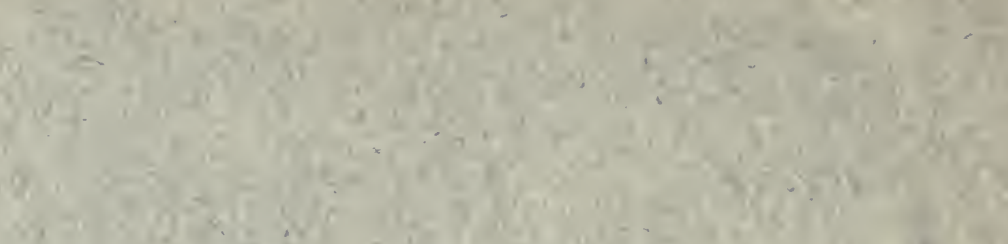

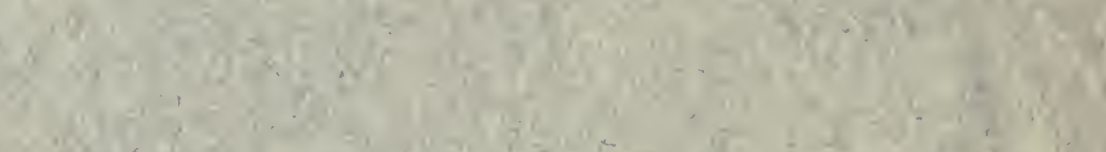

$$
\begin{aligned}
& \text { W }
\end{aligned}
$$

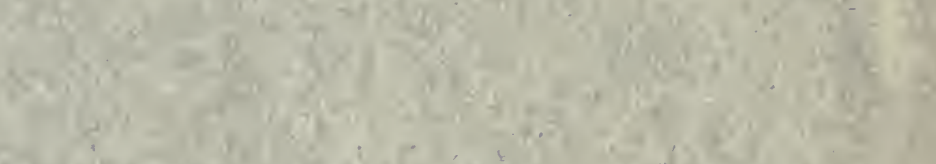

$$
\begin{aligned}
& \text { 1. }
\end{aligned}
$$

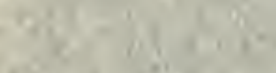

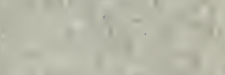

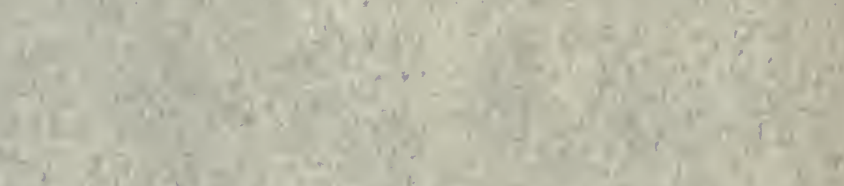

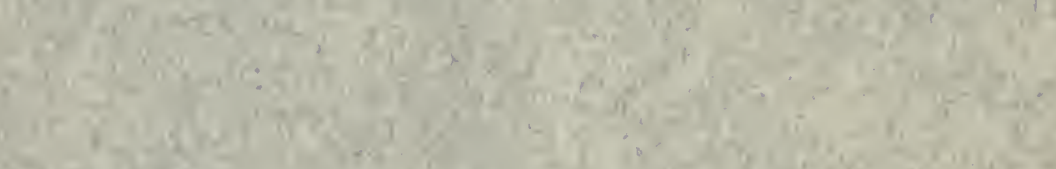

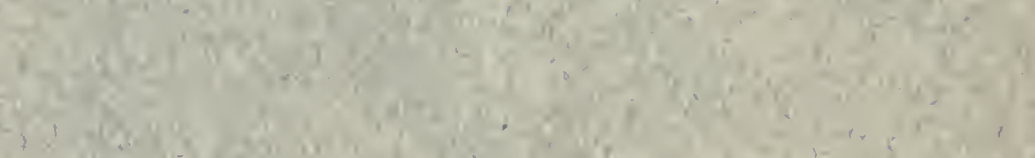

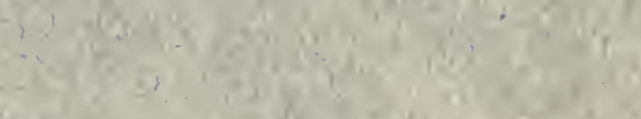

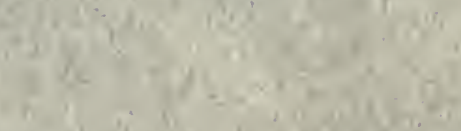

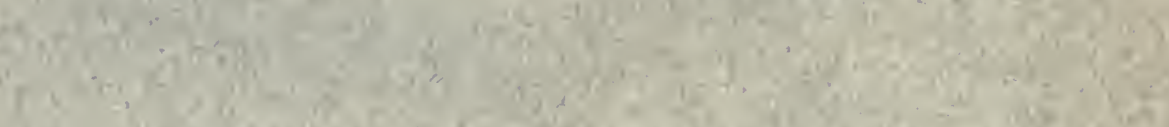

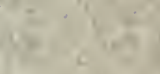

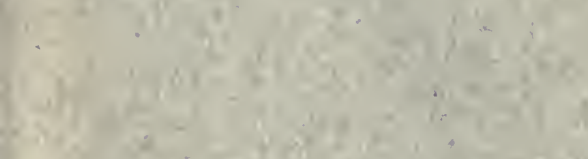

$$
\begin{aligned}
& 1+-2,-71 \\
& \sqrt{9} \\
& \text { तil }
\end{aligned}
$$

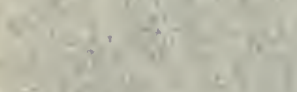

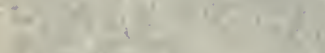



r.

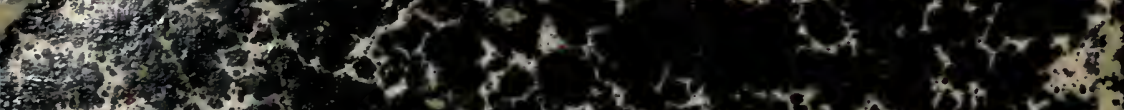
$2-2 x+0,040$ 16.

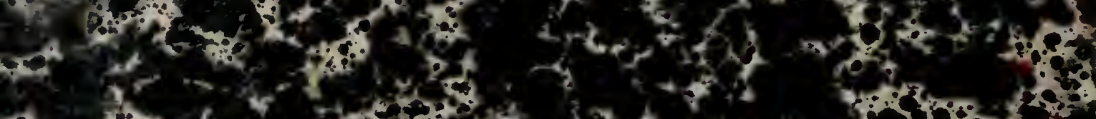
a 1 50 35. 30 ,

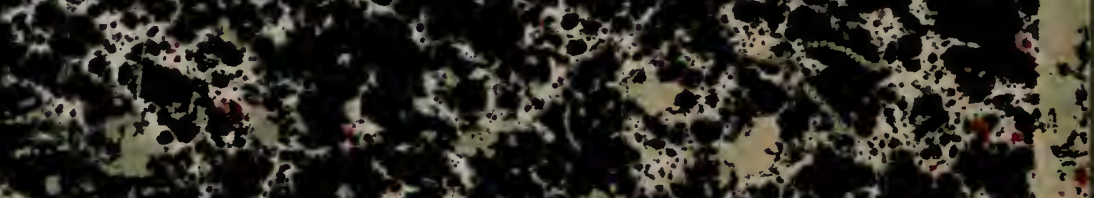

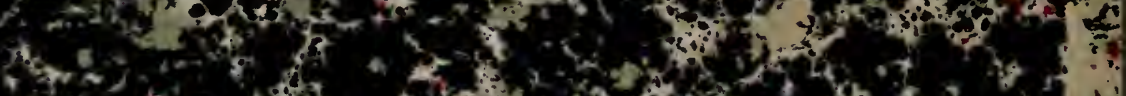

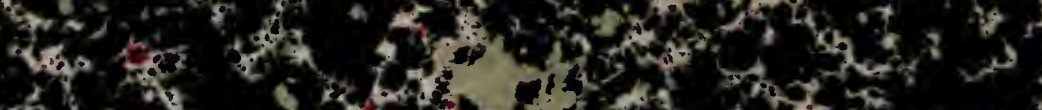

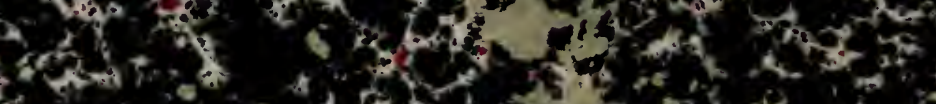

ton

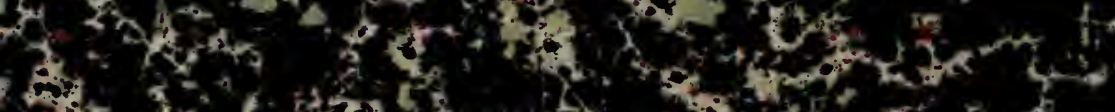

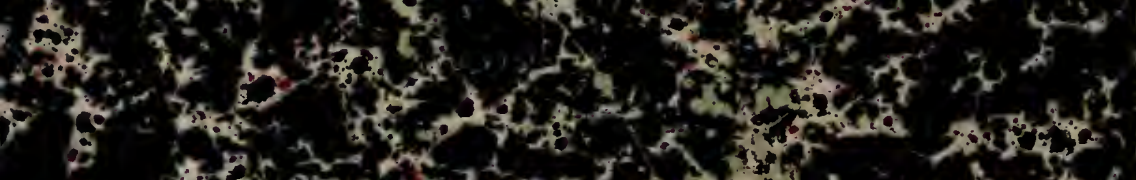

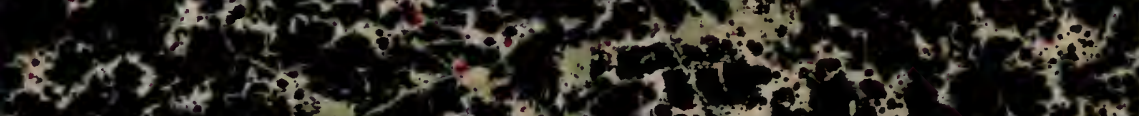
$4 x^{2}+2+10$ 1 350

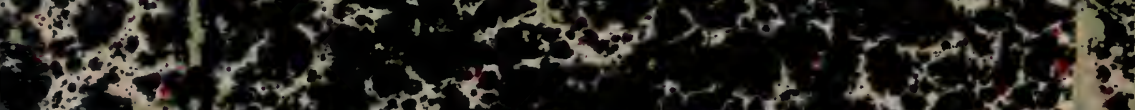

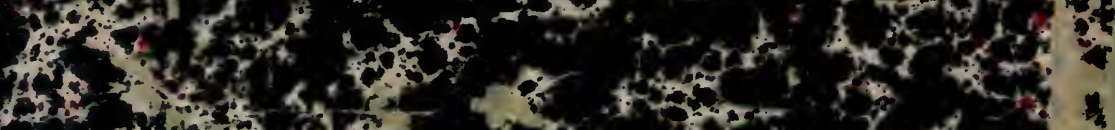

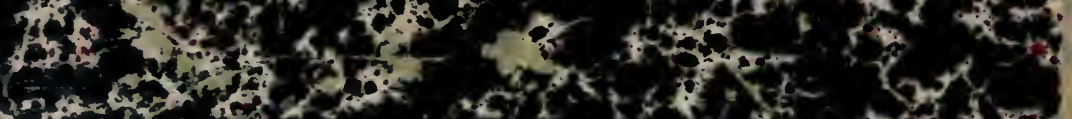

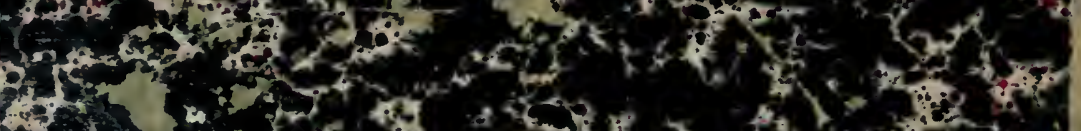

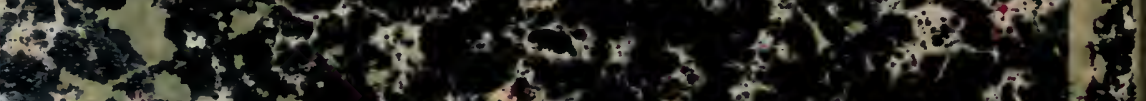
Solotis (3)

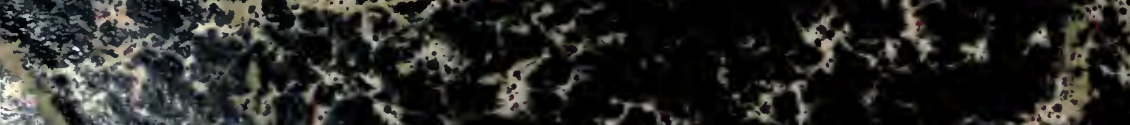

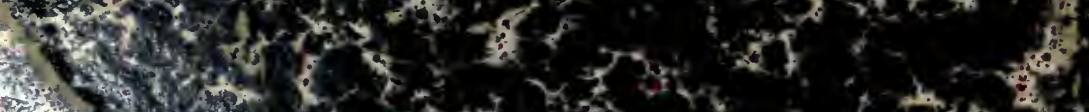

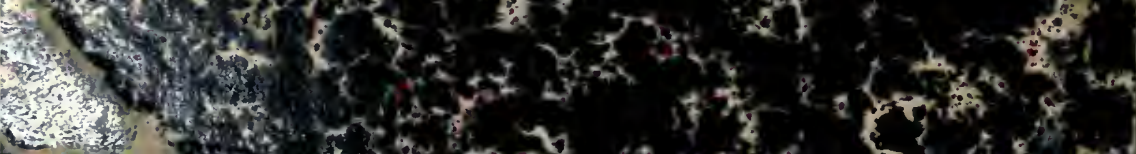

